\title{
Study of Transit Bus Duty Cycle and its Influence on Fuel Economy and Emissions of Diesel-Electric Hybrids
}

Jairo A. Sandoval Leon

West Virginia University

Follow this and additional works at: https://researchrepository.wvu.edu/etd

\section{Recommended Citation}

Sandoval Leon, Jairo A., "Study of Transit Bus Duty Cycle and its Influence on Fuel Economy and Emissions of Diesel-Electric Hybrids" (2011). Graduate Theses, Dissertations, and Problem Reports. 3051. https://researchrepository.wvu.edu/etd/3051

This Dissertation is protected by copyright and/or related rights. It has been brought to you by the The Research Repository @ WVU with permission from the rights-holder(s). You are free to use this Dissertation in any way that is permitted by the copyright and related rights legislation that applies to your use. For other uses you must obtain permission from the rights-holder(s) directly, unless additional rights are indicated by a Creative Commons license in the record and/ or on the work itself. This Dissertation has been accepted for inclusion in WVU Graduate Theses, Dissertations, and Problem Reports collection by an authorized administrator of The Research Repository @ WVU. For more information, please contact researchrepository@mail.wvu.edu. 
Study of Transit Bus Duty Cycle and its Influence on Fuel Economy and Emissions of Diesel Electric Hybrids

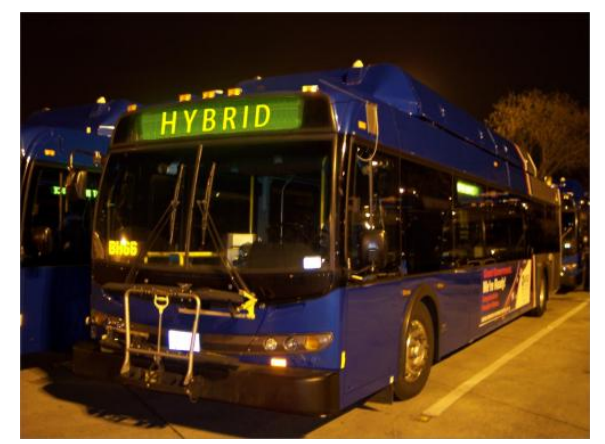

Jairo A. Sandoval León

Dissertation submitted to the College of Engineering and Mineral Resources

at West Virginia University

in partial fulfillment of the requirements

for the degree of

Doctor of Philosophy

in

Mechanical Engineering

W. Scott Wayne, Ph.D., Chair

Nigel N. Clark, Ph.D.

Hailin Li, Ph.D.

Benjamin C. Shade, Ph.D., and

John W. Zondlo, Ph.D.

Department of Mechanical and Aerospace Engineering

Morgantown, West Virginia

2011

Keywords: Transit Bus, Hybrid, Fuel Economy, Emissions, Inventory, Route, Procurement

Copyright 2011 Jairo A Sandoval León 


\section{AbSTRACT \\ Study of Transit Bus Duty Cycle and its Influence on Fuel Economy and Emissions of Diesel - Electric Hybrids}

\section{Jairo A. Sandoval León}

The Center for Alternative Fuels, Engines, and Emissions (CAFEE) of West Virginia University (WVU) is developing the Integrated Bus Information System (IBIS), an information resource on transit bus emissions for vehicle procurement purposes. IBIS provides the transit bus industry with exhaust emissions information, including an emissions database, and predictive models for fuel economy (F.E.) and emissions. Inputs for the models are in the form of drive cycle metrics, but the knowledge of such metrics is not readily available for transit agencies.

The first part of this dissertation was an effort to close the gap between engineering drive cycle metrics and the information available to transit bus operators. In cooperation with WMATA Transit, an extensive evaluation to characterize transit bus operation was performed. This evaluation was based on GPS and ECU logs of diverse bus routes. Instantaneous speed and road grade were determined for all the routes. Transit operation was classified in four main service groups: Inner-City, Urban, Suburban, and Commuter. Characterizing transit bus operation played an important role because it defined the parameters, and their ranges, to be used in F.E. and emissions models.

The second part of the dissertation studied the effects that drive cycles have over emissions and F.E. of diesel-electric hybrid buses, focusing specifically in MY 2007-2009 diesel-electric serieshybrid 40' transit buses. Using ANL's PSAT, the hybrid bus was dynamically modeled and validated against chassis dynamometer test data. As part of the vehicle dynamic model, a model was developed for fuel consumption and $\mathrm{NO}_{\mathrm{x}}$ emissions of the Cummins ISB $260 \mathrm{H}$ diesel engine. The vehicle model was simulated over a variety of duty cycles assuming zero grade, producing a database of instantaneous fuel and $\mathrm{NO}_{\mathrm{x}}$ rates, with all tests satisfying SAE J2711's restriction for state of charge.

A regression based method was devised for predicting cycle F.E., $\mathrm{CO}_{2}$, and $\mathrm{NO}_{\mathrm{x}}$, in which the inputs were average speed, percentage idle, and characteristic acceleration. Fuel consumption and $\mathrm{NO}_{\mathrm{x}}$ were broken into the idle and driving contributions. The driving portion was predicted with average speed without idle and characteristic acceleration without grade, and then aggregated with the idle contribution. The proposed approach produced excellent predictions with coefficients of determination of 0.96 for F.E., 0.99 for $\mathrm{CO}_{2}$, and 0.99 for $\mathrm{NO}_{x}$.

A tool was developed to allow transit agencies to place hybrid buses in routes that take the most advantage of the hybrid-electric capabilities and to evaluate emissions impacts in strategic planning and vehicle procurement. The selection of the best routes is based on fuel savings. Depending on the route, hybrid transit buses have the potential for saving between 0.5 and 1.2 gallons of fuel per hour per vehicle and 5 to $12 \mathrm{~kg}$ of $\mathrm{CO}_{2}$ per hour. 
To my wife Mayra Alejandra Sacanamboy Franco for her unconditional love and support and my daughter Sophia Marie for being an endless source of motivation and joy. 


\section{ACKNOWLEDGEMENTS}

I express gratitude to the United States Department of Transportation Federal Transit Administration for sponsorship of this research project and for its continued support to basic research. Funding was provided under the Transit Vehicle Exhaust Emissions Resources program.

I would like to acknowledge all of those who have helped me and encouraged me to succeed, both as a person and as a professional. I want to thank my mother (Emérita) and my grandmother (Ana Lucia) for always being there for me and for teaching me the moral values that became the foundation of my life. I give endless thanks to my wife, Mayra, for following me to a strange country (it seemed so at the time), for sharing a road that has much more to be walked, and for giving me the most wonderful baby girl. I thank my brother, César, for countless years of friendship.

My most sincere thanks and appreciation go to my advisor, Dr. Scott Wayne, whose unique character, kindness, and support have guided me through my doctoral studies. I want to thank my other committee members, Drs. Clark, Li, Shade, and Zondlo, for their valuable feedback and suggestions. Special thanks to David McKain, Ron Jarrett, Richard Atkinson, Zac Luzader, Bradley Ralston, Byron Rapp, and Michael Hildebrand at WVU CAFEE Laboratories; Drs. Mario Perhinschi and Chris Atkinson for their guidance in the development and tuning of the hybrid controller and ANN models; Dr. Andrew Nix for his many thoughts and suggestions. I must thank WVU graduates Clay Bell for working hand in hand to develop and calibrate the hybrid model, and Dr. Clint Bedick for giving me a head start with ANNs. I am grateful to the WVU Department of Transportation for sharing resources which helped me fine-tune the route data logging hardware before taking it to WMATA.

I acknowledge the many people from the transit, engines, and hybrid industries who cooperated with key information to the development of the project: Vernon Lyght (Supervisory Engineer, New Technology Testing) at WMATA, Tom Webb (Director of Business Development, Transport Systems) at BAE Systems, Rob Smith (Manager - LDD CPE) at Cummins, Robert R. Tejchma (Manager $\mathrm{E}^{\mathrm{P}}$ 40/50 Hybrid Product) at Allison Transmission, and Theresa Smith (Maintenance Engineering Specialist) and Rocky Rogers (Assistant Vice President Technical Services) at Dallas Area Rapid Transit. 


\section{TABLE OF CONTENTS}

$\begin{array}{lr}\text { Abstract } & \text { ii } \\ \text { List of Figures } & \text { ix } \\ \text { List of Tables } & \text { xiii } \\ \text { List of Equations } & \text { xv } \\ \text { Nomenclature } & \text { xviii } \\ \text { Chapter 1. Introduction } & \text { 1 } \\ \text { 1.1. Background } & \\ \text { 1.2. Objectives } & \\ \text { 1.3. Contributions } & \\ \text { 1.4. Research Approach } & \\ \text { 1.4.1. Engine Model for Emissions and Fuel Consumption } & \\ \text { 1.4.2. Vehicle Dynamic Model Development and Validation } & \\ \text { 1.4.3. Prediction of Cycle Fuel Economy and Emissions } & \\ \text { 1.5. Outline } & 9\end{array}$

Chapter 2. Literature Review _ 10

2.1. Vehicle Energy Demand ___ 10

2.1.1. Road Load _ 10

2.1.2. Driving Modes ___ 14

2.1.3. Energy Recuperation and Fuel Consumption _ـ 15

2.2. Driving Characterization__ 18

2.2.1. Speed Related Metrics ___ 18

2.2.2. Characteristic Acceleration, Aerodynamic Speed, and Kinetic Intensity ___ 21

2.3. Vehicle Duty Cycles __ 22

2.3.1. Drive Cycle Development __ 23

2.3.2. Actual Driving Patterns ___ 26

2.3.3. Existing Duty Cycles ___ 26

2.3.4. Federal Test Procedure for Light-Duty Vehicles and Light-Duty Trucks___ 34

2.4. Hybrid Architectures___ 34

2.4.1. Series Hybrid _ 35

2.4.2. Parallel Hybrid___ 35

2.4.3. 1-Mode EVT or Input Split ___ 36

2.4.4. The 2-Mode EVT__ 36

2.4.5. The 2-Mode Hybrid with Fixed Gear Ratios ___ 41 
2.5. Emissions Testing Procedures

2.5.1. Analytic Instrumentation___ 43

2.5.2. Chassis-Dynamometer Transportable Facility __ 45

2.5.3. Engine-Dynamometer Facility____ 47

2.5.4. Mobile Emissions Measurement System (MEMS)___ 47

2.6. Regression Analysis __ 48

2.6.1. Simple Least-Squares ___ 49

2.6.2. Simple Linear Regression __ 50

2.6.3. Analysis of Variance (ANOVA) _ 53

2.6.4. Multiple Regression___ 54

2.6.5. Artificial Neural Networks __ 57

2.6.6. Multivariate Adaptive Regression Splines __ 58

2.7. Emissions Predictions / Inventories and Previous Work _ 59

2.7.1. MOBILE6 and MOVES _ 59

2.7.2. Integrated Bus Information System (IBIS) _ 60

2.7.3. Engine and Vehicle Emissions Prediction___ 61

2.7.4. ANNs for Engine Emissions ___ 63

2.7.5. Hybrid - Conventional Comparison __ 66

2.7.6. Emissions Inventory __ 67

2.7.7. Road Grade __ 69

2.7.8. Hybrid Vehicle Simulation __ 70

Chapter 3. Energy and Hybrid Vehicles__ 73

3.1. Simulation Parameters _ 74

3.1.1. Rolling Resistance ___ 76

3.1.2. Rotational Inertia ___ 77

3.2. Effect of Regeneration Efficiency on Net Tractive Energy __ 78

3.3. Effect of Test Weight on Net Tractive Energy ___ 82

3.4. Effect of Grade on Tractive Energy ___ 84

3.4.1. Grade Testing with Chassis Dynamometer ___ 89

3.4.2. Maximum Speed at Constant Grade ___ 91

3.5. Summary __ 92

Chapter 4. Characterization of Transit Duty Cycles___ 94

4.1. Transit Bus Activity Data Acquisition __ 94

4.2. Data Reduction __ 97

4.3. Analysis___ 99

4.3.1. Classification of Routes by Service Type __ 108 
4.3.2. Survey of Service Type to Bus Operators

4.3.3. Distribution of Speed and Acceleration by Service Type

4.4. Summary

Chapter 5. Diesel Engine Fuel Consumption and Emissions Model

5.1. Chassis Dynamometer Test Data from MY 2006 Orion - BAE Hybrid Bus

5.2. Engine Performance Curve 122

5.3. Emissions and ECU Data Reduction 123

5.4. Analysis of Continuous Fuel Rate and Emissions 126

5.4.1. Area of the Torque-Speed Map Covered by the Test Data 126

5.4.2. Effect of Fuel Properties 127

5.4.3. Comparison between Carbon Balance and ECU Fueling Rates 128

5.5. 2D Maps or Lookup Table Approach 130

5.6. Artificial Neural Network Models Development and Validation 132

5.6.1. Time Span of the History Inputs 136

5.6.2. Number of Neurons 137

5.6.3. Order of the Transfer Functions 138

5.6.4. Number of Inputs (History Terms) 138

5.6.5. Final Results and Validation 138

5.7. Correction for Emissions Certification 142

5.8. Summary 143

Chapter 6. Vehicle Dynamic Model

6.1. Overview of the Base Vehicle: $40 \mathrm{ft}$ Orion hybrid bus with BAE Systems HybriDrive ${ }^{\circledR} \_145$

6.2. Vehicle Dynamic Model in PSAT 146

6.3. Summary of Model Assumptions and Parameters 151

6.4. Vehicle Level Validation 153

6.5. Other Considerations 158

6.5.1. State of Charge Corrections___ 158

6.5.2. Driving History or Micro-Trip Order _ 158

6.6. Summary_ 159

Chapter 7. Influence of Transit Bus Duty Cycle on Fuel Economy and Emissions _ 160

7.1. Transit Routes - Simulations and Results__ 160

7.2. Prediction Models for Fuel Consumption and Emissions___ 165

7.2.1. Fundamentals__ 165

7.2.2. Regressions _ 167

7.2.3. Results 171

7.2.4. Considerations for Limited User Inputs __ 173 
7.2.5. Limits to the Input Parameters ___ 177

7.3. Uncertainty Analysis__ 178

7.4. Predictions for Conventional Diesel Vehicle ___ 181

7.5. Hybrid Advantage and Fuel Savings __ 183

7.5.1. Hybrid Advantage _ـ 183

7.5.2. Fuel Savings___ 186

7.5.3. Uncertainty Figures __ 188

7.5.4. Transit Service Groups __ 189

7.5.5. Effect of Automatic Engine Idle-Stop __ 190

7.6. IBIS Tools__ 192

7.7. Summary 193

Chapter 8. Conclusions and Recommendations___ 196

8.1. Summary 196

8.1.1. PSAT Model for Series Diesel-Electric Hybrid Transit Bus___ 198

8.2. Conclusions_ 199

8.3. Recommendations _ـ 201

8.3.1. Route Data Logging Procedures __ 201

8.4. Suggestions for Future Research__ 202

References__ 204 


\section{LIST OF FIGURES}

Figure 1-1 Active/Ordered Diesel-Electric Hybrid Buses by Model Year (Source: APTA, 2009) 4

Figure 2-1 Driving Modes in a Section of the OCTA Cycle 15

Figure 2-2 Acceleration Bins in a Section of the OCTA Cycle 20

Figure 2-3 Central Business District (CBD) Cycle 31

Figure 2-4 Urban Dynamometer Driving Schedule (UDDS) 31

Figure 2-5 Orange County Transit Authority (OCTA) Cycle 31

Figure 2-6 King County Metro (KCM) Cycle 31

Figure 2-7 City Suburban Heavy Vehicle Cycle (CSHVC) 32

Figure 2-8 Distance-Speed target for the City Suburban Heavy Vehicle Cycle and Route. Stars denote free accelerations 32

Figure 2-9 SORT Routes. Acceleration curves based on minimum acceleration 32

Figure 2-10 SORT 3 Route with Infinite and Minimum Acceleration 32

Figure 2-11 Schematic of the 2-Mode ETV Transmission (Grewe et al., 2007) 37

Figure 2-12 EVT 1 Input Split Mode in the 2-Mode ETV Transmission 40

Figure 2-13 EVT 2 Compound Split Mode in the 2-Mode ETV Transmission 40

Figure 2-14 GM 2-Mode Hybrid System, Front-Wheel Drive (picture taken at GM booth during the SAE 2008 World Congress) 42

Figure 2-15 Statistical model for linear regression 51

Figure 2-16 Graphical interpretation of $t^{*}$ 52

Figure 2-17 Frequency Distribution of Fuel Economy Observations in Transit Buses, Ang and Fwa (1989) 62

Figure 3-1 Effect of Energy Recuperation on Mean Energy of Transit Bus, Mini-Bus, and SUV 79

Figure 3-2 Effect of Energy Recuperation on Energy at Wheels Economy of Transit Bus, Mini-Bus, and SUV 80

Figure 3-3 Increase in Energy at Wheels Economy (E.W.E.) vs. $\eta_{\text {regen }}$ 81

Figure 3-4 Effect of Test Weight and $\eta_{\text {regen }}$ on Mean Net Tractive Energy 83

Figure 3-5 Ratio of Net Tractive Energy and Test Weight to Empty Weight Values 83

Figure 3-6 Sine Wave Grade Function and Elevation 85

Figure 3-7 Square Wave Grade Function and Elevation 85

Figure 3-8 Mean Tractive Power vs. Maximum Grade for Sine and Square Grade Profiles 87

Figure 3-9 Net Mean Tractive Energy vs. Characteristic Acceleration for Sine and Square Grade Profiles 
Figure 3-10 Braking to Tractive Energy Ratio vs. Characteristic Acceleration for Sine and Square Grade Profiles 88

Figure 3-11 Ratio of Tractive Energy to Value at Zero Grade Shift

Figure 3-12 Ratio of Braking/Tractive Energy to Value at Zero Grade Shift____ 88

Figure 3-13 Ratio of Characteristic Acceleration to Value at Zero Grade Shift ___ 89

Figure 3-14 Gravity, Rolling, and Aerodynamic Loads (kW) for a Typical 40' Transit Bus Driving the

KCM Cycle 91

Figure 3-15 Net Dynamometer Electric Power at Empty and Full Weights (KCM Cycle)___ 91

Figure 3-16 Maximum Vehicle Speed of Conventional Transit Bus vs. Grade___ 92

Figure 4-1 Layout of the GPS and ECU Data Logging Unit ___ 95

Figure 4-2 Scatter-plot Matrix for the Driving Cycle Database (Logged + Standard)__ 100

Figure 4-3 Ratio of Characteristic Acceleration with and without Grade versus (a) Average Speed and

(b) Average Grade Ascent 101

Figure 4-4 Typical Distribution of Stops of Inner-City Route (Route D8) 102

Figure 4-5 Estimated vs. Measured Average Speed 105

Figure 4-6 Actual Average Speed vs. Idle 105

Figure 4-7 Residuals: Estimated vs. Measured Average Speed 105

Figure 4-8 Residuals: Actual Average Speed vs. Idle 105

Figure 4-9 Non-Revenue Time Fraction vs. Actual Idle 106

Figure 4-10 Recovery Time Fraction vs. Actual Idle 106

Figure 4-11 Distribution of Fleet Average Speed by Distance Traveled (\%) in WMATA Operation, June 2008 107

Figure 4-12 Distribution of Fleet Average Speed by Time Fractions in WMATA Operation, June 2008 107

Figure 4-13 $\tilde{a}$ without Grade vs. Average Speed 110

Figure 4-14 Percentage Idle vs. Average Speed 110

Figure 4-15 Stops per mile vs. Average Speed 111

Figure 4-16 Standard Deviation of Speed vs. Average Speed 111

Figure 4-17 Average Acceleration vs. Average Speed 111

Figure 4-18 Average Deceleration vs. Average Speed 111

Figure 4-19 Aerodynamic Speed vs. Average Speed 111

Figure 4-20 Kinetic Intensity without Grade vs. Average Speed 111

Figure 4-21 Fuel Economy vs. Average Speed 112

Figure 4-22 $\mathrm{NO}_{\mathrm{x}}$ Emissions vs. Average Speed 112

Figure 4-23 Distribution of Route Average Speed at DART 113

Figure 4-24 Speed Distribution of Service Type Centroids 115 
Figure 4-25 Box Plots of Driving Mode Fractions by Service Type 116

Figure 4-26 Distribution of Speed-Acceleration of Service Type Centroids

Figure 4-27 Distribution of Acceleration of Service Type Centroids 118

Figure 4-28 Distribution of Deceleration of Service Type Centroids 119

Figure 5-1 Cummins ISB 260H Engine Performance Curve

Figure 5-2 Torque - Speed Region Covered by the Test Data 127

Figure 5-3 Fuel Rate from Carbon Balance and ECU for a Section of the OCTA Cycle 129

Figure 5-4 Difference between Integrated ECU and Carbon Balance Fuel Rates 129

Figure 5-5 Engine Fueling Map 131

Figure 5-6 Engine Efficiency Map 131

Figure 5-7 Engine Brake-Specific Fuel Consumption Map 131

Figure 5-8 Engine $\mathrm{NO}_{\mathrm{x}}$ Rate Map 131

Figure 5-9 Engine Brake-Specific $\mathrm{NO}_{\mathrm{x}}$ Emissions Map 131

Figure 5-10 Performance of 2D Maps 133

Figure 5-11 Transfer Functions 135

Figure 5-12 General Overview of the ANNs 135

Figure 5-13 Effect of Time-Span of the History Terms on ANN Performance 136 Figure 5-14 Effect of Number of Neurons on ANN Performance 137

Figure 5-15 Effect of Number of History Terms on ANN Performance 139 Figure 5-16 Example of ANN and 2D-Map Output. Section of OCTA Cycle 140

Figure 5-17 Percent Error in Integrated Emissions for all Test Runs: $(a)$ Fuel, $(b) \mathrm{NO}_{\mathrm{x}}$ 141

Figure 5-18 Standard Errors for all Test Runs: (a) Fuel, (b) $\mathrm{NO}_{\mathrm{x}}$ 141

Figure 6-1 Generic Format of a Component in PSAT (ANL, 2008) 147

Figure 6-2 PSAT Series Hybrid Drivetrain Configuration 148

Figure 6-3 Outline of the PSAT Series Hybrid Vehicle Model in Simulink 148

Figure 6-4 Traction Motor Efficiency Map (Bell, 2011) 152

Figure 6-5 Vehicle Level Validation Results 156

Figure 6-6 Comparison of Simulation Fuel Consumption with Chassis Tests 157

Figure 7-1 Fuel Economy vs. Average Speed 163

Figure 7-2 Fuel Economy from Test and Simulation 163

Figure 7-3 $\mathrm{CO}_{2}$ Emissions vs. Average Speed 163

Figure 7-4 $\mathrm{NO}_{\mathrm{x}}$ Emissions vs. Average Speed 163

Figure 7-5 Driving Fuel Consumption 164

Figure 7-6 Driving $\mathrm{NO}_{\mathrm{x}}$ Emissions 164 Figure 7-7 Idle Fuel Consumption Rate 164

Figure 7-8 Idle $\mathrm{NO}_{\mathrm{x}}$ Emissions Rate 164 
Figure 7-9 Idle Fuel Fraction 164

Figure 7-10 Idle $\mathrm{NO}_{\mathrm{x}}$ Fraction 164

Figure 7-11 Flowchart for Cycle Fuel Economy and Emissions Calculation 167

Figure 7-12 Cross-Validation RMSE for F.E. and $\mathrm{NO}_{\mathrm{x}}$ Predictions 169

Figure 7-13 F.E. Driving Predicted versus Target Plot 170

Figure 7-14 NO $\mathrm{x}_{\text {Driving }}$ Predicted versus Target Plot 170

Figure 7-15 F.E. Driving Three-Dimensional Contour Plot 170

Figure 7-16 NO $\mathrm{x}_{\text {Driving }}$ Three-Dimensional Contour Plot 170

Figure 7-17 Predicted Fuel Economy 172

Figure 7-18 Fuel Economy Predicted versus Simulation 172

Figure 7-19 Predicted $\mathrm{CO}_{2}$ Emissions 172

Figure 7-20 $\mathrm{CO}_{2}$ Emissions Predicted versus Simulation 172

Figure 7-21 Predicted $\mathrm{NO}_{\mathrm{x}}$ Emissions 172

Figure 7-22 $\mathrm{NO}_{\mathrm{x}}$ Emissions Predicted versus Simulation 172

Figure 7-23 Comparison of Predictions with IBIS Model 173

Figure 7-24 Approximation of Idle 173

Figure 7-25 Approximation of $\tilde{a}_{n o}$ grade 173

Figure 7-26 Flowchart for Limited Inputs 174

Figure 7-27 Fuel Economy Predictions with Limited Inputs 175

Figure 7-28 Effect of Model Inputs on Fuel Economy RMSE and $\mathrm{R}^{2}$ 175

Figure 7-29 $\mathrm{NO}_{\mathrm{x}}$ Predictions with Limited Inputs 176

Figure 7-30 Effect of Model Inputs on $\mathrm{NO}_{\mathrm{x}} \mathrm{RMSE}$ and $\mathrm{R}^{2}$ 176

Figure 7-31 Graphical Representation of the Limits on Percentage Idle 178

Figure 7-32 Uncertainty Bars for Cycle Fuel Economy Under the Various Input Groups 180

Figure 7-33 Predicted Fuel Economy for Conventional Diesel Bus 182

Figure 7-34 Hybrid versus Conventional Contrasts 184

Figure 7-35 Hybrid Advantage 185

Figure 7-36 Fuel Consumption Rates and Fuel Saving Rates 187

Figure 7-37 Uncertainty Bars in Selected Results 189

Figure 7-38 Fuel Economy and Savings for the Four Bus Service Categories 190

Figure 7-39 Effect of Engine Idle-Stop 191

Figure 7-40 Sample Output from GPS Data Cleaning Tool 192

Figure 7-41 Data Input Menus for the Hybrid Savings Calculator Tool 194

Figure 7-42 Outputs of the Hybrid Savings Calculator Tool 195 


\section{LIST OF TABLES}

Table 1-1 Summary of EPA Emissions Standards for Heavy-Duty Diesel Engines, g/bhp·hr 2

Table 1-2 European Union Emission Standards for Heavy-Duty Diesel Engines, g/bhp·hr (Smoke in $\left.\mathrm{m}^{-1}\right)$ 2

Table 1-3 Summary of Estimated Emissions for MY 2007 Diesel-Electric Hybrids (U.S. FTA, 2007) 4

Table 2-1 Suggested Drag Coefficients for Commercial Vehicles, Hucho (1998) 11

Table 2-2 Observed Ranges of Acceleration Metrics 20

Table 2-3 Classification Vehicle Operation According to Instantaneous Acceleration (Kern, 2000) _ 20

Table 2-4 Classification of Driving Conditions 23

Table 2-5 Cycle and Micro-trip Metrics Considered by Dembski et al., 2005 25

Table 2-6 Properties of Drive Cycles and Routes 28

Table 2-7 Summary of Analytic Instrumentation 45

Table 2-8 Components of the Various WVU Chassis Dynamometers 46

Table 3-1 Vehicle Properties of Bus, Mini-Bus, and SUV 75

Table 3-2 Comparison of Hybrid Transit Bus Simulation Coefficients with Other Studies 76

Table 3-3 Effect of Energy Recuperation on Mean Energy of Transit Bus, Mini-Bus, and SUV 79

Table 3-4 Test Weights of Conventional and Hybrid Buses 82

Table 3-5 Effect of Test Weight and $\eta_{\text {regen }}$ on Mean Tractive Energy 82

Table 3-6 Effect of Maximum Grade and Grade Function on Characteristic Acceleration for Selected

Drive Cycles 86

Table 4-1 Logged ECU Variables 96

Table 4-2 Test Buses 96

Table 4-3 Logged Routes 96

Table 4-4 Barometer Calibration Table 98

Table 4-5 Cycle Metrics for Route Characterization 99

Table 4-6 Summary of Properties of Logged Routes 103

Table 4-7 Metrics of Service Type Centroids 109

Table 4-8 Route Classification Criterion 109

Table 4-9 Classification of WMATA Routes by Service Type 110

Table 5-1 Summary of Emissions Tests - Orion-BAE Series Hybrid Bus. (Transit Resource Center, 2007) 122

Table 5-2 Cummins ISB 260H Engine Parameters 123

Table 5-3 Fuel Properties 125

Table 5-4 Corrections to Table 5-1 for Fuel Properties and Data Reduction Calculations 128 
Table 5-5 ANN Input / Output Normalization 134

Table 5-6 Selected ANN Architectures 139

Table 5-7 Emissions and F.E. New Flyer 40’ Diesel-Hybrid Transit Buses, 2005/2008 (CAFEE, 2011)

Table 5-8 Emissions and F.E. Gillig 40' Conventional Diesel Transit Buses, 2004-5/2007

Table 6-1 Breakdown of Auxiliary (Accessory) Loads (O'Keefe and Vertin, 2002) 143

Table 6-2 Series Hybrid Bus Modeling Parameters 153

Table 6-3 OCTA Cycle Emissions from Selected $40 \mathrm{ft}$ Hybrid Transit Buses 154

Table 7-1 Fuel Consumption and Emissions Simulation Results for the Hybrid Bus Under Varying

Driving Conditions 161

Table 7-2 Regression Summary for Driving Fuel Consumption (g/mi) 169

Table 7-3 Regression Summary for Driving $\mathrm{NO}_{\mathrm{x}}$ Emissions (g/mi) 169

Table 7-4 Distribution Summary for Idle Fuel Consumption and Idle $\mathrm{NO}_{\mathrm{x}}$ Emissions (g/hr) 170

Table 7-5 Constant Absolute Uncertainties for Predictions of F.C._Driving and $N O_{x_{-} \text {Driving }}(\mathrm{g} / \mathrm{mi})$ 180

Table 7-6 Fuel Consumption and Emissions Chassis Test Results for Conventional Diesel Bus (CAFEE, 2007) 182 


\section{LIST OF EQUATIONS}

Equation 2.1 Road Force _ 10

Equation 2.2 Aerodynamic Force ___ 11

Equation 2.3 Mechanical Drag Force ___ 12

Equation 2.4 Driveline Drag Force___ 12

Equation 2.5 Rolling Resistance Force___ 12

Equation 2.6 Grade Force, $\alpha$

Equation 2.7 Grade Force, $h$

Equation 2.8 Inertia Force __ 13

Equation 2.9 Effective inertia mass of rotating components __ 13

Equation 2.10 Road Load Equation - Force __ 13

Equation 2.11 Road Load Equation - Power __ 14

Equation 2.12 Engine Power _ 14

Equation 2.13 Net Tractive Energy __ 16

Equation 2.14 Tractive Energy ___ 16

Equation 2.15 Braking Energy __ 16

Equation 2.16 Fuel Consumption Equation __ 17

Equation 2.17 Hybrid Fuel Consumed in Powered Driving___ 17

Equation 2.18 Fuel Consumption Equation __ 17

Equation 2.19 Average Speed Without Idle___ 19

Equation 2.20 Standard Deviation of Speed___ 19

Equation 2.21 Five-point Approximation of Acceleration 19

Equation 2.22 Average Acceleration and Deceleration _ 20

Equation 2.23 Characteristic Acceleration ___ 21

Equation 2.24 Characteristic Acceleration for a Time Step __ 21

Equation 2.25 Characteristic Acceleration ___ 21

Equation 2.26 Aerodynamic Speed __ 21

Equation 2.27 Aerodynamic Speed __ 22

Equation 2.28 Kinetic Intensity ___ 22

Equation 2.29 Root-Mean-Square Formula __ 24

Equation 2.30 Conversion of Test Route to Time Basis __ 33

Equation 2.31 CAFE Combined Fuel Economy__ 34

Equation 2.32 2-Mode Input Split Equations __ 37

Equation 2.33 2-Mode Transmission Ratios __ 38 
Equation 2.34 2-Mode Compound Split Equations __ 38

Equation 2.35 2-Mode Input Split Equations in Terms of N __ 39

Equation 2.36 2-Mode Compound Split Equations in Terms of N __ 39

Equation 2.37 Engine Output Torque, Thompson et al. (2002) __ 48

Equation 2.38 Correlation, $r \_49$

Equation 2.39 Least-Squares Regression Line __ 49

Equation 2.40 Population Regression Line __ 50

Equation 2.41 Statistical Model for Simple Linear Regression __ 51

Equation 2.42 Average Squared Residual __ 51

Equation 2.43 Confidence Intervals for Slope and Intercept __ 51

Equation $2.44 \mathrm{t}$ Statistic _ 52

Equation 2.45 Confidence Interval for Mean Response___ 52

Equation 2.46 Confidence Interval for Single Prediction _ 53

Equation 2.47 Sum of Squares __ 53

Equation 2.48 Mean Square Error __ 54

Equation 2.49 Correlation Coefficient, $\mathrm{R}^{2}$

Equation 2.50 F Statistic___ 54

Equation 2.51 Statistical Model for Multiple Linear Regression __ 54

Equation 2.52 Statistical Model in Matrix Form _ 55

Equation 2.53 Regression Parameters _ 55

Equation 2.54 Least-Squares Multiple Linear Regression __ 55

Equation 2.55 Mean Squared Residual___ 56

Equation 2.56 Standard Error of the Regression Parameters __ 56

Equation 2.57 Standard Error of the Mean Response___ 56

Equation 2.58 Standard Error of a Single Prediction _ 56

Equation 2.59 Tailpipe Emissions Model, Barth et al. (2005) __ 62

Equation 2.60 Fuel Consumption Model, Frey et al. (2007) __ 63

Equation 2.61 First and Second Derivatives Over a Time Period $\Delta t \_64$

Equation 2.62 Sensitivity of Fuel Consumption to Road Load, Sharer et al. (2007)___ 72

Equation $3.1 \eta_{\text {regen }}$ limit

Equation 3.2 Rolling Resistance Coefficient __ 76

Equation 3.3 Rotational Inertial Mass __ 77

Equation 3.4 Ratio of Moments of Inertia __ 77

Equation $3.5 e_{\text {trac,net }} / e_{\text {trac, net, EW }}$

Equation 3.6 Sine Wave Grade___ 84

Equation 3.7 Mean tractive power __ 86 
Equation 3.8 Maximum Speed 91

Equation 4.1 Grade Calculation 97

Equation 4.2 Fleet Emissions 106

Equation 4.3 Estimated Average Speed for Composite Service 106

Equation 5.1 Emissions Data Reduction Equations 124

Equation 5.2 ECU Data Reduction Equations 126

Equation 5.3 Fuel Economy Calculation 126

Equation 5.4 $\mathrm{CO}_{2}$ Emissions from Fueling Rate 132

Equation 5.5 ANN Transfer Functions 134

Equation 7.1 Cycle Fuel Consumption 165

Equation 7.2 Idle Component of Fuel Consumption 166

Equation 7.3 Cycle $\mathrm{NO}_{\mathrm{x}}$ Emissions 166

Equation 7.4 Fuel Economy and $\mathrm{CO}_{2}$ Emissions 166

Equation 7.5 Limits on Percentage Idle 177

Equation 7.6 Propagation of Uncertainty 179

Equation 7.7 Uncertainty in Cycle Fuel Consumption and Cycle $\mathrm{NO}_{\mathrm{x}}$ Emissions 179

Equation 7.8 Hybrid Advantage 183

Equation 7.9 Uncertainty in Hybrid Advantage 183

Equation 7.10 Volumetric Fuel Consumption Rate 186

Equation 7.11 Volumetric Fuel Saving Rate 186

Equation 7.12 $\mathrm{CO}_{2}$ Savings Rate 188 


\section{NOMENCLATURE}

$\begin{array}{ll}\text { Abbreviations } \\ \text { ANN } & \text { Artificial neural network } \\ \text { APTA } & \text { American Public Transportation Association } \\ \text { CAFEE } & \text { WVU Center for Alternative Fuels, Engines, and Emissions } \\ \text { CB } & \text { Carbon balance } \\ \text { CBD } & \text { Central Business District cycle } \\ \text { CFR } & \text { Code of Federal Regulations } \\ \text { CNG } & \text { Compressed natural gas } \\ \text { CO } & \text { Carbon monoxide } \\ \text { CO } & \text { Carbon dioxide } \\ \text { DART } & \text { Dallas Area Rapid Transit } \\ \text { DF } & \text { Dilution factor } \\ \text { DPF } & \text { Diesel particulate filter } \\ \text { ECU } & \text { Electronic control unit } \\ \text { EGR } & \text { Exhaust gas recirculation } \\ \text { EPA } & \text { U.S. Environmental Protection Agency } \\ \text { E.W.E. } & \text { Energy at wheels efficiency (km/kWh) } \\ \text { F.C. } & \text { Fuel consumption } \\ \text { F.E. } & \text { Fuel economy } \\ \text { FTA } & \text { U.S. Federal Transit Administration } \\ \text { FTP } & \text { Federal Test Procedure } \\ \text { GVWR } & \text { Gross vehicle weight rating } \\ \text { HC } & \text { Hydrocarbons } \\ \text { HFID } & \text { Heated flame ionization detector } \\ \text { IBIS } & \text { Integrated Bus Information System } \\ \text { KCM } & \text { King County Metro cycle } \\ \text { MY } & \text { Model year } \\ \text { NMHC } & \text { Non-methane hydrocarbons } \\ \text { NO } & \text { Nitric oxide } \\ \text { NO } & \\ \text { NO } & \text { Nitrogen dioxide } \\ \text { OCTA } & \text { Oxides of nitrogen } \\ \text { PM } & \text { Orange County Transit Authority cycle } \\ & \text { Particulate matter } \\ \end{array}$


PSAT Argonne National Laboratory's (ANL) Powertrain Systems Analysis Toolkit (PSAT)

SCR Selective catalytic reduction

SOC State of charge

Stdv. Standard deviation

TransLab WVU Transportable Heavy-Duty Vehicle Emissions Testing Laboratory

ULSD Ultra-low sulfur diesel

WMATA Washington Metropolitan Area Transit Autority

WVU West Virginia University

$1 / 2$ SLW $\quad$ Half seated load weight

\section{$\underline{\text { Symbols }}$}

$a$

$\tilde{a}$

$\bar{a}_{+}$

$\bar{a}_{-}$

$A_{f}$

$c_{r}$

$c_{d}$

$D$

$E_{b, A C C}^{+}$

$E_{B R}$

$\dot{E}_{\text {road }}$

Etrac

$e_{\text {trac,net }}$

$E_{\text {trac,net }}$

$g$

$h$

$H_{f}$

$I_{e}$

$I_{w}$

$k i$

\section{Acceleration}

Characteristic acceleration, Equation 2.23

Average acceleration, Equation 2.22

Average deceleration, Equation 2.22

Vehicle frontal area

Rolling resistance coefficient

Aerodynamic drag coefficient

Distance traveled

energy required by vehicle accessories or hotel loads

Braking energy

Tractive power

Tractive energy

Mean net tractive energy ( $E_{\text {trac, net }}$ per unit distance, $\mathrm{Wh} / \mathrm{km}$ )

Net energy that must be supplied by the primary power source for propulsion (at wheels)

Acceleration of gravity

Elevation or altitude

Heating value of the fuel

Moment of inertia of the engine and the rotating parts on the engine-side of the transmission

Moment of inertia of the wheels and all the rotating parts on the wheel-side of the transmission

Kinetic intensity, Equation 2.28 


$\begin{array}{ll}m & \text { Actual or test vehicle mass, also mass in general } \\ m_{e} & \text { Effective vehicle mass, } m_{e}=m+m_{r} \\ m_{f} & \text { Mass of fuel consumed } \\ m_{r} & \text { Effective inertia mass, Equation } 2.8 \\ r_{e f} & \text { Effective rolling radius } \\ r_{w} & \text { Wheel radius } \\ s & \text { Used for distance as well as standard deviation } \\ T, t & \text { Time } \\ t^{*} & \text { Student's } t \text { distribution critical value for } C \% \text { confidence } \\ \bar{U} & \text { Average vehicle speed } \\ V & \text { Vehicle speed } \\ \bar{V} & \text { Average speed, alternate of } \bar{U} \\ V_{a e r o} & \text { Aerodynamic speed, Equation } 2.26 \\ \alpha & \text { Road angle, also atomic hydrogen to carbon ratio of the fuel } \\ \Delta & \text { Change; absolute uncertainty } \\ \gamma & \text { overall gear ratio, } \omega_{\text {engine }} / \omega_{\text {wheels }} \\ \eta_{b} & \text { Engine efficiency } \\ \eta_{d r} & \text { Driveline efficiency } \\ \eta_{r e g e n} & \text { Regenerative braking efficiency } \\ \rho & \text { Density } \\ \rho_{f} & \text { Fuel density } \\ \phi & \text { Diameter } \\ \omega & \text { Angular velocity } \\ d & \text { Definition } \\ = & \end{array}$

\section{$\underline{\text { Subscripts }}$}

Driving Relative to the driving (dynamic) contribution to fuel consumption and emissions

Idle Relative to the idle (stationary) contribution to fuel consumption and emissions

NI No idle, without idle 


\section{Chapter 1. INTRODUCTION}

Academic studies and in-use data from transit agencies have shown that the performance of hybridelectric buses is highly sensitive to driving behavior.

When procuring new buses, transit agencies need to decide whether to buy conventional or hybrid technology; a decision that is affected by several factors: capital investment, fuel prices, emissions, familiarity with the technologies, and federal and/or state incentives, among others. Life cycle cost and emissions evaluations play a key role in the analysis because they provide a global and detailed perspective on each particular choice (U.S. FTA, 2007). A drawback of this approach is that it requires accurate information on duty cycles, fuel consumption, distance specific emissions, and their interdependences.

On the other hand, transit agencies may conduct fuel economy comparison tests, in which they run both conventional and alternative-technology buses on certain routes for a period of time. Such comparisons usually do not collect emissions data; they are expensive and time consuming, and, normally, are not affordable alternatives for small transit agencies. Several factors must be considered when performing fuel economy comparisons (Chiang, 2007), for example, comparison among equivalent configurations, equal service schedules, evaluation time, maintenance schedule, fuel measurement method, a well defined test plan and pre-test evaluation of the selected vehicles. Therefore, an educated approach to the effects of duty cycle on emissions and fuel economy is needed.

Engines for automotive applications are certified over the Federal Test Procedure (FTP) in the U.S. Heavy-duty diesel engines must comply with the EPA emissions standards presented in Table 1-1 (source: DieselNet.com). Prior to 2004, engines sold in the state of California had a different, more stringent, standard. For emissions certification only the engine, separate from the vehicle, is tested using an engine dynamometer test facility. Table 1-2 (source: DieselNet.com) presents the emissions regulations imposed by the European Union for heavy-duty diesel engines used in transit buses where the limits were converted from $\mathrm{g} / \mathrm{kWh}$ to $\mathrm{g} / \mathrm{bhp} \cdot \mathrm{hr}$. The dynamometer cycles used are: ECE R-49 for Euro I and Euro II, European Stationary Cycle (ESC), European Transient Cycle (ETC), and European Load Response (ELR) test for smoke opacity.

On the other hand, chassis-dynamometer vehicle tests measure fuel economy and emissions for the vehicle as a system. Several test cycles exist for both light- and heavy-duty vehicles. EPA Tier 2 and California LEVII (low emissions vehicle II) Standards require certification of passenger vehicles and light-duty trucks (EPA: up to $10,000 \mathrm{lbs}-4,536 \mathrm{~kg}-$ GVWR, California: up to $14,000 \mathrm{lbs}-6,350 \mathrm{~kg}-$ 
GVWR). For transit buses in the U.S., emissions standards are not based on chassis-dynamometer tests of complete vehicles, but engines are certified to the emissions levels required in Table 1-1.

In order to comply with the $0.01 \mathrm{~g} / \mathrm{bhp} \cdot \mathrm{hr}$ particulate matter standard, diesel engines use diesel particulate filters (DPF), which have been proven to reduce engine-out PM levels by over 95\%. The stringent PM standard also poses a challenge in the particulate measurement system; laboratories throughout the U.S. were required to update their equipment (CFR 1065, 2006). The $0.2 \mathrm{~g} / \mathrm{bhp} \cdot \mathrm{hr} \mathrm{NO}_{\mathrm{x}}$ standard is being met with the implementation of advanced combustion control, exhaust gas recirculation, and selective catalytic reduction (SCR) urea injection systems.

It is possible that in the near future the European Union will start regulating emissions of ultrafine PM, less than $0.1 \mu \mathrm{m}$ in size, by particle number measurement; which will replace or complement the current filter based mass measurement methods (UNECE-GRPE, 2006, Giechaskiel et al., 2009). For this purpose, the Particle Measurement Programme (PMP), managed by the Joint Research Centre (JRC), was established in 2001. The PMP program developed the protocols and technologies necessary to put in place such particle number regulations (Martini et al., 2009).

Table 1-1 Summary of EPA Emissions Standards for Heavy-Duty Diesel Engines, g/bhp·hr

\begin{tabular}{c|c|c|c|c|c}
\hline Engine Model Year & CO & HC & NMHC & NO $_{\mathbf{x}}$ & PM \\
\hline 1988 & 15.5 & 1.3 & & 10.7 & 0.60 \\
\hline 1990 & 15.5 & 1.3 & & 6.0 & 0.60 \\
\hline 1991 & 15.5 & 1.3 & & 5.0 & 0.25 \\
\hline 1994 & 15.5 & 1.3 & & 5.0 & 0.10 \\
\hline 1998 & 15.5 & 1.3 & & 4.0 & 0.10 \\
\hline 2004 & 15.5 & 1.3 & 0.5 & $2.5^{\mathrm{A}}$ & 0.10 \\
\hline 2007 & & & 0.14 & $1.2-1.5^{\mathrm{B}}$ & 0.01 \\
\hline 2010 & & & 0.14 & 0.2 & 0.01 \\
\hline
\end{tabular}

$\mathrm{A}-\mathrm{NMHC}+\mathrm{NO}_{\mathrm{x}}$

B - Family Emission Limit (FEL) as a phase-in towards $0.2 \mathrm{~g} / \mathrm{bhp} \cdot \mathrm{hr}$

Table 1-2 European Union Emission Standards for Heavy-Duty Diesel Engines, g/bhp·hr (Smoke in $\mathbf{m}^{-1}$ )

\begin{tabular}{|c|c|c|c|c|c|c|c|c|}
\hline Tier & Date & Test & $\mathrm{CO}$ & $\mathrm{HC}$ & NMHC & $\mathrm{NO}_{\mathrm{x}}$ & PM & Smoke \\
\hline Euro I & $1992,>114 \mathrm{hp}$ & ECE R-49 & 3.4 & 0.8 & & 6.0 & 0.27 & \\
\hline \multirow{2}{*}{ Euro II } & 1996.10 & ECE R-49 & 3.0 & 0.8 & & 5.2 & 0.19 & \\
\hline & 1998.10 & ECE R-49 & 3.0 & 0.8 & & 5.2 & 0.11 & \\
\hline \multirow{2}{*}{ Euro III } & \multirow{2}{*}{2000.10} & ESC / ELR & 1.6 & 0.5 & & 3.7 & 0.07 & 0.8 \\
\hline & & ETC & 4.1 & & 0.6 & 3.7 & 0.12 & \\
\hline \multirow{2}{*}{ Euro IV } & \multirow{2}{*}{2005.10} & ESC / ELR & 1.1 & 0.3 & & 2.6 & 0.015 & 0.5 \\
\hline & & ETC & 3.0 & & 0.4 & 2.6 & 0.022 & \\
\hline \multirow{2}{*}{ Euro V } & \multirow{2}{*}{2008.10} & ESC / ELR & 1.1 & 0.3 & & 1.5 & 0.015 & 0.5 \\
\hline & & ETC & 3.0 & & 0.4 & 1.5 & 0.022 & \\
\hline \multirow{2}{*}{ Euro VI } & \multirow{2}{*}{2013.01} & ESC & 1.1 & 0.10 & & 0.3 & 0.007 & \\
\hline & & ETC & 3.0 & 0.12 & & 0.3 & 0.007 & \\
\hline
\end{tabular}

Emissions results from chassis-dynamometer testing represent specific driving conditions set by the test schedule (speed-time or speed-distance) and are not easily extrapolated for other drive cycles, 
e.g. cycles with different average speed, stops per mile, accelerations, idle, etc. The work developed in this dissertation will provide fuel economy and emissions figures of diesel-electric hybrid transit buses under a wide range of driving conditions. A regression model was developed to assist transit agencies achieve best advantage from their hybrid buses. This dissertation focused specifically model year 2007-2009 40’ diesel-electric hybrid buses.

\subsection{Background}

Current research is under way at the Center for Alternative Fuels, Engines, and Emissions (CAFEE) of West Virginia University (WVU) with the aim of predicting overall impact on emissions footprint of hybrid and conventional transit buses given driving characteristics. The core of this research program is the Integrated Bus Information System (IBIS). IBIS is an online based system that allows evaluation of the emissions and fuel efficiency implications of existing and emerging technology transit vehicles for the purposes of bus procurement and strategic planning (APTA, 2008; Marlowe, 2009). Some of the features of IBIS are: searchable databases of transit vehicle emissions studies and predictive emissions modeling tools.

Marlowe (2009) developed two tools for IBIS. The first one is a multivariate polynomial regression tool capable of generating regression models based on distance specific emissions and drive cycle metrics. Second, he developed a tool that expands the number of inputs to the emissions database using existing tests; this tool breaks second-by-second data into micro-trips, creates new cycles, and estimates emissions generating new entries into the dataset. In the case of hybrids, given that the concatenated micro-trips are segments of overall cycles, they are not guaranteed to represent charge sustaining operation (zero delta battery state of charge, SOC). Emissions tests must be reported to zero $\triangle$ SOC (SAE J2711, SAE International, 2002) but the current IBIS approach cannot correct the new dataset inputs for SOC. Also, SOC was not measured on a continuous basis in the test programs that collected the IBIS data. Backbone models for clean diesel, CNG, and diesel-electric hybrid buses were developed.

WVU (U.S. FTA, 2007) estimated emissions and fuel consumption for CNG, B20 biodiesel, diesel, and diesel-electric hybrid buses (MY 2007, 40'). The report addressed life cycle costs of transit buses over their expected life of 12 years. A summary of the results for the diesel-electric hybrid technology is presented in Table 1-3. The cycles in Table 1-3 are presented in ascending average speed. These data show that there are noticeable trends in $\mathrm{NO}_{\mathrm{x}}, \mathrm{CO}_{2}$, and fuel economy; PM and NMHC on the other hand do not exhibit such obvious behavior. These results cannot be extrapolated to other drive cycles or test weights. 
Table 1-3 Summary of Estimated Emissions for MY 2007 Diesel-Electric Hybrids (U.S. FTA, 2007)

\begin{tabular}{l|c|c|c}
\hline \multirow{2}{*}{ Pollutant Emissions } & \multicolumn{3}{|c}{ Cycle } \\
\cline { 2 - 4 } & Manhattan & OCTA & CBD \\
\hline $\mathrm{NO}_{\mathrm{x}}(\mathrm{g} / \mathrm{mi})$ & 5.54 & 4.41 & 4.14 \\
\hline $\mathrm{PM}(\mathrm{g} / \mathrm{mi})$ & 0.013 & 0.006 & 0.017 \\
\hline $\mathrm{NMHC}(\mathrm{g} / \mathrm{mi})$ & 0.10 & 0.00 & 0.10 \\
\hline $\mathrm{CO}_{2}(\mathrm{~g} / \mathrm{mi})$ & 2,504 & 1,972 & 1,869 \\
\hline Fuel Economy $(\mathrm{mpg})$ & 3.86 & 4.90 & 5.17 \\
\hline
\end{tabular}

The penetration of hybrid technologies in the U.S. transit industry has been significant during the last decade. The American Public Transportation Association (APTA) reported that in 2005 there were nearly 500 Diesel-Electric hybrid transit buses in the U.S. (Wayne et al., 2009). Their number has growth considerably ever since, Figure 1-1 shows the distribution of existing and ordered diesel hybrids larger than $30 \mathrm{ft}$ through 2013 (APTA, 2009). Based on these data, the diesel hybrid U.S. transit bus fleet is expected to reach at least 4,300 buses by 2014 , which is almost $7 \%$ of the U.S. fleet. On the other hand the penetration of Gasoline-Electric hybrids buses remains minimal, with their number reaching less than 200 by 2014 .

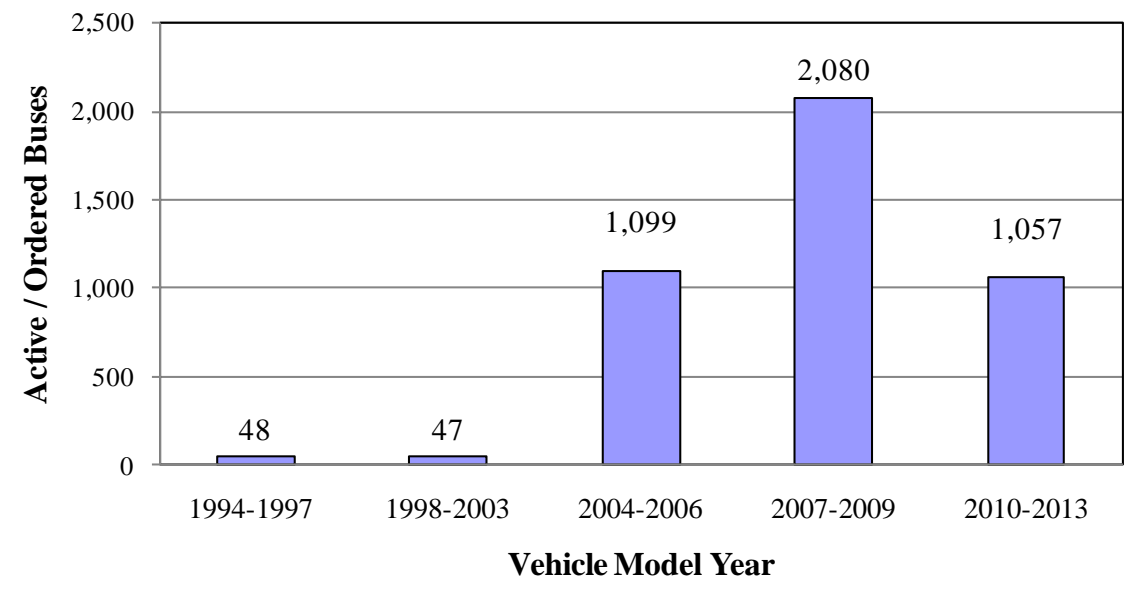

Figure 1-1 Active/Ordered Diesel-Electric Hybrid Buses by Model Year (Source: APTA, 2009)

\subsection{Objectives}

The outcome of this dissertation helps to expand the IBIS knowledge database in two areas. First, transit bus operation was characterized beyond the drive cycles available in the literature, through an evaluation of in-use routes and their properties. This study had the cooperation of the Washington Metropolitan Area Transit Authority (WMATA). Characterization of transit bus operation played an important role because it defined the parameters (and their ranges) to be used in fuel economy and 
emissions prediction models. The most significant parameters were average speed, percentage idle, and characteristic acceleration.

Second, the effect of duty cycle on diesel-electric hybrid bus emissions and fuel economy was studied, and a regression based emissions model was developed. This emissions regression model will be added to IBIS, where it will allow transit agencies to place hybrid buses in routes that take the most advantage of the hybrid-electric capabilities.

The objectives of this dissertation were identified as two overall goals and a set of tasks that lead to the completion of the goals.

\section{Overall Goals:}

1. To carry out an evaluation to characterize transit bus operation. This assessment seeks to bridge the gap between the engineering metrics of drive cycles and the information available to transit authorities about their bus operation, by developing correlations between the information available to transit agencies and the cycle metrics.

2. To develop a modeling tool to evaluate the effects of driving (duty) cycle on the performance and emissions of diesel-electric hybrid transit buses to help transit agencies place hybrids on service routes that maximize their benefits. The base vehicle was a 40' transit bus, model year 2007-2009.

\section{Tasks for Duty Cycle Characterization:}

$>$ Collect data from transit bus operation. Reduce and process the data.

$>$ Characterize and categorize driving patterns, e.g. highly transient inner city, express routes, suburban, commuter, etc.

$>$ Determine the metrics and ranges of interest.

$>$ Correlate cycle metrics with the data available to transit agencies.

$>$ Develop an interface for IBIS to estimate cycle metrics from user inputs

\section{Tasks for Hybrid Emissions Modeling:}

Determine the factors and parameters required for the model.

$>$ Obtain engine dynamometer emissions data for emissions and fuel economy models.

$>$ Construct and tune up a dynamic model for the hybrid bus in the Powertrain Systems Analysis Toolkit (PSAT) software.

$>$ Determine the emissions prediction procedure.

$>$ Project, given a drive cycle, emissions and F.E. from hybrid and conventional bus. 
Predict emissions over different routes and give an assessment as to what routes are most suitable for hybrid buses, i.e. the ones that best capitalize the hybrid system.

Develop a regression based modeling tool that allows transit agencies to evaluate on what routes to place their hybrid buses.

\subsection{Contributions}

This dissertation presents two main contributions, the first one being bridging the gap between engineering cycle metrics and the data available to the transit industry. Relationships were developed to correlate transit bus activity information available to transit agencies with engineering drive cycles and metrics. These relationships are the link between bus operation and the corresponding emissions and fuel economy figures. No scholarly articles were found related to the subject.

The second main contribution is a regression based $\mathrm{NO}_{\mathrm{x}}, \mathrm{CO}_{2}$, and fuel consumption model (an addition to IBIS) which will allow transit agencies to place hybrid buses in routes that take the most advantage of the hybrid-electric capabilities. Regressions were based on simulations of the dynamic model under conditions dictated by the drive cycle analysis.

The proposed cycle emissions and F.E. prediction approach can be extended to other vehicle technologies such as conventional diesel, $\mathrm{CNG}$, gasoline, etc.

\subsection{Research Approach}

In order to characterize transit bus activity, time-speed data was collected from a variety of transit bus routes. Road grade was estimated from topographic map and GPS location. Relationships between bus service and the engineering cycle metrics defined in the literature (e.g. average speed, standard deviation of speed, percentage idle, and kinetic intensity) were explored.

An emissions and fuel consumption model was developed for the MY 2007 diesel engine; this engine model was then integrated into the PSAT model of the hybrid bus. The engine emissions model was constructed from chassis-dynamometer test data; the model relied upon the accuracy of ECU broadcasts of engine torque and speed. Data for the engine model was from a MY 2006 Cummins ISB $260 \mathrm{H}$ engine. The model was appropriately scaled to match emissions standard of the MY 2007 engine. The model includes $\mathrm{NO}_{x}, \mathrm{CO}_{2}$, and fuel consumption.

$\mathrm{NO}_{\mathrm{x}}$ and $\mathrm{CO}_{2}$ emissions and fuel consumption were approached by introducing to the simulation tool maps and genetic algorithms. As hybrid buses are equipped with Diesel Particulate Filters (DPF) which produce extremely low levels of particulate emissions on a mass basis, PM emissions were not 
considered; emissions of $\mathrm{HC}$ and $\mathrm{CO}$ are also very low and were also neglected. The distribution of size of the emitted ultrafine particles is still a concern to public health, but particle sizing analysis was beyond the scope of this study.

Computer simulations, using PSAT as the core development software, were used to estimate fuel economy, $\mathrm{CO}_{2}$, and $\mathrm{NO}_{\mathrm{x}}$ over a variety of driving conditions. Simulations represented a series dieselelectric hybrid bus, model year 2007-2009. Chassis-dynamometer emissions test data from the WVU TransLab Database were used for calibration, improvement and validation of the simulation models.

The combination of the dynamic vehicle model, the engine emissions and fuel consumption model, and the use of regression analysis made it possible to predict emissions over different routes and to give an assessment as to what routes are most suitable for hybrid buses, i.e. the ones that best capitalize the hybrid system.

\subsubsection{Engine Model for Emissions and Fuel Consumption}

The engine emissions and fuel consumption model was based on artificial neural networks (ANNs) and 2D-steady-state maps. The general theory of ANNs was discussed in Section 2.6.5. There is extensive literature on ANN models for engine emissions, some relevant examples were presented in Sections 2.6.5 and 2.7.3.

An ANN requires being trained with sample data before it learns enough to be used. It was not possible to obtain engine dynamometer test data for an engine equivalent to the one on the hybrid bus; therefore, the author had to rely on chassis-dynamometer tests. A dataset suitable for ANNs training was produced using laboratory emissions measurements and ECU broadcasts of fuel rate, torque, and speed. The accuracy of this approach leans on several assumptions, starting for the fact that there are no direct measurements of engine speed and torque. The ECU broadcast of torque was given as percentage which was converted to engineering units with the engine's lug curve. Since the ECU does not report negative values of (percent) torque, there was no information regarding the motored (no fuel) torque curve.

As a general rule the primary inputs to the ANN are engine speed and torque with some derivative to account for transients. In more specialized cases, and depending on the availability of information, other inputs have been used, e.g. oil temperature, coolant temperature, turbocharger boost pressure, injection timing, etc.

The possibility that the test data corresponded to a different engine than the one that was to be simulated was considered; the ANN model can be scaled to represent the desired engine. As an example of this procedure, Tóth-Nagy et al. (2006) performed linear scaling of inputs and outputs of the ANN producing adequate results. Such scaling was not necessary since the engine from the test vehicle was the same as in the dynamic model. 
As mentioned before, $\mathrm{NO}_{\mathrm{x}}$ emissions were be corrected by the ratio of certification levels from the test engine (MY 2006) to the MY 2007 diesel engine. This method was used by Wayne et al. 2009 to estimate MY 2007-2009 emissions figures of transit buses based on emissions results of MY 20032005 buses.

\subsubsection{Vehicle Dynamic Model Development and Validation}

The possibility of modeling a 2-mode hybrid bus was considered but a suitable controller was not available. As the development of the vehicle dynamic model was not the purpose of this dissertation but rather a tool towards completion of the objectives, it was decided to pursue modeling of a series hybrid bus. A $40 \mathrm{ft}$ New Flyer hybrid powered with BAE Systems HybriDrive ${ }^{\circledR}$ series propulsion system was used as the target. BAE Systems provided technical specifications which allowed developing or scaling the various hybrid components. This process was developed in cooperation with Bell (2011).

The control strategy that was the most similar to the one of the test bus was PSAT's load following controller. The controller was modified to better regulate battery SOC under sustained high power conditions by limiting traction motor output until SOC was brought to a desired level. The PI engine speed controller was tuned to provide stability and improved response.

It was noted that PSAT did not maintain engine accessory loads during braking and stops. This problem was solved by an enhancement of the braking and stop controller in which accessory loads were sustained. This improvement over the default controller made it possible to create a vehicle model that better resembled the actual vehicle.

Validation of the dynamic model was performed at the vehicle level and not on a second-by-second basis. This approach was sensible because, first, the control strategies were different and, second, the objective was not to develop a model to exactly match the test bus but a model that was representative of the hybrid transit bus technology.

\subsubsection{Prediction of Cycle Fuel Economy and Emissions}

The dynamic model was simulated over 95 different routes that represented transit bus operation. The results were decomposed into the idle and driving contributions of fuel consumption and $\mathrm{NO}_{\mathrm{x}}$ emissions. The idle contribution was not zero as today's hybrid buses do not exercise engine shut-off at stops.

Polynomial least squares regressions were developed to predict the driving contribution of fuel consumption and $\mathrm{NO}_{\mathrm{x}}$ emissions. $\mathrm{CO}_{2}$ emissions were determined assuming complete fuel carbon conversion to $\mathrm{CO}_{2}$. The uncertainty on the prediction was evaluated and reported. 
The effects of grade on emissions performance of the hybrid bus were not explored, but were left as future work. This decision was based on the fact that instantaneous road grade may not be available to transit agencies in a way that could be used for the predictive models.

\subsection{Outline}

Chapter 2 presents a literature review relevant to this study. Chapter 3 evaluates the gains that can be achieved in transit vehicles from regenerative braking and studies the effects on tractive energy of road grade and test weight. Chapter 4 presents the transit bus route data collection and analysis. The diesel engine fuel consumption and emissions model is developed in Chapter 5. Chapter 6 presents the development and validation of the vehicle dynamic model. Chapter 7 evaluates the influences of transit bus duty cycle in fuel economy and emissions and the development of the regression based emissions model that will be implemented in IBIS. Finally, Chapter 8 presents the conclusions and recommendations from the work developed in the dissertation. 


\section{Chapter 2. LITERATURE REVIEW}

\subsection{Vehicle Energy Demand}

As a vehicle is driven, energy is supplied by the power unit (such as an internal combustion engine, an electric motor or a fuel-cell) to a transmission system and, finally, to the wheels. The purpose of the transmission system is to maintain the power unit within its operational and most efficient envelope at all vehicle speeds. Depending on the specifics of the transmission system, up to $20 \%$ of the energy supplied by the propulsion system is lost by friction, i.e. efficiencies of above $80 \%$ are observed. The remaining energy (transferred to the wheels) is used to accelerate and elevate the vehicle, and to overcome driveline drag forces, rolling friction at the wheels, and air resistance (Walston et al., 1976).

These terms form the road load which can be expressed as a force, $F_{\text {road }}$, or as power, $P_{\text {road }}=F_{\text {road }} \cdot V$, where $V$ is vehicle speed. The tractive power $P_{\text {road }}$ may also be called $\dot{E}_{\text {road }}$, which will be used in Section 2.1.3 to represent energy supply to the vehicle by the drivetrain.

When a vehicle makes a turn, energy is also consumed to change its momentum's direction. Since chassis dynamometer testing does not account for this fact, it will not be discussed here.

In this section we discuss in detail the road load components, the different driving modes and the opportunity for energy recuperation.

\subsubsection{Road Load}

Vehicle 3D dynamics can be simplified to longitudinal dynamics when only the forces in the longitudinal direction are of interest and all other forces can be neglected (e.g. winds, cornering, moments, etc.) With that in mind, the energy required to drive a vehicle has four components in form of forces that constitute the road load, $F_{\text {road }}$ :

Equation 2.1 Road Force

$$
F_{\text {road }}=F_{\text {aero }}+F_{\text {mech }}+F_{\text {grav }}+F_{\text {inertia }} \text {. }
$$


The road load equation (Equation 2.1) comes from Newton's second law or equilibrium of forces applied parallel to the road or in the direction of the vehicle's motion. This equation is accompanied by equilibrium of torques, which are not considered here. More information on the subject may be found in Gillespie (1992).

The components of the road load are described next:

$>$ Aerodynamic drag, $\boldsymbol{F}_{\text {aero }}$ : the aerodynamic drag corresponds to the energy used to overcome the force exerted by the airflow around the vehicle. This force is distributed along the vehicle surface, depends on air pressure distribution and shear. The aerodynamic drag is accompanied by an aerodynamic lift force which decreases the effective vehicle mass for mechanical drag purposes, however, this effect is very small (Buckley, 1995). It is customary to express the aerodynamic drag force as

\section{Equation 2.2 Aerodynamic Force}

$$
F_{\text {aero }}=\frac{1}{2} \rho_{\text {Air }} \cdot c_{d} \cdot A_{f} \cdot V_{r}^{2}
$$

where $\rho_{\text {Air }}$ is air density, $A_{f}$ is vehicle frontal area, $c_{d}$ is drag coefficient, and $V_{r}$ is wind speed relative to the vehicle. The term $1 / 2 \cdot \rho_{\text {Air }} \cdot V_{r}^{2}$ is the dynamic pressure. The drag coefficient is nearly constant for fully developed turbulent flows, a condition that is determined by the Reynolds Number, $\operatorname{Re}=V \cdot w / v$, where $w$ is the vehicle width and $v$ is the kinematic viscosity of air. The SAE Recommended Practice for Truck and Bus Wind Tunnel Testing suggests to use this approximation for $R e \geq 2 \times 10^{6}$ (SAE J1252, SAE International, 1981), which corresponds to 25 mph for an $81 / 2 \mathrm{ft}$ wide bus and $35 \mathrm{mph}$ for a mid-size sedan. If more data are available, the drag coefficient may be expressed as a function of vehicle speed, $c_{d}=c_{d}(V)$. Typical values of the drag coefficient for commercial vehicles are presented in Table 2-1.

The drag coefficient is highly dependent on vehicle shape but is also affected by the relative wind direction. This condition has been modeled, from experimental data, as a forth order polynomial with respect to yaw angle, $Y, c_{d}=c_{d}(Y)$; the coefficients are determined through coastdown procedures (SAE J2263, SAE International, 1996). The drag coefficient reaches a minimum at $0^{\circ}$ yaw and increases with yaw angle (Buckley et al., 1976).

Table 2-1 Suggested Drag Coefficients for Commercial Vehicles, Hucho (1998)

\begin{tabular}{l|c}
\hline \multicolumn{1}{c|}{ Vehicle Type } & Drag Coefficient Range \\
\hline Cars & $0.26-0.45$ \\
\hline Vans & $0.32-0.50$ \\
\hline Buses & $0.40-0.66$ \\
\hline Tractor-Trailers & $0.48-0.76$ \\
\hline Tractor-Semitrailers & $0.55-0.86$ \\
\hline
\end{tabular}


Mechanical Drag Force, $\boldsymbol{F}_{\text {mech }}$ : It consists of driveline drag or friction, $F_{\text {driveline, and rolling }}$ resistance at the wheels, $F_{\text {rolling: }}$ :

Equation 2.3 Mechanical Drag Force

$$
F_{\text {mech }}=F_{\text {driveline }}+F_{\text {rolling }}
$$

The driveline drag was shown to be linear with speed (Walston et al., 1976):

Equation 2.4 Driveline Drag Force

$$
F_{\text {driveline }}=b+c \cdot V
$$

where the coefficients $b$ and $c$ are determined through a driveline drag test; $b$ is related to coulomb damping while $c$ is to viscous damping.

The rolling resistance force is proportional to the component of the vehicle weight $(m \cdot g)$ that is perpendicular to the road surface force. Although the force is distributed among the wheels, it can be modeled as a single force:

Equation 2.5 Rolling Resistance Force

$$
F_{\text {rolling }}=c_{r} \cdot m \cdot g \cdot \cos (\alpha)
$$

where $\alpha$ is the angle between the road and the horizontal axis, $c_{r}$ is the rolling resistance coefficient, $m$ is the vehicle mass, and $g$ is the gravitational constant. The rolling resistance coefficient is determined experimentally and is affected by road surface and tire conditions. Also, $c_{r}$ may be determined as a function, usually linear, of vehicle speed. Rolling resistance depends on the construction of the tire and the compounds used.

Mechanical drag has also been modeled as a second order polynomial in speed, combining driveline and tire drag (SAE J2263, SAE International, 1996).

Grade, $\boldsymbol{F}_{\text {grav }}$ : The grade or gravitational force is the force used to increase the altitude of the vehicle $(\alpha>0)$. It is the given by the component of the vehicle weight parallel to the road. The grade force stores energy in the vehicle as potential energy and which can be released in downhill driving $(\alpha<0)$. The grade force is:

Equation 2.6 Grade Force, $\alpha$

$$
F_{\text {grav }}=m \cdot g \cdot \sin (\alpha)
$$

or alternatively,

Equation 2.7 Grade Force, $h$

$$
F_{\text {grav }}=m \cdot g \cdot \frac{d h}{d s}
$$


where $h$ is the elevation and $s$ is the distance travelled $(d s=V d t)$. The grade angle is related to the commonly used percent grade by $\alpha=\tan ^{-1}\left(\frac{\% \text { grade }}{100}\right)$.

Inertia, $\boldsymbol{F}_{\text {inertia }}$ : The inertia force includes both inertia due to vehicle mass (translational inertia) and inertia of the rotating parts (Guzzella and Sciarretta, 2007), and can be expressed as:

Equation 2.8 Inertia Force

$$
F_{\text {inertia }}=\left(m+m_{r}\right) \cdot \frac{d V}{d t}
$$

where the term in parenthesis is the effective vehicle mass, $m_{e}$, which contains the actual vehicle mass, $m$. The effective inertia mass of the rotating components, $m_{r}$, is

Equation 2.9 Effective inertia mass of rotating components

$$
m_{r}=\frac{1}{r_{w}^{2}} \cdot\left(I_{w}+\gamma^{2} I_{e}\right)
$$

where $I_{w}$ is the moment of inertia of the wheels and all the rotating parts on the wheel-side of the transmission, $I_{e}$ is the moment of inertia of the engine and the rotating parts on the engine-side of the transmission, $r_{w}$ is the wheel radius, and $\gamma$ is the overall gear ratio $\left(\gamma=\omega_{\text {engine }} / \omega_{\text {wheels }}\right)$. It is assumed that there is no wheel slip; transmission inefficiency is neglected. The mass due to rotating components is necessary for simple simulations in which only a general model of the vehicle is used. On the other hand, when the simulation has sub-models for all vehicle parts, inertia would be included in such sub-models and the term $m_{r}$ would not be necessary. For chassis dynamometer testing, $m$ includes curb and load masses; $m_{r}$ shall not be considered during emissions testing but shall be used for track coastdown data analysis (SAE J2263, SAE International, 1996).

The above components comprise the road load $\left(F_{\text {road }}\right.$, also called the traction force, $\left.F_{\text {trac }}\right)$ or vehicle's energy demand $\left(P_{\text {road }}\right)$. In addition to this energy, there are parasitic losses and auxiliary loads that need to be supplied by the vehicle's propulsion system.

Replacing Equation 2.2 to Equation 2.8 into Equation 2.1 we obtain the final form of the road load equation:

\section{Equation 2.10 Road Load Equation - Force}

$$
F_{\text {road }}=\frac{1}{2} \rho_{\text {Air }} c_{d} A_{f} V_{r}^{2}+(b+c V)+c_{r} m g \cos (\alpha)+m g \sin (\alpha)+m_{e} \frac{d V}{d t}
$$

and the tractive power: 
Equation 2.11 Road Load Equation - Power

$$
\begin{aligned}
& P_{\text {road }}=\dot{E}_{\text {road }}=\underbrace{\frac{1}{2} \rho_{\text {Air }} c_{d} A_{f} V_{r}^{2} V}_{\text {Aerodynamic }}+\underbrace{\left(b V+c V^{2}\right)}_{\text {Loads due }}+\underbrace{c_{r} m g V \cos (\alpha)}_{\text {Rolling }}+\underbrace{m g V \sin (\alpha)}_{\text {Elevation }}+\underbrace{m_{e} V \frac{d V}{d t}}_{\text {Inertial }} \\
& \text { Drag to Driveline Resistance Load Load } \\
& \text { Friction }
\end{aligned}
$$

The power demand on the engine of a conventional vehicle can be calculated by

Equation 2.12 Engine Power

$$
P_{\text {engine }}=\frac{P_{\text {road }}}{\eta_{d r}}+P_{A C C}
$$

where $\eta_{d r}$ is drivetrain efficiency and $P_{A C C}$ is the power drain of vehicle accessories and hotel loads.

\subsubsection{Driving Modes}

The vehicle operation can be classified depending on the value of the traction force (Guzzella and Sciarretta, 2007) in three modes as follows:

$>$ Traction mode if $F_{\text {road }}$ (or $P_{\text {road }}$ since only forward motion is considered) is greater than zero; the propulsion system provides energy to the vehicle.

$>$ Braking mode if $F_{\text {road }}$ (or $P_{\text {road }}$ ) is less than zero; the braking system reduces the vehicle's kinetic and potential energy while rolling and aerodynamic forces continue to act.

$>$ Coasting mode if $F_{\text {road }}$ (or $P_{\text {road }}$ ) is equal to zero; the propulsion system is disengaged from the wheels and the change in kinetic and potential energy matches the rolling and drag losses. To be more precise, in an actual vehicle the change in mechanical energy would also be providing for the friction losses in the drivetrain.

Figure 2-1 shows the different driving modes in a section of the Orange County Transit Authority (OCTA) cycle for transit buses. The coasting curve is the local solution to the first order PDE Equation 2.10 with $F_{\text {road }}=0$ and depends on the specific parameters of the bus. Observe that during the last seconds of the traction mode the vehicle is decelerating while the tractive power is still positive; if the accelerator pedal was released, the vehicle would follow a coasting curve. Instead, to follow the drive cycle further deceleration is required and the brake pedal is depressed. 


\subsubsection{Energy Recuperation and Fuel Consumption}

As mentioned above, the vehicle's propulsion system must provide energy to overcome aerodynamic and mechanical drag, to increase the elevation, and to accelerate the vehicle. The first two factors dissipate energy and the last two store energy in the vehicle, thus may be recovered to some extent. Kinetic and potential energies are mechanical forms of energy and they can be converted directly from one to another. In braking events of a conventional vehicle, the engine does not supply power but it can consume some (due to for example friction or compression braking). If the powertrain is disengaged from the wheels, the kinetic energy stored in the vehicle is dissipated at braking in two ways, one part goes to rolling and drag losses, and the other part is dissipated into heat by the braking elements. Potential energy is normally dissipated in steep downhill driving because it tends to accelerate the vehicle above safe driving speeds. In special downhill cases, such potential energy change is just enough to overcome the losses $\left(P_{\text {road }}=0\right)$ and the vehicle can be driven without drivetrain power at nearly constant speed.

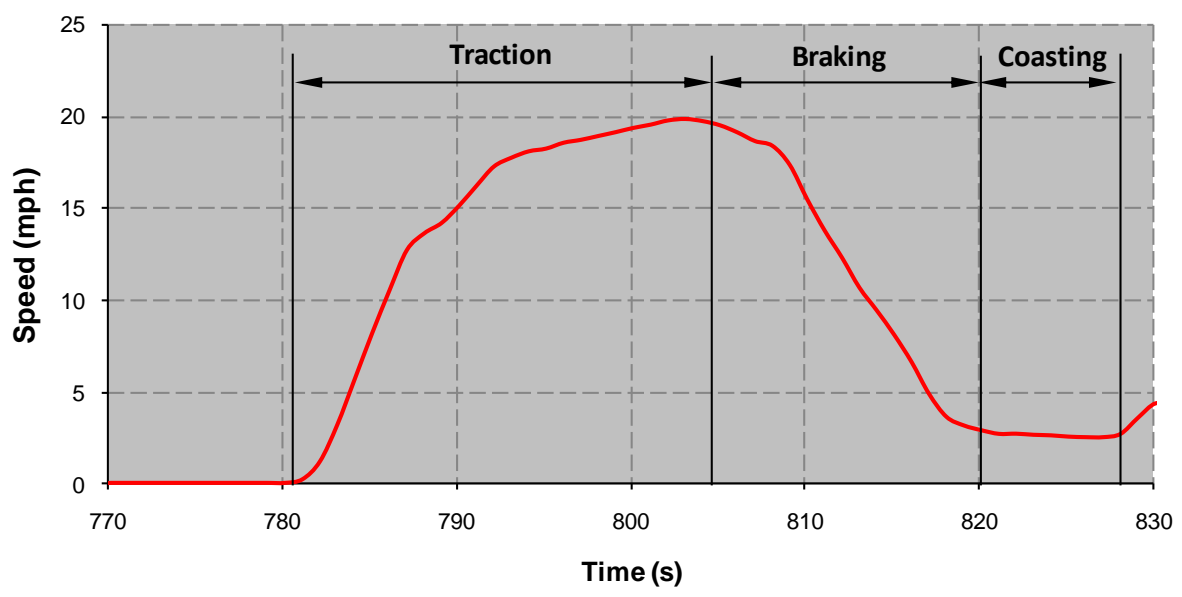

Figure 2-1 Driving Modes in a Section of the OCTA Cycle

Energy is available for recuperation through regenerative braking whenever the tractive power is negative, i.e. the vehicle's mechanical energy needs to be decreased but the aerodynamic drag and rolling resistance loads do not suffice to remove this energy, and an external force needs to be applied. This energy can be either dissipated into heat through a friction brake system or recovered for later use through, for example, an electric motor-battery system.

Energy recuperation may be assessed by means of the mean net tractive energy, $e_{t r a c, n e t}$, which represents the net energy required to drive the vehicle per unit distance travelled. Net tractive energy, $E_{\text {trac, net }}$, is defined in terms of the tractive or propulsion energy, $E_{t r a c}$, the braking energy, $E_{B R}$, and the regeneration efficiency, $\eta_{\text {regen }}$, as follows 


\section{Equation 2.13 Net Tractive Energy}

$$
E_{\text {trac, net }}=E_{\text {trac }}-\eta_{\text {regen }} \cdot E_{B R}
$$

The mean net tractive energy is $e_{\text {trac,net }}=E_{\text {trac,net }} / D$. Where $D$ is distance traveled, $D=\int_{0}^{T} V d t \cong \sum \bar{V}_{i} \cdot \Delta t_{i} ; i$ represents time interval and the over-bar represents the average value over the time interval and $T$ is the dive cycle time.

Tractive energy, $E_{t r a c}$, is equal to the summation of the positive tractive power of Equation 2.11 (Guzzella and Sciarretta, 2007, Sovran and Blaser, 2003) over the drive cycle. It can be expressed in integral form as

\section{Equation 2.14 Tractive Energy}

$$
\left.E_{\text {trac }}=\int_{0}^{T} \dot{E}_{\text {road }}\right)^{+} d t \cong \sum_{\bar{E}_{\text {road }, i}>0} \overline{\dot{E}}_{\text {road }, i} \cdot \Delta t_{i}
$$

Similarly, the braking energy is defined as the integral of negative tractive power (absolute value),

Equation 2.15 Braking Energy

$$
\left.E_{B R}=\int_{0}^{T} \mid \dot{E}_{\text {road }}\right)^{-}\left|d t \cong \sum_{\dot{E}_{\text {road }, i}<0}\right| \overline{\dot{E}}_{\text {road }, i} \mid \cdot \Delta t_{i}
$$

When looking at the vehicle as a whole, engine braking is discounted from $E_{B R}$ since it reduces the required braking effort (Sovran and Blaser, 2003).

The braking energy capture-redeployment efficiency or simply regeneration efficiency, $\eta_{\text {regen }}$, is defined as the fraction of the total available braking energy $\left(E_{B R}\right)$ that is actually delivered back to the wheels. $\eta_{\text {regen }}$ is affected by the use of friction brakes (i.e. when braking power is above the hybrid system's limits) and by the efficiencies of transmission, electric motor, power converter, and energy storage hardware; the theoretical limit being a perfect recuperation device $100 \%$ efficient $\left(\eta_{\text {regen }}=1\right)$.

Note that for conventional vehicles $\left(\eta_{\text {regen }}=0\right)$ Equation 2.13 reduces to Equation 2.14 , and $E_{\text {trac }}$, net $=E_{\text {trac }}$. Also note that tractive and braking energies depend upon vehicle parameters (e.g. $c_{d} A_{f}, c_{r}, m$, $m_{r}$ ), drive cycle; that is, all the parameters involved in the road load equation (Equation 2.10 and Equation 2.11).

Net tractive energy can be converted to fuel efficiency (gal/mi or L/100 km) with the consideration of average drivetrain and engine conversion efficiencies. In such case, it is essential to account for fuel consumption due to idle and hotel loads. This kind of analysis has been discussed by O'Keefe et al. (2007). Also, Sovran and Blaser (2003) presented a comprehensive analysis of fuel consumption and developed the following equation for drive-cycle fuel consumption, $\tilde{g}$ [volume of fuel per unit distance driven] 


\section{Equation 2.16 Fuel Consumption Equation}

$$
\tilde{g}=\frac{1}{\rho_{\text {fuel }} \cdot D}\left[\frac{\left(E_{\text {trac }}-\eta_{\text {regen }} E_{B R}\right) / \tilde{\eta}_{d r}+E_{b, A C C}^{+}}{H_{f} \eta_{b, \max }\left(\tilde{\eta}_{b} / \eta_{b, \max }\right)}+t_{b r k g} \cdot\left(\overline{\dot{m}}_{f}\right)_{b r k g}+t_{\text {idle }}\left(\overline{\dot{m}_{f}}\right)_{\text {idle }}\right],
$$

where $\rho_{f u e l}$ is fuel density, $\tilde{\eta}_{d r}$ is the energy-transfer-weighted average drivetrain efficiency, $\eta_{b, \max }$ and $\tilde{\eta}_{b}$ are maximum and fuel-consumption-weighted average brake thermal efficiencies, $E_{b, A C C}^{+}$is the energy required by vehicle accessories or hotel loads, $H_{f}$ is the heating value of the fuel, $t_{b r k g}$ is the braking time, $t_{i d l e}$ is the idle time, and $\left(\overline{\dot{m}}_{f}\right)_{b r k g}$ and $\left(\overline{\dot{m}}_{f}\right)_{\text {idle }}$ are the fueling rates at braking and idle, respectively.

In Equation 2.16 each of the terms in brackets is mass of fuel consumed; the first term corresponds to the powered driving section. Equation 2.16 assumes that when compared with the conventional vehicle the hybrid will have the same road load and will require the same tractive energy. In a followup paper, Sovran and Blaser (2006) considered the fact that due to the increased weight of the hybrid system components a hybrid vehicle would be heavier than its conventional counterpart. With that in mind, the amount $m_{f}$ of fuel consumed in powered driving would be expressed as, Sovran and Blaser (2006):

\section{Equation 2.17 Hybrid Fuel Consumed in Powered Driving}

$$
m_{f, h y b r i d}=\frac{\tilde{\eta}_{b, 0}}{\tilde{\eta}_{b}} \cdot \frac{\tilde{\eta}_{d r, 0}}{\tilde{\eta}_{d r}} \cdot \frac{E_{t r a c}}{E_{\text {trac }, 0}} \cdot\left(1-\eta_{\text {regen }} \cdot \frac{E_{B R}}{E_{\text {trac }}}\right) \cdot m_{f, 0},
$$

where the subscript 0 refers to the conventional vehicle and the non-zero parameters refer to the hybrid vehicle. Equation 2.17 denotes the benefits of hybridization. Note that, since accessory loads are considered separately, $m_{f, 0}$ in Equation 2.17 should be only fuel used for propulsion. Combining Equation 2.16 and Equation 2.17 we obtain another useful fuel consumption equation:

\section{Equation 2.18 Fuel Consumption Equation}

$$
\tilde{g}=\frac{1}{\rho_{\text {fuel }} \cdot D}\left[m_{f, h y b r i d}+\frac{E_{b, A C C}^{+}}{H_{f} \tilde{\eta}_{b}}+t_{b r k g} \cdot\left(\overline{\dot{m}}_{f}\right)_{b r k g}+t_{\text {idle }}\left(\overline{\dot{m}}_{f}\right)_{\text {idle }}\right] .
$$

The concepts described in this section have been used in the literature (e.g. Sharer et al., 2007) to evaluate performance of hybrid vehicles and their response to duty cycle. 


\subsection{Driving Characterization}

Given that vehicle fuel economy and emissions are driving pattern dependent, an analysis of the wide operative envelope of transit buses has to account for the various driving conditions encountered. For this purpose, drive cycles are studied, and their properties extracted in the form of cycle metrics. Cycle metrics describe or characterize the drive cycles. A desirable characteristic of cycle metrics is that they must be independent of vehicle properties, such as weight and architecture, among others; this property gives the metric a general character. Some metrics may involve parameters like accessory loads and regenerative braking, and even though their significance would not be general, they will serve as an indicator of the performance comparison among various vehicle technologies in a given transit application.

Several metrics have been defined and employed; among them we have average speed, percentage idle, average speed without idle, stops per mile, standard deviation of speed, maximum and average acceleration, maximum and average deceleration, aerodynamic speed, characteristic acceleration, and kinetic intensity. The object of this section is to introduce and briefly discuss such cycle metrics.

\subsubsection{Speed Related Metrics}

Idle: Idle is the time (or fraction of the time) in which the vehicle is stationary. The effect of idle is, as observed in Equation 2.16 (page 17), to increase fuel consumption and reduce fuel economy. Inner-city and urban driving are characterized by relatively large percent idle times; for example the NY Bus cycle has an idle fraction of $65 \%$. A large idle fraction is directly related to a low average velocity and a large kinetic intensity; metrics which are defined below. Idle percentage ranges from as high as $65 \%$ for stop-and-go operation (NY Bus Cycle) to nearly zero for highway driving (HWFET Cycle).

Average Speed, $\bar{V}$ or $\bar{U}$ : Average speed is defined as distance travelled divided by cycle time, $\bar{V} \stackrel{d}{=}$ $D / T$. It links idle with driving periods and has been widely used to characterize driving behaviors. Average speed ranges from $2 \mathrm{mph}$ for heavy inner city operation (HHDDT-Creep Schedule) to 50 mph for highway operation (HWFET).

$>$ Average Speed without Idle: Average speed without idle is defined as $\bar{V}_{\text {noidle }} \stackrel{d}{=} D /\left(T-t_{\text {idle }}\right)$. It excludes the segments of the drive cycle in which the vehicle is stationary and represents the dynamic behavior of the cycle. Average speed without idle ranges from $3 \mathrm{mph}$ (HHDDT-Creep) to $52 \mathrm{mph}$ (US06 Cycle). 
Average speed without idle can be calculated from average speed and percent idle:

Equation 2.19 Average Speed Without Idle

$$
\bar{V}_{\text {noidle }}=\bar{V} \cdot \frac{1}{1-\operatorname{Idle}(\%) / 100} .
$$

Standard Deviation of Speed: Standard deviation of speed represents the transient character of the drive cycle. It can be defined either with or without idle to characterize the overall cycle and its mobile section. Standard deviation of speed ranges from $2 \mathrm{mph}$ (HHDDT-Creep) to $25 \mathrm{mph}$ (US06) while standard deviation of speed without idle from $2 \mathrm{mph}$ (HHDDT-Creep) to $22 \mathrm{mph}$ (HHDDT and US06).

Standard deviation of speed is calculated by

Equation 2.20 Standard Deviation of Speed

$$
s=\left[\frac{1}{N-1} \sum_{i=1}^{N}\left(V_{i}-\bar{V}\right)^{2}\right]^{1 / 2},
$$

where $N$ is the size of the speed vector $V$. The result $s^{2}$ is an unbiased estimator of the variance of speed. A similar equation is used for standard deviation of speed without idle, where $V$ is replaced by the $V_{\text {no idle }}$ vector and $N$ by the corresponding vector size.

Acceleration: Acceleration is the derivative of speed: $a=d V / d t$. High accelerations imply elevated power demands and fueling rates; conditions that are believed to produce lower fuel economy. Both maximum acceleration and deceleration rates have been reported in the literature, together with their average values (averaged over the acceleration or deceleration periods only). Typical ranges of the acceleration metrics are presented in Table 2-2.

The acceleration rate has been calculated in this dissertation using the 5-point fourth-order accuracy $\left(\Delta t^{4}\right)$ approximation:

Equation 2.21 Five-point Approximation of Acceleration

$$
a_{i}=\frac{-V_{i+2}+8 V_{i+1}-8 V_{i-1}+V_{i-2}}{12 \Delta t} .
$$

This approximation may lead to acceleration values before the vehicle starts moving and after the vehicle stops. The calculations were performed as to avoid this situation.

Acceleration and deceleration may be classified as light, medium, and heavy (Kern, 2000). Table 2-3 shows the acceleration bins proposed by Kern (2000); if $|a|$ is less than $0.44 \mathrm{ft} / \mathrm{s}^{2}$ (and $\mathrm{V} \neq 0$ ) the vehicle is considered to be cruising. Figure 2-2 shows the acceleration bins observed in the section of the OCTA cycle shown in Figure 2-1. The section contains all the bins except for heavy deceleration. It must be noted that unlike this section most of the cycle exhibits heavy decelerations. 
Average acceleration $\bar{a}_{+}$and average deceleration $\bar{a}_{-}$are defined as

Equation 2.22 Average Acceleration and Deceleration

$$
\begin{array}{ll}
\left.\bar{a}_{+} \stackrel{d}{=} \frac{1}{T_{+}} \cdot \int_{0}^{T} a\right)^{+} d t & \left.\bar{a}_{-} \stackrel{d}{=} \frac{1}{T_{-}} \cdot \int_{0}^{T} \mid a\right)^{-} \mid d t \\
\bar{a}_{+}=\frac{1}{T_{+}} \cdot \sum_{a_{i}>0} a_{i} \cdot \Delta t_{i} & \bar{a}_{-}=\frac{1}{T_{-}} \cdot \sum_{a_{i}<0}\left|a_{i}\right| \cdot \Delta t_{i}
\end{array}
$$

where $T_{+}$is time with $a>0$ (time spent in acceleration) and $T_{-}$is time with $a<0$ (time spent in deceleration).

Table 2-2 Observed Ranges of Acceleration Metrics

\begin{tabular}{c|c|c}
\hline Metric & $\begin{array}{c}\text { Low } \\
\left(\mathbf{f t} / \mathbf{s}^{\mathbf{2}}\right)\end{array}$ & $\begin{array}{c}\text { High } \\
\left(\mathbf{f t} / \mathbf{s}^{\mathbf{2}}\right)\end{array}$ \\
\hline Max Accel. & 1.5 & 14.2 \\
\hline Max Decel. & 2.2 & 13.5 \\
\hline Average Accel. & 0.4 & 3.7 \\
\hline Average Decel. & 0.4 & 6.2 \\
\hline
\end{tabular}

Table 2-3 Classification Vehicle Operation According to Instantaneous Acceleration (Kern, 2000)

\begin{tabular}{c|c}
\hline Classification & Range $(\mathbf{~ m p h} / \mathbf{s})$ \\
\hline Cruise & $|a|<0.3$ \\
\hline Light Accel./Decel. & $0.3 \leq|a|<1$ \\
\hline Medium Accel./Decel. & $1 \leq|a|<2$ \\
\hline Heavy Accel./Decel. & $|a| \geq 2$ \\
\hline
\end{tabular}

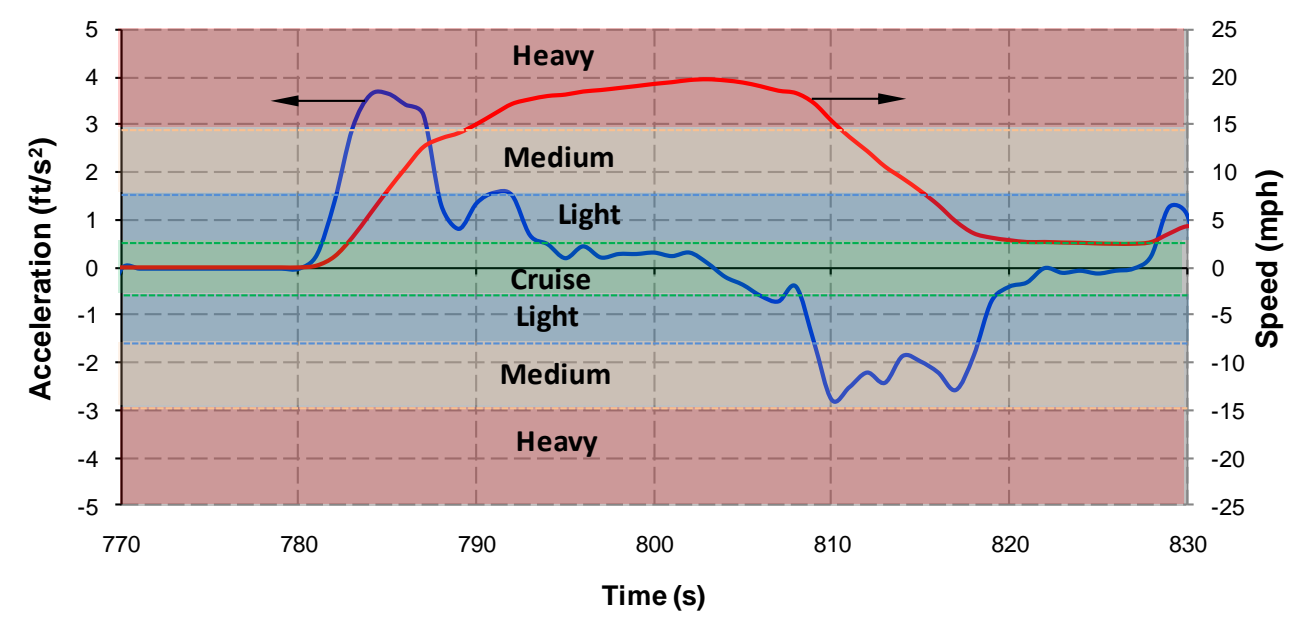

Figure 2-2 Acceleration Bins in a Section of the OCTA Cycle

Stops per unit distance: A stop is defined as when the vehicle speed goes below a predefined threshold and this condition remains for a minimum period of time. This metric is related to 
average speed such that the larger its value, the lower the average speed. Its values can be as low as 0.1 stops/mile for highway operation and as high as 24 stops/mile for heavy inner-city driving.

\subsubsection{Characteristic Acceleration, Aerodynamic Speed, and Kinetic Intensity}

O'Keefe et al. (2007) derived characteristic acceleration, aerodynamic speed, and kinetic intensity metrics, which are reviewed next. Characteristic Acceleration, $\tilde{a}$, is the integrated positive mechanical energy (kinetic and potential energy) supplied per unit mass per unit distance, and has units of $\mathrm{m} / \mathrm{s}^{2}$. The characteristic acceleration of the cycle is therefore

Equation 2.23 Characteristic Acceleration

$$
\tilde{a}=\frac{d}{D} \sum_{i=2}^{N}\left[\frac{1}{2}\left(V_{i}^{2}-V_{i-1}^{2}\right)+g\left(h_{i}-h_{i-1}\right)\right]^{+},
$$

where $N$ is the number of time steps and $h$ is elevation. Also, for a time step we have

Equation 2.24 Characteristic Acceleration for a Time Step

$$
\tilde{a}_{i} \stackrel{d}{=} \frac{1}{\Delta D_{i}}\left[\frac{1}{2}\left(V_{i}^{2}-V_{i-1}^{2}\right)+g\left(h_{i}-h_{i-1}\right)\right] .
$$

$\Delta D_{i}$ is distance traveled in the interval and its first order backward approximation is $\Delta D_{i}=\left(V_{i}+V_{i-1}\right) /$ $\left(2 \cdot \Delta t_{i}\right)$. We can also write $\tilde{a}$ as:

Equation 2.25 Characteristic Acceleration

$$
\left.\tilde{a}=\frac{1}{D} \sum_{i=2}^{N} \Delta D_{i} \cdot \tilde{a}_{i}\right)^{+} .
$$

Characteristic acceleration measures the inertial work to accelerate and/or raise the vehicle per unit mass per unit distance over the cycle. It is the positive part of specific kinetic and potential energy per distance associated with moving the vehicle over a duty cycle.

Aerodynamic Speed is defined as the square root of the ratio of average cubic speed to average speed. The squared aerodynamic speed is defined by

Equation 2.26 Aerodynamic Speed

$$
V_{\text {aero }}^{2} \stackrel{d}{=} \frac{1}{T} \int_{0}^{T} V^{3} d t / \frac{1}{T} \int_{0}^{T} V d t=\int_{0}^{T} V^{3} d t / \int_{0}^{T} V d t .
$$

It is linked to the impact of aerodynamic resistance on vehicle fuel usage. The squared aerodynamic speed can be also expressed as: 
Equation 2.27 Aerodynamic Speed

$$
V_{\text {aero }}^{2}=\frac{1}{D} \int_{0}^{D} V^{2} d x
$$

From Equation 2.27, it is clear that $V_{\text {aero }}^{2}$ is a distance averaged squared velocity and therefore adding or removing idle segments to a duty cycle does not affect aerodynamic speed.

Characteristic acceleration and aerodynamic speed appear naturally in the road load equation when the specific energy per distance is evaluated for a time step. They are also found in fuel consumption estimations similar to Equation 2.16 (page 17); specifically they are present in the $E_{\text {trac }}$ and $E_{B R}$ terms (O'Keefe et al., 2007).

Simpson (2005) suggested that average speed, aerodynamic speed, and characteristic acceleration form an orthogonal and independent coordinate set that quantifies the multiple dimensions of a driving cycle.

Kinetic Intensity (O’Keefe et al., 2007),

Equation 2.28 Kinetic Intensity

$$
k i \stackrel{d}{=} \frac{\tilde{a}}{V_{\text {aero }}^{2}},
$$

with units of one over distance, is an important factor for hybrid vehicles because a cycle energy use analysis shows that high values of kinetic intensity translate into higher fractions of $E_{B R} / E_{t r a c}$ and give room to fuel economy improvements.

Kinetic Intensity can be used to compare micro-trips and duty cycles from an energy perspective. Observed ranges for aerodynamic speed, characteristic acceleration, and kinetic intensity, along with other cycle statistics, are presented in Table 2-6 (page 28).

Another metric defined by O'Keefe et al. (2007) is $\boldsymbol{\beta}$ : the ratio of fuel consumed by non propulsion efforts (e.g. hotel loads and idle operation) to the cycle integrated positive tractive effort. This parameter differs from the others in that it is technology dependent and must be experimentally evaluated for a given test vehicle. High values of $\beta$ tend to decrease the hybrid advantage (percent reduction in fuel consumption) since more energy used in hotel loads overshadows the gains of the hybrid system.

\subsection{Vehicle Duty Cycles}

The cycle metrics listed above are defined in a way that requires presenting the driving schedule as a time-speed-elevation relationship. A Drive (or Test) Cycle can be defined as a prescribed speed trace that the vehicle must reproduce as a function of time. This trace normally consists of time-speed 
points and it may or may not include grade. A different kind of schedule is a Drive (or Test) Route, which is distance-speed based, similar to how a vehicle would be driven in the streets or in a test track. The main characteristic of drive routes is that they exercise the vehicle at full power during free acceleration sections; also, cycle time is modified depending upon vehicle performance and the total distance is maintained (Nine et al., 1999).

Both, drive cycles and routes, are used to evaluate the vehicle as a whole and are generally run in chassis-dynamometer facilities. They provide data for emissions inventories and the ability to determine improvements in fuel economy and emissions from hybrid architectures. On the other hand, engine dynamometer testing is used for engine certification and, even though extensive data exist, it is difficult to translate engine dynamometer results to vehicle fuel economy and emissions. Furthermore, engine testing data is not suitable for accurate emissions inventory prediction (Guensler et al., 1991, Nine et al., 1999). Engine test cycles are given as engine speed-torque (\%) targets, where $0 \%$ is idle and $100 \%$ is maximum load at the given speed.

Driving conditions can be classified according to vehicle speed in the following categories: innercity or stop-and-go, urban, suburban, and highway. Table 2-4 shows the ranges of the various categories as defined by the author based on Zou et al. (2004). The speed thresholds for urban, suburban, and highway driving match the urban, rural, and motorway sections of the European Transient Cycle (ETC). Several, if not all, of these conditions can be present in any particular drive cycle; for example the King County Metro (KCM) cycle (Figure 2-6, page 31) displays segments in all the driving categories. Zou et al. (2004) categorized micro-trips by peak speed; they used $40 \mathrm{mph}$ as the threshold for highway driving.

Table 2-4 Classification of Driving Conditions

\begin{tabular}{c|c}
\hline Condition & Range $(\mathbf{m p h})$ \\
\hline Inner-City & $V<15$ \\
\hline Urban & $15 \leq V<30$ \\
\hline Suburban & $30 \leq V<50$ \\
\hline Highway & $V \geq 50$ \\
\hline
\end{tabular}

\subsubsection{Drive Cycle Development}

Duty cycles representing specific driving patterns are developed from collected in-use data (timespeed-grade). The speed trace is divided into micro-trips, defined from start-to-stop, and statistical metrics of the database are calculated. Finally, using the measured micro-trips, drive cycles are generated to match the statistical metrics of the database (Dembski et al., 2005).

West Virginia University has extensive experience in drive cycle construction. In their approach the cycle is developed from micro-trip concatenation to match several metrics (average speed without 
idle, average acceleration, etc.) with the metric of the database (Nine et al., 1999, McKain et al., 2000, Wayne et al., 2002); idle segments are included afterward. Another approach could be to define a micro-trip from start-to-start, thus including idle and the driving event. Then, based on the micro-trips, a large number of candidate cycles are created and their statistics are calculated and compared with the database. For example West Virginia University developed the OCTA Cycle (Wayne et al., 2004a) by randomly concatenated micro-trips (from start to stop) into cycles of at around 35 min in duration, excluding idle which was added later. By this means, 20,000 candidate cycles were created. The candidate cycles were compared with the database according to the root-mean-square (RMS) formula (Nine et al., 1999, Wayne et al., 2004a):

Equation 2.29 Root-Mean-Square Formula

$$
R M S \stackrel{d}{=}\left[\sum_{k=1}^{n}\left(\frac{P_{k, c d}-P_{k, d b}}{P_{k, d b}}\right)^{2}\right]^{1 / 2},
$$

where $P_{k}$ represents the various parameters of interest, $c d$ is candidate, $d b$ is database, and $n$ is number of parameters. In the specific case of the OCTA cycle, the parameters were: average speed without idle, standard deviation of vehicle speed, average weight-specific kinetic energy, and its standard deviation. The candidate cycle with the lowest RMS is therefore the one that best represents the activity database. A candidate cycle would have been excluded if one of the error terms contributed to more than half of the total RMS error. Refer to Wayne et al. (2004a) for other possible reasons for excluding a candidate cycle.

By this means, the number of candidate cycles is reduced to a handful of them. This final selection is analyzed and adjusted to accelerations and deceleration rates that match dynamometer capabilities (deceleration rates no greater than $2 \mathrm{mph} / \mathrm{s}$ or $2.93 \mathrm{ft} / \mathrm{s}^{2}$ ). Extreme accelerations (or decelerations) that are characteristic of downhill (or uphill) driving are also adjusted. The latter consideration corresponds to development of drive cycles that do not include grade information and would be unnecessary when grade is measured and included into the micro-trips.

The International Association of Public Transport (UITP) developed the SORT method (Standardised On-Road Test Cycles) which is intended to promote fuel consumption testing of transit vehicles among European manufacturers and transit operators (UITP, 2004). The SORT methodology does not require collection of activity data, it proposes three standard cycles that represent urban, suburban, and intermediate transit driving conditions. The UITP document points out that the selection of a drive cycle should consider average route speed, average time spent at stops (idle), average distance between stops, and load, among others. Their methodology is based solely on average speed. Also, the document acknowledges that test results may differ from every-day fuel consumption due to factors such as driving style, number of passengers (weight), and topographic and climate conditions. 
Zhu et al. (2004) used a different method for developing a drive cycle, targeting Heavy-Duty Hybrid Electric Class 4-6 Vehicles. Their approach was to create a composite drive cycle based on existing drive cycles having the following constraints: must be an achievable speed-time trace for target applications, must represent the typical driving pattern of these applications, and is practical for testing and state-of-charge correction. These criteria were applied to numerous element and composite cycles and, ultimately, a new composite cycle was developed and selected: the Combined International Local and Commuter Cycle (CILCC). The CILCC is composed by four repeats of the International Local Cycle and one repeat of the Commuter Cycle.

Duoba et al. (2005) used yet another approach in a study that evaluated the effects of driving speed on fuel economy. They evaluated passenger cars with the UDDS (FTP-72) and HWFET cycles, and defined a scaling factor for cycle speed. The higher the scaling factor, the more aggressive the driving is, since much higher accelerations and decelerations are encountered. Their results show that, overall, more aggressive driving yields lower fuel economy for both hybrid and conventional vehicles.

Dembski et al., 2005, developed driving and duty cycles for refuse vehicles. They performed principal component analysis and k-means classification of micro-trips. In their analysis, micro-trips included idle segments; segment time and distance were used in the set of metrics. Table 2-5 lists the metrics calculated by Dembski et al., 2005, for the database. Distance traveled and sequence time resulted very influential for micro-trip classification, but they dod not necessarily serve to differentiate specific driving conditions, like suburban and highway segments.

Table 2-5 Cycle and Micro-trip Metrics Considered by Dembski et al., 2005

\begin{tabular}{l|c|l|c}
\hline \multicolumn{1}{c|}{ Metric } & Units & \multicolumn{1}{c}{ Metric } & Units \\
\hline Maximum Speed & $\mathrm{mph}$ & Average Grade & $\%$ \\
\hline Average Speed & $\mathrm{mph}$ & Mean Grade Climb & $\%$ \\
\hline Average Speed without Idle & $\mathrm{mph}$ & Mean Grade Descent & $\%$ \\
\hline Peak Acceleration & $\mathrm{ft} / \mathrm{s}^{2}$ & Percent Time Accelerating & $\%$ \\
\hline Peak Deceleration & $\mathrm{ft} / \mathrm{s}^{2}$ & Percent Distance Accelerating & $\%$ \\
\hline Average Acceleration $(*)$ & $\mathrm{ft} / \mathrm{s}^{2}$ & Percent Time Decelerating & $\%$ \\
\hline Average Deceleration $(*)$ & $\mathrm{ft} / \mathrm{s}^{2}$ & Percent Distance Decelerating & $\%$ \\
\hline Distance Traveled & $\mathrm{mi}$ & Percent Time Cruise & $\%$ \\
\hline Sequence Time & $\mathrm{s}$ & Percent Distance Cruise & $\%$ \\
\hline Time Vehicle Moving & $\mathrm{s}$ & Percent Time Grade Climb & $\%$ \\
\hline Time Vehicle Stopped & $\mathrm{s}$ & Percent Distance Grade Climb & $\%$ \\
\hline Average Distance Between Stops & $\mathrm{mi}$ & Percent Time Grade Descent & $\%$ \\
\hline Average Time of Stops & $\mathrm{s}$ & Percent Distance Grade Descent & $\%$ \\
\hline Maximum Grade Climb & $\%$ & Percent Time Grade Level & $\%$ \\
\hline Maximum Grade Descent & $\%$ & Percent Distance Grade Level & $\%$ \\
\hline
\end{tabular}

(*) Equation 2.22 (page 20) 


\subsubsection{Actual Driving Patterns}

The UITP collected over 6 million miles of data (10 million kilometers) from Voith DIWA transmissions used world-wide in transit vehicles (UITP, 2004). Characteristic values of average speed were 6-9 mph and 10-12 mph in inner city traffic of large and small cities, respectively, and 16$19 \mathrm{mph}$ in suburban traffic. Idle time was found to be as high as $45 \%$ for urban driving and $20 \%$ for suburban driving. The number of stops per distance traveled ranged from 10 stops per mile (6 stops per kilometer) in urban traffic to 3 stops per mile (2 stops per kilometer) in suburban traffic. It was found that the time spent in coasting varies between $20 \%$ and $45 \%$, but no correlation with average speed was observed.

On the other hand, GM Allison collected over 20 million fleet miles on their 2-Mode compound split parallel hybrid architecture installed in 40' and 60' transit buses, and in 45' coaches (Chiang, 2007). The GM Allison hybrid validation and field engineering team observed that the following factors would impact the hybrid bus fuel economy:

$>$ Duty cycle profile/service route statistics: average speed, stops per mile, maximum speed, driving idle time, and average acceleration,

$>$ Idle time,

$>$ Accessory usage,

$>$ Operator driving style,

$>$ Seasonal variation,

$>$ Engine baseline fuel consumption,

$>$ Engine exhaust after-treatment, and

$>$ Auxiliary brake/regenerative brake.

The information found in the literature regarding actual driving patterns is rather generic. As stated in Section 1.3, this dissertation addresses the subject by collecting and classifying transit bus data.

\subsubsection{Existing Duty Cycles}

This section presents statistics of a wide variety of drive cycles compiled by the author. Timespeed traces for selected cycles are also presented. Table 2-6 shows the most representative metrics of the compiled drive cycles, namely: distance, duration, average speed and average speed without idle segments, standard deviation of speed with and without idle, percent idle, number of stops per mile, average acceleration and deceleration, characteristic acceleration, aerodynamic speed, and kinetic intensity. A brief description of the cycles is also included. Data in Table 2-6 have been sorted 
according to average speed. Therefore, stop-and-go operation is at the top of the list while suburban and highway operation is at the bottom.

The first cycle to be discussed is the Central Business District (CBD), shown in Figure 2-3 (page 31). The CBD is a geometric or sawtooth type cycle. It was the test cycle used by the SAE J1376 recommended practice (Fuel Economy Measurement Test for Trucks and Buses, 1982). It consists of 14 repetitions of a basic cycle which exhibits acceleration, cruise, deceleration, and idle modes. The top speed is $20 \mathrm{mph}$ which may favor a particular gear and engine speed during the cruise mode. Early chassis-dynamometer emissions data is found primarily over the CBD cycle. Average speed is 12.92 $\mathrm{mph}$, idle is $17.5 \%$, maximum acceleration is $3.23 \mathrm{ft} / \mathrm{s}^{2}$, and kinetic intensity is $4.06 \mathrm{mi}^{-1}$.

The EPA Urban Dynamometer Driving Schedule (UDDS) for use in exhaust emissions tests is shown in Figure 2-4 (CFR 40: 86, App. I). The UDDS was designed to simulate gasoline-fueled heady-duty vehicle operation in urban areas. Percentage idle is $32.5 \%$, average speed is $18.9 \mathrm{mph}$, maximum acceleration is $6.42 \mathrm{ft} / \mathrm{s}^{2}$, and kinetic intensity is $0.61 \mathrm{mi}^{-1}$. The UDDS is also used as a preconditioning cycle for evaporative emissions tests.

The Orange County Transit Authority (OCTA) cycle (Wayne et al., 2004a) represents bus driving activity in Orange County, California. The OCTA cycle was developed by WVU in 2002 and has been adopted by SAE for emissions measurement of hybrid and conventional heavy-duty vehicles (SAE J2711, SAE International, 2002). It was shown that the OCTA cycle represents well the current average speed of the U.S. national bus fleet (U.S. FTA, 2007). Average speed is $12.33 \mathrm{mph}$, idle is $22.0 \%$, maximum acceleration is $5.95 \mathrm{ft} / \mathrm{s}^{2}$, and kinetic intensity is $3.59 \mathrm{mi}^{-1}$.

Another drive cycle that stands out is the King County Metro (KCM) cycle (Hayes et al., 2006). The KCM cycle, Figure 2-6, is the first cycle to include elevation information (in the form of grade). Grade was included in its simplest form: constant positive (uphill) and negative (downhill) values during three segments of the test. The cycle exhibits the characteristics of four different routes at King County Metro where grade is set to $3.5 \%$ (KCM1), 0\% (KCM2), 1.5\% (KCM3), and 5\% (KCM4). The KCM cycle includes sharp accelerations which may not be closely followed by all transit buses. Average speed is $23.43 \mathrm{mph}$, idle is $11.6 \%$, maximum acceleration is $14.18 \mathrm{ft} / \mathrm{s}^{2}$, and kinetic intensity is $0.99 \mathrm{mi}^{-1}$. The composite breakdown of the KCM cycle into the four sections is included in Table 2-6; average speed for the various sections is: 41.85, 19.61, 16.39, and $15.04 \mathrm{mph}$. 
Table 2-6 Properties of Drive Cycles and Routes

\begin{tabular}{|c|c|c|c|c|c|c|c|c|c|c|c|c|c|c|}
\hline Schedule & Description & $\begin{array}{l}\text { Distance } \\
\quad(\mathrm{mi})\end{array}$ & $\begin{array}{l}\text { Duration } \\
\quad(\mathrm{sec})\end{array}$ & $\underset{(\mathbf{m p h})}{\overline{\mathbf{U}}}$ & $\begin{array}{l}\overline{\mathbf{U}}_{\text {no idle }} \\
(\mathbf{m p h})\end{array}$ & $\underset{\text { Stdv. }}{\mathbf{U}} \underset{\text { (mph) }}{\text { St }}$ & $\begin{array}{c}\text { Stdv. } \\
\text { U } \mathbf{U}_{\text {no idle }} \\
(\mathbf{m p h})\end{array}$ & $\begin{array}{l}\text { Idle } \\
(\%)\end{array}$ & $\begin{array}{c}\text { Stops } \\
\text { per } \\
\text { mile }\end{array}$ & $\begin{array}{c}\bar{a}_{+} \\
\left(\mathbf{f t} / \mathbf{s}^{2}\right)\end{array}$ & $\begin{array}{c}\bar{a}_{-} \\
\left(\mathbf{f t} / \mathbf{s}^{2}\right)\end{array}$ & $\begin{array}{c}\tilde{a} \\
\left(\mathbf{f t} / \mathbf{s}^{2}\right)\end{array}$ & $\begin{array}{l}V_{\text {aero }} \\
(\mathbf{m p h})\end{array}$ & $\underset{\left(\mathbf{m i}^{-1}\right)}{k i}$ \\
\hline Idle & Cycle for idle emissions & 0.00 & V.D. & 0.00 & - & 0.00 & - & 100.0 & - & - & - & - & - & - \\
\hline HHDDT-Creep & Heavy Heavy-Duty Diesel Truck & 0.12 & 253 & 1.77 & 3.02 & 2.03 & 1.81 & 41.5 & 24.18 & 0.62 & 0.42 & 0.21 & 4.58 & 24.93 \\
\hline Yard & Yard operation of heavy-duty trucks & 1.08 & 1,164 & 3.34 & 6.19 & 4.66 & 4.75 & 46.0 & 12.04 & 0.91 & 1.18 & 0.40 & 10.70 & 8.65 \\
\hline New York Bus & $\begin{array}{l}\text { Very low speed bus operation in New } \\
\text { York city }\end{array}$ & 0.61 & 600 & 3.69 & 10.58 & 6.46 & 6.86 & 65.2 & 17.91 & 3.71 & 2.23 & 1.21 & 16.61 & 10.79 \\
\hline MiamiD & Miami Dade Transit bus cycle & 2.62 & 1,431 & 6.58 & 13.89 & 9.32 & 9.04 & 52.6 & 6.50 & 2.23 & 2.64 & 1.13 & 21.14 & 6.23 \\
\hline Paris & ADEME-RATP Paris Bus Cycle & 3.51 & 1,910 & 6.62 & 9.89 & 7.28 & 6.84 & 33.1 & 13.10 & 1.75 & 1.85 & 0.83 & 16.07 & 7.88 \\
\hline Manhattan & Low speed bus operation & 2.06 & 1,089 & 6.83 & 10.65 & 7.32 & 6.54 & 35.9 & 9.69 & 1.78 & 2.31 & 0.92 & 15.76 & 9.14 \\
\hline MX1 & Mexico City Schedule Mode 1 & 1.98 & 1,000 & 7.12 & 9.35 & 6.91 & 6.47 & 23.9 & 7.08 & 1.31 & 1.26 & 0.64 & 15.56 & 6.52 \\
\hline SORT1* (1) & $\begin{array}{l}\text { European geometric route for urban } \\
\text { traffic }\end{array}$ & 0.32 & 154 & 7.55 & 11.99 & 7.96 & 6.87 & 37.0 & 9.29 & 2.28 & 2.41 & 0.71 & 16.86 & 6.10 \\
\hline WMATA & $\begin{array}{l}\text { Washington Metropolitan Area Transit } \\
\text { Authority Bus Cycle }\end{array}$ & 4.26 & 1,839 & 8.34 & 13.56 & 10.32 & 10.13 & 38.5 & 6.34 & 1.51 & 1.80 & 0.77 & 23.24 & 3.50 \\
\hline WVU City & City driving cycle for heavy trucks & 3.39 & 1,430 & 8.53 & 12.38 & 10.21 & 10.18 & 31.0 & 6.19 & 1.01 & 1.25 & 0.51 & 22.66 & 2.44 \\
\hline New York Composite & Heavy-duty vehicles, New York city & 2.51 & 1,029 & 8.77 & 12.87 & 9.44 & 8.83 & 31.9 & 7.58 & 1.61 & 1.82 & 0.77 & 20.70 & 4.42 \\
\hline Nuremberg & Nuremberg Bus Route 36 & 2.67 & 1,084 & 8.86 & 12.40 & 8.90 & 8.18 & 28.5 & 8.99 & 1.88 & 1.79 & 0.80 & 19.41 & 5.20 \\
\hline CBD Truck & Central Business District & 2.18 & 850 & 9.24 & 11.18 & 7.08 & 6.24 & 17.3 & 6.41 & 0.96 & 1.85 & 0.52 & 15.44 & 5.38 \\
\hline Houston Bus & $\begin{array}{l}\text { Metropolitan Transit Authority of Harris } \\
\text { County, Houston, TX }\end{array}$ & 5.54 & 1,800 & 11.07 & 17.80 & 11.72 & 10.05 & 37.8 & 5.96 & 1.44 & 1.60 & 0.57 & 24.44 & 2.33 \\
\hline MCS & Mexico City Bus Schedule & 9.26 & 3,000 & 11.11 & 16.12 & 12.01 & 11.34 & 31.1 & 4.10 & 1.59 & 1.69 & 0.73 & 26.17 & 2.62 \\
\hline SORT2* (1) & $\begin{array}{l}\text { European geometric route for mixed } \\
\text { traffic }\end{array}$ & 0.57 & 184 & 11.18 & 16.46 & 11.30 & 10.04 & 32.1 & 5.25 & 2.00 & 2.45 & 0.62 & 24.07 & 2.62 \\
\hline ECE 15 & European urban drive cycle & 0.62 & 195 & 11.40 & 16.47 & 10.58 & 8.84 & 30.8 & 4.86 & 2.10 & 2.46 & 0.48 & 22.38 & 2.34 \\
\hline Liberty & WCDOT Bus Cycle & 6.30 & 1,882 & 12.05 & 18.10 & 13.83 & 13.33 & 33.4 & 3.97 & 1.76 & 2.16 & 0.81 & 30.77 & 2.10 \\
\hline International Local & $\begin{array}{l}\text { City-suburban drive cycle, International } \\
\text { Truck \& Engine Corporation }\end{array}$ & 2.55 & 755 & 12.18 & 14.81 & 9.74 & 8.75 & 17.7 & 2.35 & 1.92 & 1.94 & 0.28 & 21.75 & 1.44 \\
\hline OCTA & $\begin{array}{l}\text { Orange County Transit Authority Bus } \\
\text { Cycle }\end{array}$ & 6.54 & 1,909 & 12.33 & 15.35 & 10.28 & 9.23 & 19.6 & 4.74 & 1.49 & 2.09 & 0.71 & 22.10 & 3.59 \\
\hline MX2 & Mexico City Schedule Mode 2 & 3.55 & 1,000 & 12.78 & 17.77 & 12.66 & 11.58 & 28.1 & 2.82 & 1.32 & 1.40 & 0.56 & 27.31 & 1.84 \\
\hline CBD & Central Business District Cycle & 2.01 & 560 & 12.92 & 15.66 & 8.19 & 6.18 & 17.5 & 6.97 & 2.67 & 5.87 & 0.57 & 18.52 & 4.06 \\
\hline MX3 & Mexico City Schedule Mode 3 & 3.73 & 1,000 & 13.44 & 22.86 & 14.17 & 11.24 & 41.2 & 3.75 & 2.22 & 2.72 & 0.94 & 29.34 & 2.67 \\
\hline
\end{tabular}




\begin{tabular}{|c|c|c|c|c|c|c|c|c|c|c|c|c|c|c|}
\hline Schedule & Description & $\begin{array}{l}\text { Distance } \\
(\mathbf{m i})\end{array}$ & $\begin{array}{l}\text { Duration } \\
\quad(\mathrm{sec})\end{array}$ & $\underset{(\mathbf{m p h})}{\overline{\mathbf{U}}}$ & $\begin{array}{l}\overline{\mathbf{U}}_{\text {no idle }} \\
\text { (mph) }\end{array}$ & $\begin{array}{l}\text { Stdv. } \\
\text { U } \\
(\mathbf{m p h})\end{array}$ & $\begin{array}{l}\text { Stdv. } \\
\mathbf{U}_{\text {no idle }} \\
\text { (mph) }\end{array}$ & $\begin{array}{l}\text { Idle } \\
(\%)\end{array}$ & $\begin{array}{l}\text { Stops } \\
\text { per } \\
\text { mile }\end{array}$ & $\begin{array}{c}\bar{a}_{+} \\
\left(\mathbf{f t} / \mathbf{s}^{2}\right)\end{array}$ & $\begin{array}{c}\bar{a}_{-} \\
\left(\mathbf{f t} / \mathrm{s}^{2}\right)\end{array}$ & $\underset{\left(\mathbf{f t} / \mathrm{s}^{2}\right)}{\tilde{a}}$ & $\begin{array}{c}V_{\text {aero }} \\
(\mathbf{m p h})\end{array}$ & $\begin{array}{c}k i \\
\left(\mathbf{m i}^{-1}\right)\end{array}$ \\
\hline CILCC & $\begin{array}{l}\text { Composite International Truck Local } \\
\text { Cycle and Commuter }\end{array}$ & 12.32 & 3,192 & 13.89 & 16.69 & 12.41 & 11.76 & 16.8 & 2.03 & 1.77 & 2.02 & 0.28 & 28.69 & 0.84 \\
\hline Route 22 & Bus route to BOS Airport, MA & 2.05 & 530 & 13.91 & 19.62 & 12.49 & 10.39 & 29.1 & 2.93 & 1.66 & 2.12 & 0.32 & 26.18 & 1.15 \\
\hline Braunschweig & Braunschweig City Bus Cycle & 6.74 & 1,740 & 13.95 & 18.49 & 11.34 & 9.30 & 24.5 & 4.30 & 1.83 & 2.42 & 0.72 & 24.19 & 3.01 \\
\hline CSHVC & City Suburban Heavy Vehicle Cycle & 6.68 & 1,700 & 14.15 & 18.44 & 13.06 & 11.96 & 23.3 & 2.54 & 1.32 & 1.60 & 0.56 & 27.72 & 1.79 \\
\hline $\operatorname{CSHVR}^{(1)}$ & $\begin{array}{l}\text { City Suburban Heavy-Duty Vehicle } \\
\text { Route }\end{array}$ & 6.68 & V.D. & V.D. & V.D. & V.D. & V.D. & V.D. & 2.54 & V.D. & V.D. & V.D. & V.D. & V.D. \\
\hline ETC-Urban & European Transient Cycle, Segment 1 & 2.37 & 600 & 14.21 & 15.87 & 8.46 & 7.31 & 10.5 & 1.69 & 1.00 & 1.18 & 0.44 & 20.03 & 2.69 \\
\hline BEELINE & WCDOT Bus Cycle & 6.80 & 1,701 & 14.39 & 19.44 & 14.75 & 14.00 & 26.0 & 3.68 & 1.90 & 2.37 & 0.88 & 32.05 & 2.09 \\
\hline KCM 4 & Section 4 of KCM Cycle, Rte-106 & 1.16 & 278 & 15.04 & 19.72 & 10.75 & 7.68 & 23.7 & 3.44 & 1.78 & 2.07 & 1.07 & 23.46 & 4.75 \\
\hline $\begin{array}{l}\text { HHDDT-Trans } \\
\text { (Trans3) }\end{array}$ & Heavy Heavy-Duty Diesel Truck & 2.85 & 668 & 15.37 & 18.20 & 13.39 & 12.68 & 15.6 & 1.75 & 1.02 & 1.37 & 0.50 & 29.57 & 1.40 \\
\hline SORT3* (1) & $\begin{array}{l}\text { European geometric route for suburban } \\
\text { traffic }\end{array}$ & 0.90 & 206 & 15.75 & 19.19 & 12.48 & 11.12 & 18.0 & 3.33 & 1.75 & 2.50 & 0.61 & 27.11 & 2.04 \\
\hline FTP-72 Phase $2 * *$ & Last $864 \mathrm{sec}$ of the FTP-72 & 3.86 & 864 & 16.08 & 19.46 & 10.69 & 8.51 & 17.4 & 3.11 & 1.62 & 1.91 & 0.58 & 23.91 & 2.51 \\
\hline KCM 3 & Section 3 of KCM Cycle, Rte-120 & 3.30 & 725 & 16.39 & 20.84 & 12.81 & 10.76 & 21.4 & 3.64 & 1.75 & 2.89 & 0.89 & 27.59 & 2.87 \\
\hline Route 77 & Bus route to BOS Airport, MA & 4.01 & 860 & 16.77 & 19.82 & 13.58 & 12.56 & 15.4 & 3.74 & 1.71 & 2.35 & 0.51 & 30.06 & 1.39 \\
\hline JE05 & Japanese 2005 heavy-duty vehicle cycle & 8.63 & 1,829 & 16.99 & 22.47 & 15.94 & 14.59 & 24.4 & 1.62 & 1.03 & 1.08 & 0.40 & 34.98 & 0.80 \\
\hline BAC & Business Arterial Commuter & 14.04 & 2,830 & 17.85 & 23.27 & 16.21 & 14.71 & 23.3 & 3.63 & 2.26 & 5.92 & 0.47 & 36.12 & 0.89 \\
\hline UDDS (Test-D) & $\begin{array}{l}\text { EPA Heavy-Duty Urban Dynamometer } \\
\text { Driving Schedule }\end{array}$ & 5.55 & 1,060 & 18.85 & 27.91 & 19.84 & 18.16 & 32.5 & 2.52 & 1.62 & 1.89 & 0.45 & 42.52 & 0.61 \\
\hline FTP-72 (UDDS)** & $\begin{array}{l}\text { EPA emissions certification cycle for } \\
\text { light-duty vehicles and light-duty trucks. } \\
\text { Also LA-4 }\end{array}$ & 7.45 & 1,369 & 19.59 & 23.84 & 14.68 & 12.68 & 17.8 & 2.28 & 1.66 & 1.90 & 0.57 & 33.12 & 1.28 \\
\hline KCM 2 & Section 2 of KCM Cycle, Rte-174 & 2.51 & 461 & 19.61 & 23.48 & 13.39 & 11.12 & 16.5 & 2.79 & 1.65 & 3.05 & 0.87 & 30.23 & 2.33 \\
\hline WVU 5 Peak & 5-Speed Truck Cycle & 5.00 & 900 & 20.02 & 24.25 & 13.44 & 10.78 & 17.4 & 1.00 & 1.05 & 1.90 & 0.19 & 30.26 & 0.52 \\
\hline WVU 5 Mile ${ }^{(1)}$ & Route based on WVU 5 Peak cycle & 5.00 & V.D. & V.D. & V.D. & V.D. & V.D. & V.D. & 1.00 & V.D. & V.D. & V.D. & V.D. & V.D. \\
\hline FTP-75** & EPA light-duty cert. cycle & 11.04 & 1,874 & 21.21 & 25.88 & 15.93 & 13.74 & 18.0 & 1.99 & 1.68 & 1.89 & 0.57 & 35.78 & 1.09 \\
\hline $\mathrm{SC03} * *$ & EPA SFTP, air conditioning & 3.58 & 596 & 21.62 & 26.35 & 15.27 & 12.62 & 18.0 & 1.68 & 1.65 & 1.98 & 0.68 & 34.13 & 1.43 \\
\hline KCM & King County Metro Bus Cycle & 12.78 & 1,964 & 23.43 & 28.45 & 18.09 & 15.96 & 17.6 & 1.88 & 1.61 & 2.31 & 0.65 & 40.18 & 0.99 \\
\hline KCM-no grade & Removing grade & 12.78 & 1,964 & 23.43 & 28.45 & 18.09 & 15.96 & 17.6 & 1.88 & 1.61 & 2.31 & 0.54 & 40.18 & 0.83 \\
\hline FTP-72 Phase 1** & First $505 \mathrm{sec}$ of the FTP-72 & 3.59 & 505 & 25.60 & 31.45 & 18.21 & 14.94 & 18.6 & 1.39 & 1.73 & 1.88 & 0.56 & 40.76 & 0.83 \\
\hline Arterial & Arterial segment of BAC & 2.00 & 270 & 26.68 & 29.17 & 14.54 & 12.58 & 8.5 & 2.00 & 1.97 & 6.02 & 0.65 & 35.56 & 1.26 \\
\hline
\end{tabular}




\begin{tabular}{|c|c|c|c|c|c|c|c|c|c|c|c|c|c|c|}
\hline Schedule & Description & $\begin{array}{l}\text { Distance } \\
(\mathbf{m i})\end{array}$ & $\begin{array}{l}\text { Duration } \\
\text { (sec) }\end{array}$ & $\underset{(\mathbf{m p h})}{\overline{\mathbf{U}}}$ & $\begin{array}{l}\overline{\mathbf{U}}_{\text {no idle }} \\
(\mathbf{m p h})\end{array}$ & $\begin{array}{c}\text { Stdv. } \\
\text { U } \\
(\mathbf{m p h})\end{array}$ & $\begin{array}{l}\text { Stdv. } \\
\mathbf{U}_{\text {no idle }} \\
\text { (mph) }\end{array}$ & $\begin{array}{l}\text { Idle } \\
(\%)\end{array}$ & $\begin{array}{c}\text { Stops } \\
\text { per } \\
\text { mile }\end{array}$ & $\begin{array}{c}\bar{a}_{+} \\
\left(\mathbf{f t} / \mathrm{s}^{2}\right)\end{array}$ & $\begin{array}{c}\bar{a}_{-} \\
\left(\mathbf{f t} / \mathbf{s}^{2}\right)\end{array}$ & $\underset{\left(\mathbf{f t} / \mathbf{s}^{2}\right)}{\tilde{a}}$ & $\begin{array}{c}V_{\text {aero }} \\
(\mathbf{m p h})\end{array}$ & $\begin{array}{c}k i \\
\left(\mathbf{m i}^{-1}\right)\end{array}$ \\
\hline HHDDT & Heavy Heavy-Duty Diesel Truck & 26.05 & 3,004 & 31.21 & 35.61 & 23.60 & 21.88 & 12.4 & 0.50 & 0.52 & 0.58 & 0.18 & 50.70 & 0.17 \\
\hline Freeway & Freeway operation of heavy-duty trucks & 15.55 & 1,640 & 34.13 & 37.97 & 21.75 & 19.50 & 10.1 & 0.32 & 0.67 & 0.70 & 0.23 & 49.64 & 0.22 \\
\hline ETC (FIGE) & $\begin{array}{l}\text { European cycle for emission certification } \\
\text { of heavy-duty diesel vehicles }\end{array}$ & 18.28 & 1,800 & 36.56 & 37.89 & 17.81 & 16.69 & 3.5 & 0.27 & 0.68 & 0.78 & 0.22 & 46.75 & 0.25 \\
\hline EUDC Low Power & EUDC for low power vehicles & 4.11 & 400 & 36.96 & 41.07 & 16.98 & 12.30 & 10.0 & 0.24 & 1.39 & 3.34 & 0.20 & 45.88 & 0.23 \\
\hline EUDC & Extra Urban Driving Cycle & 4.32 & 400 & 38.89 & 43.22 & 19.54 & 15.40 & 10.0 & 0.23 & 1.24 & 3.04 & 0.31 & 50.77 & 0.29 \\
\hline HHDDT-Cruise & Heavy Heavy-Duty Diesel Truck & 23.07 & 2,083 & 39.88 & 43.28 & 22.01 & 19.45 & 7.9 & 0.26 & 0.38 & 0.41 & 0.14 & 52.86 & 0.12 \\
\hline KCM 1 & Section 1 of KCM Cycle, I-5 & 5.81 & 500 & 41.85 & 46.40 & 18.84 & 13.50 & 9.8 & 0.17 & 0.96 & 0.83 & 0.34 & 51.12 & 0.32 \\
\hline $\begin{array}{l}\text { ETC-Rural and } \\
\text { Motorway }\end{array}$ & $\begin{array}{l}\text { Rural and Motorway sections of the ETC } \\
\text { Cycle }\end{array}$ & 15.91 & 1,329 & 43.11 & 47.74 & 16.09 & 8.08 & 9.7 & 0.06 & 0.52 & 0.58 & 0.19 & 49.51 & 0.19 \\
\hline Commuter & Commuter segment of BAC & 4.00 & 310 & 46.49 & 49.53 & 16.57 & 11.90 & 6.1 & 0.25 & 0.93 & 6.21 & 0.15 & 52.84 & 0.14 \\
\hline US06** & EPA SFTP, aggressive driving & 8.01 & 600 & 48.05 & 51.57 & 24.50 & 21.50 & 6.8 & 0.62 & 2.21 & 2.39 & 0.69 & 62.07 & 0.44 \\
\hline \multirow[t]{3}{*}{ HWFET** } & EPA highway fuel economy & 10.26 & 765 & 48.27 & 48.52 & 10.09 & 9.49 & 0.5 & 0.10 & 0.64 & 0.72 & 0.23 & 50.88 & 0.22 \\
\hline & Minimum & 0.12 & 154 & 1.77 & 3.02 & 2.03 & 1.81 & 0.5 & 0.06 & 0.38 & 0.41 & 0.14 & 4.58 & 0.12 \\
\hline & Maximum & 26.05 & 3,192 & 48.27 & 51.57 & 24.50 & 21.88 & 65.2 & 24.18 & 3.71 & 6.21 & 1.21 & 62.07 & 24.93 \\
\hline
\end{tabular}

* $\quad$ Estimates based on minimum acceleration and deceleration rates. Actual time based metrics will vary.

** Drive cycle for passenger vehicles and light-duty trucks.

V.D. Vehicle and test weight dependent.

(1) Test Route. 


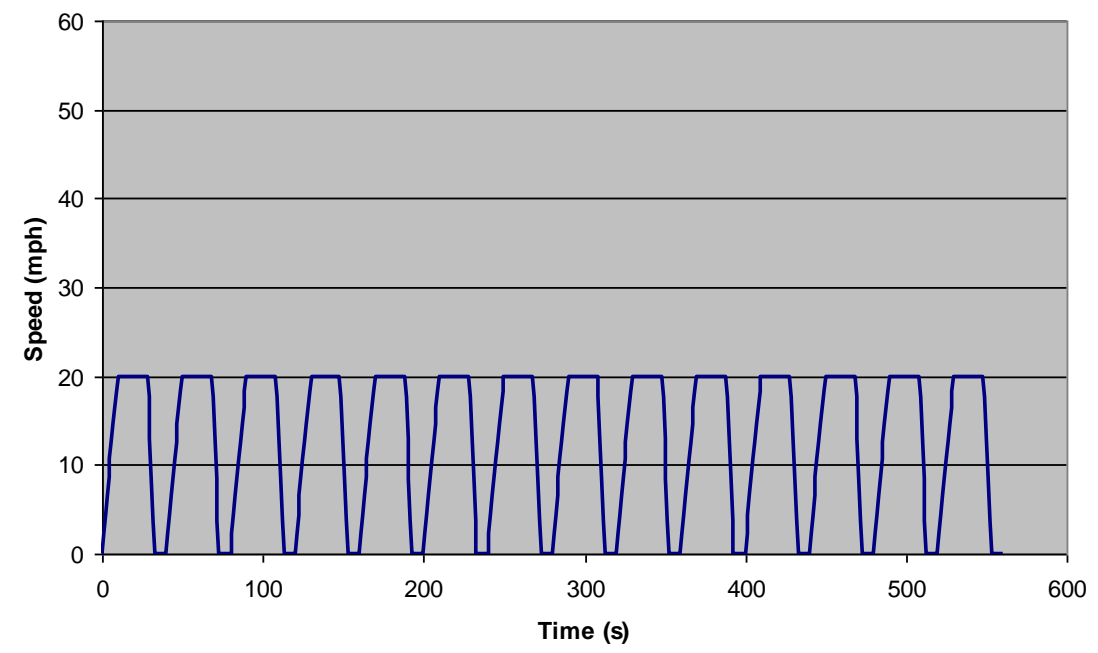

Figure 2-3 Central Business District (CBD) Cycle

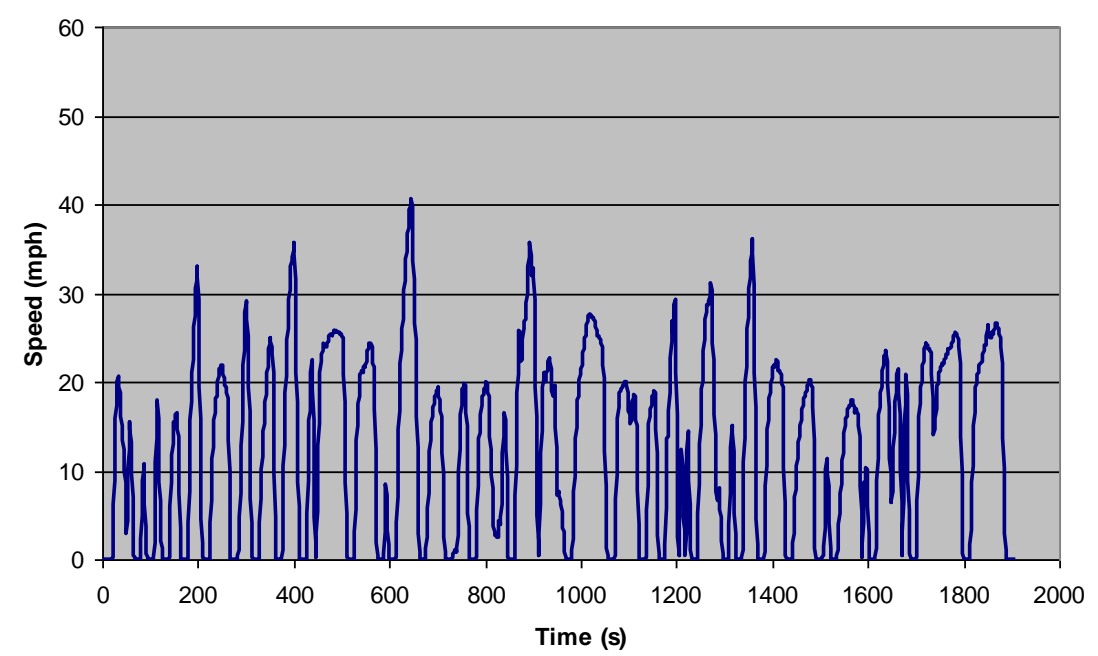

Figure 2-5 Orange County Transit Authority (OCTA) Cycle

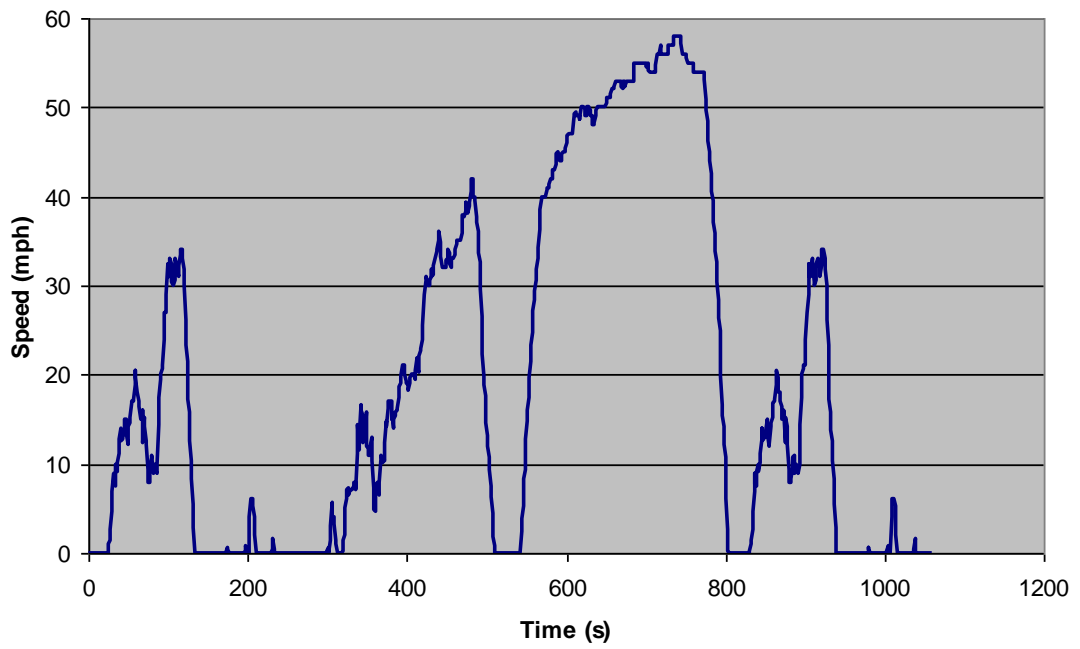

Figure 2-4 Urban Dynamometer Driving Schedule (UDDS)

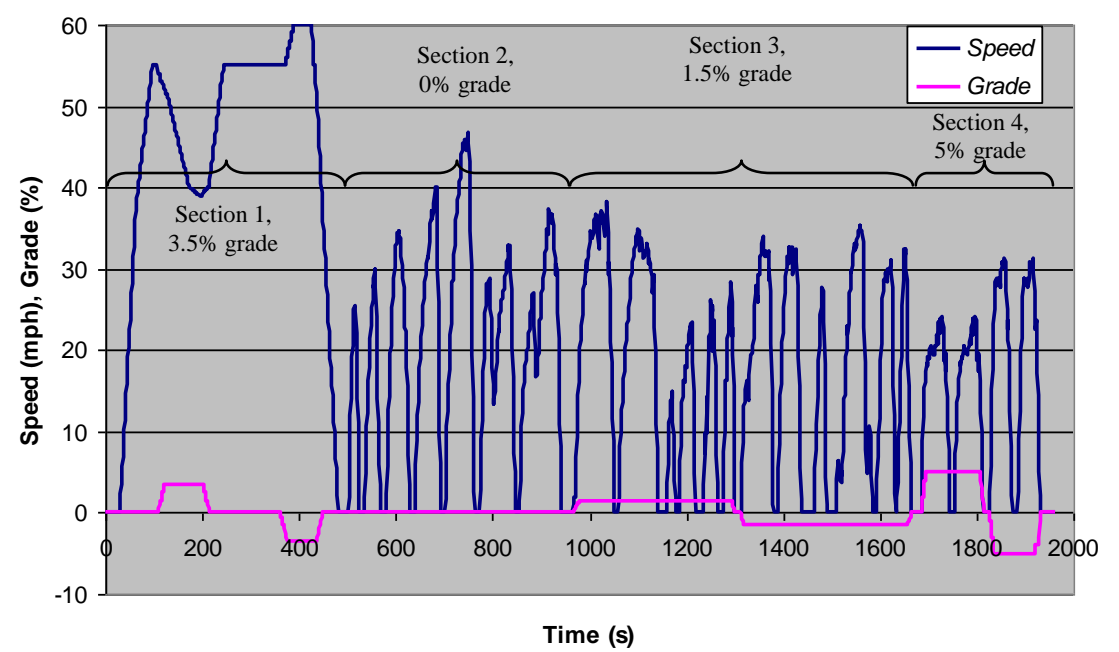

Figure 2-6 King County Metro (KCM) Cycle 


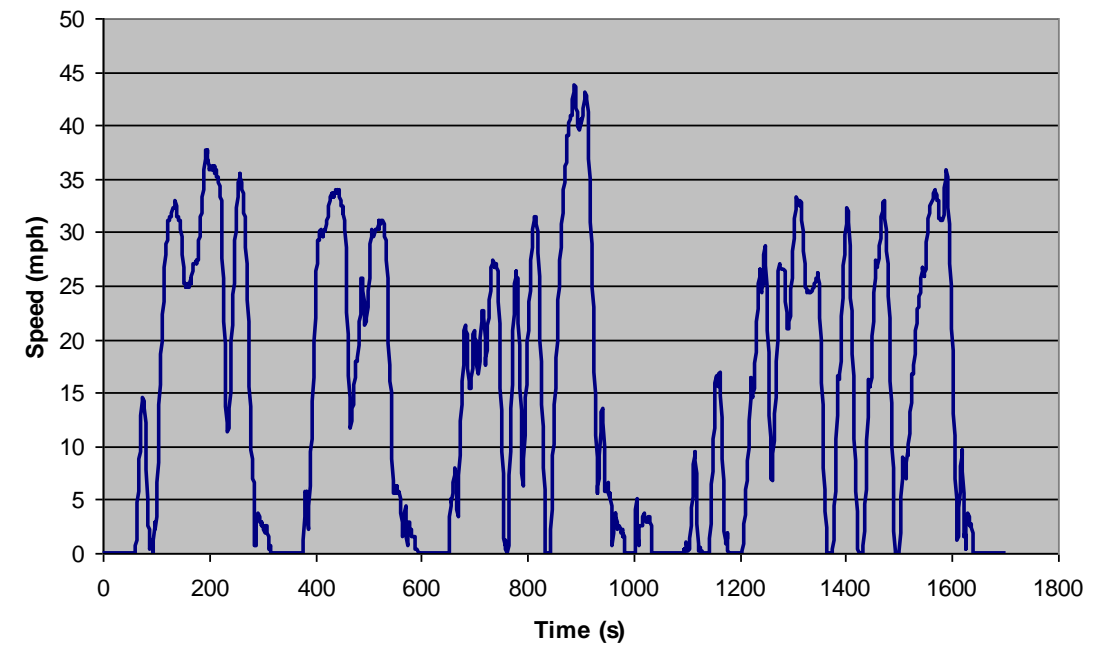

Figure 2-7 City Suburban Heavy Vehicle Cycle (CSHVC)

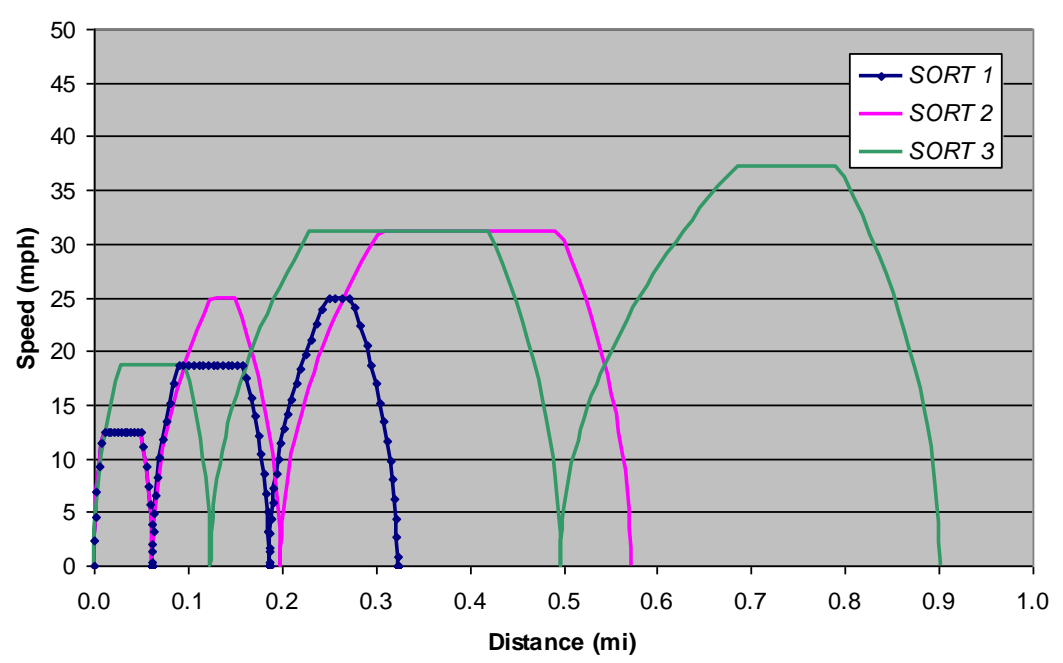

Figure 2-9 SORT Routes. Acceleration curves based on minimum acceleration

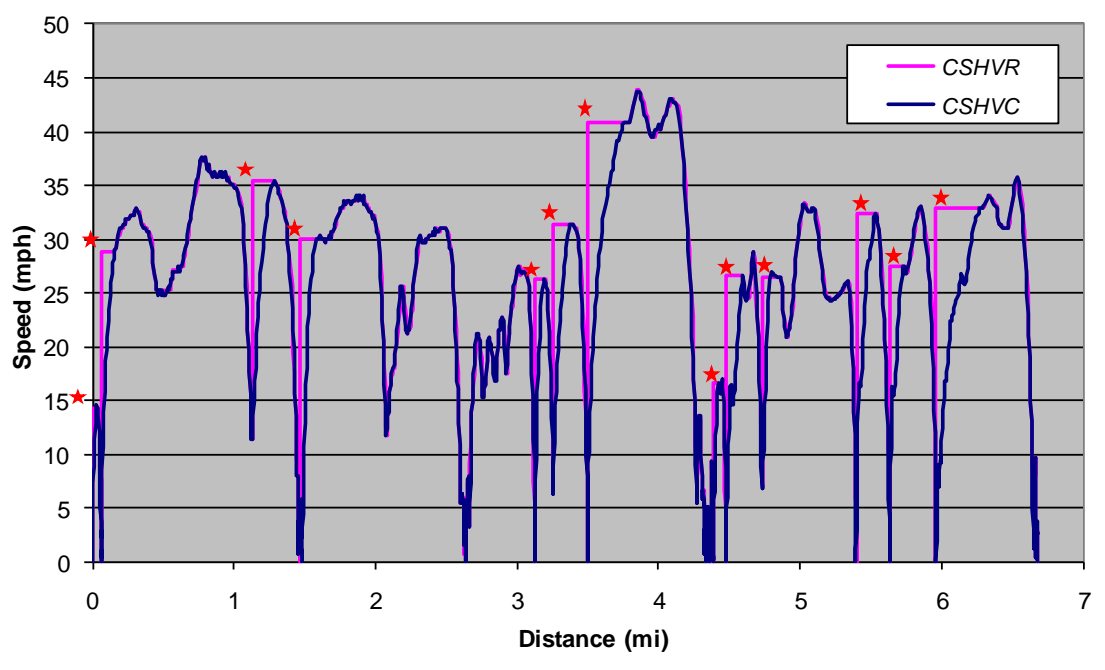

Figure 2-8 Distance-Speed target for the City Suburban Heavy Vehicle Cycle and Route. Stars denote free accelerations

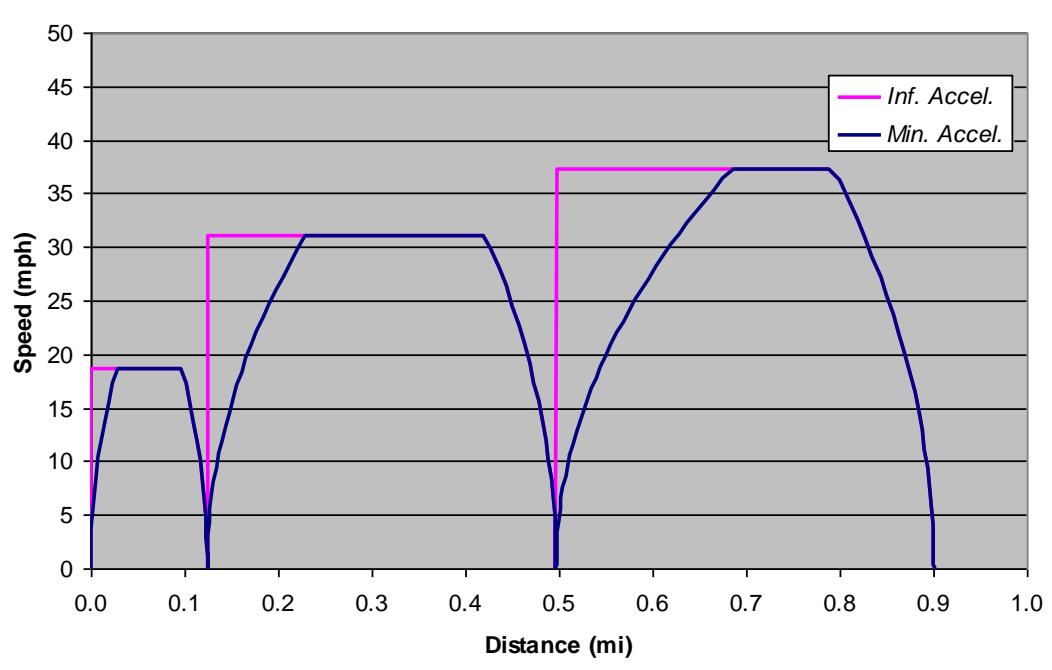

Figure 2-10 SORT 3 Route with Infinite and Minimum Acceleration 
Clark et at. (1999) developed the City Suburban Heavy Vehicle Cycle (CSHVC), WVU City, Yard, and Freeway cycles. The cycles were created by WVU for emissions characterization of class 8 heavyduty trucks. The CSHVC is accompanied by the corresponding Test Route (CSHVR). The TimeSpeed trace for the CSHVC is shown in Figure 2-7. The CSHVC represents the statistics of tractortrailer data collected under yard, city, suburban, and interstate/freeway operation (Nine et al., 1999). In the CSHVR, Figure 2-8, the truck is required to employ maximum acceleration at 13 points during the cycle. Depending on the power-to-weight ratio of the vehicle the time scale is adjusted; with more powerful vehicles completing the route in less time than low-powered vehicles.

UITP (2004) designed the SORT Routes for fuel economy measurement of transit buses. The SORT Routes are composed of geometrical modules (base cycles), which are repeated several times both to provide accuracy and to obtain the desired average speed. Each module is composed of three sections spaced by traffic stop segments. Passenger boarding stops are included in between modules. Following the ideas of the Central Business District (CBD) and Business Arterial Commuter (BAC) cycles, a section, which has a trapezoidal shape, consists of an acceleration ramp, a constant speed cruise, and a deceleration ramp.

Based upon observed typical driving conditions, SORT defined three types of operation: urban (SORT 1), suburban (SORT 3), and mixed (SORT 2). Distance-Speed targets are presented in Figure 2-9. Five trapezoidal micro-trips, characterized by their maximum (cruise) speed, were defined: 20 $\mathrm{km} / \mathrm{hr}$ (12.43 mph), $30 \mathrm{~km} / \mathrm{hr}(18.64 \mathrm{mph}), 40 \mathrm{~km} / \mathrm{hr}(24.85 \mathrm{mph}), 50 \mathrm{~km} / \mathrm{hr}(31.07 \mathrm{mph})$, and 60 $\mathrm{km} / \mathrm{hr}(37.28 \mathrm{mph})$. Minimum allowed acceleration rates range from $1.03 \mathrm{~m} / \mathrm{s}^{2}\left(3.38 \mathrm{ft} / \mathrm{s}^{2}\right)$ for the low speed urban cycle to $0.77 \mathrm{~m} / \mathrm{s}^{2}\left(2.53 \mathrm{ft} / \mathrm{s}^{2}\right)$ for the suburban cycle. All the deceleration ramps are at 0.8 $\mathrm{m} / \mathrm{s}^{2}\left(2.63 \mathrm{ft} / \mathrm{s}^{2}\right)$. The acceleration curves of Figure 2-9 are based on the minimum acceleration rates described above, but in reality, these are free accelerations and the discussion for the CSHVR above also applies.

A route, distance-speed based, may be translated to a time basis. Starting from the basic kinematic relationships: $V=d s / d t$ and $a=d V / d t$, distance can be expressed as $s=\int V d t$. Integrating over one step, $j-1$ to $j$, the elapsed time is determined, after some algebra, by

Equation 2.30 Conversion of Test Route to Time Basis

$$
\Delta t=\frac{\Delta s}{V_{j-1}+\Delta V / 2},
$$

where $\Delta t=t_{j}-t_{j-1}, \Delta s=s_{j}-s_{j-1}$, and $\Delta V=V_{j}-V_{j-1}$. Idle segments must be added in between stops since their information is lost in the distance-speed basis. The acceleration curves of Figure 2-9 are linear ramps in the time frame with slope equal to the value set for minimum acceleration. 
Figure 2-10 shows the difference between an ideal vehicle, with infinite acceleration, and a vehicle that follows the route putting up the minimum required acceleration. All real vehicles will fall within the two curves.

\subsubsection{Federal Test Procedure for Light-Duty Vehicles and Light-Duty Trucks}

The FTP-72 driving cycle (CFR 40: 86, App. I), also called Urban Dynamometer Driving Schedule (UDDS) or LA-4 cycle, reproduces urban driving as encountered in Los Angles, CA, in the late 1960s. This schedule serves as the basis for certification of light-duty vehicles and light-duty trucks. Such certification consists of three sections: $i$ ) Cold Start: collects data from the first 505 seconds of the cycle (FTP-72 Phase 1 in Table 2-6); ii) Stabilized or Transient: collects data from the rest of the cycle (FTP-72 Phase 2); and iii) Hot Start: repeats Phase 1 after a 10 min soak. The three sections described comprise the FTP-75 schedule which is the actual certification procedure. Emissions expressed in $\mathrm{g} / \mathrm{mi}$ from the three sections are weighted; cold start and hot start are weighed 0.43/0.57 respectively. This result from Phase 1 is averaged with the result from Phase 2 to obtain the composite FTP-75 emissions.

In addition to the FTP-75, light-duty vehicles are tested over the HWFET (highway driving). Fuel economy results from these two tests are combined to determine the vehicle's fuel economy to be used for Corporate Average Fuel Economy (CAFE). The corresponding CAFE combined fuel economy is determined by (Sovran and Blaser, 2006)

Equation 2.31 CAFE Combined Fuel Economy

$$
m p g_{\text {combined }}=\frac{1}{\frac{0.55}{m p g_{U D D S}}+\frac{0.45}{m p g_{H W F E T}}}
$$

The Supplemental Federal Test Procedures (SFTP) are the US06 (aggressive, high speed driving) and the SC03 (air conditioning) driving schedules. SFTP emissions are calculated as a weighted average: $0.35 \times$ FTP-75 + $0.37 \times \mathrm{SC} 03+0.28 \times \mathrm{US} 06$.

\subsection{Hybrid Architectures}

It has been demonstrated (Guzzella and Sciarretta, 2007) that hybrid architectures give the most benefits when they are able to bring the engine to its maximum efficiency (i.e. high torques and speeds) during most of the different driving conditions and events. Moreover, maximization of the 
overall energy conversion efficiency, which includes transmission, power-train, and electrical losses, is a must.

The leading architectures for transit buses are the series and the 2-Mode EVT (parallel). Two major manufacturers of series hybrid powertrains are BAE Systems and ISE Corporation. The 2-Mode EVT architecture is manufactured by Allison Transmission.

Epicyclic or planetary gear trains are the center of the Toyota power split and GM 2-Mode electrically variable transmissions (EVT) for hybrid vehicles. They have been widely used in automatic transmissions for many years; specifically, the planetary trains used in automotive automatic transmissions are of the compound type. A compound train is one in which at least one shaft holds more than one gear. In such trains, the gears are always in mesh and the different ratios, as well as reverse, are obtained by engaging and disengaging brakes on different members of the train (Norton, 2004).

All hybrid architectures can take advantage of electrifying accessories such as power steering, air conditioning, power brakes (vacuum), among others. Next, several hybrid architectures available on the market are discussed, namely: Series, Parallel, Power Split, 2-Mode, and 2-Mode with fixed gear ratios.

\subsubsection{Series Hybrid}

In the series hybrid architecture, the combustion engine is mechanically decoupled from the wheels which are driven by an electric motor. The engine drives an electric generator which can either send the current to the battery or directly to the motor. Given that the motor is the element mechanically driving the vehicle, it must be sized to provide the maximum power requirements, thus the motor is large. Engine and generator are normally sized to provide the average driving power.

Two generic control strategies have been utilized: $i$ ) Load following, where the generated power is mostly sent to the motor and the battery is used to help bring the engine to its optimal operational point, and ii) Charging based, where the engine is on when the battery depletes to a specified level and goes off when a desired maximum battery state of charge (SOC) is reached.

\subsubsection{Parallel Hybrid}

In parallel hybrid architecture, the IC engine is mechanically connected to the wheels and the electric motor is linked to the mechanical path pre or post transmission. There may also be a gearing between the motor and the main shaft. The motor can boost the engine and be used as a generator for

regenerative braking purposes; it also helps in altering engine load to improve efficiency. When 
compared with series hybrids, motors in the parallel hybrid tend to have a smaller size since the vehicle could basically be propelled with the engine alone.

\subsubsection{1-Mode EVT or Input Split}

The input split (or power split), which has one planetary gearset, is suitable for small to mid-size vehicles because the constant presence of the electrical path requires very powerful motors in larger vehicles. This condition can be somewhat relaxed with the use of an output reduction. Such additional gearing can be attached to the motor, reducing its torque requirements. The power split has one mechanical point at which the generator's speed reaches zero and a pure mechanical path is achieved; the pitfall is that such mechanical point is located beyond the operational envelope of the powertrain.

\subsubsection{The 2-Mode EVT}

When compared with conventional transit buses, the GM Allison Hybrid $\mathrm{E}^{\mathrm{P}} 40$ and $\mathrm{E}^{\mathrm{P}} 50$ Systems ${ }^{\mathrm{TM}}$ (2-Mode EVT) have achieved $20 \%$ to $40 \%$ improvement on fuel economy (Chiang, 2007). The advantage of these systems is favored at low speed (stop-and-go type operation), when the hybrid control system has more choices to efficiently meet the torque demand and has more opportunity to gain energy with regenerative braking (Chiang, 2007).

The transmission has two mechanical points located at $25 \mathrm{mph}$ and $62 \mathrm{mph}$ (AutoSpeed.com, 2006); at these speeds the transmission produces a direct drive, just like a conventional transmission. A 40' urban bus equipped with an $\mathrm{E}^{\mathrm{P}}$ System accelerates, at 35,000 lb, from 0 to $30 \mathrm{mph}$ in 10 seconds (Allison Transmission $\mathrm{E}^{\mathrm{V}}$ Drive website).

Figure 2-11 shows a schematic of the transmission. The 2-Mode EVT transmission, developed for hybrid buses by GM Allison and known as $\mathrm{E}^{\mathrm{V}}$ Drive $^{\mathrm{TM}}$, employs three planetary sets (E1, E2, and E3) and two wet-plate clutches (C1 and C2) to shift between input split (EVT 1) and compound split (EVT 2) modes. Input split means that only the first planetary, E1, produces split while the others, E2 and E3, act as gear reductions. Compound split means that the split is produced by the E1 and E2 compound gearset while E3 is a gear reduction (Chiang, 2007, Schmidt, 1999). 


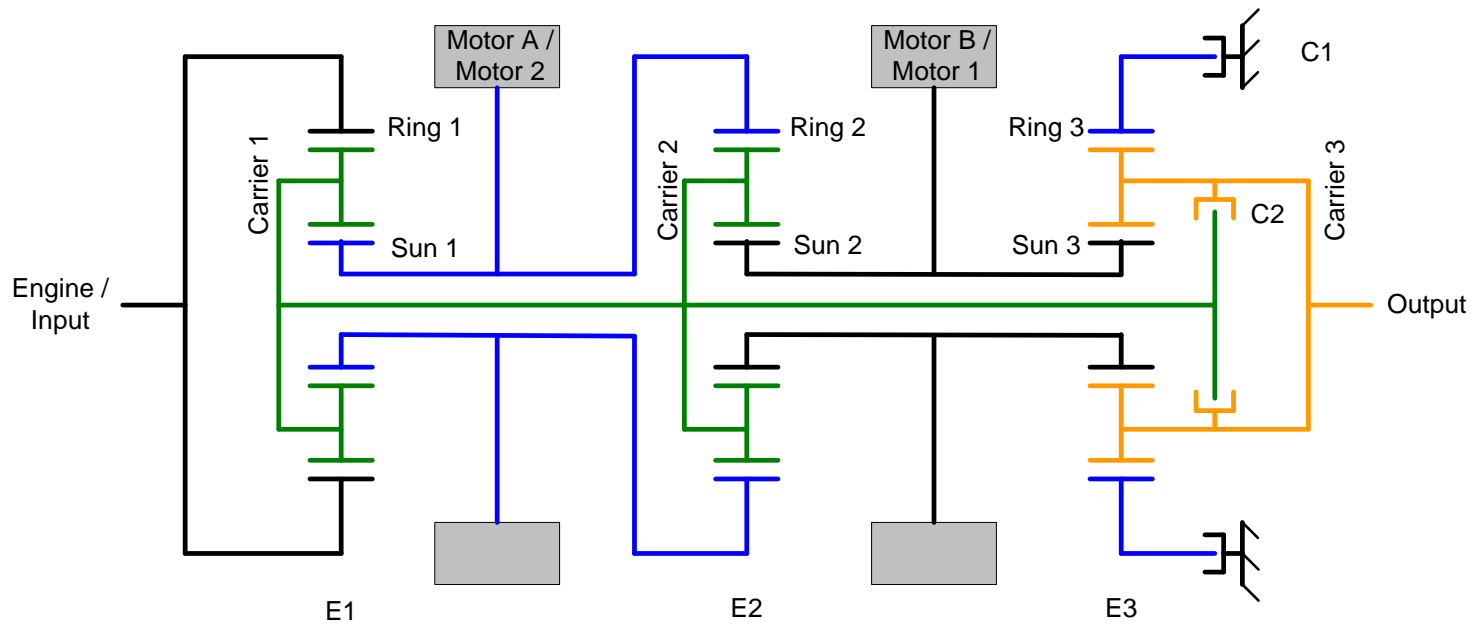

Figure 2-11 Schematic of the 2-Mode ETV Transmission (Grewe et al., 2007)

This architecture is similar to the input split with output gearing, except that in the 2-Mode EVT the output gearing is a planetary gearset (Miller, 2006) with clutches that either connect both sets (EVT 2 mode) or ground one of E2's components (EVT 1 mode). In EVT 1 mode, the engine-side epicyclic gearset E1 acts as a differential and the second planetary E2 as a torque multiplier. In EVT 2 mode, both E1 and E2 act as differentials. The third epicyclic gearset, E3, is used to improve the torque characteristics of the system. The input-split mode is utilized at low speeds or for high accelerations (Conlon, 2005). The compound-split mode is used at higher speeds (Zhang et al., 2001).

The configuration of the transmission is as follows (Grewe et al., 2007):

- $\quad$ The engine is joined with ring 1.

- Motor 1 is joined with suns 2 and 3.

- Motor 2 is connected with sun 1 and ring 2. In GM/Allison literature motor 2 is referred to as motor $\mathrm{A}$, and motor 1 as motor $\mathrm{B}$.

- Output is joined with carrier 3.

- Carrier 1 is joined with carrier 2.

- Clutch $\mathrm{C} 1$ is a brake or stationary clutch. It holds ring 3, activating input split mode and lowspeed torque multiplication.

- Clutch C2 is a rotating clutch. It connects the output shaft with carriers 1 and 2, activating the compound split mode.

Neglecting mechanical losses and inertias of the gearset, the equations for input split mode, EVT 1, are (Source: PSAT transmission model and Kim et al., 2009):

Equation 2.32 2-Mode Input Split Equations

$$
\omega_{m 1}=e \cdot \omega_{\text {out }}
$$




$$
\begin{gathered}
\omega_{m 2}=-\frac{a}{1-b c} \cdot \omega_{\text {engine }}+\frac{b d}{1-b c} \cdot \omega_{m 1} \\
J_{\text {engine }} \cdot \dot{\omega}_{\text {engine }}=\tau_{\text {engine }}-\frac{a}{1-b c} \cdot \tau_{m 2} \\
\frac{1}{e} \cdot \tau_{\text {out }}=\tau_{m 1}+\frac{b d}{1-b c} \cdot \tau_{m 2}
\end{gathered}
$$

In Equation $2.32 \mathrm{~m} 1$ and $m 2$ are motor 1 and motor 2 respectively, $\omega$ is angular speed, $\tau$ is torque, and $J$ is moment of inertia. Engine speed is calculated as the integral of $\dot{\omega}_{\text {engine. Additionally, the }}$ transmission ratios $a$ through $e$ are defined as:

Equation 2.33 2-Mode Transmission Ratios

$$
\begin{gathered}
a=\frac{N_{R 1}}{N_{S 1}} \\
b=\frac{N_{S 1}+N_{R 1}}{N_{S 1}} \\
c=\frac{N_{R 2}}{N_{S 2}+N_{R 2}} \\
d=\frac{N_{S 2}}{N_{S 2}+N_{R 2}} \\
e=\frac{N_{S 3}+N_{R 3}}{N_{S 3}}
\end{gathered}
$$

In Equation $2.33 \mathrm{~N}$ is number of teeth, $R$ is ring gear, $S$ is sun gear, and the numbers correspond to the three planetaries: E1, E2, and E3. These angular velocity equations can be determined using one of several methods, e.g. bond graphs (Kim et al., 2009), lever diagrams (Conlon, 2005), or Kane's method (Zhang et al., 2001).

Similarly, the equations for compound split mode, EVT 2, are:

\section{Equation 2.34 2-Mode Compound Split Equations}

$$
\begin{gathered}
\omega_{m 1}=\frac{a c}{d} \cdot \omega_{\text {engine }}+\frac{1-b c}{d} \cdot \omega_{\text {out }} \\
\omega_{m 2}=-a \cdot \omega_{\text {engine }}+b \cdot \omega_{\text {out }} \\
J_{\text {engine }} \cdot \dot{\omega}_{\text {engine }}=\tau_{\text {engine }}+\frac{a c}{d} \cdot \tau_{m 1}-a \cdot \tau_{m 2} \\
\tau_{\text {out }}=\frac{1-b c}{d} \cdot \tau_{m 1}+b \cdot \tau_{m 2}
\end{gathered}
$$

Replacing the gear ratios of Equation 2.33 into Equation 2.32 and Equation 2.34, we obtain the input and compound equations in terms of the number of teeth N. For input split (Source: PSAT transmission model): 
Equation 2.35 2-Mode Input Split Equations in Terms of $\mathbf{N}$

$$
\begin{gathered}
\omega_{m 1}=\left(1+\frac{N_{R 3}}{N_{S 3}}\right) \cdot \omega_{\text {out }} \\
\omega_{m 2}=\left(\frac{N_{R 1} N_{R 2}+N_{R 1} N_{S 2}}{N_{R 1} N_{R 2}-N_{S 1} N_{S 2}}\right) \cdot \omega_{\text {engine }}+\left(1-\frac{N_{R 1} N_{R 2}+N_{R 1} N_{S 2}}{N_{R 1} N_{R 2}-N_{S 1} N_{S 2}}\right) \cdot \omega_{m 1} \\
J_{\text {engine }} \cdot \dot{\omega}_{\text {engine }}=\tau_{\text {engine }}+\left(\frac{N_{R 1} N_{R 2}+N_{R 1} N_{S 2}}{N_{R 1} N_{R 2}-N_{S 1} N_{S 2}}\right) \cdot \tau_{m 2} \\
\frac{1}{1+N_{R 3} / N_{S 3}} \cdot \tau_{\text {out }}=\tau_{m 1}+\left(1-\frac{N_{R 1} N_{R 2}+N_{R 1} N_{S 2}}{N_{R 1} N_{R 2}-N_{S 1} N_{S 2}}\right) \cdot \tau_{m 2}
\end{gathered}
$$

And for compound split:

\section{Equation 2.36 2-Mode Compound Split Equations in Terms of $\mathbf{N}$}

$$
\begin{gathered}
\omega_{m 1}=\left(\frac{N_{R 1} N_{R 2}}{N_{S 1} N_{S 2}}\right) \cdot \omega_{\text {engine }}+\left(1-\frac{N_{R 1} N_{R 2}}{N_{S 1} N_{S 2}}\right) \cdot \omega_{\text {out }} \\
\omega_{m 2}=-\left(\frac{N_{R 1}}{N_{S 1}}\right) \cdot \omega_{\text {engine }}+\left(1+\frac{N_{R 1}}{N_{S 1}}\right) \cdot \omega_{\text {out }} \\
J_{\text {engine }} \cdot \dot{\omega}_{\text {engine }}=\tau_{\text {engine }}+\left(\frac{N_{R 1} N_{R 2}}{N_{S 1} N_{S 2}}\right) \cdot \tau_{m 1}-\left(\frac{N_{R 1}}{N_{S 1}}\right) \cdot \tau_{m 2} \\
\tau_{\text {out }}=\left(1-\frac{N_{R 1} N_{R 2}}{N_{S 1} N_{S 2}}\right) \cdot \tau_{m 1}+\left(1+\frac{N_{R 1}}{N_{S 1}}\right) \cdot \tau_{m 2}
\end{gathered}
$$

Next the input split and compound split modes are described based on Grewe et al. (2007).

Input Split Mode EVT 1: In EVT 1, clutch C1 is on, grounding ring 3, and C2 is opened. EVT 1 includes electric launch and engine starting. Figure 2-12 shows the corresponding schematic. The speed of motor 1 is governed by the output shaft and the transmission ratio $e$ (Equation $2.32 a$ ). Engine speed is controlled by output speed and by velocity and torque of motor 2. During engine powered driving $\mathrm{m} 2$ acts as a generator while $\mathrm{m} 1$ acts as a motor. Engine power is split into a direct mechanical path and an electrical path ( $\mathrm{m} 2$ to $\mathrm{m} 1$ ). During regenerative braking motor 1 acts as a generator.

EVT 1 has a mechanical point (\#1), where motor 2 is stationary, producing mechanical transference of engine power (no split). Under these conditions the speed relations of Equation 2.32 reduce to $\omega_{\text {engine }}=b d / a \cdot \omega_{m 1}=b d e / a \cdot \omega_{\text {out }}$; the transmission ratio is fixed at $b d e / a$. 


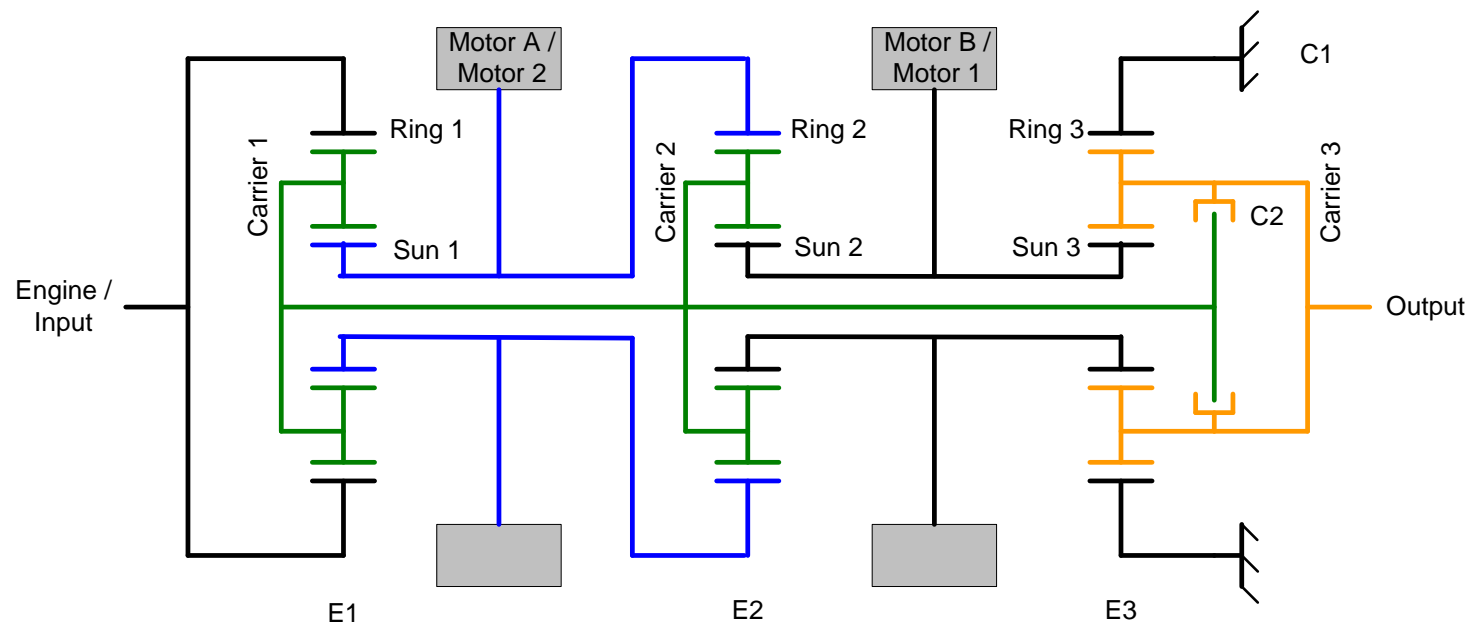

Figure 2-12 EVT 1 Input Split Mode in the 2-Mode ETV Transmission

Compound Split Mode EVT 2: In EVT 2, clutch C1 is opened while clutch C2 is closed linking the output shaft with carriers 1 and 2. Figure 2-13 shows the corresponding schematic. EVT 2 provides two additional mechanical points (\#2 and \#3); in \#2 motor 2 is stationary whereas in \#3 motor 1 is stationary. From Equation 2.34, the transmission ratios are b/a for point \#2 and $(b c-1)$ / ac for \#3. Between points \#2 and \#3, motor 1 operates as a generator while motor 2 is motoring; after point \# 3 the action is reversed.

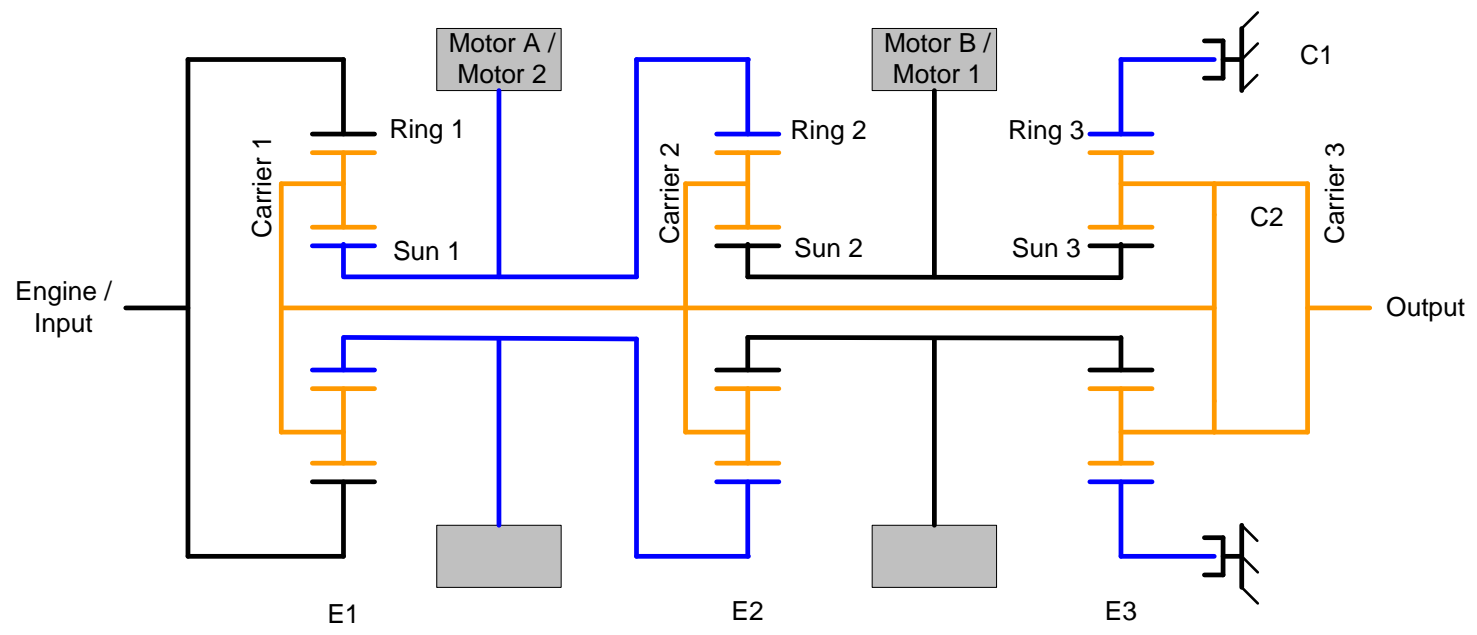

Figure 2-13 EVT 2 Compound Split Mode in the 2-Mode ETV Transmission

Synchronous Mode Shift: The GM-Allison EVT transmission was designed such that mechanical points \#1 and \#2 occur simultaneously. From the discussion above one can state that $b d e / a=b / a$ and therefore $d e=1$ must be satisfied; this implies that the ring to sun ratios of E2 and E3 must be identical $\left(N_{R 2} / N_{S 2}=N_{R 3} / N_{S 3}\right)$. At the synchronous speed the transmission can be simultaneously 
in EVT 1 and EVT 2 modes. Shift from EVT 1 to EVT 2 can be performed anytime the angular speeds of clutch C2 and carriers 1 and 2 are synchronized, i.e. have zero relative speed. This is attained by commanding the speed of motor 2. Mode 1 is used at low speed and mode 2 at high speeds. Mode shift is usually performed at the low speed mechanical point. Mode shifting is performed in a synchronous way at an intermediate vehicle speed, when motor 2 reaches zero velocity (mechanical point) and the clutches reverse action.

Degrees of Freedom: In each of its modes, the 2-Mode EVT is described by 4 linear equations (two for speed and two for torque) with 8 unknowns (speed and torque of engine, motor 1, motor 2, and output). The system has four degrees of freedom. Output speed can be removed since it is given by vehicle speed and differential ratio. By the same token, output torque is given by driver's demand and could be removed. Effectively, there are two degrees of freedom for controlling the system: one speed and one torque. The governing speed can be either $\omega_{m 2}$ or $\omega_{\text {engine }}$ in input split, while in compound split it can be either $\omega_{m l}, \omega_{m 2}$, or $\omega_{\text {engine }}$. Regardless of mode, the governing torque can be any one of $\tau_{m 1}, \tau_{m 2}$, and $\tau_{\text {engine. }}$. On the other hand, output torque can be determined by setting $\tau_{m l}$ and $\tau_{\text {engine }}$ in input split, while in compound split it is determined by setting any two of $\tau_{m 1}, \tau_{m 2}$, and $\tau_{\text {engine }}$.

Engine Operation: The engine operating point is optimized to maximize fuel efficiency. Conlon (2005) explains that "in general, this means that the engine is operated along a lower speed constraint line at low speeds, then along a torque constraint line passing through the best brakespecific-fuel-consumption points at higher powers. The reduction in engine losses must be traded off against the holding torque and spin losses of the electric machines. In practice, this generally means that the best system efficiency point lies at somewhat lower torques and higher speeds than the optimum engine efficiency trajectory."

\subsubsection{The 2-Mode Hybrid with Fixed Gear Ratios}

Developed for SUVs by GM, the 2-Mode Hybrid transmission includes 4 fixed gear ratios attained by clutch-locking different parts of the transmission (Grewe et al., 2007). Compared with the 2-Mode EVT with one fixed gear ratio, the 2-Mode Hybrid adds 3 fixed gear ratios and 2 clutches. The rear wheel drive version uses 3 planetary gearsets with the third one acting as a final drive, whereas the front wheel drive version uses only two planetaries and the final drive is through conventional gearing. Four balancing effects have been identified, namely: $i$ ) the additional clutches increase the spin losses of the transmission. ii) The use of fixed gears reduces losses through the electrical path and allows more efficient regenerative braking at high speeds. iii) The use of fixed gears slightly drifts engine 
operative conditions from best efficiency. $i v$ ) The use of fixed gears increases transmission output torque which allows a reduced final drive ratio, reducing spin losses.

Overall, the 2-Mode Hybrid transmission provides increased acceleration and better fuel economy over severe driving conditions (e.g. the US06 driving schedule). For less severe driving, modeling of both the 2-Mode EVT and the 2-mode Hybrid yielded similar fuel economy results (Grewe et al., 2007). The 2-Mode Hybrid architecture is intended for use in full-size SUVs that require a demanding towing capacity with inherently higher cruising and grade loads; furthermore, it reduces the motor continuous power requirements required in equivalent 2-Mode EVTs. Figure 2-14 shows a cutaway and the components of the GM 2-Mode Hybrid transmission installed in the 2008 Saturn VUE (picture taken at GM booth during the SAE 2008 World Congress); input shaft is in the front and output shaft is in the back. The 2-Mode Hybrid transmission is governed in its input and compound split modes by the equations of the 2-Mode EVT presented above.

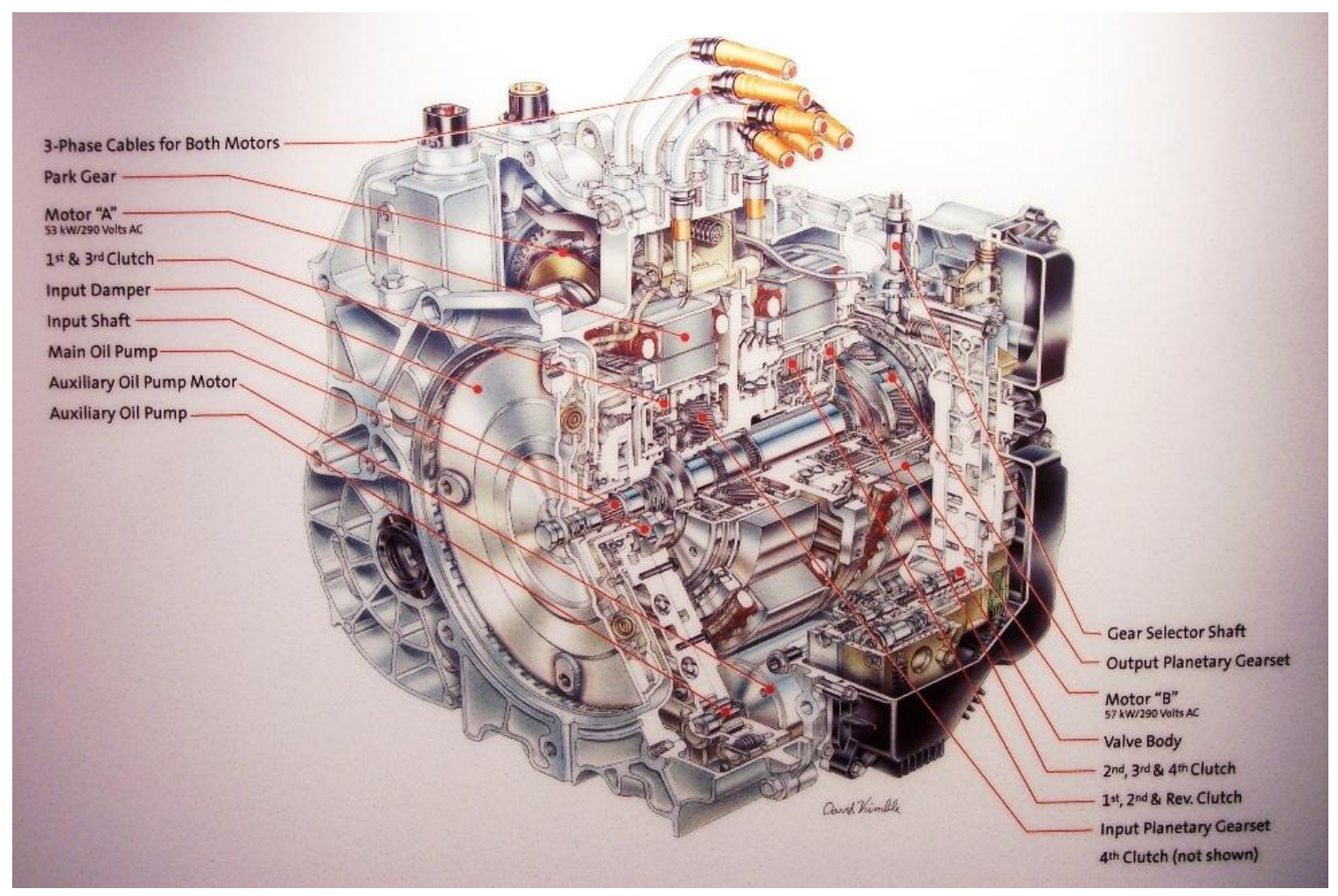

Figure 2-14 GM 2-Mode Hybrid System, Front-Wheel Drive (picture taken at GM booth during the SAE 2008 World Congress) 


\subsection{Emissions Testing Procedures}

Emissions testing laboratories in the U.S. are regulated by the requirements of CFR 40 Parts $86^{\mathrm{a}}$ and 1065 (Engine Testing Procedures). West Virginia University has chassis and engine dynamometer test facilities designed according to Subparts $\mathrm{B}^{\mathrm{b}}$ and $\mathrm{N}^{\mathrm{c}}$ of Part 86, and Part 1065 . WVU recently upgraded its test facilities to comply with regulations pertaining to testing of 2007 and newer engines/vehicles (CFR 40 Part 1065).

\subsubsection{Analytic Instrumentation}

The tail pipe exhaust is conducted to the full scale dilution tunnel where it is mixed with HEPA filtered dilution air. The sampling plane is located at least 10 tunnel diameters downstream from the inlet, where heated sampling probes draw the diluted exhaust. Heated lines conduct the diluted exhaust to the analytic instruments for continuous $\mathrm{HC}, \mathrm{NO}, \mathrm{NO}_{\mathrm{x}}, \mathrm{CO}$, and $\mathrm{CO}_{2}$ measurement, and collection in bags for specialized tests. All of the analyzers are checked for drift and they are calibrated (using a gas divider) before each test. Background tests are conducted before, during, or after testing. Background emissions are subtracted from the diluted stream to obtain truly tailpipe emissions. During the test, background and diluted exhaust bags are also filled, providing composite emissions that can be compared with instantaneous results.

The analytic equipment is described next.

Hydrocarbons, HC: A heated flame ionization detector (HFID) continuously measures HC emissions. The instrument has a burner in which a regulated sample gas flow passes through a flame. The flame is sustained by flows of air and a premixed hydrogen/diluent fuel gas. The hydrocarbons in the sample are combusted producing positive and negative ions. Ions are collected in polarized electrodes, causing current to flow. Current flow is proportional to hydrocarbon concentration in the sample. Concentrations are reported wither on methane $\left(\mathrm{CH}_{4}\right)$ or propane $\left(\mathrm{C}_{3} \mathrm{H}_{8}\right)$ equivalent emissions. Zero gas is zero air. Zero air is purified air with less than $0.1 \mathrm{ppm}$ $\mathrm{HC}$.

Oxides of Nitrogen, $\mathrm{NO}_{x}$ : Two heated wet chemiluminescent analyzers continuously measure nitric oxide $(\mathrm{NO})$ and $\mathrm{NO}_{\mathrm{x}}$. $\mathrm{NO}$ reacts with ozone $\left(\mathrm{O}_{3}\right)$ producing nitrogen dioxide $\left(\mathrm{NO}_{2}\right)$ and

\footnotetext{
${ }^{a}$ Part 86: Control of Emissions from New and In-Use Highway Vehicles and Engines.

${ }^{\mathrm{b}}$ Subpart B: Emissions Regulations for 1977 and Later Year New Light-Duty Vehicles and New Light-Duty Trucks and New Otto-Cycle Complete Heavy-Duty Vehicles; Test Procedures.

${ }^{c}$ Subpart N: Emissions Regulations for New Otto-Cycle and Diesel Heavy-Duty Engines; Gaseous and Particulate Exhaust Test Procedures.
} 
oxygen $\left(\mathrm{O}_{2}\right)$. Some $10 \%$ of the $\mathrm{NO}_{2}$ is electronically-excited and will revert to its non-excited state with the emission of photons (chemiluminescent reaction). The amount of photons thus produced is proportional to the NO concentration of the sample. Ozone is produced from an oxygen cylinder and ultraviolet radiation. $\mathrm{NO}_{\mathrm{x}}$ is determined by dissociation of the $\mathrm{NO}_{2}$ into $\mathrm{NO}$ prior to entering the reaction chamber. $\mathrm{NO}_{2}$ is determined by subtraction. Zero gas is nitrogen.

Carbon Monoxide, CO, and Carbon Dioxide, $\mathrm{CO}_{2}$ : Non-dispersive infrared (NDIR) analyzers continuously measure $\mathrm{CO}$ and $\mathrm{CO}_{2}$. The instrument consists of two infrared light sources, a chopper, a measuring cell filled with the gas sample, a reference cell filled with reference (nonabsorbing) gas, and a detector. The infrared light intermittently enters the measuring cell where it is partially absorbed by the sample gas. The detector consists of two sealed chambers connected by a flexible metal diaphragm and filled with a gas mixture containing the component of interest. In the chamber, the gas absorbs infrared energy, its temperature increases, and its pressure rises. The detector senses pressure difference between the chambers. The difference in energy of the beam due to the presence of the component of interest in the sample gas causes the pressure in the sample chamber to increase less than the reference chamber pressure. The differential pressure causes the diaphragm to flex changing the capacitance of a two-plate variable capacitor. Capacitance change is proportional to $\mathrm{CO} / \mathrm{CO}_{2}$ concentration in the sample. Intermittency of the beam being blocked by the chopper equalizes the pressure in both chambers. Zero gas is nitrogen. Two analyzers are used for $\mathrm{CO}$ emissions, low range and high range.

Other gases: Gases like non-methane hydrocarbons (NMHC), aldehyde, alcohols, and other toxic emissions are determined by gas chromatography. Sample bags are collected and sent to WVU where the mean concentration is measured.

Particulate Matter (PM): PM is measured gravimetrically. The PM sample is conducted through a secondary dilution tunnel where the double diluted exhaust is kept at a temperature no higher than $125^{\circ} \mathrm{F}\left(50{ }^{\circ} \mathrm{C}\right)$. The sample is filtered through primary and secondary $70 \mathrm{~mm}$ fluorocarbon coated glass fiber filters. Filtration efficiency of the filters is $98 \%$ for particles 0.1 micron and larger. Filters are pre- and post- weighted on a microbalance in a clean room, conditioned to $50 \%$ $\mathrm{RH}, 70^{\circ} \mathrm{F}\left(25^{\circ} \mathrm{C}\right)$, for at least one hour.

A Tapered Element Oscillating Microbalance (TEOM) is available for continuous measurement of PM emissions. The TEOM is easily affected by exhaust humidity and several studies have shown that its accuracy is limited, especially with the new trap-equipped engines.

The following instrument verification/audit procedures are standard ( $\mathrm{Wu}$ et al., 2009): propane injections to verify CVS flow rates, $\mathrm{H}_{2} \mathrm{O}$ interference for $\mathrm{CO}$ and $\mathrm{CO}_{2}$ analyzers, $\mathrm{CO}_{2}$ interference for 
$\mathrm{CO}$ analyzers, $\mathrm{O}_{2}$ interference for $\mathrm{HC}$ analyzer, $\mathrm{CO}_{2}$ and $\mathrm{H}_{2} \mathrm{O}$ quench for $\mathrm{NO} / \mathrm{NO}_{\mathrm{x}}$ analyzer, and $\mathrm{NO}_{2}$ to NO converter efficiency.

Test results are converted to engineering units and processed according to CFR 40 Part 86 Subpart N (86.1342) and Part 1065 Subpart G.

Table 2-7 Summary of Analytic Instrumentation

\begin{tabular}{c|l|c}
\hline Component & Method & Zero gas \\
\hline NO/NO & Chemiluminescent & $\mathrm{N}_{2}$ \\
\hline $\mathbf{H C}$ & HFID & Zero Air \\
\hline $\mathrm{CO} / \mathrm{CO}_{2}$ & NDIR & $\mathrm{N}_{2}$ \\
\hline
\end{tabular}

\subsubsection{Chassis-Dynamometer Transportable Facility}

The WVU Transportable Heavy-Duty Vehicle Emissions Testing Laboratory (TransLab) was developed under contract with the U.S. DOE. The TransLab can operate medium to heavy-duty vehicles and collects gaseous and particulate emissions. The following is a brief description of the components and operation of the laboratory. The description of the pre 2007 compliant equipment is taken from Nix et al. (2009). Specifications of the CFR 1065 (2007) laboratory are taken from Wu et al. (2009).

\section{Pre-2007 Laboratory}

The laboratory has four major components: i) Heavy-duty chassis dynamometer for vehicle weights between 20,000 lb to 65,000 lb. ii) Medium-duty chassis dynamometer for vehicle weights between $6,000 \mathrm{lb}$ to $22,000 \mathrm{lb}$. iii) Analytical instrumentation trailer housing full scale dilution tunnel, gaseous and particulate analysis equipment, control system, and data acquisition system. iv) Critical flow venturi-constant volume sampling (CFV-CVS) blower system.

In the heavy-duty dynamometer, the test vehicle is supported on free-spinning rollers and hub adapters are put in place of the external drive tires. Drive shafts connect the hub adapters to the dynamometer units, one on each side of the vehicle. Inertia loads are simulated by flywheels set to the desired test weight. Aerodynamic drag and rolling resistance losses are simulated by two 300 hp eddy current power absorbers. Two $20 \mathrm{hp}$ variable speed motors supply restricted motoring to help overcome frictional losses in the dynamometer driveline. Aerodynamic and rolling drag loads are applied in closed loop control.

The medium-duty dynamometer differs from the heavy-duty dynamometer in that it applies load through the rollers. Table 2-8 presents component sizes of the various WVU chassis dynamometers. The $\mathrm{NO}_{\mathrm{x}}$ analyzers are heated (wet) chemiluminescent. 
Table 2-8 Components of the Various WVU Chassis Dynamometers

\begin{tabular}{|c|c|c|c|}
\hline & $\begin{array}{c}\text { Pre 2007 } \\
\text { Heavy-Duty }\end{array}$ & $\begin{array}{c}\text { Pre 2007 } \\
\text { Medium-Duty }\end{array}$ & $\begin{array}{c}\text { CFR-1065 Compliant } \\
\text { Laboratory }\end{array}$ \\
\hline Vehicle weight, $\mathrm{lb}$ & 20,000 to 65,000 & 6,000 to 22,000 & Did not change \\
\hline $\begin{array}{lll}\text { Eddy } & \text { current } & \text { power } \\
\text { absorbers, } & \text { hp } & \end{array}$ & $2 \times 300$ & $1 \times 400$ & Did not change \\
\hline Variable speed motors, hp & $2 \times 20$ & $1 \times 25$ & Did not change \\
\hline Blower & \multicolumn{2}{|c|}{ CFV-CVS } & SSV-CVS \\
\hline Dilution tunnel & \multicolumn{2}{|c|}{$\emptyset=18 \mathrm{in}, \mathrm{L}=20 \mathrm{ft}$} & $\varnothing=18$ in, $L=20 \mathrm{ft}$ \\
\hline Secondary dilution tunnel & \multicolumn{2}{|c|}{$\varnothing=3$ in, $L=30$ in } & $\varnothing=7 / 8$ in, $L=84$ in \\
\hline
\end{tabular}

\section{$>$ CFR-1065 Compliant Laboratory}

The dynamometers were not updated and they are shared between the two laboratories. On the other hand, a brand new analytical/controls trailer was constructed. The laboratory has two dilution tunnels, one for legacy-high PM diesel vehicles and one for clean-low PM vehicles. The blower system is a subsonic venturi-constant volume sampler (SSV-CVS).

The $\mathrm{NO}_{\mathrm{x}}$ analyzers are both heated (wet) and cold (dry) chemiluminescent. The PM sample is directed through a cyclone separator, which removes mechanically generated coarse particles, before it enters the filter. Detailed information on detection limits of the various analyzers, and the laboratory in general, can be found in Wu et al. (2009).

\section{Energy Management}

When the vehicle is accelerating in a level road, Equation 2.11 predicts that the loads are inertia ( $m V d V / d t)$, rolling resistance $\left(c_{r} m g V \cos (\alpha)\right)$, aerodynamic $\operatorname{drag}\left(0.5 \rho c_{d} A_{f} V_{r}^{2} V\right)$; loads that are overcome by the vehicle's power source. The inertial load is applied by the flywheels, which at the same time store kinetic energy that is redeployed at deceleration. The rolling and aerodynamic loads are imposed by the power absorbers. If, in addition, the vehicle is driving uphill, an extra load consisting of grade resistance is present $(m g V \sin (\alpha))$ which is imposed by the power absorbers. Even though, when in the road this grade energy is stored in the vehicle as potential energy, in the chassis laboratory, unlike the inertial load, the grade load is not stored. On the other hand, if the vehicle is driving downhill, motoring is provided by the decreasing vehicle's potential energy, which offsets the load in the engine. In the chassis-dynamometer, this motoring is provided by the variable speed motors.

When the vehicle is decelerating in a level road, aerodynamic and rolling losses are still present and would be simulated by the power absorbers; the flywheels redeploy the stored kinetic energy, part 
of this energy is used to overcome aerodynamic and rolling losses. The remaining kinetic energy is absorbed by braking (conventional or regenerative) and engine brake.

\subsubsection{Engine-Dynamometer Facility}

The engine dynamometer test cell has also been recently updated to comply with CFR 1065 . The following description corresponds to the pre-2007 laboratory.

As with the transportable laboratory, the full engine exhaust is directed to the dilution tunnel. The blower system is CFV-CVS. Inlet air to the engine and dilution air are controlled for temperature and humidity. The analytical instrumentation as described in Section 2.5.1.

\subsubsection{Mobile Emissions Measurement System (MEMS)}

The WVU Mobile Emissions Measurement System (MEMS) provides a portable and accurate way of measuring in-use emissions from heavy-duty vehicles. The drawback of MEMS is that the size of its equipment is enough as to interfere with the normal operation of transit vehicles. Therefore, its use in collecting emissions data from transit buses is restricted by functionality. MEMS have been used in studies of emissions and fuel consumption of tractor trucks where the equipment is carried in the trailer. Emissions results can be reported in time-specific $(\mathrm{g} / \mathrm{s})$, distance-specific $(\mathrm{g} / \mathrm{mi}$ or $\mathrm{g} / \mathrm{km})$, or brake-specific $(\mathrm{g} / \mathrm{kW}-\mathrm{hr})$ units. Engine power can be determined from the electronic control unit (ECU) broadcasts of engine speed and torque. Torque is computed in the ECU from engine speed and fueling rate. Details of the MEMS can be found in Krishnamurthy (2006).

Thompson et al. (2002) presented a method to infer load and power from diesel engines for on-road emissions monitoring. This method can also be used in chassis-dynamometer testing when engine load if of interest, e.g. brake-specific mass emissions. It would help in obtaining data for engine emission maps when such data is not available from engine dynamometer testing but vehicles with the engines of interest have been tested.

Brake-specific emissions require estimation, over a period of time, of the mass emitted and the work delivered to the engine output shaft (Thompson et al., 2002). The ECU does not report engine torque in engineering units $(\mathrm{N}-\mathrm{m}$ or $\mathrm{ft}-\mathrm{lb})$; instead, torque is reported as a percentage of the maximum torque at a given speed. Torque is not reported at the engine output shaft; it is reported as indicated torque, which is torque in the cylinders and includes the friction load (SAE J1939-71: percent load at current speed and actual engine-percent torque). The actual output torque will be indicated torque minus friction losses. The standard that regulates ECU communication is the SAE J1939-71; it provides the following useful definitions of the parameters that the ECU broadcasts related to torque: 
Percent Load at Current Speed: "The ratio of actual engine percent torque (indicated) to maximum indicated torque available at the current engine speed, clipped to zero torque during engine braking."

Actual Engine - Percent Torque: "The calculated output torque of the engine. The data is transmitted in indicated torque as a percent of reference engine torque. The engine percent torque value will not be less than zero and it includes the torque developed in the cylinders required to overcome friction."

Nominal Friction - Percent Torque: "The calculated torque that indicates the amount of torque required by the basic engine itself added by the loss torque of accessories. It contains the frictional and thermodynamic loss of the engine itself, and the losses of fuel, oil and cooling pumps. The data is transmitted in indicated torque as a percent of reference engine torque.

The realization can be done by a map dependent on engine speed and engine temperature and an offset value for additional loss torques."

The ECU computes torque from fueling rate and engine speed (Thompson et al., 2002). The method proposed by Thompson et al. (2002) requires the engine lug torque curve supplied by the manufacturer and a curbside no-load test. The curbside no-load tests serves to determine the percent load required to overcome internal friction. It was shown that ECU engine speed is very accurate, with an average absolute percent difference, when compared with measured laboratory speed, of $0.4 \%$ to $0.7 \%$ on the FTP cycle. The equation for engine output torque is

Equation 2.37 Engine Output Torque, Thompson et al. (2002)

$$
T=T_{\max } \cdot\left(\frac{E C U_{\%}-E C U_{\text {no-load }}}{E C U_{\% \max }-E C U_{\text {no-load }}}\right) .
$$

Where $T_{\max }$ is the lug curve torque, $E C U_{\%}$ is the percent load signal, $E C U_{n o-l o a d}$ is the no-load signal, and $E C U_{\% \max }$ is the associated lug curve percent load. All the parameters in Equation 2.37 are function of engine speed. It is assumed that internal friction is a function of speed only and the contribution of engine load is neglected. Thompson et al. (2002) report an error of 5\% in FTP cycle work (integrated power).

\subsection{Regression Analysis}

Regression analysis will be used in this dissertation in two main instances. First, to develop an engine emissions and fuel consumption model which will be integrated into the vehicle model. And 
second, to compile the outcome of all the obtained results in a form usable in IBIS. This section briefly describes the main techniques which will be considered: Least Squares, Neural Networks, and Multivariate Adaptive Regression Splines (MARS). Some important definitions are also presented.

Correlation, $\boldsymbol{r}$, measures the linear association between two or more variables; that is, how closely the data points follow a straight line (Moore \& McCabe, 2006). The correlation $r$ between variables $x$ and $y$ is

\section{Equation 2.38 Correlation, $r$}

$$
r=\frac{d}{n-1} \sum_{i=1}^{n}\left(\frac{x_{i}-\bar{x}}{s_{x}}\right) \cdot\left(\frac{y_{i}-\bar{y}}{s_{y}}\right),
$$

where $n$ is number of data points, $s$ is standard deviation, and over bar is mean value; $s_{x}^{2}=\frac{1}{n-1} \sum_{i=1}^{n}\left(x_{i}-\bar{x}\right)^{2}, \quad s_{y}^{2}=\frac{1}{n-1} \sum_{i=1}^{n}\left(y_{i}-\bar{y}\right)^{2} . \quad$ The quantities in parenthesis are standardized observations. The familiar correlation coefficient, $r^{2}$ (usually $R^{2}$,), is the square of $r$ in Equation 2.38. Correlation $r$ is always between -1 and $1 ; r>0$ represents a positive association and $r<0$ represents a negative association. $r^{2}$ is always between 0 and 1 , where values closer to the upper limit represent a stronger linear association. It must be noted that correlation is severely affected by outliers.

\subsubsection{Simple Least-Squares}

A regression line that describes or predicts the relationship between two variables, say $x$ and $y$, can be produced by Least-Squares regression. The regression has the form $y=b_{0}+b_{1} x$, where $\boldsymbol{b}_{\mathbf{0}}$ is the intercept and $\boldsymbol{b}_{1}$ is the slope. The best line to fit the data is a line that has the minimum separation in the vertical direction to all the points. The least-squares regression line minimizes the sum of the squares of the vertical distances between the data and the line.

The least-squares regression line of $y$ on $x$ is (Moore \& McCabe, 2006)

Equation 2.39 Least-Squares Regression Line

$$
\begin{aligned}
& \hat{y}=b_{0}+b_{1} x \\
& b_{1}=r \frac{s_{y}}{s_{x}} \\
& b_{0}=\bar{y}-b_{1} \bar{x}
\end{aligned}
$$

The parameters in Equation 2.39 were defined above. For example, $\bar{x}$ is the mean of all the $x$ values in the data set and $s_{x}$ their standard deviation. Note that correlation and slope share the same sign. 
A note of caution goes to extrapolation; predictions outside of the data region are often unreliable. Least-squares correlations are severely affected by outliers. Outliers in the $x$ direction have a strong influence in the regression line because they tend to pull the line toward themselves. A similar effect is observed with outliers in $y$.

The $R^{2}$ value "is the fraction of the variation in the values of $y$ that is explained by the least-squares regression" (Moore \& McCabe, 2006). Therefore, $R^{2}$ percent of the change of $y$ due to changes in $x$ is accounted for by the linear regression; the remaining $1-R^{2}$ percent corresponds to vertical scatter of the observed responses, that is, differences between observed values $(y)$ and predicted values $(\hat{y})$. In terms of variances (Var) of $y$ and $\hat{y}, R^{2}$ can be expressed as $R^{2}=\operatorname{Var}(\hat{y}) / \operatorname{Var}(y)$, which indicates that the variance in least-squares predicted values is always smaller than the variance of observed values. Finally, $R^{2} \cdot \operatorname{Var}(y)$ can be understood as the variance that the responses would have if there were no scatter in the data.

Linear regression can be applied to other types of relationships, e.g. logarithmic, if the data are transformed, e.g. apply the log function, such that the transformed data represent a linear trend.

Another measure of dispersion are the residuals, defined as the difference between observed and

predicted values: residual $=e_{i}=y_{i}-\hat{y}_{i}$. Residual plots are of common use in statistics. A good regression would yield no specific (unstructured) pattern in the residual plot. Further verification should be made by normal quantile plots of response variable and residuals, which give a good check for normality.

\subsubsection{Simple Linear Regression}

The previous section presented the least-squares line for $y$ on $x$ where a dataset of $(x, y)$ points was available; say through experiments. The points in the dataset are truly a sample of the population of all possible $(x, y)$ values. In this sense, the least-squares line is an estimate of the true regression line for the population (Moore \& McCabe, 2006), where the larger the number of data points, the better the estimate and the narrower the confidence interval.

Population parameters are labeled by Greek letters; so the population regression line is

\section{Equation 2.40 Population Regression Line}

$$
\mu_{y}=\beta_{0}+\beta_{1} x
$$

which reminds that the coefficients in the fitted line, Equation 2.39, are estimates of the $\beta$ 's.

In the statistical model for simple linear regression, it is assumed that the response $y$ is normally distributed for each value of $x$ ( $y$ is a random variable). In Equation 2.40, $\mu_{y}$ is the mean of the population for each value of $x$ and describes how the mean response changes with $x$. It is also assumed 
that the standard deviation $\sigma$ is constant with $x$. Figure 2-15 (Moore \& McCabe, 2006) compiles these ideas; the height of the normal curves represents the probabilities of each $y$ value to occur for a given $x$.

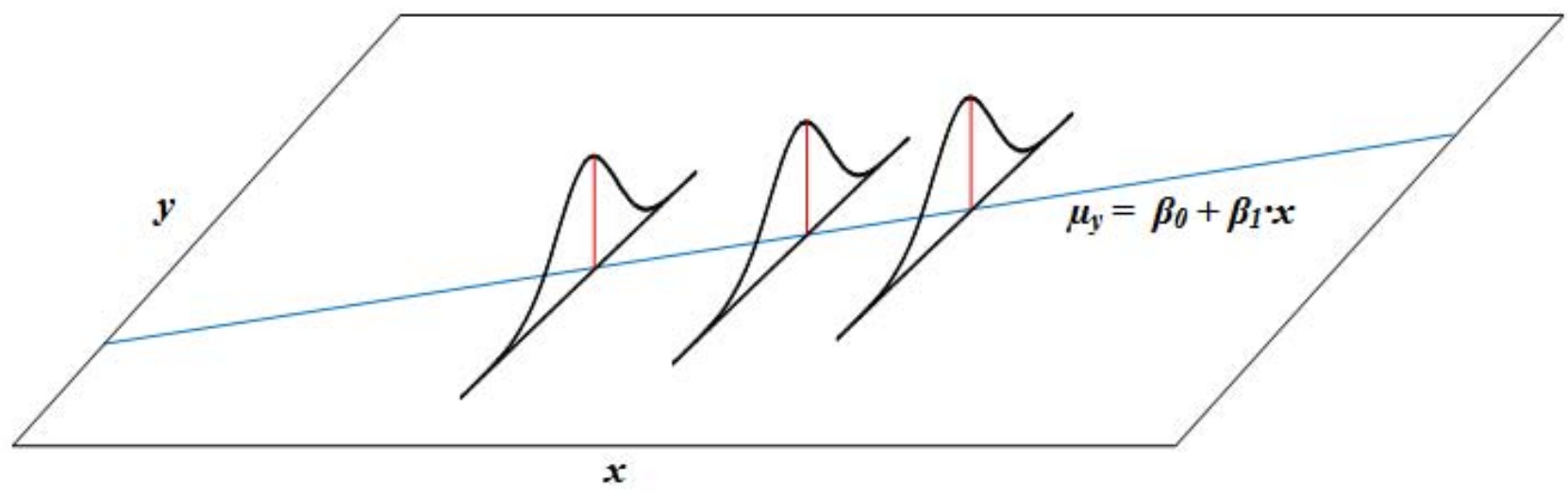

Figure 2-15 Statistical model for linear regression

The statistical model for the response $y_{i}$ is described mathematically by a mean value $\left(\beta_{0}+\beta_{1} \cdot x_{i}\right)$ plus a deviation, noise or residual $\left(\varepsilon_{i}\right)$. Residuals are likelihood deviations from the mean; they are independent and normally distributed, with zero mean and standard deviation $\sigma, \mathrm{N}(0, \sigma)$. The statistical model for the observed response is

\section{Equation 2.41 Statistical Model for Simple Linear Regression}

$$
y_{i}=\beta_{0}+\beta_{1} \cdot x_{i}+\varepsilon_{i} .
$$

The regression parameters $\left(\beta_{0}, \beta_{1}\right.$, and $\left.\sigma\right)$ are obtained from Section $2.6 .1 ; b_{0}$ and $b_{1}$ are unbiased estimators of $\beta_{0}$ and $\beta_{1}$. They are normally (or approximately normally) distributed with means $\beta_{0}$ and $\beta_{1}$. This means that $\hat{y}$ is also an unbiased estimator of $\mu_{y}$. The residuals $e_{i}$ correspond to the model residuals $\varepsilon_{i}$ and $\sigma^{2}$ is estimated from the average squared residual $s^{2}$ :

Equation 2.42 Average Squared Residual

$$
s^{2}=\frac{1}{n-2} \sum e_{i}^{2}=\frac{1}{n-2} \sum\left(y_{i}-\hat{y}_{i}\right)^{2} .
$$

The degrees of freedom (number of independent elements) are $n-2$ because two were already used for $b_{0}$ and $b_{1}$.

Confidence intervals, with a $C$ percentage (say 95\%), for $\beta_{0}$ and $\beta_{1}$ are

Equation 2.43 Confidence Intervals for Slope and Intercept

$$
\begin{aligned}
& \beta_{0}: b_{0} \pm t^{*} \cdot S E_{b 0} \text { and } \\
& \beta_{1}: b_{1} \pm t^{*} \cdot S E_{b 1} .
\end{aligned}
$$


$S E$ are standard errors given by $S E_{b_{0}}=s \sqrt{\frac{1}{n}+\frac{\bar{x}^{2}}{\sum\left(x_{i}-\bar{x}\right)^{2}}}$ and $S E_{b_{1}}=\frac{s}{\sqrt{\sum\left(x_{i}-\bar{x}\right)^{2}}}$; the critical value $t^{*}$ (see Figure 2-16) is solved from the $t$ distribution with $n-2$ degrees of freedom, $t(n-2)$, where $C$ is the area between $-t^{*}$ and $t^{*}$. $t^{*}$ can be calculated from a table of $t$ distribution percentage points.

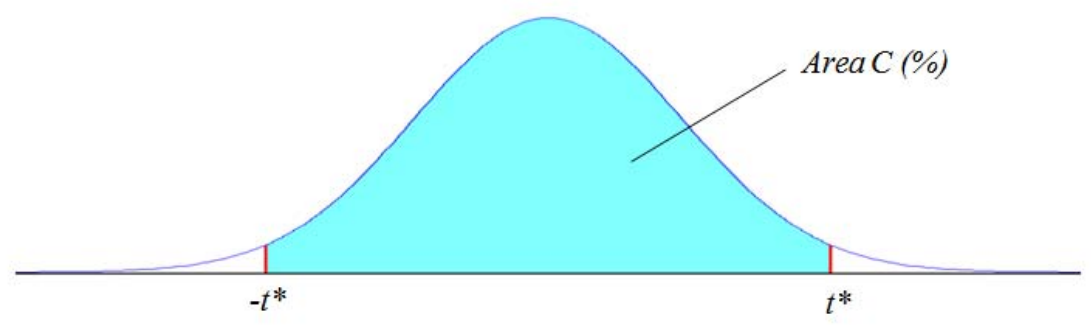

Figure 2-16 Graphical interpretation of $t^{*}$

If a significance test for the null hypothesis $H_{0}: \beta_{1}=0$ is positive, there is no linear relation and $\mu_{y}$ $=\beta_{0}$. Here, the test statistic is

Equation 2.44 t Statistic

$$
t=\frac{b_{1}}{S E_{b_{1}}}
$$

On the other hand, if the null hypothesis is rejected, a statistically significant linear relationship is then demonstrated. The statistic $t$ is also used to test for $H_{0}: \rho=0$, where $\rho$ is the population correlation is estimated from the sample correlation $r$. The statistic can be calculated alternatively by $t=\frac{r \sqrt{n-2}}{\sqrt{1-r^{2}}}$.

The hypothesis $H_{0}: \beta_{1}=0$ is tested based on the $\boldsymbol{P}$-value (Moore \& McCabe, 2006): "the probability, computed assuming that $H_{0}$ is true, that the test statistic would take a value as extreme or more extreme than that actually observed is called the P-value of the test. The smaller the P-value, the stronger the evidence against $H_{0}$ provided by the data." In other words, the chance of obtaining a statistic with value " $t$ " or larger is " $P$-value". The P-value is calculated with the $t(n-2)$ distribution and the value of $t$. The traditional level for statistical significance is $\mathbf{0 . 0 5}$.

The $C(\%)$ confidence interval for the mean response $\mu_{y}$ for any $x=x *$ is

Equation 2.45 Confidence Interval for Mean Response

$$
\hat{\mu}_{y} \pm t * S E_{\hat{\mu}}
$$


where $\hat{\mu}_{y}=b_{0}+b_{1} x^{*}$ and $S E_{\hat{\mu}}=s \sqrt{\frac{1}{n}+\frac{\left(x^{*}-\bar{x}\right)^{2}}{\sum\left(x_{i}-\bar{x}\right)^{2}}}$. Note that since $S E_{\hat{\mu}}$ is dependent on $x^{*}$, the confidence limits of the regression line are not static but vary with $x$. Usually, the interval is narrower near the mean $x, \bar{x}$, and increases as you move away from $\bar{x}$.

Equation 2.45 gives the confidence interval for the average response at a given $x=x^{*}$. Often times you would be interested in the confidence interval of a single prediction, that is, where the possible response would lie. The $C(\%)$ confidence interval provides this information for any $x=x *$,

Equation 2.46 Confidence Interval for Single Prediction

$$
\hat{y} \pm t * S E_{\hat{y}} .
$$

Where $\hat{y}=\hat{\mu}_{y}$ is the best guess for the predicted value and the standard error for the individual prediction is $S E_{\hat{y}}=s \sqrt{1+\frac{1}{n}+\frac{\left(x^{*}-\bar{x}\right)^{2}}{\sum\left(x_{i}-\bar{x}\right)^{2}}}$. The prediction interval is much wider than the confidence interval for mean response; for instance the ratio is $S E_{\hat{y}} / S E_{\hat{\mu}}$.

The concepts illustrated in this section can be extended to nonlinear models.

\subsubsection{Analysis of Variance (ANOVA)}

ANOVA expresses the variation in the response, $y_{i}-\bar{y}$, as two contributions, one from the effect of varying $x\left(\hat{y}_{i}-\bar{y}\right)$ and one from the scatter of data $\left(y_{i}-\hat{y}_{i}\right)$, that is, $\left(y_{i}-\bar{y}\right)=\left(\hat{y}_{i}-\bar{y}\right)+\left(y_{i}-\hat{y}_{i}\right)$. Then, the variation is expressed as a sum of squares (SS) where the left hand side is called total (SST), the first term of the RHS is model (SSM) and the last term is error (SSE) or residual. We have

Equation 2.47 Sum of Squares

$$
\begin{aligned}
& S S T=S S M+S S E \\
& S S T=\sum\left(y_{i}-\bar{y}\right)^{2} \\
& S S M=\sum\left(\hat{y}_{i}-\bar{y}\right)^{2} \\
& S S E=\sum\left(y_{i}-\hat{y}_{i}\right)^{2}
\end{aligned}
$$

It is also observed that the total degrees of freedom (DFT) are the sum of the degrees of freedom of the model (DFM) and the degrees of freedom of error (DFE), thus $D F T=D F M+D F E$. In simple regression, $\mathrm{DFM}=1$ (one independent variable), $\mathrm{DFE}=n-2($ two $\beta \mathrm{s})$, and DFT $=n-1$. The mean square (MS) is defined as the ratio of the sum of squares to the degrees of freedom, and as such there are MST, MSM, and MSE. A mean square is an average squared deviation. 
Then, $M S T=\frac{S S T}{D F T}=\frac{\sum\left(y_{i}-\bar{y}\right)^{2}}{n-1}$ which is the sample variance $s_{y}^{2}$. Furthermore, $M S E$ turns out to be the estimate of the variance of the residuals in the regression model, $s^{2}$ from Equation 2.42,

Equation 2.48 Mean Square Error

$$
s^{2}=M S E=\frac{S S E}{D F E}=\frac{\sum\left(y_{i}-\hat{y}_{i}\right)^{2}}{n-2} .
$$

The correlation coefficient $R^{2}$ may also be expressed in terms of sum of squares,

Equation 2.49 Correlation Coefficient, $\mathbf{R}^{2}$

$$
R^{2}=\frac{S S M}{S S T}
$$

The ANOVA test statistic for the null hypothesis $H_{0}: \beta_{1}=0$ is called the $F$ statistic

Equation 2.50 F Statistic

$$
F=\frac{M S M}{M S E} .
$$

Large values of $F(\mathrm{MSM} \gg \mathrm{MSE})$ indicate that $\beta_{1} \neq 0$. "The $\mathrm{P}$-value is the probability that a random variable having the $F(1, n-2)$ distribution is greater than or equal to the calculated value of the $F$ statistic" (Moore \& McCabe, 2006). The one in $F(1, n-2)$ is for DFM, and the $n-2$ for DFE. The $F$ statistic is related to the $t$ statistic defined above (Equation 2.44): $F=t^{2}$.

The ANOVA table gives degrees of freedom, sum of squares, as well as the mean squares for model, error, and total variations.

\subsubsection{Multiple Regression}

Consider the response variable $y$ and $p$ explanatory variables $x_{j}, j=1,2, \ldots, p$, with $n$ observations (sample size $n$ ). Similarly to the model for simple linear regression of Equation 2.41, the statistical model for multiple linear regression is (Rawlings et al., 1998)

Equation 2.51 Statistical Model for Multiple Linear Regression

$$
\begin{aligned}
& y_{i}=\beta_{0}+\sum_{j=1}^{p} \beta_{j} x_{i j}+\varepsilon_{i} \\
& y_{i}=\beta_{0}+\beta_{1} x_{i 1}+\beta_{2} x_{i 2}+\cdots+\beta_{p} x_{i p}+\varepsilon_{i}
\end{aligned}
$$


The subscript $i$ represents each individual observation and $i=1,2, \ldots, n$. There are $(p+1) \beta_{j}$ parameters in the model, $\beta_{0}$ to $\beta_{p}$. It is required that the number of observations be greater than the number of parameters, i.e. $n>p+1$.

The model can be represented in matrix notation as follows. Let $\underline{Y}$ be a column vector with the $n$ observations, $\underline{\beta}$ be a column vector with the $p+1$ unknown parameters, $\underline{X}$ be a $n \times p+1$ matrix of the dependent variables where the $i^{\text {th }}$ row has the form $\left[1, x_{i 1}, x_{i 2}, \ldots, x_{i p}\right]$, and $\underline{\varepsilon}$ be a column vector with the residuals. Subsequently, the model in matrix form is

\section{Equation 2.52 Statistical Model in Matrix Form}

$$
\begin{gathered}
\underline{Y}=\underline{X} \cdot \underline{\beta}+\underline{\varepsilon}, \text { or } \\
{\left[\begin{array}{c}
y_{1} \\
y_{2} \\
\vdots \\
y_{n}
\end{array}\right]=\left[\begin{array}{ccccc}
1 & x_{11} & x_{12} & \cdots & x_{1 p} \\
1 & x_{21} & x_{22} & \cdots & x_{2 p} \\
\vdots & \vdots & \vdots & & \vdots \\
1 & x_{n 1} & x_{n 2} & \cdots & x_{n p}
\end{array}\right]\left[\begin{array}{c}
\beta_{0} \\
\beta_{1} \\
\vdots \\
\beta_{p}
\end{array}\right]+\left[\begin{array}{c}
\varepsilon_{1} \\
\varepsilon_{2} \\
\vdots \\
\varepsilon_{n}
\end{array}\right]}
\end{gathered}
$$

As before, it is assumed that the deviations or residuals are normally distributed and have unknown standard deviation $\sigma$. The regression parameters $\left(\beta_{\mathrm{j}}\right)$ are obtained from the unbiased least squares estimators (Rawlings et al., 1998) $b_{j}$ (see Equation 2.39)

\section{Equation 2.53 Regression Parameters}

$$
\underline{b}=\left(\underline{X}^{\prime} \underline{X}\right)^{-1}\left(\underline{X}^{\prime} \underline{Y}\right)
$$

where $\underline{b}$ is a column vector $(p+1 \times 1), \underline{b}=\left[b_{0}, b_{1}, \ldots, b_{p}\right]^{\prime}$. In the $\underline{X}^{\prime} \underline{X}$ matrix $(p+1 \times p+1)$, the diagonal elements are the sum of squares of each explanatory variable and the off-diagonal elements are sums of products between explanatory variables. The column vector $\underline{X}^{\prime} \underline{Y}(p+1 \times 1)$ contains the sums of products for each observation $i$ between the dependent variable and the independent variables, $\underline{X}^{\prime} \underline{Y}=\left[\Sigma y_{i}, \Sigma x_{i 1} y_{i}, \ldots, \Sigma x_{i p} y_{i}\right]^{\prime}$. A unique solution exists if $\underline{X}^{\prime} \underline{X}$ has inverse, which is guaranteed if $\underline{X}$ has full column rank; that is, there are no linear dependencies among the explanatory variables, thus there are no redundant explanatory variables.

The least-squares multiple linear regression for the sample is

Equation 2.54 Least-Squares Multiple Linear Regression

$$
\begin{aligned}
& \hat{y}=b_{0}+b_{1} x_{1}+b_{2} x_{2}+\cdots+b_{p} x_{p} \\
& \hat{y}=\underline{x} \underline{b}
\end{aligned}
$$

Where $\underline{x}=\left[1, x_{1}, x_{2}, \ldots, x_{p}\right]$ is a row vector $(1 \times p+1)$. Equation 2.39 and Equation 2.41 are special cases, with $p=1$, of Equation 2.52 to Equation 2.54 .

The mean response is $\mu_{y}=\beta_{0}+\beta_{1} x_{1}+\beta_{2} x_{2}+\cdots+\beta_{p} x_{p}$ and $\hat{y}$ is an unbiased estimator of $\mu_{y} . \sigma^{2}$ is estimated from the mean squared residual (see Equation 2.42) 
Equation 2.55 Mean Squared Residual

$$
s^{2}=\frac{1}{n-(p+1)} \sum e_{i}^{2}=\frac{1}{n-(p+1)} \sum\left(y_{i}-\hat{y}_{i}\right)^{2} .
$$

Sometimes, $s$ is referred as root mean square error in statistical software.

Confidence intervals for $\beta_{j}$ are, as in Equation 2.43, $\beta_{j}: b_{j} \pm t^{*} \cdot S E_{b_{j}}$; the number of degrees of freedom for error is $n-(p+1)$. The standard error of the parameters is

Equation 2.56 Standard Error of the Regression Parameters

$$
S E_{b_{j}}=\sqrt{s^{2} c_{j j}}
$$

where $c_{j j}$ is the $(j+1)$ th diagonal element of $\left(\underline{X}^{\prime} \underline{X}\right)^{-1}$. This approach is called univariate confidence intervals. For the hypothesis $H_{0}: \beta_{j}=0$, the $t$ statistic (Equation 2.44) $t=\frac{b_{j}}{S E_{b_{j}}}$ is used.

The confidence interval for the mean response for any $\underline{x}^{*}=\left[1, x_{1}^{*}, x_{2}{ }^{*}, \ldots, x_{p}{ }^{*}\right]$ is (Equation 2.45) $\hat{\mu}_{y} \pm t * S E_{\hat{\mu}}$. The mean response is $\hat{\mu}_{y}=\underline{x}^{*} \underline{b}$ and the standard error of $\hat{\mu}_{y}$ is

Equation 2.57 Standard Error of the Mean Response

$$
S E_{\hat{\mu}}=\sqrt{s^{2} \cdot \underline{x}^{* \prime}\left(\underline{X}^{\prime} \underline{X}\right)^{-1} \underline{x}^{*}}
$$

The confidence interval for a single prediction is (Equation 2.46) $\hat{y} \pm t * S E_{\hat{y}}$, with $\hat{y}=\hat{\mu}_{y}=\underline{x}^{*} \underline{b}$. The standard error is

Equation 2.58 Standard Error of a Single Prediction

$$
S E_{\hat{\mu}}=\sqrt{s^{2} \cdot\left(1+\underline{x}^{* \prime}\left(\underline{X}^{\prime} \underline{X}\right)^{-1} \underline{x} *\right)} .
$$

The ANOVA $F$ statistic tests the hypothesis $H_{0}: \beta_{1}=\beta_{2}=\ldots=\beta_{p}=0$; the alternative hypothesis is $H_{a}$ : at least one $\beta_{j}$ is not 0 . Here, DFM $=p$ and $\mathrm{DFE}=n-p-1(D F T=D F M+D F E=n-1)$. The squared multiple correlation is also $R^{2}=S S M / S S T$ (Equation 2.49) and represents the proportion of the variation explained by the multiple linear regression model.

Factor interactions are not considered in multiple linear regression, only main effects are accounted for. On the other hand, nonlinear and polynomial models can include and evaluate factor interactions. Equations equivalent to the ones presented for linear regression exist for polynomial and nonlinear models.

Moore \& McCabe (2006) recommend the following procedure for performing a multiple regression analysis: 
Preliminary Analysis: examine carefully each of the explanatory and response variables. Look at means, standard deviations, minimum and maximum values. Use stemplots or histograms to examine the distributions and identify any possible outliers. Use normal quantile plots to identify if there distributions look normal (they are not required to be). Note and check for accuracy of extreme values.

$>$ Relationships between pairs of variables: get a better understanding of the data by examining relationship between all pairs of variables using scatterplots and correlations. Observe P-values.

$>$ Regression on subsets of variables: run several regressions on subsets of variables. Verify that the DOF in the output of the statistical program match the actual data; this ensures that the model was defined properly. In the ANOVA tables, check $r^{2}$ values, P-values, estimates of $\sigma\left(s^{2}=M S E\right)$. For the parameter estimates $(\beta$ or $b)$, check the $\mathrm{t}$ statistics $\left(t=b / S E_{b}\right)$ and corresponding $\mathrm{P}$-values for statistical significance of the regression coefficients. Variables without statistical significance indicate a weak correlation.

Note that a statistically significant result does not mean that the regression produces good results; the correlation coefficient sheds light on this point.

If two variables showed correlation in the previous step, then maybe only one variable will suffice at this stage and the other one could be eliminated. This will be revealed by the significance tests.

Residuals: examine the normal quantile residual plots, one for each explanatory variable, for outliers and linearity (thus normality of the residuals).

$>$ Refine the model: remove variables that contribute the less and run the regressions again. Compare $R^{2}$ and $t$ values with the previous ones; observe how the results have changed.

$>$ Regression using all variables: explore if a regression equation including all the explanatory variables gives better correlation coefficient. Identify the variables with statistical significance.

\subsubsection{Artificial Neural Networks}

Artificial neural networks (ANN) use a network of interconnected computing units (neurons) that can learn the input/output relationship of a complex system through training with sampled data (Zhou, 2003). ANNs exploit the principles of nonlinearity and parallelism as seen in the human brain.

Neural networks can be classified (Zhou, 2003) in feed-forward networks and recurrent networks according to the data flow architecture. In feed-forward networks data flows forward between layers of neurons. Recurrent networks have feedback loops which make them especially useful in optimization problems. Neural Networks can be single layer or multilayer. Multilayer feed-forward networks are also to referred as multilayer perceptrons (MLP). MLPs consist of input nodes, output nodes, intermediate (hidden) layers, and links that connect neurons between layers. 
The neurons in the hidden layers perform nonlinear transformations (mapping) between inputs and outputs. The mapping process is tuned by weights associated with the links; weights are adjusted through training. The nonlinear response is obtained by transfer or activation functions in the hidden neurons.

For the problem at hand, the recommended learning/training process is supervised learning. In supervised learning a set of input/output training data is used and the ANN's response is compared to the target output by estimation of a performance measure. Performance measures may be for example a mean squared error, or the instantaneous squared error for all the neurons at the output layer. The weights are adjusted according to an error correction rule, reducing the approximation errors. Backpropagation is a standard training technique in which the errors in the output layer are feed-backwards and the weights are adjusted according to error gradients.

Once the network is trained, it must be validated with a different dataset to assess how well it performs with unseen data. Two common validation techniques are simple validation and crossvalidation. Simple validation calculates the average error over all validation points. Cross-validation performs various partitions of the full dataset in training/validation subsets and trains the same number of ANNs. The network with the minimum validation error is selected.

Another common type of neural networks is the Radial Basis Function neural network. Radial basis networks are a special case of nonlinear feed-forward networks, in which the transfer function is symmetric with respect to a center. Radial basis networks are easier to train than MLPs.

Training can be improved by methods like adaptive learning rate, momentum models, and optimization-based methods (Zhou, 2003). These methods target issues of training efficiency and local minima.

A critical aspect in the design of ANNs is the number of hidden layers and the number of neurons per layer. If there are too many neurons, the system is overfitted and the response becomes erratic. If, on the other hand, there are too few neurons, the system is underfitted and the performance is poor. Several approaches have been developed to address these issues (Zhou, 2003), for example network pruning and resampling.

Neural networks have been used in a variety of applications from control and optimization to regression and complex system modeling. Specific examples of ANNs for emissions modeling is presented in Section 2.7.4 “ANNs for Engine Emissions”.

\subsubsection{Multivariate Adaptive Regression Splines}

MARS is a statistical regression method for high dimensional data, developed by Friedman (1991). MARS is a nonparametric method, meaning that the functional relationship between inputs and outputs 
is not predefined; the form of the relation is inferred from the data. The input space is partitioned and the model adjusts itself for best performance over the various regions. In MARS, the basis functions are hinge functions such as $\max \left\{0, x-x^{*}\right\}$, where $x^{*}$ is a partition point. MARS can be seen as a piece-wise linear regressor. The explanatory variables, factor interactions, and the partitions are selected automatically during the computations. An overfitted model is first created and then pruned to improve generalization. Nonlinearities are handled as multiplication of factors, where each factor has its own hinge function.

Krishnamurthy (2006) used MARS for $\mathrm{NO}_{\mathrm{x}}$ emissions modeling of heavy-duty trucks. The MARS algorithm does not come standard in $\mathrm{MATLAB}^{\circledR}$ software, but public domain user defined toolboxes (e.g. ENTOOL) exist with its implementation.

\subsection{Emissions Predictions / Inventories and Previous Work}

An important part of this dissertation is prediction of tailpipe emissions. This section discusses several methods used for emissions prediction and inventory. A review is presented of various methods that have been used in the literature to predict emissions. The majority of literature on the subject was found in journal papers, Master's Thesis and Ph.D. Dissertations from West Virginia University.

\subsubsection{MOBILE6 and MOVES}

MOBILE6 is a computer model developed by EPA used to predict emissions from on-road motor vehicles (Heirigs et al., 2001) for calendar years 1952 to 2050. MOBILE6 incorporates all types of onhighway vehicles, including motorcycles, light-duty cars and trucks, heavy-duty trucks, and buses. It accounts for fuel type, vehicle model year, vehicle operating parameters (speed, off-cycle effects, air conditioning, and soak time), and environmental parameters (temperature, humidity, cloud cover or fraction of light, and altitude), amidst other considerations.

MOBILE6 is activity based; the models are based on emission factors or base emission rates (g/mi), where these emission rates are function of vehicle mileage. The pollutant species considered are $\mathrm{HC}, \mathrm{CO}, \mathrm{NO}_{\mathrm{x}}, \mathrm{PM}$ (MOBILE6.1), and greenhouse gases (M6.3/NGM1). Base emissions data are collected from chassis-dynamometer FTP-72 tests. MOBILE6 applies correction factors to account for varying average speed, ambient temperature, and fuel. It also uses statistical activity data to determine 
travel fractions from the various vehicle types and vehicle age distributions. Finally, fleet average grams per mile emissions are reported.

MOBILE 6 divides driving in four groups: Freeway, Arterial/collector, Local roadway, and Freeway ramp Driving (EPA, 2004). Distribution of vehicle miles traveled is thus divided among these four categories. Transit and urban buses are classified under the category labeled HDBT; gasoline (HDGB) and diesel (HDDBT) transit buses are considered.

EPA's Motor Vehicle Emission Simulator (MOVES) is currently under development, which is intended to be a replacement for the MOBILE6 and NONROAD applications (EPA, 2009). MOVES builds upon previous models, provides more flexibility and includes the newest emissions measurements from light-duty vehicles. Its newest release, Draft MOVES2009, includes models for all criteria pollutants.

On the other hand, the California Air Resources Board (CARB) has developed the emissions inventory models EMission FACtors (EMFAC) and OFFROAD. These models estimate $\mathrm{CO}_{2}$ and $\mathrm{CH}_{4}$ emissions. EMFAC deals with motor vehicles, like passenger cars and heavy-duty trucks, operating in California; OFFROAD deals with off-road equipment, like agricultural, construction, off-road recreation, lawn, and garden.

\subsubsection{Integrated Bus Information System (IBIS)}

West Virginia University, under a contract with the Federal Transit Administration (FTA), is developing the web-based Integrated Bus Information System (IBIS). The purpose of IBIS it to provide assistance to transit agency managers in evaluating the impacts of vehicle, fuel, and emissions reduction technologies, both in existing fleets and for fleet planning. Based on chassis dynamometer testing of transit buses at WMATA over 16 driving schedules in 2006, speed correction factors and empirical correlations have been developed to relate driving cycle effects to tailpipe emissions. IBIS estimates the emissions inventory of a transit bus fleet by allowing the user to specify fleet composition and service spectrum within each bus group in the fleet (Marlowe, 2009). Inputs are vehicle parameters, duty cycle characteristics, and external operating conditions, such as grade or season. ULSD and CNG fueled buses are considered.

Marlowe (2009) published his Master's Thesis on the work he developed for IBIS. Marlowe created a multivariate polynomial regression tool (PolyTool) for fuel economy and emissions. PolyTool was used to generate backbone regression models of conventional ULSD and CNG buses, and diesel-electric hybrid buses. In order to expand the experimental emissions dataset, a tool was developed (Cycle Generator Tool) to extract emissions and fuel consumption data from existing emissions tests in the form of micro-trips. Cycle Generator Tool concatenates micro-trips to create new cycles representing different overall driving conditions than those of the cycles used in testing, 
and thus generates new inputs to the database. The concatenation tool used genetic algorithms. Drive cycles were characterized by $i$ ) average speed, $i$ ) standard deviation of speed, iii) kinetic intensity, $i v$ ) percentage idle, and $v$ ) number of stops per mile. Average speed, alone or paired with one of the other metrics, is used to predict emissions. Acceptable limits, based on 12 standard drive cycles, were explored for the four cycle metrics (ii to $v$ ) as a function of average speed. Multiplicative and additive correction factors are applied to backbone models to account for variations in vehicle configurations and technologies. Uncertainty, variability, and confidence intervals were not addressed. The Cycle Generator Tool did not have a way to account for hybrid state of charge within micro-trips.

Khan's (2009) Ph.D. Dissertation developed fuel consumption and emissions correlations for 40' transit diesel buses where the predictor is average speed. He included fuel consumption correction factors for passenger weight and road grade.

\subsubsection{Engine and Vehicle Emissions Prediction}

Ang and Fwa (1989) used regression techniques to study the fuel economy characteristics of a fleet of diesel transit buses in Singapore. The study was performed under actual operating conditions, where fuel consumption was measured on a daily basis. Statistical methods were used to establish the effects of the following: service route, vehicle model, engine overhaul, vehicle speed and load, and number of stops, among others. Average speed and loaded weight were observed to explain two thirds of the variations in fuel consumption. The differences of fuel economy between peak and non-peak operations were estimated. The study involved 188 buses, serving 15 routes; data were collected for one week. Figure 2-17 shows the frequency distribution of the fuel economy measurements; mean = $5.89 \mathrm{mpg}$, Standard Deviation $=0.93 \mathrm{mpg}, \min =3.11 \mathrm{mpg}, \max =11.29 \mathrm{mpg}$. Average speed of the routes ranged from 8.8 to $15.4 \mathrm{mi} / \mathrm{hr}$. The numbers of stops (traffic lights and service stops) were calculated for each route, the values ranged from 5.3 to 9.3 stops per mile; note that these are not actual stops.

Ergeneman et al. (1999) used emission maps to evaluate fuel consumption and emissions from a dual CNG/diesel transit bus. The maps were based on engine speed and torque while emission rates based on brake-specific power.

Kosto (2001) modeled a gasoline-electric hybrid car comparing its emissions with an equivalent conventional powertrain. Kosto developed a transient model for the gasoline engine, which input was fuel-air ratio, as well as a transient (temperature dependent) catalyst model. The modeling platform was the ADVISOR vehicle simulation software. Emissions of $\mathrm{HC}, \mathrm{NO}_{\mathrm{x}}$, and $\mathrm{CO}$ were predicted. Cold- and hot-engine emissions were considered though coolant temperature correction factors. 


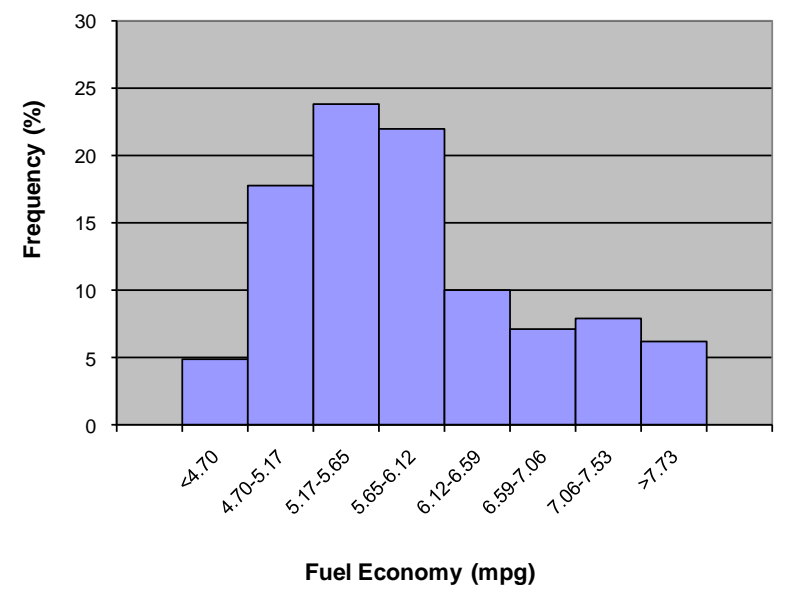

Figure 2-17 Frequency Distribution of Fuel Economy Observations in Transit Buses, Ang and Fwa (1989)

Barth et al. (2005) developed modal emissions and fuel consumption models for heavy-duty diesel trucks. The models were implemented to provide emission inventories for California. Modal emissions models are based on vehicle operating modes: idle, acceleration, deceleration, cruise, and transitions; the models predict second-by-second emission rates. Several models were developed to characterize distinctive categories of vehicles and technologies. The emissions model is composed of six modules: $i$ ) engine power demand, ii) engine speed iii) fuel rate, iv) engine control module, $v$ ) engine-out emissions, and $v i$ ) after-treatment pass fraction. Tailpipe emissions (g/s) are modeled as a function of fuel rate $(\mathrm{g} / \mathrm{s})$, engine-out emission indices $\left(\mathrm{g}_{\text {emissions }} / \mathrm{g}_{\text {fuel }}\right)$, and emission after-treatment pass fraction:

Equation 2.59 Tailpipe Emissions Model, Barth et al. (2005)

Tailpipe Emissions $=($ fuel rate $) \cdot\left(\frac{g_{\text {emissions }}}{g_{\text {fuel }}}\right) \cdot($ after - treatment pass fraction $)$.

After-treatment pass fraction is defined as the ratio of tailpipe to engine-out emissions. Fuel rate is a function of power demand and engine speed.

Emissions data collected with the University of California Riverside's Mobile (on-board) Emissions Research Laboratory (MEL) were used to calibrate the models. The effects of grade and off-cycle ignition timing were investigated. Also, the report presents a literature review survey, as well as key references, on the following topics: policy and emission standards, heavy-duty truck physical attributes, driving activity, drive cycle development, emissions characterization, combustion modeling, and heavy-duty diesel emissions modeling.

Krishnamurthy (2006) predicted $\mathrm{NO}_{\mathrm{x}}$ emissions for heavy-duty diesel engines using inputs from the electronic control unit (ECU) broadcast. The ECU parameters used were engine speed, engine 
torque, injection timing, fueling rate, manifold air temperature and pressure, and coolant and oil temperatures. The prediction was accomplished using the Multivariate Adaptive Regression Splines (MARS) method (Section 2.6.6). $\mathrm{NO}_{\mathrm{x}}$ data were collected with the on-road emissions testing capabilities of the MEMS. The vehicles were tested over urban and highway routes. The prediction uncertainty of the models was at most $\pm 20 \%$ which was considered highly successful.

Frey et al. (2007) developed a Vehicle Specific Power (VSP) approach for fuel consumption modeling of diesel buses and hydrogen fuel cell buses. Relative errors fewer than $10 \%$ were observed when the model was compared with experimental observations. VSP is tractive power (Equation 2.11) per unit mass of the vehicle and has units of $\mathrm{m}^{2} / \mathrm{s}^{3}$ on the SI system. The spectrum of VSP values was binned on 8 modes; the first mode consists of negative or zero VPS values and the last mode of VSP $\geq$ $13 \mathrm{~m}^{2} / \mathrm{s}^{3}$. Cycle fuel consumption (gal) is calculated as

Equation 2.60 Fuel Consumption Model, Frey et al. (2007)

$$
\text { Fuel Consumption }=\sum_{k}(\text { fuel consumption rate, gal/s })_{k} \cdot(\text { Time speed in mode, } s)_{k} \text {. }
$$

Where $k$ is VPS mode. Fuel consumption rates are obtained from chassis dynamometer or on-board emissions tests. The same method was used to estimate emissions. Two downsides can be seen on this approach, first, it cannot be directly applied to hybrids because in hybrids road load is not directly linked to engine load; second, the effects of transients are neglected.

Section 2.7.6 "Emissions Inventory” below includes other vehicle emissions modeling approaches.

\subsubsection{ANNs for Engine Emissions}

Thompson et al. (2000) used Artificial Neural Networks (ANNs) for emissions prediction of a 1993 Navistar V8 7.3 L T444E diesel engine. Prediction accuracy was to within 5\% of measured data. Inputs to the ANN models, at a sampling rate of $10 \mathrm{~Hz}$, where engine speed, accelerator petal position, intake manifold air temperature and pressure, exhaust temperature, oil and coolant temperature, injection pressure, injection pulse width, and start of injection. The training data were set of custom randomly designed transient cycles amounting to a total of 6,270 seconds. The validation data were two runs of the FTP and two custom cycles, for a total of 3,697 seconds. Emissions data were time shifted to account for analyzer response and transport delay; $\mathrm{NO}_{\mathrm{x}}$ and $\mathrm{CO}_{2}$ where shifted by crosscorrelation with power, while all the others were shifted by visual inspection. Torque measured by the dynamometer was corrected by dynamic effects or inertia to produce instantaneous engine output torque.

Four ANNs where developed: torque, gaseous emissions $\left(\mathrm{NO}_{\mathrm{x}}, \mathrm{CO}_{2}, \mathrm{CO}, \mathrm{HC}\right.$,), $\mathrm{PM}$, and opacity. The torque model did not require coolant and oil temperature inputs. Emissions and PM neural nets 
had two hidden layers; with 15 and 5 neurons in the first and second layer, respectively. The torque and opacity ANNs had a single hidden layer with 15 neurons. The model consisted of a preprocessor, the ANN, and a post-processor. The preprocessor filters (with an infinite impulse response, IIR, filter) and normalizes input data to improve numerical stability. The ANN incorporates a tapped delay line in the input to maintain a receding history window of 5-10 s; the tapped delay helps in accounting for the effect of turbocharger lag. The post-processor performs anti-normalization and filtering, including bound checks and IIR filter.

Jarrett and Clark (2002) performed emissions modeling of a 1999 Cummins ISM 370 diesel engine through ANNs. The purpose of the study was the determination of the most relevant parameters for prediction of emissions with ANNs; the effect of training with data from different cycles was also revised. The models predicted continuous $\mathrm{CO}_{2}, \mathrm{CO}, \mathrm{NO}_{\mathrm{x}}$, and $\mathrm{PM}$ emissions. The dynamometer cycles used where the FTP, the European Stationary Cycle (ESC), the European Transient Cycle (ETC), and random cycles from Thompson et al. (2000). For $\mathrm{CO}_{2}$ and $\mathrm{NO}_{\mathrm{x}}$ emissions, torque and speed were found to be the most dominant parameters, while derivatives of these were more significant for $\mathrm{CO}$ and PM. In the case of $\mathrm{CO}_{2}$ and $\mathrm{NO}_{x}$, although the weights of torque and speed were higher than the ones for the derivatives, the derivative inputs were significant and contributed to improve performance of the model. Training with FTP data produced the highest correlation coefficients for $\mathrm{NO}_{\mathrm{x}}$ prediction, with $\mathrm{R}^{2}$ values over 0.8 . Prediction of $\mathrm{CO}$ was fairly poor.

Inputs to the model were torque, speed, and their respective first and second derivatives over 1,5 , and $10 \mathrm{~s}$. The derivatives of variable $x$ over a time period $\Delta t$ are expressed as:

Equation 2.61 First and Second Derivatives Over a Time Period $\Delta t$

$$
\begin{aligned}
& \dot{x}_{\Delta t}=\frac{x_{t}-x_{t-\Delta t}}{\Delta t} \\
& \ddot{x}_{\Delta t}=\frac{\dot{x}_{t}-\dot{x}_{t-\Delta t}}{\Delta t}
\end{aligned}
$$

Even though the original data was logged at $5 \mathrm{~Hz}$, it was averaged to $1 \mathrm{~Hz}$ for the analysis. Delay in emissions measurement was corrected by cross-correlation. An axial dispersion function was applied to torque and speed signals. The ANN was back propagation multi-layer with three hidden layers in parallel and twenty neurons per layer. Activation functions for the three hidden layers were symmetric logistic, hyperbolic tangent, and hyperbolic tangent $[1.5 \mathrm{x}]$. The choice of activation functions was based on the ability of these to model linear response as well as nonlinearities. The same network design was later used by Tóth-Nagy et al. (2006) but with only torque, speed, and first derivatives as inputs.

Tóth-Nagy et al. (2006) discussed artificial neural networks (ANN) for $\mathrm{CO}_{2}$ and $\mathrm{NO}_{\mathrm{x}}$ emissions simulation of conventional and hybrid vehicles. The ANN models were trained with data from engine dynamometer tests of two heavy-duty diesel engines, i) Cummins ISM 370, 1999, 10.8 L, 370 hp, and 
ii) Navistar T444E, 1994, 7.3 L, $175 \mathrm{hp}$. Inputs to the models were engine speed and torque and their first derivatives. The ISM 370 model was scaled to $435 \mathrm{hp}$; this was done by scaling the input torque with the maximum torque ratio of both engines and multiplying the output by the inverse of the ratio. This process assumes that emissions scale with power. Alternatively, the ANN was fed with unscaled torque inputs of the $435 \mathrm{hp}$ engine; a matched comparison of the results from both methods produced correlation coefficients $\left(\mathrm{R}^{2}\right)$ greater than 0.99 for both $\mathrm{CO}_{2}$ and $\mathrm{NO}_{\mathrm{x}}$. The $\mathrm{ANN}$ proved to be extremely robust in extrapolating and the unscaled approach was used in final simulations with the ADVISOR vehicle simulation software.

They used back-propagation training, 6 input nodes, 3 hidden layers, and 20 nodes per hidden layer. The inputs were engine speed, engine torque, and their derivatives over 5 and 10 seconds. Each hidden layer had a different activation function: symmetric logistic, hyperbolic tangent, and hyperbolic tangent $[1.5 \mathrm{x}]$. The input layer had a linear activation function while the output layer had a symmetric activation function.

Once the model was implemented in ADVISOR, the operation of a tractor truck and a series hybrid-electric bus was studied. The bus model was exercised over the Manhattan, NY Bus, and CBD cycles. The results were compared with chassis test results from a similar vehicle. The model underpredicted for the Manhattan (-11.9\% $\left.\mathrm{CO}_{2},-5.1 \% \mathrm{NO}_{\mathrm{x}}\right)$ and $\mathrm{NY} \mathrm{Bus}\left(-35.7 \% \mathrm{CO}_{2},-29.8 \% \mathrm{NO}_{\mathrm{x}}\right)$ cycles, and over predicted for the CBD cycle $\left(+10.6 \% \mathrm{CO}_{2},+5.4 \% \mathrm{NO}_{\mathrm{x}}\right)$. The researchers concluded that the main factor in the error was the hybrid control strategy. The truck model was exercised under various weights. It was found that accessory loads also have a considerable impact on simulation results.

Perhinschi et al. (2007) developed ANN models to predict emissions from medium-duty vehicles based on chassis-dynamometer emissions tests. Inputs to the models were vehicle speed, acceleration, torque at drive axle, and exhaust temperature. Four different types of networks were developed and compared: adaptive linear, single hidden layer with sigmoid activation function, nonlinear polynomial (Sigma Pi), and Gaussian radial basis function (RBF). Accuracy of the results was improved by defining the model as the sum a linear regression model and the ANN. The parameters of the linear regression model varied depending on the pollutant in hand; for example, it was based on speed and acceleration for $\mathrm{NO}_{\mathrm{x}}$ and only on speed for $\mathrm{CO}_{2}$. Overall, the $R B F+$ Linear regression model performed better.

Bedick (2009) developed a single-hidden-layer feed-forward ANN heavy-duty diesel engine model that predicted $\mathrm{NO}_{\mathrm{x}}$ emission rate, exhaust temperature, and volumetric flow. Inputs to the model were engine speed and torque (with derivatives over 1 and $10 \mathrm{sec}$ ), oil temperature (with derivative over 10 $\mathrm{sec}$ ), and turbocharger boost pressure (with derivative over $1 \mathrm{sec}$ ). The activation function of the 28 hidden layer neurons was the logistic sigmoid function, logsig in MATLAB ${ }^{\circledR}: \log \operatorname{sig}(n)=(1-$ $\left.e^{-n}\right)^{-1}$, where $n$ is the neuron's weighted input. The three output neurons had a linear activation 
function: $f(m)=m$, where $m$ is the weighted output of the hidden layer. Input and output parameters were normalized between $[0,1]$ or $[-1,1]$ depending on the variable.

Network training (weighting factor optimization) was accomplished by the Levenberg-Marquardt method (trainlm command in MATLAB ${ }^{\circledR}$ ) with a learning rate value of 0.1 . The trained network was converted into a Simulink block. A composite training cycle was used, including sections from the FTP cycle, the ACES cycle (Transient, Cruise, and Creep modes), and the steady-state marine ICOMIA cycle.

Bedick employed a $\mathrm{NO}_{\mathrm{x}}$ sharpening model which took the output of the ANN, corrected it for delay and diffusion, and estimated truly engine out emissions. The procedure was implemented because the goal of the project was the optimization of an SCR catalyst and $\mathrm{NO}_{\mathrm{x}}$ entering the catalyst was a main concern. Nevertheless, $\mathrm{NO}_{\mathrm{x}}$ sharpening is not relevant to this dissertation.

\subsubsection{Hybrid-Conventional Comparison}

Several studies have evaluated fuel economy and emissions performance of hybrid buses compared with conventional buses. The following sources are a sample:

Bass and Alferman (2003) compared fuel economy advantages of the Allison two-mode hybrid buses. The comparisons were performed on a chassis dynamometer at Environment Canada facilities; the cycles evaluated were Manhattan, CBD, and UDDS. The cycle parameters that were found to have a significant effect of fuel economy are average speed, stops per mile, and idle. The fuel economy benefit is higher at lower average speeds and higher stopping frequencies. The authors recognize that reduction of parasitic losses, like idle and accessory loads, is a key for hybrid fuel economy. Also, cycles that allow the energy storage system to be more utilized (mitigating engine load) are good for fuel economy (e.g. cycles with high stopping frequencies and low average speeds).

Ribeiro and D'Agosto (2004) explored potential improvements to Brazilian transit bus emissions from using alternative fuels and new hybrid technologies. Fuel economy of series hybrid-drive buses was compared with equivalent conventional diesels in urban service. Field tests revealed fuel economy gains exceeding $20 \%$.

Holmén et al. (2005) compared PM emissions between diesel-electric Allison hybrid and conventional diesel transit buses (model year 2003). The year-long study, carried out by University of Connecticut, used on-board emissions testing and three different fuel/after-treatment configurations. The configurations were: $i$ ) diesel fuel $1+$ DOC, $i$ ) ULSD + DOC, and iii) ULSD + DPF. The study found that the hybrid buses do not offer significant particulate emissions benefits over the conventional diesel buses. 
The U.S. FTA (2005) presented an overview of the status of electric drive technologies (plug-in, hybrid-electric, and fuel cell) for transit applications. The report examined the benefits of electric drive buses, the barriers for higher deployment, and potential steps to alleviate these barriers. The report reviewed technical aspects of hybrids, emissions and fuel economy results, capital and operating costs, performance and durability issues, and the regulatory status. It was observed that hybrid-electric buses had improved fuel economy, $10 \%$ to $50 \%$, depending on system architecture and duty cycle. Capital costs of hybrids were estimated to be $60 \%$ to $80 \%$ higher than comparable diesel buses.

O'Keefe et al. (2007) defined kinetic intensity and hybrid advantage which in principle give the ability to assess a duty cycle's suitability for hybrid vehicle usage. Their work also targeted the estimation of fuel consumption for a given vehicle over a target duty cycle and the effect of accessory loads.

\subsubsection{Emissions Inventory}

With the purpose of using continuous emissions data for vehicle emissions inventory models, Ramamurthy (1999) correlated emissions data with axle power for trucks and buses tested with WVU's TransLab. Four transit buses were tested; they were 1994 and 1996 diesels with DDC S50 and Cummins M11 engines. The buses were exercised on the CBD cycle, the WVU 5 peak cycle, and the WVU 5 mile route. Emissions data are time shifted and dispersed when compared with axle power because the measurement systems for axle power and emissions differ in that the exhaust has to travel through the dilution tunnel, sampling lines, and analyzers. Gaseous emissions were time-aligned with axle power. A dispersion function was applied to axle power as if the signal traveled with the exhaust gases. Ramamurthy developed linear regression models for gaseous emissions as a function of axle power. $\mathrm{NO}_{\mathrm{x}}$ and $\mathrm{CO}_{2}$ predictions were reliable, while $\mathrm{CO}$ prediction was not as reliable. The models can be combined with vehicle activity to estimate contributions to emissions inventories.

Kern (2000) studied heavy-duty diesel emissions inventory and prediction. Using data collected by WVU's TransLab for over 8 years a method was developed to obtain emissions factors in grams per mile. Emissions were categorized (binned) by vehicle instantaneous speed and acceleration. Given a drive cycle, time fractions in each of the speed-acceleration bins were calculated and the fractions were coupled with the emissions bins (in grams per second) to estimate total emissions. Prediction errors were as low as 5\%. Kern estimated continuous PM emissions by proportioning continuous $\mathrm{CO}$ data and gravimetric PM. Kern did a thorough review of the parameters affecting diesel emissions, namely, vehicle class, driving schedule, weight, fuel type, exhaust after-treatment, vehicle age, terrain, and injection timing. 
Recognizing that emissions inventories did not consider the influence of terrain and road grade, Andrei (2001) studied the effect of grade on diesel missions. Simulations were performed for a 1998 class- 8 tractor truck, a 1995 class-6 box truck, and a 1996 transit bus over different grades at a constant speed. $\mathrm{NO}_{\mathrm{x}}, \mathrm{CO}_{2}, \mathrm{CO}$, and $\mathrm{PM}$ emissions were predicted as a function of tractive (or axle) power. Grade was found to have a significant effect on $\mathrm{CO}$ and $\mathrm{PM}$, and a moderate effect on $\mathrm{CO}_{2}$ and $\mathrm{NO}_{\mathrm{x}}$. The roads considered consisted of flat land, $\pm 2 \%$ grade, $\pm 5 \%$ grade, and sinusoidal terrains with maximum grade between $0 \%$ and $7 \%$. Also, speed correction factors were developed for the 3 vehicles considered to account for the effect of average speed on emissions.

Gajendran (2005) developed an emissions inventory prediction methodology for heavy-duty diesel trucks based on emissions factor tables (grams per second). Emissions factors were defined on the basis of instantaneous vehicle speed and acceleration. The effects of drive cycle, vehicle weight, and off-cycle injection strategy were studied in detail. The main interest was on $\mathrm{NO}_{\mathrm{x}}$ emissions, but $\mathrm{HC}$ and $\mathrm{CO}$ were also considered. A drive cycle with high average speed (36.94 $\mathrm{mph}$ ) was developed to extend the database which was short on high speed information; the cycle was named Inventory Highway Cycle (IHC).

Vora (2006) studied the effect of weight and duty cycle on emissions of heavy duty trucks. Vora used two-dimensional linear correlations to predict emissions rates of one cycle from emissions of other cycles. A method for emissions prediction as a function of drive cycle was proposed; the method is based on known emissions rates. Cycle metrics are the predictive parameters: average speed, average acceleration, and average inertial power $(V \cdot d V / d t$ in Equation 2.11, page 14). The method calculates time specific emissions $(\mathrm{g} / \mathrm{min})$ on the new cycle as a linear combination of the emissions on the known cycles and cycle properties; the new drive cycle is perceived as a linear combination of the known cycles. When two cycle metrics are used, the method requires emissions to be known on three cycles (in this study: Idle, HHDDT-Cruise, and HHDDT-Transient). For analysis of weight, vehicles were tested at 30,000 lbs, 56,000 lbs, and 66,000 lbs; it was not possible to establish a correlation between $\mathrm{NO}_{\mathrm{x}}$ emissions and weight over the wide range studied with only 3 levels of weight.

Strimer (2006) used WVU's MEMS to study the effect of varying terrain and weight on emissions and fuel economy of a 1996 diesel tractor truck. It was found that distance-specific $\mathrm{NO}_{\mathrm{x}}$ emissions increased about half the percentage increase in test weight and that fuel economy decreased about 0.5 $\mathrm{mi} / \mathrm{gal}$ for every $15,000 \mathrm{lbs}$ in added test weight. Driving in mountainous terrain caused fuel economy to decrease by around $1.2 \mathrm{mi} / \mathrm{gal}$ when compared with relatively flat terrain. In order to report brakespecific emissions, engine power was calculated from ECU broadcasts; alternatively, tractive power from the road load equation was used. The drawbacks of the latter approach are that it relied on estimated road grade and it did not account for powertrain losses.

The U.S. FTA (2007) compiled a report on transit bus life cycle cost and emissions estimation of MY 2007 diesel, biodiesel, CNG, and diesel-electric hybrid buses. The life cycle analysis considered 
capital and operational cost; costs were presented on lifetime, per mile, per passenger mile basis. Greenhouse gas (GHG) emissions were computed as well-to-tank and tank-to-wheels components. $\mathrm{PM}, \mathrm{NO}_{\mathrm{x}}$, and NMHC emissions were considered. Given the absence of emissions data for MY 2007 buses, emissions were estimated based on a ratio of certification levels and existing emissions tests results. All parameters were predicted at the national average speed of $12.72 \mathrm{mph}(20.47 \mathrm{~km} / \mathrm{h})$. Fuel consumption and GHGs were adjusted by $10 \%$ to account for idling and hotel loads. A summary of the unadjusted emissions results for the diesel-electric hybrid technology is presented in Table 1-3.

Khan (2009) studied 23 drive cycles and concluded that the main predictor metric for fuel consumption and emissions is average speed; with other metrics like standard deviation of speed, percent idle, characteristic acceleration, kinetic intensity, and stops per mile being closely related or predicted by average speed. Khan also studied correlations between average speed and emissions data with chassis-dynamometer data collected on 13 buses (6 lean-burn CNGs, 3 diesel-hybrids, 2 retrofitted diesels, and 2 clean diesels). In order to fill the gap in the data for cycles with higher speeds, sections of the KCM cycle were used to generate cycles with speeds above $25 \mathrm{mph}$ (31.51 $\mathrm{mph}, 34.13 \mathrm{mph}$, and $38.29 \mathrm{mph}$ with less than $10 \%$ idle). Then, the corresponding figures for emissions and fuel consumption were computed using continuous data from the chassis tests. The road-load equation and a simple model for drivetrain efficiency and auxiliary loads were used to study the effects of passenger weight and road grade on fuel consumption and emissions. The analysis of grade assumed a sinusoidal road profile. No correlation was found between weight and emissions of $\mathrm{NO}_{\mathrm{x}}, \mathrm{CO}, \mathrm{HC}$, and PM.

\subsubsection{Road Grade}

Smith (2001) evaluated the effects of grade on the performance and design of heavy-duty hybrids. A sine wave grade profile was superimposed into various drive cycles. It was concluded that the phase shift of the sine wave has important repercussions in state of charge, power requirements, and charge sustaining operation. Power requirements for constant speed climb were determined from the road load equation.

Conley (2002) worked on the optimal determination of the components of a hybrid electric SUV. The approach was based on real world vehicle activity (speed and grade) and maximization of regenerative braking. Road grade was determined from barometric pressure and GPS speed data using the hydrostatic equation $(d P / d z=-\rho \cdot g)$. Electric motors and energy storage systems were sized for a post transmission parallel configuration.

Moynahan (2005) developed a framework to verify engine power from ECU data for on-road emissions testing of a tractor truck. The procedure, based on tractive power (road load), also permitted 
estimation of driveline efficiency. Moynahan calculated road graded based on atmospheric pressure measurements and ECU vehicle speed. Altitude measurements from GPS system were found to be scrambled, such that the initial and final altitudes of a loop were different (as high as $14 \mathrm{~m}, 46 \mathrm{ft}$ ). This fact rendered little value to GPS elevation data. Altitude from pressure was smoothed with a central 10 second moving average; if the elevation-change for a given time period was above a threshold (35 ft, $0.2 \mathrm{~s}$ ), the reading was neglected.

Hannon et al. (2007) used distance based elevation profiles on chassis dynamometer testing of brake components. The elevation profiles were obtained by driving the routes of interest with a combination of GPS unit and altimeter. The software on the chassis dynamometer was then programmed to receive the elevation-distance profile.

Khan (2009) used a sine wave grade profile superimposed on existing drive cycles. He developed a fuel consumption correction factor for grade. The correction factor was a function of average speed and maximum grade attained. The correction factor ranged from about $5 \%$ for $2 \%$ grade to about $30 \%$ for $5 \%$ grade.

\subsubsection{Hybrid Vehicle Simulation}

Smith (2001) performed Microsoft Excel based fuel economy simulations of Class 2B, Class 6, and Class 8 hybrid vehicles. Optimum engine size, motor size, and control strategy parameters were determined for series and parallel architectures under a variety of operations (from low to freeway speed). The model was power based and did not account for torque or engine maps. The effect of grade was also considered.

Ciccarelli and Toossi (2002) performed simulation studies to evaluate potential fuel savings and reduced emissions from hybrid buses on Long Beach City, CA, buses. Conventional diesel, dieselelectric hybrid (series and parallel), and gas turbine-hybrid architectures were compared. Fuel economy was estimated by simulation, using ADVISOR. Emissions results were taken from 1997 to 1999 model year conventional diesel and series diesel-electric hybrid buses tested at the WVU's TransLab under a contract with the Northeast Advanced Vehicle Consortium (NAVC). Emissions were modeled for gas turbine buses but not for diesels. The selection of the Long Beach Transit bus routes used in the study was based on volume of riders and number of stops. The drive cycles used for fuel economy modeling were: CBD, Colorado 16th St. Mall, and Long Beach Routes 1 and 192. The researchers recognized that hybrid architectures and control strategies can be selected for optimal operation under specific driving patterns or road conditions. Optimized operation was viewed as maximum fuel economy, minimal emissions, or a combination of both. 
In modeling the 40' New Flyer diesel buses, Ciccarelli and Toossi (2002) used the parameters shown in Table 3-2, and the following: gear ratios $=3.49,1.86,1.41,1.00,0.75$, rear axle ratio $=4.04$, and engine peak power $=275 \mathrm{hp} 9(205 \mathrm{~kW})$, among others.

O'Keefe and Vertin (2002) presented an analysis of hybrid electric systems for transit buses. Simulations predicted that fuel economy can be improved 1.7 times over the baseline vehicle. The researchers concluded that the keys to increasing fuel economy are hybridization, weight reduction, and auxiliary load reduction. A parametric analysis was performed to estimate the effects on fuel economy of vehicle weight, mechanical and electrical auxiliary loads, drive cycle, rolling resistance, and aerodynamic drag. The study considered $\mathrm{CNG}$ and diesel fueled transit buses with conventional and series-hybrid architectures. An energy audit analysis was presented, in which energy use and losses were broken up. Losses included engine, auxiliary loads, drivetrain, generator, energy storage system, motor/controller, friction braking, aerodynamic drag, and rolling resistance. The regenerative braking capture efficiency was estimated to be as high as $49 \%$. The effects of air conditioning were modeled; the models considered solar loading, cabin thermal-fluid, and transient air conditioning. When run over a steady-state high speed cycle (Commuter cycle), the series-hybrid transit bus was observed to lose its fuel economy advantage over the conventional bus.

The vehicle modeled by O'Keefe and Vertin (2002) was a 40' series diesel hybrid transit bus; they obtained fuel economy of $3.32 \mathrm{mpg}$ with $\mathrm{A} / \mathrm{C}$ on and $4.26 \mathrm{mpg}$ with $\mathrm{A} / \mathrm{C}$ off over the $\mathrm{CBD}$ cycle. The model considered: weight $=15,940 \mathrm{~kg}$; Auxiliary loads $=23 \mathrm{~kW}$ mechanical and $9 \mathrm{~kW}$ electrical with $\mathrm{A} / \mathrm{C}$ on, and $9 \mathrm{~kW}$ mechanical and $9 \mathrm{~kW}$ electrical with $\mathrm{A} / \mathrm{C}$ off; engine $=230 \mathrm{hp} 7.3 \mathrm{~L}$ Navistar T444E/DDC S30, 44\% peak efficiency; Transmission = single reduction direct drive, final drive ratio of 6.34 ; electric motor $=187 \mathrm{~kW} \mathrm{AC,} 92 \%$ peak efficiency; battery $=46$ modules, 85 Amp-hr, 3400 lbs.

Rajagopalan et al. (2003) developed a fuzzy logic controller for parallel hybrid vehicles. The controller accounts for emissions while selecting the optimal torque split between the IC engine and the electric motor. The optimum torque point was based on road load, SOC, engine curves, and the relative importance of emissions and fuel consumption. A cost function was defined as a weighted sum of normalized emissions $\left(\mathrm{CO}, \mathrm{HC}\right.$, and $\left.\mathrm{NO}_{\mathrm{x}}\right)$ and efficiency; the relative importance was given by weighting factors. In the architecture studied, engine speed was set by the fixed transmission ratios and the controller did not perform speed or shift control. Emissions were provided by maps (lookup tables) indexed by engine speed and torque. Additionally, an engine scaling model called the Willan's Line Model was implemented into the NREL's ADVISOR software. The scaling model was based on torque and used data from similar engines to produce an engine-specific fuel-use map starting from basic engine information (e.g. stroke and bore); the model did not scale emissions.

Sharer et al. (2007) used computational models to study the impacts of duty cycle aggressiveness and speed of hybrid and conventional cars (2004 Toyota Prius and Ford Focus). It was observed that 
hybrids are more sensitive to drive cycle variations than conventional vehicles. Simulations were performed using PSAT software. Drive cycle aggressiveness was assessed by a cycle scaling factor which scales the speed trace. The drive cycles of choice were the light-duty UDDS (or FTP-72) and the HWFET. The models were validated against hot-start chassis-dynamometer tests; the hybrid model was considered validated if fuel consumption and delta SOC were predicted consistently within $5 \%$ on various cycles. Torques in engine, motor, and generator were also compared. Sensitivity of fuel consumption to road load was defined as

Equation 2.62 Sensitivity of Fuel Consumption to Road Load, Sharer et al. (2007)

$$
\Gamma_{\text {load }}^{f u e l}=\frac{\Delta \text { Energy }- \text { Fuel }}{\Delta \text { Energy }- \text { Road Load }} ;
$$

where the difference is calculated against the un-scaled cycle. 


\section{Chapter 3. ENERGY AND HYBRID VEHICLES}

The purpose of this chapter is to evaluate the gains than can be achieved in transit vehicles from regenerative braking. The results are presented in terms of energy at the hub or wheels of the vehicle. This analysis uses the theory presented in Section 2.1 where one of the main parameters is the regeneration efficiency, $\eta_{\text {regen }}$. In this dissertation, regeneration efficiency is defined as captureredeployment efficiency or fraction of available braking energy that is redelivered to the wheels (Sovran and Blaser, 2003 and 2006).

Although sometimes regeneration efficiency has been defined in terms of energy recuperated at the battery (e.g. Sharer et al., 2007), it is more appropriate to use energy at wheels because per Equation 2.13 the amount of energy that must be supplied by the primary power source for propulsion, $E_{\text {trac,net }}$, is calculated by subtracting recovered energy $\left(\eta_{\text {regen }} \cdot E_{B R}\right)$ from tractive energy $\left(E_{\text {trac }}\right)$, that is $E_{\text {trac } \text { net }}=E_{\text {trac }}$ $-\eta_{\text {regen }} \cdot E_{B R}$. The net tractive energy term also appears in the equations for fuel consumption, Equation 2.16 and Equation 2.17.

NAVC (2000) discussed regenerative braking concluding that not all the kinetic energy can be captured by the batteries. This is because braking is limited by traction of the tires, which makes deceleration rates higher than accelerations. Recovery of kinetic energy is limited by the capacity of the hybrid drive system to accept energy. Since hybrid systems are usually designed for acceleration performance, the energy that the system cannot take must be dissipated by the service brakes. Also, for rear wheel drive buses, braking energy available in the front wheels could not be recovered (the opposite is true for front wheel drive systems.). Other sources of losses are the batteries, electric motor/generators, power inverter, differential, and transmission. Yet another limitation for regeneration is the energy capacity ( $\mathrm{kWh}$ rating) of the battery, since in sustained descents the energy storage may become full. O'Keefe and Vertin (2002) reported regenerative capture efficiency of about $40 \%$ for a series hybrid transit bus.

Hybrid vehicles have the added extra mass of the hybrid components (electric motors, batteries, etc.), making them generally heavier than the conventional counterparts (Sovran and Blaser, 2003 and 2006). This effect is offset in part by the use of a downsized combustion engine. The ratio of hybrid weights to conventional weight, $m / m_{0}$, serves as the indicator parameter of how much the mass of the vehicle has increased; the subscript 0 refers to the conventional counterpart and no subscript means 
hybrid. The increased mass is reflected in higher propulsion energy: the term $E_{\text {trac }} / E_{\text {trac }, 0} \geq 1$ in Equation 2.17. This increase in propulsion energy is accompanied by increased braking energy, since more mass needs to be decelerated.

For light duty vehicles An and Santini (2004) report values of $m / m_{0}$ ranging from 1.03 to 1.07 ; Sovran and Blaser (2006) used a reference value of 1.05. On the heavy-duty side, the curb weight of a MY $200640 \mathrm{ft}$ New Flyer diesel transit bus is 28,500 lbs $(12,927.4 \mathrm{~kg})$ and the hybrid counterpart weights $29,900 \mathrm{lbs}(13,562.4 \mathrm{~kg})$; giving a value of $\mathrm{m} / \mathrm{m}_{0}$ equal to 1.05 . It is interesting to note that both heavy and light duty modern hybrid applications have nearly the same 5\% increase in mass.

The regeneration efficiency can be estimated from the fuel consumption Equation 2.16 and advanced simulations or test data. Knowing fuel consumption values for equivalent hybrid and conventional vehicles, one can write the ratio of hybrid to conventional fuel consumption. If the effects of idle and braking fuel consumption, accessory load, and differences in driveline and engine efficiencies are neglected, one would obtain an upper limit for $\eta_{\text {regen }}$. Such limit is

Equation 3.1 $\eta_{\text {regen }}$ limit

$$
\eta_{\text {regen }}<\frac{1}{E_{B R} / E_{\text {trac }}}\left(1-\frac{\tilde{g} / \widetilde{g}_{0}}{E_{\text {trac }} / E_{\text {trac }, 0}}\right)
$$

$\eta_{\text {regen }}$ is lower than the hypothesized value because usually the hybrids have better engine efficiency, lower idle fuel consumption, and lower accessory loads, among others.

The outline of the chapter is as follows: the effects of regeneration efficiency, vehicle weight, and road grade on tractive energy are studied in Sections 3.2, 3.3, and 3.4, respectively. Section 3.1 presents the simulation parameters that are used in the calculations.

\subsection{Simulation Parameters}

Before studying the effects mentioned above, it is important to define the values of the parameters like rolling resistance and aerodynamic drag coefficients. The evaluation will consider, for comparative purposes, one light-duty passenger vehicle, one 22' mini-bus, and a 40' transit bus. The SUV and the mini-bus are expected to have the same shape in the hybrid configuration, and as such the frontal areas are the same. In the case of the bus, the hybrid batteries are mounted on the roof, yielding a taller vehicle, with increased frontal area. The parameters for the conventional bus, the hybrid bus, the mini-bus, and the SUV are listed in Table 3-1. 
For chassis dynamometer testing the test weight is usually "half seated load weight" ( $1 / 2 \mathrm{SLW})$, which is curb weight plus half seated load weight plus the driver, with $150 \mathrm{lbs} /$ person (SAE J2711, SAE International, 2002; O'Keefe and Vertin, 2002). The 1/2SLW will be used in the simulations.

For the SUV, the baseline vehicle is the MY 2009 Honda CR-V LX 2WD' drag coefficient is taken from PSAT (vehicle file "veh_1200_266_044_SUV") and, as an estimate, it is not intended to exactly represent the CR-V. Test weight is curb weight plus 300 pounds (CFR 40: 86.082-2 and 86.129-94).

The baseline vehicle for the mini-bus is a MY 2008 Goshen Coach GCII ${ }^{\mathrm{e}}, 21$ passengers. The load is half seated load (11) plus driver. The aerodynamic drag coefficient was taken from Zia (2009) who studied coastdown procedures of USPS delivery trucks.

For the $40 \mathrm{ft}$ bus, the baseline vehicle is a MY 2006 New Flyer. The hybrid is a MY 2006 New Flyer hybrid with the GM-Allison Hybrid Drive E40. Load is half seated load (20) plus driver. Vehicle information is courtesy of the Washington Metropolitan Area Transit Authority (WMATA), Bus Engineering Department.

Table 3-1 Vehicle Properties of Bus, Mini-Bus, and SUV

\begin{tabular}{|c|c|c|c|c|c|}
\hline Parameter & Units & Conventional Bus & Hybrid Bus $^{\mathrm{f}}$ & Mini-Bus & SUV \\
\hline$G V W R$ & $\mathrm{~kg} / \mathrm{lb}$ & $18,416 / 40,600$ & $18,416 / 40,600$ & $6,373 / 14,050$ & - \\
\hline$m_{\text {curb }}$ & $\mathrm{kg} / \mathrm{lb}$ & $12,927 / 28,500$ & $13,562 / 29,900$ & $4,876 / 10,750$ & $1,536 / 3,386$ \\
\hline$m_{\text {load }}$ & $\mathrm{kg} / \mathrm{lb}$ & $1,429 / 3,150$ & $\leftarrow$ & $816 / 1,800$ & $136 / 300$ \\
\hline$m_{r} / m_{\text {curb }}$ & $\%$ & 3.0 & - & 3.0 & 3.0 \\
\hline$m_{r}$ & $\mathrm{~kg} / \mathrm{lb}$ & $388 / 855$ & $\leftarrow$ & $147 / 323$ & $46 / 102$ \\
\hline$m=m_{\text {curb }}+m_{\text {load }}$ & $\mathrm{kg} / \mathrm{lb}$ & $14,356 / 31,650$ & $14,991 / 33,050$ & $5,692 / 12,550$ & $1,672 / 3,686$ \\
\hline$m_{e}=m+m_{r}$ & $\mathrm{~kg} / \mathrm{lb}$ & $14,744 / 32,505$ & $15,379 / 33,905$ & $5,839 / 12,873$ & $1,718 / 3,788$ \\
\hline Length & $\mathrm{m} / \mathrm{ft}$ & $12.62 / 41.4$ & $12.44 / 40.8$ & $6.71 / 22$ & $4.54 / 14.9$ \\
\hline Width & $\mathrm{m} / \mathrm{in}$ & $2.59 / 102$ & $2.59 / 102$ & $2.39 / 94$ & $1.82 / 71.6$ \\
\hline Height & $\mathrm{m} / \mathrm{in}$ & $3.11 / 122.5$ & $3.38 / 133$ & $2.92 / 115$ & $1.68 / 66.1$ \\
\hline Ground Clearance & $\mathrm{m} / \mathrm{in}$ & $0.36 / 14$ & $0.36 / 14$ & $0.30 / 12$ & $0.17 / 6.7$ \\
\hline$c_{d}$ & - & 0.78 & $\leftarrow$ & 0.67 & 0.42 \\
\hline$A_{f}$ & $\mathrm{~m}^{2} / \mathrm{ft}^{2}$ & $7.14 / 76.85$ & $7.83 / 84.29$ & $6.25 / 67.24$ & $2.74 / 29.54$ \\
\hline$c_{d} \cdot A_{f}$ & $\mathrm{~m}^{2} / \mathrm{ft}^{2}$ & $5.57 / 59.94$ & $6.11 / 65.75$ & $4.19 / 45.05$ & $1.13 / 12.16$ \\
\hline$c_{r, 0}{ }^{A}$ & - & 0.008 & $\leftarrow$ & 0.009 & 0.009 \\
\hline$c_{r, 1}{ }^{A}$ & $\mathrm{~s} / \mathrm{m}$ & $1.2 \times 10^{-4}$ & $\leftarrow$ & $1.2 \times 10^{-4}$ & $1.2 \times 10^{-4}$ \\
\hline Weight Front / Rear & $\%$ & - & - & - & $58 / 42$ \\
\hline Tires & & $6 \times$ B 305/70R22.5 & $6 \times$ B 305/70R22.5 & $6 \times$ LT225/75R16 & $4 \times 225 / 65 \mathrm{R} 17$ \\
\hline Towing Capacity & $\mathrm{kg} / \mathrm{lb}$ & - & - & - & $680 / 1,500$ \\
\hline Capacity & & $\begin{array}{l}39 \text { seated }+20 \\
\text { standing }\end{array}$ & $\begin{array}{l}39 \text { seated }+20 \\
\text { standing }\end{array}$ & 21 seated & 5 seated \\
\hline
\end{tabular}

A - Equation 3.2

\footnotetext{
${ }^{\mathrm{d}} \underline{\text { http://automobiles.honda.com/cr-v/specifications.aspx }}$

${ }^{\mathrm{e}}$ http://www.goshencoach.com/GCII.aspx

${ }^{\mathrm{f}}$ Vehicle properties listed in this table are not the ones used in the final models. Please refer to Table 6-2 (page 152) for the definitive values.
} 
Table 3-2 Comparison of Hybrid Transit Bus Simulation Coefficients with Other Studies

\begin{tabular}{l|c|c|c}
\hline Parameter & This study $^{\mathbf{g}}$ & O'Keefe and Vertin (2002) $^{\prime}$ & Ciccarelli and Toossi (2002) \\
\hline$m_{c u r b}(\mathrm{~kg})$ & 13,562 & 14,170 & 13,900 \\
\hline$c_{d} \cdot A_{f}\left(\mathrm{~m}^{2}\right)$ & $0.78 \cdot 7.83=6.11$ & $0.79 \cdot 8.05=6.36$ & $0.79 \cdot 6.4=5.06$ \\
\hline$c_{r, 0}$ & 0.008 & 0.00938 & 0.008 \\
\hline$c_{r, 1}(\mathrm{~s} / \mathrm{m})$ & $1.2 \times 10^{-4}$ & - & 0 \\
\hline Architecture & Parallel & Series & Series \\
\hline
\end{tabular}

Frontal areas in Table 3-1 have been calculated as the product of vehicle width and effective height (height minus clearance). For comparative purposes, Table 3-2 presents the simulation coefficients used in other transit bus studies; the numbers show good agreement.

\subsubsection{Rolling Resistance}

The rolling resistance coefficient $c_{r}$ is affected by many parameters (Genta, 1997) and among them we can mention: vehicle speed, tire structure and materials, wear, temperature, inflation pressure and load, aspect ratio and radius, road conditions, sideslip angle, and tractive and braking forces.

The simplest rolling resistance model considers only the effect of speed in a first or second order polynomial:

\section{Equation 3.2 Rolling Resistance Coefficient}

$$
c_{r}=c_{r, 0}+c_{r, 1} \cdot V+c_{r, 2} \cdot V^{2},
$$

where $V$ is in $\mathrm{m} / \mathrm{s}, c_{r, 1}$ in $\mathrm{s} / \mathrm{m}$ and $c_{r, 2}$ in $\mathrm{s}^{2} / \mathrm{m}^{2}$. Genta (1997) reports $c_{r, 0}=0.013, c_{r, 1}=0 \mathrm{~s} / \mathrm{m}, c_{r, 2}=$ $6.5 \times 10^{-6} \mathrm{~s}^{2} / \mathrm{m}^{2}$ for a 14-inch radial tire. Values for $c_{r, 0}$ from 0.005 to 0.008 for industrial tires which exhibit an almost negligible effect of speed $\left(c_{r, 1} \approx c_{r, 2} \approx 0\right)$ are also reported.

An important parameter related to the tires is the effective rolling radius $r_{e f}$, defined as the ratio of vehicle speed to wheel angular velocity, $r_{e f}=V / \omega$. Under no load conditions or free rolling $r_{e f}$ lies between the unloaded radius $r_{w}$ and the loaded radius $r_{l}$. Under braking $r_{\text {ef }}$ grows toward the unloaded radius and under traction decreases towards the loaded radius. For the sake of simplicity, this dynamic effect can be ignored and effective and loaded radius will be assumed equal and constant: $r_{e f} / r_{w}=r_{l} /$ $r_{w}=0.95$. The loaded radius $r_{l}$ relates forces and torques; the effective radius $r_{e f}$ relates linear and angular speed. Since they are assumed equal, the effective radius will be used in place of the loaded radius.

\footnotetext{
${ }^{\mathrm{g}}$ Vehicle properties listed in this table are not the ones used in the final models. Please refer to Table 6-2 (page 152) for the definitive values.
} 


\subsubsection{Rotational Inertia}

Equation 2.9 introduced the concept of effective inertia mass of the rotating components as $m_{r}=\frac{I_{w}}{r_{w}^{2}}+\frac{\gamma^{2} I_{e}}{r_{w}^{2}}$. In Equation 2.9, the term $r_{w}{ }^{2}$ is actually $r_{e f} \cdot r_{l}$ because one radius comes from torque/force and the other one from linear/rotational speed. With the above assumptions, the rotational inertial mass is

Equation 3.3 Rotational Inertial Mass

$$
m_{r}=\frac{I_{w}}{r_{e f}^{2}}+\frac{\gamma^{2} I_{e}}{r_{e f}^{2}}
$$

SAE J2264 (1995) suggests a value for rotational inertial mass in light-duty vehicles of 3\% the mass of the vehicle.

The mass moment of inertia of the bus wheels is approximated as follows. Assuming a cylindrical shape, the moment of inertia of a cylinder is $I_{w}=1 / 2 m \cdot R^{2}$. Thus the ratio of moments of inertia of bus to car wheels would be

\section{Equation 3.4 Ratio of Moments of Inertia}

$$
I_{w, \text { bus }} / I_{w, \text { car }}=\left(m_{w, \text { bus }} / m_{w, \text { car }}\right) \cdot\left(r_{w, \text { bus }} / r_{w, \text { car }}\right)^{2},
$$

that is, the wheel weight ratio times the squared radius ratio. Now consider a thin ring with all the mass concentrated in the outside diameter, the mass moment of inertia is $I_{w}=m \cdot R^{2}$ (twice that of the cylinder). If both wheels are assumed either a cylinder or a ring, the ratio $I_{w, b u s} / I_{w, c a r}$ remains unchanged, as long as the weight distribution is equivalent. Therefore, the moment of inertia of bus tires can be roughly estimated if the geometry and a reference value for $I_{w, c a r}$ are known.

For 205/70R15 car wheel with steel rim, $m_{w, c a r}=20.76 \mathrm{~kg}$ and $r_{w, c a r}=0.330 \mathrm{~m}$. A heavy-duty $305 / 70 / \mathrm{R} 22.5$ wheel with steel rim has $m_{w, b u s}=77.5 \mathrm{~kg}$ and $r_{w, b u s}=0.495 \mathrm{~m}$. Replacing in Equation 3.4: $I_{w, \text { bus }} / I_{w, \text { car }}=(77.5 \mathrm{~kg} / 20.76 \mathrm{~kg}) \cdot(0.495 / 0.330)^{2}=3.73 \cdot(1.5)^{2}=3.73 \cdot 2.25 ; I_{w, \text { bus }} / I_{w, \text { car }} \approx 8.4$.

A reference value of $I_{w, c a r} \approx 1 \mathrm{~kg} \cdot \mathrm{m}^{2}$ was obtained from PSAT for light-duty wheels. Using $I_{w, c a r}$ $=1 \mathrm{~kg} \cdot \mathrm{m}^{2}$, the inertia of the bus wheel would be: $I_{w, b u s} \approx 8.4 \cdot 1 \mathrm{~kg} \cdot \mathrm{m}^{2}=8.4 \mathrm{~kg} \cdot \mathrm{m}^{2}$. Now, if $I_{w, c a r}$ was $1.5 \mathrm{~kg} \cdot \mathrm{m}^{2}, I_{w, \text { bus }} \approx 8.4 \cdot 1.5 \mathrm{~kg} \cdot \mathrm{m}^{2}=12.6 \mathrm{~kg} \cdot \mathrm{m}^{2}$. An intermediate value of $\boldsymbol{I}_{\boldsymbol{w}, \text { bus }}=\mathbf{1 1} \mathbf{~ k g} \cdot \mathbf{m}^{2}$ per wheel will be used in this dissertation. 


\subsection{Effect of Regeneration Efficiency on Net Tractive Energy}

Net tractive energy was defined in Equation 2.13 as the net energy used for propulsion during a duty cycle:

$$
E_{\text {trac,net }}=E_{\text {trac }}-\eta_{\text {regen }} \cdot E_{B R} \text {. }
$$

Alternatively, the mean net tractive energy $(\mathrm{Wh} / \mathrm{km})$, which is $E_{\text {trac, net }}$ per unit distance, was defined:

$$
e_{\text {trac,net }}=\frac{E_{\text {trac,net }}}{D} \text {. }
$$

In Equation 2.13, the first term in the RHS is the total positive propulsion energy, i.e. the tractive energy; the second term is the fraction of braking energy recovered. These terms were defined in Equation 2.14 and Equation 2.15:

$$
\begin{gathered}
\left.E_{\text {trac }}=\int_{0}^{T} \dot{E}_{\text {road }}\right)^{+} d t \cong \sum_{\bar{E}_{\text {road }, i}>0} \overline{\dot{E}}_{\text {road }, i} \cdot \Delta t_{i}, \\
\left.E_{B R}=\int_{0}^{T} \mid \dot{E}_{\text {road }}\right)^{-}\left|d t \cong \sum_{\bar{E}_{\text {road }, i}<0}\right| \overline{\dot{E}}_{\text {road }, i} \mid \cdot \Delta t_{i} .
\end{gathered}
$$

Where $\dot{E}_{\text {road }}$ is the tractive power of Equation 2.11:

$$
\dot{E}_{\text {road }}=\frac{1}{2} \rho_{\text {Air }} c_{d} A_{f} V_{r}^{2} V+\left(b V+c V^{2}\right)+c_{r} m g V \cos (\alpha)+m g V \sin (\alpha)+m_{e} V \frac{d V}{d t} .
$$

The inverse of $e_{\text {trac,net }}$ is called the Energy at Wheels Efficiency (E.W.E., $\mathrm{km} / \mathrm{kWh}$ ) and, similarly to fuel economy, represents the distance traveled per unit of mechanical energy at the wheels (or axle to be more precise). Energy usage results will be presented alternatively as $e_{\text {trac,net }}$ or E.W.E. Emphasis is placed here in the "at wheels" part so that E.W.E. is differentiated from the overall energy efficiency used in hybrid and electric vehicles which has the same units of $\mathrm{km} / \mathrm{kWh}$.

Using the above equations and the parameters from Table 3-1, a comparison of tractive energy usage for the SUV, mini-bus, and transit bus was performed. For each vehicle class, three scenarios were considered: i) conventional powertrain, ii) hybrid powertrain with $50 \%$ capture-redeployment efficiency $\left(\eta_{\text {regen }}=0.5\right)$, and $\left.i i i\right)$ hybrid powertrain with perfect recuperation $\left(\eta_{\text {regen }}=1\right)$. The following assumptions apply: $i$ ) air-standard conditions, $i i)$ winds are not considered $\left(V_{r}=V\right)$, iii) driveline drag is neglected ( $b=c=0)$, $i v$ ) the SUV is exercised over the FTP-72 and SC03 schedules, the mini-bus over the OCTA (Figure 2-5) and CBD (Figure 2-3), and the transit bus over the KCM (Figure 2-6), OCTA, and CBD cycles.

Figure 3-1 and Table 3-3 show a break-up of the energy usage $(\mathrm{Wh} / \mathrm{km})$ to overcome inertia (yellow shade), rolling resistance (purple), and aerodynamic drag (cyan). In Figure 3-1 the mean net 
tractive energy $e_{\text {trac,net }}$ is the total height of each column. Figure 3-2 presents the corresponding E.W.E. $(\mathrm{km} / \mathrm{kWh})$.

The results of Table 3-3 show that the rolling resistance component $e_{\text {mech }}$ is higher in the CBD cycle than in the OCTA cycle. This is because in the OCTA cycle most of the operation is at low speed (below $20 \mathrm{mph}$ ); analysis of the drive cycles shows that the CBD has a large portion of cruise operation at $20 \mathrm{mph}(\approx 60 \%$ of the time), and nearly $40 \%$ of the driving time is below $20 \mathrm{mph}$, whereas in the OCTA cycle a larger portion of the driving time $(60 \%)$ is below $20 \mathrm{mph}$. The inertial component, $e_{\text {inertia }}$, is higher in the OCTA cycle than in the CBD due to the more transient character of the first; this character is made clear when the characteristic acceleration (supplied kinetic energy per unit distance, Section 2.2.2) of both cycles is compared (Table 2-6): $0.71 \mathrm{ft} / \mathrm{s}^{2}$ for OCTA and $0.57 \mathrm{ft} / \mathrm{s}^{2}$ for CBD. The transient character of the drive cycle is also measured in part by the standard deviation of speed without idle. Aerodynamic losses are greater in the KCM because of the higher speeds.

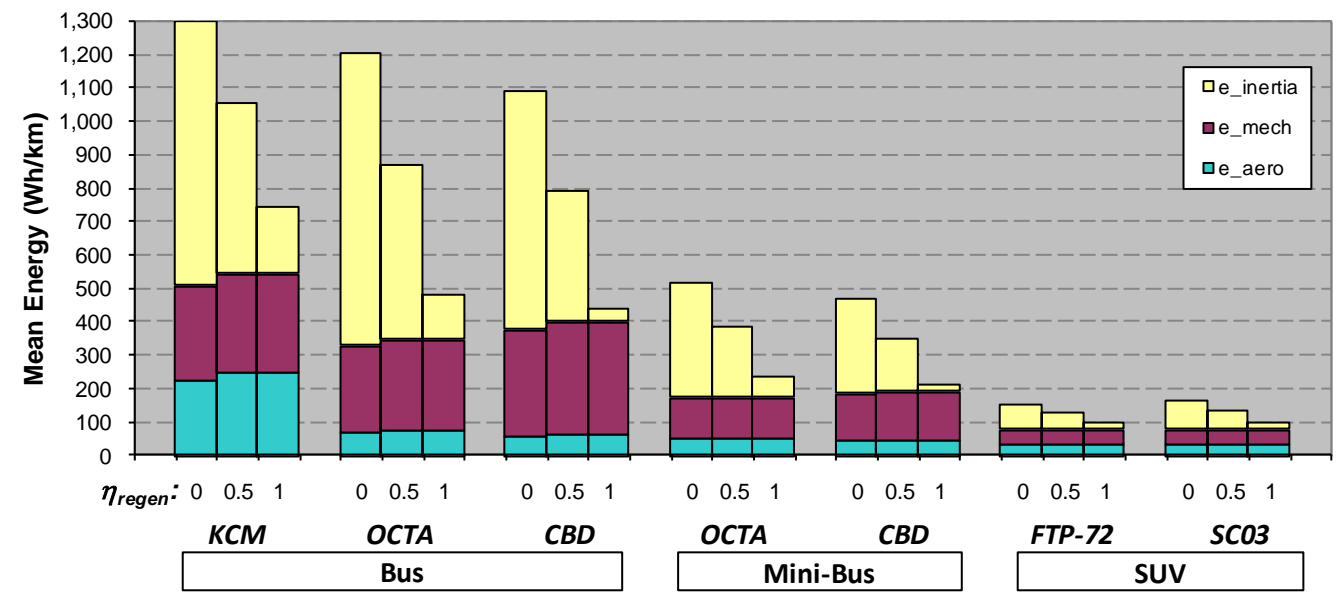

Figure 3-1 Effect of Energy Recuperation on Mean Energy of Transit Bus, Mini-Bus, and SUV

Table 3-3 Effect of Energy Recuperation on Mean Energy of Transit Bus, Mini-Bus, and SUV

\begin{tabular}{|c|c|c|c|c|c|c|c|c|c|}
\hline$\eta_{\text {regen }}$ & $\mathbf{0}$ & 0.5 & 1 & $\mathbf{0}$ & 0.5 & 1 & 0 & 0.5 & 1 \\
\hline & \multicolumn{9}{|c|}{ Transit Bus } \\
\hline & \multicolumn{3}{|c|}{ OCTA } & \multicolumn{3}{|c|}{$C B D$} & \multicolumn{3}{|c|}{$K C M$} \\
\hline$e_{\text {aero }}(\mathrm{Wh} / \mathrm{km})$ & 70.1 & 76.9 & 76.9 & 60.8 & 66.7 & 66.7 & 224.8 & 247.0 & 247.0 \\
\hline$e_{\text {mech }}(\mathrm{Wh} / \mathrm{km})$ & 258.7 & 270.1 & 270.1 & 316.2 & 330.2 & 330.2 & 284.5 & 297.9 & 297.9 \\
\hline$e_{\text {inertia }}(\mathrm{Wh} / \mathrm{km})$ & 870.5 & 517.6 & 127.2 & 708.5 & 390.0 & 40.9 & 790.8 & 509.5 & 195.2 \\
\hline \multirow[t]{3}{*}{$e_{\text {trac, net }}(\mathrm{Wh} / \mathbf{k m})$} & $1,199.4$ & 864.7 & 474.3 & $1,085.6$ & 786.9 & 437.9 & $1,300.1$ & $1,054.4$ & 740.1 \\
\hline & \multicolumn{6}{|c|}{ Mini-Bus } & & & \\
\hline & \multicolumn{3}{|c|}{ OCTA } & \multicolumn{3}{|c|}{$C B D$} & & & \\
\hline$e_{\text {aero }}(\mathrm{Wh} / \mathrm{km})$ & 53.7 & 53.7 & 53.7 & 45.8 & 45.8 & 45.8 & & & \\
\hline$e_{\text {mech }}(\mathrm{Wh} / \mathrm{km})$ & 115.8 & 120.8 & 120.8 & 139.3 & 145.3 & 145.3 & & & \\
\hline$e_{\text {inertia }}(\mathrm{Wh} / \mathrm{km})$ & 342.1 & 207.7 & 59.0 & 280.6 & 155.8 & 19.2 & & & \\
\hline$e_{\text {trac,net }}(\mathrm{Wh} / \mathbf{k m})$ & 511.6 & 382.1 & 233.4 & 465.7 & 346.8 & 210.3 & & & \\
\hline
\end{tabular}




\begin{tabular}{|c|r|r|r|r|r|r|}
\hline & \multicolumn{6}{|c|}{ SUV } \\
\cline { 2 - 7 } & \multicolumn{2}{|c|}{ FTP-72 } & \multicolumn{3}{|c|}{ SC03 } \\
\hline$e_{\text {aero }}(\mathrm{Wh} / \mathrm{km})$ & 36.4 & 36.4 & 36.4 & 36.4 & 36.2 & 36.2 \\
\hline$e_{\text {mech }}(\mathrm{Wh} / \mathrm{km})$ & 37.3 & 39.0 & 39.0 & 37.0 & 38.4 & 38.4 \\
\hline$e_{\text {inertia }}(\mathrm{Wh} / \mathrm{km})$ & 74.7 & 48.3 & 18.4 & 87.8 & 57.3 & 22.3 \\
\hline $\boldsymbol{e}_{\text {trac, } \text {, } e \text { t }}(\mathbf{W h} / \mathbf{k m})$ & $\mathbf{1 4 8 . 5}$ & $\mathbf{1 2 3 . 7}$ & $\mathbf{9 3 . 8}$ & $\mathbf{1 6 1 . 3}$ & $\mathbf{1 3 1 . 9}$ & $\mathbf{9 6 . 9}$ \\
\hline
\end{tabular}

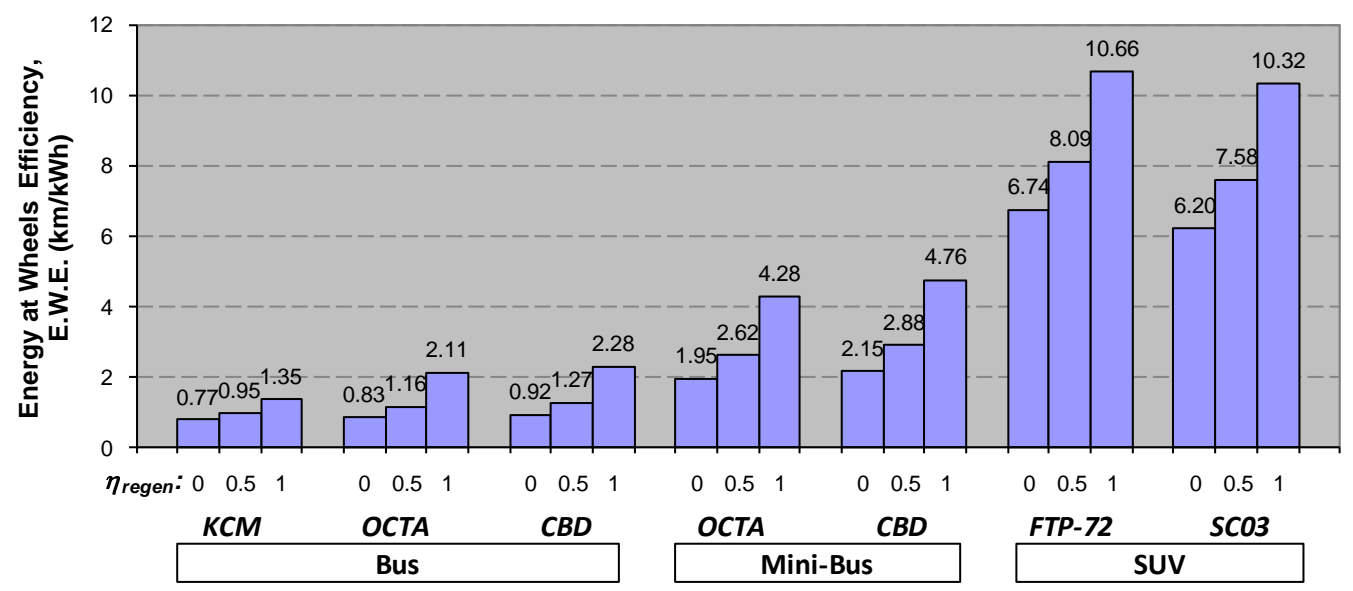

Figure 3-2 Effect of Energy Recuperation on Energy at Wheels Economy of Transit Bus, Mini-Bus, and SUV

Regarding the aerodynamic losses, it is observed that the $9.7 \%$ increase in frontal area of the hybrid bus $\left(7.14 \mathrm{~m}^{2}\right.$ to $\left.7.83 \mathrm{~m}^{2}\right)$ is reflected in the same percentage increase in aerodynamic load $e_{\text {aero. As }}$ expected, no change is observed for the mini-bus. A slight change in $e_{\text {aero }}$ is noted for the SUV in the $\mathrm{SC} 03$ cycle; this is is due to fact that here $e_{\text {aero }}$ is only summed for positive tractive power and the change in vehicle mass affects the integrals.

Regenerative braking has an impressive effect on the mean net tractive energy $e_{\text {trac, net }}$. In the case of the transit bus driving the OCTA cycle, regeneration efficiency of $50 \%$ reduces $e_{\text {trac,net }}$ by $28 \%$ (1119.4 to $864.7 \mathrm{Wh} / \mathrm{km})$ and the ideal regeneration efficiency by $60 \%$ (1119.4 to $474.3 \mathrm{Wh} / \mathrm{km})$. Similar conclusions can be drawn from Figure 3-2, where E.W.E. (km/kWh) could be more than doubled in the ideal case $(0.83$ to $2.11 \mathrm{~km} / \mathrm{kWh})$ and increased by nearly $40 \%$ with $\eta_{\text {regen }}=50 \%(0.83$ to $1.16 \mathrm{~km} / \mathrm{kWh}$ ).

As can be noted in Figure 3-1, not all the supplied inertia can be recovered, i.e. $e_{\text {inertia }}$ is not zero when $\eta_{\text {regen }}=1$. This inertial energy is transformed in aerodynamic and rolling losses during the braking sections. Therefore, when $\eta_{\text {regen }}=1, e_{\text {trac,net }}$ is the energy used to overcome the dissipative forces of the tractive power: aerodynamic and rolling resistance (plus transmission losses if they were included).

It is observed that mean net tractive energy $e_{\text {trac,net }}$ of passenger vehicles (represented here by the SUV) is one order of magnitude lower than that of transit buses. The opposite is true for E.W.E. This 
means that if, for example, a passenger vehicle travels ten miles for a given amount of energy input, a transit bus would travel just one mile with the same tractive energy; also that, ignoring other factors, a transit bus with more than 10 passengers will pollute less than the same number of people driving their cars. These findings are in line with the order of magnitude difference in vehicle mass (Table 3-1).

Figure 3-3 combines the data from Figure 3-2 into a continuous graph showing the percentage increase in E.W.E (compared to E.W.E. of the conventional baseline powertrain) as the regeneration efficiency is varied from 0 to 1 . It is observed that the benefits from energy recuperation are much higher in heavy-duty vehicles; which means that improvements on $\eta_{\text {regen }}$ will be a key factor in future development of the hybrid technology. Note that even though fuel economy figures, in miles per gallon, can be inflated in plug-in hybrids, the distance traveled per unit energy (E.W.E.) has an upper limit dictated by $\eta_{\text {regen }}=1$. All remaining improvements have to be made by decreasing fuel consumption due to hotel loads, during braking and idle (see Equation 2.18), and through improvements in engine efficiency.

Note that the KCM cycle offers less room for improvement through regeneration; in high speed cycles a larger fraction of tractive energy is spent in friction (aerodynamic and rolling losses). This is reflected in the term $1-\eta_{\text {regen }} \cdot\left(E_{B R} / E_{\text {trac }}\right)$ in Equation 2.17 for fuel consumed in powered driving; the ratio of braking to tractive energy is around 0.45 in the $\mathrm{KCM}$ cycle, while it is 0.60 in the OCTA and CBD.

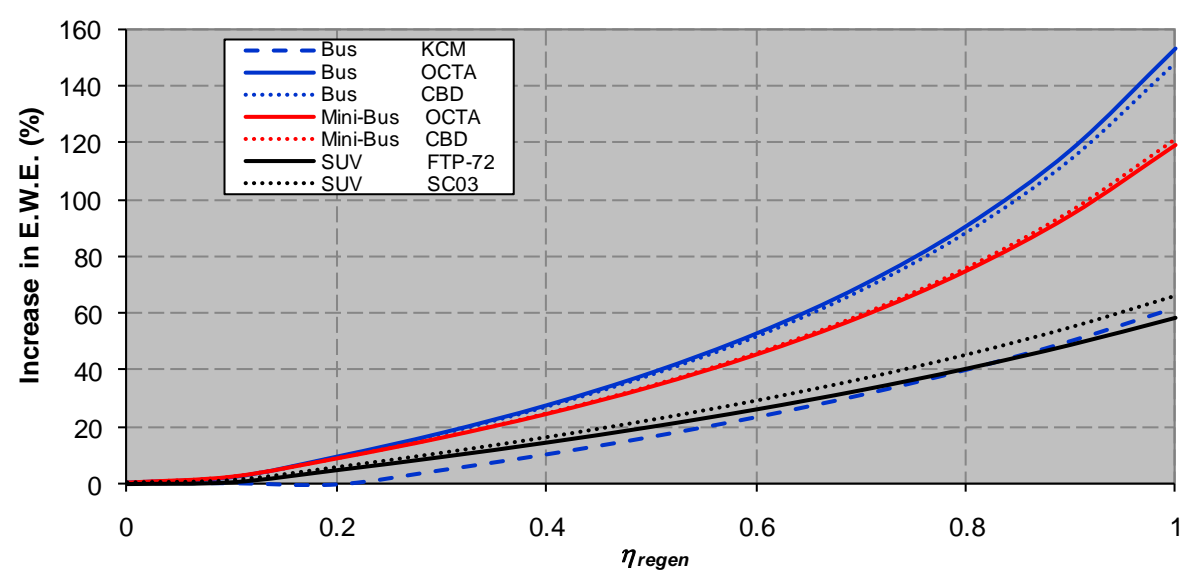

Figure 3-3 Increase in Energy at Wheels Economy (E.W.E.) vs. $\eta_{\text {regen }}$

Using fuel consumption data from Khan (2009) for MY 2005 and 2006 conventional and hybrid 40' diesel transit buses and Equation 3.1, the upper regeneration efficiency limit of Equation 3.1 was found to be $36 \%$ for the OCTA, $41 \%$ for the CBD, and $26 \%$ for the KCM-no grade cycles. 


\subsection{Effect of Test Weight on Net Tractive Energy}

The previous section explored mean net tractive energy and energy at wheels efficiency for a range of vehicle classes and the effect of regeneration efficiency. This section explores the effect of test weight on tractive energy of the 40' transit bus (Table 3-1).

Test weight is varied from empty weight to full weight. Empty weight is assumed to be curb weight plus driver's weight; full weight is GVWR plus driver's weight. These values are presented in Table 3-4; half weight is the average of empty and full weights. Note how the traditional half seated load weight $(1 / 2 \mathrm{SLW})$ is roughly halfway between empty and half weight loads. At $150 \mathrm{lb}$ per person, fully loaded weight is nearly 2,000 lbs below GVWR.

Table 3-4 Test Weights of Conventional and Hybrid Buses

\begin{tabular}{c|c|c}
\hline Test Weight, $\boldsymbol{m}$ & $\begin{array}{c}\text { Conventional } \\
(\mathbf{k g} / \mathbf{l b})\end{array}$ & $\begin{array}{c}\text { Hybrid } \\
\text { (kg / lb) }\end{array}$ \\
\hline Empty Weight & $12,995 / 28,650$ & $13,631 / 30,050$ \\
\hline 1/2SLW & $14,356 / 31,650$ & $14,991 / 33,050$ \\
\hline Half weight & $15,740 / 34,700$ & $16,057 / 35,400$ \\
\hline $\begin{array}{c}\text { Fully Loaded } \\
\text { 60 people }\end{array}$ & $17,010 / 37,500$ & $17,645 / 38,900$ \\
\hline Full Weight & $18,484 / 40,750$ & $18,484 / 40,750$ \\
\hline
\end{tabular}

Table 3-5 Effect of Test Weight and $\eta_{\text {regen }}$ on Mean Tractive Energy

\begin{tabular}{|c|c|c|c|c|c|c|}
\hline Test Weight & Empty & Half & Full & Empty & Half & Full \\
\hline \multirow[b]{2}{*}{$(\mathrm{Wh} / \mathrm{km})$} & \multicolumn{6}{|c|}{$\eta_{\text {regen }}=\mathbf{0}$} \\
\hline & \multicolumn{3}{|c|}{$O C T A$} & \multicolumn{3}{|c|}{$C B D$} \\
\hline$e_{\text {trac }, \text { net }}=e_{\text {trac }}$ & $1,094.5$ & $1,306.0$ & $1,517.6$ & 990.3 & $1,182.6$ & $1,374.9$ \\
\hline$e_{B R}$ & 678.8 & 822.1 & 965.5 & 607.5 & 732.8 & 858.0 \\
\hline$e_{\text {aero }}$ & 70.1 & 69.9 & 69.7 & 60.8 & 60.8 & 60.8 \\
\hline$e_{\text {mech }}$ & 234.2 & 282.6 & 331.0 & 286.3 & 346.7 & 407.2 \\
\hline \multirow{3}{*}{$e_{\text {inertia }}$} & 790.2 & 953.5 & $1,116.9$ & 643.1 & 775.0 & 906.9 \\
\hline & \multicolumn{6}{|c|}{$\eta_{\text {regen }}=0.5$} \\
\hline & \multicolumn{3}{|c|}{$O C T A$} & \multicolumn{3}{|c|}{$C B D$} \\
\hline$e_{\text {trac }}$ & $1,150.3$ & $1,337.3$ & $1,524.3$ & $1,040.7$ & $1,210.8$ & $1,380.8$ \\
\hline$e_{B R}$ & 709.8 & 836.5 & 963.3 & 636.1 & 746.8 & 857.6 \\
\hline$e_{\text {aero }}$ & 77.2 & 76.8 & 76.6 & 66.7 & 66.7 & 66.7 \\
\hline$e_{m e c h}$ & 246.6 & 288.9 & 331.5 & 300.3 & 353.7 & 407.2 \\
\hline$e_{\text {inertia }}$ & 471.6 & 553.3 & 634.6 & 355.7 & 416.9 & 478.1 \\
\hline \multirow[t]{3}{*}{$e_{\text {trac, } n e t}$} & 795.4 & 919.0 & $1,042.7$ & 722.7 & 837.3 & 952.0 \\
\hline & \multicolumn{6}{|c|}{$\eta_{\text {regen }}=1$} \\
\hline & \multicolumn{3}{|c|}{ OCTA } & \multicolumn{3}{|c|}{$C B D$} \\
\hline$e_{\text {aero }}$ & 77.2 & 76.8 & 76.6 & 66.7 & 66.7 & 66.7 \\
\hline$e_{m e c h}$ & 246.6 & 288.9 & 331.5 & 300.3 & 353.7 & 407.2 \\
\hline$e_{\text {inertia }}$ & 116.7 & 135.0 & 153.0 & 37.6 & 43.5 & 49.3 \\
\hline$e_{\text {trac, } n e t}$ & 440.5 & 500.8 & 561.0 & 404.6 & 463.9 & 523.2 \\
\hline
\end{tabular}


Table 3-5 shows the simulation results. The table presents distance specific tractive, braking, aerodynamic drag, rolling resistance, and inertial energies; $e_{\text {trac,net }}$ was calculated per Equation 2.13. The simulations were carried out for the OCTA and CBD schedules. The test weights of the conventional bus were used for $\eta_{\text {regen }}=0$; the weights of the hybrid for $\eta_{\text {regen }}>0$. Tractive and braking energies $\left(e_{t r a c}\right.$ and $\left.e_{B R}\right)$ are a function of test weight, vehicle properties, and drive cycle; therefore since for the hybrid bus $\eta_{\text {regen }}$ was assumed independent of vehicle weight, $e_{t r a c}$ and $e_{B R}$ are the same for all values of $\eta_{\text {regen }}$.

Figure 3-4 presents a plot of test weight versus $e_{\text {trac,net }}$ for $\eta_{\text {regen }}=0,0.5$, and 1 . The figure is conclusive to show that for a given drive cycle $e_{\text {trac,net }}$ is a perfect linear function of test weight. The correlation coefficients were calculated for all the curves, obtaining $r^{2}=1.000$. Here again, the predicted energy savings of regenerative braking are pretty significant.

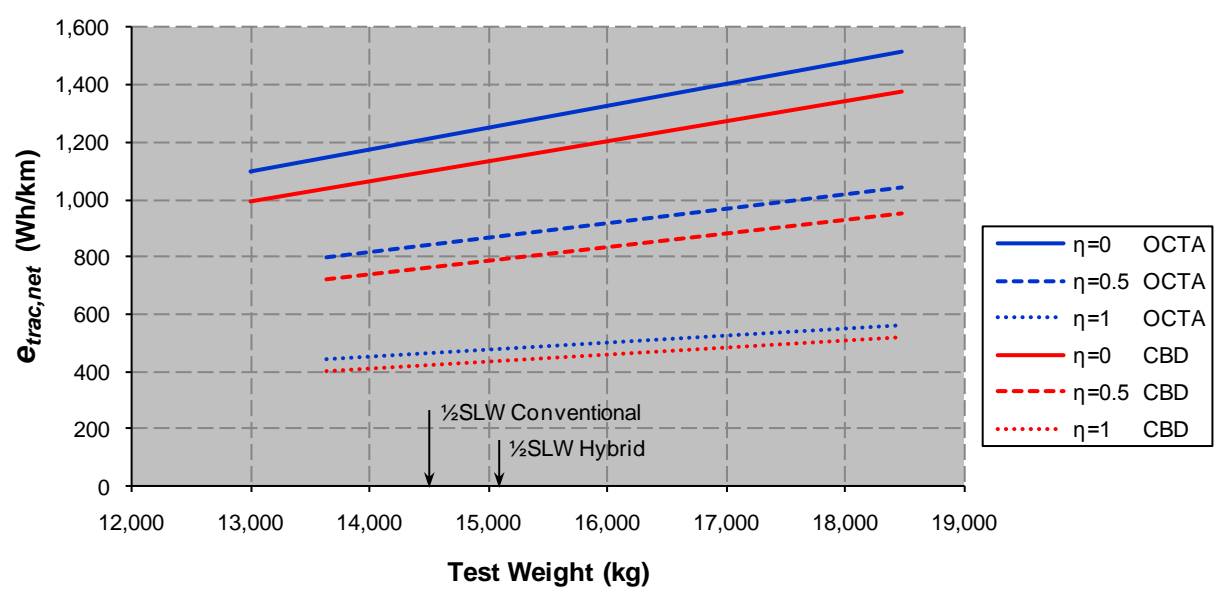

Figure 3-4 Effect of Test Weight and $\eta_{\text {regen }}$ on Mean Net Tractive Energy

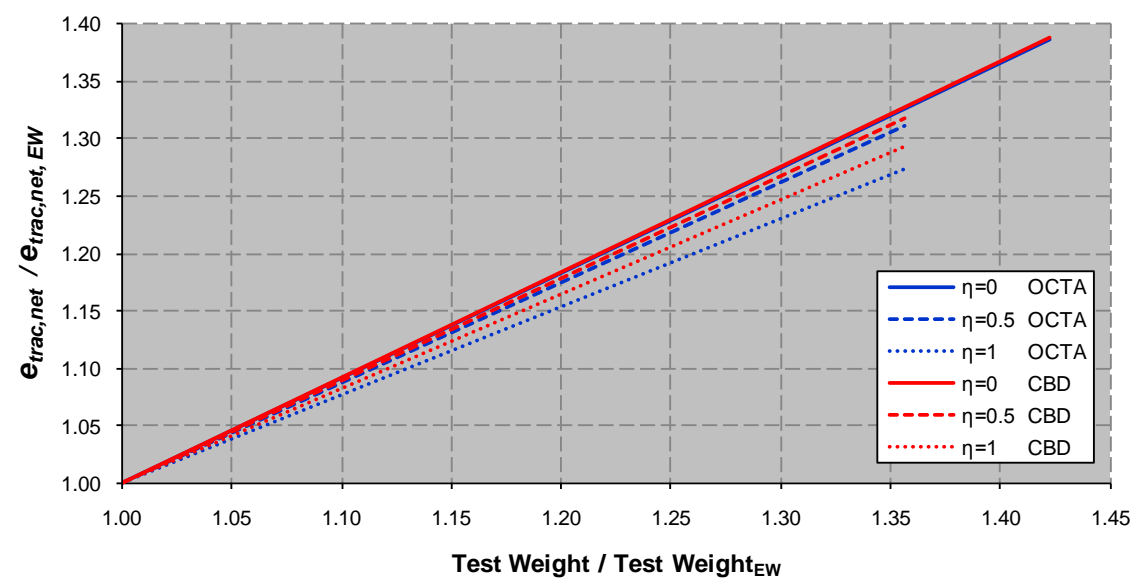

Figure 3-5 Ratio of Net Tractive Energy and Test Weight to Empty Weight Values 
Figure 3-5 shows $e_{\text {trac,net }}$ vs. test weight divided by the corresponding values for empty weights, that is $e_{\text {trac,net }} / e_{\text {trac, net,EW }}$ and $m / m_{E W}$. The subscript EW indicates reference to empty weight. This figure shows the increase in net tractive energy due to an increase in test weight; for example, for the conventional bus $\left(\eta_{\text {regen }}=0\right)$ a $14 \%$ increase in test weight, above empty weight, is reflected in a $13 \%$ increase in net tractive energy.

The ratio of loaded to empty $e_{\text {trac,net }}$ can be expressed, after some algebra, as:

Equation $3.5 e_{\text {trac,net }} / e_{\text {trac, net,EW }}$

$$
\frac{e_{\text {trac }, \text { net }}}{e_{\text {trac }, \text { net }, E W}}=\frac{e_{\text {trac }}}{e_{\text {trac }, E W}} \cdot \frac{1-\eta_{\text {regen }} \cdot e_{B R} / e_{\text {trac }}}{1-\eta_{\text {regen }} \cdot\left(e_{B R} / e_{\text {trac }}\right)_{E W}} .
$$

From Equation 3.5, it is clear that the key parameters are mean tractive energy, $e_{t r a c}$, and the ratio of braking to tractive energy, $e_{B R} / e_{t r a c}$. Mean tractive energy $\left(e_{t r a c}\right)$ varies linearly with test weight for a given drive cycle; the ratio $e_{B R} / e_{t r a c}$ is almost insensitive to test weight with an increase of no more than $2.5 \%$ from empty to full weight.

Once other factors like engine and transmission efficiency are considered (Equation 2.16), the effect of weight on fuel consumption will not look nearly as linear (Clark et al., 2007a). To contrast the influence of these factors, Clark et al. (2007a) report for a 40' transit bus with a MY 2003 DDC S50, $272 \mathrm{hp}$, diesel engine that increase in test weight above empty weight of $14 \%$ and $32 \%$ represented increase in fuel consumption of $6 \%$ and 19\% respectively (OCTA cycle). In regard to the above discussion, the $13 \%$ increase in tractive energy (14\% increase weight) is achieved by a $6 \%$ increase in fuel consumption. Also, for the $32 \%$ increase in test weight, the increase in mean net tractive energy for the OCTA cycle is $29 \%$; this increase in $e_{\text {trac, net }}$ was achieved by a $19 \%$ increase in fuel consumption. This corroborates that the engine is more efficient at higher loads.

\subsection{Effect of Grade on Tractive Energy}

Smith (2001) and Khan (2009) proposed evaluating road grade as a sine wave where grade is a function of distance. The grade profile is then superimposed on standard drive cycles. The general sinusoidal grade function is shown Figure 3-7 and can be expressed as:

Equation 3.6 Sine Wave Grade

$$
\text { Grade }=G_{0} \sin \left(2 \pi \cdot \frac{s-D_{\phi}}{D_{0}}\right) .
$$

Where $G_{0}$ is the maximum (and minimum) road grade (\%), $s$ is distance, $D_{0}$ is the distance traveled for one cycle or hill, and $D_{\phi}$ is the phase shift of the wave. The top of the hill $\left(h_{\max }\right)$ is reached at $D_{\phi}+$ 
$D_{0} / 2 ; h_{\max }$ is determined by integration: $h_{\max }=\int_{D_{\phi}}^{D_{\phi}+D_{0} / 2} \sin \alpha d s$, where $\alpha=\tan ^{-1}($ Grade $/ 100)$. For practical purposes $D_{\phi} \in\left[0, D_{0}\right)$.

Alternatively, a square wave function can be used to define grade. Figure 3-7 shows this function and the corresponding elevation profile. Grade is $G_{0}$ until $D_{\phi}+D_{0} / 2$ when is jumps to $-G_{0}$ until $D_{\phi}+$ $D_{0}$, where it completes one cycle. This concept was used in the KCM cycle where each section has a different value of $G_{0}$.

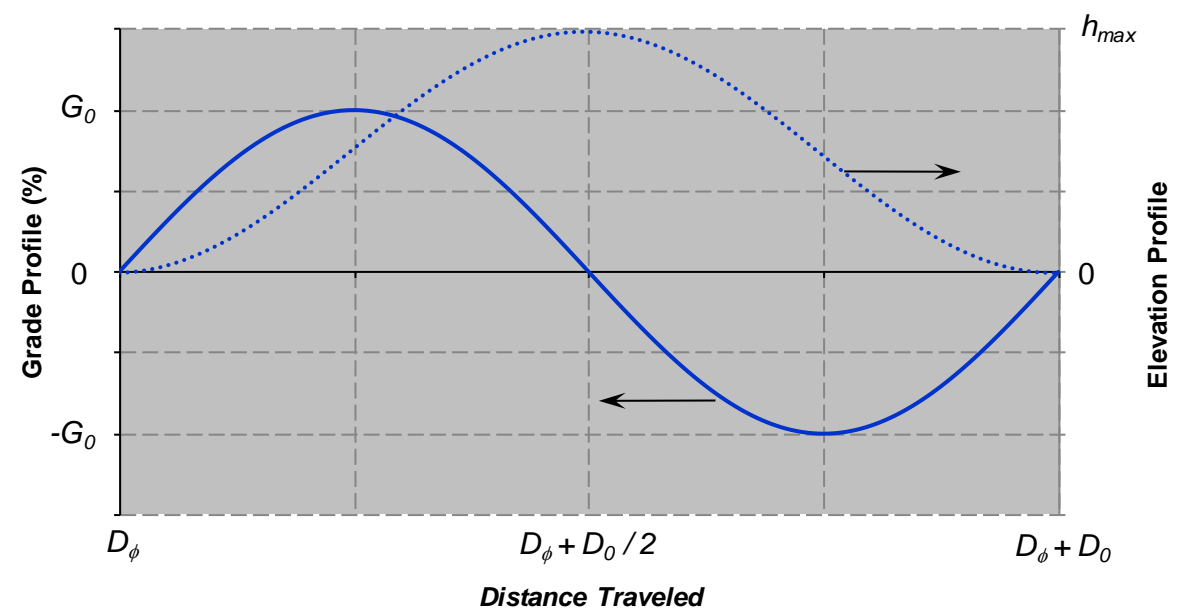

Figure 3-6 Sine Wave Grade Function and Elevation

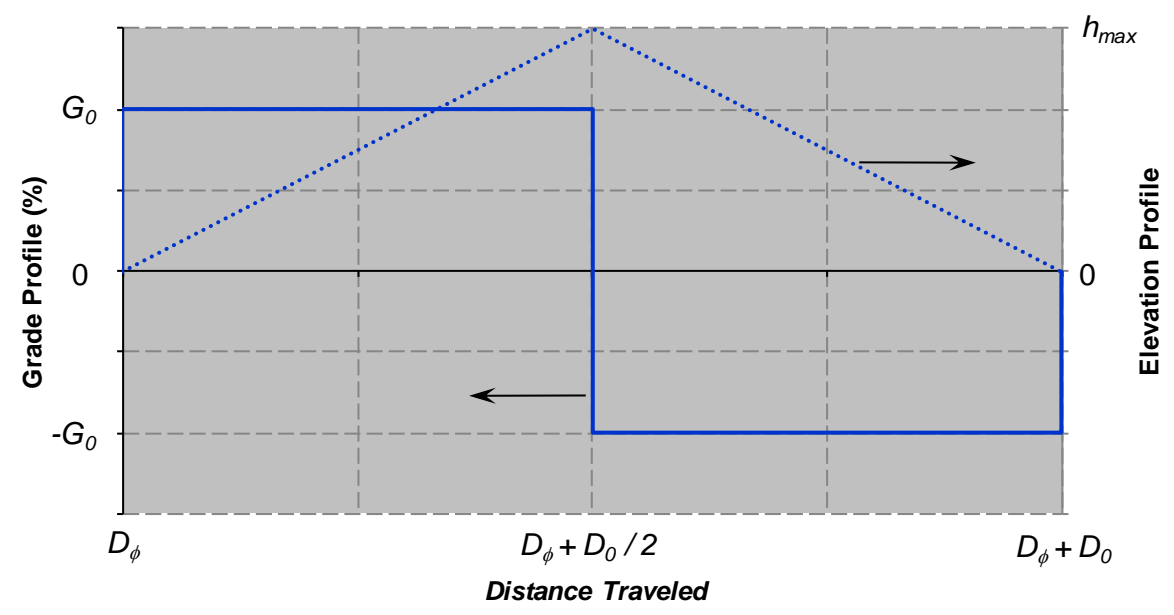

Figure 3-7 Square Wave Grade Function and Elevation

An analysis of variable maximum road grade $G_{0}$ was performed with values from 0 (flat terrain) to $10 \%$ grade. This is equivalent to driving the route in increasingly higher hills. Values selected for $D_{0}$ and $D_{\phi}$ were $0.5 \mathrm{mi}(0.805 \mathrm{~km})$ and 0 , respectively. The conventional transit bus of Table 3-1 was used for the analysis. The OCTA $(\bar{V}=12.3 \mathrm{mph}), \mathrm{CBD}(\bar{V}=12.9 \mathrm{mph})$, and $\mathrm{KCM}(\bar{V}=23.4 \mathrm{mph})$ cycles were selected for simulation. A summary of the results is presented in Table 3-6 and Figure 3-8 to Figure 3-10. Table 3-6 shows values of characteristic acceleration (ã) as a function of $G_{0}$; recall that 
$\tilde{a}$ (Equation 2.23) is a cycle property and is not influenced by vehicle parameters. In general, ã is dictated by the speed trace and the grade profile; in this case, only grade effects are changing.

Table 3-6 Effect of Maximum Grade and Grade Function on Characteristic Acceleration for Selected Drive Cycles

\begin{tabular}{|c|c|c|c|c|c|c|c|c|c|c|c|}
\hline$G_{0}$ & 0 & 1 & 2 & 3 & 4 & 5 & 6 & 7 & 8 & 9 & 10 \\
\hline & \multicolumn{11}{|c|}{ Characteristic Acceleration $\tilde{a}\left(\mathrm{ft} / \mathrm{s}^{2}\right)$} \\
\hline \multicolumn{12}{|l|}{ OCTA } \\
\hline Sine Wave & 0.715 & 0.713 & 0.734 & 0.769 & 0.812 & 0.863 & 0.921 & 0.985 & 1.052 & 1.123 & 1.196 \\
\hline Square Wav & 0.715 & 0.725 & 0.774 & 0.845 & 0.929 & 1.027 & 1.136 & 1.252 & 1.374 & 1.500 & 1.630 \\
\hline \multicolumn{12}{|l|}{$C B D$} \\
\hline Sine Wave & 0.568 & 0.636 & 0.707 & 0.778 & 0.849 & 0.919 & 0.990 & 1.061 & 1.131 & 1.205 & 1.283 \\
\hline Square Wave & 0.568 & 0.679 & 0.793 & 0.907 & 1.021 & 1.135 & 1.249 & 1.362 & 1.477 & 1.601 & 1.737 \\
\hline \multicolumn{12}{|l|}{ KCM } \\
\hline Sine Wave & 0.544 & 0.577 & 0.621 & 0.677 & 0.740 & 0.808 & 0.883 & 0.963 & 1.047 & 1.133 & 1.221 \\
\hline Square Wave & 0.544 & 0.593 & 0.665 & 0.757 & 0.861 & 0.975 & 1.103 & 1.236 & 1.374 & 1.514 & 1.658 \\
\hline
\end{tabular}

Figure 3-8 presents the mean tractive power $\bar{P}_{\text {trac }}$ as a function of $G_{0}$. It is observed that for $G_{0} \leq 1$ the effect of grade is negligible. For a given value of $G_{0}$ the square wave function is much more aggressive than the sine wave. Taking into account that the available tractive power of a conventional bus is nearly $150 \mathrm{~kW}$ (Khan, 2009, $275 \mathrm{hp}$ engine, 75\% transmission efficiency), it is clear that the bus will not be able to follow closely the speed trace of the KCM cycle at high grades, especially in the case of a square grade profile. The mean tractive power $\bar{P}_{\text {trac }}$ is

Equation 3.7 Mean tractive power

$$
\bar{P}_{\text {trac }}=\frac{E_{\text {trac }}}{t_{\text {traction }}},
$$

where $t_{\text {traction }}$ is the traction time (time where $P_{\text {road }}>0$ ). Note how the OCTA and CBD curves diverge into two paths, one for each grade function (sine and square). This behavior suggests that the effect of grade is mostly dependent on grade function and cycle average speed (OCTA and CBD average speeds are very close).

Figure 3-9 shows mean tractive energy $e_{\text {trac,net }}$ plotted vs. characteristic acceleration. It is observed that the curves are clustered by drive cycle; and presumably by average speed. The effect of grade on tractive energy is considerable. On the other hand, Figure 3-10 presents the ratio of braking to tractive energy $e_{B R} / e_{t r a c}$; it is noted that the ratio is mostly drive cycle dependent and that, as expected, the low speed cycle (OCTA and CBD) have higher values than the high speed cycle (KCM). As $G_{0}$ increases, the braking energy increases more rapidly than does the tractive energy, making $e_{B R} / e_{t r a c}$ rise.

For the sine grade profile with $G_{0}=5 \%$, characteristic acceleration increased by $21 \%$ in the OCTA ( 0.715 to $\left.0.863 \mathrm{ft} / \mathrm{s}^{2}\right), 62 \%$ in the CBD $\left(0.568\right.$ to $\left.0.919 \mathrm{ft} / \mathrm{s}^{2}\right)$, and $49 \%$ in the $\mathrm{KCM}$ (0.544 to 0.808 
$\left.\mathrm{ft} / \mathrm{s}^{2}\right)$. At the same time, mean net tractive energy increased by $10 \%$ in the OCTA $(1,199$ to 1,319 $\mathrm{Wh} / \mathrm{km}), 26 \%$ in the CBD (1,086 to $1,371 \mathrm{Wh} / \mathrm{km})$, and $16 \%$ in the $\mathrm{KCM}(1,230$ to $1,422 \mathrm{Wh} / \mathrm{km}) ; e_{B R}$ / $e_{\text {trac }}$ increased by $5 \%$ in the OCTA (0.625 to 0.657$), 13 \%$ in the CBD (0.617 to 0.696$)$, and $14 \%$ in the $\operatorname{KCM}(0.433$ to 0.493$)$.

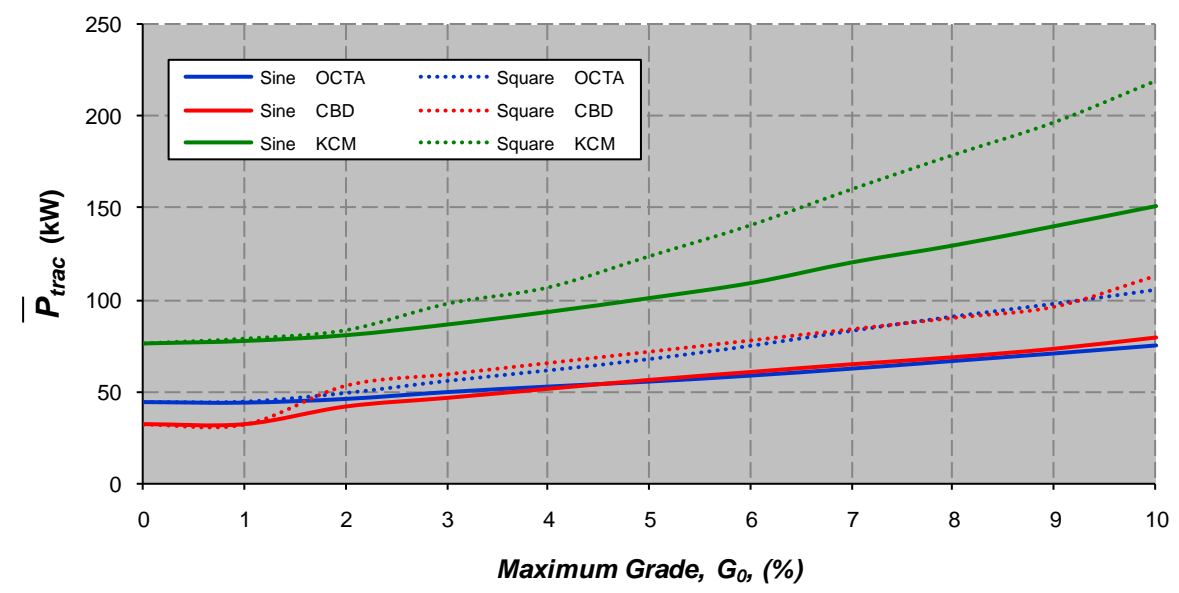

Figure 3-8 Mean Tractive Power vs. Maximum Grade for Sine and Square Grade Profiles

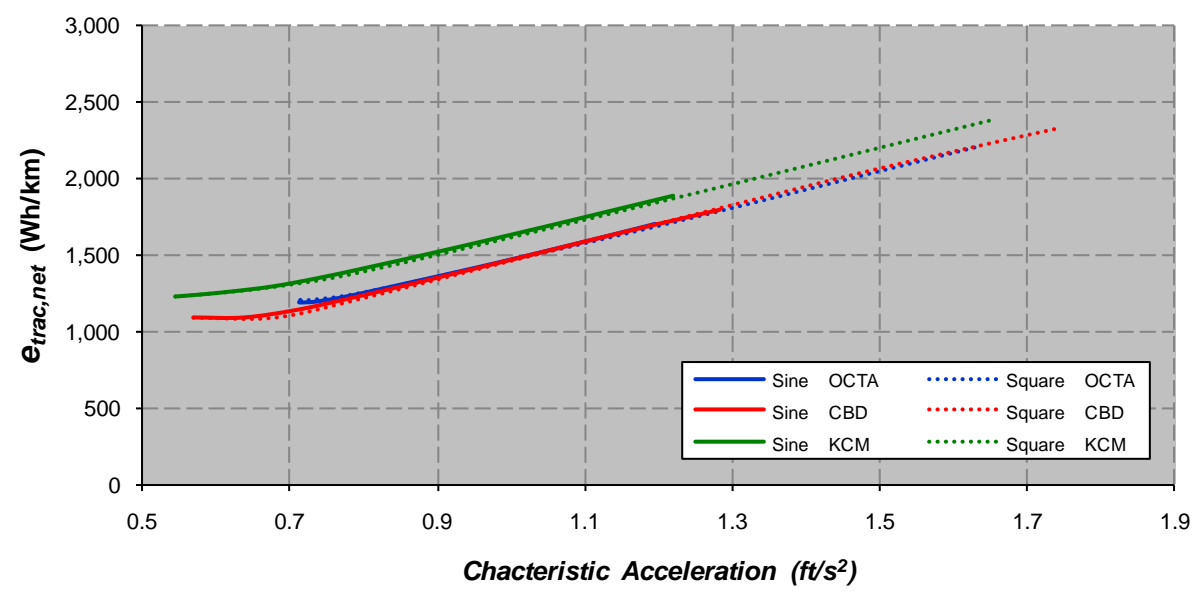

Figure 3-9 Net Mean Tractive Energy vs. Characteristic Acceleration for Sine and Square Grade Profiles 


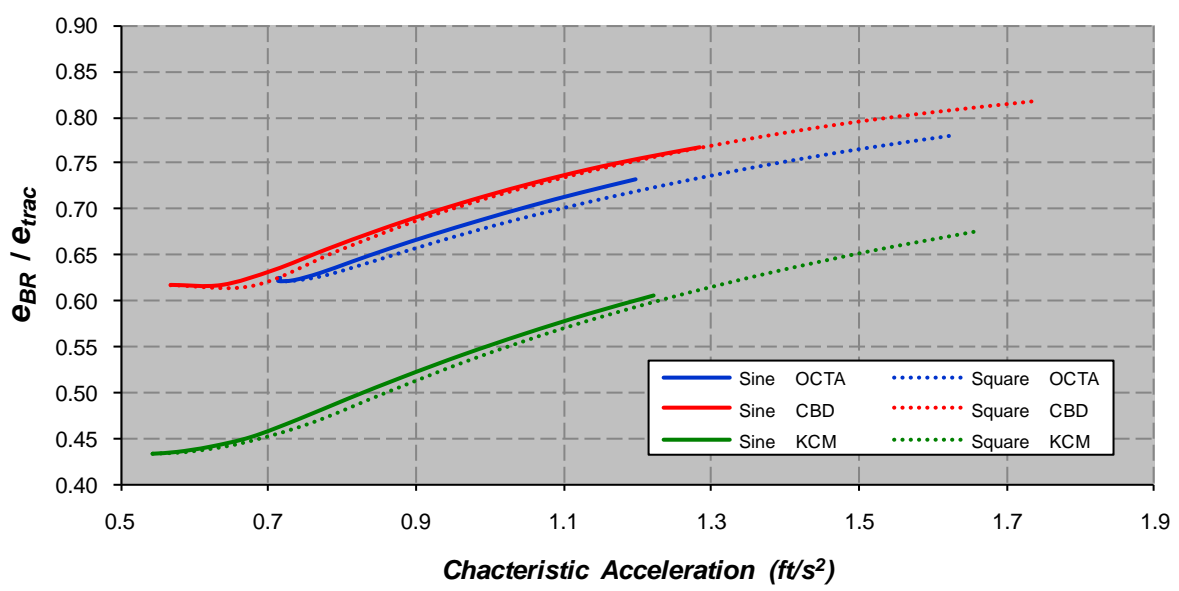

Figure 3-10 Braking to Tractive Energy Ratio vs. Characteristic Acceleration for Sine and Square Grade Profiles

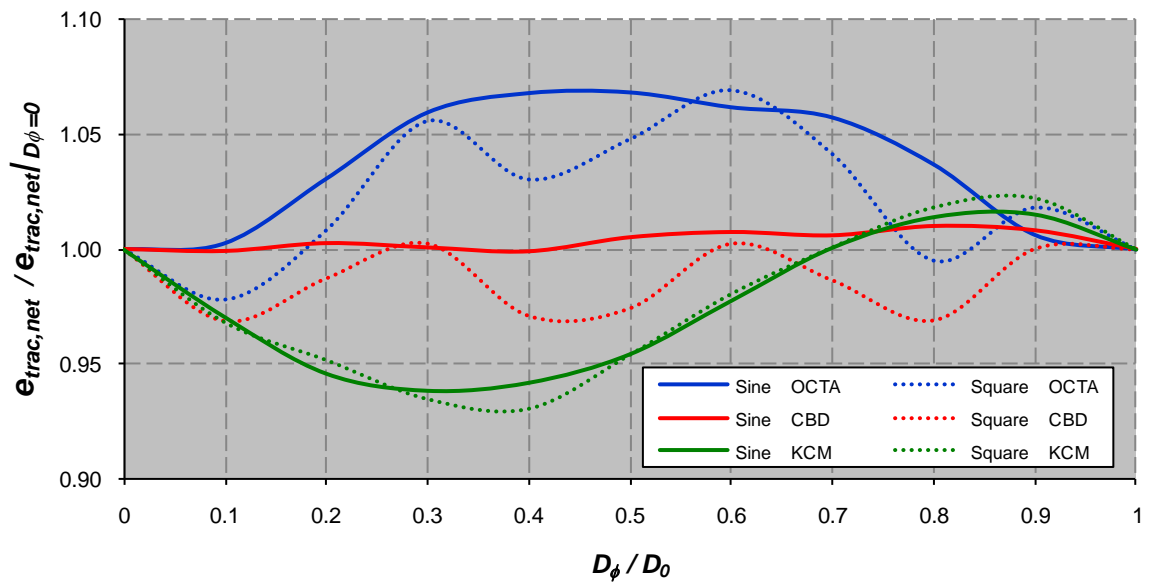

Figure 3-11 Ratio of Tractive Energy to Value at Zero Grade Shift

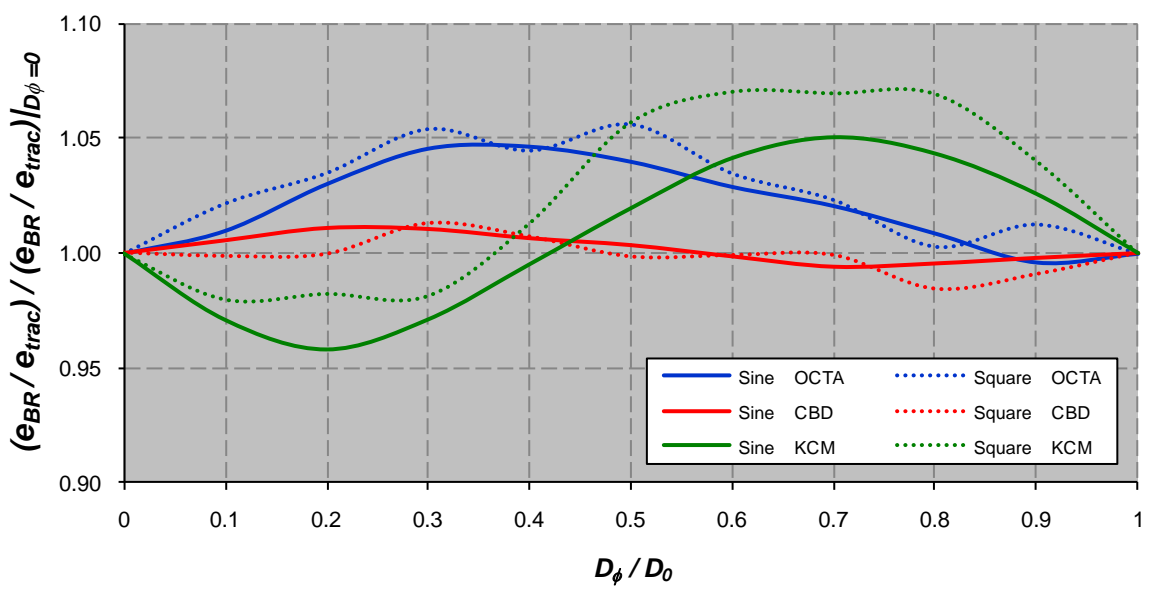

Figure 3-12 Ratio of Braking/Tractive Energy to Value at Zero Grade Shift 


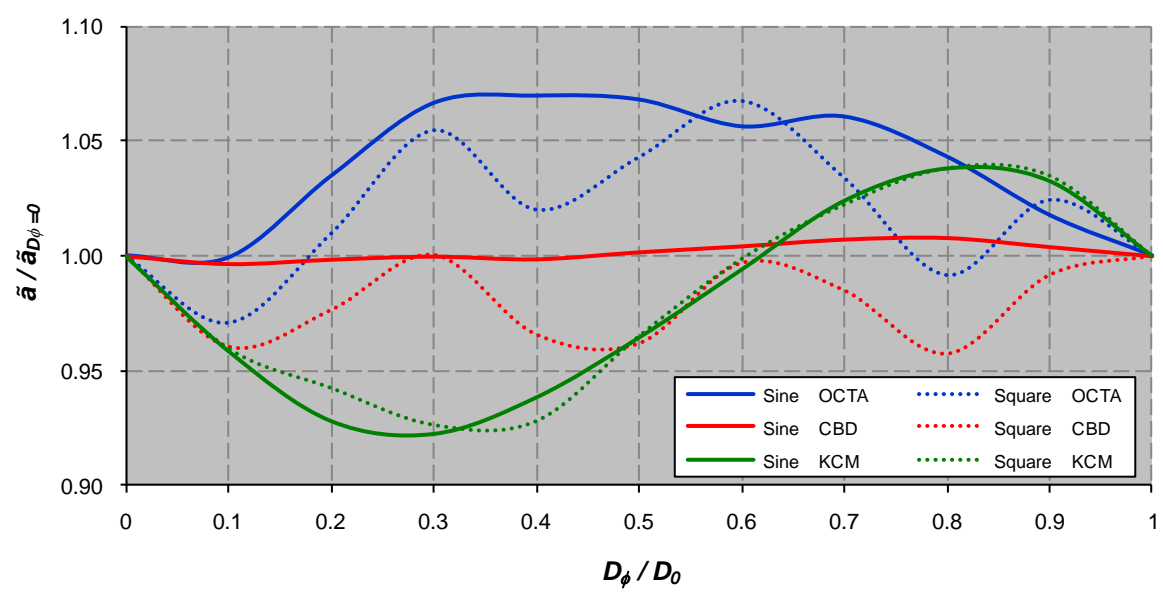

Figure 3-13 Ratio of Characteristic Acceleration to Value at Zero Grade Shift

Next the effect of the phase shift $D_{\phi}$ of the grade wave was examined. Building upon the previous analysis, the phase shift ratio $D_{\phi} / D_{0}$ was varied from 0 to 1 while $G_{0}$ was kept at $5 \%$. The results are presented in Figure 3-11 to Figure 3-13. For $0<D_{\phi} / D_{0}<0.5$ the cycle starts downhill, for $0.5<D_{\phi} /$ $D_{0}<1$ the cycle starts uphill, for $D_{\phi} / D_{0}=0$ the cycle starts at the bottom of the hill; for $D_{\phi} / D_{0}=0.5$ it starts at the top of the hill. Figure 3-11 shows net mean tractive energy, Figure 3-12 shows $e_{B R} / e_{\text {trac }}$, and Figure 3-13 presents characteristic acceleration, all with reference to the value at zero phase shift.

The results for the sine grade function are discussed next. For the OCTA cycle, tractive energy increases by as much as $7 \%$ when compared to zero shift; meaning that comparing $5 \%$ sine grade with flat terrain, the increase in $e_{\text {trac, net }}$ of the conventional transit bus can range from $10 \%(1,319 \mathrm{Wh} / \mathrm{km})$ to $18 \%(1,409 \mathrm{Wh} / \mathrm{km})$. In the OCTA cycle, standard deviation is $2.8 \%$ for a and $e_{\text {trac,net }}$, and $1.8 \%$ for $e_{B R} / e_{t r a c}$. The standard deviation is a measure of the variability on the expected effect of phase shift. Standard deviation in the CBD is $0.4 \%$ for ã and $e_{\text {trac,net }}$, and $0.6 \%$ for $e_{B R} / e_{\text {trac }}$. In the KCM cycle, standard deviation is $4.4 \%$ for ã, $3.0 \%$ for $e_{t r a c, n e t}$, and $3.4 \%$ for $e_{B R} / e_{t r a c}$.

\subsubsection{Grade Testing with Chassis Dynamometer}

The presence of grade in a cycle poses a different kind on demand in a chassis dynamometer than do cycles with flat terrain. When the terrain is flat, the dynamometer will pose a load on the vehicle simulating aerodynamic and rolling resistance, while flywheels simulate vehicle inertia. During acceleration the inertial masses load the drivetrain; they provide motoring during deceleration. If grade is to be included, a similar way of storing and releasing the potential energy $(m \cdot g \cdot h)$ should be considered. If not, the dynamometer should have the capacity of providing the extra retarding and motoring loads. 
Usually, the retarding load is not an issue because the total load is limited by vehicle maximum power and, in the case of WVU's TransLab, the 2-300 hp-eddy current power absorbers have been designed for that condition. On the other hand, the eddy current absorbers are incapable of motoring. For testing grade, either an AC or DC electric motor/generator dynamometer or variable speed motors installed in parallel to the eddy current dyno would be needed. The TransLab has $2-20 \mathrm{hp}-$ variable speed motors.

The author was interested in evaluating grade in emissions testing of transit buses with the TransLab. Consider the KCM cycle introduced in Figure 2-6 and Table 2-6; the cycle has four sections (KCM1 to KCM4), three with elevation and one on flat terrain.

Figure 3-14 shows gravity, rolling resistance, and aerodynamic loads over the KCM cycle for the conventional transit bus of Table 3-1; this is the net electric dynamometer power. Positive values are retarding loads on the vehicle; negative values are motoring loads. Excess motoring load (red line, negative value) is dissipated by conventional and/or regenerative braking, or it can be used to accelerate the vehicle reducing the load on the engine (downhill driving). In the TransLab, inertia loads are simulated by the flywheel set and the remaining loads (aerodynamic, rolling, and grade) are simulated by the power absorbers and the variable speed motors. Note that the gravitational motoring load reaches $130 \mathrm{~kW}$ in section 1 (40 kW net, $-3.5 \%$ grade), $35 \mathrm{~kW}$ in section 3 (5 kW net, $-1.5 \%$ grade), and $100 \mathrm{~kW}$ in section 4 (70 kW net, $-5 \%$ grade).

Available motoring power in the TransLab (neglecting transmission efficiencies) from the variable speed motors was then analyzed. It was observed that the powering capabilities of the laboratory are limited. Sections 1 (3.5\% grade) and 4 (5\% grade) could not be executed while section 3 (1.5\% grade) would be just within the limits of the laboratory. Motoring power was calculated from: $P_{\text {motoring }}(\mathrm{kW})$ $=V(\mathrm{~m} / \mathrm{s}) / r_{e f}(\mathrm{~m}) * \tau(\mathrm{Nm}) / 1000$, where $V$ is vehicle speed, $r_{e f}$ is effective wheel radius $\left(r_{e f} \approx 0.470\right.$ $\mathrm{m}$ ), and $\tau$ is motoring torque, $\tau=2$ motors $* 50 \mathrm{ft}-\mathrm{lb} /$ motor $* 3.5$ (differential ratio) $=350 \mathrm{ft}-\mathrm{lb}$. The theoretical motoring power at $60 \mathrm{mph}$ is $27 \mathrm{~kW}(36 \mathrm{hp})$.

Figure 3-15 shows the net dynamometer electric power that would need to be simulated during the KCM cycle both at empty and full weights. The maximum motoring power at full weight is nearly 100 $\mathrm{kW}(134 \mathrm{hp})$

Conventional and hybrid 60-foot articulated diesel transit buses were tested over the KCM cycle in NREL's ReFUEL Laboratory (Hayes et al., 2006). The laboratory was suited for vehicles up to 80,000 lb (36,287 kg) and equipped with a DC electric motor with $283 \mathrm{~kW}(380 \mathrm{hp})$ absorbing and $268 \mathrm{~kW}$ (360 hp) motoring ratings. The hybrid bus had 23\% lower fuel consumption compared with the conventional bus. Comparing fuel consumption ( $\mathrm{gal} / \mathrm{mi}$ ) for the KCM cycle with and without grade, it was observed that fuel consumption was $15 \%$ greater with grade for the conventional bus and only $5 \%$ greater for the hybrid. 


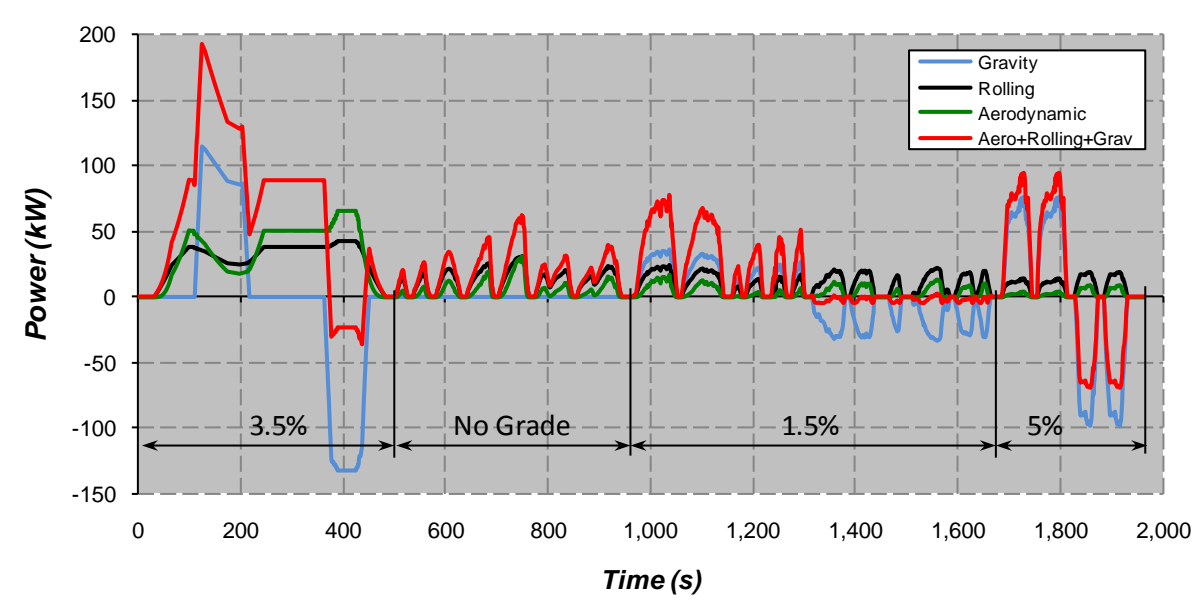

Figure 3-14 Gravity, Rolling, and Aerodynamic Loads (kW) for a Typical 40' Transit Bus Driving the KCM Cycle

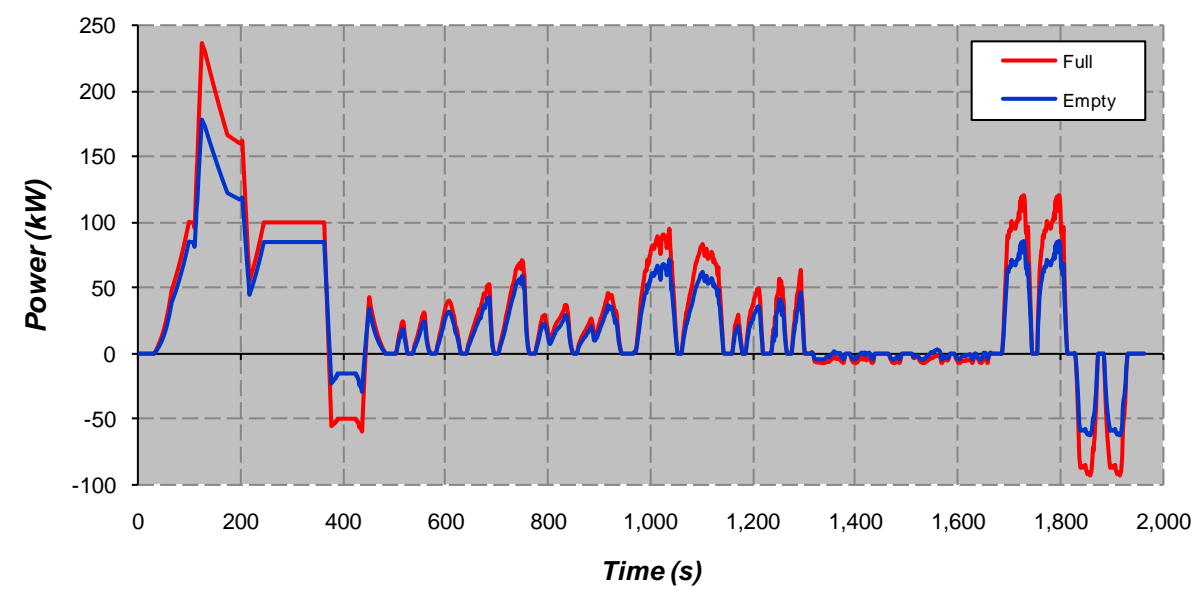

Figure 3-15 Net Dynamometer Electric Power at Empty and Full Weights (KCM Cycle)

\subsubsection{Maximum Speed at Constant Grade}

The maximum speed attainable at a given slope can be determined from the road load equation (Equation 2.11) as the root of the following polynomial on speed:

Equation 3.8 Maximum Speed

$$
\left(0.5 \rho_{\text {Air }} c_{d} A_{f}\right) V_{\max }^{3}+c V_{\max }^{2}+\left[m g\left(c_{r} \cos \alpha+\sin \alpha\right)+b\right] V_{\max }-P_{\text {road, } \max }=0,
$$

where $P_{\text {road,max }}$ is maximum engine power discounting transmission efficiency and auxiliary load.

Figure 3-16 shows maximum speed of the conventional bus (Table 3-1) as a function of grade for empty, $1 / 2$ SLW, and fully loaded weights (Table 3-4). Tractive power $P_{\text {road,max }}$ was estimated with: maximum engine power $=202 \mathrm{~kW}(275 \mathrm{hp})$, transmission efficiency $=0.75$, and auxiliary loads $=13.4$ 
$\mathrm{hp}(10 \mathrm{~kW})$. It is concluded that maximum speed is almost insensitive to weight for flat terrain. This is because aerodynamic drag is dominant (cubic term in the previous equation). The effect of grade on maximum speed is very significant. Consider the $1 / 2 \mathrm{SLW}$ scenario: from $67 \mathrm{mph}$ on flat terrain, maximum speed drops to $40 \mathrm{mph}$ at $4 \%$ grade, $30 \mathrm{mph}$ at $6 \%$ grade, and $20 \mathrm{mph}$ at $10 \%$ grade. Similar figures were reported by Smith (2001) for Class 8 trucks.

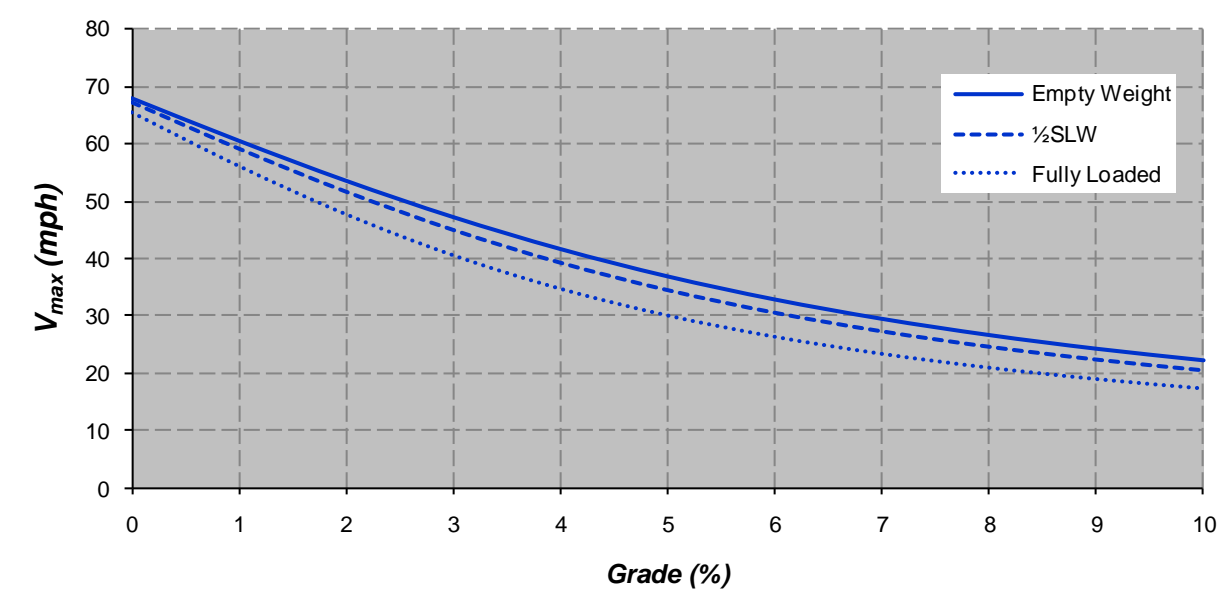

Figure 3-16 Maximum Vehicle Speed of Conventional Transit Bus vs. Grade

\subsection{Summary}

The results of this chapter, e.g. Figure 3-4 and Figure 3-9, suggest that it is more appropriate to explore correlations of fuel consumption (gal/mi or L/100 km), rather than fuel economy (mi/gal), with vehicle and drive cycle parameters, such as test weight, average speed, and characteristic acceleration.

The simulation parameters for conventional and hybrid $40^{\prime}$ transit buses were introduced. The moment of inertia of the bus wheels was estimated to be around $11 \mathrm{~kg} \cdot \mathrm{m}^{2}$.

The fuel consumption Equation 2.16 was used to estimate an upper limit for regeneration efficiency from knowledge of fuel consumption data for hybrid and conventional vehicles. This limit is presented in Equation 3.1; it is dependent on hybrid to conventional fuel consumption $(\tilde{g})$ and tractive energy $\left(E_{\text {trac }}\right)$ ratios, and hybrid braking to tractive energy $\left(E_{B R} / E_{\text {trac }}\right)$. Using fuel economy data from Khan (2009), it was concluded that for current hybrid buses the regeneration efficiency is between $20 \%$ and $40 \%$, with a strong dependency on duty cycle.

These bounds on $\eta_{\text {regen }}$ neglect effects of idle and braking fuel consumption, accessory load, and differences in driveline and engine efficiencies. Simulation results of in-use transit routes from Chapter 7 below show that these bounds are inaccurate because they neglect the effects just mentioned. 
The results of Chapter 7 indicate that $\eta_{\text {regen }}$ might range between $45 \%$ and $70 \%$ for series hybrid transit buses.

Tractive energy per unit distance was decomposed on aerodynamic, rolling, and inertial efforts. These values were estimated for a range of vehicle classes from light duty vehicles to transit buses over various drive cycles. The concept of energy at wheels efficiency (E.W.E.) was introduced; it is similar to fuel economy and represents the distance traveled per unit energy spent. Regeneration efficiency was varied from 0 to 1 and the relative impact on E.W.E. was observed.

The analysis showed the effects of energy recuperation alone. Hybrids also benefit from engine downsizing, which in turn allows it to operate in more efficient regimes.

The effect of weight on net mean tractive energy $e_{\text {trac,net }}$ was studied; weight has a linear effect on $e_{\text {trac, net }}$ both for conventional and hybrid vehicles.

The presence of grade significantly changes the operational envelope of the vehicle. During uphill sections the engine works harder whereas it works less in downhill sections. Overall, more fuel is consumed. Sine and square grade profiles superimposed on existing drive cycles were considered; the parameters are maximum grade $G_{0}$, hill length $D_{0}$, and phase shift $D_{\phi}$. The grade profile thus defined directly alters the cycle's characteristic acceleration, $\tilde{a}$, with the square wave having the strongest effect.

For a more in-depth analysis of grade effects, it would be worthwhile exploring the impact of changes in the value of the hill distance $D_{0}$. In this analysis a value of $D_{0}=0.5 \mathrm{mi}(0.805 \mathrm{~km})$ was selected. In the KCM cycle $D_{0}$ changes from section to section: $2.67 \mathrm{mi}$ in section 1 ( $3.5 \%$ grade), $3.30 \mathrm{mi}$ in section 3 (1.5\% grade), and $1.16 \mathrm{mi}$ in section 4 (5\% grade). Dimensional analysis shows that $\tilde{a} / \bar{U}^{2}$ has units of one over distance, thus the dimensionless group $D_{0} \cdot \tilde{\mathrm{a}} / \bar{U}^{2}$ could be used to explore such effects. The group ã / $\bar{U}^{2}$ resembles kinetic intensity (Equation 2.28: $\tilde{a} / \bar{V}_{\text {aero }}^{2}$ ) and the dimensionless group $D_{0} \cdot \tilde{\mathrm{a}} / \bar{V}_{\text {aero }}^{2}$ might also be appropriate.

The arbitrary selection of a grade phase shift $D_{\phi}$ introduces variability in the energy requirements. This effect was measured in terms of standard deviations of the parameters.

Finally, the possibility of including grade in emissions testing with the TransLab was explored. It was concluded that the laboratory is not suited for providing the motoring loads encountered in sections with negative grade. 


\section{Chapter 4. ChaRACTERIZATION OF TRANSIT DUTY CYClES}

The first part of this dissertation was aimed to developing a tool to estimate the metrics of bus duty cycles from data provided by transit agencies. Transit bus operation was characterized in cooperation with WMATA where GPS and ECU data were logged on a variety of routes. The logs amount to 2,900 miles and 230 hours of operation, and were designed to obtain the whole activity range, with average speeds ranging from $6.7 \mathrm{mph}$ on their slowest route to $25.9 \mathrm{mph}$ on their fastest route. WMATA also provided their estimates of route average speed, calculated from service time and route distance.

Data collection, data reduction, and data analysis are presented in this chapter. Knowledge of average speed can be used to predict other cycle metrics. Percentage idle and characteristic acceleration can be inferred but not with accuracy, as the correlation of these metrics with average speed are low.

\subsection{Transit Bus Activity Data Acquisition}

Bus activity data was collected with the collaboration of WMATA during the spring of 2009. Data logging took place from the Landover and Bladensburg divisions. At the time, WMATA had a fleet of about 1,480 buses; the Landover and Bladensburg divisions housed 167 and 273 buses, respectively. The Landover division serves Maryland and some parts of the District of Columbia (DC) whereas Bladensburg serves mainly the DC area. Vehicle speed and road grade were the primary variables of concern, but engine operating conditions were also recorded.

The data acquisition system is shown in Figure 4-1. The system recorded $(i)$ position and speed through a GPS antenna, (ii) ECU data through an ECU J1939 interface, and (iii) barometric pressure through a static pressure probe protruding from the top-front of the vehicle. Data was logged using a DL-1 data logger from Race Technology, which includes internal accelerometers that are used in conjunction with the GPS readings for an accurate measurement of vehicle speed. An Omega PX02 barometric pressure transducer, which provided a 0 to 5 volts DC output to the DL- 1 system, was used to provide an estimate of elevation changes and road grade. The vehicle's 12 volt system was used to power the electronic equipment. 


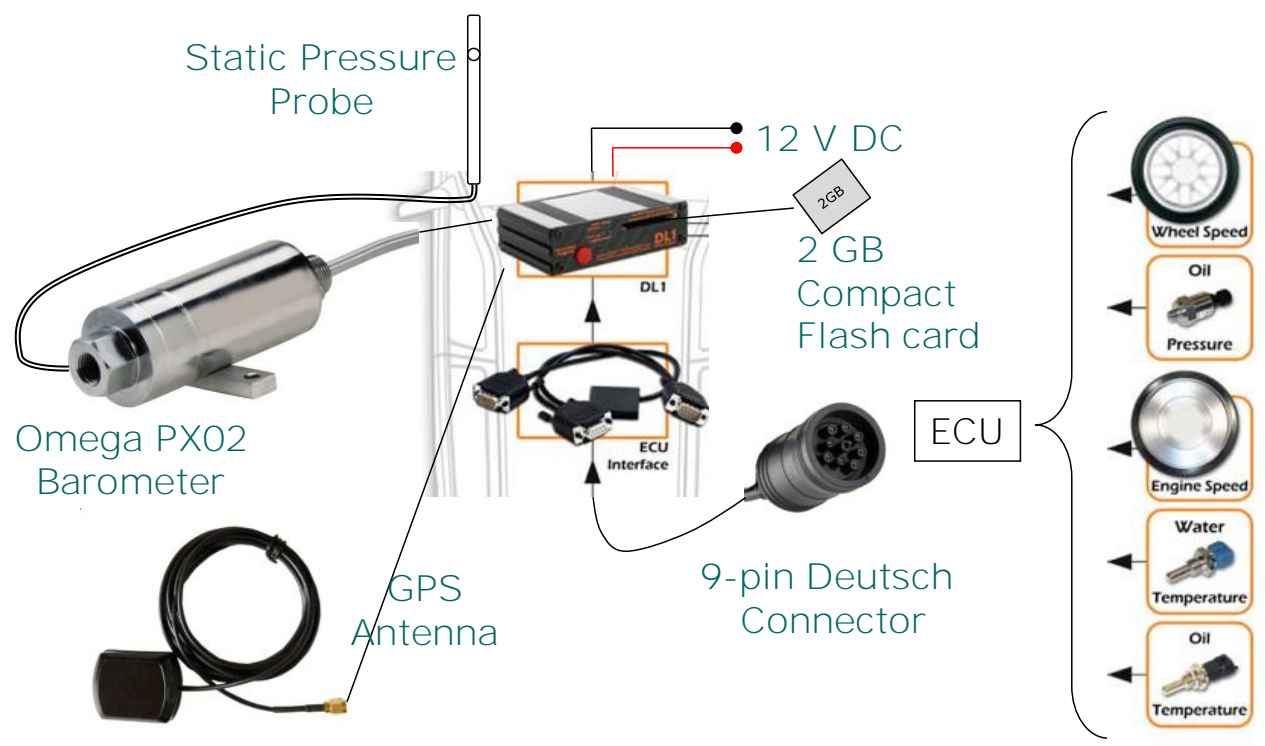

Figure 4-1 Layout of the GPS and ECU Data Logging Unit

Two data logging units were built and there was a third data logger available that recorded GPS information alone. The data logging systems did not interfere with normal vehicle operation and schedule. Each system was self sustaining and did not need to be monitored during driving. The systems were started before the buses leaved the depot and data were removed once the buses were back.

Table 4-1 displays the logged ECU variables. A sample ECU signal from the various buses was taken to set the ECU-interface. It was observed that certain buses did not report some of the desired ECU signals, for example the hybrids did not output accelerator pedal position while the CNGs did not report fuel rate.

Table 4-2 lists the eight test buses used for duty cycle logs. The list includes two articulated buses, one diesel-electric hybrid, two diesels, and three CNGs. The articulated buses were 60 footers and the others were 40 footers. Table 4-3 lists the 19 lines that were monitored.

Run-to-run variations, day-to-day variations as well as morning-to-afternoon variations were considered. Three lines were studied in consecutive runs to evaluate any possible effects of run-to-run variations. Similarly four lines were studied in different days and at the same time of day to evaluate possible day-to-day variations. Twelve lines were evaluated in morning and in afternoon-evening service to estimate possible differences from time of day. 
Table 4-1 Logged ECU Variables

\begin{tabular}{l|c|c|c|c|c|c|c}
\hline \multicolumn{1}{c|}{ ECU Signal } & Units & $\begin{array}{c}\text { Suspect } \\
\text { Parameter } \\
\text { Number (SPN) }\end{array}$ & $\begin{array}{c}\text { Parameter } \\
\text { Group } \\
\text { Number (PGN) }\end{array}$ & $\begin{array}{c}\text { Start } \\
\text { Bit }\end{array}$ & $\begin{array}{c}\text { Length } \\
\text { (bits) }\end{array}$ & Scaling & Offset \\
\hline Accelerator Pedal Position & $\%$ & 91 & F003 & 8 & 8 & $1 / 2.5$ & 0 \\
\hline Actual Engine - Percent Torque & $\%$ & 513 & F004 & 16 & 8 & 1 & -125 \\
\hline Actual Gear Ratio & - & 526 & F005 & 8 & 16 & $1 / 1000$ & 0 \\
\hline Barometric Pressure & $\mathrm{kPa}$ & 108 & FEF5 & 0 & 8 & $1 / 2$ & 0 \\
\hline Brake Switch & $\mathrm{boolean}$ & 597 & FEF1 & 28 & 2 & 1 & 0 \\
\hline Current Gear & - & 523 & F005 & 24 & 8 & 1 & -125 \\
\hline Engine Coolant Temperature & ${ }^{\circ} \mathrm{C}$ & 110 & FEEE & 0 & 8 & 1 & -40 \\
\hline Engine Speed (ECU) & $\mathrm{rpm}$ & 190 & F004 & 24 & 16 & $1 / 8$ & 0 \\
\hline Fuel Rate & $\mathrm{L} / \mathrm{h}$ & 183 & FEF2 & 0 & 16 & $1 / 20$ & 1 \\
\hline Intake Manifold 1 Temperature & ${ }^{\circ} \mathrm{C}$ & 105 & FEF6 & 16 & 8 & 1 & -40 \\
\hline Percent Load at Current Speed & $\%$ & 92 & F003 & 16 & 8 & 1 & 0 \\
\hline Wheel-Based Vehicle Speed & $\mathrm{kph}$ & 84 & FEF1 & 8 & 16 & $1 / 256$ & 0 \\
\hline
\end{tabular}

Table 4-2 Test Buses

\begin{tabular}{l|c|c|c|c|l|c}
\hline \multicolumn{1}{c|}{ Description } & Model Year & Bus \# & Fuel & $\begin{array}{c}\text { Curb Weight } \\
(\mathbf{l b s})\end{array}$ & \multicolumn{1}{|c}{ Engine } & $\begin{array}{c}\text { Engine } \\
\text { Power (hp) }\end{array}$ \\
\hline Ikarus Articulated & 1995 & 5225 & Diesel & 44,060 & Cummins SM-11-E & 330 \\
\hline NABI Articulated & 2008 & 5415 & CNG & 47,826 & Cummins ISL G & 320 \\
\hline New Flyer & 2002 & 2405 & CNG & 29,500 & Cummins C8.3 G & 280 \\
\hline New Flyer & 2006 & 6177 & Diesel & 28,500 & Cummins ISM & 280 \\
\hline New Flyer & 2007 & 2812 & CNG & 30,150 & Cummins LGas-280 & 280 \\
\hline New Flyer Hybrid & 2006 & 6002 & Diesel & 29,900 & Cummins ISL & 280 \\
\hline Orion V & 1997 & 4391 & Diesel & 29,300 & DDC Series 50, DDEC-IV & 275 \\
\hline Orion VII & 2005 & 2626 & CNG & 31,650 & John Deere Powertech 6081 & 250 \\
\hline
\end{tabular}

Table 4-3 Logged Routes

\begin{tabular}{l|c|c}
\hline \multicolumn{1}{c|}{ Line } & Route & Bus \# \\
\hline Anacostia-Eckington & P6 & 2626 \\
\hline Annapolis Road & T18 & 6177 \\
\hline Benning Road-H Street & X2 & 5225 \\
\hline Benning Road-Potomac Park & X1, X3 & 2405 \\
\hline Bowie State University, Pointer Ridge & B21, B22, C28 & 6177 \\
\hline Bowie-Belair & B24, B25 & 6002 \\
\hline Capitol Hts-Benning Hts & $\mathrm{U} 8$ & 5415 \\
\hline College Park & $82,83,86$ & 2405,2626 \\
\hline Crofton-New Carrollton & B29 & 4391 \\
\hline Eastover-Addison Road & P12 & 6177,6002 \\
\hline Georgia Avenue Metro Extra & 79 & 2812 \\
\hline Greenbelt-BWI Airport Express & B30 & 4391 \\
\hline Hospital Center & D8 & 2626 \\
\hline Marlow Heights-Temple Hills & H11, H12, H13 & 6177,6002 \\
\hline Martin Luther King Jr Highway & A12 & 6002 \\
\hline Minnesota Ave-M Street & V7, V9 & 2405,2626 \\
\hline North Capitol Street & 80 & 2405 \\
\hline Prince George's-Silver Spring & F4, F6 & 6002 \\
\hline Rhode Island Ave-New Carrollton & 84,85 & 6177 \\
\hline
\end{tabular}




\subsection{Data Reduction}

Raw log files were pre-processed to correct sections where the GPS lost all the satellites and produced rubbish data. When these sections were short, speed and position (longitude and latitude) were filled in assuming a linear variation between the nearest good points. When the sections were long they had to be removed. Additionally, all engine-off segments were removed.

The primary outputs of data reduction were vehicle speed and road grade. The following paragraphs describe the data processing considerations.

Two measures of vehicle speed were obtained: wheel based and GPS based. Comparison of GPS and wheel speeds showed that GPS speed can divert considerably from wheel speed, especially in inner-city driving due to obstruction of the satellites. It was concluded that wheel based speed is more reliable and is preferred over GPS speed. Nevertheless, if wheel speed is unavailable GPS speed measurement produces reasonably good results.

Wheel speed values below $0.2 \mathrm{mph}$ were set to zero; this threshold was $1 \mathrm{mph}$ in the case of GPS speed. In order to remove noise, a low pass filter was applied, with a $2 \mathrm{~Hz}$ cut-off for wheel speed and $0.33 \mathrm{~Hz}$ cut-off for GPS speed. Due to the GPS inaccuracies at idle, micro-trips with speeds below 5 $\mathrm{mph}$ were removed because it was not easy to determine if these were real or fictitious. This artifact was not necessary when wheel speed was used, thus crawling motions were effectively recorded by wheel speed. In order to maintain consistency between data from both types of measurement systems, it was decided to remove all micro-trips with $V_{\max }$ below $5 \mathrm{mph}$. These micro-trips represent less than $1 / 3^{\text {th }}$ of a percent of the distance traveled.

The author observed that, when satellite signal was good, the difference between wheel and GPS speeds was less than $1 \%$, and even though it could have been possible to apply some sort of averaging (Race Technology DL1 reports GPS speed as three-dimensional speed which is compatible with wheel speed), it was decided to use wheel speed alone. Differences of up to $10 \mathrm{mph}$ were noticed when satellite signal was poor.

Grade was determined from altitude $(h)$ and horizontal distance $(x)$ :

\section{Equation 4.1 Grade Calculation}

$$
\text { Grade }_{j}=100 \cdot \frac{\Delta h}{\Delta x},
$$

where the subscript $j$ denotes time step, $\Delta h$ is elevation change $\left(\Delta h=h_{j}-h_{j-1}\right)$, and $\Delta x$ is the run or horizontal distance travelled, which was determined from GPS longitude and latitude using the spherical law of cosines (Veness, 2010). Care had to be exercised since at low speeds the sensitivity of the GPS becomes an issue making $\Delta x \rightarrow 0 \Rightarrow$ Grade $\rightarrow \infty$. It was observed that the resolution for $\Delta x$ provided by the GPS and the geodesic distance method was of about $0.4 \mathrm{~m}$ for raw data, corresponding 
to a speed of about $4 \mathrm{~m} / \mathrm{s}$ or $9 \mathrm{mph}$. Having into account the resolution and to avoid the division by zero, grade was kept constant at speeds below $5 \mathrm{mph}$ and a limit of $10 \%$ was imposed on grade values.

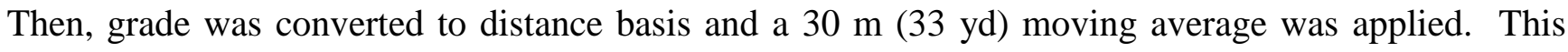
smoothing tended to shift the final value of elevation, thus a distance traveled based linear elevation correction was performed such that initial and final elevation values of each micro-trip were matched with the reference values.

Three measures of altitude were obtained: GPS reported altitude, altitude from barometric pressure, and altitude from topographic map and GPS location. It was concluded that the most reliable measure was that from the topographic map, thus topographic based grade was used as output. The topographic map was obtained from the National Elevation Dataset (NED, U.S. Geological Survey, 2010) with a resolution or grid spacing of $1 / 3$ arc second (about 10 meters). GPS reported longitude and latitude values were used to interpolate elevation from the NED map (Dembski et al., 2005). As expected, some wobbles were observed whenever the road had a bridge crossing a creek or any other depression. These wobbles were reduced with a $0.05 \mathrm{~Hz}$ cut-off low-pass filter.

As other studies have reported (Conley, 2002, Moynahan, 2005), GPS altitude showed random variations that could not be distinguished from real elevation changes, rendering GPS altitude of little use. Similarly, altitude from barometric pressure (Conley, 2002) presented inexistent elevation changes. The author believes these are due to gusts of wind that affect the static pressure reading by dynamic pressure. Barometer readings were good predictions of elevation changes at highway speeds (where the air flow is well oriented with the static pressure probe) but were poor predictors at low speeds. Noise in the pressure signal was removed by first applying a $0.1 \mathrm{~Hz}$ cut-off low-pass filter and then a 20 second moving average filter. Barometer output voltage was converted to engineering units with the calibration shown in Table 4-4.

Table 4-4 Barometer Calibration Table

\begin{tabular}{l|c|c}
\hline Data logger & Box 1 & Box 2 \\
\hline Model & PX02-D0-16A5T & PX02-D0-16A5T \\
\hline Serial No. & 246918 & 247011 \\
\hline Calib. Date & $9 / 12 / 2008$ & $9 / 12 / 2008$ \\
\hline $\begin{array}{c}\text { Pressure } \\
\text { (in Hg) }\end{array}$ & $\begin{array}{c}\text { Reading } \\
\text { (Vdc) }\end{array}$ & $\begin{array}{c}\text { Reading } \\
\text { (Vdc) }\end{array}$ \\
\hline 16.00 & 0.004 & 0.005 \\
\hline 24.00 & 2.509 & 2.515 \\
\hline 32.00 & 5.018 & 5.018 \\
\hline 24.00 & 2.510 & 2.517 \\
\hline 16.00 & 0.005 & 0.006 \\
\hline
\end{tabular}

The final output of the data reduction algorithm consisted of the time (sec), speed (mph), and grade $(\%)$ vectors, with a resolution of 0.1 seconds $(10 \mathrm{~Hz})$. 


\subsection{Analysis}

The first step in analyzing the bus activity was to calculate the cycle metrics corresponding to each of the logs. Following the work of Dembski et al., 2005 (Table 2-5 in page 25), the cycle metrics listed in Table 4-5 were calculated.

Average grade ascent and descent were calculated with Equation 4.1 given the total elevation gain $\left(\Delta h=\Delta s \cdot \sin \alpha_{j}\right.$, where $\Delta s=\int_{j-1}^{j} V \cdot d t$ and $\left.\left.\alpha_{j}=\operatorname{atan}_{[\operatorname{grade}} / 100\right]\right)$ and run $\left(\Delta x=\Delta s \cdot \cos \alpha_{j}\right)$ for ascent and descent sections, respectively. Average grade of the duty cycle was calculated with total elevation gain and total run.

The distribution of stops was characterized by the 5-point summary: [minimum, quartile 1, median, quartile 3, maximum]. The average time of stops was determined by total stop time divided by number of stop sections. The number of stop sections is one more than the number of stops (accounting for the stop segment before the first micro-trip).

The distribution of acceleration was characterized using the groups of Table 2-3 (page 20): cruise, light, medium, and heavy. Similarly the distribution of instantaneous speed was characterized using the bins of Table 2-4 (page 23): inner-city, urban, suburban, and highway.

Table 4-5 Cycle Metrics for Route Characterization

\begin{tabular}{l|c|l|c}
\hline \multicolumn{1}{c|}{ Metric } & Units & \multicolumn{1}{c}{ Metric } & Units \\
\hline Percentage of Idle & $\%$ & Kinetic Intensity w/o Grade & $\mathrm{mi}^{-1}$ \\
\hline Stops per mile & $\mathrm{mi}^{-1}$ & Average Grade & $\%$ \\
\hline Average Speed $(\bar{U})$ & $\mathrm{mph}$ & Average Grade Ascent & $\%$ \\
\hline Average Speed w/o idle & $\mathrm{mph}$ & Average Grade Descent & $\%$ \\
\hline Aerodynamic Speed & $\mathrm{mph}$ & Average Time of Stops & $\mathrm{s}$ \\
\hline Standard Deviation of Speed & $\mathrm{mph}$ & Distribution of Stops & $\%$ \\
\hline Standard Deviation of Speed w/o Idle & $\mathrm{mph}$ & Percent Time in Acceleration Bins & $\%$ \\
\hline Average Acceleration $\left(\bar{a}_{+}\right)$ & $\mathrm{ft} / \mathrm{s}^{2}$ & Percent Time in Deceleration Bins & $\%$ \\
\hline Average Deceleration $\left(\bar{a}_{-}\right)$ & $\mathrm{ft} / \mathrm{s}^{2}$ & Percent Time of Accel., Cruise, and Decel. & $\%$ \\
\hline Characteristic Acceleration $(\tilde{a})$ & $\mathrm{ft} / \mathrm{s}^{2}$ & Percent Time in Speed Bins & $\%$ \\
\hline Characteristic Acceleration w/o Grade & $\mathrm{ft} / \mathrm{s}^{2}$ & Percent Time in Speed/Acceleration Bins & $\%$ \\
\hline Kinetic Intensity $(k i)$ & $\mathrm{mi}^{-1}$ & Percent Time in Speed/Accel.-Decel. Bins & $\%$ \\
\hline
\end{tabular}

Correlations between pairs of metrics were investigated and Figure 4-2 shows the scatter-plot matrix with the corresponding correlation coefficients. In the figure and the subsequent analysis, characteristic acceleration and kinetic intensity correspond to the no-grade case as most emissions studies and cycles do not include grade.

The data showed that average speed is strongly correlated with average speed without idle, aerodynamic speed, and standard deviation of speed. Average speed is mildly correlated with stops per mile and kinetic intensity. Low correlations exist between average speed and percentage idle, characteristic acceleration, and average Accel./ Decel. The coefficient of determination was used to 
decide how strong the correlations where: greater than $80 \%$ was taken as a strong correlation, between $50 \%$ and $80 \%$ was taken as a mild correlation, and below $50 \%$ as a low correlation.

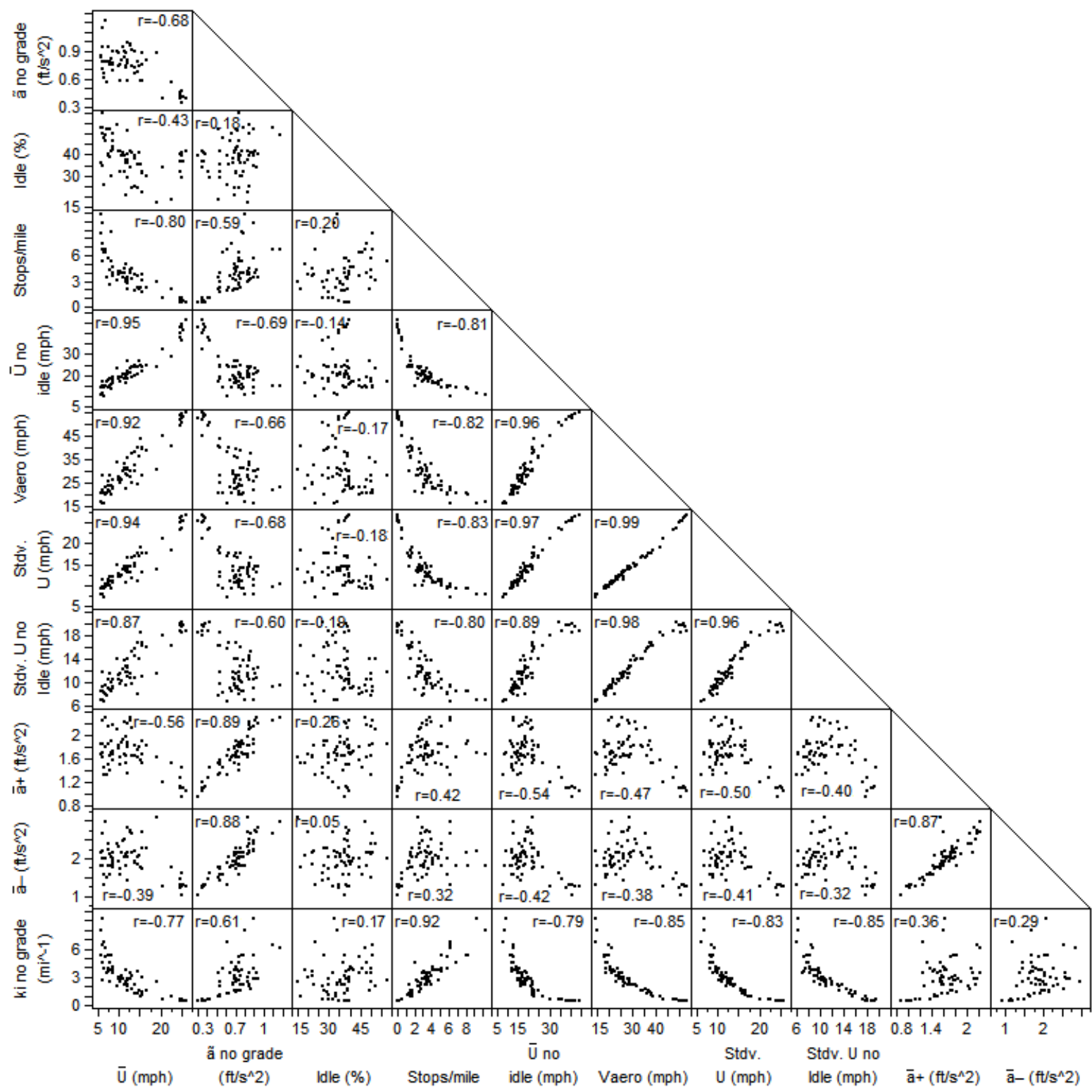

Figure 4-2 Scatter-plot Matrix for the Driving Cycle Database (Logged + Standard)

More important than these thresholds is the fact that out of the four metrics paired with average speed in the current IBIS models to predict emissions (namely: percentage idle, stops per mile, standard deviation of speed, and kinetic intensity) only percentage idle is nearly independent of average speed. This indicates that the other three metrics offer little information (or prediction power) beyond what average speed provides. 
On the other hand, characteristic acceleration (or alternatively average acceleration) is fairly uncorrelated with average speed, which makes it a good predictor candidate for emissions and fuel economy. Characteristic acceleration (Equation 2.23, page 21) represents the distance averaged supplied mechanical energy per unit mass.

The terrain was not very mountainous and average grade ascent values were below $2.5 \%$. Road grade produced an increase in characteristic acceleration, $\tilde{a}$, ranging from 5 to $15 \%$ for the majority of the routes. The effect is higher as average speed increases, thus commuter routes presented the greatest increase, of around $20 \%$. The same arguments are valid for kinetic intensity. These values can be used to evaluate the effect of grade on fuel economy and emissions.

Figure 4-3 shows a simple linear regression between the ratio ã to ã without grade against average speed and average grade ascent. The linear fit for average grade ascent is not statistically significant while it is significant for average speed. On the other hand, in a bilinear model (prediction with the two variables) the effect of average grade ascent does turn out to be significant; that is, the effect of grade on characteristic acceleration is dependent on average speed. It must be noted that in all the routes the overall average grade was zero since the routes started and ended at the bus depot. These findings can be used in evaluating the effect of grade on emissions and fuel economy.

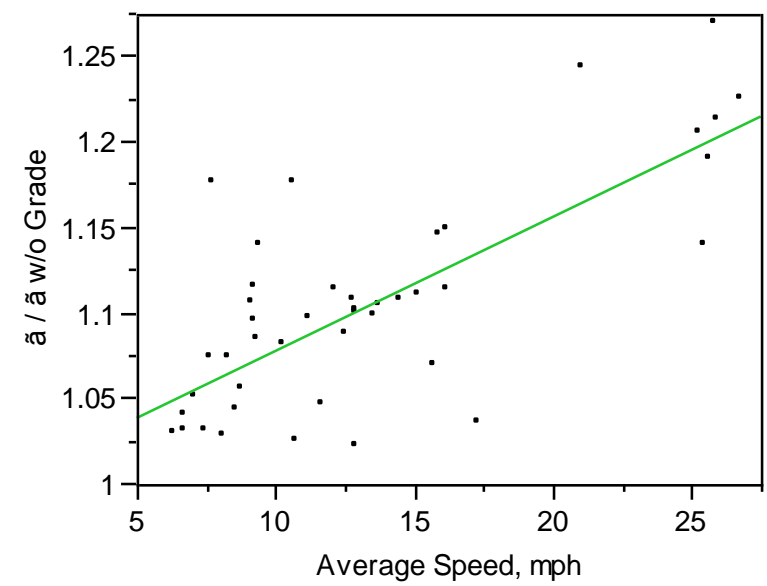

(a)

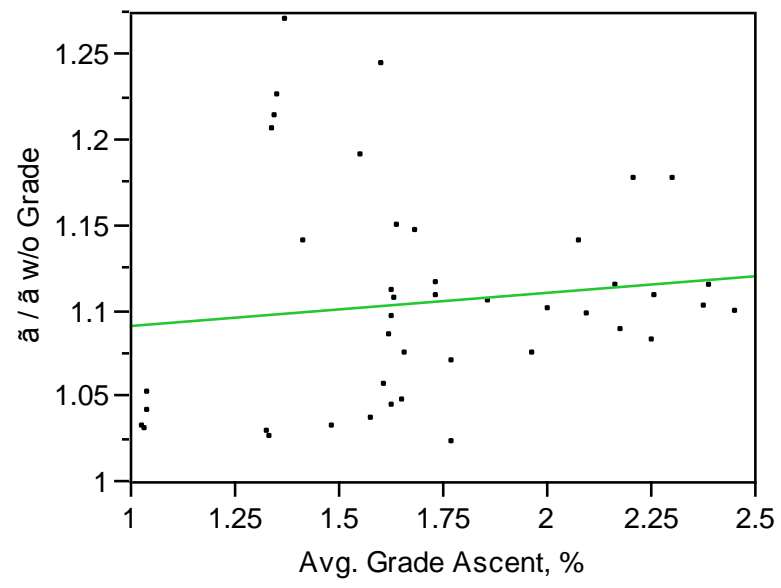

(b)

Figure 4-3 Ratio of Characteristic Acceleration with and without Grade versus (a) Average Speed and (b) Average Grade Ascent

To evaluate the time of stops the average time of a stop and the 5-number summary of the distribution (minimum, lower quartile, median, upper quartile, and maximum) were calculated for all the routes. The average stop time ranged from 20 seconds to 3 minutes, while the median time of stop ranged from 8 to 40 seconds. The shortest stops were observed in suburban routes (probably at stop signs) while commuter routes had the longest stops. The distribution of stops is skewed to the right with a few very long stops and many short stops. As an example, Figure 4-4 shows the distribution of 
stops of an inner-city route (Route D8); the five-number summary of the distribution is $[0.6,10.8,17$, 37.8, 534.1].

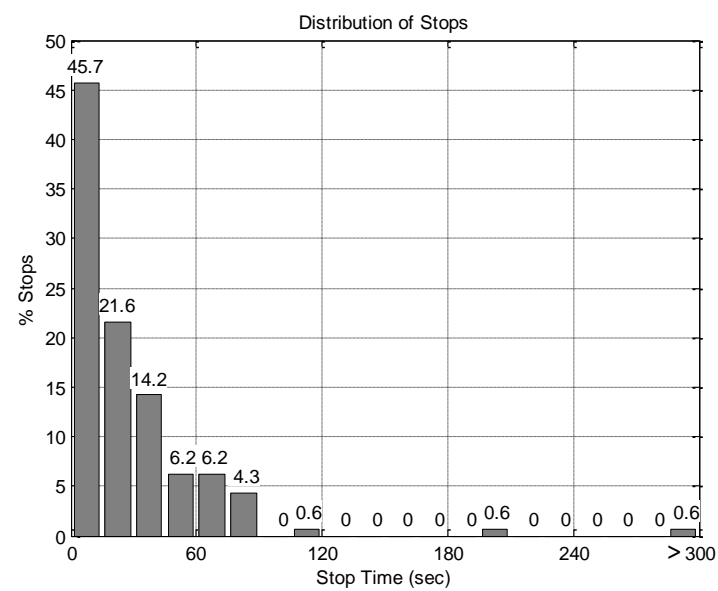

Figure 4-4 Typical Distribution of Stops of Inner-City Route (Route D8)

The properties of the logged routes are summarized in Table 4-6 (sorted alphabetically as in Table 4-3, page 96). Minimum and maximum values of the metrics are included at the bottom of the table. It is worthwhile noting that percentage idle ranges from $28 \%$ to 59\%. Regarding AM to PM differences, it was observed that, in general, morning service (AM) displayed higher average speeds than afternoon and evening (PM) service. This may be due to the low traffic encountered by the buses very early in the morning. Some routes showed marked differences in average speed while in others the effect of service time was not relevant.

It was uncertain if the values of average speed estimated by the transit agency were close to the measured values. For each route a transit authority knows the distance of one round trip and the time programmed for a bus to service the route. These two parameters are an indicator of route average speed, but due to dead heading operation the actual average speed is different from the predictor. The effect of dead heading is smaller as the duration of the route increases: it will have less dead heading and more revenue service. Figure 4-5 presents the correlation of actual average speed by estimated average speed, performed with $\mathrm{JMP}^{\circledR}$ software. The shaded region is the $95 \%$ confidence interval on the mean; the regression equation is included in the figure. It is observed that estimated average speed is just below the mean actual average speed (nearly 3\% lower in average). Figure 4-7 is a plot of the residuals where no specific pattern is visible. Point deviations from the estimated value can be as high as $5 \mathrm{mph}$. It can be concluded that in general the value of average speed estimated by the transit agency is a good approximation to the expected value. 
Table 4-6 Summary of Properties of Logged Routes

\begin{tabular}{|c|c|c|c|c|c|c|c|c|c|c|c|c|c|c|c|c|c|c|c|c|}
\hline Line & Route & $\begin{array}{c}\mathbf{A M} / \\
\mathbf{P M}\end{array}$ & $\begin{array}{l}\text { Dist. } \\
\text { (mi) }\end{array}$ & $\begin{array}{c}\text { Durat. } \\
\text { (hr) }\end{array}$ & $\underset{(\mathbf{m p h})}{\overline{\mathbf{U}}}$ & $\begin{array}{l}\overline{\mathbf{U}}_{\text {no idle }} \\
(\mathbf{m p h})\end{array}$ & $\begin{array}{c}V_{\text {aero }} \\
(\mathbf{m p h})\end{array}$ & $\underset{(\mathbf{m p h})}{\mathbf{U}}$ & $\begin{array}{c}\text { Stdv. } \\
\mathbf{U}_{\text {no idle }} \\
(\mathbf{m p h})\end{array}$ & $\begin{array}{l}\text { Idle } \\
(\%)\end{array}$ & $\begin{array}{c}\text { Stops } \\
\text { per } \\
\text { mile }\end{array}$ & $\begin{array}{c}\bar{a}_{+} \\
\left(\mathbf{f t} / \mathbf{s}^{2}\right)\end{array}$ & $\begin{array}{c}\bar{a}_{-} \\
\left(\mathbf{f t} / \mathbf{s}^{2}\right)\end{array}$ & $\begin{array}{c}\tilde{a}_{\text {no grade }} \\
\left(\mathbf{f t} / \mathbf{s}^{2}\right)\end{array}$ & $\begin{array}{c}\tilde{a} \\
\left(\mathbf{f t} / \mathbf{s}^{2}\right)\end{array}$ & $\begin{array}{c}k i_{\mathrm{no}} \\
\text { grade } \\
\left(\mathbf{m i}^{-1}\right)\end{array}$ & $\begin{array}{c}k i \\
\left(\mathbf{m i}^{-1}\right)\end{array}$ & $\begin{array}{c}\text { Avg. } \\
\text { Time } \\
\text { of a } \\
\text { Stop } \\
\text { (s) }\end{array}$ & $\begin{array}{l}\text { Med. } \\
\text { Stop } \\
\text { (s) }\end{array}$ & $\begin{array}{c}\text { Avg. } \\
\text { Grade } \\
\text { Ascent } \\
(\%)\end{array}$ \\
\hline Anacostia & P6 & $\mathrm{AM}$ & 61.20 & 8.27 & 7.4 & 14.4 & 22.3 & 9.8 & 9.3 & 48.8 & 6.60 & 2.27 & 2.92 & 1.20 & 1.24 & 5.93 & 6.11 & 35.8 & 18.8 & 1.5 \\
\hline Annapolis Rd & $\mathrm{T} 18$ & PM & 41.24 & 3.05 & 13.5 & 20.2 & 27.1 & 12.8 & 10.6 & 33.0 & 4.07 & 2.27 & 2.68 & 0.96 & 1.06 & 3.21 & 3.53 & 21.5 & 9.2 & 2.5 \\
\hline Benning Rd-H & $\mathrm{X} 2$ & $\mathrm{PM}$ & 43.57 & 6.90 & 6.3 & 13.3 & 19.7 & 8.7 & 8.2 & 52.4 & 8.42 & 1.82 & 2.10 & 0.81 & 0.84 & 5.15 & 5.30 & 35.4 & 24.2 & 1.0 \\
\hline Benning Rd-H & $\mathrm{X} 2$ & $\mathrm{AM}$ & 62.91 & 8.93 & 7.0 & 13.4 & 19.8 & 8.9 & 8.1 & 47.5 & 6.25 & 1.44 & 1.54 & 0.60 & 0.63 & 3.76 & 3.94 & 38.8 & 21.1 & 1.0 \\
\hline Benning-Potom. & $\mathrm{X} 1, \mathrm{X} 3$ & PM & 22.59 & 3.40 & 6.6 & 16.2 & 27.5 & 10.8 & 11.4 & 59.0 & 5.18 & 1.83 & 1.94 & 0.78 & 0.80 & 2.52 & 2.60 & 61.2 & 21.7 & 1.0 \\
\hline Bowie State & B21, B22, C28 & PM & 59.68 & 2.84 & 21.0 & 31.6 & 44.6 & 20.8 & 17.8 & 33.4 & 0.90 & 1.16 & 1.24 & 0.38 & 0.47 & 0.47 & 0.58 & 62.1 & 12.2 & 1.6 \\
\hline Bowie State & B21, B22, C28 & $\mathrm{AM}$ & 117.02 & 4.56 & 25.6 & 35.7 & 50.2 & 23.4 & 20.1 & 28.2 & 0.86 & 1.41 & 1.57 & 0.46 & 0.55 & 0.45 & 0.53 & 45.5 & 11.3 & 1.6 \\
\hline Bowie-Belair & B24, B25 & $\mathrm{PM}$ & 29.41 & 2.29 & 12.8 & 26.1 & 35.2 & 16.2 & 13.8 & 50.8 & 1.94 & 1.81 & 2.11 & 0.75 & 0.76 & 1.48 & 1.51 & 72.3 & 9.0 & 1.8 \\
\hline Bowie-Belair & B24, B25 & $\mathrm{AM}$ & 149.42 & 9.51 & 15.7 & 26.3 & 35.5 & 16.7 & 13.7 & 40.3 & 1.86 & 1.78 & 2.05 & 0.71 & 0.76 & 1.39 & 1.48 & 49.5 & 10.7 & 1.8 \\
\hline Capitol Hts & U8 & $\mathrm{PM}$ & 20.04 & 2.33 & 8.6 & 17.8 & 27.4 & 11.8 & 11.2 & 51.7 & 4.14 & 1.62 & 2.09 & 0.74 & 0.77 & 2.42 & 2.52 & 51.7 & 20.9 & 1.6 \\
\hline College Park & $82,83,86$ & PM & 32.12 & 2.87 & 11.2 & 18.4 & 25.4 & 11.9 & 10.1 & 39.2 & 3.83 & 1.84 & 2.13 & 0.78 & 0.86 & 2.98 & 3.27 & 32.7 & 16.7 & 2.1 \\
\hline College Park & $82,83,86$ & PM & 119.47 & 10.23 & 11.7 & 19.6 & 29.2 & 13.4 & 12.1 & 40.5 & 3.20 & 1.91 & 2.45 & 0.93 & 0.97 & 2.68 & 2.80 & 38.9 & 13.7 & 1.7 \\
\hline College Park & 82,83 & $\mathrm{PM}$ & 89.94 & 7.01 & 12.8 & 19.9 & 30.5 & 13.9 & 12.6 & 35.4 & 3.06 & 1.78 & 2.12 & 0.78 & 0.86 & 2.06 & 2.26 & 32.4 & 13.4 & 2.0 \\
\hline College Park & $82,83,86$ & $\mathrm{AM}$ & 98.34 & 5.71 & 17.2 & 24.3 & 38.0 & 17.3 & 15.8 & 29.0 & 2.09 & 1.79 & 2.25 & 0.79 & 0.82 & 1.34 & 1.39 & 28.8 & 14.0 & 1.6 \\
\hline Crofton ... & B29 & PM & 59.42 & 2.34 & 25.4 & 37.1 & 48.5 & 23.0 & 18.4 & 31.4 & 0.84 & 1.24 & 1.43 & 0.44 & 0.50 & 0.46 & 0.52 & 51.7 & 18.3 & 1.4 \\
\hline Eastover-Addis. & $\mathrm{P} 12$ & PM & 106.14 & 8.25 & 12.9 & 21.3 & 30.3 & 14.1 & 12.2 & 39.7 & 3.34 & 2.18 & 2.79 & 0.96 & 1.06 & 2.58 & 2.84 & 33.2 & 10.2 & 2.4 \\
\hline Eastover-Addis. & P12 & $\mathrm{PM}$ & 74.51 & 4.61 & 16.2 & 23.2 & 39.0 & 17.4 & 16.4 & 30.4 & 2.91 & 1.88 & 2.24 & 0.73 & 0.82 & 1.18 & 1.32 & 23.1 & 12.9 & 2.2 \\
\hline Georgia Ave & 79 & $\mathrm{PM}$ & 64.29 & 7.05 & 9.1 & 15.6 & 21.6 & 10.2 & 8.7 & 41.7 & 4.45 & 1.65 & 1.80 & 0.73 & 0.81 & 3.84 & 4.24 & 36.9 & 25.8 & 1.6 \\
\hline Georgia Ave & 79 & $\mathrm{AM}$ & 34.47 & 3.73 & 9.2 & 17.0 & 22.6 & 10.7 & 8.8 & 45.8 & 3.51 & 1.56 & 1.73 & 0.68 & 0.75 & 3.25 & 3.62 & 50.4 & 26.5 & 1.7 \\
\hline Georgia Ave & 79 & $\mathrm{AM}$ & 64.62 & 6.94 & 9.3 & 16.4 & 22.5 & 10.6 & 9.0 & 43.3 & 4.50 & 1.69 & 1.96 & 0.75 & 0.82 & 3.65 & 3.96 & 37.0 & 23.9 & 1.6 \\
\hline Greenbelt-BWI & B30 & $\mathrm{AM}$ & 147.40 & 5.83 & 25.3 & 42.0 & 53.3 & 25.6 & 19.7 & 39.8 & 0.41 & 1.09 & 1.25 & 0.41 & 0.49 & 0.35 & 0.42 & 134.6 & 21.2 & 1.3 \\
\hline Greenbelt-BWI & B30 & PM & 202.52 & 7.86 & 25.8 & 42.0 & 52.0 & 25.0 & 18.4 & 38.7 & 0.31 & 0.93 & 1.00 & 0.33 & 0.42 & 0.30 & 0.38 & 170.9 & 28.0 & 1.4 \\
\hline Greenbelt-BWI & B30 & $\mathrm{PM}$ & 100.89 & 3.77 & 26.7 & 45.5 & 54.6 & 26.5 & 18.5 & 41.2 & 0.33 & 1.03 & 1.20 & 0.38 & 0.47 & 0.31 & 0.38 & 164.7 & 37.5 & 1.4 \\
\hline Hospital Center & D8 & $\mathrm{PM}$ & 29.05 & 3.49 & 8.3 & 13.6 & 21.0 & 9.6 & 8.8 & 38.9 & 5.54 & 1.84 & 2.21 & 0.92 & 0.99 & 5.12 & 5.50 & 30.2 & 17.0 & 2.0 \\
\hline Marlow Heights & H11, H12, H13 & $\mathrm{AM}$ & 86.93 & 5.76 & 15.1 & 23.0 & 39.6 & 17.3 & 16.5 & 34.5 & 2.08 & 1.73 & 2.00 & 0.66 & 0.73 & 1.03 & 1.15 & 39.4 & 10.7 & 1.6 \\
\hline Marlow Heights & H11, H12, H13 & $\mathrm{PM}$ & 74.03 & 4.67 & 15.8 & 24.2 & 43.0 & 18.8 & 18.3 & 34.6 & 1.99 & 1.56 & 1.71 & 0.56 & 0.64 & 0.74 & 0.85 & 39.3 & 8.3 & 1.7 \\
\hline Marlow Heights & H11, H12, H13 & $\mathrm{PM}$ & 98.14 & 6.08 & 16.1 & 23.4 & 39.2 & 17.1 & 16.0 & 30.9 & 1.56 & 1.51 & 1.68 & 0.56 & 0.65 & 0.90 & 1.04 & 43.9 & 8.4 & 1.6 \\
\hline Martin Luther & A12 & $\mathrm{AM}$ & 65.34 & 5.22 & 12.5 & 19.8 & 29.8 & 13.7 & 12.4 & 36.8 & 2.82 & 1.94 & 2.06 & 0.86 & 0.93 & 2.36 & 2.57 & 37.4 & 10.9 & 2.2 \\
\hline
\end{tabular}


Chapter 4 - Characterization of Transit Duty Cycles

\begin{tabular}{|c|c|c|c|c|c|c|c|c|c|c|c|c|c|c|c|c|c|c|c|c|}
\hline Line & Route & $\begin{array}{c}\mathbf{A M} / \\
\mathbf{P M}\end{array}$ & $\begin{array}{l}\text { Dist. } \\
\text { (mi) }\end{array}$ & $\begin{array}{c}\text { Durat. } \\
\text { (hr) }\end{array}$ & $\begin{array}{c}\overline{\mathbf{U}} \\
(\mathbf{m p h})\end{array}$ & $\begin{array}{l}\overline{\mathbf{U}}_{\text {no idle }} \\
(\mathbf{m p h})\end{array}$ & $\begin{array}{c}V_{\text {aero }} \\
(\mathbf{m p h})\end{array}$ & $\underset{(\mathbf{m p h})}{\mathbf{U}}$ & $\begin{array}{c}\text { Stdv. } \\
\text { Unn idle } \\
\text { (mph) }\end{array}$ & $\begin{array}{l}\text { Idle } \\
(\%)\end{array}$ & $\begin{array}{c}\text { Stops } \\
\text { per } \\
\text { mile }\end{array}$ & $\begin{array}{c}\bar{a}_{+} \\
\left(\mathbf{f t} / \mathbf{s}^{2}\right)\end{array}$ & $\begin{array}{c}\bar{a}_{-} \\
\left(\mathbf{f t} / \mathbf{s}^{2}\right)\end{array}$ & $\begin{array}{c}\tilde{a}_{\text {no grade }} \\
\left(\mathbf{f t} / \mathbf{s}^{2}\right)\end{array}$ & $\begin{array}{c}\tilde{a} \\
\left(\mathbf{f t} / \mathbf{s}^{2}\right)\end{array}$ & $\begin{array}{c}k i_{\text {no }} \\
\text { grade } \\
\left(\mathbf{m i}^{-1}\right)\end{array}$ & $\begin{array}{c}k i \\
\left(\mathbf{m i}^{-1}\right)\end{array}$ & $\begin{array}{l}\text { Avg. } \\
\text { Time } \\
\text { of a } \\
\text { Stop } \\
\text { (s) }\end{array}$ & $\begin{array}{c}\text { Med. } \\
\text { Stop } \\
\text { (s) }\end{array}$ & $\begin{array}{c}\text { Avg. } \\
\text { Grade } \\
\text { Ascent } \\
(\%)\end{array}$ \\
\hline Martin Luther & A12 & $\mathrm{PM}$ & 116.26 & 9.13 & 12.7 & 20.2 & 27.5 & 13.0 & 10.8 & 36.9 & 2.51 & 1.86 & 1.98 & 0.77 & 0.85 & 2.49 & 2.75 & 41.4 & 11.9 & 2.3 \\
\hline Minnesota Ave & V7, V9 & $\mathrm{AM}$ & 77.30 & 9.60 & 8.0 & 16.7 & 24.9 & 11.0 & 10.3 & 51.7 & 5.49 & 2.13 & 2.37 & 0.93 & 0.96 & 3.69 & 3.79 & 42.1 & 14.2 & 1.3 \\
\hline Minnesota Ave & V7, V9 & PM & 44.71 & 5.10 & 8.8 & 14.9 & 23.4 & 10.5 & 9.8 & 41.1 & 5.14 & 1.67 & 2.00 & 0.81 & 0.85 & 3.63 & 3.83 & 32.7 & 20.7 & 1.6 \\
\hline Minnesota Ave & V7, V9 & $\mathrm{AM}$ & 42.46 & 3.96 & 10.7 & 18.1 & 27.0 & 12.3 & 11.1 & 40.8 & 3.93 & 1.80 & 2.29 & 0.86 & 0.88 & 2.88 & 2.95 & 34.6 & 15.2 & 1.3 \\
\hline North Capitol & 80 & PM & 92.81 & 12.15 & 7.6 & 14.2 & 19.6 & 9.1 & 7.8 & 46.1 & 6.24 & 1.77 & 1.98 & 0.78 & 0.83 & 4.95 & 5.31 & 34.8 & 17.2 & 1.7 \\
\hline North Capitol & 80 & $\mathrm{AM}$ & 8.47 & 1.09 & 7.8 & 15.9 & 20.3 & 9.5 & 7.4 & 51.3 & 3.31 & 1.30 & 1.45 & 0.55 & 0.65 & 3.27 & 3.85 & 69.4 & 23.1 & 2.2 \\
\hline Prince George's & $\mathrm{F} 4, \mathrm{~F} 6$ & PM & 16.11 & 1.57 & 10.3 & 22.3 & 33.2 & 14.5 & 13.8 & 54.0 & 2.98 & 2.07 & 2.37 & 0.88 & 0.95 & 1.95 & 2.11 & 62.2 & 17.6 & 2.3 \\
\hline Prince George's & $\mathrm{F} 4, \mathrm{~F} 6$ & $\mathrm{AM}$ & 98.24 & 9.23 & 10.6 & 18.0 & 25.6 & 11.8 & 10.2 & 40.8 & 3.57 & 1.75 & 1.89 & 0.72 & 0.85 & 2.69 & 3.16 & 38.5 & 11.2 & 2.3 \\
\hline Prince George's & $\mathrm{F} 4, \mathrm{~F} 6$ & PM & 96.90 & 8.02 & 12.1 & 19.7 & 32.8 & 14.3 & 13.5 & 38.6 & 4.26 & 2.19 & 2.45 & 0.88 & 0.98 & 2.01 & 2.24 & 26.9 & 11.0 & 2.4 \\
\hline Rhode Island & 84,85 & PM & 89.09 & 9.49 & 9.4 & 18.5 & 25.5 & 11.8 & 10.2 & 49.3 & 3.82 & 2.14 & 2.13 & 0.88 & 1.00 & 3.32 & 3.78 & 49.4 & 10.8 & 2.1 \\
\hline Rhode Island & 84,85 & $\mathrm{AM}$ & 71.03 & 5.18 & 13.7 & 23.0 & 36.6 & 16.2 & 15.1 & 40.4 & 3.07 & 2.10 & 2.64 & 0.89 & 0.99 & 1.64 & 1.80 & 34.4 & 10.2 & 1.9 \\
\hline Minimum & & & & & 6.3 & 13.3 & 19.6 & 8.7 & 7.4 & 28.2 & 0.31 & 0.93 & 1.00 & 0.33 & 0.42 & 0.30 & 0.38 & 21.5 & 8.3 & 1.0 \\
\hline Maximum & & & & & 26.7 & 45.5 & 54.6 & 26.5 & 20.1 & 59.0 & 8.42 & 2.27 & 2.92 & 1.20 & 1.24 & 5.93 & 6.11 & 170.9 & 37.5 & 2.5 \\
\hline
\end{tabular}




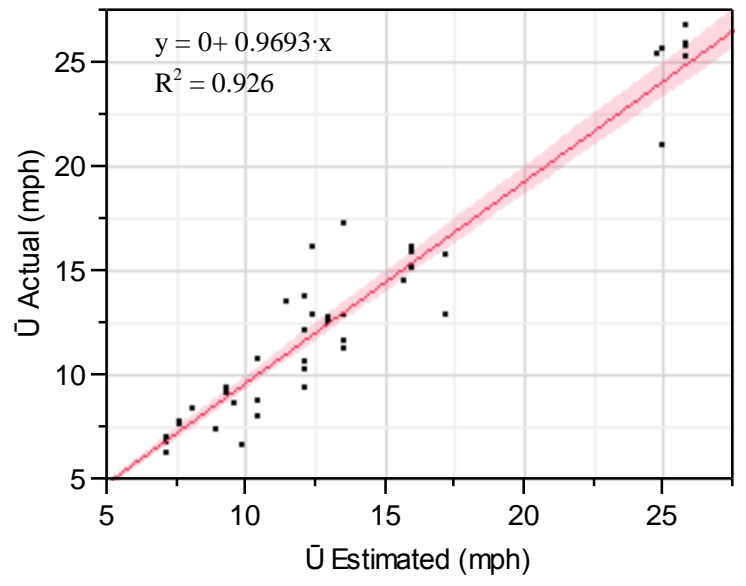

Figure 4-5 Estimated vs. Measured Average Speed

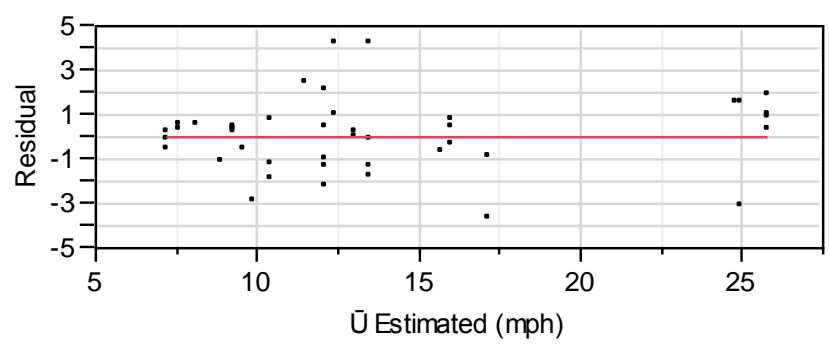

Figure 4-7 Residuals: Estimated vs. Measured Average Speed

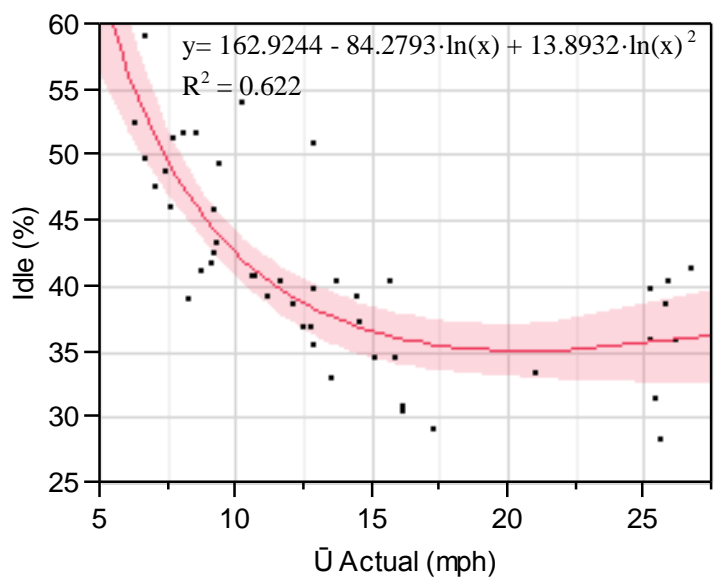

Figure 4-6 Actual Average Speed vs. Idle

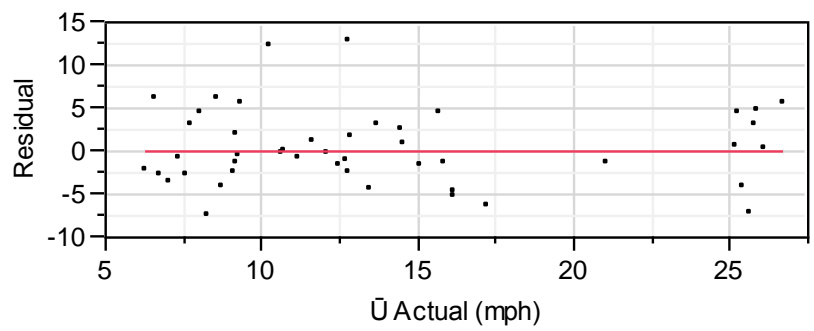

Figure 4-8 Residuals: Actual Average Speed vs. Idle

Figure 4-6 presents the correlation of actual idle by actual average speed; Figure 4-8 presents the residuals. A second order polynomial has been fit for the range 5-30 $\mathrm{mph}$; there is a mild correlation between the two with $\mathrm{R}^{2}=0.622$. The shaded area is the $95 \%$ confidence interval in the mean; the equation for the confidence interval is complex, but it can be simplified to a constant value of $\pm 2.5 \%$ without losing a great deal of accuracy. Using this information, once the transit agency provides an estimation of average speed it would be possible to predict percentage idle (a likely value). This pair (average speed and \% idle) can be used to predict emissions and fuel consumption with more confidence than using average speed alone.

Figure 4-9 and Figure 4-10 are scatterplots of actual idle (\%) by non-revenue time fraction and recovery time fraction, respectively. Recovery time is the time between a completed a trip and the start of the next trip; it is reserved for traffic and loading delays. An analysis of variance shows no statistical evidence of correlation between non-revenue time fraction $(F=0.1234$, p-value $=0.7277)$ or recovery time fraction $(F=0.4239$, p-value $=0.5196)$ and actual idle. This suggests that the best estimator of percentage idle is average speed. The author observed that most standard cycles have lower values of percentage idle than the ones encountered at WMATA, and this is why the correlation of Figure 4-2 is so low. Most standard cycles exclude dead-heading and idling at the bus depot, thus 
their percentage of idle is lower than the WMATA logs which included all the engine-on periods instead of revenue service only.

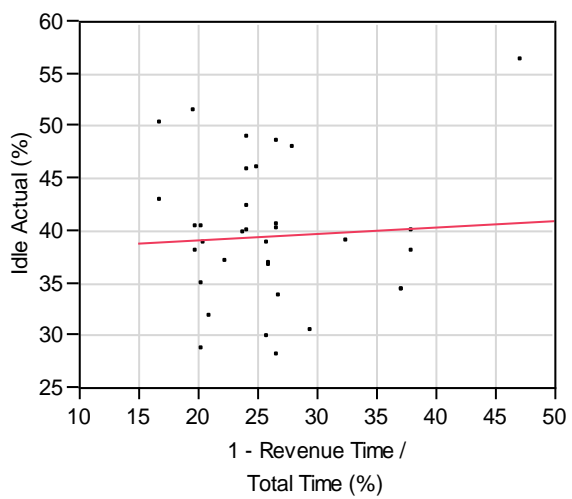

Figure 4-9 Non-Revenue Time Fraction vs. Actual Idle

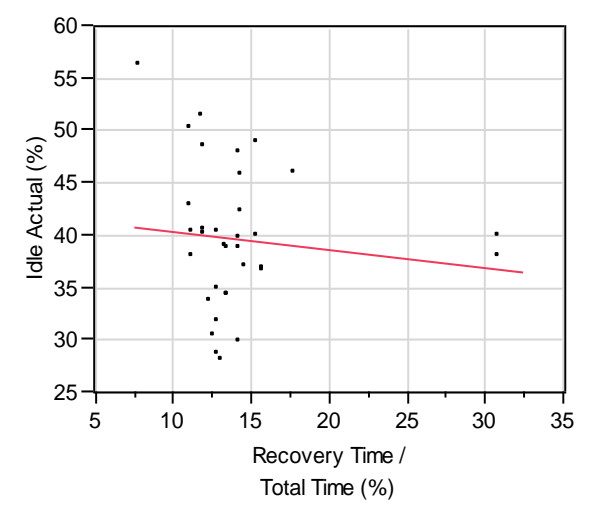

Figure 4-10 Recovery Time Fraction vs. Actual Idle

From data supplied by WMATA dated June 2008, Figure 4-11 presents the distribution of average speed by distance traveled in each average speed bin. This distribution can be used to estimate vehicle miles traveled in each mode, and thus predict fleet fuel consumption and emissions (Wayne et al., 2009):

\section{Equation 4.2 Fleet Emissions}

$$
\begin{gathered}
\text { Emissions [tons }]=\sum_{i=1}^{n}(\text { emissions rate })_{i}[\mathrm{~g} / \mathrm{mi}] \cdot \mathrm{VMT}_{i}[\mathrm{mi}] / 1000 \\
\text { Fuel Economy }[\mathrm{mpg}]=100 / \sum_{i=1}^{n}\left(\% \text { Dist }_{i} / \mathrm{mpg}_{i}\right)
\end{gathered}
$$

The fleet's overall average speed was $12.0 \mathrm{mph}$, value that is calculated, as described below, with the time fractions in each mode, rather than with the distance traveled fractions of Figure 4-11. The distribution of average speed by fractions of service time is shown in Figure 4-12. For composite service (serving two or more routes, or even the whole fleet) overall average speed (based on average speed of the individual routes) is calculated from the time fraction spent in each route. If $n$ routes were served, the overall average speed is:

\section{Equation 4.3 Estimated Average Speed for Composite Service}

$$
\bar{U}_{\text {overall }}=\sum_{i=1}^{n} \frac{t_{i}}{t_{\text {total }}} \cdot \bar{U}_{i},
$$

where $t_{i}$ is the time spent in route $i, \bar{U}_{i}$ is the average speed of route $i$, and $t_{\text {total }}$ is the total service time $\left(t_{\text {total }}=\sum_{i=1}^{n} t_{i}\right)$. Note that the factor $t_{i} \cdot \bar{U}_{i}$ is the distance traveled of route $i$. In Figure 4-12 each bin's 
$\bar{U}_{i}$ would be the center of the bin. Equation 4.3 is equivalent to dividing total mileage by total service time.

The distribution of average speed can be observed from various angles from which we have looked at distance traveled and time spent per bin. There is yet another approach and it is looking at counts of routes in the speed bins (i.e. frequency). This method is simple and is useful to get a first glimpse of the distribution, but for calculations, the distance traveled method is more accurate to estimate overall emissions while accounting for the various speed bins; the time spent method is best if the overall average speed is desired, giving a single point emissions and fuel economy value.

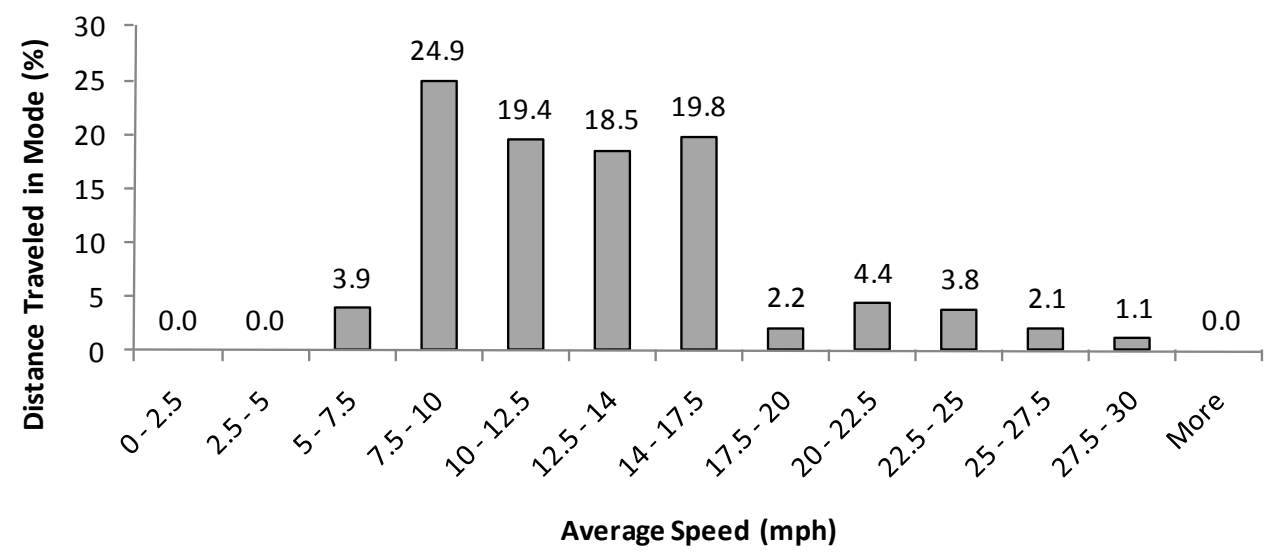

Figure 4-11 Distribution of Fleet Average Speed by Distance Traveled (\%) in WMATA Operation, June 2008

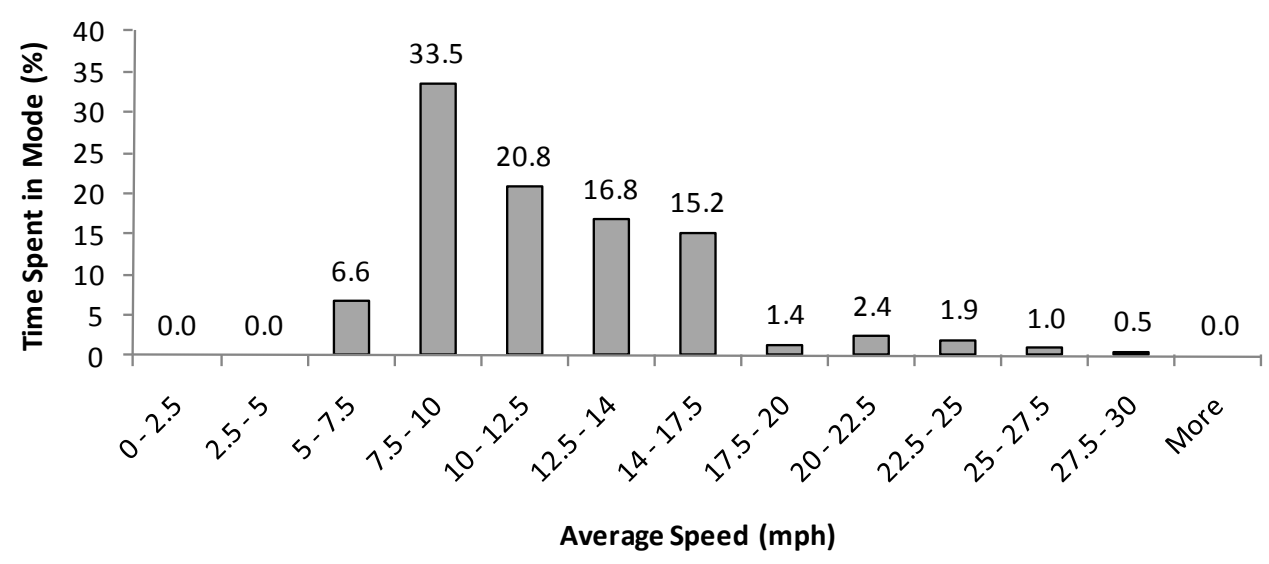

Figure 4-12 Distribution of Fleet Average Speed by Time Fractions in WMATA Operation, June 2008

IBIS was used to predict $\mathrm{NO}_{\mathrm{x}}$ emissions and fuel economy for the WMATA distribution (assuming a MY 2005 diesel electric-hybrid bus as reference and using the correlation for \% idle of Figure 4-6). The distance method yielded $4.62 \mathrm{mpg}$ and $8.40 \mathrm{~g}_{\mathrm{NOx}} / \mathrm{mi}$, while the overall average speed method (time spent) yielded $4.57 \mathrm{mpg}$ and $8.51 \mathrm{~g}_{\mathrm{NOx}} / \mathrm{mi}$. The second method differs from the first (more 
accurate) method by about $1 \%$. This difference is within the margin of error of the IBIS predictions and therefore the use of the overall speed method seems to be the most practical for its simplicity.

\subsubsection{Classification of Routes by Service Type}

The author proposed classification of the routes into four categories according to service type:

- Inner-City Service: Routes operating in heavy inner-city traffic with very low average speed. Average speed below $10 \mathrm{mph}$.

- Urban Service: Mostly urban/city routes with periods of both heavy and low traffic. Speed limit up to $30 \mathrm{mph}$. Average speed between 10 and $14 \mathrm{mph}$.

- Suburban Service: Mostly suburban routes with relatively high distance between stops. Speed limit up to $50 \mathrm{mph}$. Average speed between 14 and $20 \mathrm{mph}$.

- Commuter Service: Routes that go on highways/freeways with intercity/town commutes. Speed limit above $50 \mathrm{mph}$. Average speed above $20 \mathrm{mph}$. Commuter routes may originate from a suburban park-n-ride, operate on freeway for a long distance, and then become a downtown circulator to drop off passengers at employment centers.

In addition to these four categories the author considered Express and Bus-Rapid-Transit (BRT) service. It was found that routes from these two categories can be placed into the other four groups depending on their average speed.

The idea of grouping the routes into service types is that routes within the same category will have similar emissions and fuel economy. The service type categories will be useful whenever average speed data are not available to the IBIS user.

The speed ranges above were determined by k-means analysis of the duty cycle database. The analysis included the logged routes plus some of the standard cycles (Bee-Line, Braunschweig, Houston, KCM no grade, Liberty, Manhattan, Mexico City, MiamiD, New York Composite, Nuremberg, OCTA, Paris, and WMATA). Cycles of the geometric type (e.g. CBD or Arterial) were excluded from the analysis since they do not represent real driving conditions.

The k-means clustering algorithm randomly selects centers for the k clusters (4 in this case) and measures the Euclidean distance between each individual route and the centers. The centers are changed and the routes are moved between clusters until the sum of distances is minimized. The metrics were normalized prior to the analysis.

If all the metrics of Table 4-5 are used in the classification, the classifier differentiates the dynamic behavior of the routes (i.e. accelerations, cruise, etc.) but such dynamic behavior does not necessarily differentiate their emissions. For instance, consider a route with high average speed without idle and high idle, which would have a low value of average speed (say, in the Inner-City category). This route 
is dynamically similar to routes with higher average speeds (e.g. Urban routes with less idle) thus would be classified to the higher average speed cluster. On the other hand, since emissions and fuel efficiency are reported in grams per mile and gallons per mile $(1 / \mathrm{mpg})$, respectively, this route will have higher emissions values than the routes of the cluster it was assigned to. This is because the distance travelled (the denominator) is lower than the other routes (with higher average speed); the bus is polluting and consuming fuel but it is not moving, thus distance based emissions are higher and the emissions characteristics of the route will be more closely matched by routes with similar average speed.

The dynamic clustering just described is appropriate for electric and hybrid vehicles that do not pollute while idling. The issue being that $\mathrm{A} / \mathrm{C}$ and heat shall be functioning at idle and hybrid buses need the engine running at idle to either maintain battery state of charge if the accessories are electrically driven or to drive them directly.

Therefore, average speed must have more weight than the other metrics in the clustering logic for the clusters to reasonably represent emissions and fuel economy characteristics. A sensitivity analysis was performed in which the appropriate weight for average speed was determined, and in the end the classifier sorted by average speed. The centroids of the four categories are presented in Table 4-7 and the classification criterion (from k-means analysis) is shown in Table 4-8.

The logged routes were classified and the results are presented in Table 4-9. The table also shows the average speed that WMATA had estimated for each route, the depot, and the number of repetitions. The data in the table shows that the Bladensburg depot served mainly Inner-City routes while the Landover depot served mostly Urban and Suburban routes.

Table 4-7 Metrics of Service Type Centroids

\begin{tabular}{c|c|c|c|c|c|c|c|c|c|c}
\hline Group & $\begin{array}{c}\overline{\mathbf{U}} \\
(\mathbf{m p h})\end{array}$ & $\begin{array}{c}\text { Idle } \\
(\boldsymbol{\%})\end{array}$ & $\begin{array}{c}\text { Stops } \\
\text { / mile }\end{array}$ & $\begin{array}{c}\text { Stdv. U } \\
(\mathbf{m p h})\end{array}$ & $\begin{array}{c}\boldsymbol{k i} \mathbf{w} / \mathbf{o} \\
\mathbf{G r a d e}\left(\mathbf{m i}^{-\mathbf{1}}\right)\end{array}$ & $\begin{array}{c}\tilde{\mathbf{a}} \mathbf{w} / \mathbf{o} \\
\mathbf{G r a d e}\left(\mathbf{f t} / \mathbf{s}^{\mathbf{2}}\right)\end{array}$ & $\begin{array}{c}\overline{\boldsymbol{a}}_{+} \\
\left(\mathbf{f t} / \mathbf{s}^{\mathbf{2}}\right)\end{array}$ & $\begin{array}{c}\overline{\boldsymbol{a}}_{-} \\
\left(\mathbf{f t} / \mathbf{s}^{\mathbf{2}}\right)\end{array}$ & $\begin{array}{c}\overline{\mathbf{U}}_{\text {no idle }} \\
(\mathbf{m p h})\end{array}$ & $\begin{array}{c}\boldsymbol{V}_{\text {aero }} \\
(\mathbf{m p h})\end{array}$ \\
\hline Inner-City & 7.93 & 44.10 & 5.83 & 9.64 & 4.58 & 0.80 & 1.72 & 1.95 & 14.41 & 21.39 \\
\hline Urban & 12.23 & 37.02 & 3.47 & 13.21 & 2.44 & 0.80 & 1.83 & 2.16 & 19.63 & 28.71 \\
\hline Suburban & 15.89 & 30.14 & 2.45 & 15.59 & 1.69 & 0.73 & 1.68 & 2.11 & 22.87 & 34.56 \\
\hline Commuter & 25.06 & 34.23 & 0.69 & 23.80 & 0.42 & 0.41 & 1.16 & 1.31 & 38.58 & 50.11 \\
\hline
\end{tabular}

Table 4-8 Route Classification Criterion

\begin{tabular}{c|c}
\hline Service Type & Average Speed (mph) \\
\hline Inner-City & $\overline{\mathrm{U}} \leq 10$ \\
\hline Urban & $10<\overline{\mathrm{U}} \leq 14$ \\
\hline Suburban & $14<\overline{\mathrm{U}} \leq 20$ \\
\hline Commuter & $\overline{\mathrm{U}}>20$
\end{tabular}


Table 4-9 Classification of WMATA Routes by Service Type

\begin{tabular}{l|c|c|c|c|c|c}
\hline \multicolumn{1}{c|}{ Line } & Route & Service Type & $\begin{array}{c}\text { Average } \\
\text { Speed } \\
(\mathbf{m p h})\end{array}$ & $\begin{array}{c}\text { WMATA } \\
\text { Estimated } \\
(\mathbf{m p h})\end{array}$ & Depot & Rep. \\
\hline Anacostia-Eckington & P6 & Inner-City & 7.4 & 8.9 & Bladensburg & 1 \\
\hline Annapolis Road & T18 & Urban & 13.5 & 11.5 & Landover & 1 \\
\hline Benning Road-H Street & X2 & Inner-City & $6.3-7.0$ & 7.2 & Bladensburg & 2 \\
\hline Benning Road-Potomac Park & X1, X3 & Inner-City & 6.6 & 9.9 & Bladensburg & 1 \\
\hline Bowie State University, Pointer Ridge & B21, B22, C28 & Commuter & $21.0-25.6$ & 25.0 & Landover & 2 \\
\hline Bowie-Belair & B24, B25 & Urban / Suburban & $12.8-15.7$ & 17.2 & Landover & 2 \\
\hline Capitol Hts-Benning Hts & U8 & Inner-City & 8.6 & 9.6 & Bladensburg & 1 \\
\hline College Park & $82,83,86$ & Urban / Suburban & $11.2-17.2$ & 13.5 & Bladensburg & 4 \\
\hline Crofton-New Carrollton & B29 & Commuter & 25.4 & 24.8 & Landover & 1 \\
\hline Eastover-Addison Road & P12 & Urban / Suburban & $12.9-16.2$ & 12.4 & Landover & 2 \\
\hline Georgia Avenue Metro Extra & 79 & Inner-City & $9.1-9.3$ & 9.3 & Bladensburg & 3 \\
\hline Greenbelt-BWI Airport Express & B30 & Commuter & $25.3-26.7$ & 25.8 & Landover & 3 \\
\hline Hospital Center & D8 & Inner-City & 8.3 & 8.1 & Bladensburg & 1 \\
\hline Marlow Heights-Temple Hills & H11, H12, H13 & Suburban & $15.1-16.1$ & 16.0 & Landover & 3 \\
\hline Martin Luther King Jr Highway & A12 & Urban & $12.5-12.7$ & 13.0 & Landover & 2 \\
\hline Minnesota Ave-M Street & V7, V9 & Inner-City / Urban & $8.0-10.7$ & 10.4 & Bladensburg & 3 \\
\hline North Capitol Street & 80 & Inner-City & $7.6-7.8$ & 7.6 & Bladensburg & 2 \\
\hline Prince George's-Silver Spring & F4, F6 & Urban & $10.3-12.1$ & 12.1 & Landover & 3 \\
\hline Rhode Island Ave-New Carrollton & 84,85 & Inner-City / Urban & $9.4-13.7$ & 12.1 & Landover & 2 \\
\hline
\end{tabular}

Regarding WMATA's overall service (Figure 4-12), the transit agency equally serves Inner-City and Urban routes with $40.1 \%$ and $37.5 \%$ of service time, respectively. Suburban service amounts to $16.6 \%$ and Commuter service to $5.8 \%$ of service time.

The following figures show the various metrics plotted versus average speed and marked according to the assigned cluster. Figure 4-13 shows characteristic acceleration without grade, Figure 4-14 shows percentage idle, Figure 4-15 stops per mile, Figure 4-16 standard deviation of speed, Figure 4-17 average acceleration, Figure 4-18 average deceleration, Figure 4-19 aerodynamic speed, and Figure 4-20 kinetic intensity without grade.

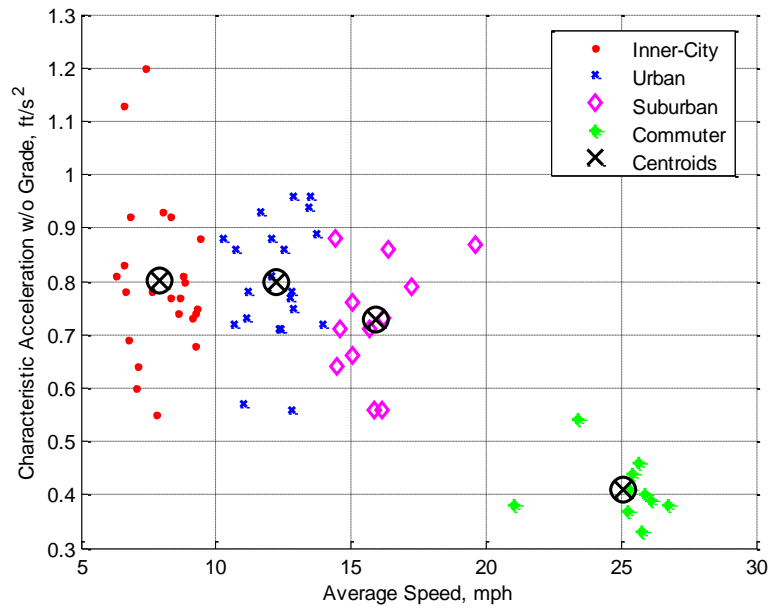

Figure 4-13 $\tilde{a}$ without Grade vs. Average Speed

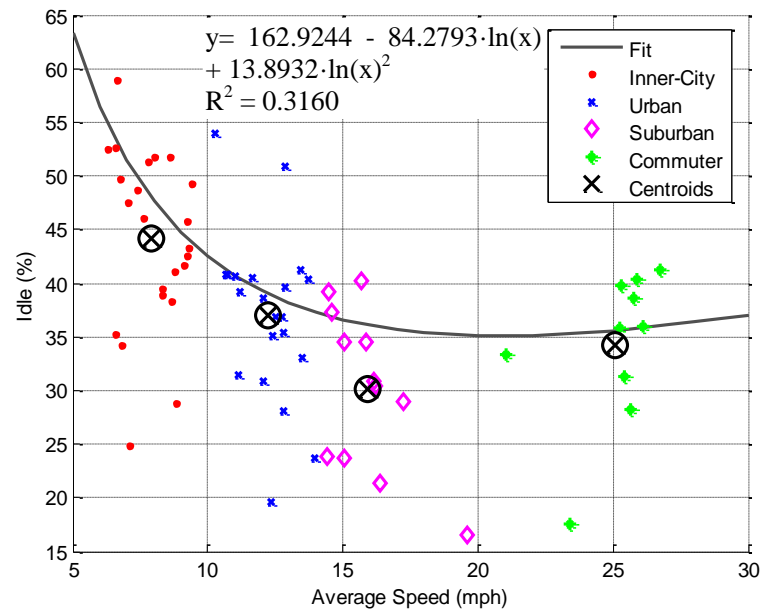

Figure 4-14 Percentage Idle vs. Average Speed 


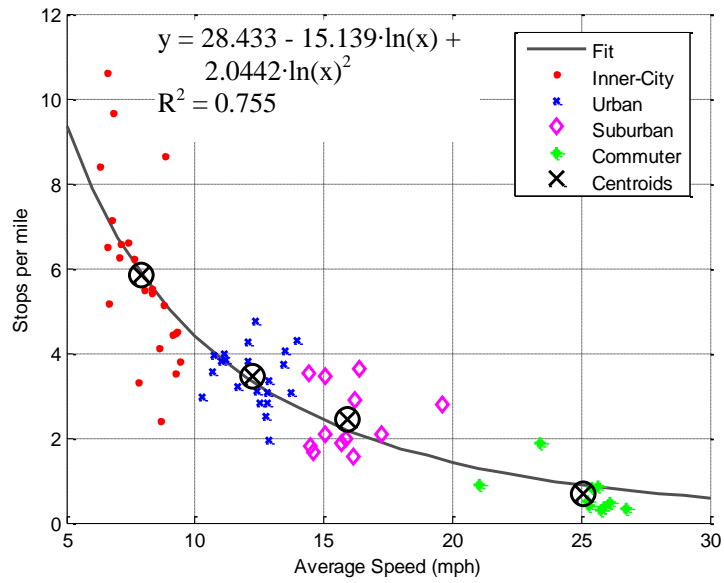

Figure 4-15 Stops per mile vs. Average Speed

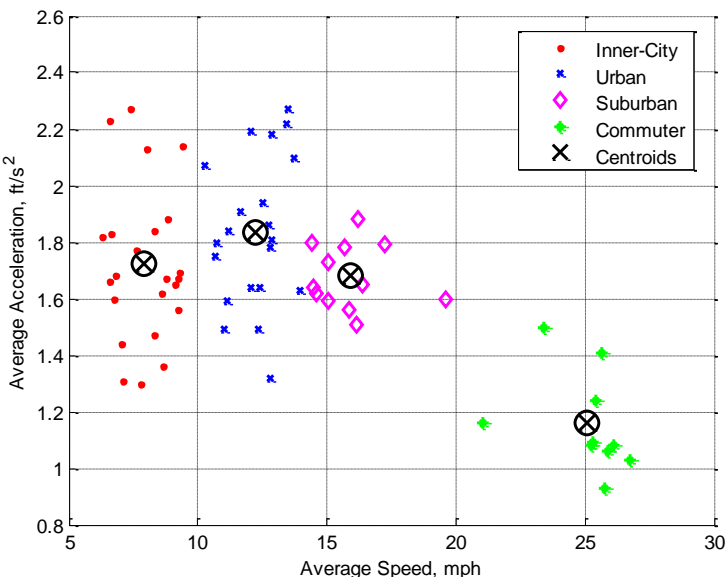

Figure 4-17 Average Acceleration vs. Average Speed

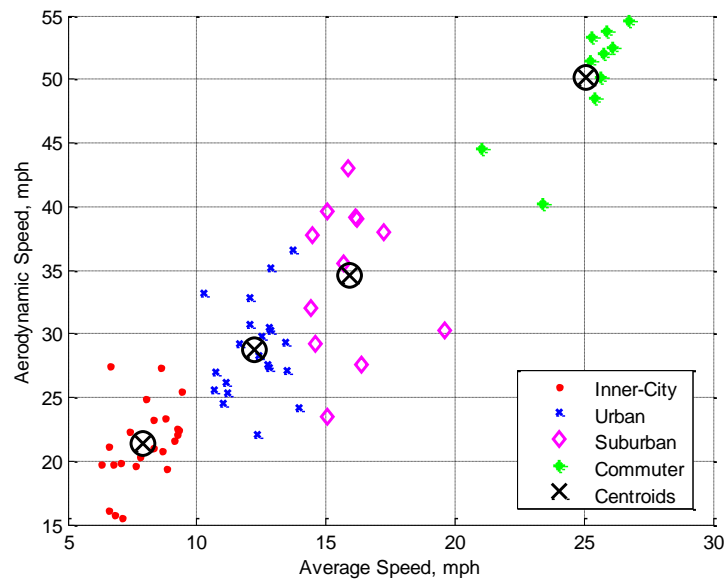

Figure 4-19 Aerodynamic Speed vs. Average Speed

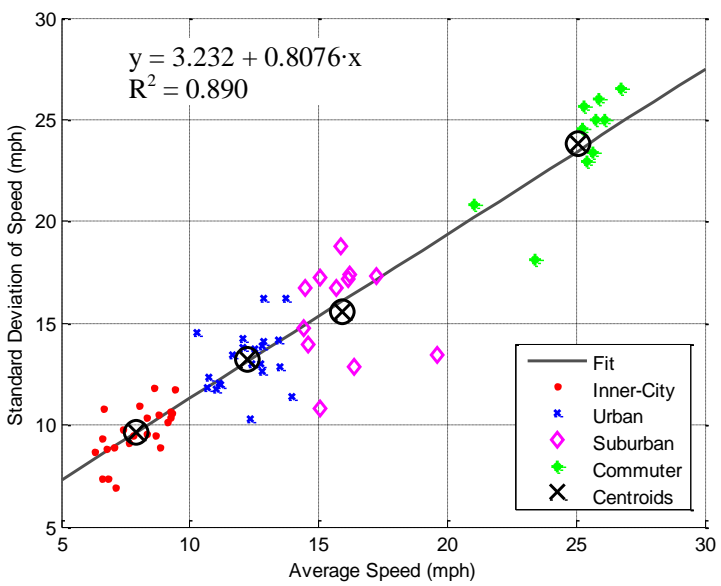

Figure 4-16 Standard Deviation of Speed vs. Average Speed

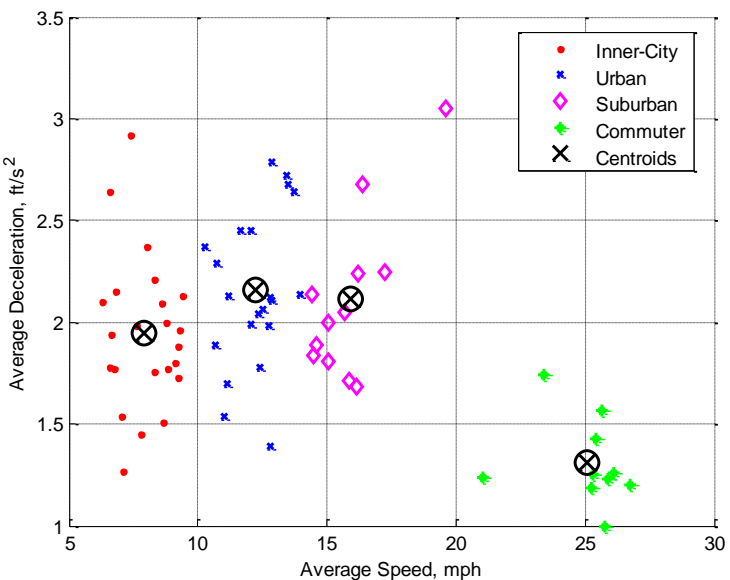

Figure 4-18 Average Deceleration vs. Average Speed

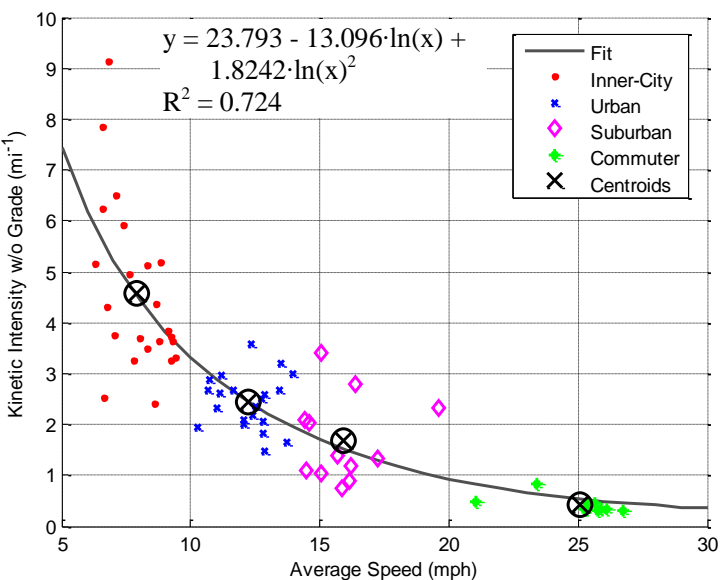

Figure 4-20 Kinetic Intensity without Grade vs. Average Speed 
As in Figure 4-2, there are correlations between average speed and many of the other metrics. Regression curves were developed for the four predictor metrics used in the IBIS models: the regessions for percentage idle, stops per mile, standard deviation of speed, and kinetic intensity are shown Figure 4-14, Figure 4-15, Figure 4-16, and Figure 4-20, respectively. The percentage idle regression curve has a low coefficient of determination but the author believes that the fit is a good representation of the values of percentage idle observed in the field. Given a value of average speed, these regression equations can be used to obtain estimates of the predictor metrics, which in turn allow estimation of fuel economy and emissions in IBIS.

Fuel economy (Figure 4-21) and $\mathrm{NO}_{\mathrm{x}}$ emissions (Figure 4-22) were evaluated using IBIS. The inputs were all five predictor metrics: average speed, percentage idle, stops per mile, standard deviation of speed, and kinetic intensity. The simulation assumed a MY 2005 diesel-electric hybrid bus. The figures show that emissions of the centroids are in good agreement with the emissions of their cluster. Moreover, emissions predicted with the metric regression equations from above are in good agreement with the overall trend and the individual routes.

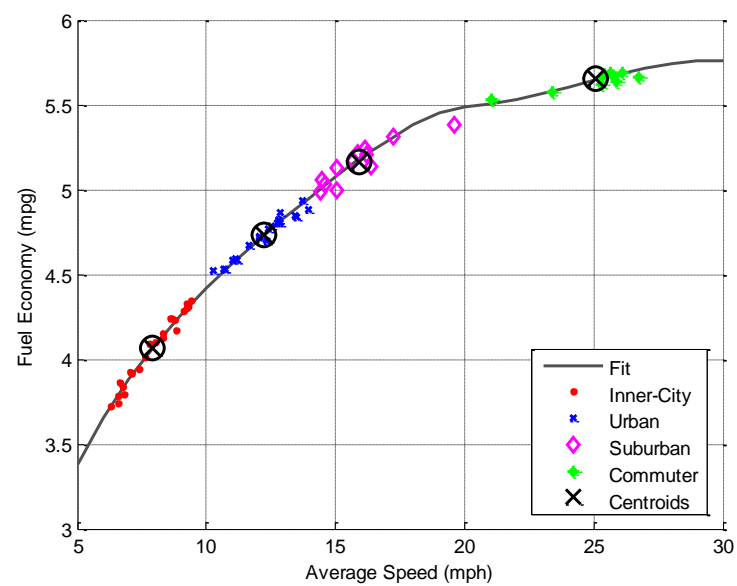

Figure 4-21 Fuel Economy vs. Average Speed

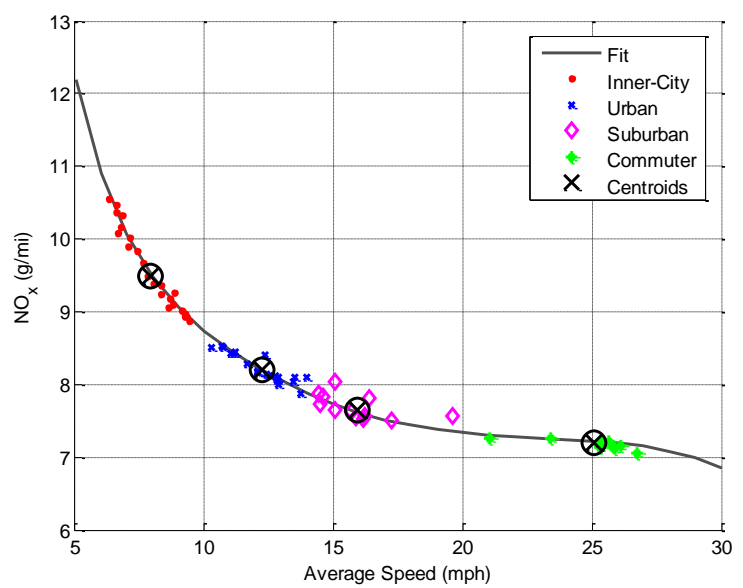

Figure 4-22 $\mathrm{NO}_{\mathrm{x}}$ Emissions vs. Average Speed

\subsubsection{Survey of Service Type to Bus Operators}

The author surveyed a handful of transit agencies inquiring about the average speed of their various service types. The following agencies were contacted: King County Metro (KCM), Orange County Transportation Authority (OCTA), New York City Transit (NYCT), Long Beach Transit, WMATA, and Dallas Area Rapid Transit (DART).

$\mathrm{KCM}$ reported that their system average speed was $13 \mathrm{mph}$, that their Inner-City service had 8 $\mathrm{mph}$, and that their Express service had $17 \mathrm{mph}$. OCTA reported that their overall average speed was 
$13 \mathrm{mph}$. Long Beach Transit had an overall average speed of $12.5 \mathrm{mph}$, ranging from 9 to $16 \mathrm{mph}$. The operation at WMATA had an overall average speed of $12 \mathrm{mph}$, ranging from 7 to $28 \mathrm{mph}$. NYCT reported that their Inner-City service ran at 5-6 $\mathrm{mph}$, Urban service at 7-8 $\mathrm{mph}$, Suburban at 10-12 mph, Commuter service at 12-15 mph, and BRT service at 7-8 mph. Average speed from New York City is significantly lower than the speed from the other surveyed agencies. In general, the clusters of Table 4-7 and Table 4-8 have good agreement with the values reported by the transit agencies.

The centers of the clusters also agree with data provided by Dallas Area Rapid Transit (DART) in that the routes they classify as urban have a mean average speed of $12 \mathrm{mph}$ and a median of $11.4 \mathrm{mph}$. The system's average speed was $12.5 \mathrm{mph}$. DART identified a group of routes called cross-town, that do not travel through the Central Business District (CBD); these routes correspond for the most part to the Urban category (mean $12.4 \mathrm{mph}$, median $12.2 \mathrm{mph}$ ). Similarly, DART's Rail Feeder routes also correspond to Urban service (mean $11.9 \mathrm{mph}$, median $11.5 \mathrm{mph}$ ). The last category of service identified by DART is from routes that link suburban park-and-ride stations with the CBD traveling on the freeway. These routes correspond mostly to the Suburban category but include some Commuter and some Urban (mean $\bar{U}$ is $18.6 \mathrm{mph}$, median $17.9 \mathrm{mph}$ ).

The distribution of transit bus operation from DART is shown in Figure 4-23. DART's operation is heavily concentrated in Urban service $(72.1 \%)$, followed by Suburban (13.4\%), Inner-City (8.8\%) and Commuter (with only 2.7\%). It must be noted that neither of the surveyed agencies had operation below $5 \mathrm{mph}$ or above $30 \mathrm{mph}$.

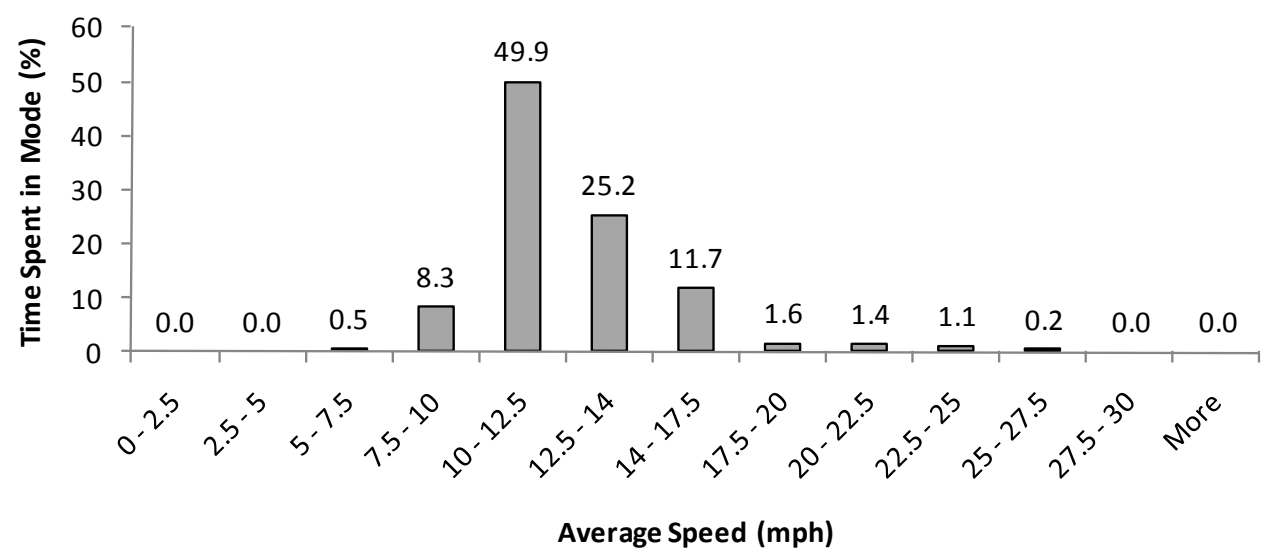

Figure 4-23 Distribution of Route Average Speed at DART 


\subsubsection{Distribution of Speed and Acceleration by Service Type}

The distributions of acceleration and speed were calculated for all the routes. It was observed that routes within the same group had similar distributions. The acceleration and speed distributions of the centroids of the four categories are shown next.

Figure 4-24 presents the distribution of speed for the centroids of the four service types. Inner-City corresponds to speeds below $15 \mathrm{mph}$, Urban between 15 and $30 \mathrm{mph}$, Suburban between 30 and 50 mph, and Highway above $50 \mathrm{mph}$ (Table 2-4, page 23). Inner-City service spends most of the time $(54 \%)$ at low speeds, followed by urban speed taking nearly $40 \%$ of the time. As the average speed of the route increases, the distribution shifts towards higher speeds and the fractions of suburban and highway speeds increase. Suburban routes spent most of the time at urban and inner-city speeds but their fraction of suburban and highway speeds are higher compared to Urban routes. Figure 4-12 (page 107) and Figure 4-23 (page 113) presented the distribution of average route speed from two transit agencies and they showed that nearly $75 \%$ of transit bus service corresponds to Inner-City and Urban service (average speeds between 5 and $14 \mathrm{mph}$ ). These two types of service are represented by the distributions of Figure 4-24 (a) and (b), as well as (a) and (b) in the subsequent figures (Figure 4-26 to Figure 4-28). 


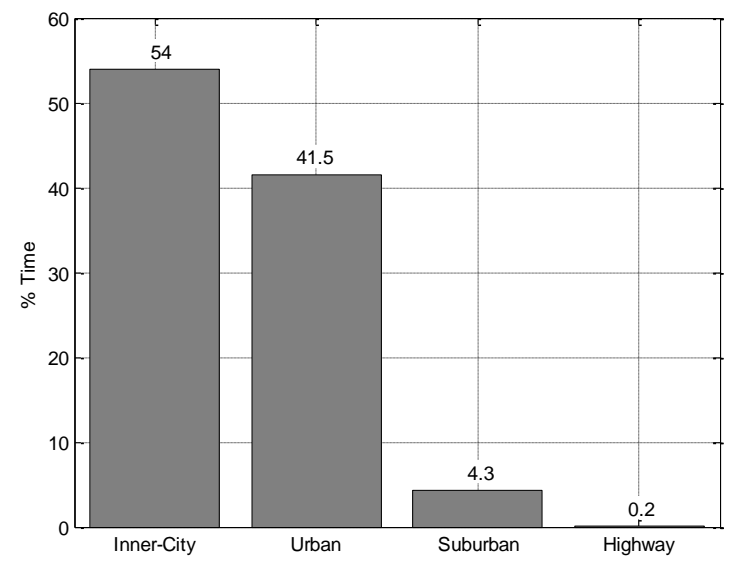

(a) Inner-City Service

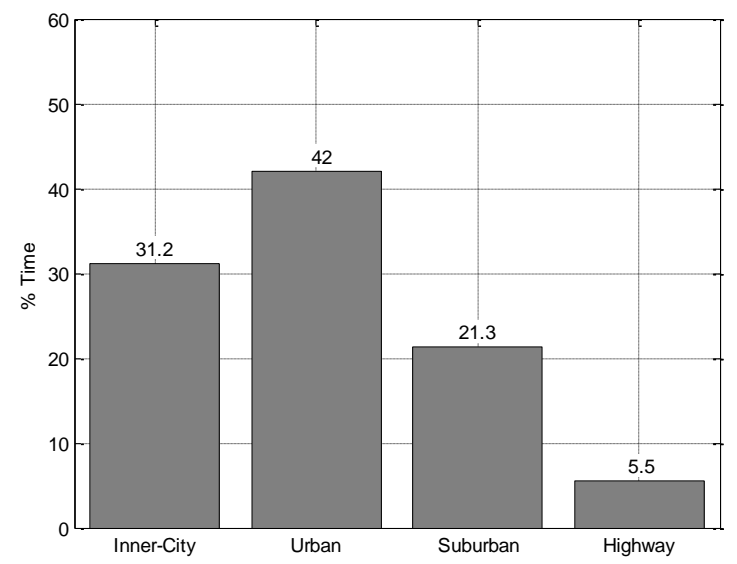

(c) Suburban Service

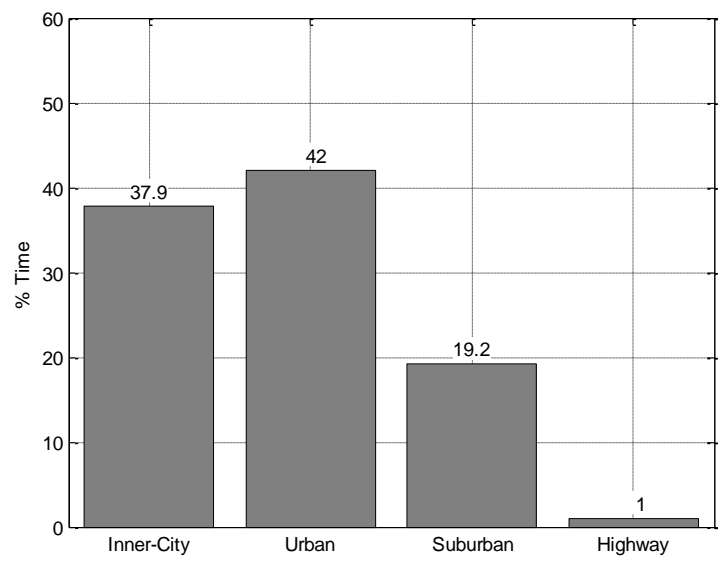

(b) Urban Service

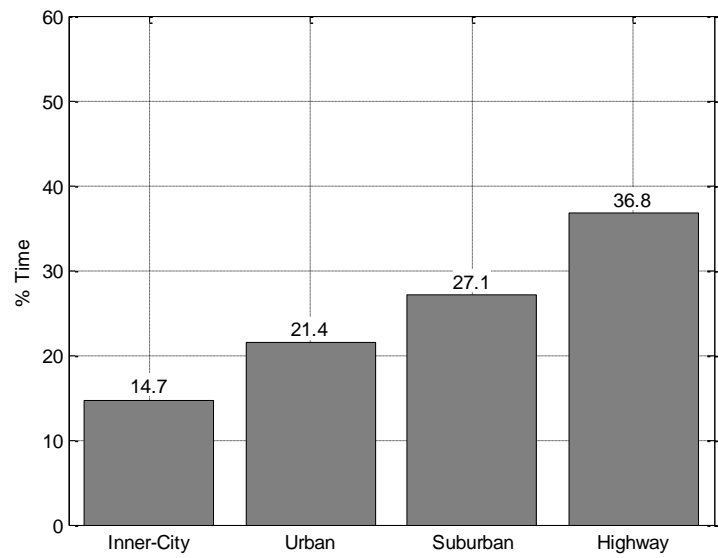

(d) Commuter Service

Figure 4-24 Speed Distribution of Service Type Centroids

Figure 4-25 shows the box plots of the driving modes for the four service types. The variability of the data is apparent in the figure. The red mark in the middle is the median, the blue box encloses the quartiles (middle half of the data), the whiskers extend to extreme data points, and the red "+" marks are data points away from the closest quartile by more than 1.5 times the interquartile distance (these would be outliers if the distribution were normal, which is not the case). The circled crosses $(x)$ represent the centroids of the clusters. It is observed that the fraction of urban driving is very similar for all but Commuter service. 


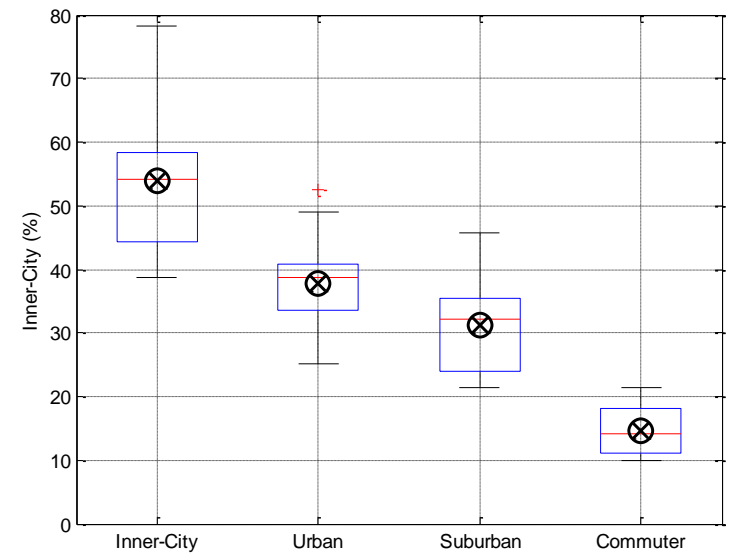

(a) Inner-City Driving

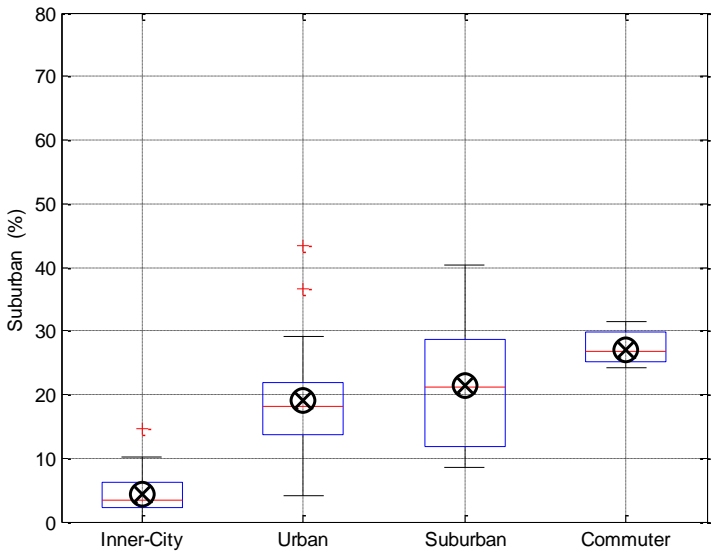

(c) Suburban Driving

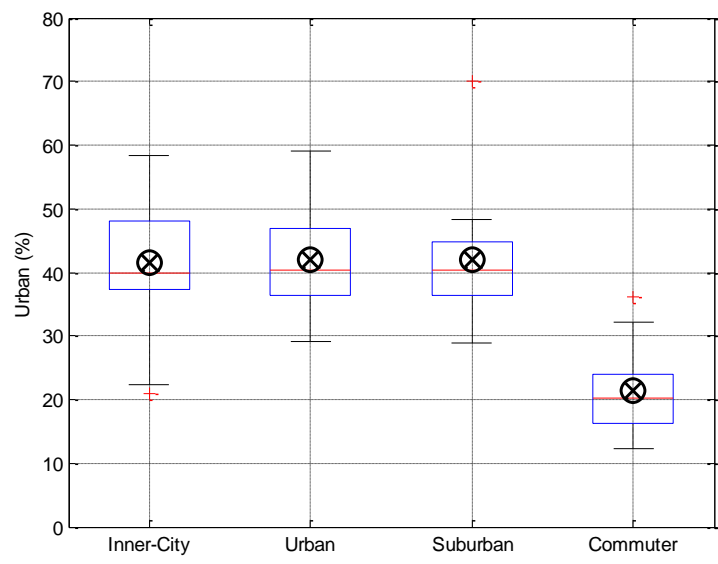

(b) Urban Driving

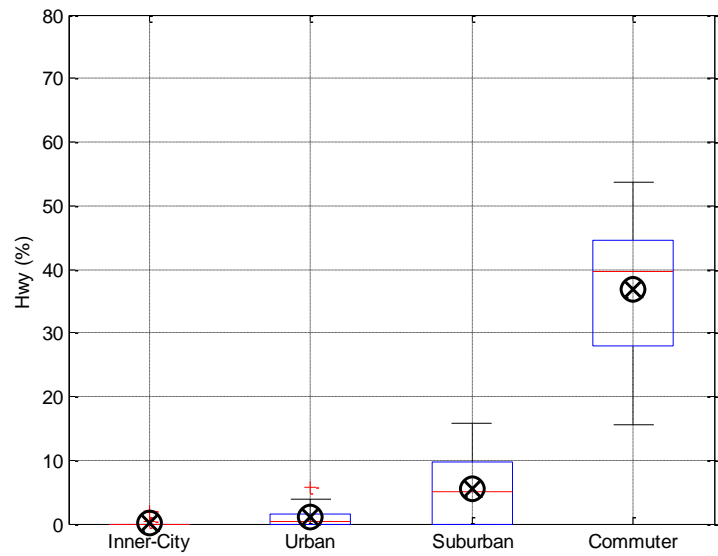

(d) Highway Driving

Figure 4-25 Box Plots of Driving Mode Fractions by Service Type

Figure 4-26 has broken the speed groups into accelerations to further explore the characteristics of each driving mode. Acceleration at inner-city speeds exhibits dominance of heavy and medium accelerations, urban speeds are dominated by medium and light accelerations, suburban speeds are dominated by light accelerations, and highway speeds have mostly cruise accelerations. That is, at low speeds accelerations are high and they decrease in intensity as speed increases.

Figure 4-27 and Figure 4-28 show the distribution of accelerations and decelerations, respectively, for the various service types. For Inner-City, Urban, and Suburban service most accelerations are medium and light, whereas Commuter service is composed primarily of light and cruise accelerations. Decelerations show some degree of uniformity for all but Commuter service, which as in its accelerations, exhibits primarily light and cruise decelerations. 


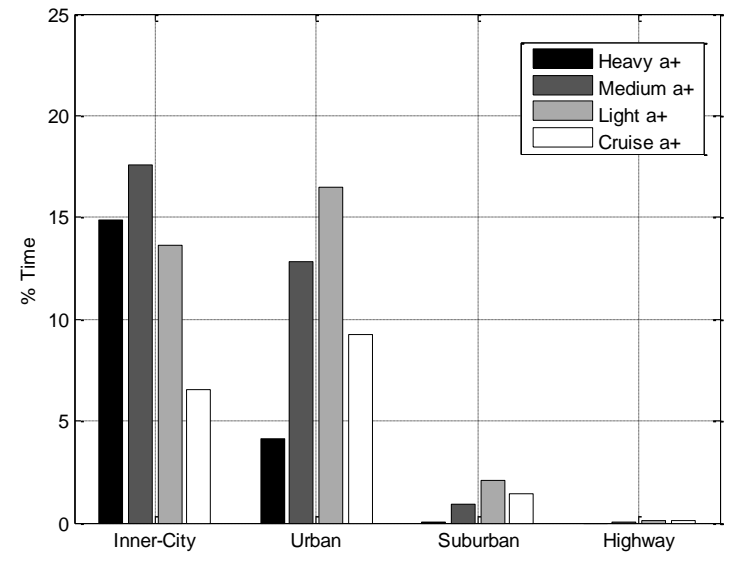

(a) Inner-City Service

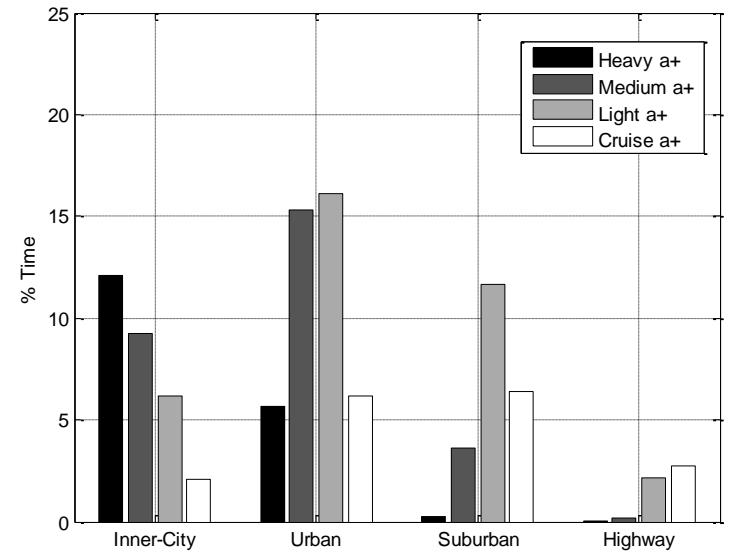

(c) Suburban Service

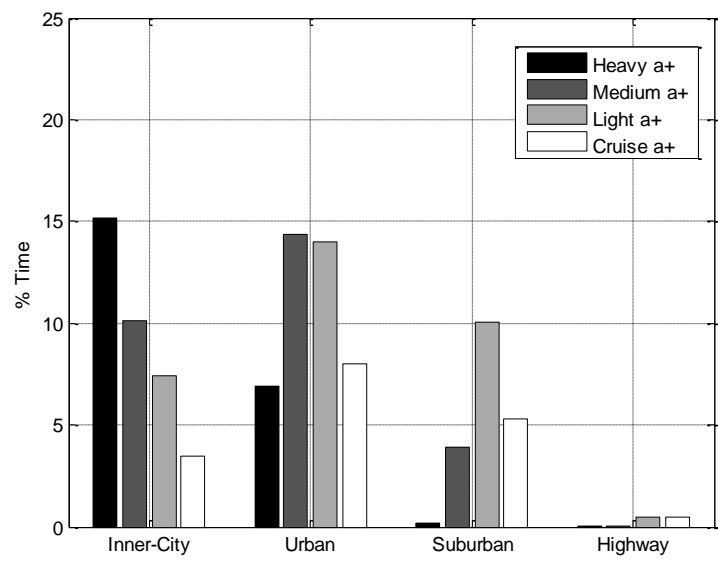

(b) Urban Service

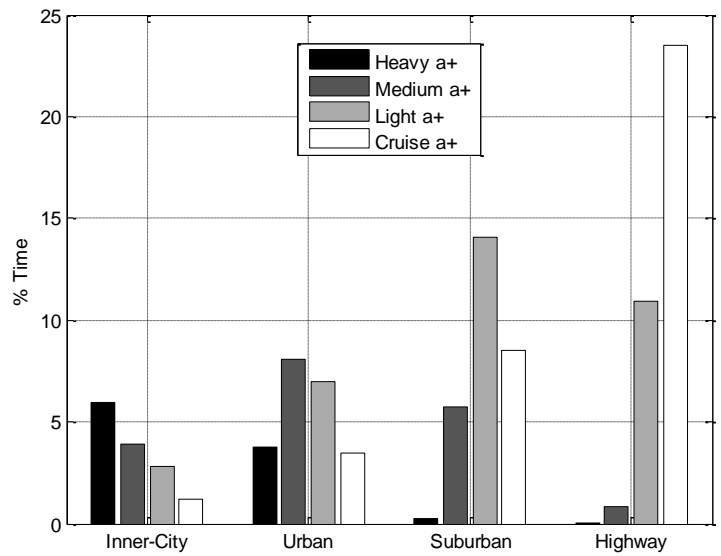

(d) Commuter Service

Figure 4-26 Distribution of Speed-Acceleration of Service Type Centroids 


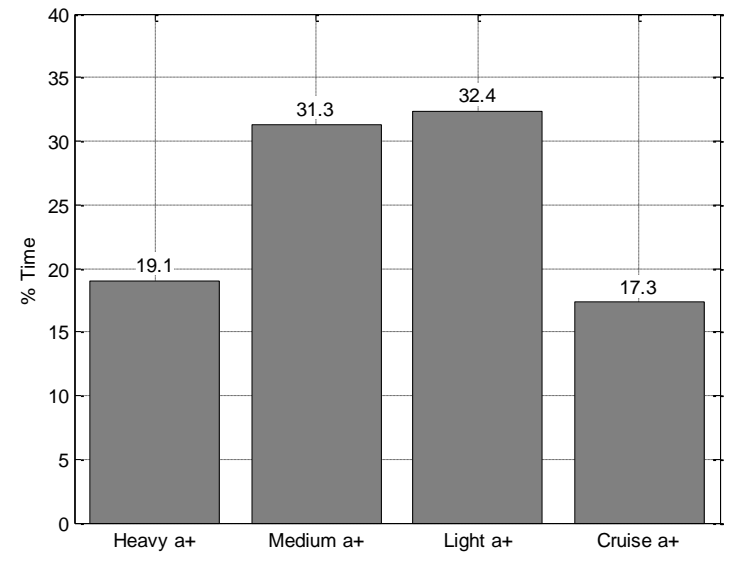

(a) Inner-City Service

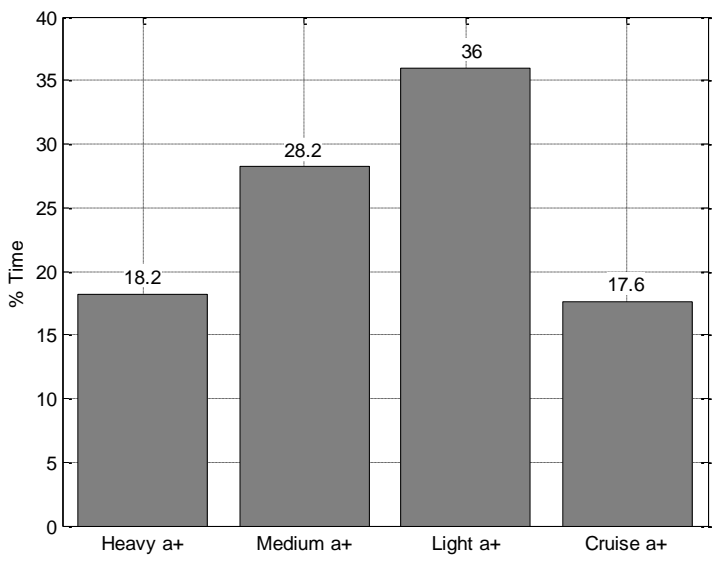

(c) Suburban Service

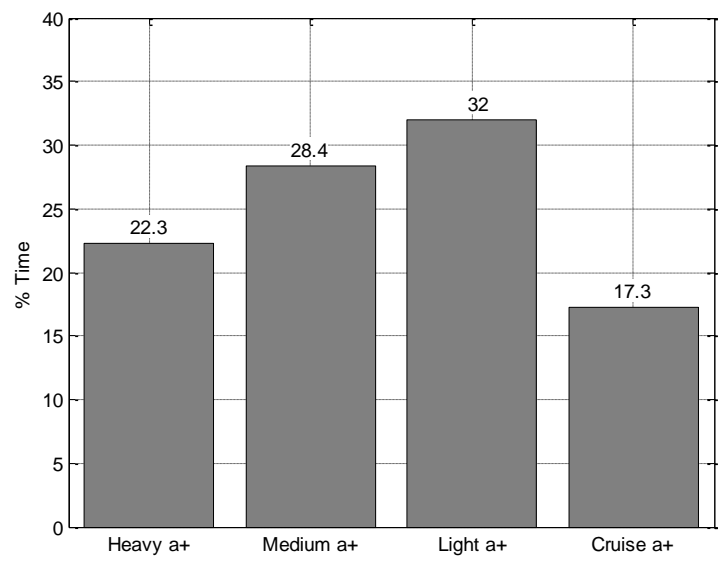

(b) Urban Service

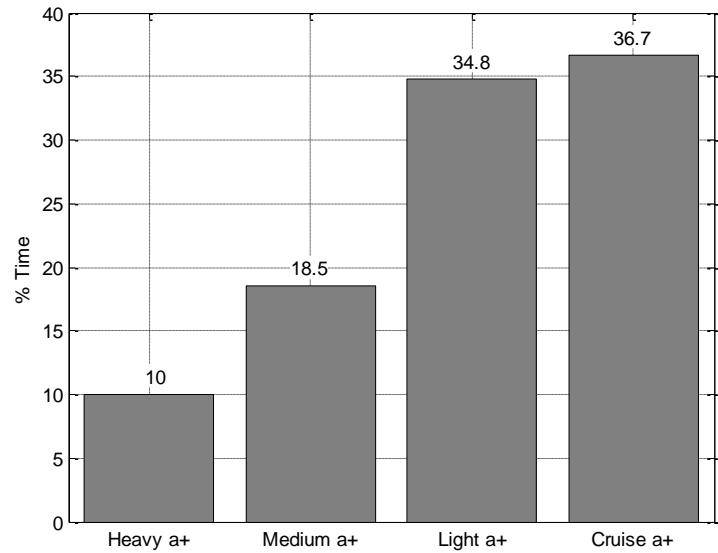

(d) Commuter Service

Figure 4-27 Distribution of Acceleration of Service Type Centroids 


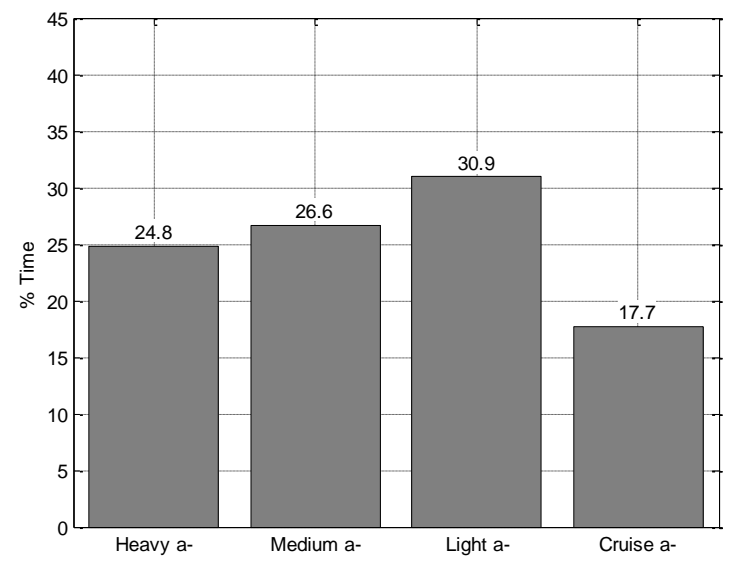

(a) Inner-City Service

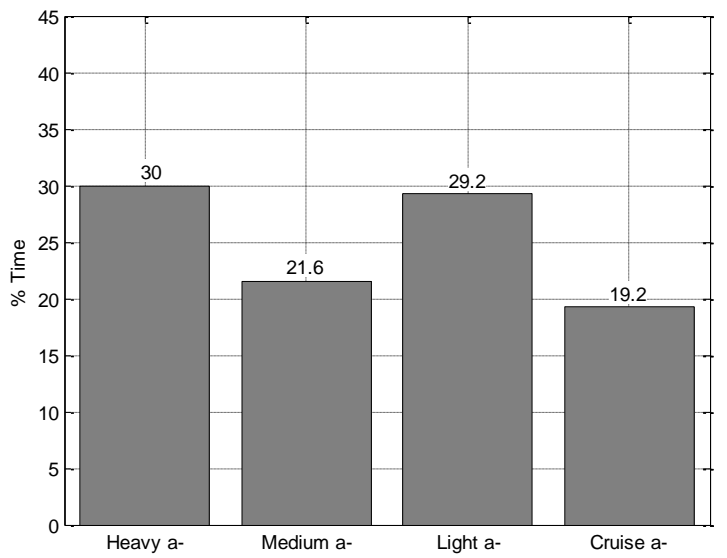

(c) Suburban Service

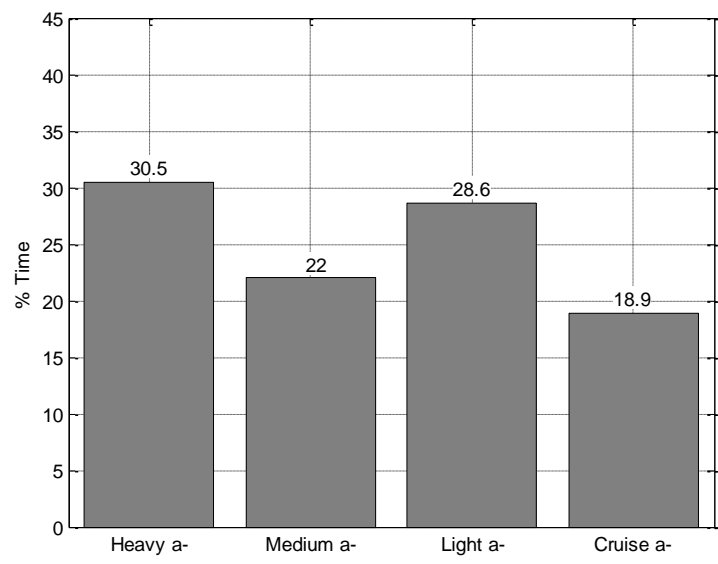

(b) Urban Service

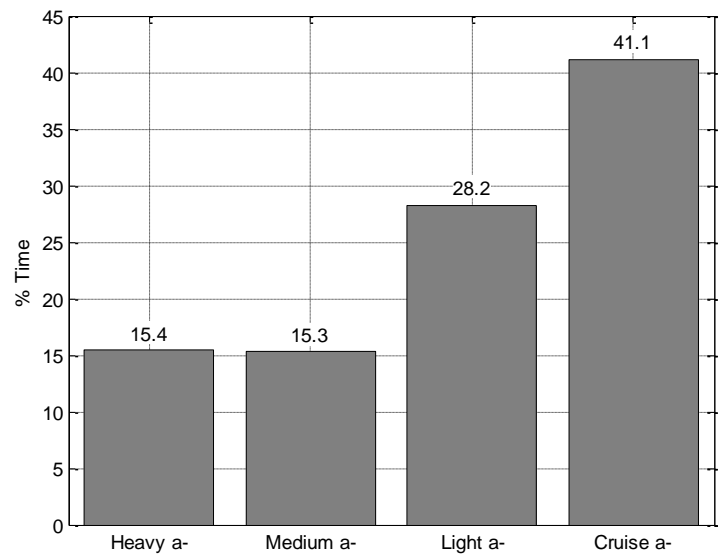

(d) Commuter Service

Figure 4-28 Distribution of Deceleration of Service Type Centroids

\subsection{Summary}

In this chapter, in-use transit bus duty cycles we characterized and transit bus operation was classified by average speed into four service categories: Inner-City ( $\bar{U} \leq 10)$, Urban $(10<\bar{U} \leq 14)$, Suburban $(14<\bar{U} \leq 20)$, and Commuter $(\bar{U}>20)$.

The ranges observed for the cycle metrics were presented at the bottom of Table 4-6. The metricvalues that best represent each service type were determined by k-means analysis and were presented in Table 4-7 (page 109). Regression curves were developed for various cycle metrics as a function of average speed: percentage idle (Figure 4-14), stops per mile (Figure 4-15), standard deviation of speed (Figure 4-16), and kinetic intensity (Figure 4-20). These regressions were used to predict fuel economy and $\mathrm{NO}_{\mathrm{x}}$ emissions in IBIS (assuming a MY 2005 diesel-electric hybrid bus), and it was 
determined that the predicted values were good representations of the overall duty cycle database. Fuel economy and $\mathrm{NO}_{\mathrm{x}}$ emissions predictions of the logged routes and the regression fit were presented in Figure 4-21 and Figure 4-22, respectively.

A handful of transit agencies were surveyed and their operation agreed with the proposed service type classification. It was determined that there is not a significant difference between predicting emissions for the overall bus fleet (using fleet average speed), and predicting for individual routes and then determining the composite fleet emissions. This result is important in that it provides confidence in the simplified approach (overall average speed).

Correlations between pairs of metrics were explored. It was determined that average speed is well correlated with most of the other metrics, except for characteristic acceleration and percentage idle. The author concluded that the three nearly independent metrics (average speed, characteristic acceleration, and percentage idle) should be explored as predictor metrics for emissions and fuel consumption.

It was determined that the estimations of route average speed provided by the transit agency were in good agreement with the actual average speed values, and that the effects of day-to-day and run-torun variations were mild. It is recommended that when evaluating fleet emissions, agency estimated average speed values are used directly as inputs to the IBIS models. In the case that no average speed values are known by the agency, the values for the service type centroids are recommended, once the agency has determined its dominant service type. 


\section{Chapter 5. DiESEl Engine Fuel CONSUMPTION AND EMissions MODEL}

The author chose to use Artificial Neural Networks (ANNs) and 2D lookup tables to develop the fueling and $\mathrm{NO}_{\mathrm{x}}$ emissions engine models. A general description of ANNs was presented in Section 2.6.5 and specific examples of ANNs for emissions modeling can be found in Section 2.7.4.

The engine to be modeled was a MY 2007-2009 Cummins ISB 260H (1.2-1.5 g/bhp $\left.\cdot h r \mathrm{NO}_{\mathrm{x}}\right)$. No data was available to the author from engine dynamometer tests of such engine, but the WVU TransLab had tested a transit bus with an engine of the same class but MY 2006 (2.5 g/bhp·hr NO $\mathrm{NO}_{\mathrm{x}}$. The chassis dynamometer tests had recorded engine ECU broadcast (engine speed and percent torque) and this provided a suitable source of data.

The engine broadcast was matched with the results from the emissions bench, obtaining a dataset of [time, speed, torque, fueling rate, and $\mathrm{NO}_{\mathrm{x}}$ emissions] that was used to develop the ANN models.

The following sections summarize the chassis dynamometer tests, engine lug curve, development of the fueling and $\mathrm{NO}_{\mathrm{x}}$ emissions maps (2D lookup tables), and development and validation of the ANNs.

\subsection{Chassis Dynamometer Test Data from MY 2006 Orion - BAE Hybrid Bus}

WVU TransLab tested a MY 2006 40' Orion hybrid bus (Transit Resource Center, 2007). The bus belonged to Liberty Lines Transit / Westchester County Department of Transportation. The hybrid configuration was a series architecture powered with the BAE Systems HybriDrive ${ }^{\circledR}$ propulsion system. The bus was tested over 6 different drive cycles: Beeline, Manhattan, New York Bus, OCTA, UDDS, and WMATA. Table 5-1 summarizes the fuel economy and emissions results.

Curb weight was 33,440 lb (15,170 kg), GVWR was 42,540 lb (19,295 kg), test weight was 38,540 lb $(17,480 \mathrm{~kg})$, the test fuel was ULSD\#1, and all the reported tests were warm-start tests. The vehicle was equipped with oxidation catalyst (DOC) and particulate trap (DPF). The diesel engine was a 2006 Cummins ISB $260 \mathrm{H}$.

The advantage of using these data to develop the engine models is that the test data correspond to in-use engine operation and not to an engine certification test. Thus the data truly represents the 
engine operational envelope that is expected during service in the series-hybrid transit bus application. The disadvantage is that one must rely upon engine torque and speed broadcasted by the ECU. Conversion of ECU torque to engineering units was much simpler than in previous research programs (e.g. Thompson et al., 2002) because the engine torque channel (SPN 513 of SAE J1939: Actual Engine - Percent Torque) is a percentage and can be directly converted to engineering units with the engine lug torque.

Table 5-1 Summary of Emissions Tests - Orion-BAE Series Hybrid Bus. (Transit Resource Center, 2007)

\begin{tabular}{|c|c|c|c|c|c|c|c|c|c|c|}
\hline Cycle & Repeat & $\begin{array}{l}\overline{\mathbf{U}}_{\text {no idle }} \\
\text { (mph) }\end{array}$ & $\begin{array}{c}\overline{\mathbf{U}} \\
(\mathbf{m p h})\end{array}$ & $\begin{array}{c}\text { Time } \\
\text { (s) }\end{array}$ & $\begin{array}{c}\text { Fuel } \\
\text { Economy } \\
(\mathrm{mpg})\end{array}$ & $\begin{array}{l}\text { Fuel } \\
\text { Cons. } \\
\text { (g/mi) }\end{array}$ & $\begin{array}{c}\mathrm{NO}_{\mathrm{x}} \\
(\mathrm{g} / \mathrm{mi})\end{array}$ & $\begin{array}{c}\mathrm{CO}_{2} \\
(\mathrm{~g} / \mathrm{mi})\end{array}$ & $\begin{array}{c}\mathbf{P M} \\
(\mathrm{g} / \mathrm{mi})\end{array}$ & $\begin{array}{l}\text { Dist. } \\
\text { (mi) }\end{array}$ \\
\hline \multirow{6}{*}{ Beeline } & 1 & 21.8 & 15.2 & 1,722 & 4.62 & 668 & 9.85 & 2,092 & 0.006 & 7.26 \\
\hline & 2 & 21.8 & 15.1 & 1,722 & 4.73 & 651 & 9.51 & 2,039 & 0.011 & 7.22 \\
\hline & 3 & 22.2 & 15.2 & 1,722 & 4.81 & 642 & 9.23 & 2,008 & 0.007 & 7.26 \\
\hline & 4 & 21.9 & 15.1 & 1,722 & 4.87 & 633 & 9.32 & 1,982 & 0.004 & 7.22 \\
\hline & 5 & 21.9 & 15.0 & 1,722 & 4.80 & 643 & 9.45 & 2,012 & 0.007 & 7.20 \\
\hline & 6 & 21.9 & 15.1 & 1,722 & 4.83 & 639 & 9.34 & 2,000 & 0.005 & 7.23 \\
\hline \multirow{3}{*}{ Manhattan } & 1 & 11.8 & 7.3 & 1,097 & 4.05 & 760 & 13.46 & 2,384 & 0.007 & 2.24 \\
\hline & 2 & 11.9 & 7.3 & 1,097 & 3.90 & 792 & 13.35 & 2,476 & - & 2.23 \\
\hline & 3 & 11.9 & 7.3 & 1,097 & 3.94 & 782 & 12.76 & 2,452 & - & 2.24 \\
\hline \multirow{3}{*}{$\begin{array}{c}\text { New York } \\
\text { Bus }\end{array}$} & 1 & 11.4 & 3.8 & 598 & 2.19 & 1,417 & 23.28 & 4,403 & 0.021 & 0.64 \\
\hline & 2 & 11.2 & 3.8 & 598 & 2.30 & 1,342 & 22.43 & 4,199 & 0.035 & 0.64 \\
\hline & 3 & 11.3 & 3.7 & 598 & 2.24 & 1,377 & 23.42 & 4,304 & 0.014 & 0.62 \\
\hline \multirow{3}{*}{ OCTA } & 1 & 17.8 & 13.0 & 1,948 & 5.15 & 599 & 9.98 & 1,875 & 0.004 & 7.05 \\
\hline & 2 & 17.7 & 13.0 & 1,948 & 5.23 & 590 & 9.79 & 1,847 & 0.004 & 7.05 \\
\hline & 3 & 17.8 & 13.0 & 1,948 & 5.29 & 583 & 9.73 & 1,826 & 0.003 & 7.05 \\
\hline \multirow{3}{*}{ UDDS } & 1 & 30.5 & 20.1 & 1,059 & 5.77 & 534 & 7.59 & 1,672 & 0.012 & 5.91 \\
\hline & 2 & 30.7 & 20.3 & 1,059 & 5.95 & 518 & 7.43 & 1,623 & 0.011 & 5.97 \\
\hline & 3 & 30.8 & 20.3 & 1,059 & 5.75 & 536 & 7.36 & 1,679 & 0.008 & 5.97 \\
\hline \multirow{3}{*}{ WMATA } & 1 & 15.9 & 9.0 & 1,837 & 4.26 & 724 & 11.91 & 2,268 & 0.002 & 4.62 \\
\hline & 2 & 15.8 & 9.0 & 1,837 & 4.52 & 683 & 10.91 & 2,138 & 0.003 & 4.60 \\
\hline & 3 & 15.5 & 9.0 & 1,837 & 4.62 & 667 & 11.16 & 2,091 & 0.001 & 4.60 \\
\hline
\end{tabular}

\subsection{Engine Performance Curve}

Engine ECU parameters of interest were logged: engine speed (rpm), current torque to maximum available torque ratio $(\%)$, fuel rate $(\mathrm{L} / \mathrm{s})$, and vehicle velocity $(\mathrm{km} / \mathrm{h})$. The torque ratio is a ratio between the engine torque output and the maximum torque at the current speed. Therefore, the engine performance curve had to be obtained from the engine manufacturer. Figure 5-1 was built from this information showing the maximum torque and the maximum power curves in $\mathrm{Nm}$ and $\mathrm{kW}$ respectively. 


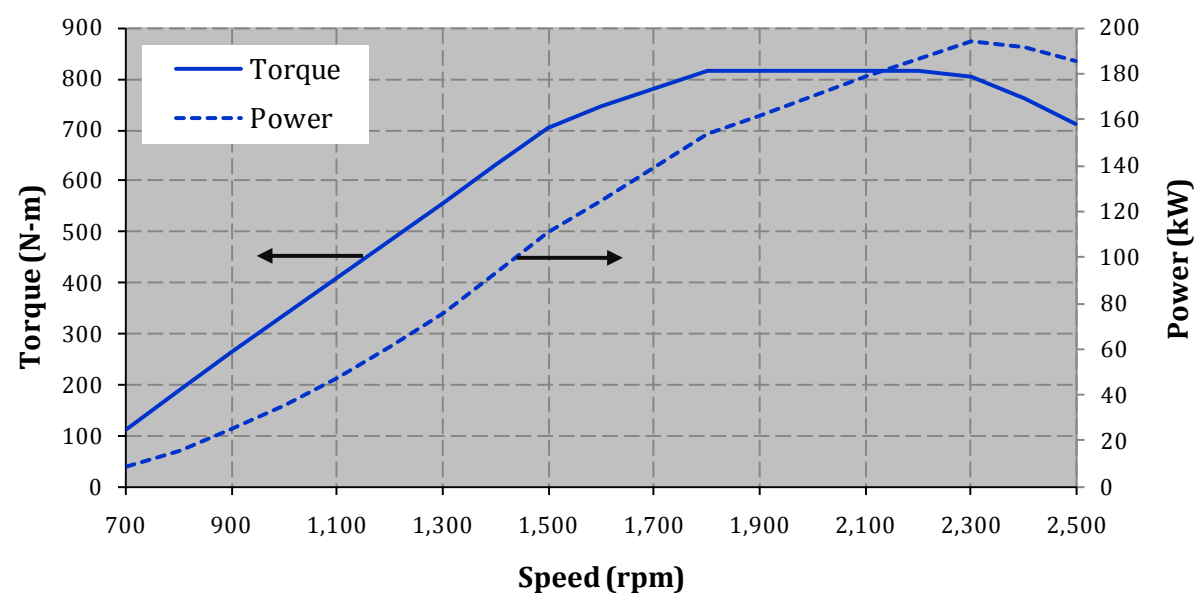

Figure 5-1 Cummins ISB 260H Engine Performance Curve

Engine peak torque is $815 \mathrm{Nm}(600 \mathrm{ft}-\mathrm{lb})$ at $1,800 \mathrm{rpm}$, peak power is $194 \mathrm{~kW}$ (260 hp) at 2,300 $\mathrm{rpm}$, and governed speed is 2,500 rpm. Table 5-2 is a list of relevant engine parameters.

Table 5-2 Cummins ISB 260H Engine Parameters

\begin{tabular}{l|l}
\hline \multicolumn{1}{c|}{ Parameter } & \multicolumn{1}{c}{ Input } \\
\hline Peak Power & 260 HP $(194 \mathrm{KW}) @ 2300 \mathrm{rpm}$ \\
\hline Peak Torque & $600 \mathrm{ft}-\mathrm{lb}(815 \mathrm{~N}-\mathrm{m}) @ 1800 \mathrm{rpm}$ \\
\hline Fuel System & Bosch Electronic \\
\hline Cylinders & 6 \\
\hline Bore & $4.02 \mathrm{in}(102 \mathrm{~mm})$ \\
\hline Stroke & 4.72 in $(120 \mathrm{~mm})$ \\
\hline & U.S. EPA 2004 \\
Emission Certification & HC+ NO $: 2.5 \mathrm{~g} / \mathrm{bhp} \cdot \mathrm{hr}$ \\
& CO: $0.6 \mathrm{~g} / \mathrm{bhp} \cdot \mathrm{hr}$ \\
& PM: $0.04 \mathrm{~g} / \mathrm{bhp} \cdot \mathrm{hr}$ \\
\hline Aspiration & Turbocharged and Charge Air Cooled \\
\hline Displacement & 359 in $^{3}(5.9 \mathrm{~L})$ \\
\hline
\end{tabular}

\subsection{Emissions and ECU Data Reduction}

The primary objective of the engine models was to predict tailpipe-out $\mathrm{CO}_{2}$ and $\mathrm{NO}_{\mathrm{x}}$ emissions and instantaneous fuel consumption, all in grams per second, based on engine speed and torque. The emissions measurements from the analytical bench do not exactly correspond to tailpipe-out values due to diffusion and dispersion effects (Madireddy and Clark, 2006). Diffusion and dispersion effects are due to mixing of the charge in the tunnel and due to analyzer time response; these effects were not considered since there was not enough information to reconstruct instantaneous tailpipe-out emissions.

There is a time delay in the concentration measurements as the exhaust travels the dilution tunnel and sampling system; similarly there is a delay in the tunnel flow rate measurement. The delays were 
corrected through cross-correlation with hub power. ECU data were time-aligned as well by crosscorrelation between engine power and hub power.

Continuous emissions concentration measurements and tunnel flow were extracted from the TransLab Server at $1 \mathrm{~Hz}$ using the ContData software, and were converted to $10 \mathrm{~Hz}$ using cubic splines. Engine-out emissions were calculated by correcting species concentrations for background concentrations per CFR 40 Part 86 Subpart N Sections 86.1319-90 and 86.1342-90. The equations for emissions mass flow rate calculation are:

\section{Equation 5.1 Emissions Data Reduction Equations}

$$
\begin{aligned}
& H C_{\text {mass flow }, i}=\frac{1}{10^{6}} \cdot\left[H C_{e, i}-H C_{b} \cdot\left(1-\frac{1}{D F_{i}}\right)\right] \cdot V_{\text {mix }, i} \cdot \text { Density } H C \\
& C O_{\text {mass flow }, i}=\frac{K_{w, i}}{10^{6}} \cdot\left[C O_{e, i}-C O_{b} \cdot\left(1-\frac{1}{D F_{i}}\right)\right] \cdot V_{m i x, i} \cdot \text { Density } C O \\
& C O_{2 \text { mass flow }, i}=\frac{K_{w, i}}{10^{6}} \cdot\left[C O_{2 e, i}-C O_{2 b} \cdot\left(1-\frac{1}{D F_{i}}\right)\right] \cdot V_{m i x, i} \cdot \text { Density } C O_{2} \\
& N O_{x \text { mass flow }, i}=\frac{K_{H}}{10^{6}} \cdot\left[N O_{x e, i}-N O_{x b} \cdot\left(1-\frac{1}{D F_{i}}\right)\right] \cdot V_{m i x, i} \cdot \text { Density } \mathrm{NO}_{2} \\
& \text { Fuel }_{\text {mass flow } C B, i}=\frac{1}{\text { Fuel }_{\text {Carbon }}} \cdot\left(0.273 \cdot \mathrm{CO}_{2 \text { mass flow }, i}+0.429 \cdot \mathrm{CO}_{\text {mass flow }, i}\right. \\
& \left.+\frac{12.011}{12.011+1.008 \cdot \alpha} \cdot H C_{\text {mass flow }, i}\right) \\
& V_{m i x, i}=\frac{K_{v} \cdot P_{v}}{\sqrt{T_{v}}} \\
& K_{w, i}=1-\frac{\alpha}{200} \cdot \frac{C O_{2 e, i}}{10^{4}}-\frac{1.608 \cdot H}{7000+1.608 \cdot H} \\
& K_{H}=\frac{1}{1-0.0026 \cdot(H-75)} \\
& D F_{i}=\frac{D F_{\text {Constant }}}{\left(C O_{2 e, i}+C O_{e, i}+H C_{e, i}\right) / 10^{4}} \\
& D F_{\text {Constant }}=100 \cdot \frac{x}{x+y / 2+3.76 \cdot(x+y / 4-z / 2)} \\
& x=\text { Fuel }_{\text {Carbon }} / 12.011 ; \quad y=\text { Fuel }_{\text {Hydrogen }} / 1.008 ; \quad z=\text { Fuel }_{\text {Oxygen }} / 15.999 \\
& C O_{e, i}=\left[1-(0.01+0.005 \cdot \alpha) \cdot\left(C O_{2 e, i} / 10^{4}\right)-0.000323 \cdot \mathrm{R}\right] \cdot C O_{\text {eraw }, i}
\end{aligned}
$$


The variables in Equation 5.1 are defined as follows. The first four equations define mass flow rates in grams per second of $\mathrm{HC}, \mathrm{CO}, \mathrm{CO}_{2}$, and $\mathrm{NO}_{\mathrm{x}}$, respectively; subscript $i$ denotes values at a given time step. $\mathrm{HC}_{e}, \mathrm{CO}_{e_{-} \text {raw }}, \mathrm{CO}_{2 \_e}$, and $\mathrm{NO}_{x_{-} e}$ are measured concentrations in ppm; $\mathrm{HC}_{b}, \mathrm{CO}_{b}, \mathrm{CO}_{2 \_}$, and $N O_{x_{-} b}$ are background concentrations in ppm. $\mathrm{HC}$ concentration is in carbon equivalent ppm. $C O_{e}$ is the $\mathrm{CO}$ concentration $\left(\mathrm{CO}_{e_{-} \text {raw }}, \mathrm{ppm}\right)$ corrected for water vapor and $\mathrm{CO}_{2}$ extraction. $\mathrm{DF}$ is dilution factor and $V_{\text {mix }}$ is dilute exhaust volumetric flow rate (at standard conditions of $20^{\circ} \mathrm{C}$ and $101.3 \mathrm{kPa}$ ). $K_{v}$ is the venturi calibration coefficient, $P_{v}$ is venturi absolute inlet pressure, and $T_{v}$ is venturi absolute inlet temperature.

Densities at standard conditions are $0.57665 \mathrm{~kg} / \mathrm{m}^{3}$ for $\mathrm{HC}, 1.164 \mathrm{~kg} / \mathrm{m}^{3}$ for CO, $1.830 \mathrm{~kg} / \mathrm{m}^{3}$ for $\mathrm{CO}_{2}$, and $1.913 \mathrm{~kg} / \mathrm{m}^{3}$ for $\mathrm{NO}_{2} . K_{w}$ is dry-to-wet correction factor and $K_{H}$ is humidity correction factor. $\alpha$ is the atomic hydrogen to carbon ratio of the fuel. $H$ is average absolute humidity of the intake air in grains of water per pound of dry air, and $R$ is the relative humidity (\%). Fuel Carbon $_{\text {, Fuel }}$ Hydrogen, and Fuel $_{\text {Oxygen }}$ are the fuel mass fractions of carbon, hydrogen, and oxygen, respectively. With the fuel properties listed below, $D F_{\text {Constant }}$ turns out to be 13.45 .

A fuel analysis was not performed in the Westchester County test program. Thus, fuel properties were assumed from Wayne et al. (2004b) and are listed in Table 5-3.

Table 5-3 Fuel Properties

\begin{tabular}{l|l}
\hline \multicolumn{1}{c|}{ Parameter } & \multicolumn{1}{c}{ Input } \\
\hline Density $\left(\rho_{\text {Fuel }}\right)$ & $0.830 \mathrm{~kg} / \mathrm{L}$ \\
\hline Carbon Mass Fraction $\left(\right.$ Fuel $\left._{\text {Carbon }}\right)$ & 0.8619 \\
\hline Hydrogen Mass Fraction $\left(\right.$ Fuel $\left._{\text {Hydrogen }}\right)$ & 0.1346 \\
\hline Oxygen Mass Fraction $\left(\right.$ Fuel $\left._{\text {Oxygen }}\right)$ & $1-0.8619-0.1346=0.0035$ \\
\hline Atomic Hydrogen to Carbon Ratio $(\alpha)$ & $(0.1346 / 1.008) /(0.8619 / 12.011)=1.8608$ \\
\hline \multirow{2}{*}{ Lower Heating Value $\left(\right.$ LHV $\left._{\text {Fuel }}\right)$} & $18,447.4 \mathrm{BTU} / \mathrm{lb}$ \\
\hline
\end{tabular}

Percent torque (Torque\%,i) broadcast by the ECU was converted to engineering units (Torque in $\mathrm{Nm}$ ) following Equation $5.2(a)$ and $(b)$, where Speed $_{i}$ is engine speed (rpm) and the maximum torque at current speed (Torque max,$i_{i}$ in $\mathrm{Nm}$ ) is obtained from the performance curve of Figure 5-1. Volumetric fuel rate $\left(\dot{V}_{F u e l, i}\right)$ broadcast by the ECU was converted to mass flow rate $\left(\dot{m}_{F u e l, i}\right)$ using the fuel density value of Table 5-3. Engine power $\left(P_{\text {engine }}\right)$ was calculated from Equation $5.2(d)$ with the appropriate conversion factors. Engine efficiency $\left(\eta_{\text {engine }}\right)$ in \% was calculated from Equation $5.2(e)$, again applying the appropriate conversion factors. In the engine efficiency calculation, $\dot{m}_{\text {Fuel }}$ could be from either carbon balance or ECU.

Equation 5.3 recalls the equations used to calculate cycle fuel economy and fuel volume; $m_{\text {Fuel }}$ is the integral of fuel rate, $\rho_{\text {Fuel }}$ is fuel density, and $D$ is cycle distance. 
Equation 5.2 ECU Data Reduction Equations

$$
\begin{gathered}
\text { Torque }_{i}=\text { Torque }_{\%, i} / 100 \cdot \text { Torque }_{\text {max }, i} \\
\text { Torque }_{\max , i}=f\left(\text { Speed }_{i}\right) \\
\dot{m}_{\text {Fuel }, i}=\dot{V}_{\text {Fuel }, i} \cdot \rho_{\text {Fuel }} \\
P_{\text {engine }, i}=\text { Torque }_{i} \cdot \text { Speed }_{i} \\
\eta_{\text {engine }}=\frac{P_{\text {engine }}}{\dot{m}_{\text {Fuel }} \cdot \text { LHV }_{\text {Fuel }}} \cdot 100
\end{gathered}
$$

Equation 5.3 Fuel Economy Calculation

$$
\begin{gathered}
V_{\text {Fuel }}=\frac{m_{\text {Fuel }}}{\rho_{\text {Fuel }}} \\
\text { F.E. }=\frac{D}{V_{\text {Fuel }}}
\end{gathered}
$$

\subsection{Analysis of Continuous Fuel Rate and Emissions}

The following sections present a discussion of the reduced dataset and the implications of assumed fuel properties, and carbon balance versus ECU fueling, among others.

All runs were inspected visually for anomalies. It was observed that some of the test runs presented drops in the engine speed-torque signals. These sections were removed so they did not introduce flawed entries to the models.

\subsubsection{Area of the Torque-Speed Map Covered by the Test Data}

The region of the torque-speed domain covered by the test data is shown in Figure 5-2. The figure shows the number of data points in cells of size $10 \mathrm{rpm} \times 5 \mathrm{Nm}$ with cells having 10 or more points being at the top of the scale; the top line corresponds to the engine lug curve. The total number of valid data points was 289 thousand or about 8 hours of testing. The portion of the data that represented idle conditions was $23 \%$ (engine speed below $900 \mathrm{rpm}$ ).

The covered region represents the operation of the Cummins ISB $260 \mathrm{H}$ engine in the BAE hybrid bus and serves as a good baseline for the development of the engine fueling and $\mathrm{NO}_{\mathrm{x}}$ models. 


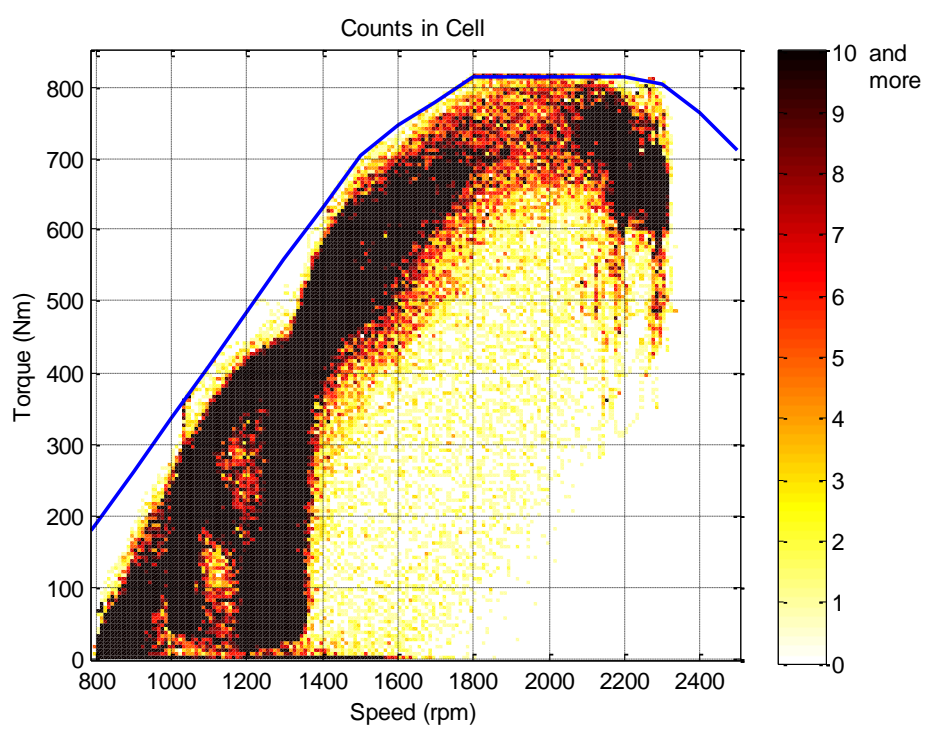

Figure 5-2 Torque - Speed Region Covered by the Test Data

\subsubsection{Effect of Fuel Properties}

The assumed fuel properties produced a difference when the integrated results were compared with the records in the TransLab database (shown in Table 5-1). The greater difference occurred in fuel economy which showed values $3 \%$ to $4 \%$ higher; this was due to a higher value for fuel density (2\% greater) used here and to the carbon mass fraction being $1 \%$ higher. The higher carbon content reduces the calculated fuel mass (Equation $5.1 e$ ), then the higher fuel density further reduces the calculated fuel volume, with the combined effect being a higher fuel economy.

The difference in the hydrogen to carbon ratio $(\alpha)$ also accounted for nearly $1 \%$ lower $\mathrm{CO}_{2}$ emissions, while the effect on $\mathrm{NO}_{\mathrm{x}}$ emissions was negligible. These variations due to the unavailability of accurate fuel properties should bring to the attention of the reader the importance of collecting and analyzing the test fuel, especially if slight differences in vehicle performance are to be detected. There is clearly an uncertainty in fuel economy due to uncertain fuel properties of almost $5 \%$.

Table 5-4 shows the integrated emissions and fuel economy values calculated with the data reduction procedure and fuel properties used in this dissertation. The performance of the engine models developed in the following sections will be benchmarked against these values. 
Table 5-4 Corrections to Table 5-1 for Fuel Properties and Data Reduction Calculations

\begin{tabular}{|c|c|c|c|c|c|}
\hline Cycle & Repeat & $\begin{array}{c}\text { Fuel } \\
\text { Economy } \\
(\mathrm{mpg})\end{array}$ & $\begin{array}{l}\text { Fuel } \\
\text { Cons. } \\
\text { (g/mi) }\end{array}$ & $\begin{array}{c}\mathrm{NO}_{\mathrm{x}} \\
(\mathrm{g} / \mathrm{mi})\end{array}$ & $\begin{array}{c}\mathrm{CO}_{2} \\
(\mathrm{~g} / \mathrm{mi})\end{array}$ \\
\hline \multirow{6}{*}{ Beeline } & 1 & 4.79 & 656 & 9.87 & 2,071 \\
\hline & 2 & 4.91 & 640 & 9.58 & 2,019 \\
\hline & 3 & 4.99 & 630 & 9.37 & 1,989 \\
\hline & 4 & 5.07 & 620 & 9.39 & 1,958 \\
\hline & 5 & 4.99 & 631 & 9.48 & 1,990 \\
\hline & 6 & 5.01 & 627 & 9.40 & 1,980 \\
\hline \multirow{3}{*}{ Manhattan } & 1 & 4.19 & 749 & 13.55 & 2,369 \\
\hline & 2 & 4.03 & 781 & 13.49 & 2,461 \\
\hline & 3 & 4.08 & 771 & 12.85 & 2,434 \\
\hline \multirow{3}{*}{ New York Bus } & 1 & 2.24 & 1,398 & 23.75 & 4,429 \\
\hline & 2 & 2.34 & 1,349 & 22.85 & 4,236 \\
\hline & 3 & 2.28 & 1,389 & 23.90 & 4,358 \\
\hline \multirow{3}{*}{ OCTA } & 1 & 5.34 & 589 & 10.00 & 1,859 \\
\hline & 2 & 5.41 & 580 & 9.82 & 1,832 \\
\hline & 3 & 5.47 & 574 & 9.77 & 1,813 \\
\hline \multirow{3}{*}{ UDDS } & 1 & 5.99 & 524 & 7.62 & 1,657 \\
\hline & 2 & 6.17 & 510 & 7.46 & 1,609 \\
\hline & 3 & 5.96 & 527 & 7.39 & 1,663 \\
\hline \multirow{3}{*}{ WMATA } & 1 & 4.39 & 717 & 11.99 & 2,261 \\
\hline & 2 & 4.66 & 674 & 10.98 & 2,127 \\
\hline & 3 & 4.77 & 659 & 11.22 & 2,079 \\
\hline
\end{tabular}

\subsubsection{Comparison between Carbon Balance and ECU Fueling Rates}

The dispersion of emissions that occurs in the dilution tunnel makes that, even after time alignment, measured values do not correspond to instantaneous tailpipe emissions; integrated values, on the other hand, are not affected. For instance, a sudden power demand is measured with a lower peak on fueling (carbon balance, Equation $5.1 e$ ) than the actual value, and once the power demand is finished, the engine cuts fueling but the measured fuel rate will drop slowly. Figure 5-3 shows an example of this situation for a section of the OCTA cycle; carbon balance fueling clearly exhibits the dispersion effects and can differ by more than $50 \%$ versus the ECU value.

If the integrated fueling rate reported by the ECU, which is thought to conserve the true transient nature of the events, compares well with carbon balance results, one could use ECU fueling to develop the prediction models.

The difference between integrated ECU and Carbon Balance fuel rates is shown in Figure 5-4 for each of the tests, where the reference is Carbon Balance. A good agreement is observed and most of the runs show less than $2 \%$ difference; the overall average difference (sum of all tests) is $1.7 \%$. This difference is lower than the results shown in the previous section for the effect of fuel properties. It is 
concluded that ECU fuel rate can be used with confidence, and it will be used as the basis for the engine fueling models.

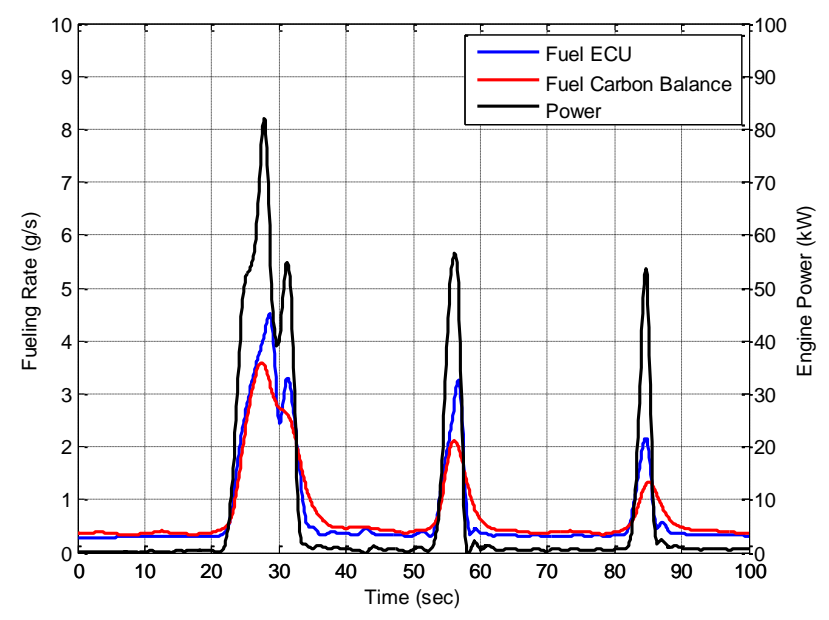

Figure 5-3 Fuel Rate from Carbon Balance and ECU for a Section of the OCTA Cycle

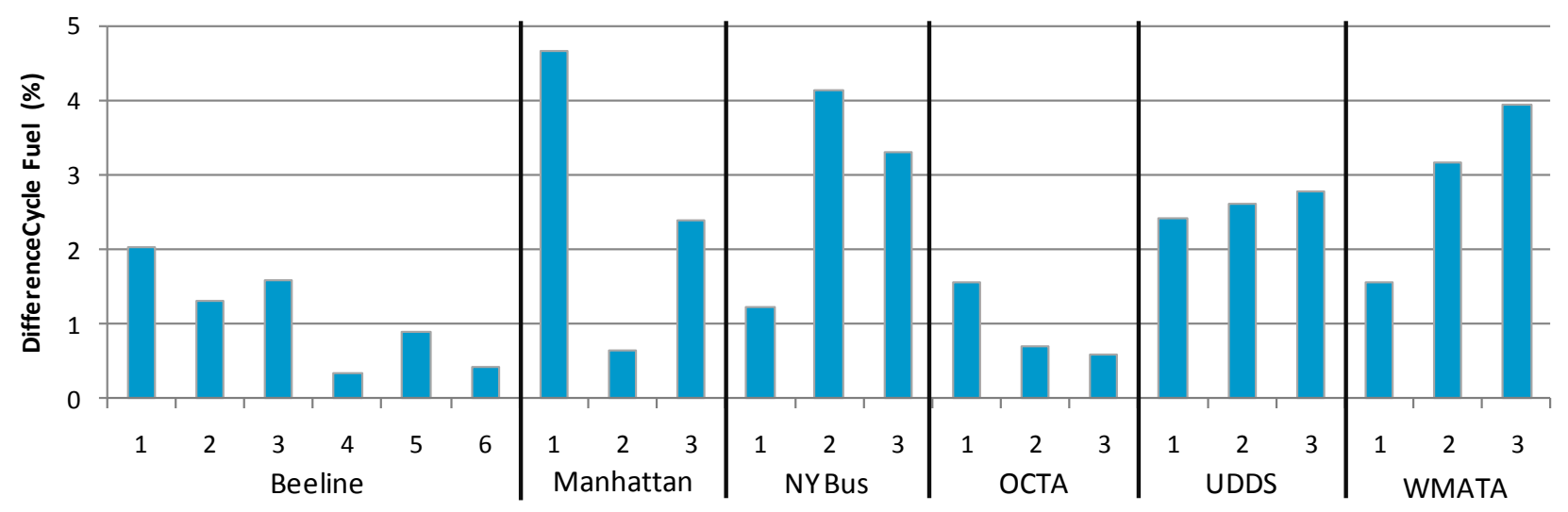

Figure 5-4 Difference between Integrated ECU and Carbon Balance Fuel Rates

A similar dispersion effect is observed in the measured $\mathrm{NO}_{\mathrm{x}}$ rate but no corrections will be performed. The dispersion and diffusion effects on $\mathrm{NO}_{\mathrm{x}}$ may not be as significant as those for fuel rate (primarily coming from $\mathrm{CO}_{2}$ ); this is likely since tailpipe $\mathrm{CO}_{2}$ is considerably mixed with the $\mathrm{CO}_{2}$ in the dilution air while the amount of $\mathrm{NO}_{\mathrm{x}}$ in the dilution air is low. 


\subsection{D Maps or Lookup Table Approach}

Before developing any ANN models let's explore first the lookup table approach. This approach is the default of PSAT and is widely known to work well for fuel rates but not so well for emissions.

The 2D maps are based on engine speed and torque as the inputs, and any torque-speed pair within the domain can be interpolated from the surface to predict fueling rate or $\mathrm{NO}_{\mathrm{x}}$ rate (or in general any species for which the maps are developed).

The maps were developed in the MATLAB ${ }^{\circledR}$ environment using the gridfit function from MATLAB ${ }^{\circledR}$ Central. The surface smoothness was adjusted with the smoothness parameter. Decreasing the smoothness parameter produced higher $\mathrm{R}^{2}$ values for the training data but predictions for other inputs were worsened. It was also observed that a finer grid produced better results than a coarser grid.

Regarding the issue of carbon balance versus ECU fueling, the maps with ECU fueling produced better results. Since the $\mathrm{NO}_{\mathrm{x}}$ data were not corrected for dispersion, it was decided to remove the decreasing torque sections (those following a power event) in an attempt to reduce the effect of dispersion. The $\mathrm{NO}_{\mathrm{x}}$ maps thus generated produced slightly better results than without ( $1 \%$ higher $\mathrm{R}^{2}$ ).

Figure 5-5 presents the fuel rate map. The map was generated with a smoothness parameter of 2 and a grid size of $50 \mathrm{rpm} \times 10 \mathrm{Nm}$; the $\mathrm{R}^{2}$ value was 0.982 . From the fueling map, the efficiency and brake-specific fuel consumption (BSFC) maps were calculated and are shown in Figure 5-6 and Figure 5-7, respectively.

Originally, the efficiency curves showed that for a constant speed the peak efficiency occurred at maximum torque. This was because the lack of data at torques near the lug curve (see Figure 5-2 in page 127) made the curve fitting algorithm underestimate fueling rates in this region. This situation was corrected by manually increasing the fueling rates in the region up to $20 \%$.

After this correction and provided the source of the data, the shape of the curves seemed reasonable and their values appropriate. The estimates for best engine efficiency and brake-specific fuel consumption are $43 \%$ and $200 \mathrm{~g} / \mathrm{kWh}(150 \mathrm{~g} / \mathrm{bhp} \cdot \mathrm{hr})$, respectively.

The $\mathrm{NO}_{\mathrm{x}}$ rate map is shown in Figure 5-8. The map was generated with a smoothness parameter of 0.5 and a grid size of $10 \mathrm{rpm} \times 5 \mathrm{Nm}$; the $\mathrm{R}^{2}$ value was 0.902 . The brake-specific $\mathrm{NO}_{\mathrm{x}}$ map is presented in Figure 5-9. The brake specific fuel and $\mathrm{NO}_{\mathrm{x}}$ maps can be converted to units of horsepower multiplying by the fraction $(1 \mathrm{~kW} / 1.341 \mathrm{hp})$. 


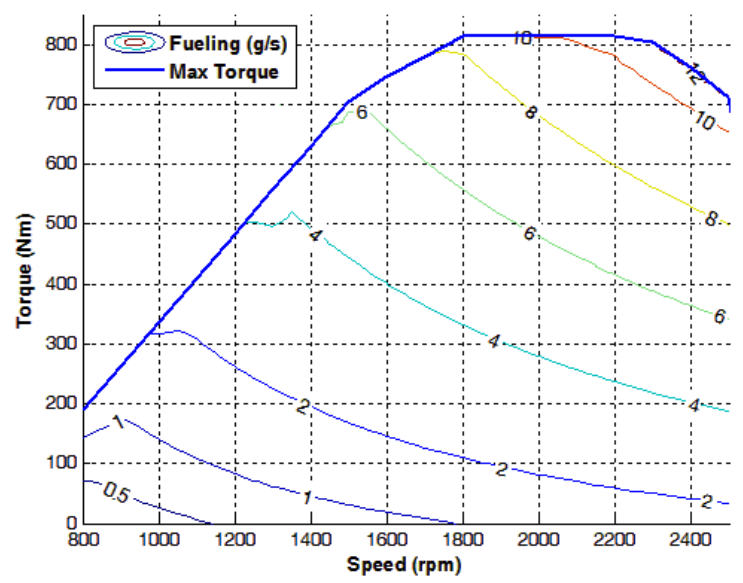

Figure 5-5 Engine Fueling Map

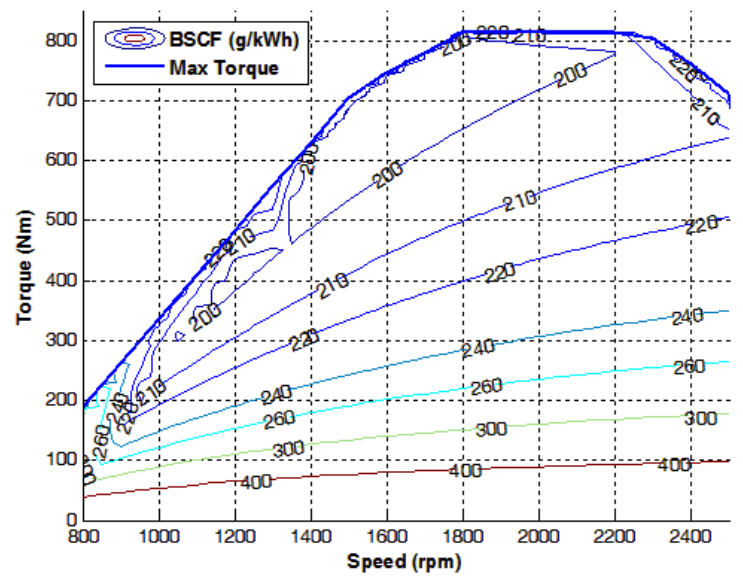

Figure 5-7 Engine Brake-Specific Fuel Consumption Map

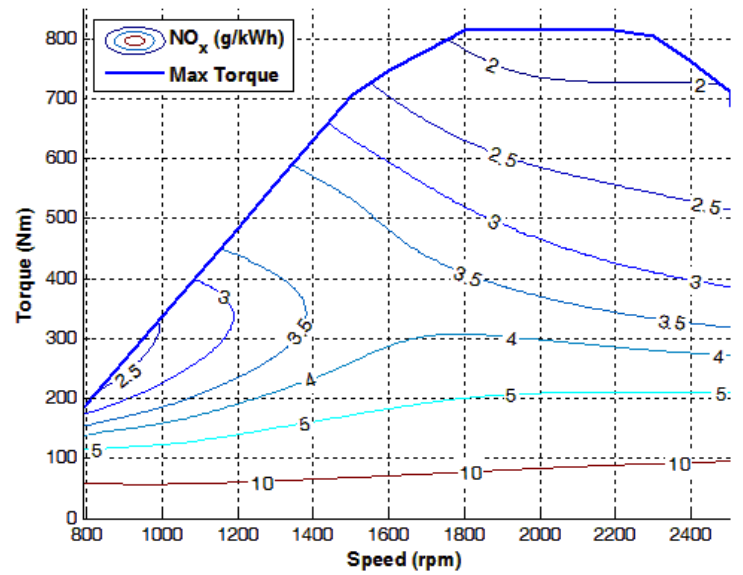

Figure 5-9 Engine Brake-Specific $\mathrm{NO}_{\mathrm{x}}$ Emissions Map

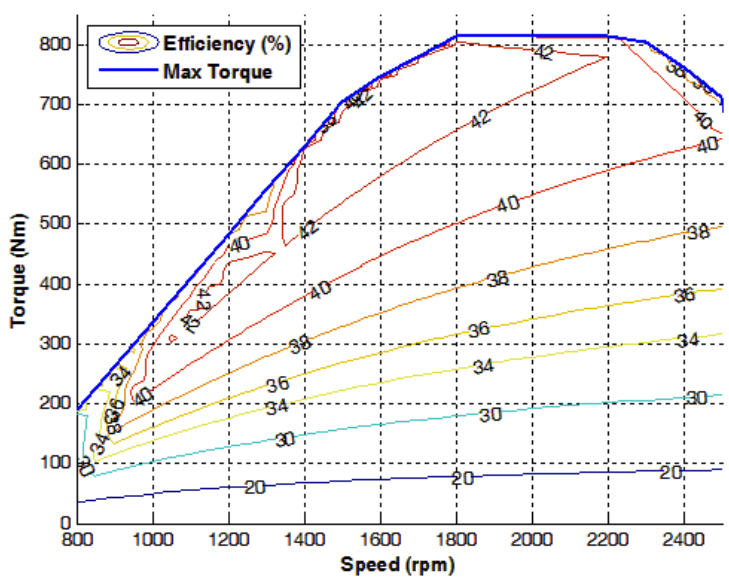

Figure 5-6 Engine Efficiency Map

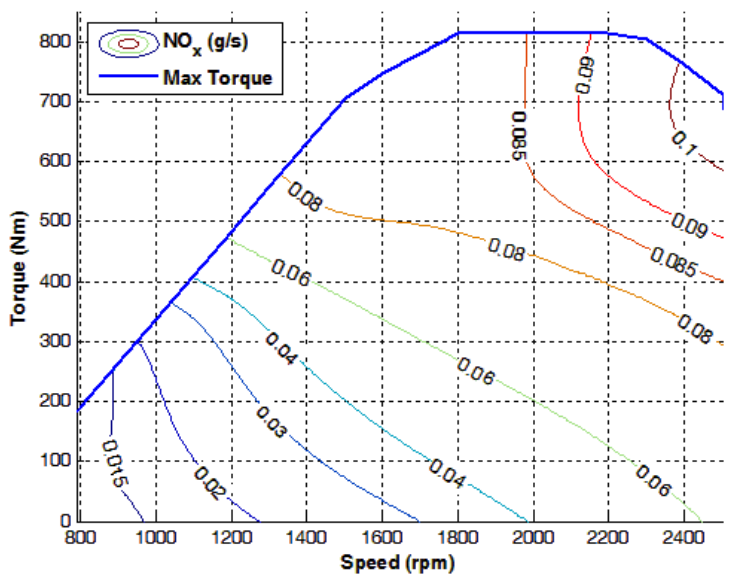

Figure 5-8 Engine $\mathrm{NO}_{\mathrm{x}}$ Rate Map 
Performance of the 2D maps was evaluated and the results are shown in Figure 5-10: (a) is percent error in cycle fuel, $(b)$ is $\%$ error in cycle $\mathrm{NO}_{\mathrm{x}}$, and $(c)$ is $\%$ error in cycle $\mathrm{CO}_{2}$. These results are equivalent to the integrated PSAT output when the engine is exercised over the duty cycles prescribed by the chassis tests. $\mathrm{CO}_{2}$ emissions were calculated following Equation $5.2 e$ and neglecting the $\mathrm{HC}$ and $\mathrm{CO}$ contributions:

Equation 5.4 $\mathrm{CO}_{2}$ Emissions from Fueling Rate

$$
\mathrm{CO}_{2 \text { mass flow }, i}=\text { Fuel }_{\text {mass flow }, i} \cdot \text { Fuel }_{\text {Carbon }} \cdot \frac{44}{12}
$$

Errors of fuel consumption predicted by the 2D fuel map were very low: cycle fuel consumption had a maximum error of $2.3 \%$ and a minimum of $-1.0 \%$, average of the errors was $0.4 \%$ and the overall error (all the runs added together) was $0.1 \%$. Performance of the $\mathrm{NO}_{\mathrm{x}}$ map and the calculation for $\mathrm{CO}_{2}$ were not as good as for the fueling map. Cycle $\mathrm{NO}_{\mathrm{x}}$ had a maximum error of 7.4\%, a minimum of $-2.2 \%$, average of $1.9 \%$ and overall $1.1 \%$. Cycle $\mathrm{CO}_{2}$ was consistently overpredicted with a maximum error of $6.5 \%$, a minimum of $0.1 \%$, an average of $2.5 \%$, and overall $2.0 \%$. Given that the fueling map was based on ECU fueling, the difference between carbon balance and ECU fueling rates of Figure 5-4 carries up to the $\mathrm{CO}_{2}$ error shown in Figure 5-10 (c).

The results of this section show that there is still room for improvement, especially in the case of $\mathrm{NO}_{\mathrm{x}}$ and $\mathrm{CO}_{2}$ prediction. The case of $\mathrm{CO}_{2}$ will not be addressed because for consistency $\mathrm{CO}_{2}$ emissions should be calculated from the carbon in the fuel as shown in Equation 5.4.

\subsection{Artificial Neural Network Models Development and Validation}

The ANN architecture chosen for modeling of fuel consumption and $\mathrm{NO}_{\mathrm{x}}$ was a multiple layer feed-forward network and was modeled in the MATLAB ${ }^{\circledR}$ environment. Training was performed using the Levenberg-Marquardt back-propagation algorithm (trainlm). Single hidden layer networks were also explored but they did not produce sufficient accuracy over the wide operative range of the engine.

ANN training used random division (dividerand in MATLAB $^{\circledR}$ ) of the training data as follows: $60 \%$ for training, $20 \%$ for validation, and $20 \%$ for testing. The validation data are used to stop training if performance does not improve for a specified number of epochs. The testing set is used to check proper generalization. Neither validation nor testing sets are used to adjust the synaptic weights. Given the random division of the training data and the dependency of initial conditions (initial synaptic weights), slightly different results were obtained every time that the training algorithm was run. 


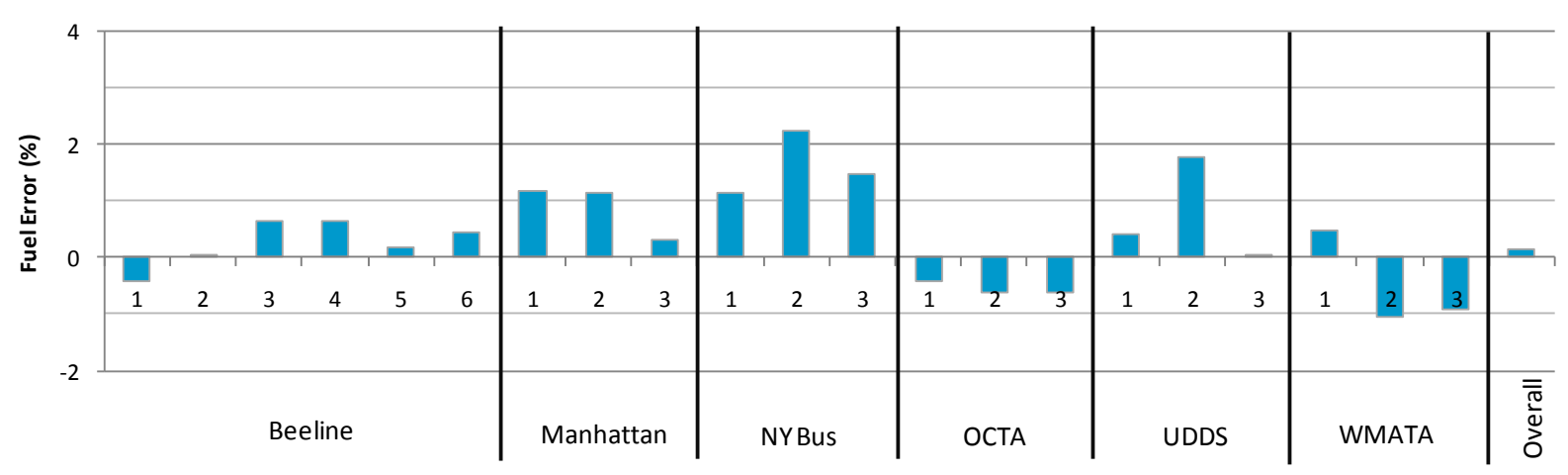

(a) Percent Error in Cycle Fuel

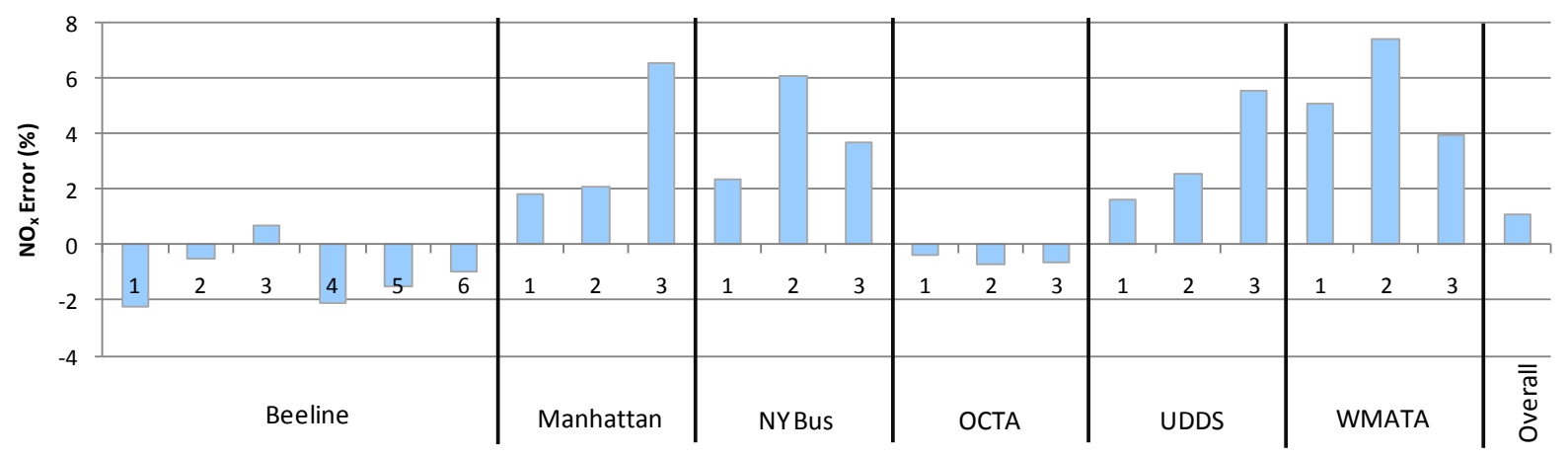

(b) Percent Error in Cycle $\mathrm{NO}_{\mathrm{x}}$

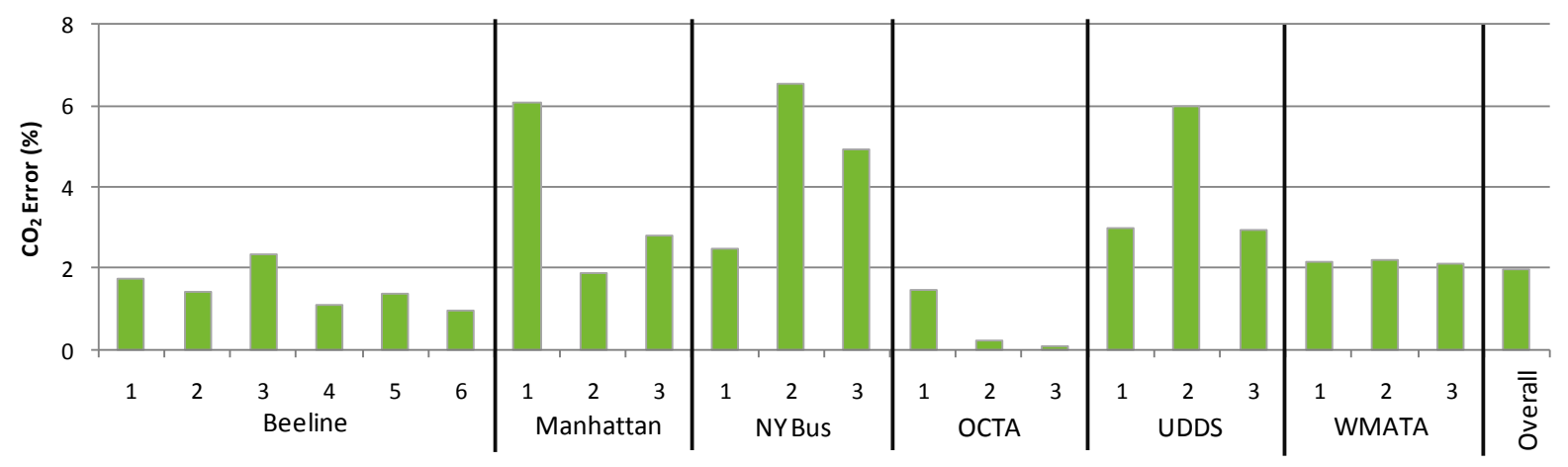

(c) Percent Error in Cycle $\mathrm{CO}_{2}$

Figure 5-10 Performance of 2D Maps

Inputs and outputs to the neural network were normalized as shown in Table 5-5. Torque inputs do not have negative values as the Cummins engine only reports positive torques. ANN outputs were then de-normalized into engineering units.

Bedik (2006) used a normalized standard error to compare performance of the ANN during training and validation under various groups of inputs. In this dissertation, the mean square error of Equation 2.48 (page 54) was used to measure performance during ANN training: 


$$
s^{2}=M S E=\frac{S S E}{D F E}=\frac{\sum\left(\hat{y}_{i}-y_{i}\right)^{2}}{n-2},
$$

where $y$ corresponds to target output and $\hat{y}$ to predicted output. The summation is carried out for all the $n$ points in the dataset. This method was used by Thompson et al. (2000). Alternatively, the correlation coefficient of Equation 2.49 (page 54), $R^{2}=S S M / S S T$, provides an estimate of performance. The drawback of $R^{2}$ is that even if a perfect correlation exists the ANN may be under- or over-predicting. Also, percent error in integrated cycle emissions was considered to assess accuracy.

Table 5-5 ANN Input / Output Normalization

\begin{tabular}{l|c|c|c}
\hline \multicolumn{1}{c|}{ Parameter } & Units & Range & Normalized Range \\
\hline Speed & $\mathrm{Rpm}$ & $700-2,350$ & $0-1$ \\
\hline Torque & $\mathrm{Nm}$ & $0-815$ & $0-1$ \\
\hline Fuel Rate & $\mathrm{g} / \mathrm{s}$ & $0-10$ & $0-1$ \\
\hline $\mathrm{NO}_{\mathrm{x}}$ & $\mathrm{g} / \mathrm{s}$ & $0-0.14$ & $0-1$ \\
\hline
\end{tabular}

The transfer functions studied were radial basis (radbas), logarithmic sigmoid (logsig), and hyperbolic tangent sigmoid (tansig). Equation 5.5 presents the functional form of the three functions and Figure 5-11 shows a plot in the range of -1 to 1 . Note that the transfer functions are bounded between -1 and 1 , providing stability to the neural net. Given the normalization of Table 5-5, the working input range is the interval $[0,1]$. The logsig function's limited range for inputs between 0 and 1 resulted in poor results when compared to the other two functions.

Equation 5.5 ANN Transfer Functions

$$
\begin{aligned}
& \operatorname{radbas}(n)=e^{-n^{\wedge} 2} \\
& \operatorname{logsig}(n)=\frac{1}{1+e^{-n}} \\
& \operatorname{tansig}(n)=\frac{2}{1+e^{-2 n}}-1
\end{aligned}
$$

The following considerations were explored in determining the best ANN configuration: number of neurons per hidden layer, order of the transfer functions within the ANN, time span of the history inputs, and number of inputs to the ANN. In order to evaluate the variability of the results, three experiments or simulations were performed for each configuration.

The configurations studied had 3 hidden layers, the first two layers had radbas or tansig transfer functions whereas the third layer (output layer) had a linear operator. Inputs were normalized torque, normalized speed, and their delayed values (history terms) over $\Delta T_{1}, \Delta T_{2}$, and $\Delta T_{3}$ seconds for a total of 8 inputs. Negative outputs were avoided by applying a zero threshold to the output. 


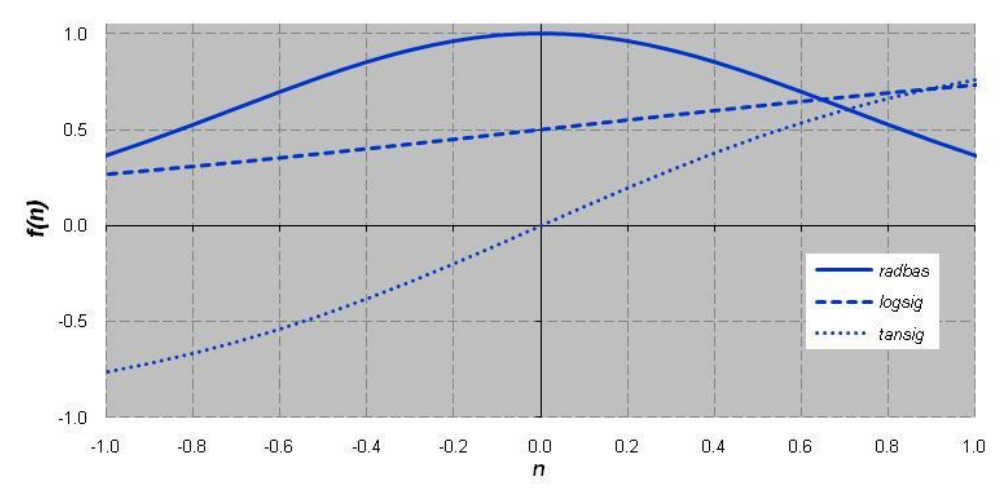

Figure 5-11 Transfer Functions

Figure 5-12 shows a general overview of the ANNs; having two hidden layers and the output layer. Each neuron in the first hidden layer is connected to the inputs; similarly each neuron in the following layers is connected to the outputs of all the neurons in the previous layer. The inputs to any given neuron are weighted and have a bias term ( $\mathbf{w}$ and $\mathbf{b}$ in Figure 5-12); parameters that are adjusted during the training process. Since two different ANNs were developed (fuel and $\mathrm{NO}_{\mathrm{x}}$ ), each ANN produced one output and the output layer was composed of one neuron.

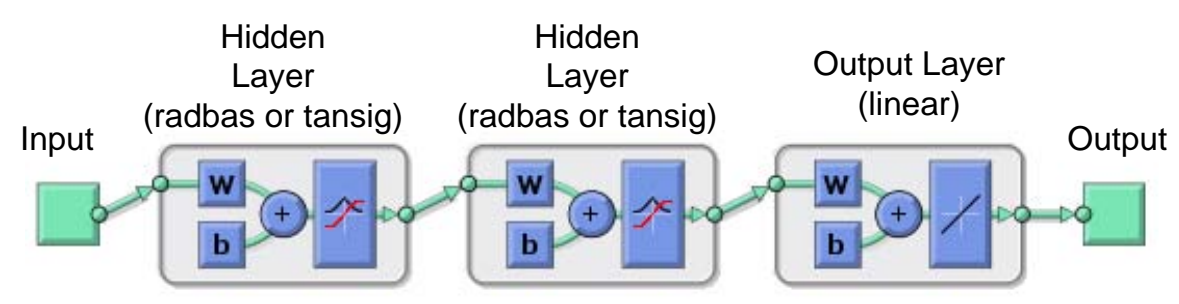

Figure 5-12 General Overview of the ANNs

For ANN training, values for learning rate and momentum coefficient were 0.1 and 0.3 , respectively. The momentum coefficient adjusts the weights with considerations of previous values of these, which improves convergence and accuracy by avoiding local minima. The maximum number of iterations allowed was 200 .

The training datasets were selected as follows: $(i)$ input data were scaled (Table 5-5) and matched with the corresponding history terms in torque and speed $\left(\Delta T_{1}, \Delta T_{2}\right.$, and $\left.\Delta T_{3}\right)$, (ii) the speed-torque domain was parceled in cells of $10 \mathrm{rpm} \times 5 \mathrm{Nm}$ and the data points (from step $i$ ) on each cell were identified, and (iii) 5 points from each cell were randomly selected to make up the training set. If the number of points in a cell was less than 5 , all the points were selected.

The training datasets thus created consisted of a total of 46,000 points (1.3 hours of test data) representing $16 \%$ of the overall dataset. The training set generation procedure assured that the networks were supplied with an even representation of all the regions in the map, and would avoid overfitting specific regions of the map. 


\subsubsection{Time Span of the History Inputs}

The history terms of 1, 5, and 10 seconds suggested by Jarrett and Clark (2002) were used as a starting point. Then, history terms of $0.1,1$, and 5 seconds were used to train the ANNs and performance was compared.

Figure 5-13 shows the effect of using two different sets of history terms, in the first one $\Delta T_{1}=1 \mathrm{~s}$, $\Delta T_{2}=5 \mathrm{~s}$, and $\Delta T_{3}=10 \mathrm{~s}$, and in the second one the values of $[0.1,1,5]$ were used. Figures $(a)$ and $(b)$ are for the fuel model, figures $(c)$ and $(d)$ are for the $\mathrm{NO}_{\mathrm{x}}$ model; figures $(a)$ and $(c)$ present the normalized mean squared error (NMSE), i.e. MSE calculated with normalized outputs; figures $(b)$ and (d) show the $\mathrm{R}^{2}$ value. Error bars are the $95 \%$ confidence intervals of three simulations. Each pair of columns corresponds to a combination of the transfer functions, where $\mathrm{R}$ stands for radbas and $\mathrm{T}$ for tansig, e.g. R-T corresponds to a radbas layer followed by a tansig layer. These experiments were carried out with 20 neurons in the first hidden layer and 15 in the second one.

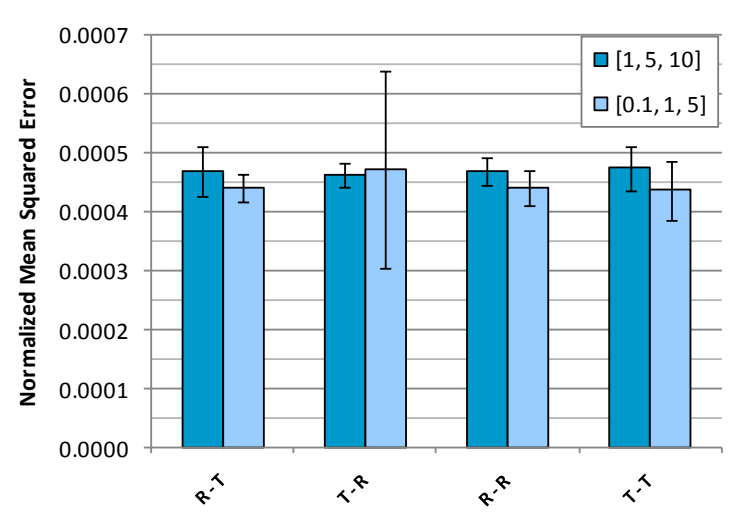

(a) Normalized Mean Squared Error for Fuel

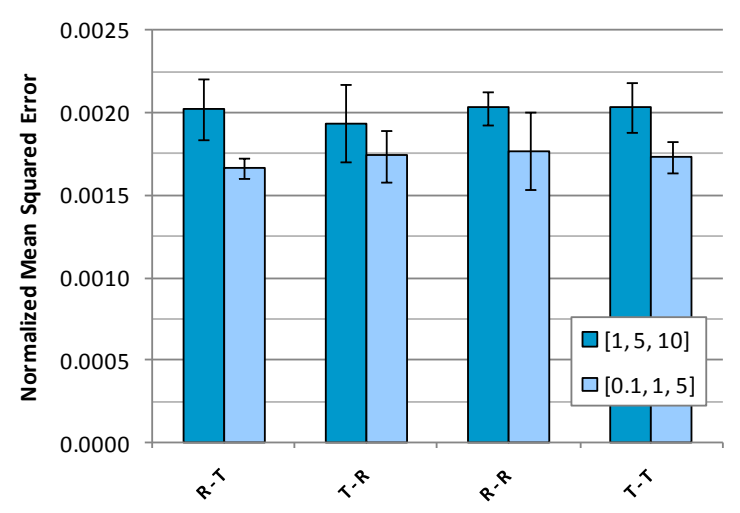

(c) Normalized Mean Squared Error for $\mathrm{NO}_{\mathrm{x}}$

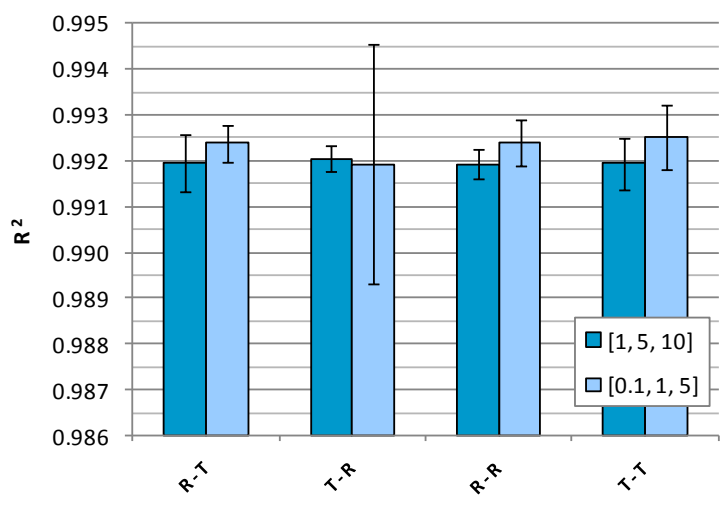

(b) Coefficient of Determination for Fuel

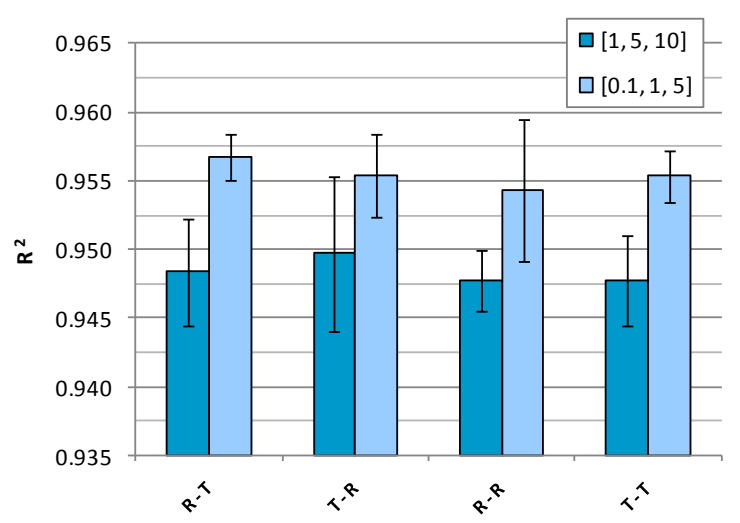

(d) Coefficient of Determination for $\mathrm{NO}_{\mathrm{x}}$

Figure 5-13 Effect of Time-Span of the History Terms on ANN Performance 
For the case of fuel, the change of time-span did not show improvement with statistical significance for either of the combinations of $\mathrm{R}$ and $\mathrm{T}$. For the case of $\mathrm{NO}_{\mathrm{x}}$, improvement was seen with statistical significance, e.g. for the radbas-tansig setting $R^{2}$ increased from $0.948 \pm 0.004$ to 0.957 \pm 0.002 .

The results showed that, when compared with the baseline values, the history terms at $0.1,1$, and 5 seconds yield better performance for $\mathrm{NO}_{\mathrm{x}}$ but not necessarily for fuel. These values were retained as the history terms of choice for both ANNs models.

\subsubsection{Number of Neurons}

Experiments were performed to evaluate the effect that the number of neurons had on performance of the ANNs. Figure 5-14 shows the results starting with 5 neurons per layer [5, 5] up to 25 neurons per layer [25, 25].

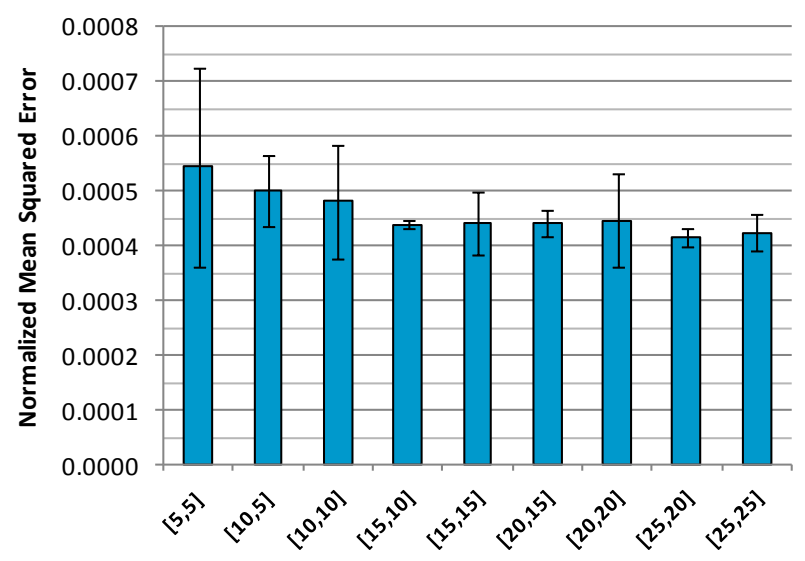

(a) Normalized Mean Squared Error for Fuel

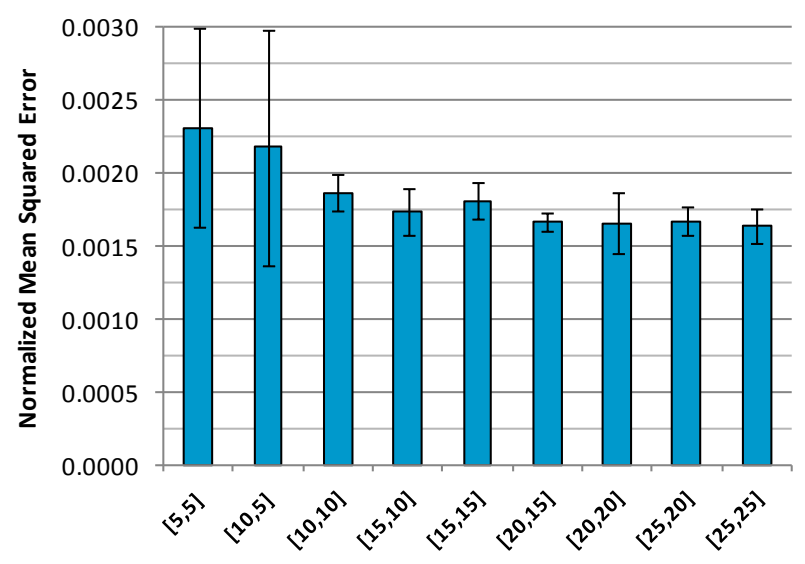

(c) Normalized Mean Squared Error for $\mathrm{NO}_{\mathrm{x}}$

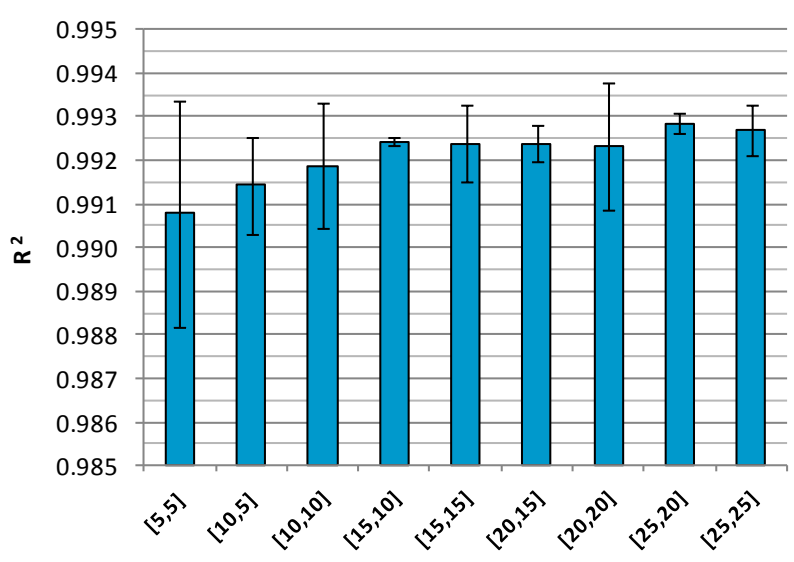

(b) Coefficient of Determination for Fuel

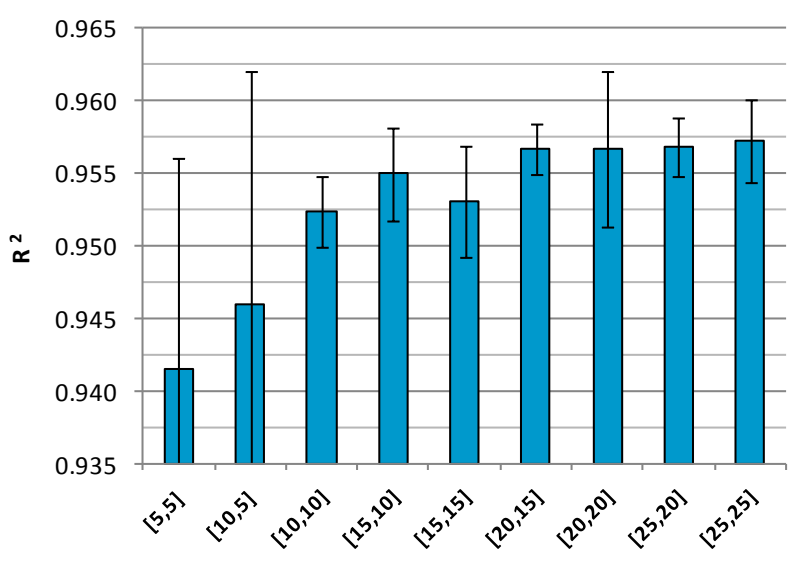

(d) Coefficient of Determination for $\mathrm{NO}_{\mathrm{x}}$

Figure 5-14 Effect of Number of Neurons on ANN Performance 
The limited number of experiments (3) made that slight variations from repetition to repetition were converted into large error bars, thus the statistical significance of comparisons was low. A tendency was observed where performance improved and then leveled up. The pairs [15, 10] and [20, 15] were compared and the first one was selected for the fuel ANN while the second one was selected for the $\mathrm{NO}_{\mathrm{x}} \mathrm{ANN}$. The $[20,15]$ pair showed lower errors in individual cycles than the smaller network and better tracking of the target $\mathrm{NO}_{\mathrm{x}}$ signal.

The effect of adding an extra layer was also evaluated but it was observed that the added layer worsened the generalization capabilities of the models.

\subsubsection{Order of the Transfer Functions}

The effect of the ordering of the radbas and tansig transfer functions within the network can be observed in Figure 5-13. The figure shows that performance of the four combinations of $\mathrm{R}$ and $\mathrm{T}$ is equivalent. It cannot be concluded (with statistical significance) that there is an effect on performance due to the order of the transfer functions.

\subsubsection{Number of Inputs (History Terms)}

The effect of the number of inputs was evaluated for the R-T setting with $[15,10]$ neurons for fuel and $[20,15]$ neurons for $\mathrm{NO}_{\mathrm{x}}$. The baseline inputs were as before, torque and speed at current time, with history terms at $0.1,1$, and 5 seconds; these are referred as $[t, t-0.1, t-1, t-5]$ where $t$ is current time in seconds. The following combinations were explored: (i) the baseline combination, (ii) $[t, t-0.1$, $t-1],(i i i)[t, t-1, t-5],(i v)[t, t-0.1],(v)[t, t-1]$, and (vi) [t].

Figure 5-15 summarizes the results. It was concluded that reducing the number of history terms did have an effect on performance. For fuel, the effect was not too strong if one history term was removed but it was significant when two terms were removed. $\mathrm{NO}_{\mathrm{x}}$ prediction was much more sensitive than fuel prediction to the number of history terms: $\mathrm{R}^{2}$ of $\mathrm{NO}_{\mathrm{x}}$ was reduced from $0.957 \pm$ 0.002 for the baseline set to $0.922 \pm 0.001$ when only current values [ $\mathrm{t}$ ] of speed and torque were used; while for fuel the reduction was only from $0.992 \pm 0.000$ to $0.988 \pm 0.002$.

\subsubsection{Final Results and Validation}

Following the previous analysis, the architectures for the fuel and $\mathrm{NO}_{\mathrm{x}}$ ANNs were selected and are summarized in Table 5-6. The total number of parameters in the neural networks shown in Table 5-6 
was obtained as follows: ( $i$ ) first layer: (number of inputs +1$) \times($ number of neurons in layer), and (ii) second and following layers: (number of neurons in previous layer +1$) \times($ number of neurons in layer). For example, in the case of $\mathrm{NO}_{\mathrm{x}}$ we have $(8+1) \times 20+(20+1) \times 15+(15+1) \times 1=511$.

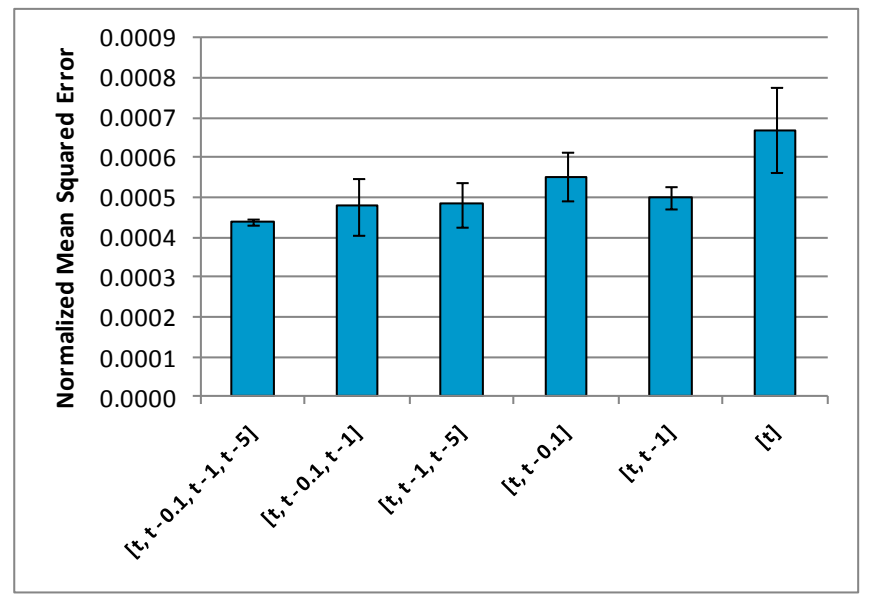

(a) Normalized Mean Squared Error for Fuel

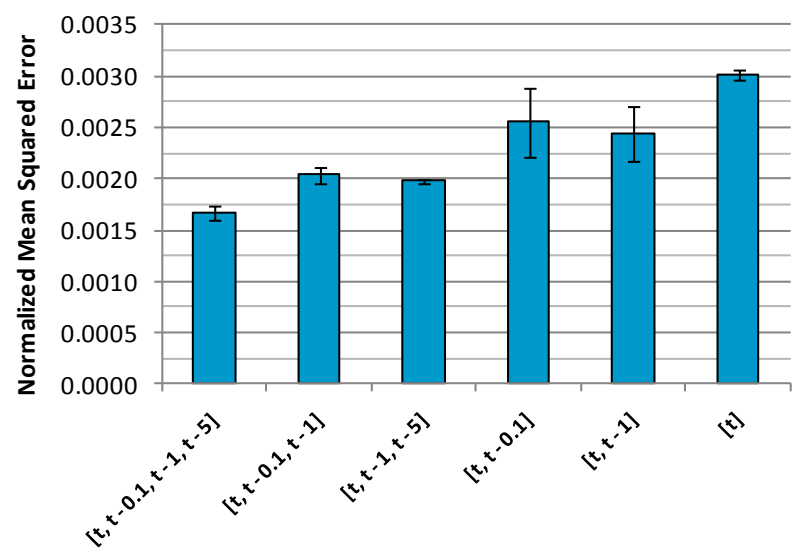

(c) Normalized Mean Squared Error for $\mathrm{NO}_{\mathrm{x}}$

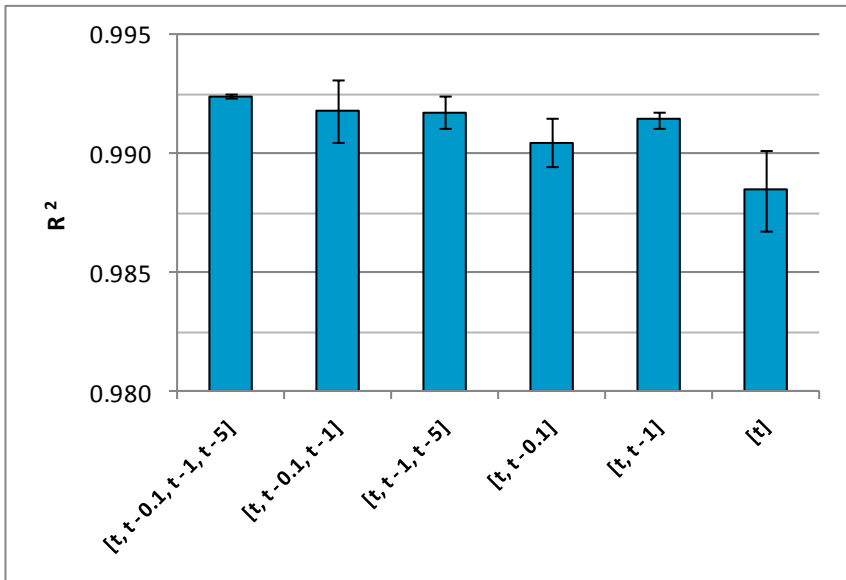

(b) Coefficient of Determination for Fuel

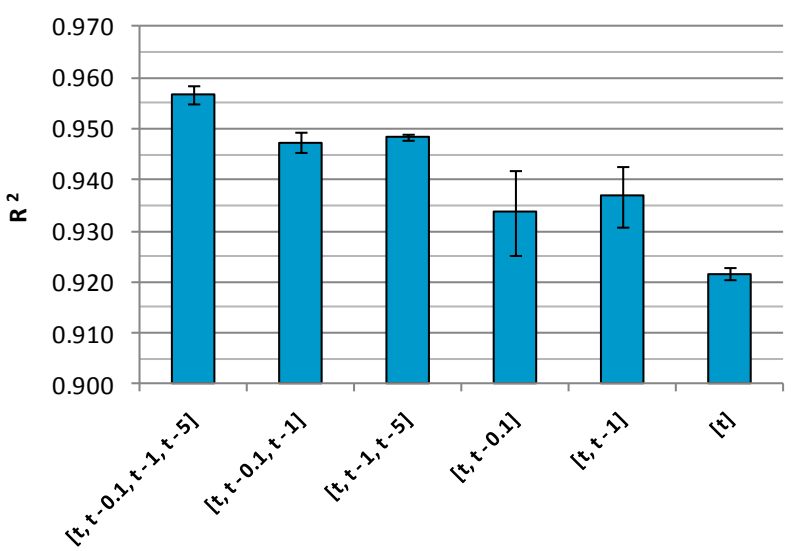

(d) Coefficient of Determination for $\mathrm{NO}_{\mathrm{x}}$

Figure 5-15 Effect of Number of History Terms on ANN Performance

Table 5-6 Selected ANN Architectures

\begin{tabular}{l|c|c}
\hline \multicolumn{1}{c|}{ Parameter } & Fuel & $\mathbf{N O}_{\mathbf{x}}$ \\
\hline Hidden Layers & 2 & 2 \\
\hline No. of Neurons & {$[15,10]$} & {$[20,15]$} \\
\hline Transfer Functions & [radbas, tansig] & {$[$ radbas, tansig] } \\
\hline Inputs & Speed and Torque & Speed and Torque \\
\hline History Terms (s) & {$[0.1,1,5]$} & {$[0.1,1,5]$} \\
\hline No. of Inputs & 8 & 8 \\
\hline No. of Outputs & 1 & 1 \\
\hline No. of Parameters & 306 & 511 \\
\hline
\end{tabular}

The coefficients of determination ( $\mathrm{R}^{2}$ values) for the overall dataset were $99.2 \%$ for fuel and $95.7 \%$ $\mathrm{NO}_{\mathrm{x}}$, which are considerably accurate; these were improved compared with the 2D map approach 
which had $98.2 \%$ and $90.3 \%$, the highest improvement being in the $\mathrm{NO}_{\mathrm{x}}$ results. Standard errors were also improved from $0.33 \mathrm{~g} / \mathrm{s}$ (fuel) and $0.009 \mathrm{~g} / \mathrm{s}\left(\mathrm{NO}_{\mathrm{x}}\right)$ with the $2 \mathrm{D}$ maps to $0.21 \mathrm{~g} / \mathrm{s}$ (fuel) and 0.006 $\mathrm{g} / \mathrm{s}\left(\mathrm{NO}_{\mathrm{x}}\right)$ with the ANNs.

Figure 5-16 compares measured and predicted values of fuel rate, $\mathrm{CO}_{2}$, and $\mathrm{NO}_{\mathrm{x}}$ for a section of the OCTA cycle. The figure shows that the ANNs give a better tracking of the target signals.
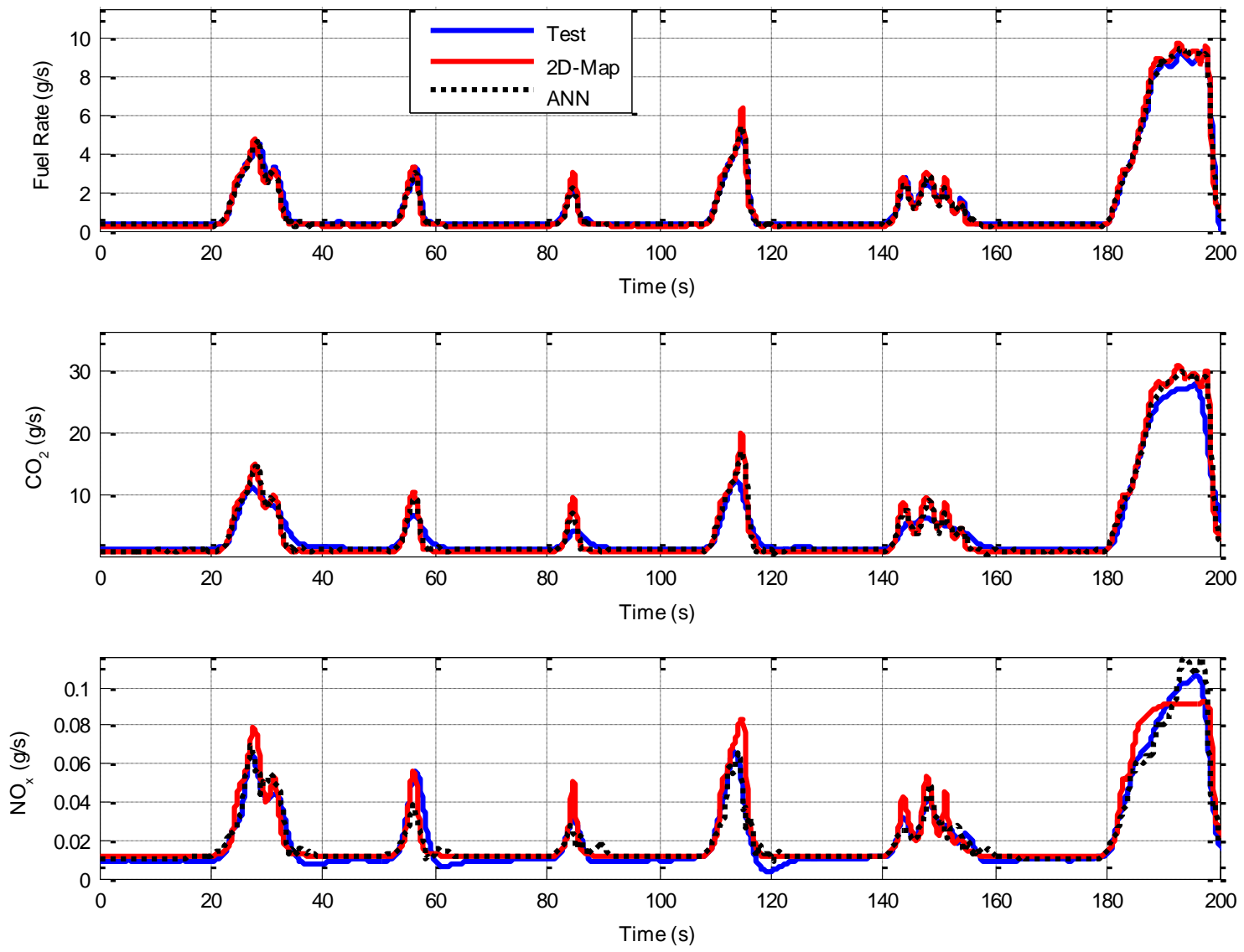

Figure 5-16 Example of ANN and 2D-Map Output. Section of OCTA Cycle

The two neural nets were contrasted against all the available test runs and the output from the $2 \mathrm{D}$ Maps. The results are summarized in Figure 5-17 for percentage error and in Figure 5-18 for standard error (root mean square error). The cycle error of Figure 5-17 shows that the fuel ANN performs better than the $\mathrm{NO}_{\mathrm{x}} \mathrm{ANN}$ with overall smaller errors, which was also true for the 2D-Maps. Also, when compared with the 2D-Map results, the ANNs considerably reduced cycle error.

Analysis of Figure 5-17 (b) for $\mathrm{NO}_{\mathrm{x}} \%$ error reveals that $85 \%$ of the results are within $3 \%$ accuracy range and $95 \%$ of them within a 5.5\% accuracy range. Similar analysis of Figure 5-18 $(b)$ reveals that $90 \%$ of the results have a standard error below $7 \mathrm{mg} / \mathrm{s}$ of $\mathrm{NO}_{\mathrm{x}}$. The distribution of $\%$ error of fuel reveals that $85 \%$ of the results are within the $0.5 \%$ accuracy range and $95 \%$ within $0.8 \%$ accuracy. 


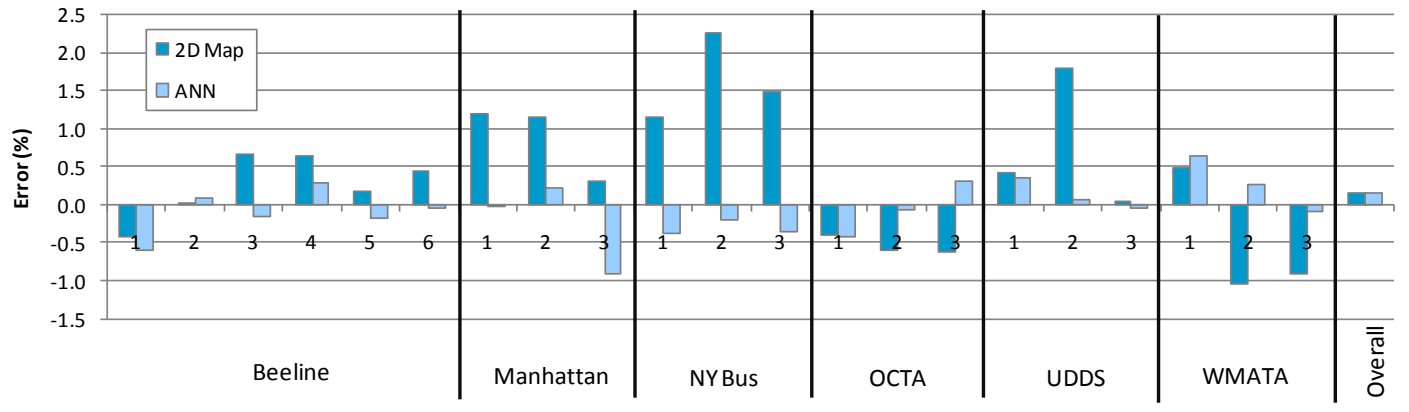

(a) Cycle Percent Error of Fuel

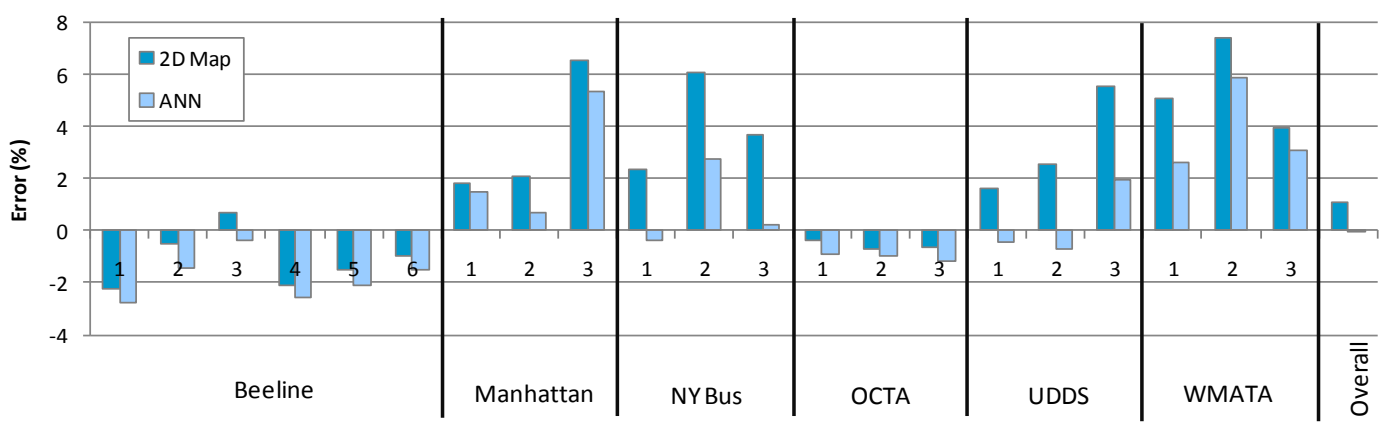

(b) Cycle Percent Error of $\mathrm{NO}_{\mathrm{x}}$

Figure 5-17 Percent Error in Integrated Emissions for all Test Runs: (a) Fuel, (b) $\mathrm{NO}_{\mathrm{x}}$

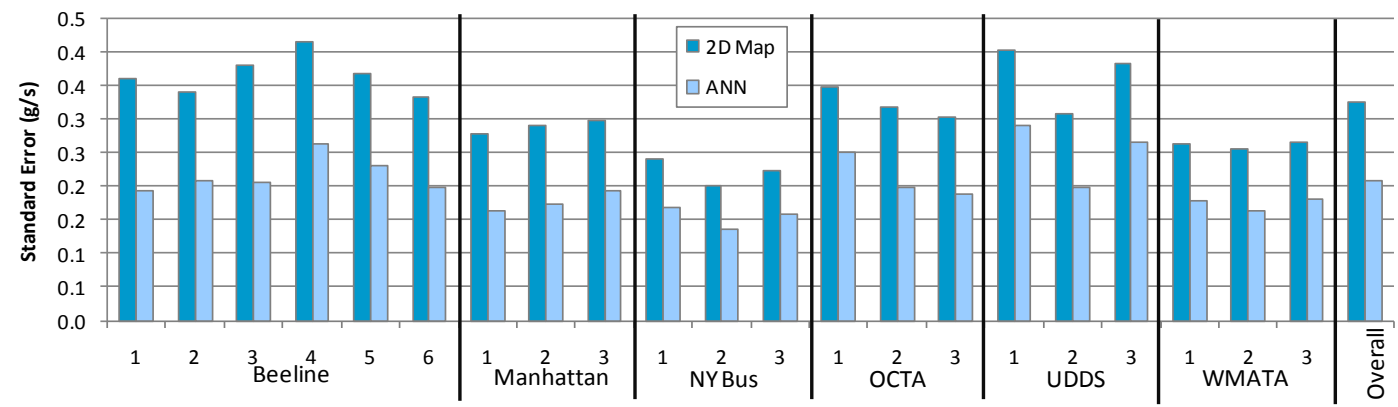

(a) Standard Error of Fuel, g/s

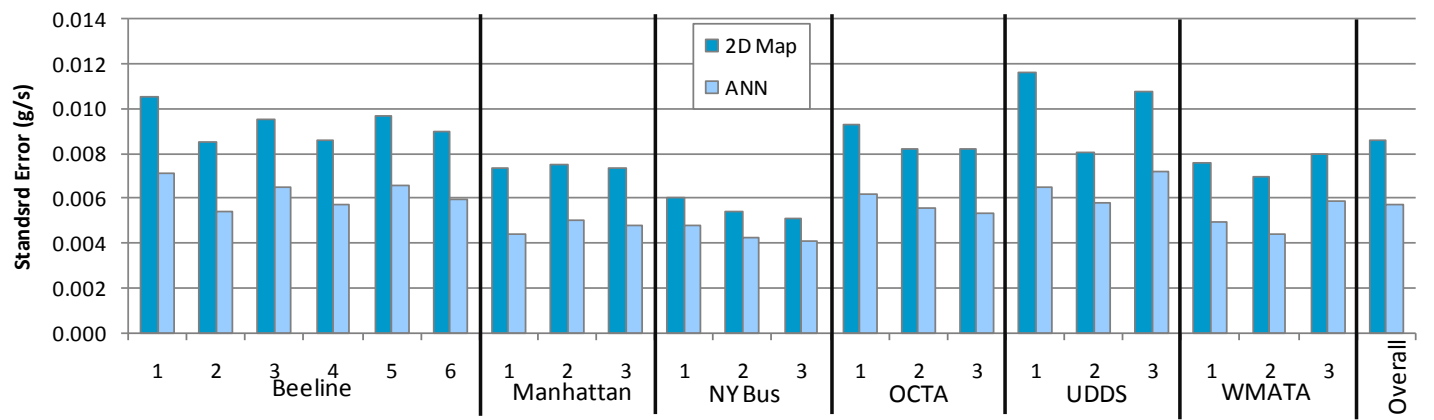

(b) Standard Error of $\mathrm{NO}_{\mathrm{x}}, \mathrm{g} / \mathrm{s}$

Figure 5-18 Standard Errors for all Test Runs: $(a)$ Fuel, $(b) \mathrm{NO}_{\mathrm{x}}$ 
Further validation of the ANN models will be performed later with the engine model integrated into the hybrid bus model to predict fuel economy and grams per mile of $\mathrm{NO}_{\mathrm{x}}$ and $\mathrm{CO}_{2}$.

\subsection{Correction for Emissions Certification}

Depending on the engine manufacturer's emissions credit trading, for a given year some engines are certified lower and others at higher emissions limits. The target EPA certification level of the engine model for this dissertation was 2007 but the only data available to the author to develop the models was from the ISB $260 \mathrm{H}$ discussed above with an $\mathrm{EPA} \mathrm{NO}$ certification level of $2.5 \mathrm{~g} / \mathrm{bhp} \cdot \mathrm{hr}$. A correction to the models is needed in order to account for the difference in certification levels.

Table 5-7 shows a summary of chassis dynamometer tests performed by the WVU TransLab on transit buses comparing 2004-2006 and 2007-2009 diesel-electric hybrid buses (CAFEE, 2011). The table shows vehicle weight, engine EPA certification level and test results on the OCTA and WMATA cycles. At the far right of the table are fuel consumption and $\mathrm{NO}_{\mathrm{x}}$ emissions normalized by test weight. Assuming that the differences on the normalized values are solely due to the engine (i.e. neglecting other factors like transmission efficiency, hybrid drive performance, etc.), it can be concluded that, for these buses, $\mathrm{NO}_{\mathrm{x}}$ reduction with the new engine is of the order of $15 \%$ to $23 \%$. $\mathrm{NO}_{\mathrm{x}}$ reduction was not of the order of the certification levels but much lower; this could be explained because the operational envelope of the engine in the transit buses is substantially different than operation over the Federal Test Procedure (FTP) used for certification. Regarding fuel consumption, and again assuming other things equal, the normalized values show a very small difference $(+2 \%$ in OCTA, $-1 \%$ in WMATA). One would expect an increase in fuel consumption due to the $\mathrm{NO}_{\mathrm{x}}$-fuel efficiency trade-off, but the data shows no evidence of this with statistical confidence.

Table 5-7 Emissions and F.E. New Flyer 40' Diesel-Hybrid Transit Buses, 2005/2008 (CAFEE, 2011)

\begin{tabular}{|c|c|c|c|c|c|c|c|c|c|c|}
\hline $\begin{array}{c}\text { Engine } \\
\text { MY }\end{array}$ & $\begin{array}{c}\text { Curb } \\
\text { Weight } \\
\text { (lb) }\end{array}$ & $\begin{array}{c}\text { GVWR } \\
\text { (lb) }\end{array}$ & $\begin{array}{c}\text { Test } \\
\text { Weight } \\
\text { (lb) }\end{array}$ & $\begin{array}{c}\mathrm{NO}_{\mathrm{x}} \\
\text { Cert. } \\
(\mathrm{g} / \mathrm{bhp} \cdot \mathbf{h r})\end{array}$ & Cycle & $\begin{array}{c}\text { F.E. } \\
\text { (mpg) }\end{array}$ & $\begin{array}{c}\text { Fuel } \\
\text { (g/mi) }\end{array}$ & $\begin{array}{c}\mathrm{NO}_{\mathrm{x}} \\
(\mathrm{g} / \mathbf{m i})\end{array}$ & $\begin{array}{c}\text { Fuel } \\
\text { (g/1000 } \\
\text { lb-mi) }\end{array}$ & $\begin{array}{c}\mathrm{NO}_{\mathrm{x}} \\
(\mathrm{g} / \mathbf{1 0 0 0} \\
\text { lb-mi) }\end{array}$ \\
\hline 2005 & 30,960 & 40,600 & 34,110 & 2.5 & OCTA & 4.81 & 653 & 9.7 & 19.1 & 0.286 \\
\hline \multirow{2}{*}{2008} & \multirow{2}{*}{33,660} & \multirow{2}{*}{42,540} & \multirow{2}{*}{36,810} & \multirow{2}{*}{1.0} & OCTA & 4.37 & 719 & 8.9 & 19.5 & 0.242 \\
\hline & & & & & WMATA & 3.88 & 811 & 9.1 & 22.0 & 0.247 \\
\hline
\end{tabular}

Table 5-8 shows results from a study that compared conventional transit buses (CAFEE, 2010). The results shown in the table are the average of four buses on each model year tested twice under each 
cycle. The newer buses showed better fuel economy but the differences were not statistically significant (to 95\% confidence). The fuel economy improvement can be attributed to the 5\% weight reduction. On the other hand, the reductions in $\mathrm{NO}_{\mathrm{x}}(\mathrm{g} / \mathrm{mi})$ of $41 \%$ in the OCTA cycle and $45 \%$ in the UDDS cycle had statistical significance. The weight-normalized results show a slight increase in fuel consumption and a significant reduction in $\mathrm{NO}_{\mathrm{x}}$ emissions: $38 \%$ in the OCTA cycle and $42 \%$ in the UDDS cycle.

Table 5-8 Emissions and F.E. Gillig 40' Conventional Diesel Transit Buses, 2004-5/2007

\begin{tabular}{|c|c|c|c|c|c|c|c|c|c|c|}
\hline $\begin{array}{c}\text { Engine } \\
\text { MY }\end{array}$ & $\begin{array}{c}\text { Curb } \\
\text { Weight } \\
\text { (lb) }\end{array}$ & $\begin{array}{c}\text { GVWR } \\
\text { (lb) }\end{array}$ & $\begin{array}{c}\text { Test } \\
\text { Weight } \\
\text { (lb) }\end{array}$ & $\begin{array}{c}\text { NO }_{\mathrm{x}} \\
\text { Cert. } \\
\text { (g/bhp·hr) }\end{array}$ & Cycle & $\begin{array}{c}\text { F.E. } \\
\text { (mpg) }\end{array}$ & $\begin{array}{c}\text { Fuel } \\
(\mathrm{g} / \mathrm{mi})\end{array}$ & $\begin{array}{c}\mathrm{NO}_{\mathbf{x}} \\
(\mathrm{g} / \mathrm{mi})\end{array}$ & $\begin{array}{c}\text { Fuel } \\
\text { (g/1000 } \\
\text { lb-mi) }\end{array}$ & $\begin{array}{c}\mathrm{NO}_{\mathrm{x}} \\
(\mathrm{g} / 1000 \\
\mathrm{lb}-\mathrm{mi}) \\
\end{array}$ \\
\hline \multirow{2}{*}{ 2004-2005 } & \multirow{2}{*}{29,230} & \multirow{2}{*}{39,500} & \multirow{2}{*}{33,123} & \multirow{2}{*}{2.8} & OCTA & 4.00 & 796 & 13.6 & 24.0 & 0.409 \\
\hline & & & & & UDDS & 5.26 & 605 & 9.8 & 18.3 & 0.297 \\
\hline \multirow{2}{*}{2007} & \multirow{2}{*}{27,860} & \multirow{2}{*}{39,600} & \multirow{2}{*}{31,363} & \multirow{2}{*}{1.25} & OCTA & 4.14 & 770 & 8.0 & 24.5 & 0.254 \\
\hline & & & & & UDDS & 5.49 & 580 & 5.4 & 18.8 & 0.173 \\
\hline \multirow{2}{*}{\multicolumn{3}{|c|}{ Difference }} & \multirow{2}{*}{$-5 \%$} & \multirow{2}{*}{$-55 \%$} & OCTA & $+3 \%$ & $-3 \%$ & $-41 \%$ & $+2 \%$ & $-38 \%$ \\
\hline & & & & & UDDS & $+4 \%$ & $-4 \%$ & $-45 \%$ & $+1 \%$ & $-42 \%$ \\
\hline
\end{tabular}

As a result, and based on the data presented above, the effect of emission certification levels was treated as a factor to multiply the output of the models developed. This approach is simple but, based on the difficulty of assessing the effects on various regions of the engine map, it will produce results that are both reasonable and in-line with the findings of emissions studies of 2007-2009 engines.

No correction was made for fueling because the data did not show a significant effect. A conservative correction factor of $\mathbf{0 . 8 5}$ (15\% reduction) was selected for $\mathrm{NO}_{\mathrm{x}}$. This value was chosen based on Table 5-7 because the engine in the series hybrid had emissions at the levels of the $2005 \mathrm{MY}$ engine shown in the table and it is reasonable to expect the same order of emissions reduction with the 2007 MY engine. The 2004-2005 MY engines of Table 5-8 had higher $\mathrm{NO}_{\mathrm{x}}$ levels than the engines in the hybrid buses and that accounts for the higher reductions of Table 5-8.

\subsection{Summary}

The models for fuel consumption and $\mathrm{NO}_{\mathrm{x}}$ emissions of the diesel engine were developed in this chapter. Source data for the models were introduced along with the data reduction procedures. The fuel models were based on ECU data while the $\mathrm{NO}_{\mathrm{x}}$ models incorporated analyzer measurements. Two different sets of models were developed, namely 2D maps and ANNs. The 2D maps performed satisfactorily for fuel consumption and the ANNs, which incorporate history terms, showed considerable improvements in $\mathrm{NO}_{\mathrm{x}}$ predictions. An analysis of data from various transit bus emissions 
studies revealed that an appropriate reduction in $\mathrm{NO}_{\mathrm{x}}$ emissions between engine model years 20032006 and 2007-2009 was of the order of 15\%. The models were used in the following chapter as part of the vehicle dynamic model. 


\section{Chapter 6. VEHICLE DYNAMIC MODEL}

This Chapter describes the vehicle dynamic model and its components. The vehicle model, developed in PSAT simulation software, was based on a $40 \mathrm{ft}$ Orion series-hybrid bus powered with a BAE Systems HybriDrive ${ }^{\circledR}$ propulsion system. It must be noted that the vehicle model developed in this Dissertation cannot be said to represent the performance of the BAE HybriDrive ${ }^{\circledR}$, and that BAE's system was used as a baseline for the models of the various components.

For this dissertation a model that resembles the New Flyer 40' hybrid transit buses, with BAE HybriDrive ${ }^{\circledR}$, was be developed in PSAT from data available publicly in the literature and component parameters supplied by BAE (BAE Systems, 2010). This chapter presents results of model validation against chassis-dynamometer data from the TransLab. It must be noted that there was limited information regarding performance of the electric system which did not allow the electric components to be individually validated. Instead, validations were carried out for overall vehicle performance: fuel economy, and $\mathrm{NO}_{\mathrm{x}}$ and $\mathrm{CO}_{2}$ emissions. Also, it was not practical to try to mimic the performance of the BAE HybriDrive ${ }^{\circledR}$ controller and a modified version of PSAT's load following controller was used.

First, the base vehicle is introduced; then the model is described, and, finally, the model is validated against chassis-dynamometers tests.

\subsection{Overview of the Base Vehicle: $40 \mathrm{ft}$ Orion hybrid bus with BAE Systems HybriDrive ${ }^{\circledR}$}

The base vehicle (see Section 5.1, page 121) was a MY $200640 \mathrm{ft}$ Orion transit bus. The bus had a series architecture where the engine is connected to a generator which provides electrical power for the drive motor and batteries. A brief description of the main components is presented next. The information below was obtained from BAE Systems (BAE Systems, 2010) for their MY 2007 system. 
Traction Motor: the traction motor is a $320 \mathrm{hp}(238 \mathrm{~kW})$ peak AC induction motor with 2,700 ft$\mathrm{lb}(3,660 \mathrm{Nm})$ of torque at zero speed. Maximum speed is 3,200 rpm. Weight is $450 \mathrm{lb}(210 \mathrm{~kg})$. The motor has a single-gear direct-drive to the differential and is oil cooled.

Traction Generator: the traction generator is a $260 \mathrm{hp}(194 \mathrm{~kW})$ continuous brushless permanent magnet motor. Maximum speed is 3,000 rpm and weight is $260 \mathrm{lb}(120 \mathrm{~kg})$. The generator is directly mounted into the engine's output shaft and is air cooled.

D Engine: The diesel engine is a Cummins ISB 260H, 6-cylinder, 5.9 litre, ULSD fuel with DPF (Table 5-2, page 123).

Battery System: The hybrid batteries are lead acid technology with a nominal voltage of $600 \mathrm{~V}$. Peak power: $268 \mathrm{hp}(200 \mathrm{~kW})$.

> Propulsion Control: the propulsion integral control handles engine speed, DPF monitoring and protection, battery management, etc. Peak power: $320 \mathrm{hp}(238 \mathrm{~kW})$. The controller weights $180 \mathrm{lb}$ $(80 \mathrm{~kg})$ and is oil cooled.

The bus curb weight was 33,440 lb (15,170 kg), GVWR was 42,540 lb (19,295 kg), and test weight was $38,540 \mathrm{lb}(17,480 \mathrm{~kg})$. The tested weight corresponded to half passenger load and not to half seated load. Frontal area was $80 \mathrm{ft}^{2}\left(7.4 \mathrm{~m}^{2}\right)$, tire diameter was 38.5 in $(0.98 \mathrm{~m})$. Simulated rolling resistance and drag coefficients were 0.0086 and 0.79 , respectively.

\subsection{Vehicle Dynamic Model in PSAT}

PSAT is a forward-looking simulator based on Simulink models (blocks) for vehicle components and control strategies. Forward-looking means that the model is resolved just like actual vehicles work: it starts with a driver's command. Backward-looking models start from the wheels up the powertrain (Rousseau and Pasquier, 2001).

In PSAT, the driver model reads the difference between target and actual speed sending a torque demand, based on the road load equation, to the vehicle controller. Then, the vehicle controller decides how to provide the desired torque demand and sends commands to the various component controllers which in turn translate the demands into component specific inputs, e.g. throttle position or motor current. The components supply torque and the vehicle moves producing a speed output. In the next simulation step, the driver again compares the output speed with the trace speed and sends out a new acceleration or deceleration (torque) request.

A generic PSAT component is shown in Figure 6-1. The ports in the top carry input commands (e.g. gear number, torque request) and output sensor signals (e.g. rotational speed). The second ports 
are efforts such as output torques and voltages; the last ports are flows such as output speeds and currents. Inputs to the model are on the left side and outputs are on right side. The model can be as simple as a lookup table or as complex as a neural network or a physical model. The model is accompanied by signal conditioning block, constraints block, and files for initialization, calculations, and scaling. The constraints block defines the performance limits of the model: the signal conditioning block reads the bus and retransforms the signals into model specific commands (e.g. torque command to throttle position).

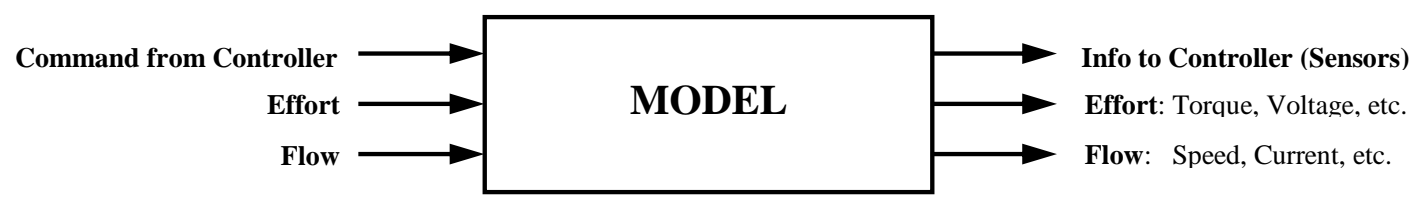

Figure 6-1 Generic Format of a Component in PSAT (ANL, 2008)

Figure 6-2 shows the global drivetrain configuration of a hybrid vehicle with a series configuration. The figure corresponds to the "ser_eng_2wd_p3" configuration in PSAT. Figure 6-3 presents the vehicle model in Simulink: three main groups are observed, namely vehicle or powertrain controller, component controller, and powertrain model. The relevant components are (ANL, 2008):

>Vehicle: the vehicle model calculates vehicle speed based on torque inputs and vehicle properties. There are two families of models, one that estimates aerodynamic and rolling losses as a second order polynomial called foflf 2 and one that uses the parameters of the road load equation (e.g. frontal area, drag and rolling resistance coefficients, etc.) called equation. Speed is solved as the integral of acceleration, where acceleration is solved from Newton's law: $a=$ net force / effective mass.

Wheel: Parameters for the wheel axle model are: maximum brake torque of the vehicle, number of wheels, wheel effective radius, inertia, and mass. The wheel model includes the brake calculations and transforms drivetrain inertia into an effective mass (see Equation 2.9, page 13). The equation model incorporates rolling resistance while the foflf2 model does not (it is included in the vehicle model).

D Differential: The parameters of the differential block are gear ratio, inertia, mass, and an efficiency map based on torque and speed. If no other information is available, a constant efficiency map is usually satisfactory; efficiency values ranging from $95 \%$ to $98 \%$ are common.

$>$ Gearbox: The gearbox of the series-hybrid bus (not shown in the figure) used consists of a single gear reduction. The PSAT gearbox model accounts for efficiency, inertia, and torque multiplication. For simplicity, differential and transmission ratios were combined into a single overall ratio and efficiency in the differential model. Therefore, the gearbox model was not necessary. 


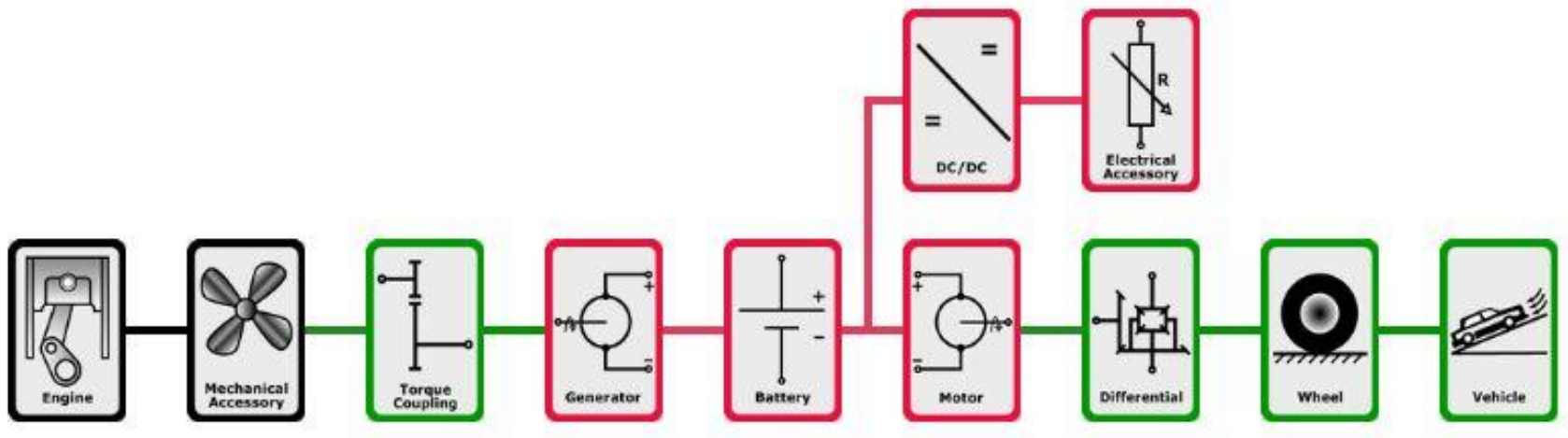

Figure 6-2 PSAT Series Hybrid Drivetrain Configuration

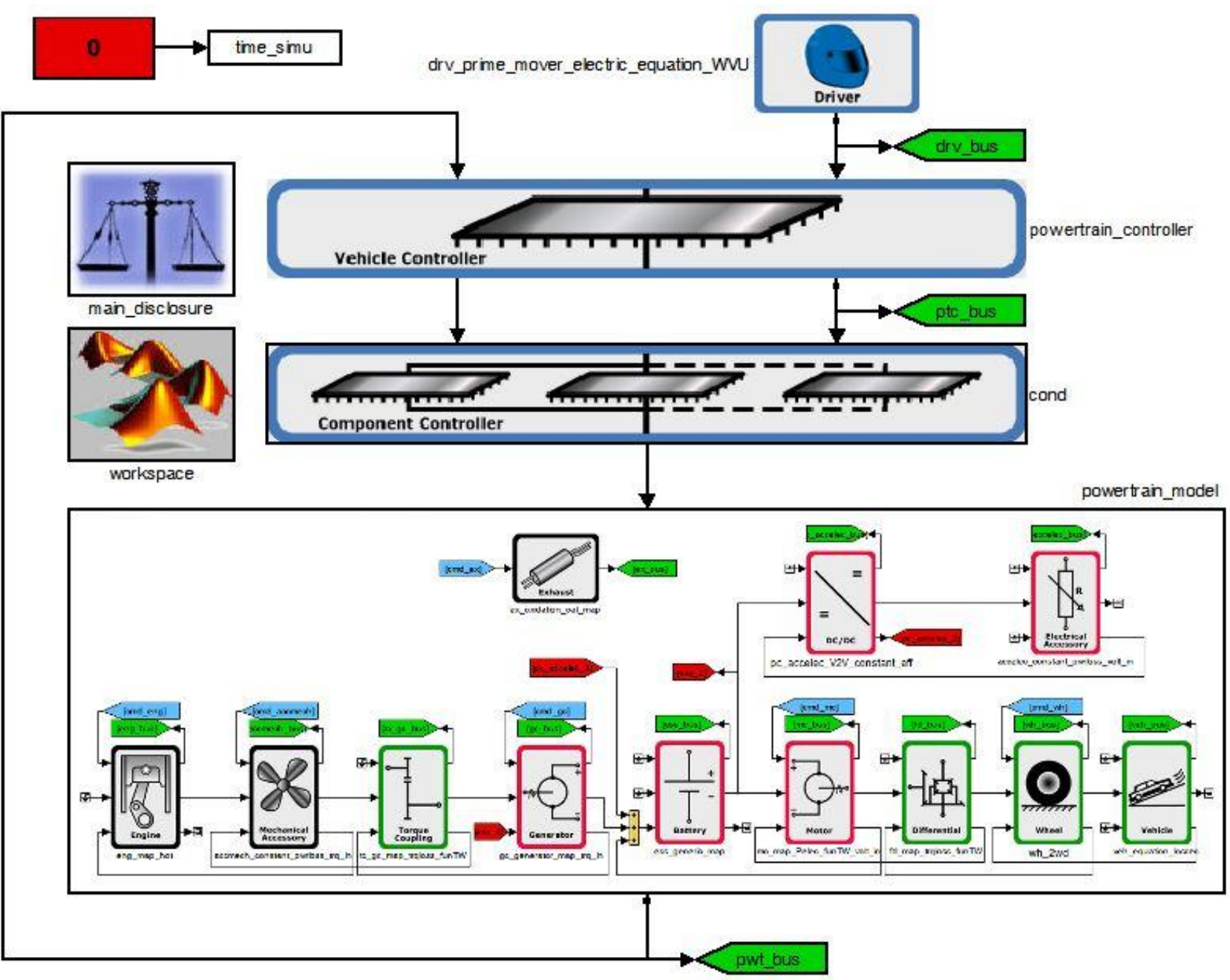

Figure 6-3 Outline of the PSAT Series Hybrid Vehicle Model in Simulink 
Torque Coupling: The torque coupling model was not necessary because the generator is directly mounted into the engine output shaft. Therefore, for modeling purposes, 100\% efficiency, 1:1 ratio and zero inertia were input in this block.

- Engine: The PSAT engine model is based on lookup tables for torque, fuel consumption and, when available, emissions. Fuel rate is a function of speed and brake torque. Controller torque demand is normalized to maximum torque at current speed and is then referred to as $T_{c m d}$; torque is calculated by $T_{\text {out }}=\left(1-T_{c m d}\right) \cdot T_{\min }+T_{c m d} \cdot T_{\max }$, where $T_{\min }$ and $T_{\max }$ are motoring and maximum brake torque, respectively.

The model is divided into four blocks: torque, thermal, fuel rate, and emissions. All of the blocks are based on lookup tables. The thermal block calculates energy lost to the exhaust and surroundings; engine and exhaust flow temperatures are set constant. Emissions lookup tables neglect the transient nature of emissions. The default model does not have flow rate calculations but there is a gas flow rate output used by the aftertreatment model. Parameters are: maximum and motoring torque maps, fueling rate (or efficiency) map, emissions rate maps, fuel properties (heating value, density, carbon weight fraction, etc.), and inertia. The engine model may be linearly scaled by power and efficiency.

Dxhaust aftertreatment: the diesel engine uses an oxidation catalyst model; gasoline engines use a three-way catalyst. The model calculates $\mathrm{HC}$ and $\mathrm{CO}$ efficiencies based on catalyst temperature and efficiency maps. Tailpipe emissions are then estimated from engine out emissions and efficiency. PM is also accounted for by a constant efficiency. The catalyst model calculates instantaneous $\mathrm{CO}_{2}$ emissions based on a carbon balance of $\mathrm{HC}, \mathrm{CO}$, fuel carbon. For this dissertation the engine model will output tailpipe emissions, hence catalyst efficiencies were set to zero.

Mechanical accessory: mechanical accessories are modeled as a constant power loss. The load is only applied when engine speed is above idle. This restriction is due to the oversimplified control algorithm default of PSAT. For this dissertation, the models were modified to include mechanical accessory loads not only in motored driving but also during braking and stops.

$>$ Electrical accessory: electrical accessories are also modeled as a constant power loss which is applied as long as the key is on.

$>$ Electric motor/generators: Torque is calculated according to the percent torque demand and the maximum torque at the current speed. Current is calculated as I = Power (electrical) / Voltage. Parameters for the model are: continuous and peak torque maps, efficiency maps (function of speed and torque), inertia, time response, and maximum current.

Power converter (electric motors and electrical accessories): There are a handful of models varying in the inputs and outputs, all having a constant efficiency. Consider for example the voltage input/output (V2V model); current to the battery is calculated from voltage, efficiency, and input 
current. Parameters are the efficiency and a constant voltage output. When additional information is available, a variable efficiency model can be used, where efficiency is expressed as a function (lookup table) of voltage and power. The series configuration used in this dissertation did not include a power converter and power converter losses were assumed to take place in the battery model.

Battery (Energy Storage): The battery model is a generic map that accommodates lithium-ion (Liion), nickel-cadmium (NiCd), nickel-metal hydride ( $\mathrm{NiMH})$, nickel-zinc (NiZn), and lead-acid $(\mathrm{Pb})$ batteries. The battery is composed of cells which are arranged in series to form modules, modules are arranged in series to form strings, and strings are arranged in parallel to form the battery. The model is divided into three blocks for SOC, voltage, and temperature calculations. The battery is modeled as a charge reservoir whose parameters are functions of SOC; all calculations are made at the cell level and then translated at the battery level. Open circuit voltage and internal resistance are functions of SOC and temperature. A simple thermal model simulates heat losses and air cooling; losses are estimated from internal resistance, current, voltage, and a coulombic efficiency table.

Parameters are: number of cells per module, number of modules per string, and number of strings in parallel; open circuit voltage, charging resistance, and discharging resistance are defined for a cell as functions of temperature and SOC. Thermal properties are defined for a module.

Since little data was available for the BAE HybriDrive ${ }^{\circledR}$ battery, the battery model was taken from the Allison buses which have a $600 \mathrm{~V}$ nominal, 19 amp-hr, NiMH battery pack (Chiang, 2007). Two sub-packs in series form a $600 \mathrm{~V}$ string (module) and three strings in parallel provide the required storage capacity. Each sub-pack has a variable speed cooled fan for thermal control. A sub-pack contains 40 cells (SAE-UK, 2007); the nominal voltage of a cell, with 80 cells per module, is $600 \mathrm{~V} /(2 \cdot 40)=7.5 \mathrm{~V}$.

Driver: models the accelerator and brake pedals through a PI controller which compares desired vehicle speed with current speed and outputs a torque request. Since the driver block calculates torque requirements, it must match the parameters on the Vehicle model. There are provisions for both types of vehicle models. The proportional gain targets the transient speed trace while the integral gain targets the steady state offset. The total output torque request is the sum of the PI torque (due to error) and the torque required to overcome road load.

For this dissertation, a modified version PSAT's normal driver was used. The modification consisted of amplifying the gains by a factor of 10 for speeds below $5 \mathrm{mph}$. The modified driver model produced satisfactory results for both low and high speeds.

Control strategy: The controller receives demands and, based on operational parameters and constraints, makes decisions generating commands to the various components. The control strategy is divided into propelling, braking and shifting, or it can be defined as a global strategy 
that performs all three functions. Two different data buses are used: one for powertrain parameters and one for controller parameters. There may be a block to handle transients such as gear shifts in a manual transmission.

The default controllers available for the series architecture were load following and thermostat. The load following controller operates the engine under the optimum efficiency curve. The batteries aid acceleration events whenever SOC is above the SOC target and are recharged when $\mathrm{SOC}$ is below the SOC target. The thermostat controller operates the engine at the maximum efficiency point if power demands are moderate; under high power demands, the engine operates at maximum power. A separate controller supervises braking and regeneration.

The load following controller was used in this dissertation. The braking controller was modified to sustain mechanical accessory loads during braking and stops.

Differential, motor, and generator models were developed by Bell (2011).

\subsection{Summary of Model Assumptions and Parameters}

The vehicle properties listed in Table 3-1 (page 75) were modified to match those of the base vehicle described in Section 6.1. A summary of the modeling parameters of the diesel-electric series hybrid bus is presented in Table 6-2. The following is a list of the assumptions and a description of the various vehicle components:

$>$ Grade effects are neglected.

$>$ Vehicle linear dynamics are considered: inertia, rolling resistance and aerodynamic drag forces (Equation 2.11, page 14).

Motor: The traction motor model was built to match the parameters listed in Section 6.1. Figure 6-4 shows the efficiency maps (torque and power) and the maximum torque/power curves. Rotational inertia of motor and generator was estimated through scaling to be approximately 1 $\mathrm{kg} \cdot \mathrm{m}^{2}$.

> Generator: Generator efficiency was assumed constant at 93\%. The generator model was modified, from that described in Section 6.1, to receive continuously the engine's peak power of $260 \mathrm{hp}(194 \mathrm{~kW})$. This allowed the vehicle to sustain a top speed of $65 \mathrm{mph}$ (BAE Systems, 2010).

Battery: The battery model used was for a NiMH battery, which is more representative of state of the art transit bus battery systems than the lead-acid battery described in Section 6.1. As mentioned in the previous section, the NiMH battery model was based on the battery of transit 
buses with the Allison Hybrid $E^{\mathrm{P}}$ System. Peak charging power (regenerative braking) was 120 kW (160 hp) and peak discharging power (power assist) was $170 \mathrm{~kW}(230 \mathrm{hp})$.

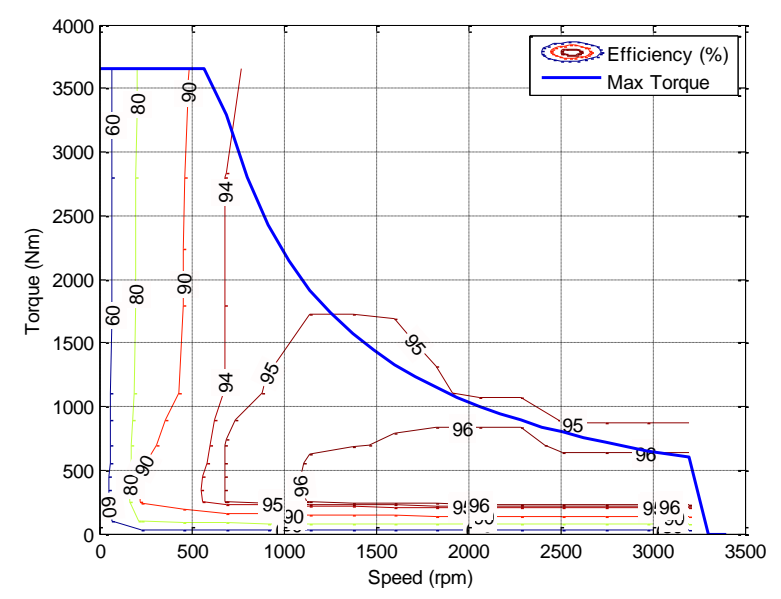

(a) Torque

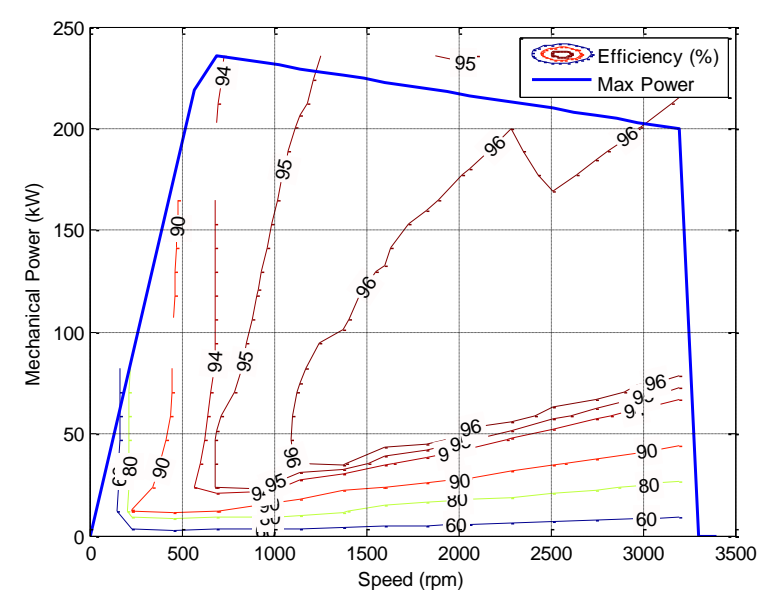

(b) Power

Figure 6-4 Traction Motor Efficiency Map (Bell, 2011)

Differential: A combined final-drive and single-speed transmission ratio of 4.9 and an efficiency of $98 \%$ were selected (Bell, 2011). At maximum traction motor torque the selected ratio provides a maximum acceleration of $6.6 \mathrm{ft} / \mathrm{s}^{2}\left(4.5 \mathrm{mph} / \mathrm{s}\right.$ or $\left.2 \mathrm{~m} / \mathrm{s}^{2}\right)$, and at maximum traction motor speed the ratio provides a top vehicle speed of $72 \mathrm{mph}$ (Bell, 2011).

Engine: The engine model was the ISB 260H model developed in Chapter 5, with 2D-Map for fuel rate and $\mathrm{ANN}$ for $\mathrm{NO}_{\mathrm{x}}$ emissions. Rotational inertia of the engine was estimated through scaling to be approximately $0.8 \mathrm{~kg} \cdot \mathrm{m}^{2}$. Maximum efficiency was $43 \%$.

Driver: A modified version of PSAT's normal driver was used. The proportional and integral gains were 1,000 and 0.5 , respectively. To provide better response at low speeds, the gains were increased 10 fold at speeds below $5 \mathrm{mph}$.

Wheel: The 6 wheels were B 305 / 70 R22.5 with a diameter of 39 in (0.99 m). Rolling resistance coefficient was 0.0086; Inertia was $11 \mathrm{~kg} \cdot \mathrm{m}^{2}$ per wheel (see Section 3.1.2, page 77). A correction factor for tire loading of 0.95 was applied to wheel diameter. Maximum vehicle braking torque was $15,000 \mathrm{Nm}(11,000 \mathrm{ft}-\mathrm{lb})$.

Vehicle: The parameters for vehicle model were those described in Section 6.1.

$>$ Controller: The vehicle controller was a modified version of PSAT's load following controller. Electric only and engine stop options were disabled. The proportional and integral gains for engine speed control were 4 and $1 \times 10^{-4}$, respectively. 
The controller was modified to maintain the mechanical accessory loads during vehicle idle and braking; in the original controller, mechanical accessory loads and engine power were set to zero under those conditions.

The controller was also modified to maintain battery state of charge and regulate motor power output at highway speeds. The original controller allowed full battery depletion when, for a sustained period, vehicle output request was beyond the engine capabilities.

Mechanical and Electrical Accessory Loads: O'Keefe and Vertin (2002), see Table 3-2 in page 76, modeled a 40' Orion VI transit bus with the following: engine $=275 \mathrm{hp}, 8.5 \mathrm{~L}$ DDC S50, 44\% peak efficiency; 5 speed transmission, and auxiliary loads of $17 \mathrm{~kW}$ with $\mathrm{A} / \mathrm{C}$ off and $31 \mathrm{~kW}$ with $\mathrm{A} / \mathrm{C}$ on. Fuel economy over the CBD cycle was $3.2 \mathrm{mpg}$ with A/C on and $4.0 \mathrm{mpg}$ with A/C off. Auxiliary load break down is shown in Table 6-1.

Evaluation of test data for engine power under idle and braking conditions showed a considerable cycle-to-cycle variation, ranging from $8 \mathrm{~kW}$ to $20 \mathrm{~kW}$. The lack of hybrid system performance records did not allow discerning if the battery was being charged during these periods nor if the full engine load was due to accessories. The average and median of all the runs were both $13 \mathrm{~kW}$.

Table 6-1 Breakdown of Auxiliary (Accessory) Loads (O'Keefe and Vertin, 2002)

\begin{tabular}{l|c|c|c}
\hline \multirow{2}{*}{ Component } & Conventional & \multicolumn{2}{|c}{ Series Hybrid } \\
\cline { 2 - 4 } & $\begin{array}{c}\text { Mechanical } \\
\text { Load (kW) }\end{array}$ & $\begin{array}{c}\text { Electrical } \\
\text { Load (kW) }\end{array}$ & $\begin{array}{c}\text { Mechanical } \\
\text { Load (kW) }\end{array}$ \\
\hline Air Compressor & 1 & - & 0.75 \\
\hline Alternator & 1 & - & - \\
\hline Engine Fan & 14 & - & 7.5 \\
\hline HVAC load & 14 & - & 14 \\
\hline Hydraulic Pump & 1 & - & 0.75 \\
\hline Coolant Pump & - & 9 & - \\
\hline
\end{tabular}

In order to establish an adequate value for auxiliary loads, the author performed a sensitivity analysis where the effects of auxiliary loads on fuel economy and $\mathrm{NO}_{\mathrm{x}}$ emissions were evaluated. It was concluded that an appropriate value for the combined mechanical and accessory loads was $12 \mathrm{~kW}(16 \mathrm{hp})$. This value is $1 / 3$ lower than the $18 \mathrm{~kW}(24 \mathrm{hp})$ reported by O'Keefe and Vertin (2002), but, provided the set of data available to this author, it was the most adequate one.

\subsection{Vehicle Level Validation}

The series-hybrid bus model was exercised over the Beeline, Manhattan, New York Bus, OCTA, UDDS, and WMATA cycles and the results were compared with the test results of Table 5-4 (page 128). The simulation results were compared with the average of each cycle. Given the differences 
between cycle target and actual vehicle speed during chassis-dynamometer testing, the average test speed trace of each cycle was used for simulation. Battery SOC correction was considered in the simulations by running each cycle back to back and using the results from the last run where $\Delta \mathrm{SOC}$ was below $1 \%$.

Table 6-2 Series Hybrid Bus Modeling Parameters

\begin{tabular}{|c|c|}
\hline Component & Details \\
\hline Vehicle & $\begin{array}{l}G V W R: 42,540 \mathrm{lb}(19,295 \mathrm{~kg}) \\
\text { Curb weight: } 33,440 \mathrm{lb}(15,170 \mathrm{~kg}) \\
\text { Half seated load weight }(1 / 2 \mathrm{SLW}): 36,440 \mathrm{lb}(16,530 \mathrm{~kg}) \\
\text { Test weight }{ }^{\mathrm{h}}: 38,540 \mathrm{lb}(17,480 \mathrm{~kg}) \\
\text { Length, Width, Height: } 40.8 \mathrm{ft}, 102 \mathrm{in}, 133 \text { in (approximately) } \\
\text { Ground clearance: } 14 \mathrm{in} \\
\text { Drag coefficient: } 0.79 \\
\text { Frontal area: } 80 \mathrm{ft}^{2}\left(7.43 \mathrm{~m}^{2}\right) \\
\text { Capacity: } 39 \text { seated }+20 \text { standing }^{2}\end{array}$ \\
\hline Wheel & $\begin{array}{l}\text { Rolling resistance coefficients: } c_{r}=c_{r, 0}+c_{r, l} \cdot V \\
c_{r, 0}=0.0086, c_{r, l}=0 \mathrm{~s} / \mathrm{m} \\
\text { Tires: } 6 \times \mathrm{B} 305 / 70 \mathrm{R} 22.5 \\
\text { Effective radius: } 19.5 \text { in }(0.495 \mathrm{~m}) \\
\text { Inertia: } 11 \mathrm{~kg} \cdot \mathrm{m}^{2} \text { per wheel } \\
\text { Weight: } 77.5 \mathrm{~kg} \text { per wheel }\end{array}$ \\
\hline $\begin{array}{l}\text { Differential / } \\
\text { Transmission }\end{array}$ & $\begin{array}{l}\text { Ratio: } 4.9 \\
\text { Efficiency: } 98 \%\end{array}$ \\
\hline Engine & $\begin{array}{l}\text { Model: Cummins ISB } 260 \mathrm{H} \\
\text { Power: } 260 \mathrm{hp}(194 \mathrm{~kW}) @ 2,300 \mathrm{rpm} \\
\text { Torque: } 600 \mathrm{ft}-1 \mathrm{~b}(815 \mathrm{Nm}) @ 1,800 \mathrm{rpm} \\
\text { Cylinders: } 6 \\
\text { Idle speed: } 800 \mathrm{rpm} \\
\text { Governed speed: } 2,500 \mathrm{rpm} \\
\text { Turbocharged and charge air cooled } \\
\text { Cooled EGR, variable-geometry-turbocharger, high pressure common rail fuel system, and DPF }\end{array}$ \\
\hline Loads & $\begin{array}{l}\text { Mechanical: } 8 \mathrm{hp}(6 \mathrm{~kW}) \\
\text { Electrical: } 8 \mathrm{hp}(6 \mathrm{~kW})\end{array}$ \\
\hline Motor & $\begin{array}{l}\text { Power: } 320 \mathrm{hp}(238 \mathrm{~kW}) \\
\text { Peak Efficiency: } 96 \% \\
\text { Torque: } 2700 \mathrm{ft}-1 \mathrm{~b} .(3660 \mathrm{~N}-\mathrm{m}) \text { @ zero speed } \\
\text { Speed: } 0-3200 \mathrm{rpm}\end{array}$ \\
\hline Generator & $\begin{array}{l}\text { Power: } 260 \mathrm{hp}(194 \mathrm{~kW}) \\
\text { Efficiency: } 93 \%\end{array}$ \\
\hline
\end{tabular}

\footnotetext{
${ }^{\mathrm{h}}$ The test weight of 38,540 $\mathrm{lb}$ was used during chassis-dynamometer testing of the base vehicle and was used here to adjust the model. It corresponds to half of the total passenger load and not the half seated load weight ( $1 / 2$ SLW) recommended by SAE J2711 (SAE International, 2002). In the following chapters the $1 / 2$ SLW of $36,440 \mathrm{lb}$ will be used as vehicle test weight.
} 


\begin{tabular}{l|l}
\hline Component & \multicolumn{1}{c}{ Details } \\
\hline & Type: NiMH \\
& Nominal voltage: 600 \\
& Peak charging power: $160 \mathrm{hp}(120 \mathrm{~kW})$ \\
& Peak discharging power: $230 \mathrm{hp}(170 \mathrm{~kW})$ \\
& Cells in sub-pack: 40 \\
& Sub-packs in series (form a string): 2 \\
& Strings in parallel: 3 \\
& Variable speed cooling fan for thermal control \\
& Nominal cell voltage: 7.5 \\
\hline
\end{tabular}

As mentioned earlier, the different control strategies did not allow comparison of instantaneous fuel and $\mathrm{NO}_{\mathrm{x}}$ rates because engine operation was very different in the simulations when compared with the tests. Therefore, only cycle integrated values were compared, as shown in Figure 6-5. Figure 6-5 compares simulation and test results for fuel economy, $\mathrm{NO}_{\mathrm{x}}$, and $\mathrm{CO}_{2}$. The fuel economy results were within the 95\%-confidence error bars except for the Beeline and New York Bus cycles, which had lower and higher values, $-7 \%$ and $+11 \%$ respectively. $\mathrm{NO}_{\mathrm{x}}$ errors were all within $5 \%$ of the test values. $\mathrm{CO}_{2}$ emissions were in line with the fuel economy results where only the Beeline and New York Bus cycles were outside the $95 \%$ confidence limits.

Ultimately, engine fuel rate was modeled using the 2D-Map and not the ANN model. Simulations using the fuel ANN resulted in lower fuel consumption and higher efficiency than expected, such that fuel economy values were consistently higher and the cycle errors were not improved. It was then decided to use the simpler 2D-Map model for fuel over the more complex ANN model. On the other hand, and as expected due to the transient nature of $\mathrm{NO}_{\mathrm{x}}$, the $\mathrm{NO}_{\mathrm{x}} 2 \mathrm{D}-\mathrm{Map}$ performed poorly versus the ANN model with an average cycle error of $13 \%$ while the ANN average cycle error was $3 \%$. The ANN model was used for $\mathrm{NO}_{\mathrm{x}}$ emissions.

Average engine efficiency ranged from $37 \%$ to $40 \%$ in the simulations; values that were in line with the tests. Engine cycle work values also showed good correlation.

A comparison between the OCTA cycle simulation results and selected chassis-dynamometer test of $40 \mathrm{ft}$ hybrid buses is shown in Table 6-3 and Figure 6-6. Input F corresponds to the base vehicle that served to develop the model. Inputs $\mathrm{A}, \mathrm{B}$, and $\mathrm{C}$ correspond to tests of three buses of the same model year and technology (CAFEE, 2007). Inputs D and E are from tests of New Flyer/ Allison hybrids tested by WVU (CAFEE, 2011). Inputs G, F, and I are simulation results: G is a simulation representing the base vehicle, $\mathrm{H}$ is a simulation with half seated load weight (Table 6-2), and $\mathrm{I}$ is a simulation including the correction for model year of Section 5.7. 


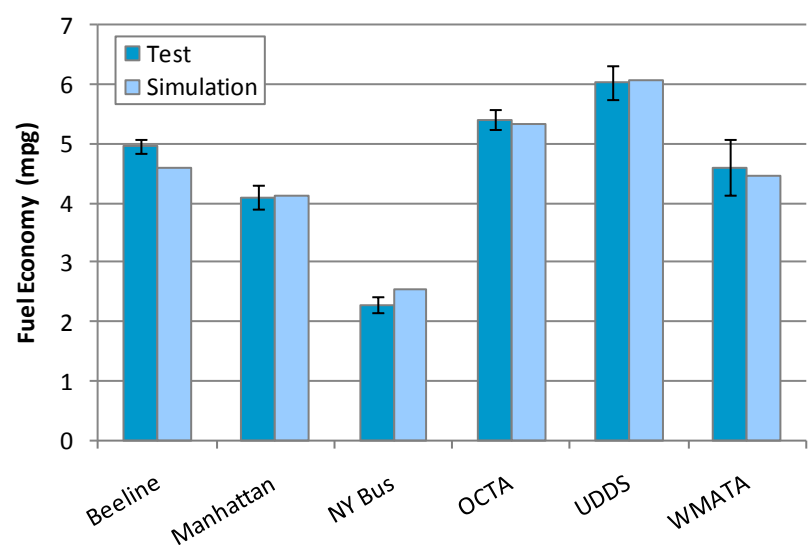

(a) Fuel Economy

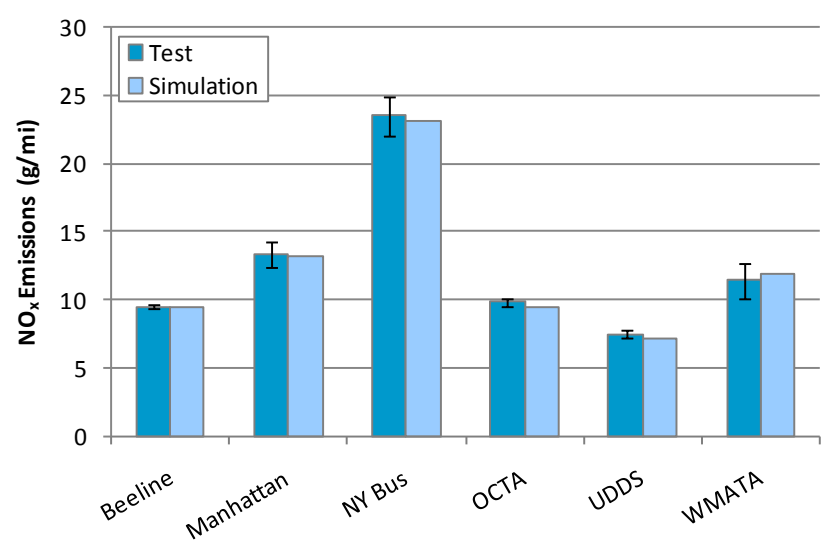

(b) $\mathrm{NO}_{\mathrm{x}}$ Emissions

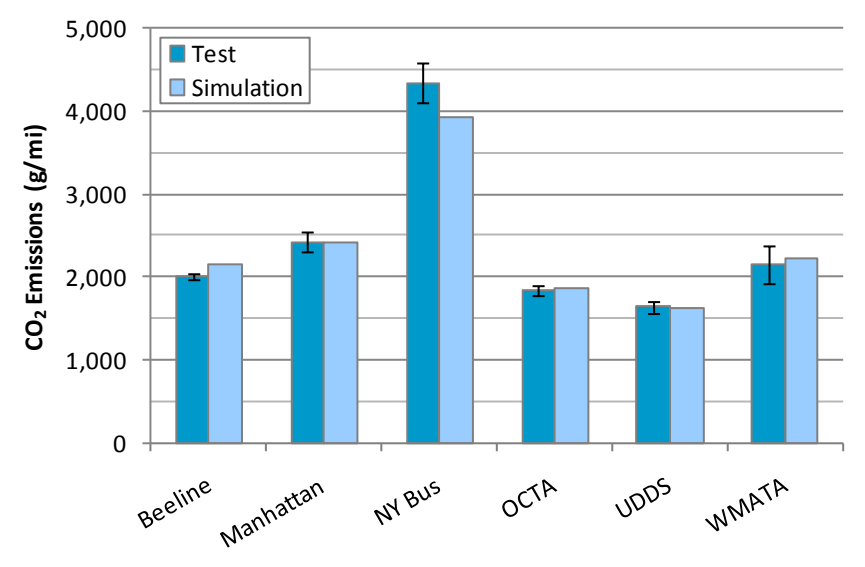

(c) $\mathrm{CO}_{2}$ Emissions

Figure 6-5 Vehicle Level Validation Results

Figure 6-6 (a) shows distance-weight specific diesel fuel consumption and it is observed that the model $(\mathrm{G}$ and $\mathrm{H})$ compare very well with the base vehicle $(\mathrm{F})$ while the New Flyer /Allison buses (parallel architecture) exhibit higher fuel consumption.

Figure 6-6 (b) shows the distance-weight specific $\mathrm{NO}_{\mathrm{x}}$ emissions, where it is observed that the model compares well with the base vehicle. In contrast with the fuel consumption figure, it is observed that in general the parallel architecture exhibits slightly lower $\mathrm{NO}_{\mathrm{x}}$ emissions than the series architecture. The predicted value for the series bus with an engine of model year 2007 to 2009 is shown at the far right of the figure (input I). 
Table 6-3 OCTA Cycle Emissions from Selected $40 \mathrm{ft}$ Hybrid Transit Buses

\begin{tabular}{|c|c|c|c|c|c|c|c|c|}
\hline Legend & Data Source & Make / Model & Engine & $\begin{array}{c}\text { Test } \\
\text { Weight } \\
\text { (lb) }\end{array}$ & $\begin{array}{c}\text { F.E. } \\
(\mathbf{m p g})\end{array}$ & $\begin{array}{c}\text { Fuel } \\
\text { (g/mi) }\end{array}$ & $\begin{array}{c}N_{(\mathrm{x}} \\
(\mathrm{g} / \mathrm{mi})\end{array}$ & $\begin{array}{c}\mathrm{CO}_{2} \\
(\mathrm{~g} / \mathrm{mi})\end{array}$ \\
\hline A & \multirow{3}{*}{ CAFEE, 2007} & 005 New Flyer/Allison & Cummins ISL 280 & 35,860 & .30 & 81 & 8.0 & 1,8 \\
\hline $\mathrm{B}$ & & 2005 New Flyer/Allison & Cummins ISL 280 & 36,160 & 5.12 & 602 & 8.8 & 1,886 \\
\hline $\mathrm{C}$ & & 2005 New Flyer/Allison & Cummins ISL 280 & 36,280 & 4.90 & 629 & 8.8 & 1,972 \\
\hline $\mathrm{D}$ & \multirow{2}{*}{ CAFEE, 2011} & 2005 New F & 280 & 34,1 & 4.91 & 661 & 9.7 & 2,102 \\
\hline $\mathrm{E}$ & & 2008 New F & ISL 280 & 36,810 & 4.46 & 729 & 8.9 & 2,318 \\
\hline $\mathrm{F}$ & $\begin{array}{c}\text { Transit } \\
\text { Resource } \\
\text { Center, } 2007 \\
\end{array}$ & 2006 Orion VII/BAE & Cummins ISB-260H & 38,540 & 5.22 & 591 & 9.8 & 1,849 \\
\hline $\mathrm{G}$ & del & $2004-20$ & ISB $260 \mathrm{H}$ & 38,540 & $\overline{5.32}$ & 590 & 9.4 & 1,866 \\
\hline $\mathrm{H}$ & & 2004-2006 Series-Hybrid & Cummins ISB 260H & 36,440 & 5.56 & 565 & 9.3 & 1,787 \\
\hline I & This model & 2007 Series-Hybrid & Cummins ISB $260 \mathrm{H}$ & 38,540 & 5.32 & 590 & 8.0 & 1,866 \\
\hline
\end{tabular}

* Simulation at $1 / 2$ SLW (Table 6-2)

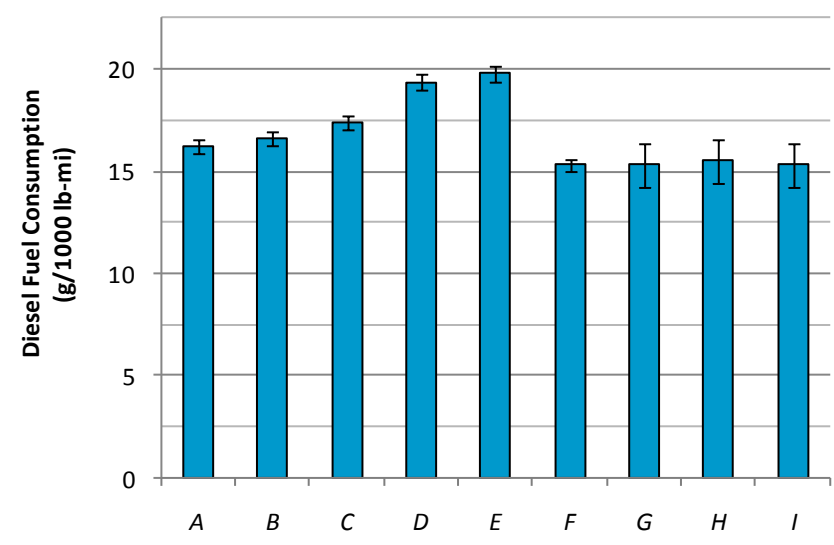

(a) Distance-Weight Specific Fuel Consumption

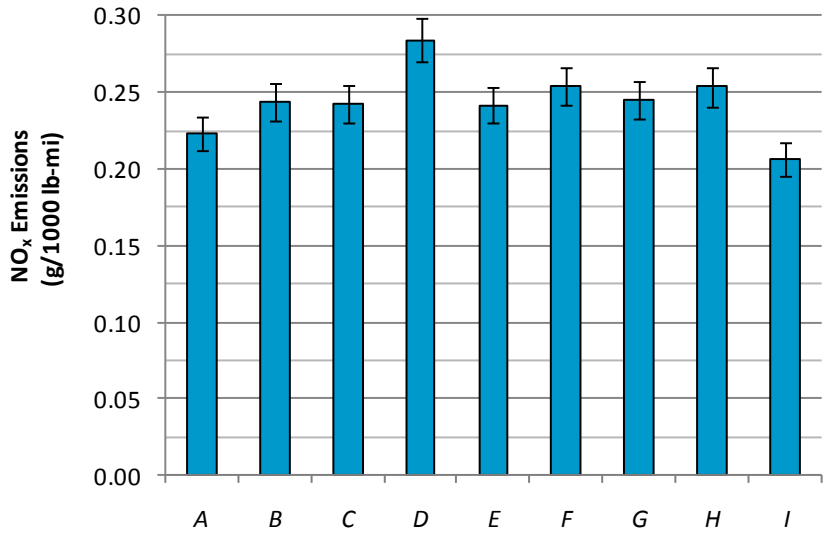

(b) Distance-Weight Specific $\mathrm{NO}_{\mathrm{x}}$ Emissions

Figure 6-6 Comparison of Simulation Fuel Consumption with Chassis Tests

Wayne et al. (2009) and U.S. FTA (2007) predicted OCTA cycle emissions and fuel economy for MY 2007 hybrids. They predicted fuel economy between 4.9 and $6.5 \mathrm{mpg}, 4.5 \mathrm{~g} / \mathrm{mi}$ of $\mathrm{NO}_{\mathrm{x}}$, and 1,600 to $2,000 \mathrm{~g} / \mathrm{mi}$ of $\mathrm{CO}_{2}$. While those fuel economy and $\mathrm{CO}_{2}$ values are in good agreement with the results from the model, their $\mathrm{NO}_{\mathrm{x}}$ prediction was lower than the $8.0 \mathrm{~g} / \mathrm{mi}$ estimated here. 


\subsection{Other Considerations}

\subsubsection{State of Charge Corrections}

The improved control strategy was able to maintain battery SOC around the 60\% SOC target. However, in order to avoid the necessity of SOC corrections, in compliance with SAE J2711 (SAE International, 2002), the ratio of net change in battery energy to total cycle energy must be less than $1 \%$. A group of simulations was run to explore whether this condition was satisfied and it was found that, indeed, it was.

The simulation software had the option of performing continuous repeats of the test cycle to allow the vehicle to settle into a stable SOC value. Data from the last cycle is then used for analysis. When no repetitions were done, the percentage was between $0.5 \%$ and $0.8 \%$, and with one repetition the percentage dropped to less than $0.1 \%$. The net effect of the repetition on fuel consumption and $\mathrm{NO}_{\mathrm{x}}$ emissions was around $1 \%$. On the other hand, for short cycles one repetition was performed in order to produce consistent results; this was not necessary for cycles longer than one hour.

\subsubsection{Driving History or Micro-Trip Order}

Marlowe (2009) used micro-trip concatenation to expand the amount of data for the emissions regression models. In Marlowe's work, it was not certain if this approach was appropriate such that the cycles thus created represented emissions and fuel economy when the hybrid vehicle were tested on that time-speed trace.

This author explored the possible impact of driving history in cycle emissions and fuel economy using the OCTA cycle. The order of the micro-trips on the original cycle was inverted, i.e. the last trip went first and the first trip went last, and simulation results of both original and inverted cycles were compared. One repetition of the cycle was performed to assure zero $\triangle \mathrm{SOC}$.

It was found that there was no change in fuel consumption $(<0.1 \%)$ and that the change in $\mathrm{NO}_{\mathrm{x}}$ emissions was less than $1 \%$. It was so concluded that, as long as zero $\triangle \mathrm{SOC}$ is satisfied and the length of the new cycle is reasonably long (at least 30 minutes), micro-trips from test or simulation can be used, as suggested by Marolowe (2009), with confidence to reproduce emissions of new cycles with different overall properties than the original test sequences. 


\subsection{Summary}

Results from the development of a dynamic model and hybrid controller for the hybrid bus were presented in this chapter. Model assumptions and a description of the various elements of the model were also presented.

From Chapter 5, error in the engine model on fuel consumption and $\mathrm{NO}_{\mathrm{x}}$ emissions was estimated to be $1 \%$ and $6 \%$, respectively; analysis of the vehicle level validation results (all the components of the vehicle model working together) show that the overall model uncertainties are $7 \%$ for fuel consumption and $5 \%$ for $\mathrm{NO}_{\mathrm{x}}$ emissions.

In conclusion, predictions from the vehicle dynamic model are very good. The model can be used with confidence as substitute for chassis-dynamometer tests of a 2007-2009 $40 \mathrm{ft}$ Diesel-Electric Series Hybrid transit bus. The effect of varying driving conditions on fuel economy and emissions is explored next. 


\section{Chapter 7. Influence of Transit Bus Duty CyCle ON Fuel ECONOMY AND EMISSIONS}

One of the objectives of this dissertation was the development of a regression-based emissions model to allow transit agencies to place hybrid buses on routes that take the most advantage of the hybrid-electric capabilities. Ignoring elevation changes, this chapter used the routes of Chapter 4 and the dynamic model of Chapter 6 to explore the effects of duty cycles on fuel economy and emissions $\left(\mathrm{NO}_{\mathrm{x}}\right.$ and $\left.\mathrm{CO}_{2}\right)$ of MY 2007-2009 40-ft diesel-electric series hybrid buses.

In order to produce more accurate predictions than previous studies (Marlowe, 2009), the contributions of idle segments and driving segments were studied independently. Idle fuel consumption (F.C.) and emissions were found to be nearly independent of cycle properties. Driving F.C. and emissions, on the other hand, were correlated with cycle properties and were expressed as a

polynomial function of average speed without idle $\left(\overline{\mathrm{U}}_{\text {no idle }}\right)$ and characteristic acceleration ( $\left.\tilde{a}_{\text {no grade }}\right)$. Considering the idle fraction, idle emissions and F.C. were then coupled with the driving contributions to obtain the overall cycle values.

This approach was also applied to develop a prediction model for a conventional bus. The benefit of the hybrid bus was presented in terms of volumetric fuel savings rate (gallons per hour) and hybrid advantage (H.A.). H.A. is the percent reduction in distance specific fuel consumption compared to a conventional vehicle serving the same route (O'Keefe et al., 2007).

The chapter concludes with a brief presentation of two tools developed for IBIS. The first tool makes the post processing of GPS logs of transit routes. The second tool is an implementation of the developed fuel economy, $\mathrm{NO}_{\mathrm{x}}$ and $\mathrm{CO}_{2}$ emissions, and fuel savings predictive methods.

\subsection{Transit Routes - Simulations and Results}

In order to characterize fuel economy and emissions of the hybrid transit bus under a wide range of operation or duty cycles, the vehicle dynamic model was simulated with a vehicle activity database consisting of 95 routes. The database includes the in-use routes summarized in Table 4-6, a selection of the standard cycles of Table 2-6, bus route logs collected by WVU at METRO Transit (Texas), CT Transit (Connecticut), Lynx (Florida), and the WVU Coliseum. The set of cycles selected from Table 2-6 was considered by the author to be representative of real transit bus operation; in contrast to other 
cycles like the CBD of Figure 2-3 which do not. A commuter route (WMATA Commuter) representing WMATA's high speed operation (Sandoval and Wayne, 2010) was simulated as well.

Table 7-1 presents a partial summary of the results: the first column is cycle name, the second set of columns contains cycle metrics, and the last set of columns presents cycle fuel economy, $\mathrm{CO}_{2}$ and $\mathrm{NO}_{\mathrm{x}}$ emissions, and fuel consumption. Fuel consumption and $\mathrm{NO}_{\mathrm{x}}$ emissions were decomposed into the driving and idle contributions; idle contributions are presented in a time basis. Cycle average engine efficiency ranged from $36 \%$ to $41 \%$. The hybrid powertrain regenerative braking captureredeployment efficiency $\left(\eta_{\text {regen }}\right)$ ranged from $45 \%$ to $70 \%$ with an average of $60 \%$.

The vehicle was simulated with its half seated load weight $(1 / 2 \mathrm{SLW}$, Table $6-2)$ of $36,440 \mathrm{lb}$. The effect of grade was not included in the analysis as it is unlikely that transit agencies would have grade metrics (Table 4-5) for their routes.

Table 7-1 Fuel Consumption and Emissions Simulation Results for the Hybrid Bus Under Varying Driving Conditions

\begin{tabular}{|c|c|c|c|c|c|c|c|c|c|c|c|c|c|}
\hline Line/Cycle Name ${ }^{\mathrm{i}}$ & $\begin{array}{l}\text { Dist. } \\
\text { (mi) }\end{array}$ & $\underset{(\mathbf{m p h})}{\overline{\mathbf{U}}}$ & $\begin{array}{l}\overline{\mathbf{U}}_{\text {no idle }} \\
(\mathbf{m p h})\end{array}$ & $\begin{array}{l}\text { Idle } \\
(\%)\end{array}$ & $\begin{array}{c}\tilde{a}_{\text {no grade }} \\
\left(\mathbf{f t} / \mathbf{s}^{2}\right)\end{array}$ & $\begin{array}{c}\text { F.E. } \\
\text { (mpg) }\end{array}$ & $\underset{(\mathrm{g} / \mathrm{mi})}{\mathrm{CO}_{2}}$ & $\underset{(\mathrm{g} / \mathrm{mi})}{\mathbf{N O}_{\mathbf{x}}}$ & $\begin{array}{c}\text { Fuel } \\
\text { (g/mi) }\end{array}$ & $\begin{array}{c}\text { Fuel } \\
\text { Driving } \\
\text { (g/mi) }\end{array}$ & $\begin{array}{c}\mathrm{NO}_{\mathrm{x}} \\
\text { Driving } \\
\text { (g/mi) }\end{array}$ & $\begin{array}{l}\text { Fuel } \\
\text { Idle } \\
(\mathrm{g} / \mathrm{s})\end{array}$ & $\begin{array}{l}\mathrm{NO}_{\mathrm{x}} \\
\text { Idle } \\
(\mathrm{g} / \mathrm{s})\end{array}$ \\
\hline \multicolumn{14}{|c|}{ WMATA Logs } \\
\hline Anacostia & 60.9 & 7.4 & 14.6 & 49.7 & 1.13 & 3.81 & 2,604 & 12.07 & 824 & 625 & 8.35 & 0.82 & 0.015 \\
\hline Annapolis Rd & 41.1 & 13.5 & 20.4 & 34.1 & 0.95 & 4.96 & 2,001 & 8.30 & 633 & 559 & 6.89 & 0.81 & 0.015 \\
\hline Benning Rd-H & 43.6 & 6.3 & 13.7 & 54.0 & 0.81 & 4.11 & 2,417 & 12.70 & 765 & 516 & 7.99 & 0.81 & 0.015 \\
\hline Benning Rd-H & 62.9 & 7.0 & 13.7 & 48.7 & 0.60 & 4.65 & 2,134 & 11.08 & 675 & 473 & 7.28 & 0.81 & 0.015 \\
\hline Benning-Potom. & 22.5 & 6.6 & 16.6 & 60.0 & 0.77 & 4.03 & 2,465 & 11.94 & 780 & 514 & 6.98 & 0.82 & 0.015 \\
\hline Bowie State & 59.5 & 21.0 & 31.7 & 33.8 & 0.38 & 6.56 & 1,515 & 5.94 & 479 & 432 & 5.06 & 0.82 & 0.015 \\
\hline Bowie State & 116.5 & 25.5 & 35.8 & 28.7 & 0.45 & 5.89 & 1,685 & 5.83 & 533 & 500 & 5.21 & 0.82 & 0.015 \\
\hline Bowie-Belair & 29.3 & 12.8 & 26.3 & 51.3 & 0.74 & 5.11 & 1,943 & 8.01 & 615 & 496 & 5.82 & 0.82 & 0.015 \\
\hline Bowie-Belair & 149.0 & 15.7 & 26.5 & 40.8 & 0.71 & 5.57 & 1,783 & 7.12 & 564 & 487 & 5.69 & 0.82 & 0.015 \\
\hline Capitol Hts & 20.0 & 8.6 & 18.0 & 52.4 & 0.70 & 4.68 & 2,120 & 10.11 & 671 & 490 & 6.76 & 0.82 & 0.015 \\
\hline College Park & 32.0 & 11.2 & 18.7 & 40.4 & 0.78 & 5.26 & 1,888 & 8.62 & 597 & 492 & 6.63 & 0.81 & 0.015 \\
\hline College Park & 89.5 & 12.8 & 20.0 & 36.2 & 0.70 & 5.59 & 1,777 & 7.91 & 562 & 479 & 6.34 & 0.82 & 0.015 \\
\hline College Park & 97.7 & 17.1 & 24.4 & 29.7 & 0.70 & 5.53 & 1,795 & 6.90 & 568 & 517 & 5.94 & 0.82 & 0.015 \\
\hline College Park & 118.7 & 11.6 & 19.8 & 41.3 & 0.85 & 4.90 & 2,028 & 8.71 & 642 & 537 & 6.76 & 0.82 & 0.015 \\
\hline Crofton ... & 59.2 & 25.4 & 37.3 & 31.9 & 0.43 & 6.05 & 1,641 & 5.51 & 519 & 482 & 4.82 & 0.82 & 0.015 \\
\hline Eastover-Addis. & 74.2 & 16.1 & 23.4 & 31.3 & 0.73 & 5.35 & 1,856 & 7.32 & 587 & 530 & 6.24 & 0.81 & 0.015 \\
\hline Eastover-Addis. & 105.8 & 12.8 & 21.5 & 40.5 & 0.96 & 4.73 & 2,101 & 8.52 & 665 & 572 & 6.78 & 0.82 & 0.015 \\
\hline Georgia Ave & 34.3 & 9.2 & 17.2 & 46.4 & 0.63 & 5.35 & 1,855 & 9.40 & 587 & 438 & 6.63 & 0.82 & 0.015 \\
\hline Georgia Ave & 64.0 & 9.1 & 15.8 & 42.6 & 0.69 & 5.27 & 1,883 & 9.57 & 596 & 458 & 6.99 & 0.82 & 0.015 \\
\hline Georgia Ave & 64.4 & 9.3 & 16.6 & 44.2 & 0.71 & 5.18 & 1,915 & 9.57 & 606 & 466 & 6.95 & 0.82 & 0.015 \\
\hline Greenbelt-BWI & 100.3 & 26.6 & 45.5 & 41.5 & 0.36 & 5.55 & 1,788 & 5.54 & 566 & 520 & 4.69 & 0.82 & 0.015 \\
\hline Greenbelt-BWI & 146.5 & 25.1 & 41.9 & 40.1 & 0.39 & 5.59 & 1,776 & 5.60 & 562 & 515 & 4.73 & 0.82 & 0.015 \\
\hline Greenbelt-BWI & 194.6 & 25.4 & 42.2 & 39.8 & 0.32 & 6.12 & 1,624 & 5.39 & 514 & 467 & 4.54 & 0.82 & 0.015 \\
\hline
\end{tabular}

${ }^{\text {i }}$ See Table 2-6 (page 28) and Table 4-6 (page 103) as references for the cycles and routes 


\begin{tabular}{|c|c|c|c|c|c|c|c|c|c|c|c|c|c|}
\hline Line/Cycle Name ${ }^{\mathrm{i}}$ & $\begin{array}{l}\text { Dist. } \\
\text { (mi) }\end{array}$ & $\underset{(\mathbf{m p h})}{\overline{\mathbf{U}}}$ & $\begin{array}{l}\overline{\mathbf{U}}_{\text {no idle }} \\
\text { (mph) }\end{array}$ & $\begin{array}{l}\text { Idle } \\
(\%)\end{array}$ & $\begin{array}{c}\tilde{a}_{\text {no grade }} \\
\left(\mathbf{f t} / \mathbf{s}^{2}\right)\end{array}$ & $\begin{array}{c}\text { F.E. } \\
(\mathbf{m p g})\end{array}$ & $\begin{array}{c}\mathrm{CO}_{2} \\
(\mathrm{~g} / \mathrm{mi})\end{array}$ & $\begin{array}{c}\mathrm{NO}_{\mathrm{x}} \\
(\mathrm{g} / \mathrm{mi})\end{array}$ & $\begin{array}{c}\text { Fuel } \\
(\mathrm{g} / \mathrm{mi})\end{array}$ & $\begin{array}{c}\text { Fuel } \\
\text { Driving } \\
\text { (g/mi) }\end{array}$ & $\begin{array}{c}\mathrm{NO}_{\mathrm{x}} \\
\text { Driving } \\
(\mathrm{g} / \mathrm{mi})\end{array}$ & $\begin{array}{l}\text { Fuel } \\
\text { Idle } \\
(\mathrm{g} / \mathrm{s})\end{array}$ & $\begin{array}{l}\text { NO }_{x} \\
\text { Idle } \\
(\mathrm{g} / \mathrm{s})\end{array}$ \\
\hline Hospital Center & 28.9 & 8.3 & 13.8 & 40.1 & 0.86 & 4.67 & 2,127 & 10.53 & 673 & 531 & 7.87 & 0.81 & 0.015 \\
\hline Marlow Heights & 73.7 & 15.8 & 24.4 & 35.2 & 0.55 & 5.57 & 1,783 & 7.02 & 564 & 498 & 5.79 & 0.82 & 0.015 \\
\hline Marlow Heights & 86.5 & 15.0 & 23.2 & 35.2 & 0.65 & 5.43 & 1,829 & 7.48 & 579 & 509 & 6.19 & 0.82 & 0.015 \\
\hline Marlow Heights & 97.8 & 16.1 & 23.4 & 31.4 & 0.55 & 6.00 & 1,654 & 6.96 & 524 & 466 & 5.89 & 0.82 & 0.015 \\
\hline Martin Luther & 65.1 & 12.5 & 20.0 & 37.6 & 0.85 & 5.27 & 1,884 & 8.12 & 596 & 507 & 6.46 & 0.82 & 0.015 \\
\hline Martin Luther & 115.8 & 12.7 & 20.3 & 37.5 & 0.76 & 5.65 & 1,757 & 7.89 & 556 & 469 & 6.26 & 0.82 & 0.015 \\
\hline Minnesota Ave & 42.2 & 10.7 & 18.3 & 41.7 & 0.80 & 5.06 & 1,961 & 8.96 & 620 & 505 & 6.81 & 0.82 & 0.015 \\
\hline Minnesota Ave & 44.5 & 8.7 & 15.0 & 42.0 & 0.74 & 4.84 & 2,053 & 9.98 & 649 & 508 & 7.32 & 0.82 & 0.015 \\
\hline Minnesota Ave & 77.1 & 8.0 & 17.1 & 53.0 & 0.93 & 4.31 & 2,304 & 10.79 & 729 & 536 & 7.17 & 0.81 & 0.015 \\
\hline North Capitol & 8.4 & 7.7 & 16.2 & 52.1 & 0.55 & 5.00 & 1,984 & 10.22 & 628 & 430 & 6.54 & 0.82 & 0.015 \\
\hline North Capitol & 92.7 & 7.6 & 14.5 & 47.5 & 0.77 & 4.59 & 2,164 & 10.86 & 685 & 504 & 7.43 & 0.81 & 0.015 \\
\hline Prince Georges & 16.1 & 10.2 & 22.6 & 54.7 & 0.87 & 4.56 & 2,179 & 9.23 & 689 & 531 & 6.31 & 0.82 & 0.015 \\
\hline Prince Georges & 96.7 & 12.1 & 20.0 & 39.6 & 0.87 & 4.84 & 2,053 & 8.61 & 650 & 553 & 6.79 & 0.82 & 0.015 \\
\hline Prince Georges & 97.9 & 10.6 & 18.2 & 41.6 & 0.71 & 5.38 & 1,845 & 8.75 & 584 & 468 & 6.59 & 0.82 & 0.015 \\
\hline Rhode Island & 70.6 & 13.6 & 23.2 & 41.3 & 0.88 & 4.59 & 2,163 & 8.42 & 685 & 580 & 6.54 & 0.95 & 0.017 \\
\hline Rhode Island & 88.8 & 9.4 & 18.7 & 50.0 & 0.88 & 4.76 & 2,085 & 9.62 & 660 & 502 & 6.68 & 0.82 & 0.015 \\
\hline \multicolumn{14}{|c|}{ Other Logs and Cycles } \\
\hline BEELINE & 6.7 & 14.2 & 19.9 & 28.5 & 0.86 & 5.14 & 1,931 & 7.85 & 611 & 556 & 6.76 & 0.76 & 0.015 \\
\hline Braunschweig & 6.7 & 13.9 & 18.9 & 26.2 & 0.71 & 5.93 & 1,673 & 7.72 & 530 & 476 & 6.66 & 0.79 & 0.016 \\
\hline Houston Bus & 5.5 & 11.0 & 18.3 & 39.8 & 0.56 & 5.85 & 1,696 & 8.19 & 537 & 433 & 6.20 & 0.80 & 0.015 \\
\hline Houston Logs 1 & 44.8 & 12.3 & 19.7 & 37.5 & 0.65 & 5.33 & 1,862 & 8.02 & 589 & 500 & 6.34 & 0.81 & 0.015 \\
\hline Houston Logs 2 & 117.0 & 13.0 & 19.3 & 32.5 & 0.61 & 5.99 & 1,659 & 7.55 & 525 & 452 & 6.17 & 0.81 & 0.015 \\
\hline Houston Logs 3 & 133.7 & 11.9 & 18.4 & 35.5 & 0.73 & 5.35 & 1,855 & 8.23 & 587 & 500 & 6.57 & 0.81 & 0.015 \\
\hline $\mathrm{KCM}$ & 12.7 & 23.3 & 28.6 & 18.4 & 0.52 & 6.41 & 1,548 & 6.30 & 490 & 467 & 5.86 & 0.81 & 0.016 \\
\hline KCM 2 & 2.5 & 19.5 & 23.6 & 17.4 & 0.84 & 5.59 & 1,777 & 7.26 & 562 & 536 & 6.75 & 0.81 & 0.016 \\
\hline KCM 3 & 3.3 & 16.2 & 21.0 & 22.6 & 0.81 & 5.48 & 1,811 & 7.85 & 573 & 533 & 7.07 & 0.81 & 0.016 \\
\hline KCM 4 & 1.2 & 15.0 & 19.8 & 24.5 & 0.70 & 6.12 & 1,623 & 7.17 & 514 & 466 & 6.26 & 0.82 & 0.016 \\
\hline Liberty & 6.2 & 11.9 & 18.6 & 35.7 & 0.80 & 5.14 & 1,933 & 8.48 & 612 & 529 & 6.87 & 0.77 & 0.015 \\
\hline Manhattan & 2.1 & 6.8 & 11.0 & 37.8 & 0.91 & 4.19 & 2,368 & 11.79 & 749 & 591 & 8.69 & 0.79 & 0.016 \\
\hline MCS & 9.2 & 11.1 & 16.4 & 32.5 & 0.72 & 5.14 & 1,934 & 8.57 & 612 & 526 & 6.95 & 0.81 & 0.015 \\
\hline MiamiD & 2.6 & 6.6 & 14.2 & 53.9 & 1.13 & 3.68 & 2,695 & 12.42 & 853 & 611 & 7.91 & 0.82 & 0.015 \\
\hline MX1 & 2.0 & 7.1 & 9.5 & 25.4 & 0.64 & 4.60 & 2,159 & 11.12 & 683 & 578 & 9.11 & 0.81 & 0.016 \\
\hline MX2 & 3.5 & 12.7 & 18.0 & 29.1 & 0.54 & 6.04 & 1,643 & 7.65 & 520 & 452 & 6.39 & 0.82 & 0.015 \\
\hline MX3 & 3.7 & 13.4 & 23.4 & 42.9 & 0.93 & 4.75 & 2,092 & 8.11 & 662 & 568 & 6.35 & 0.81 & 0.015 \\
\hline New York Bus & 0.6 & 3.7 & 10.9 & 66.3 & 1.18 & 2.50 & 3,964 & 19.19 & 1,254 & 722 & 9.20 & 0.81 & 0.015 \\
\hline New York Comp. & 2.5 & 8.7 & 13.2 & 34.5 & 0.70 & 4.82 & 2,059 & 10.32 & 651 & 533 & 8.10 & 0.82 & 0.016 \\
\hline Nuremberg & 2.7 & 8.8 & 12.9 & 31.2 & 0.79 & 4.92 & 2,017 & 10.16 & 638 & 536 & 8.19 & 0.80 & 0.016 \\
\hline OCTA & 6.5 & 12.3 & 16.3 & 24.5 & 0.71 & 5.81 & 1,709 & 7.96 & 541 & 488 & 6.86 & 0.74 & 0.015 \\
\hline Paris & 3.5 & 6.6 & 10.3 & 35.9 & 0.81 & 4.17 & 2,382 & 12.70 & 754 & 600 & 9.68 & 0.79 & 0.015 \\
\hline UDDS & 5.5 & 18.7 & 28.5 & 34.4 & 0.43 & 6.04 & 1,644 & 6.38 & 520 & 467 & 5.37 & 0.80 & 0.015 \\
\hline WMATA & 4.2 & 8.3 & 14.1 & 41.1 & 0.77 & 4.66 & 2,132 & 10.35 & 675 & 535 & 7.64 & 0.79 & 0.015 \\
\hline $\begin{array}{l}\text { WMATA } \\
\text { Commuter }\end{array}$ & 10.1 & 26.0 & 41.0 & 36.5 & 0.37 & 5.96 & 1,665 & 5.57 & 527 & 485 & 4.81 & 0.82 & 0.015 \\
\hline
\end{tabular}


The following figures provide a graphical representation of the results. Figure 7-1, Figure 7-3, and Figure 7-4 present fuel economy, $\mathrm{CO}_{2}$ emissions, and $\mathrm{NO}_{\mathrm{x}}$ emissions, respectively, plotted against average speed. The values from chassis-dynamometer tests of the series hybrid bus are also shown (Table 5-4). Recall that the test entries were used to develop and correlate the vehicle dynamic model; test data served as an independent check against validity of the results from both simulation and regression analysis.

Figure 7-2 compares fuel economy from the series hybrid (simulation and test) against chassis tests of a MY 2006 2-mode hybrid bus (CAFEE, 2007). It is observed that the simulations are attune with tests of the 2-mode bus and a closer look reveals that, for the same cycles, the series bus out performs the 2 -mode by up to $10 \%$.

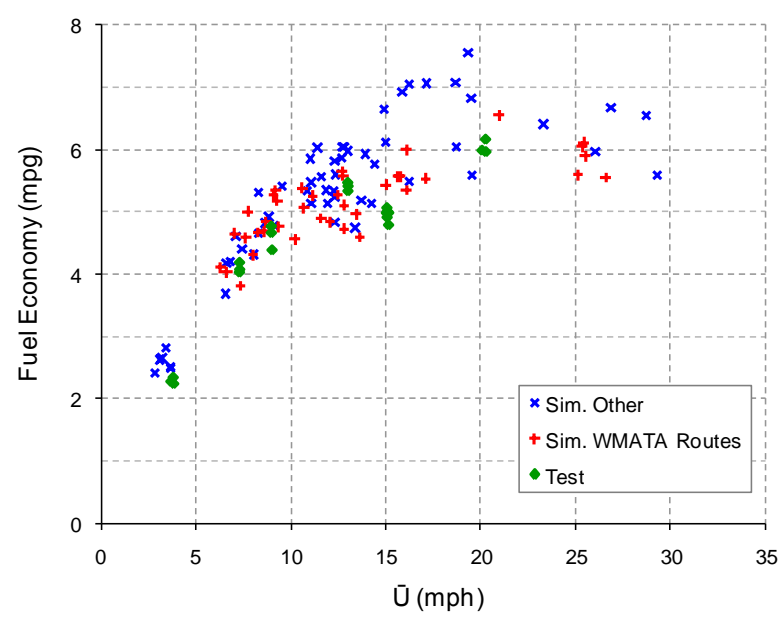

Figure 7-1 Fuel Economy vs. Average Speed

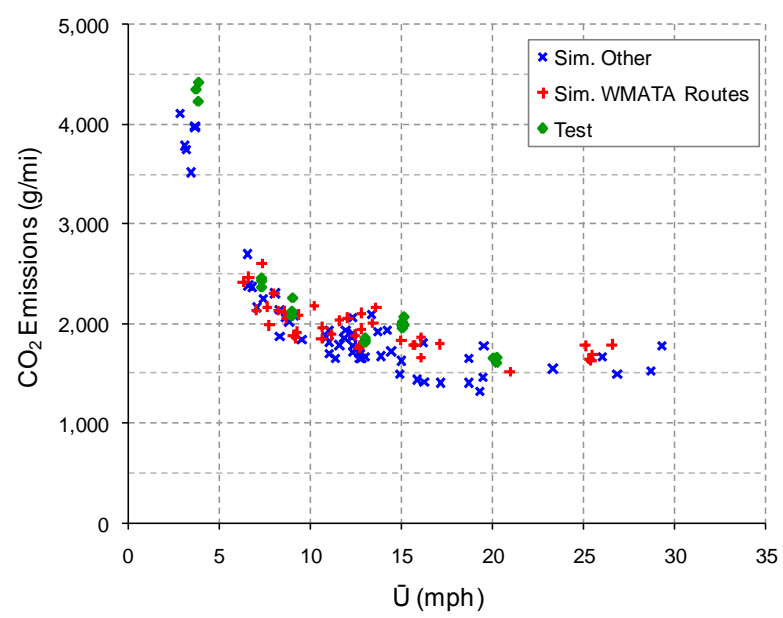

Figure 7-3 $\mathrm{CO}_{2}$ Emissions vs. Average Speed

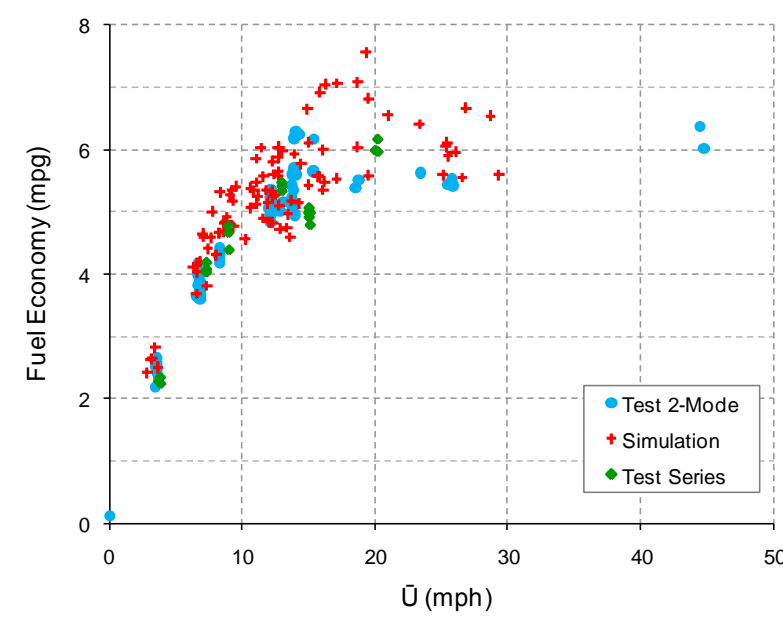

Figure 7-2 Fuel Economy from Test and Simulation

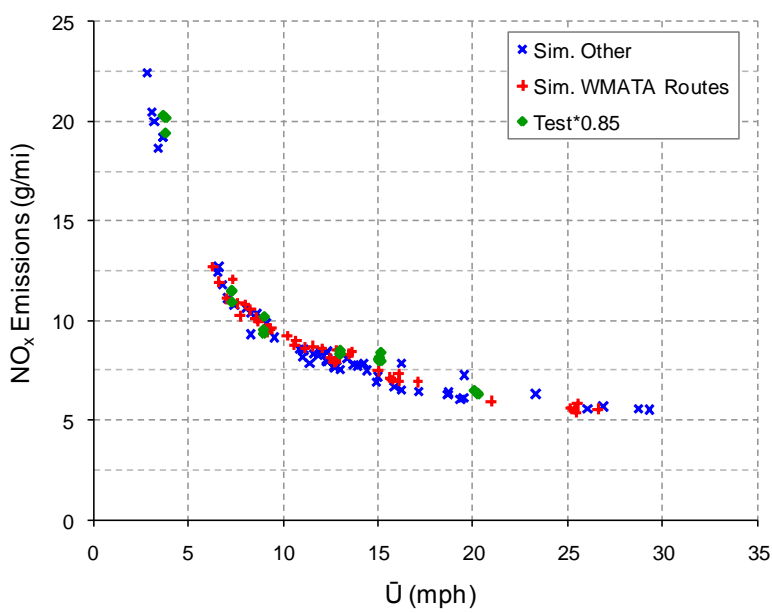

Figure 7-4 $\mathrm{NO}_{\mathrm{x}}$ Emissions vs. Average Speed 


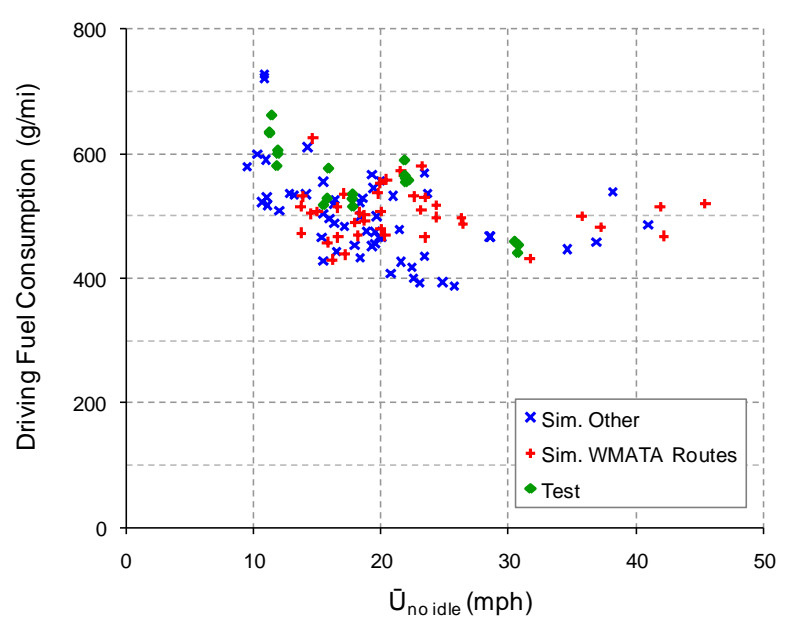

Figure 7-5 Driving Fuel Consumption

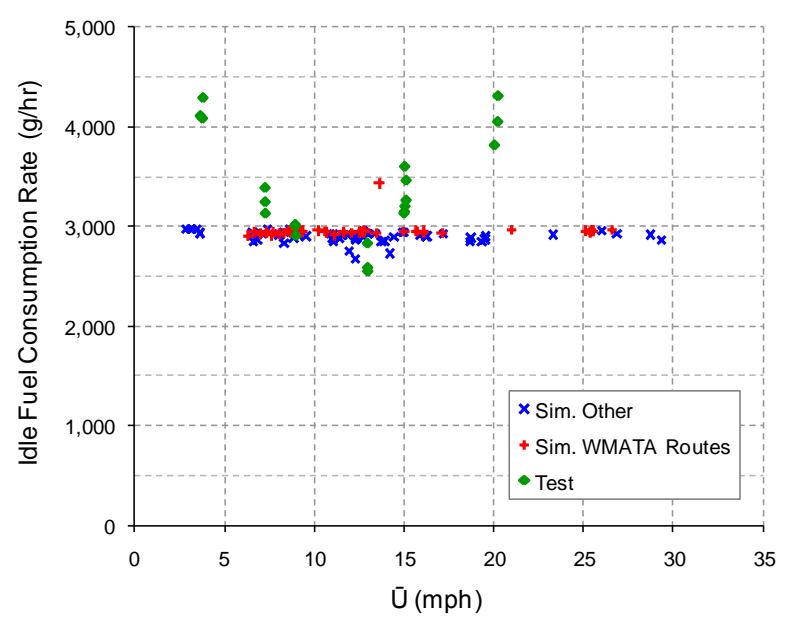

Figure 7-7 Idle Fuel Consumption Rate

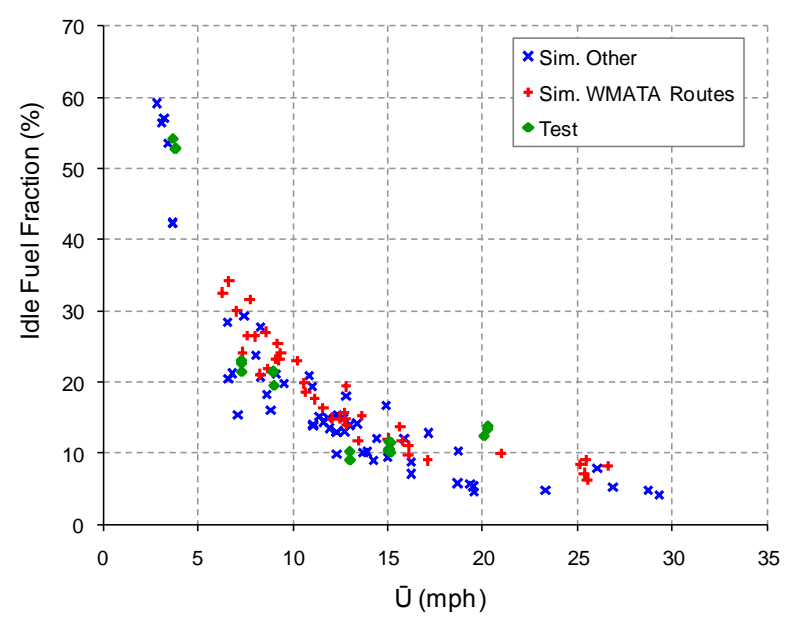

Figure 7-9 Idle Fuel Fraction

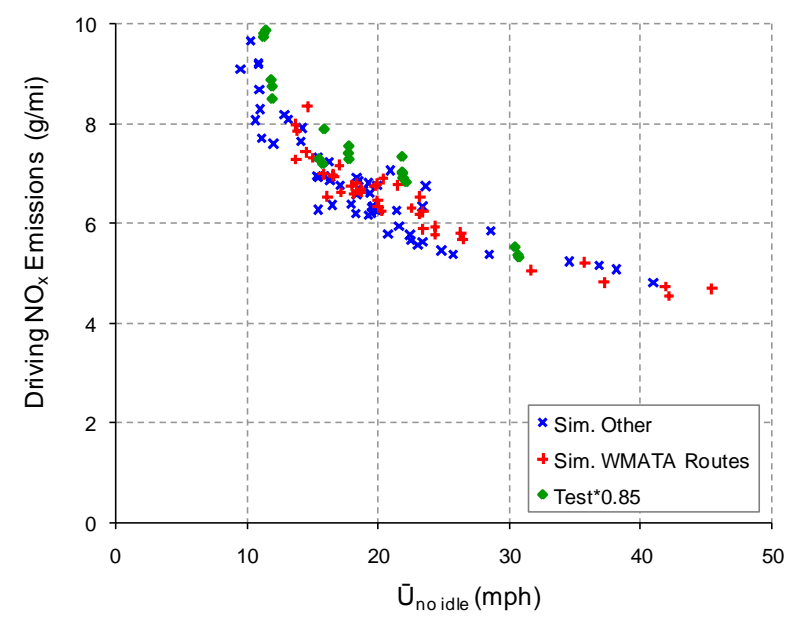

Figure 7-6 Driving $\mathrm{NO}_{\mathrm{x}}$ Emissions

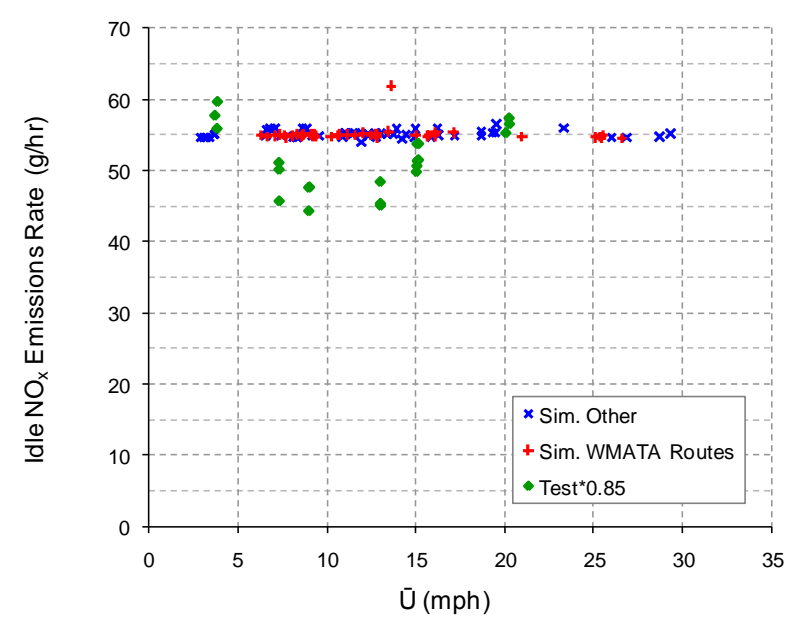

Figure 7-8 Idle $\mathrm{NO}_{\mathrm{x}}$ Emissions Rate

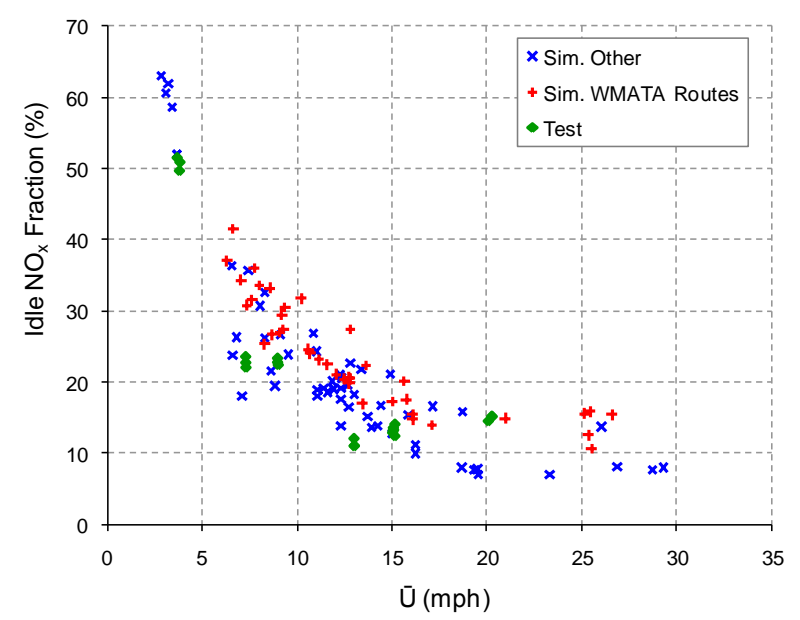

Figure 7-10 Idle $\mathrm{NO}_{\mathrm{x}}$ Fraction 
The contributions of the driving segments to fuel consumption and $\mathrm{NO}_{\mathrm{x}}$ emissions are shown in Figure 7-5 and Figure 7-6, respectively. The figures show $\bar{U}_{\text {no idle }}$ in the abscissa, but, as will be shown below, $\tilde{a}_{\text {no grade }}$ is also an explanatory factor.

Figure 7-7 and Figure 7-8 present idle fuel and idle $\mathrm{NO}_{\mathrm{x}}$ rates, respectively. It is observed that there is no correlation between these rates and average speed. The fixedness of these rates is an artifact of the computer model with constant accessory loads, whilst the test data show a higher variation. For comparison with simulation, test $\mathrm{NO}_{\mathrm{x}}$ emissions have been scaled to $85 \%$ to account for the certification limit correction (Section 5.7).

Another interesting result, presented in Figure 7-9, is the fraction of fuel consumed during idle segments. It can be as high as 50\% in the New York Cycle and for most routes is between 20 and $30 \%$. Similarly, the fraction of $\mathrm{NO}_{\mathrm{x}}$ produced during idle is presented in Figure 7-10. As the fuel idle fraction, the $\mathrm{NO}_{\mathrm{x}}$ fraction can make up a significant amount of the total $\mathrm{NO}_{\mathrm{x}}$ emissions.

\subsection{Prediction Models for Fuel Consumption and Emissions}

A method was developed for the emissions predictive models in which emissions and fuel consumption were decomposed in their driving and idle components. Idle and driving components were predicted and then aggregated to present the overall emissions, fuel consumption, and fuel economy. The idea of decomposing the various components of fuel consumption was borrowed from Sovran and Blaser (2003), who represented fuel consumption as three contributions: driving, braking, and idle (Equation 2.16).

\subsubsection{Fundamentals}

Consider one of the duty cycles presented in Table 7-1. By decomposition of the continuous data, the total amount of fuel consumed $\left(m_{f u e l}\right)$ can be broken up into the driving and idle components, $m_{\text {Driving }}$ and $m_{\text {Idle }}$ (in grams). An appropriate vehicle speed threshold can be selected to contrast idle from driving.

Cycle fuel consumption (F.C.) is usually presented as mass or volume of fuel consumed per unit distance travelled, it can be calculated in a mass basis as:

Equation 7.1 Cycle Fuel Consumption

$$
F . C .=\frac{m_{\text {Driving }}+m_{\text {Idle }}}{D}
$$


where cycle distance $(D)$ is the integral of vehicle speed. Equation $7.1(a)$ can be manipulated to express it in terms of driving fuel consumption, F.C. Driving, and idle fuel consumption rate, $\overline{\dot{m}}_{f \text { Idle }}$, in Figure 7-5 and Figure 7-7. Defining the driving and idle components of cycle fuel consumption as F.C. Driving $=m_{\text {Driving }} / D$ and F.C.Idle $=m_{\text {Idle }} / D$, Equation $7.1(\mathrm{a})$ is written as:

Equation 7.1

$$
F . C .=F . C_{\cdot_{\text {Driving }}}+F \cdot C_{\cdot_{\text {Idle }}} \cdot
$$

The idle component of fuel consumption can be rearranged in terms of percentage idle (Idle), average speed $(\bar{U})$, and the rate of idle fuel consumption $\overline{\dot{m}}_{f \text { Idle }}$ as follows:

$$
F . C ._{\text {Idle }}=\frac{m_{\text {Idle }}}{t_{\text {Idle }}} \cdot \frac{t_{\text {Idle }}}{T} \cdot \frac{T}{D} .
$$

The first term on the RHS is $\overline{\dot{m}}_{f \text { Idle }}$, the second term is the idle fraction (Idle / 100) and the last one is the inverse of $\bar{U}$. Substituting we obtain:

Equation 7.2 Idle Component of Fuel Consumption

$$
F . C ._{\text {Idle }}=\overline{\dot{m}}_{f \text { Idle }} \cdot \frac{\text { Idle } / 100}{\overline{\mathrm{U}}} .
$$

F.C.Idle has units of $\mathrm{g} / \mathrm{mi}$ if $\overline{\dot{m}}_{f \text { Idle }}$ is in $\mathrm{g} / \mathrm{hr}$ and $\bar{U}$ in $\mathrm{mph}$.

Equivalent expressions can be developed for cycle $\mathrm{NO}_{\mathrm{x}}$ emissions $(\mathrm{g} / \mathrm{mi})$ by replacing mass of fuel consumed by mass of $\mathrm{NO}_{\mathrm{x}}$ emissions:

Equation 7.3 Cycle $\mathrm{NO}_{\mathrm{x}}$ Emissions

$$
\begin{gathered}
N O_{x}=N O_{x \text { Driving }}+N O_{x \text { Idle }} \\
N O_{x \text { Idle }}=\overline{\dot{m}}_{N O_{x} \text { Idle }} \cdot \frac{\text { Idle } / 100}{\overline{\mathrm{U}}}
\end{gathered}
$$

Fuel economy (mpg) is calculated from F.C. and fuel density. $\mathrm{CO}_{2}$ emissions (g/mi) are calculated by carbon fuel balance (neglecting the $\mathrm{HC}$ and $\mathrm{CO}$ contributions). We have:

Equation 7.4 Fuel Economy and $\mathrm{CO}_{2}$ Emissions

$$
\begin{gathered}
\text { F.E. }=\rho_{\text {fuel }} / F . C . \\
C O_{2}=\text { F.C. } \cdot \text { Fuel }_{\text {Carbon }} \cdot 44 / 12
\end{gathered}
$$

Fuel density (in g/gal) and carbon content are taken from Table 5-3. Estimation of $\mathrm{CO}_{2}$ emissions from F.C. takes out the need for a $\mathrm{CO}_{2}$ regression.

Figure 7-11 depicts the flowchart of the emissions and fuel economy calculation. First the three cycle metrics $\left(\bar{U}, I d l e\right.$, and $\left.\tilde{a}_{n o \text { grade }}\right)$ are entered and validated against the model's range. Then, $\bar{U}_{\text {no idle }}$ 
is calculated and the regressions for F.C.Driving and $N O_{x \text { Driving }}$ are applied. Simultaneously, F.C.Idle and $N O_{x}$ Idle are calculated. Subsequently, the driving and idle components are aggregated to compute F.C. and $\mathrm{NO}_{x}$. Finally, F.E. and $\mathrm{CO}_{2}$ are calculated and the results displayed.

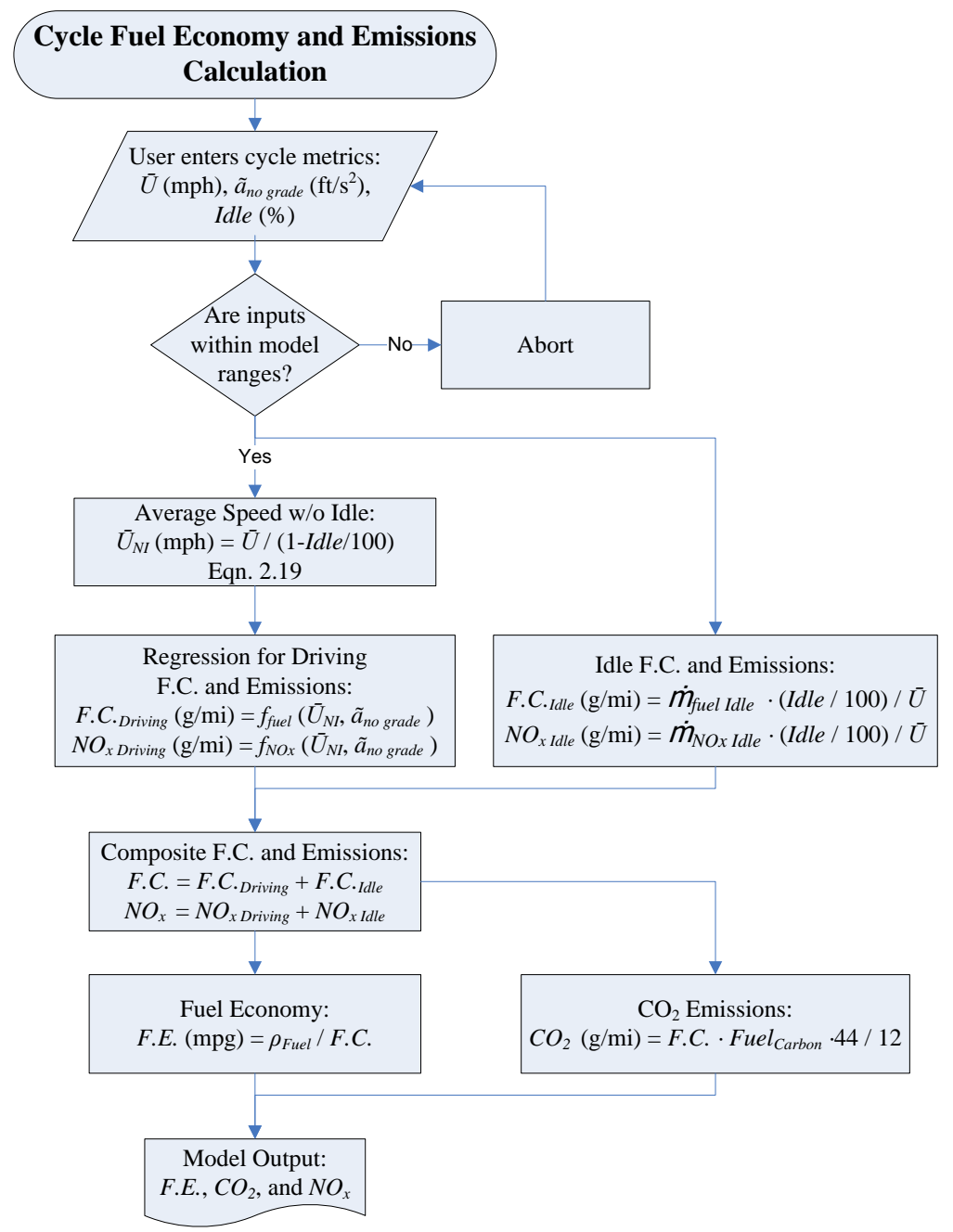

Figure 7-11 Flowchart for Cycle Fuel Economy and Emissions Calculation

\subsubsection{Regressions}

Nonlinear least squares multiple regressions for driving fuel consumption and $\mathrm{NO}_{\mathrm{x}}$ were computed with $\mathrm{JMP}^{\circledR}$ and MATLAB ${ }^{\circledR}$. In order to assure a low generalization error, a $k$-fold cross-validation study was performed to determine an adequate ratio for the training/validation datasets (Duda et al., 2000). $k$ is the number of random partitions to the input data; e.g. $k=4$ makes four partitions each containing $25 \%$ of the data. One partition is used for validation and the remaining $k-1$ partitions are used for training. The model is trained $k$ times until all $k$ partitions are used for validation. 
Performance is evaluated under the validation sets and the mean of the $k$ errors is reported. The selected performance measure was the root mean squared error (RMSE), an estimate of the average residual.

Figure 7-12 presents the results (for 100 repetitions) where $50 \%(k=4)$ to $95 \%(k=20)$ of the data were used for training. The fact that the error does not decrease indefinitely but is rather bounded in the lower limit tells us that there is not a substantial amount of over fitting. This is due to the model having only 6 parameters to be adjusted. It can also be concluded that the models possess good generalization capabilities. The standard value of $k=10$ (Duda et al., 2000) was used to develop the final models.

The regression equations were a fourth order polynomial on $\bar{U}_{N I}(\mathrm{mph})$ with a linear term on $\tilde{a}_{\text {no_grade }}\left(\mathrm{ft} / \mathrm{s}^{2}\right)$. Table 7-2 and Table 7-3 present the JMP ${ }^{\circledR}$ regression summaries for F.C.Driving and $N O_{x \_ \text {Driving }}$ including ANOVA and parameter estimates (see Section 2.6).

Target versus predicted plots for F.C. Driving and $N O_{x}$ Driving are shown in Figure 7-13 and Figure $7-14$, respectively. The coefficients of determination were 0.88 for fuel consumption and 0.97 for $\mathrm{NO}_{\mathrm{x}}$. The figures show predicted values under three datasets: training, validation, and chassisdynamometer tests. In these parity plots (predicted versus target), the continuous line represents the ideal model where predicted = target: points above the line are over predicted and points below the line are under predicted. The dashed curves represent the $95 \%$ confidence bounds on the mean response.

Three-dimensional contour plots for fuel and $\mathrm{NO}_{\mathrm{x}}$ regressions are presented in Figure 7-15 and Figure 7-16, respectively, including the training points. The distributions of idle fuel consumption (Figure 7-7) and idle $\mathrm{NO}_{\mathrm{x}}$ emissions (Figure 7-8) were evaluated, obtaining the means, standard errors, and confidence intervals on the means; parameters shown in Table 7-4. From the table, $\overline{\dot{m}}_{f \text { Idle }}=2,926$ $\mathrm{g} / \mathrm{hr}$ and $\overline{\dot{m}}_{N O_{x} \text { Idle }}=55.1 \mathrm{~g} / \mathrm{hr}$.

As mentioned above, the test points serve as an additional validation check of the results as they were used in developing the vehicle dynamic model but had no other direct influence on the construction of the regression models. As could be expected, predictions for the test points were not as good as for the simulation points. It must be noted that the deviations were not excessively significant and were for the most part of the same order of magnitude than for the simulation points. 


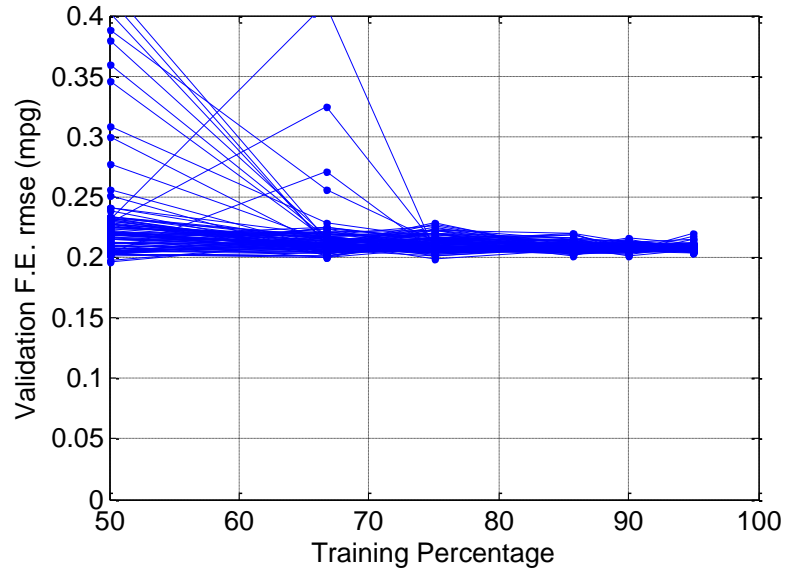

(a) Fuel Economy

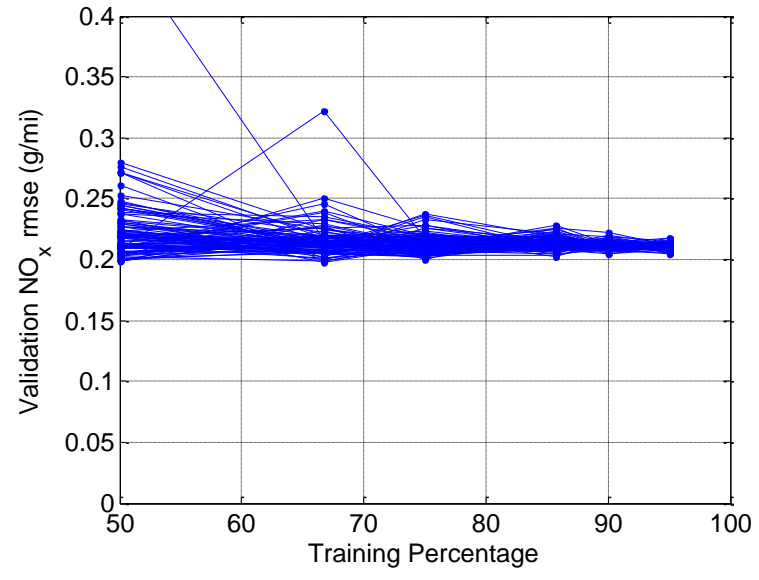

(b) $\mathrm{NO}_{\mathrm{x}}$

Figure 7-12 Cross-Validation RMSE for F.E. and $\mathrm{NO}_{\mathrm{x}}$ Predictions

Table 7-2 Regression Summary for Driving Fuel Consumption (g/mi)

\begin{tabular}{|c|c|c|c|c|}
\hline \multicolumn{5}{|c|}{ Analysis of Variance } \\
\hline Source & DF & Sum of Squares & Mean Square & F Ratio \\
\hline Model & 5 & $2.24 \mathrm{E}+05$ & $4.47 \mathrm{E}+04$ & 112 \\
\hline Error & 79 & $3.16 \mathrm{E}+04$ & 399 & Prob > F \\
\hline C. Total & 84 & $2.55 \mathrm{E}+05$ & & $<.0001$ \\
\hline \multicolumn{5}{|c|}{ Parameter Estimates } \\
\hline Term & Estimate & Std Error & t Statistic & P-value \\
\hline Intercept & 211 & 23 & 9.0 & $<.0001$ \\
\hline $\bar{U}_{N I}$ & 2.79 & 0.89 & 3.1 & 0.0024 \\
\hline$\left(\bar{U}_{N I}-20.3\right)^{2}$ & 0.649 & 0.065 & 10.0 & $<.0001$ \\
\hline$\left(\bar{U}_{N I}-20.3\right)^{3}$ & $-4.63 \mathrm{E}-02$ & $8.67 \mathrm{E}-03$ & -5.3 & $<.0001$ \\
\hline$\left(\bar{U}_{N I}-20.3\right)^{4}$ & $1.00 \mathrm{E}-03$ & $3.23 \mathrm{E}-04$ & 3.1 & 0.0026 \\
\hline$\tilde{a}_{\text {no grade }}$ & 304 & 15 & 20.6 & $<.0001$ \\
\hline
\end{tabular}

Table 7-3 Regression Summary for Driving $\mathrm{NO}_{\mathrm{x}}$ Emissions (g/mi)

\begin{tabular}{l|r|r|r|r}
\hline \multicolumn{5}{|c}{ Analysis of Variance } \\
\hline Source & \multicolumn{1}{|c|}{ DF } & Sum of Squares & Mean Square & F Ratio \\
\hline Model & 5 & 76.9 & 15.3768 & 602 \\
\hline Error & 79 & 2.0 & 0.0255 & Prob > F \\
\hline C. Total & 84 & 78.9 & & $<.0001$ \\
\hline \multicolumn{5}{|c}{ Parameter Estimates } \\
\hline Term & Estimate & Std Error & t Statistic & P-value \\
\hline Intercept & 6.86 & 0.19 & 36.8 & $<.0001$ \\
\hline $\bar{U}_{N I}$ & -0.0960 & 0.0071 & -13.5 & $<.0001$ \\
\hline$\left(\bar{U}_{N I}-20.72\right)^{2}$ & $8.85 \mathrm{E}-03$ & $5.19 \mathrm{E}-04$ & 17.0 & $<.0001$ \\
\hline$\left(\bar{U}_{N I}-20.72\right)^{3}$ & $-4.39 \mathrm{E}-04$ & $6.93 \mathrm{E}-05$ & -6.3 & $<.0001$ \\
\hline$\left(\bar{U}_{N I}-20.72\right)^{4}$ & $6.86 \mathrm{E}-06$ & $2.58 \mathrm{E}-06$ & 2.7 & 0.0095 \\
\hline$\tilde{a}_{n o \text { grade }}$ & 2.01 & 0.12 & 17.0 & $<.0001$ \\
\hline
\end{tabular}




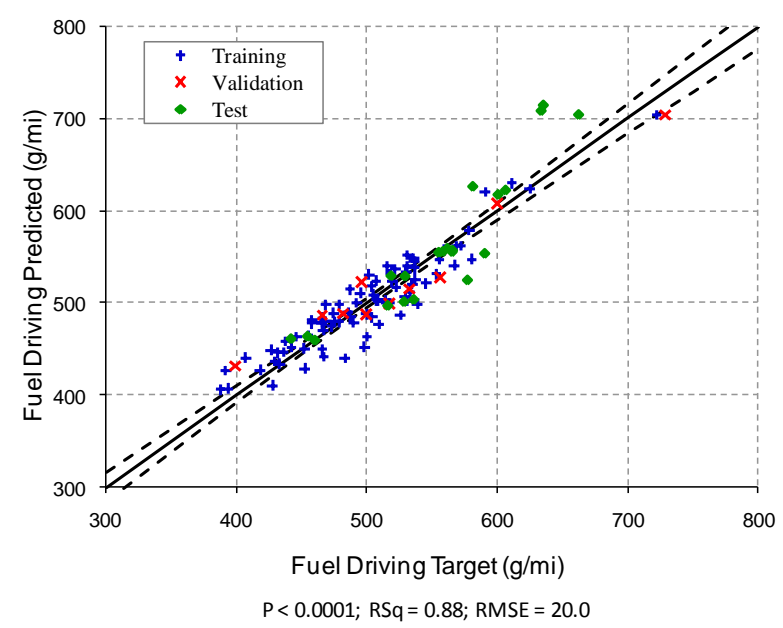

Figure 7-13 F.E.Driving Predicted versus Target Plot

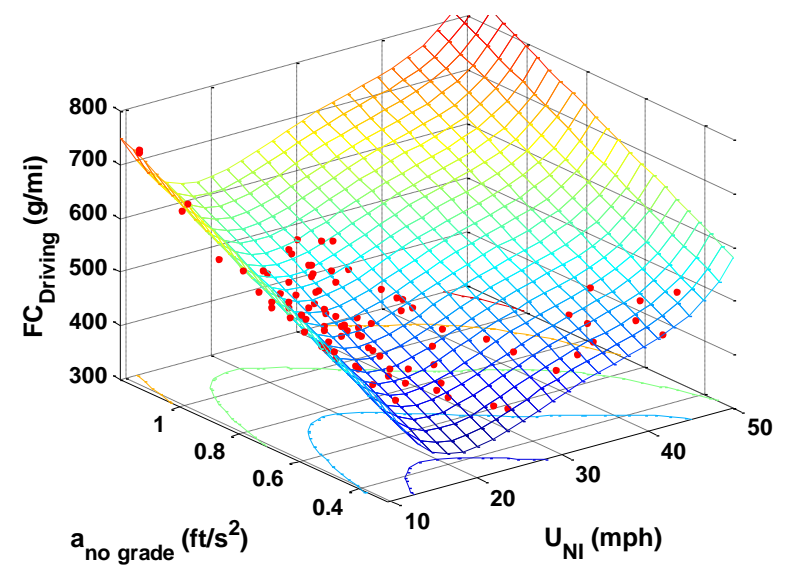

Figure 7-15 F.E.Driving Three-Dimensional Contour Plot

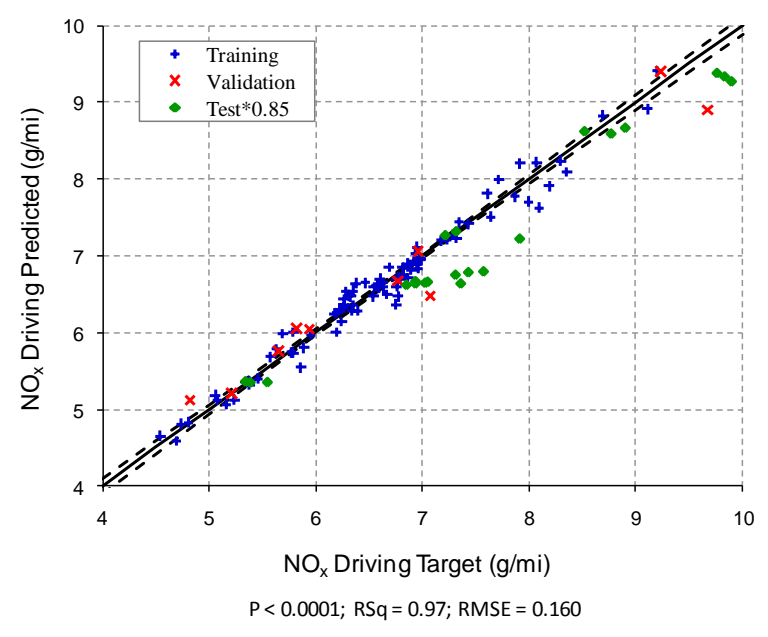

Figure 7-14 NO $\mathrm{O}_{x \text { Driving }}$ Predicted versus Target Plot

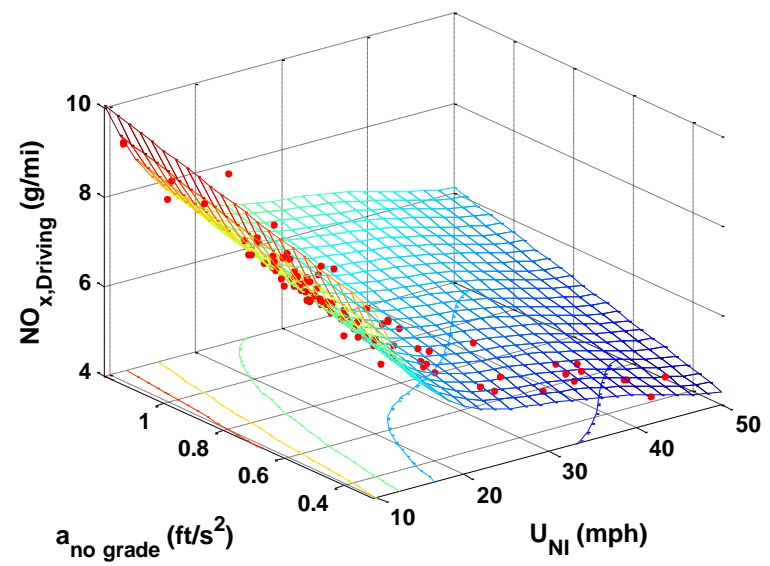

Figure 7-16 $N O_{x \text { Driving }}$ Three-Dimensional Contour Plot

Table 7-4 Distribution Summary for Idle Fuel Consumption and Idle $\mathrm{NO}_{\mathrm{x}}$ Emissions (g/hr)

\begin{tabular}{|c|c|c|}
\hline Parameter & Fuel & $\mathbf{N O}_{\mathrm{x}}$ \\
\hline Mean & 2,926 & 55.1 \\
\hline Std Dev & 73.8 & 0.84 \\
\hline Std Error Mean & 8.0 & 0.09 \\
\hline $\mathrm{t}^{*}(95 \%)$ & \multicolumn{2}{|c|}{1.989} \\
\hline $\mathrm{N}$ & \multicolumn{2}{|c|}{85} \\
\hline Lower 95\% Mean & 2,910 & 55.0 \\
\hline Upper 95\% Mean & 2,942 & 55.3 \\
\hline
\end{tabular}




\subsubsection{Results}

The methods described above were implemented to predict fuel economy, $\mathrm{CO}_{2}$ and $\mathrm{NO}_{\mathrm{x}}$. Model inputs were $\bar{U}$, Idle, and $\tilde{a}_{n o}$ grade (Figure 7-11). The following figures show the predictions compared to the simulation values and serve to assess the goodness of the model. Predictions are also compared with the chassis-dynamometer test data.

Figure 7-17 shows predicted and simulation fuel economy as a function of average speed; it is observed that the predictions are reasonably close to the simulation values. The relationship between simulation and predicted fuel economy is shown in Figure 7-18. In this parity plot, as well in the following ones, the predictions are shown for the training set, the validation set, and the test data; the dashed curves represent the $95 \%$ confidence bounds on the mean response (Equation 2.45). A high F.E. coefficient of determination (0.96) was obtained, as well as a slope (linear fit) close to unity (0.94).

When compared to the test data, fuel economy predictions are high for low speed operation (lower left corner of the figure which corresponds to the New York Cycle), having as much as 15\% error. For all other duty cycles, the predictions agree with the test data. Overall, it can be concluded that the F.E. prediction approach produces adequate predictions. Other thing to note is the extent of the model regarding vehicle operation: $\bar{U}_{N I}$ ranging from $8 \mathrm{mph}$ to $50 \mathrm{mph}$, and $\tilde{a}_{\text {no grade }}$ ranging from $0.3 \mathrm{ft} / \mathrm{s}^{2}$ and $1.2 \mathrm{ft} / \mathrm{s}^{2}$. The model is able to cope with any amount of idle as long as $\bar{U}_{N I}$ and $\tilde{a}_{\text {no grade }}$ are within range (see Section 7.2.5 for more information).

$\mathrm{CO}_{2}$ predictions are presented is Figure 7-19 and Figure 7-20. The model shows a good match with the simulation values: $\mathrm{R}^{2}$ is 0.99 and the paired slope is 0.97 . Predicted $\mathrm{NO}_{\mathrm{x}}$ emissions are shown in Figure 7-21 and Figure 7-22. The narrow spread of $\mathrm{NO}_{\mathrm{x}}$ for a given $\bar{U}$ suggests that fuel economy is more sensitive to cycle properties (idle and characteristic acceleration) than $\mathrm{NO}_{\mathrm{x}}$. The coefficient of determination was again high (0.998) and the slope close to unity (1.01).

Figure 7-23 shows a comparison of the predicted fuel economy with the current IBIS model for a MY 2005 two-mode diesel-electric hybrid bus. The inputs used in IBIS were average speed, percentage idle, standard deviation of speed, stops per mile, and kinetic intensity. As the figure illustrates, IBIS predictions are located over a smooth surface with a narrow band. IBIS predictions may be improved if the methodology proposed here were implemented into the various models. This statement is validated in Section 7.4 below where a model is developed for a conventional diesel transit bus using the same dataset that was used to produce the equivalent IBIS model. 


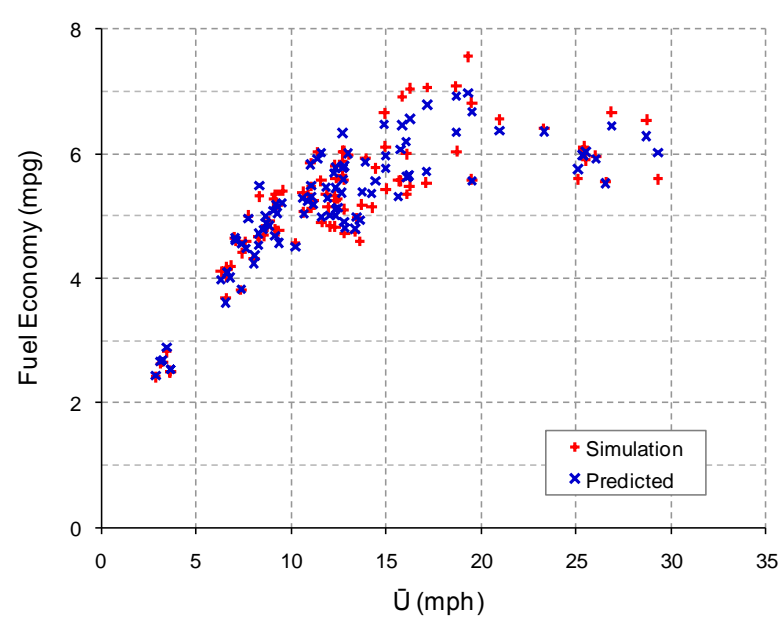

Figure 7-17 Predicted Fuel Economy

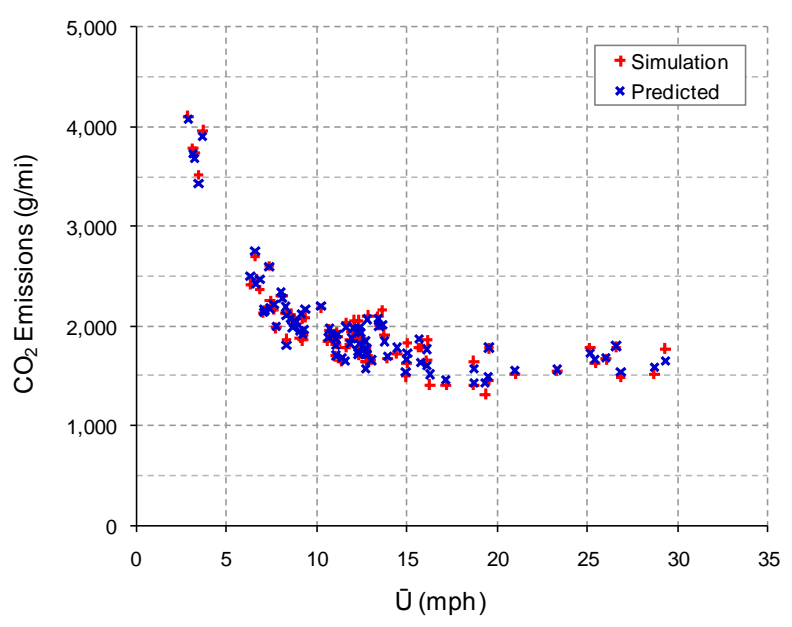

Figure 7-19 Predicted $\mathrm{CO}_{2}$ Emissions

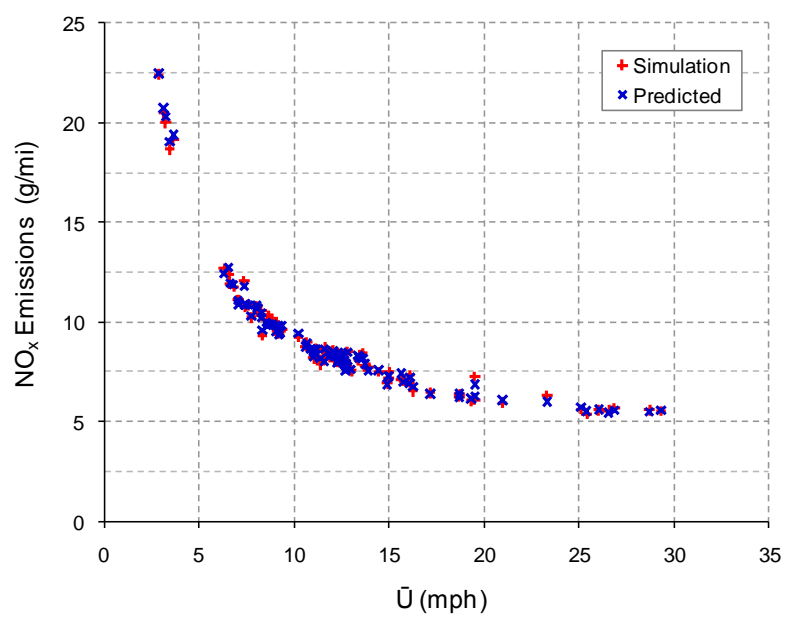

Figure 7-21 Predicted $\mathrm{NO}_{\mathrm{x}}$ Emissions

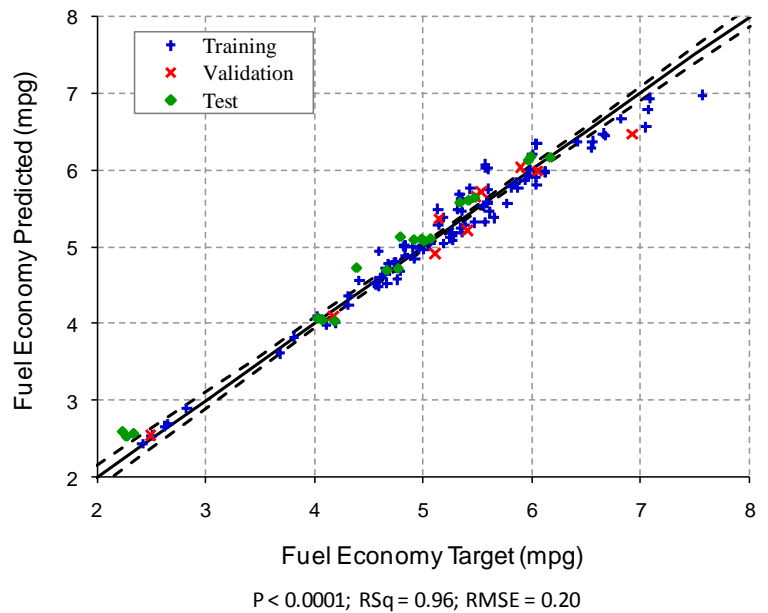

Figure 7-18 Fuel Economy Predicted versus Simulation

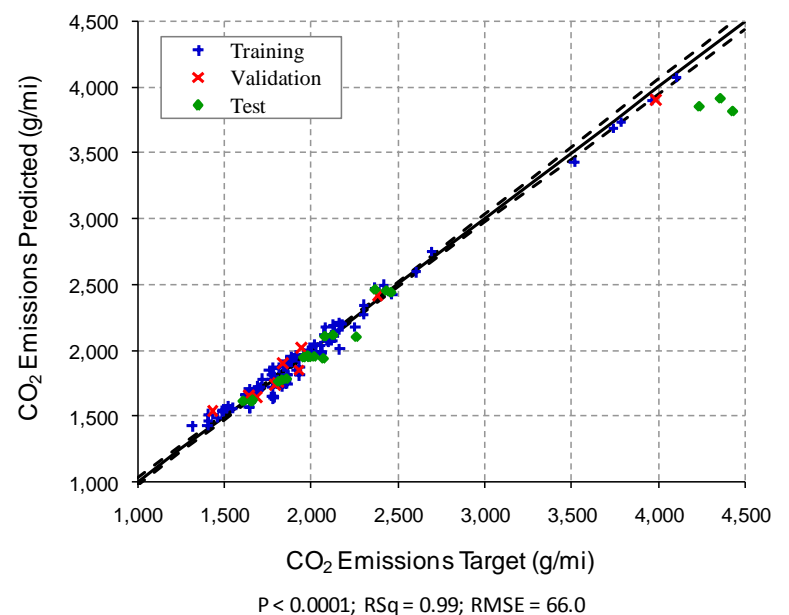

Figure 7-20 $\mathrm{CO}_{2}$ Emissions Predicted versus Simulation

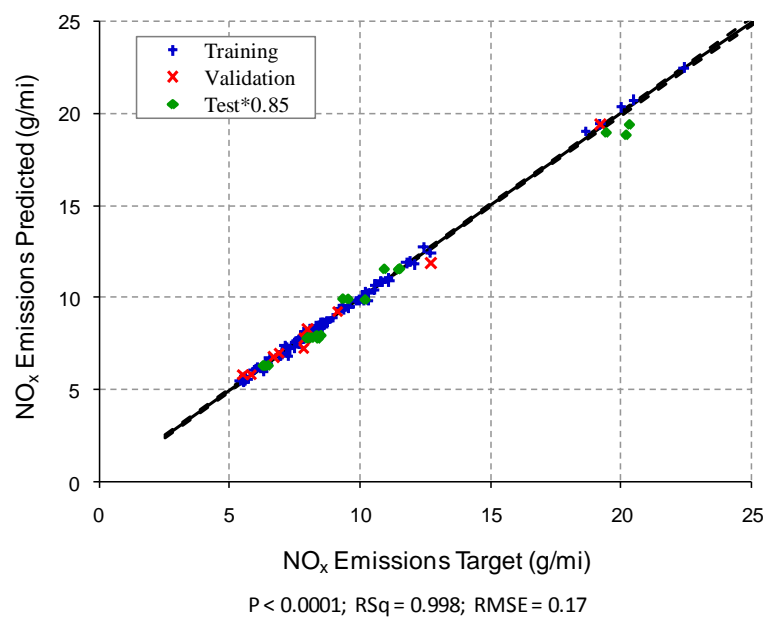

Figure 7-22 NO $_{\mathrm{x}}$ Emissions Predicted versus Simulation 


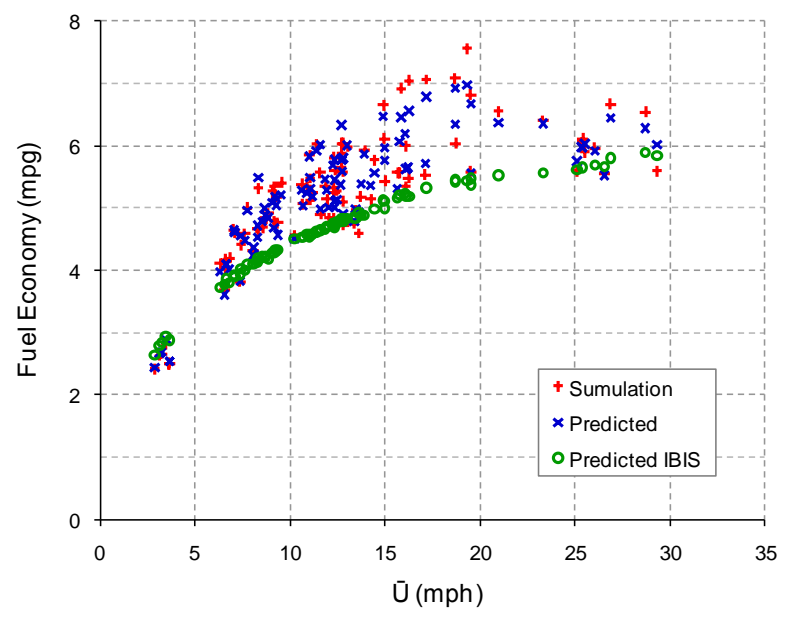

Figure 7-23 Comparison of Predictions with IBIS Model

\subsubsection{Considerations for Limited User Inputs}

It was considered that in some cases the users of the model would not have knowledge of all three required inputs. Average speed is a required input, but regressions can be developed to estimate Idle and $\tilde{a}_{n o}$ grade. Figure 7-24 presents the regression curve for Idle as a function of $\bar{U}$ and Figure 7-25 presents the regression curve for $\tilde{a}_{n o}$ grade as a function of $\bar{U}_{N I}$. The correlations are not strong but they provide the best guess for the unknown parameters.

Figure 7-26 presents a flowchart describing the process where the fit is used when the parameter is unknown. Once the three inputs are obtained they are used as described in the emissions calculation flowchart of Figure 7-11.

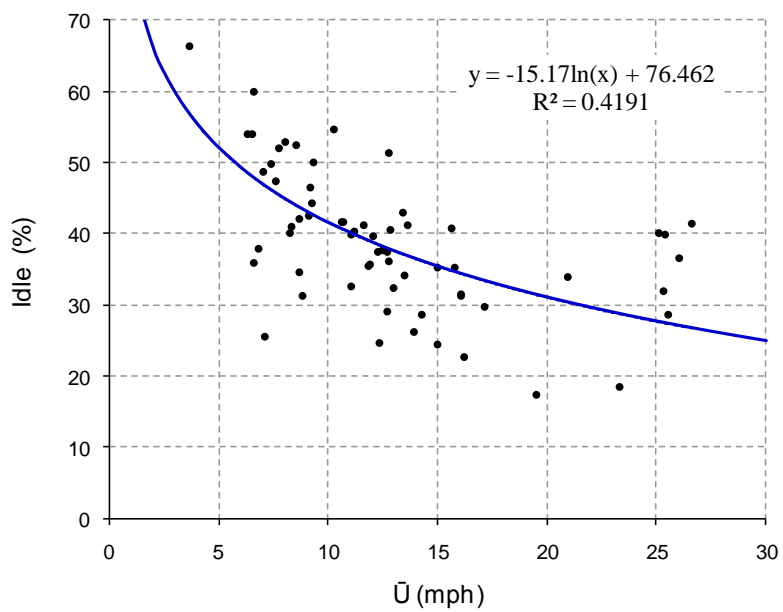

Figure 7-24 Approximation of Idle

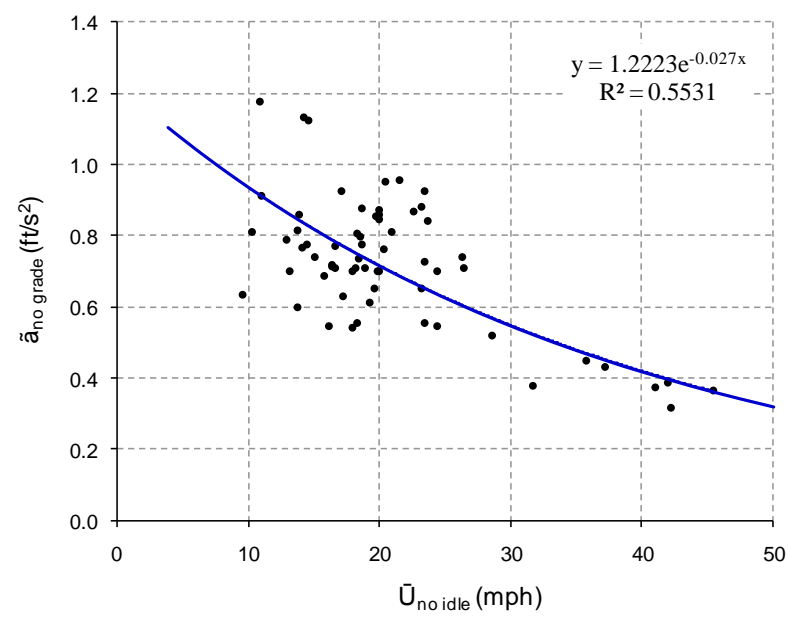

Figure 7-25 Approximation of $\tilde{a}_{n o}$ grade 


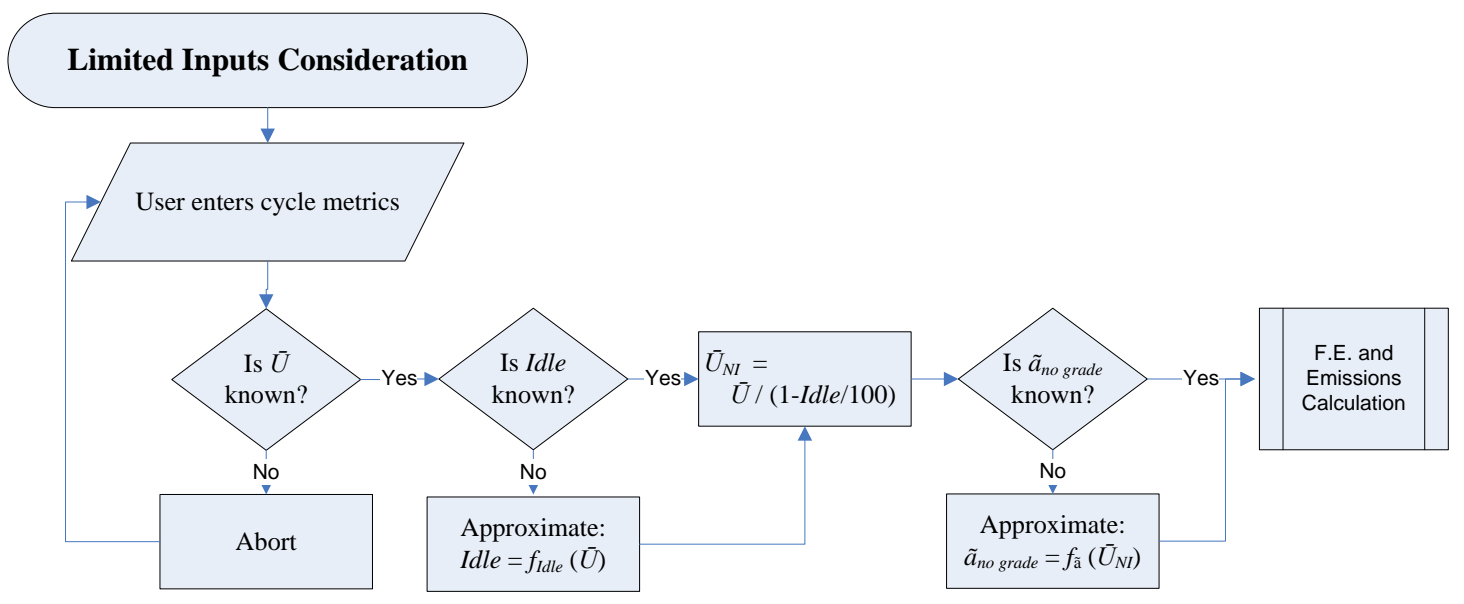

Figure 7-26 Flowchart for Limited Inputs

The three cases of limited inputs were studied: $\bar{U}$ and $\tilde{a}_{n o}$ grade are entered, $\bar{U}$ and Idle are entered, and $\bar{U}$ alone is entered. Predicted F.E. and $\mathrm{NO}_{\mathrm{x}}$ emissions for the three cases are presented in Figure 7-27 and Figure 7-29. Figure 7-28 and Figure 7-30 show the effect of the model inputs in the root mean square error (RMSE or mean squared residual, Equation 2.48) and changes in the coefficient of determination $\left(\mathrm{R}^{2}\right)$ from reducing model inputs.

It is observed that fuel economy predictions are still fairly satisfactory if $\bar{U}$ and $\tilde{a}_{n o}$ grade are known (Figure 7-27 $a$ and Figure 7-28). On the other hand, if $\tilde{a}_{n o}$ grade is unknown, the knowledge of Idle does not provide significant predictive power over predictions with $\bar{U}$ alone. In the $\left(\bar{U}, \tilde{a}_{n o}\right.$ grade $)$ case F.E. RMSE increases by about $60 \%$ over the baseline case (all parameters known); in the other two cases the F.E. RMSE doubles with respect to the baseline. Regarding the coefficient of determination (Figure 7-28 b), the results also show a marked sensitivity: it drops from 0.96 to 0.89 and then to 0.80 and 0.77 .

Since the variability in $\mathrm{NO}_{\mathrm{x}}$ emissions is lower compared with that of fuel economy, $\mathrm{NO}_{\mathrm{x}}$ is not greatly affected by model inputs. $\mathrm{NO}_{\mathrm{x}}$ predictions are not affected when Idle is unknown, $\left(\bar{U}, \tilde{a}_{n o}\right.$ grade $)$ case. This is corroborated by the RMSE and $\mathrm{R}^{2}$ values (Figure 7-30). $\mathrm{NO}_{\mathrm{x}}$ predictions for the final two cases are still very close to the simulation values with $\mathrm{R}^{2}$ above 0.98 . 


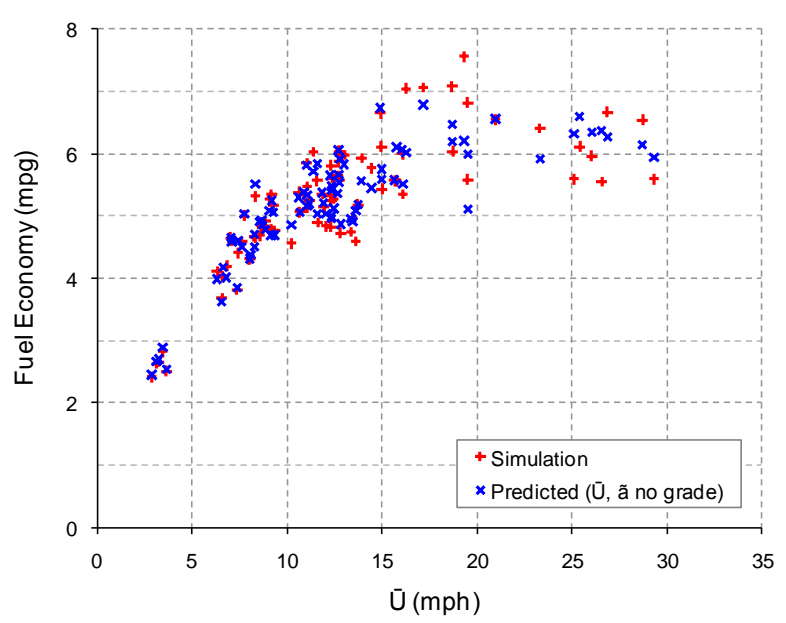

(a) $\bar{U}$ and $\tilde{a}_{\text {no grade }}$

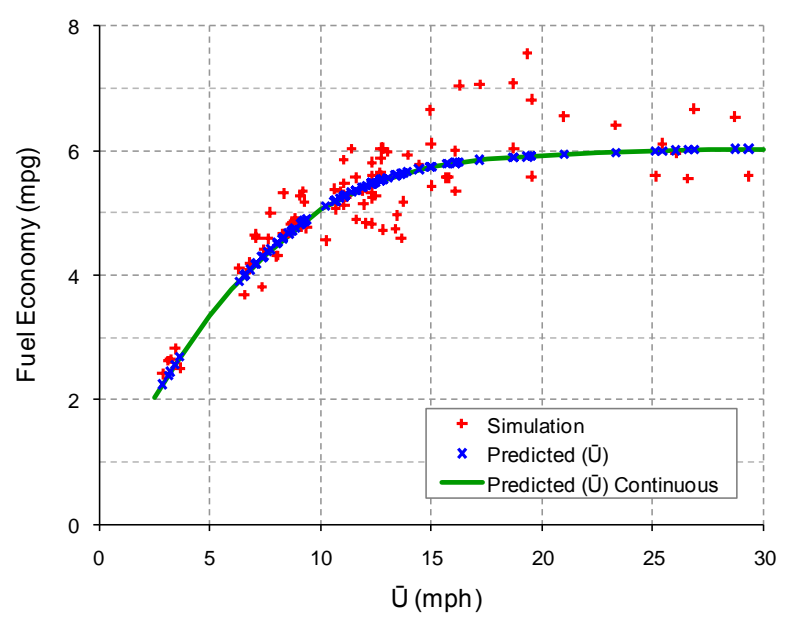

(c) $\bar{U}$

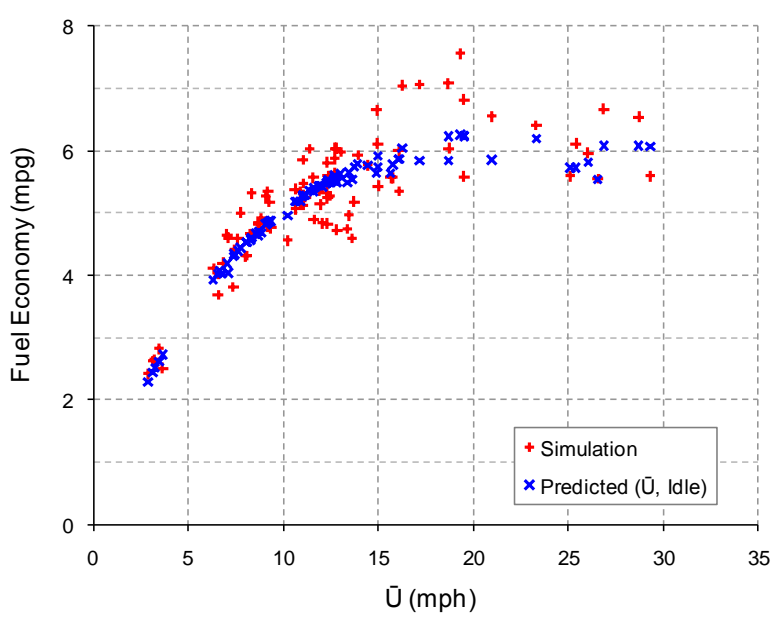

(b) $\bar{U}$ and Idle

Figure 7-27 Fuel Economy Predictions with Limited Inputs

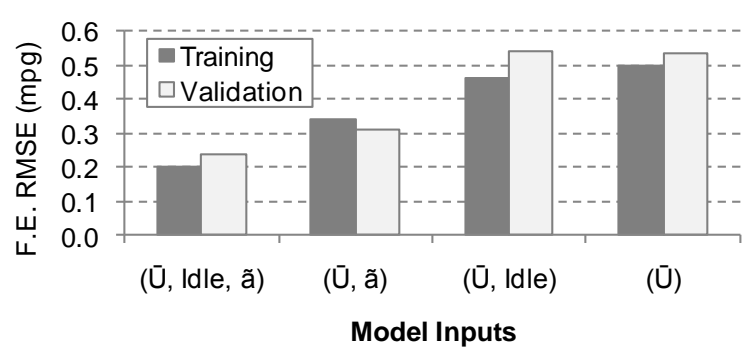

(a) RMSE

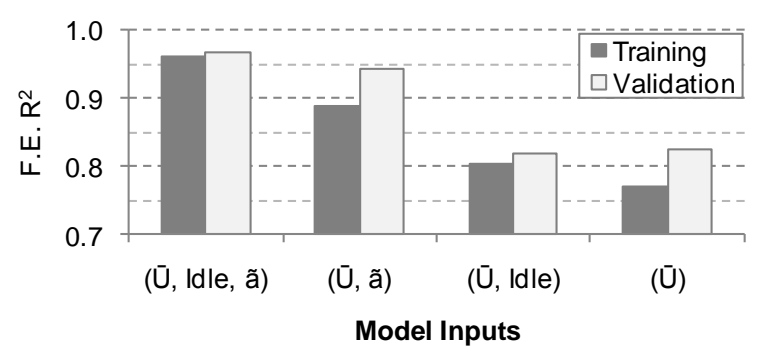

(b) $\mathbf{R}^{2}$

Figure 7-28 Effect of Model Inputs on Fuel Economy RMSE and $\mathbf{R}^{2}$ 


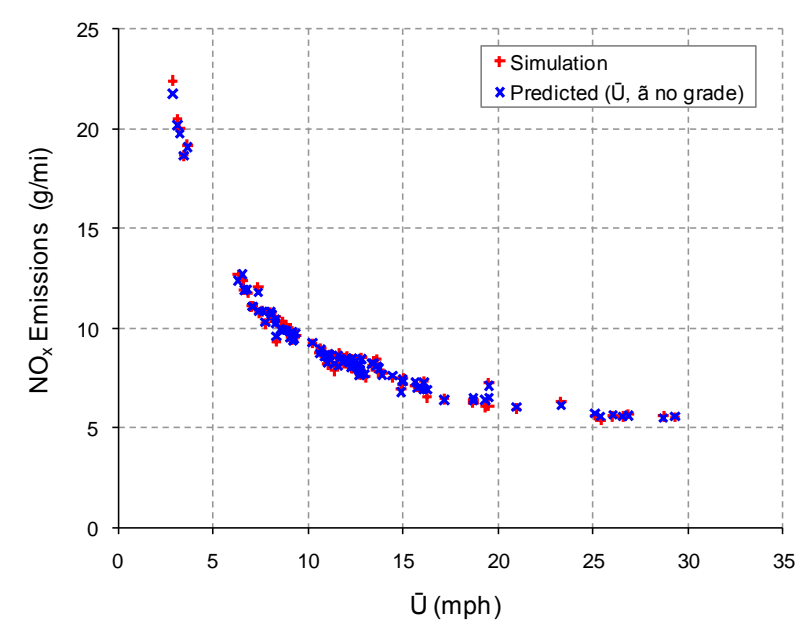

(a) $\bar{U}$ and $\tilde{a}_{\text {no grade }}$

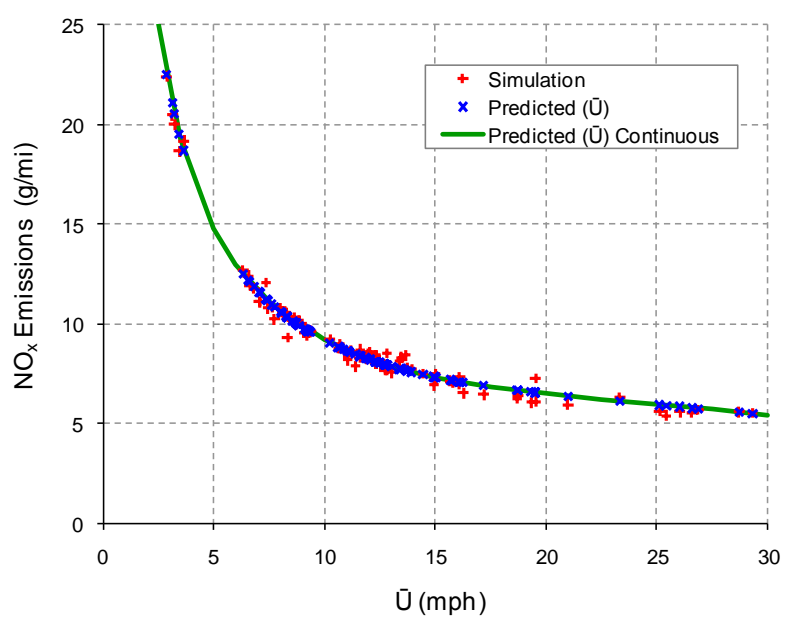

(c) $\bar{U}$

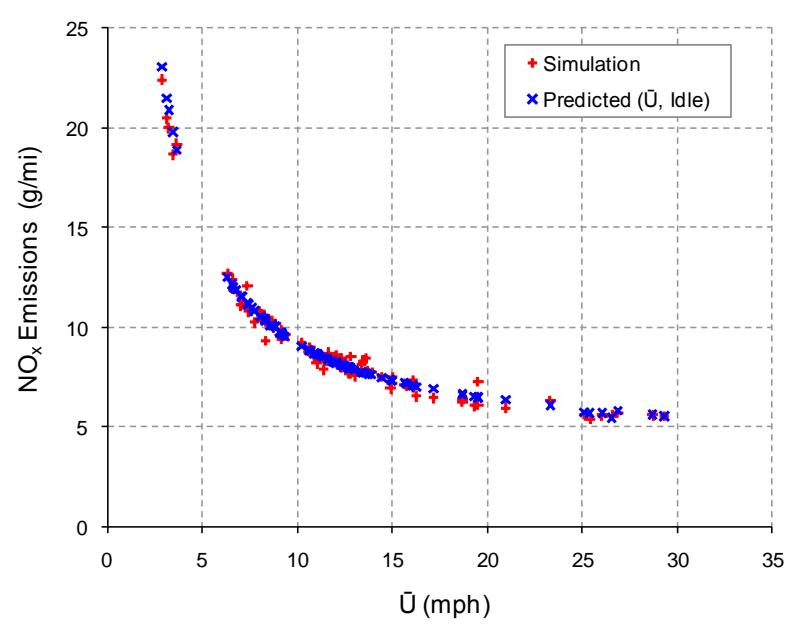

(b) $\bar{U}$ and Idle

Figure 7-29 $\mathrm{NO}_{\mathrm{x}}$ Predictions with Limited Inputs

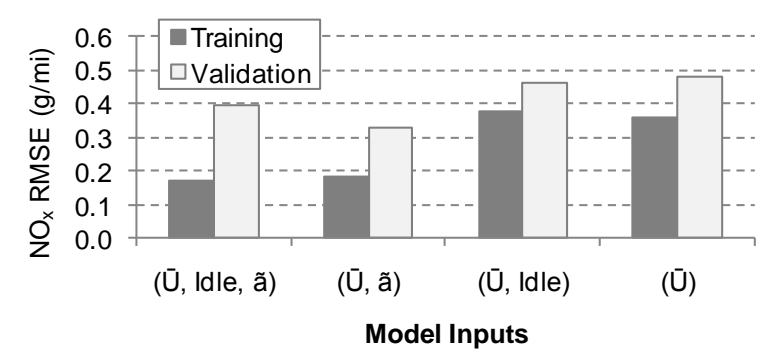

(a) RMSE

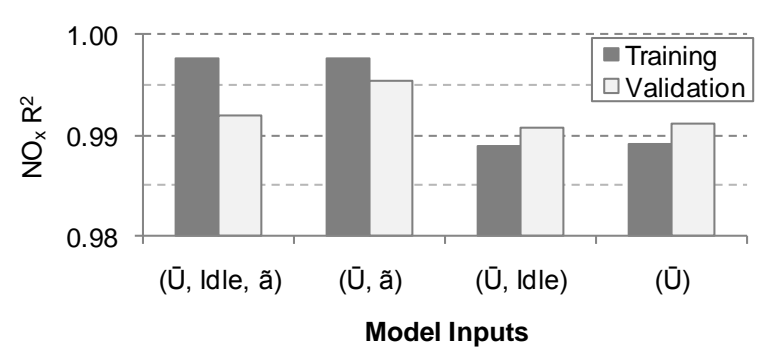

(b) $\mathbf{R}^{2}$

Figure 7-30 Effect of Model Inputs on $\mathrm{NO}_{\mathbf{x}} \mathrm{RMSE}$ and $\mathrm{R}^{2}$ 
Now consider the information needed from the user to determine the model inputs. As studied in Chapter 4 (Figure 4-5), route average speed is readily determined from transit agency records and is a very good predictor of actual speed since run-to-run variations are not very significant. Even if a single run of a particular route has an average speed considerably different from the rest of the runs, when all the runs of the route are considered together, the effect of the outlier run will be small in the route's average speed.

On the other hand, determination of both percentage idle (Section 2.2.1) and characteristic acceleration (Equation 2.23) requires knowledge of the time-speed record (and grade if applicable). Even with today's state of the art transit information systems which constantly report GPS location, it is unlikely that continuous time-speed records are available and/or reliable. Therefore, vehicle instrumentation with GPS and/or ECU monitors, such as the system described in Section 4.1, is needed to adequately compute Idle and $\tilde{a}_{n o}$ grade. A drawback of this approach is that post processing (data cleansing) of the acquired velocities can be quite intensive and time consuming.

Having into account that the main concern for a transit agency when using the prediction model is an accurate estimation of fuel economy, and as revealed by comparison of Figure 7-17 and Figure 7-27 $c$, it is highly desirable to use the full regression model rather than the model with $\bar{U}$ alone.

\subsubsection{Limits to the Input Parameters}

If Idle is unknown, the limits of $\bar{U}$ are 2.5 to $30 \mathrm{mph}$. This is because the estimation of Idle (Figure 7-24) had data within this range and estimation beyond such limits is not sensible. For a given average speed input, percentage idle is limited by the regressions in $\bar{U}_{N I}$ (Figure 7-15 and Figure 7-16) with a range from 8 to $50 \mathrm{mph}$. Recalling that the three parameters are linked by Equation 2.19: $\bar{U}_{N I}=\bar{U} /(1$ - Idle/100), the limits of Idle can be expressed as follows:

Equation 7.5 Limits on Percentage Idle

$$
\begin{aligned}
& I d l e_{\min }=\max \left[0, \quad 100 \cdot\left(1-\bar{U} / \bar{U}_{N I, \text { min }}\right)\right] \\
& \text { Idle }_{\max }=100 \cdot\left(1-\bar{U} / \bar{U}_{N I, \text { max }}\right)
\end{aligned}
$$

Where $\bar{U}>0$ and $\bar{U} \leq \bar{U}_{N I \text {, max }}$. In the present models: $\bar{U}_{N I,-\min }=8 \mathrm{mph}$ and $\bar{U}_{N I, \_m a x}=50 \mathrm{mph}$. Figure 7-31 provides a graphical representation of the relationships in Equation 7.5. 


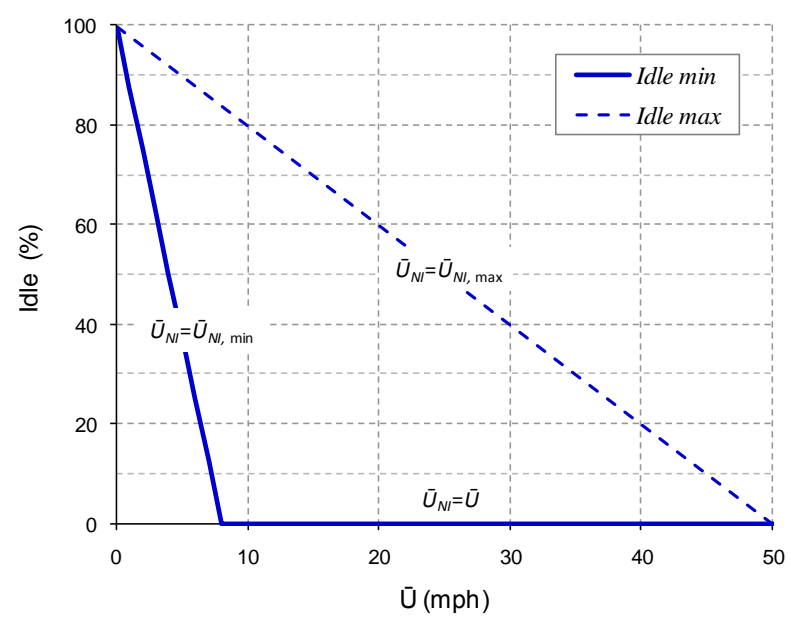

Figure 7-31 Graphical Representation of the Limits on Percentage Idle

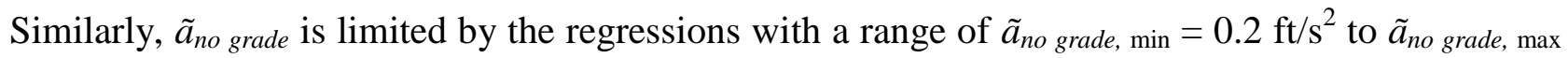
$=1.2 \mathrm{ft} / \mathrm{s}^{2}$. There is a natural trend depicted in Figure 7-25 where high speed service exhibits lower $\tilde{a}$ than low speed service. For example for $\bar{U}_{N I}$ around $45 \mathrm{mph}$ there were no observations of $\tilde{a}_{n o}$ grade in the higher end $\left(\sim 1.2 \mathrm{ft} / \mathrm{s}^{2}\right)$, nor were observations with $\bar{U}_{N I}$ of $10 \mathrm{mph}$ and $\tilde{a}_{\text {no grade }}$ of $0.2 \mathrm{ft} / \mathrm{s}^{2}$. With this in mind, one could further impose a band in characteristic acceleration as a function of $\bar{U}_{N I}$. Such band was not be considered here.

Since characteristic acceleration is independent of idle fraction, it is best to let the model use the estimate of Figure 7-25 whenever $\tilde{a}_{n o \_g r a d e}$ is unknown. The drawback, as shown below, is that uncertainties are doubled and conclusions regarding best average speed for optimized fuel savings cannot be drawn.

\subsection{Uncertainty Analysis}

This section studies uncertainty in the prediction model and how uncertainty is changed by having limited user inputs. Since the predictive model is decomposed in driving and idle contributions, uncertainty on the output will be dependent on the uncertainties of the two components and their functional relationships.

Once uncertainties of driving and idle fuel consumption and $\mathrm{NO}_{\mathrm{x}}$ are determined (later in this section), uncertainties on the model outputs are estimated by propagation analysis (Fox et al., 2004). Consider the variable $R$ calculated from measurements (or estimations) of the variables $x_{1}, x_{2}, \ldots, x_{n}$, 
each with absolute uncertainty $\Delta x_{1}, \Delta x_{2}, \ldots, \Delta x_{n}$, where $R$ is expressed mathematically as $R=R\left(x_{1}, x_{2}\right.$, $\left.\ldots, x_{n}\right)$. The absolute uncertainty on $R$ is determined by:

Equation 7.6 Propagation of Uncertainty

$$
\Delta R= \pm\left[\left(\frac{\partial R}{x_{1}} \cdot \Delta x_{1}\right)^{2}+\left(\frac{\partial R}{x_{2}} \cdot \Delta x_{2}\right)^{2}+\ldots+\left(\frac{\partial R}{x_{n}} \cdot \Delta x_{n}\right)^{2}\right]^{1 / 2}
$$

Applying Equation 7.6 to cycle fuel consumption (Equation 7.1 $b$ and Equation 7.2) and cycle $\mathrm{NO}_{\mathrm{x}}$ emissions (Equation 7.3) we obtain:

Equation 7.7 Uncertainty in Cycle Fuel Consumption and Cycle $\mathrm{NO}_{\mathrm{x}}$ Emissions

$$
\begin{aligned}
& \Delta F . C .= \pm\left[\left(\Delta F . C_{\text {Driving }}\right)^{2}+\left(\frac{\text { Idle } / 100}{\bar{U}} \cdot \Delta \overline{\dot{m}}_{\text {f Idle }}\right)^{2}\right]^{1 / 2} \\
& \Delta N O_{x}= \pm\left[\left(\Delta N O_{x \text { Driving }}\right)^{2}+\left(\frac{\text { Idle } / 100}{\bar{U}} \cdot \Delta \overline{\dot{m}}_{N O_{x} \text { Idle }}\right)^{2}\right]^{1 / 2}
\end{aligned}
$$

From Equation 7.4 and Equation 7.6, the uncertainties in fuel economy and $\mathrm{CO}_{2}$ emissions are

$$
\begin{gathered}
\Delta F . E .= \pm\left(\frac{\rho_{\text {fuel }}}{F . C^{2}}\right) \cdot \Delta F . C .= \pm\left(\frac{F . E^{2}}{\rho_{\text {fuel }}}\right) \cdot \Delta F . C . \\
\Delta C O_{2}= \pm\left(\text { Fuel }_{\text {Carbon }} \cdot 44 / 12\right) \cdot \Delta F . C .
\end{gathered}
$$

The equations above neglect uncertainties in $\bar{U}$, Idle, $\rho_{\text {fuel }}$ and Fuel $_{\text {Carbon }}$.

Lets first resolve the uncertainties in mean idle fuel consumption $\left(\overline{\dot{m}}_{f}\right.$ Idle $)$ and mean idle $\mathrm{NO}_{\mathrm{x}}$ emissions $\left(\overline{\dot{m}}_{N O_{x} \text { Idle }}\right)$. These uncertainties are the margins of error on the means and are readily determined from the standard statistical analysis presented in Table 7-4; they are $\Delta \overline{\dot{m}}_{f \text { Idle }}=15.9 \mathrm{~g} / \mathrm{hr}$ and $\Delta \overline{\dot{m}}_{N O_{x} \text { Idle }}=0.18 \mathrm{~g} / \mathrm{h}$.

Equation 2.45 illustrates the confidence interval for the mean response of a linear regression; this uncertainty is not constant as it is a function of the independent variable. Nearly $1 / 3^{\text {rd }}$ of the observations are bounded by this $95 \%$ confidence error band on the mean response. The exact uncertainties on the polynomial regressions for F.C. Driving and $N O_{x}$ Driving, on the other hand, are involved functions of $\bar{U}_{N I}$ and $\tilde{a}_{n o}$ grade and are not practical to explore for the purposes of this dissertation.

A simplified approach was used in which a constant percentage error on $F$. $C$._Driving and $N O_{x_{-} D r i v i n g}$ was determined such that a prescribed percentage $(33 \%)$ of the F.E. and $\mathrm{NO}_{\mathrm{x}}$ residuals were bounded 
by the estimated uncertainties. That is, a constant absolute error in the F.C. Driving and NO $O_{x}$ Driving predictions that yields $33 \%$ of the observations within the estimated confidence intervals $F . E$. $\pm \Delta F . E$. and $N O_{x} \pm \Delta N O_{x}$. These uncertainties are equivalent to a $95 \%$ confidence interval in the mean response.

The constant absolute errors were determined and are presented in Table 7-5. The uncertainty bars for fuel economy are depicted in the target-by-predicted plots of Figure 7-32. Uncertainties under each group of inputs were calculated using the equations above and the absolute error on the predicted values of driving fuel consumption $(\Delta F . C$._Driving $)$ from Table 7-5. The uncertainty was dominated by the driving contributions while the effect of idle rate uncertainties was marginal.

Table 7-5 Constant Absolute Uncertainties for Predictions of F.C._Driving and $\mathrm{NO}_{x_{-} \text {Driving }}(\mathrm{g} / \mathrm{mi})$

\begin{tabular}{l|r|c}
\hline Case & Fuel & NO $_{\mathbf{x}}$ \\
\hline$\left(\bar{U}\right.$, Idle,$\left.\tilde{a}_{\text {no grade }}\right)$ & 11.0 & 0.060 \\
\hline$\left(\bar{U}, \tilde{a}_{\text {no grade }}\right)$ & 14.0 & 0.059 \\
\hline$(\bar{U}$, Idle $)$ & 18.7 & 0.118 \\
\hline$(\bar{U})$ & 22.9 & 0.137 \\
\hline
\end{tabular}

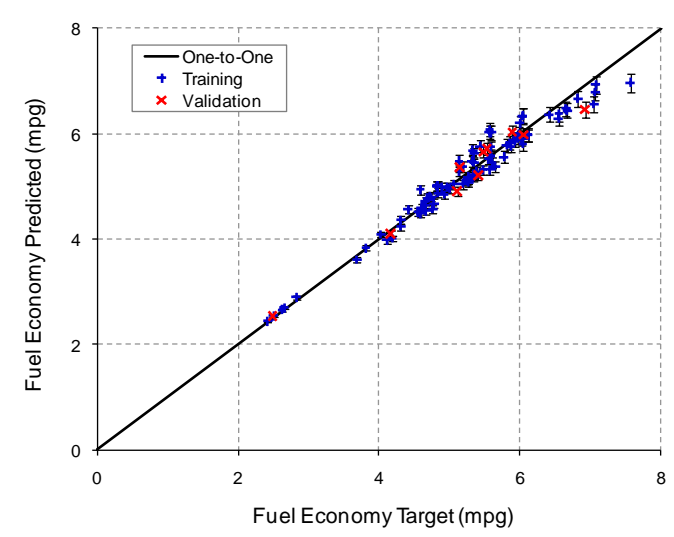

(a) $\bar{U}$, Idle, and $\tilde{a}_{\text {no grade }}$

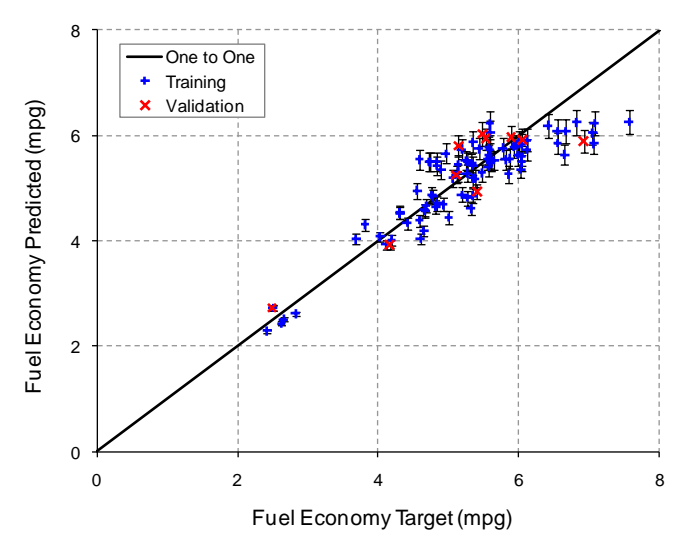

(c) $\bar{U}$ and Idle

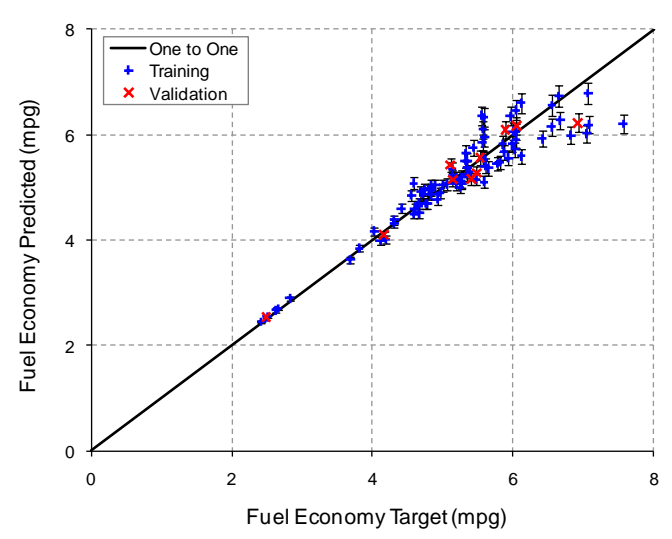

(b) $\bar{U}$ and $\tilde{a}_{\text {no grade }}$

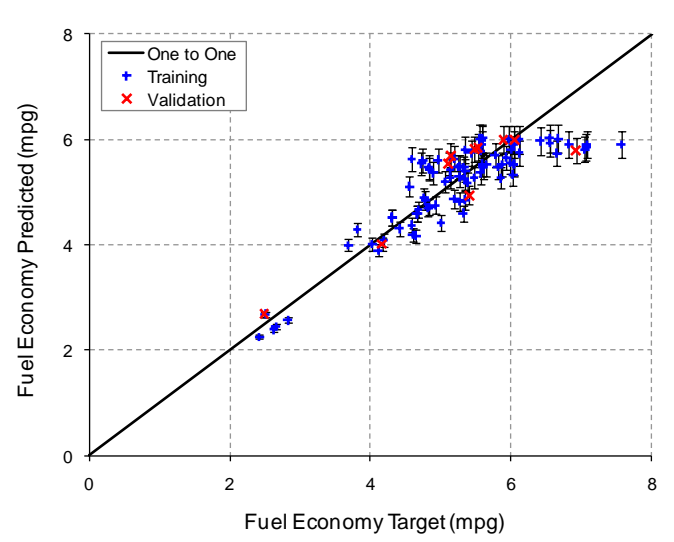

(d) $\bar{U}$

Figure 7-32 Uncertainty Bars for Cycle Fuel Economy Under the Various Input Groups 


\subsection{Predictions for Conventional Diesel Vehicle}

A prediction model for a conventional diesel counterpart to the hybrid bus was required because the fuel economy results were to be presented as reductions in fuel consumption. The author considered several options to produce data for the conventional vehicle model: $(i)$ to collect chassisdynamometer data for a MY 2007-2009 bus, (ii) to develop and validate a computational model for a MY 2007-2009 bus and use it as was used the hybrid bus model to procure the data, and (iii) to obtain chassis-dynamometer data for a MY 2003-2006 bus and to scale $\mathrm{NO}_{\mathrm{x}}$ emissions to account for the engine certification level, neglecting variations in fuel consumption.

While it was acknowledged that the first two options would produce more accurate results, the third option was the one that adjusted to the time constraints of the project. Chassis-dynamometer test data were available for a MY $200640 \mathrm{ft}$ New Flyer bus (CAFEE, 2007) under a total of 16 duty cycles. These emissions data were time aligned and processed to extract the cycle metrics and the driving and idle contributions to fuel consumption and $\mathrm{NO}_{\mathrm{x}}$ emissions shown in Table 7-6. $\mathrm{NO}_{\mathrm{x}}$ emissions were corrected for certification level by considering a $15 \%$ reduction.

The 20 data points available for development of the regressions were a small amount to produce adequate results. Tu (2011) applied the tool developed by Marlowe (2009), which breaks the datasets intro micro-trips and concatenates them randomly to produce new cycles, to the dataset of Table 7-6 in order to expand the number of data points by virtual cycles and populate the search domain. IBIS' prediction model for MY 2003-2006 conventional diesel buses is based on the virtual cycle dataset.

Those virtual cycles were used in conjunction with the data of Table 7-6 to generate the regression model for the conventional vehicle applying the methods described above: regressions for F.C._Driving and $N O_{x_{-} \text {Driving }}$, and the average idle contributions $\overline{\dot{m}}_{f \text { Idle }}$ and $\overline{\dot{m}}_{N O_{x} \text { Idle }}$. The constant absolute errors for F.C._Driving and $N O_{x_{-} \text {Driving }}$ were of the same order of magnitude as the ones from the hybrid model.

A $k$-fold cross validation study was also performed and the results were similar to those of the hybrid's model. The regressions were developed reserving $10 \%$ of the data for validation. Figure 7-33 presents the predicted fuel economy and a comparison with IBIS predictions. Although there is a higher variation than for the hybrid model, the results are very satisfactory with coefficients of determination of 0.97 for fuel economy and 0.96 for $\mathrm{NO}_{\mathrm{x}}$. Performance for the test entries is remarkable.

Additional scatter is presented in the parity plot of Figure 7-33 $b$ for the IBIS predicted values versus a lower scatter for the proposed regression model. This confirms that separation of idle and driving contributions may provide more accurate predictions that the current IBIS models which treat idle and driving as a bulk. 
Table 7-6 Fuel Consumption and Emissions Chassis Test Results for Conventional Diesel Bus (CAFEE, 2007)

\begin{tabular}{|c|c|c|c|c|c|c|c|c|c|c|c|c|c|}
\hline Line/Cycle Name & $\begin{array}{l}\text { Dist. } \\
\text { (mi) }\end{array}$ & $\underset{(\mathbf{m p h})}{\overline{\mathbf{U}}}$ & $\begin{array}{l}\overline{\mathbf{U}}_{\text {no idle }} \\
\text { (mph) }\end{array}$ & $\begin{array}{l}\text { Idle } \\
(\%)\end{array}$ & $\begin{array}{c}\tilde{a}_{\text {no grade }} \\
\left(\mathbf{f t} / \mathrm{s}^{2}\right)\end{array}$ & $\begin{array}{c}\text { F.E. } \\
\text { (mpg) }\end{array}$ & $\underset{(\mathrm{g} / \mathrm{mi})}{\mathrm{CO}_{2}}$ & $\underset{(\mathbf{g} / \mathbf{m i})}{\mathrm{NO}_{\mathbf{x}}}$ & $\begin{array}{c}\text { Fuel } \\
\text { (g/mi) }\end{array}$ & $\begin{array}{c}\text { Fuel } \\
\text { Driving } \\
\text { (g/mi) }\end{array}$ & $\begin{array}{c}\mathrm{NO}_{\mathrm{x}} \\
\text { Driving } \\
(\mathrm{g} / \mathrm{mi})\end{array}$ & $\begin{array}{l}\text { Fuel } \\
\text { Idle } \\
(\mathrm{g} / \mathrm{s})\end{array}$ & $\begin{array}{l}\mathrm{NO}_{\mathrm{x}} \\
\text { Idle } \\
(\mathrm{g} / \mathrm{s})\end{array}$ \\
\hline Arterial & 2.0 & 24.8 & 28.8 & 13.8 & 0.68 & 5.01 & 1,978 & 5.59 & 627 & 606 & 5.45 & 1.06 & 0.007 \\
\hline Arterial & 2.0 & 24.5 & 28.5 & 13.8 & 0.67 & 5.02 & 1,973 & 5.79 & 626 & 602 & 5.63 & 1.16 & 0.008 \\
\hline BEELINE & 6.7 & 13.9 & 19.4 & 28.3 & 0.85 & 3.85 & 2,570 & 7.07 & 815 & 722 & 6.65 & 1.27 & 0.006 \\
\hline Braunschweig & 6.6 & 13.6 & 17.8 & 23.7 & 0.72 & 4.27 & 2,318 & 7.33 & 735 & 659 & 6.88 & 1.22 & 0.007 \\
\hline CBD & 2.0 & 12.8 & 15.0 & 14.8 & 0.65 & 4.11 & 2,407 & 7.81 & 765 & 719 & 7.34 & 1.09 & 0.011 \\
\hline CSHVC & 6.5 & 13.9 & 18.3 & 24.4 & 0.55 & 5.20 & 1,904 & 5.38 & 604 & 526 & 5.11 & 1.23 & 0.004 \\
\hline Commuter & 3.9 & 43.1 & 48.3 & 10.7 & 0.22 & 5.72 & 1,732 & 4.49 & 549 & 538 & 4.43 & 1.28 & 0.007 \\
\hline Commuter & 4.0 & 43.5 & 48.2 & 9.8 & 0.22 & 5.95 & 1,665 & 4.23 & 528 & 520 & 4.19 & 1.06 & 0.006 \\
\hline ETC Urban & 2.3 & 14.0 & 16.1 & 13.0 & 0.46 & 5.52 & 1,791 & 6.13 & 569 & 526 & 5.89 & 1.28 & 0.007 \\
\hline $\mathrm{KCM}$ & 12.6 & 23.1 & 27.6 & 16.3 & 0.51 & 5.26 & 1,883 & 5.42 & 597 & 565 & 5.24 & 1.26 & 0.007 \\
\hline Manhattan & 2.1 & 6.8 & 10.5 & 35.5 & 0.86 & 2.79 & 3,547 & 10.31 & 1,128 & 894 & 9.25 & 1.24 & 0.006 \\
\hline New York Bus & 0.6 & 3.6 & 9.6 & 62.5 & 1.14 & 1.50 & 6,601 & 17.85 & 2,099 & 1,266 & 13.50 & 1.34 & 0.007 \\
\hline New York Comp. & 2.4 & 8.5 & 12.9 & 33.7 & 0.47 & 4.05 & 2,439 & 6.73 & 776 & 594 & 6.03 & 1.28 & 0.005 \\
\hline OCTA & 6.5 & 12.0 & 15.7 & 23.7 & 0.70 & 4.13 & 2,400 & 7.16 & 762 & 678 & 6.77 & 1.17 & 0.006 \\
\hline OCTA & 6.5 & 11.9 & 15.6 & 23.8 & 0.70 & 4.09 & 2,423 & 7.52 & 769 & 683 & 7.03 & 1.20 & 0.007 \\
\hline Paris & 3.5 & 6.5 & 9.9 & 34.1 & 0.76 & 2.94 & 3,367 & 9.33 & 1,070 & 838 & 8.43 & 1.23 & 0.005 \\
\hline HHDDT-Trans & 2.8 & 15.1 & 17.9 & 15.3 & 0.50 & 5.26 & 1,879 & 5.67 & 597 & 550 & 5.43 & 1.29 & 0.007 \\
\hline UDDS & 5.4 & 18.4 & 27.7 & 33.5 & 0.40 & 5.04 & 1,962 & 5.46 & 623 & 536 & 5.05 & 1.32 & 0.006 \\
\hline WMATA & 4.1 & 8.1 & 13.6 & 40.4 & 0.76 & 3.26 & 3,030 & 8.10 & 963 & 744 & 7.22 & 1.22 & 0.005 \\
\hline WMATA & 4.2 & 8.2 & 13.6 & 39.7 & 0.76 & 3.26 & 3,033 & 7.71 & 964 & 745 & 6.85 & 1.26 & 0.005 \\
\hline
\end{tabular}

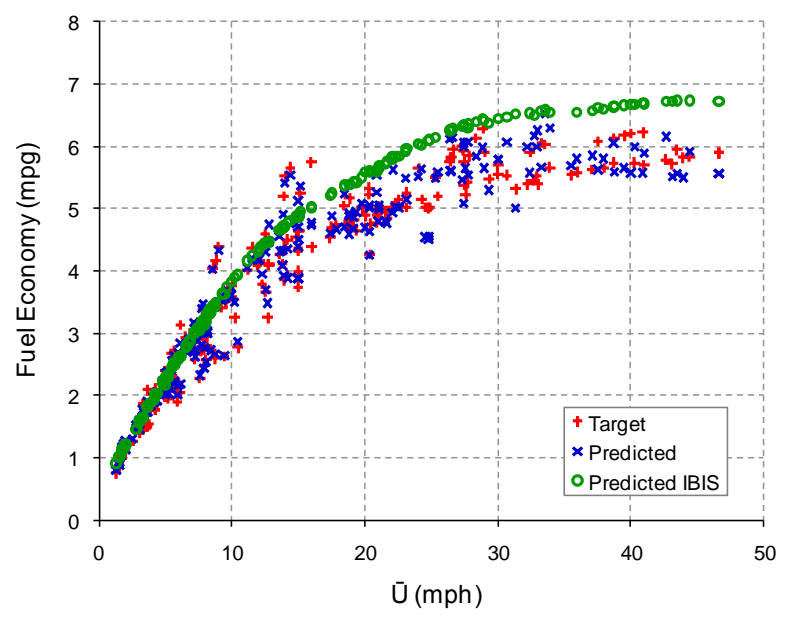

(a) F.E. versus $\bar{U}$

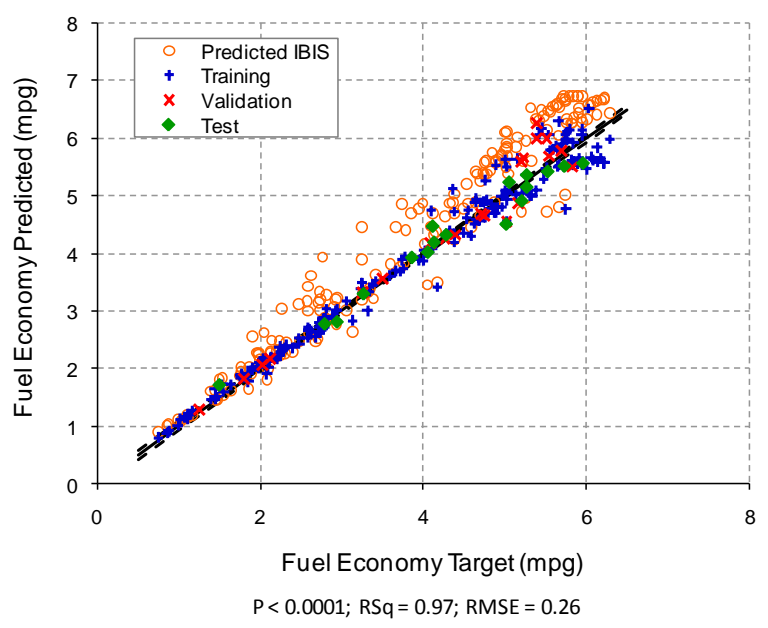

(b) Predicted versus Target Plot

Figure 7-33 Predicted Fuel Economy for Conventional Diesel Bus 


\subsection{Hybrid Advantage and Fuel Savings}

The benefit of the hybrid buses over their conventional counterparts can be expressed in terms of hybrid advantage (H.A.). The term hybrid advantage was coined by O'Keefe et al. (2007) to designate the percentage reduction in fuel consumption (gallons per mile or grams per mile), which is swiftly converted to fuel savings with the total vehicle miles traveled (VMT).

It is also possible to present the hybrid benefit as fuel savings per unit time (gallons per hour) and this option may be more relevant when comparing routes. As routes with different speeds travel different distances, and, although the H.A. may be higher for low speed routes, the potential savings could be better for faster routes as their travel is higher and consume more fuel.

\subsubsection{Hybrid Advantage}

Hybrid advantage can be stated in terms of F.C. or F.E.:

Equation 7.8 Hybrid Advantage

$$
\begin{aligned}
& H . A .=\frac{F \cdot C \cdot \cdot_{C_{C o n v}}-F \cdot C_{\cdot_{H y b}}}{F \cdot C \cdot \cdot_{\cdot_{C o n v}}} \cdot 100 \% \\
& H . A .=\frac{F \cdot E_{\cdot_{H y b}}-F \cdot E_{\cdot_{C o n v}}}{F \cdot E_{\cdot H y b}} \cdot 100 \%
\end{aligned}
$$

Since it is fuel economy what is reported by the model, Equation $7.8 b$ is preferred. Applying the error propagation of Equation 7.6 to H.A., the uncertainty in hybrid advantage is:

\section{Equation 7.9 Uncertainty in Hybrid Advantage}

$$
\Delta H . A .= \pm 100 \cdot\left[\left(\frac{F \cdot E_{\cdot C o n v}}{F \cdot E_{\cdot H y b}{ }^{2}} \cdot \Delta F \cdot E_{\cdot H y b}\right)^{2}+\left(\frac{\Delta F \cdot E_{\cdot C o n v}}{F \cdot E_{\cdot H y b}}\right)^{2}\right]^{1 / 2}
$$

Figure 7-34 contrasts the predictions of conventional and hybrid buses under the test cycle database. Figure 7-34 $a$ shows fuel economy versus average speed where a hybrid benefit is clearly noted. Figure 7-34 $b$ presents the fuel economy parity plot with the correlation expressed as a second order polynomial. Carbon dioxide emissions are presented in Figure 7-34 $c$ as a function of average speed and in Figure 7-34 $d$ in a parity plot. $\mathrm{CO}_{2}$ reductions are roughly between $10 \%$ and $30 \%$. Finally, Figure 7-34 $e$ shows the predictions for oxides of nitrogen. 


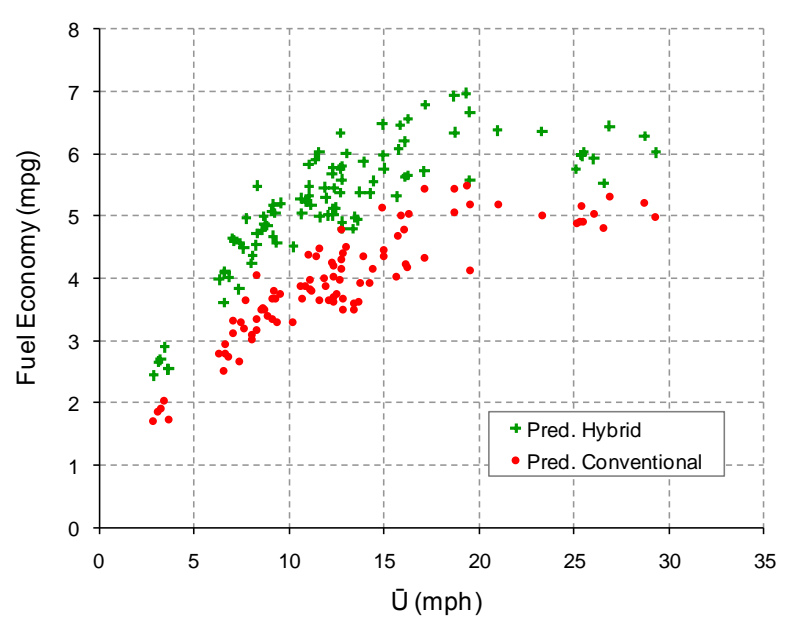

(a) Fuel Economy versus Average Speed

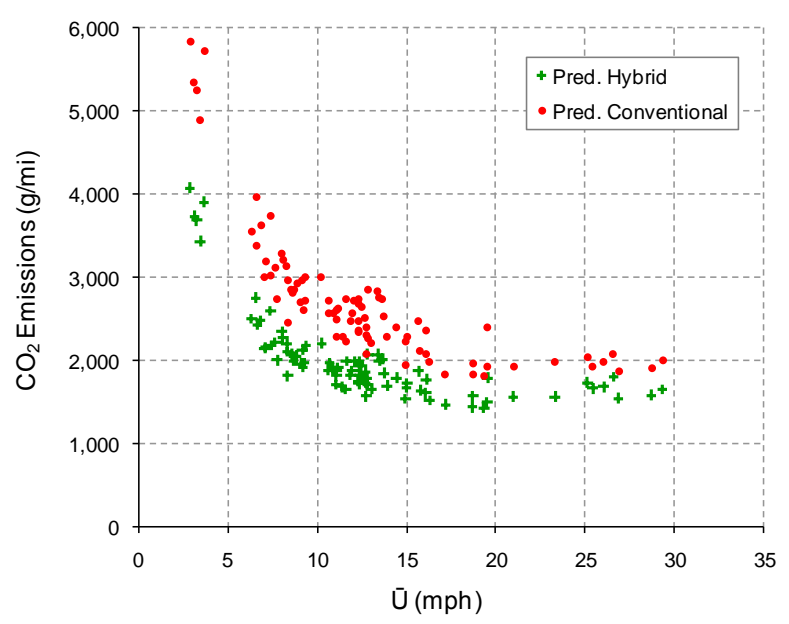

(c) $\mathrm{CO}_{2}$ Emissions versus Average Speed

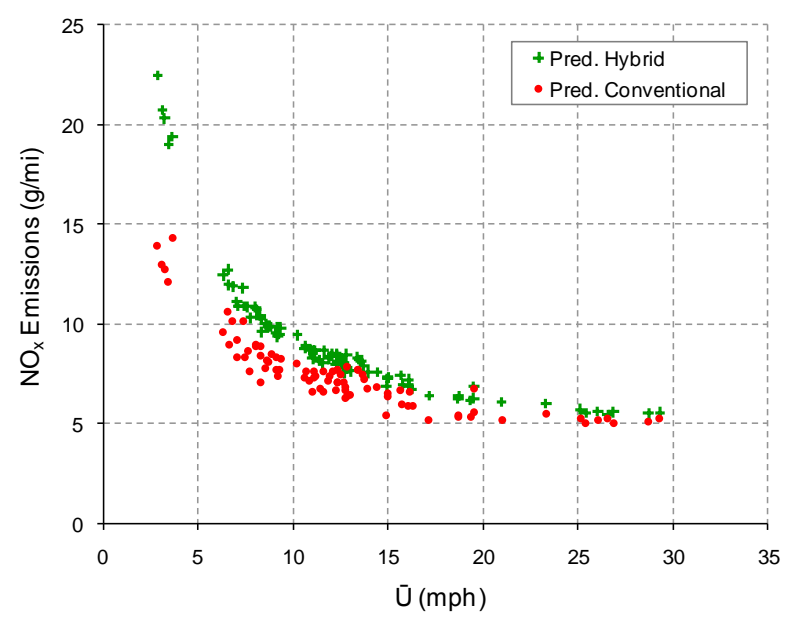

(e) $\mathrm{NO}_{\mathrm{x}}$ Emissions versus Average Speed

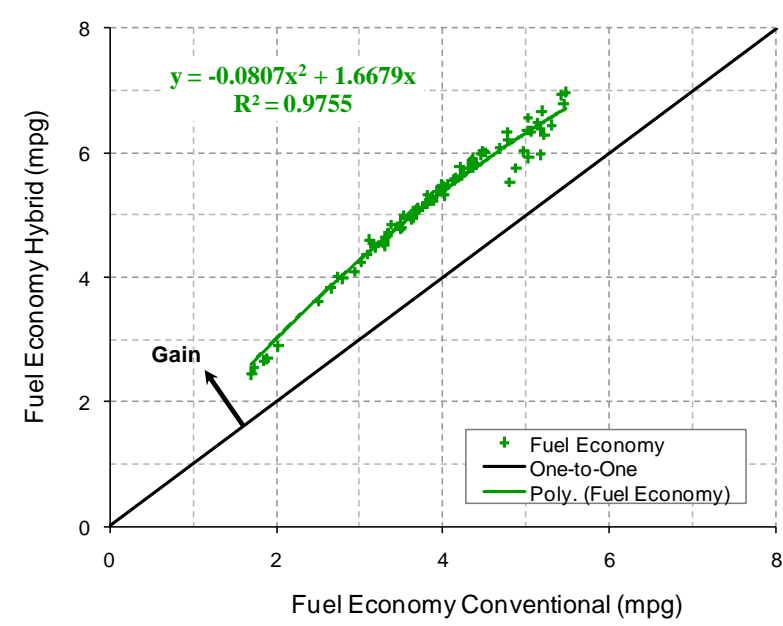

(b) F.E. Conventional versus Hybrid

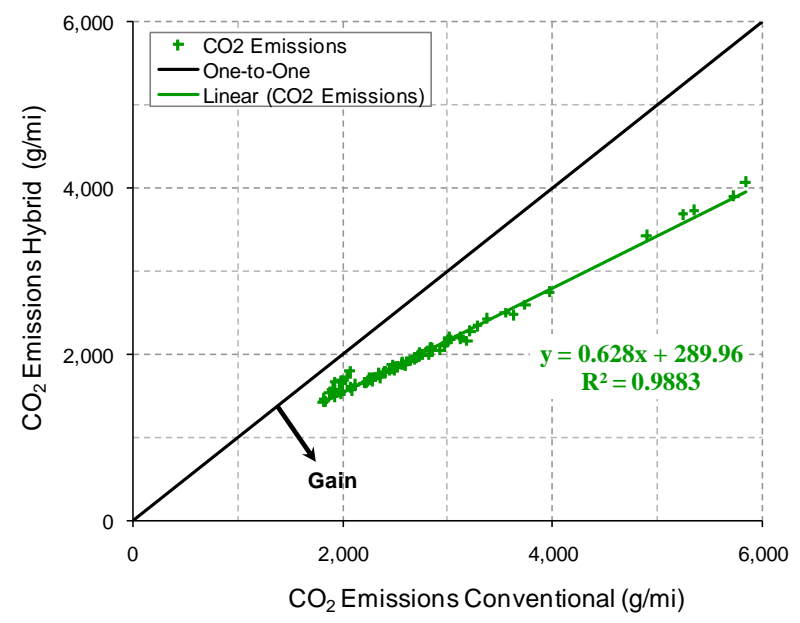

(d) $\mathrm{CO}_{2}$ Emissions Conventional versus Hybrid

Figure 7-34 Hybrid versus Conventional Contrasts 
The $\mathrm{NO}_{\mathrm{x}}$ data do not reveal a net benefit from hybridization but that hybrid buses have higher $\mathrm{NO}_{\mathrm{x}}$ emissions when compared to their conventional counterparts. On average hybrid $\mathrm{NO}_{\mathrm{x}}$ emissions predictions are $17 \%$ higher than for the conventional diesel. This result agrees with chassisdynamometer emissions tests of transit buses collected by WVU's TransLab (CAFEE, 2010, and CAFEE, 2011); data summarized in Table 5-7 and Table 5-8. This fact can be explained by the lower loads experienced by the engine on the hybrid bus, as lower loads are associated with higher brakespecific $\mathrm{NO}_{\mathrm{x}}$ (see the brake-specific $\mathrm{NO}_{\mathrm{x}}$ map of Figure 5-9). Considering that starting in 2010 EPA's $\mathrm{NO}_{\mathrm{x}}$ certification level dropped to $0.2 \mathrm{~g} / \mathrm{bhp} \cdot \mathrm{hr}$, the observed effect in $\mathrm{NO}_{\mathrm{x}}$ emissions should raise no concerns.

Hybrid advantage is presented in Figure 7-35. Figure 7-35 $a$ shows the change in H.A. with average speed; it can be observed how the low speed cycles exhibit the highest advantages. Figure 7-35 $b$ presents H.A. versus kinetic intensity, $k i$. For the routes used in this evaluation, H.A. ranged from $13 \%$ to $32 \%$ with a median of $26 \%$. The highest percentage gains are obtained for routes with low fuel economy (the slowest routes). With respect to $k i$ (Equation 2.28), and as suggested theoretically by O'Keefe et al. (2007), H.A. increases as $k i$ increases. It is also observed that H.A. does not increase indefinitely but rather reaches a plateau (30\% to 35\% in this application). If the regression line of Figure 7-35 $a$ were to be extrapolated to the limit $\bar{U} \rightarrow 0$ a maximum value of 34\% would be reached.

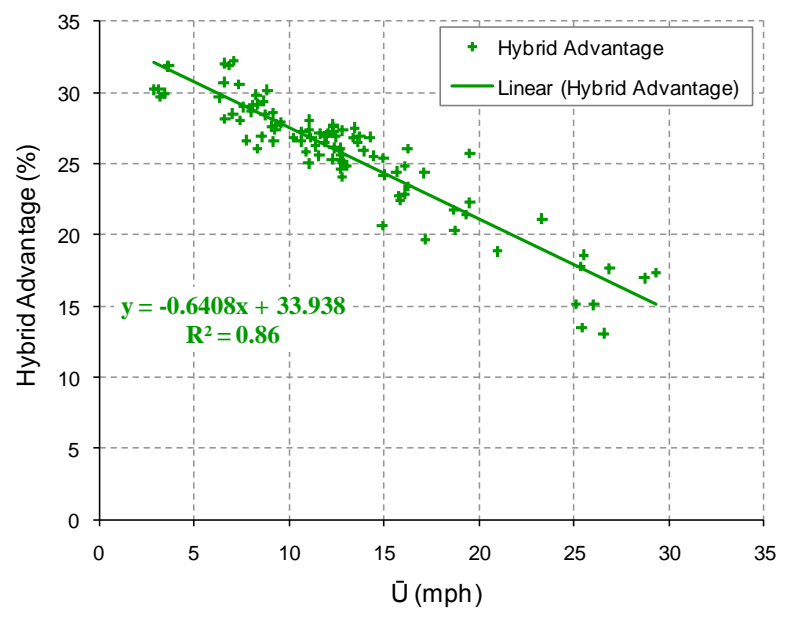

(a) Hybrid Advantage versus Average Speed

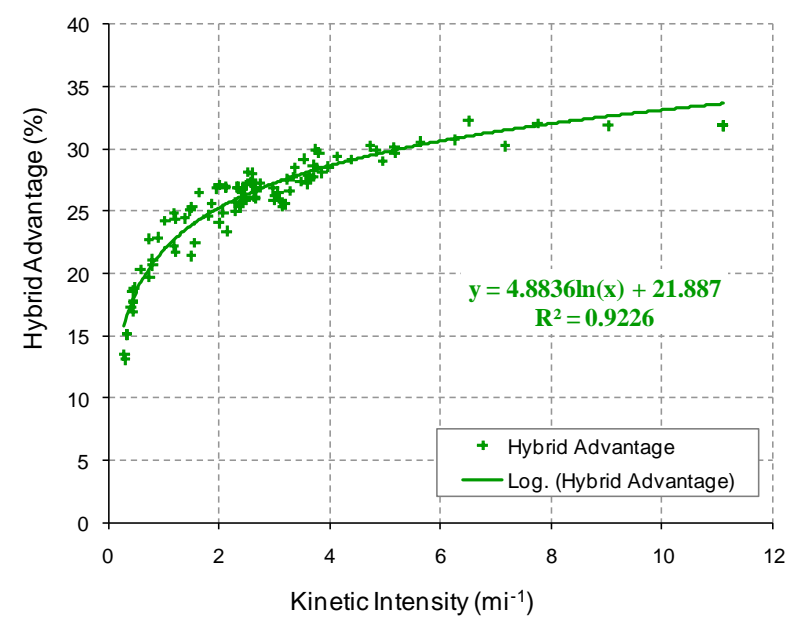

(b) Hybrid Advantage versus Kinetic Intensity

Figure 7-35 Hybrid Advantage 


\subsubsection{Fuel Savings}

The fact that the slowest routes exhibit the highest values of H.A. does not necessarily imply that these routes would yield the highest fuel savings. If we take into account that H.A. is a reduction in distance specific fuel consumption we conclude that the distance traveled has to be considered to estimate fuel savings in a time basis, say per hour of operation.

It is therefore valuable to present fuel consumption results and savings in terms of fuel usage per unit time. The volumetric fuel consumption rate, $F^{*} . C$., and its uncertainty are expressed in terms of fuel economy and average speed:

Equation 7.10 Volumetric Fuel Consumption Rate

$$
\begin{gathered}
F^{*} . C .(\mathrm{gal} / \mathrm{hr})=\frac{\bar{U}(\mathrm{mi} / \mathrm{hr})}{F . E .(m i / g a l)}=\frac{F . C \cdot(\mathrm{g} / \mathrm{mi})}{\rho_{\text {fuel }}(\mathrm{g} / \mathrm{gal})} \cdot \bar{U}(\mathrm{mi} / \mathrm{hr}) \\
\stackrel{*}{\Delta F . C .}= \pm \frac{\bar{U}}{F . E_{.}^{2}} \cdot \Delta F . E .
\end{gathered}
$$

The fuel saving rate is the difference between the conventional and hybrid volumetric fuel consumption rates:

Equation 7.11 Volumetric Fuel Saving Rate

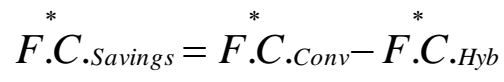

$$
\begin{aligned}
& \Delta F^{*} \cdot \text { C. Savings }=\left[\left(\Delta F^{*} \cdot C \cdot \text { Conv }\right)^{2}+\left(\Delta F^{*} \cdot C \cdot H y b\right)^{2}\right]^{1 / 2}
\end{aligned}
$$

Fuel consumption rates and fuel saving rates are presented in Figure 7-36. Figure 7-36 a compares conventional and hybrid fuel consumption rates where it is observed that, for the same service time, high speed cycles consume three to four times more fuel than inner-city cycles. Figure 7-36 $b$ and c show fuel saving rates against average speed in the first figure and against kinetic intensity in the second.

Contrasting Figure 7-35 $a$ with Figure 7-36 $b$ it is concluded that although hybrid advantage is highest at low speeds the highest saving rates are observed at middle to high speeds. That is, even when at low speed the relative benefit of hybridization is highest it does not translate in the largest savings; this is because routes at higher speeds which have a smaller relative advantage (H.A.) consume more fuel and their absolute advantage is higher. This only considers savings from fuel and 
further conclusions require an assessment of the total bus operational costs, including maintenance and capital costs, among others.

It is also noted that for a given speed there is substantial variability in the savings rate which cannot be explained by $\bar{U}$ alone. This variability can be explained jointly by $\bar{U}$ and $\tilde{a}_{n o}$ grade as shown in the regression surface of Figure 7-36 $d$. Being $\bar{U}$ constant, the highest fuel savings correspond to routes with the highest values of characteristic acceleration.

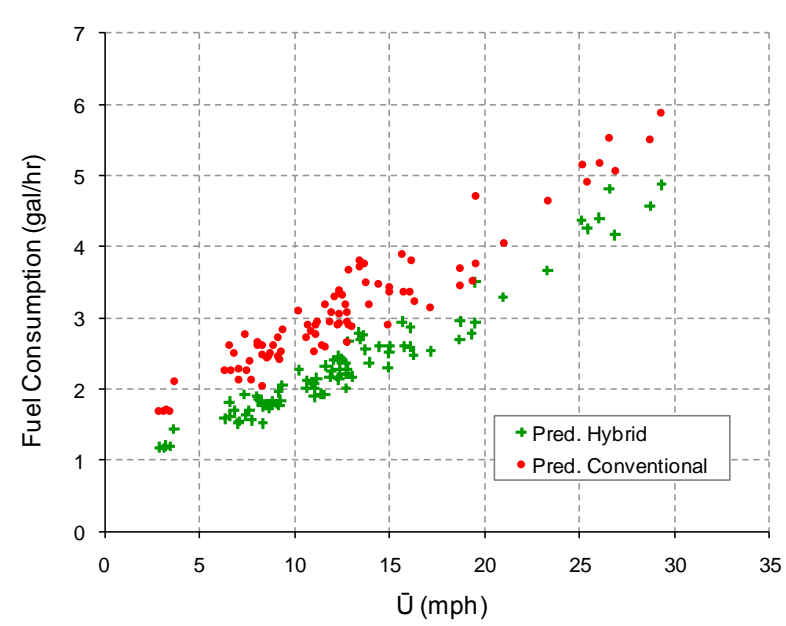

(a) Hybrid and Conventional

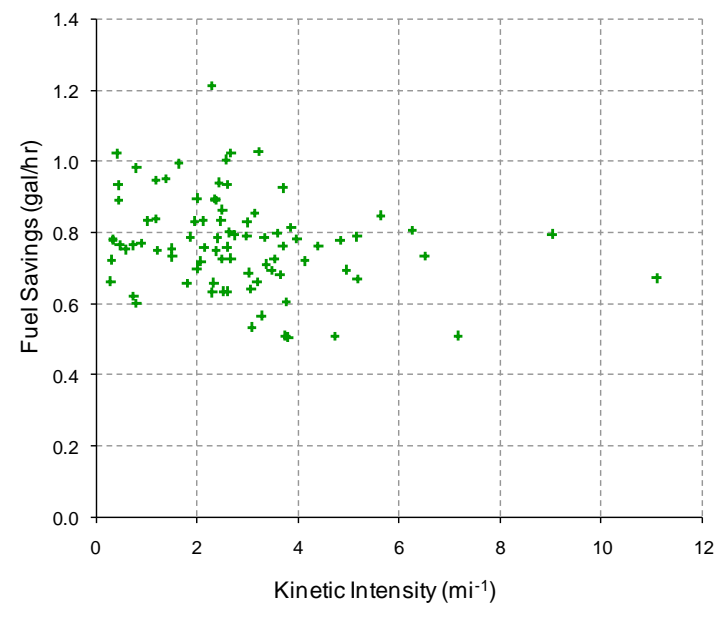

(c) Savings versus Kinetic Intensity

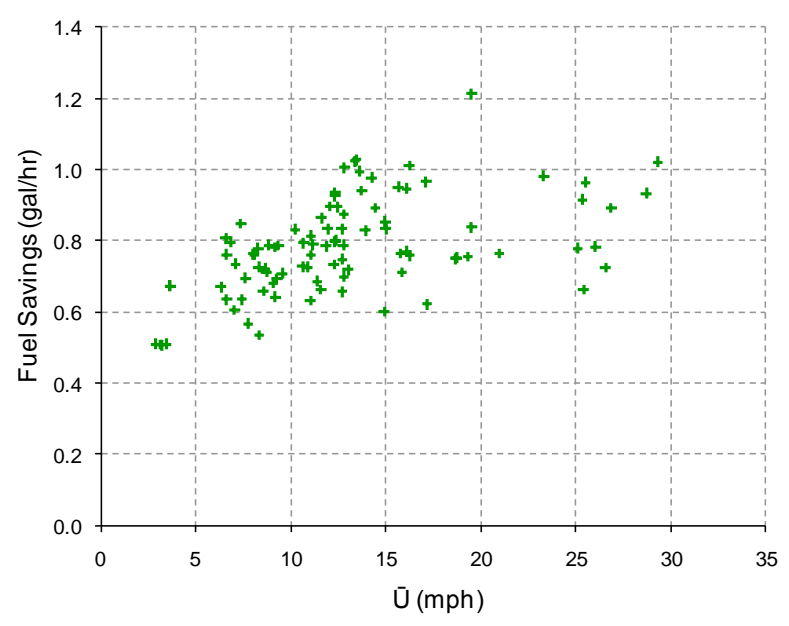

(b) Savings versus Average Speed

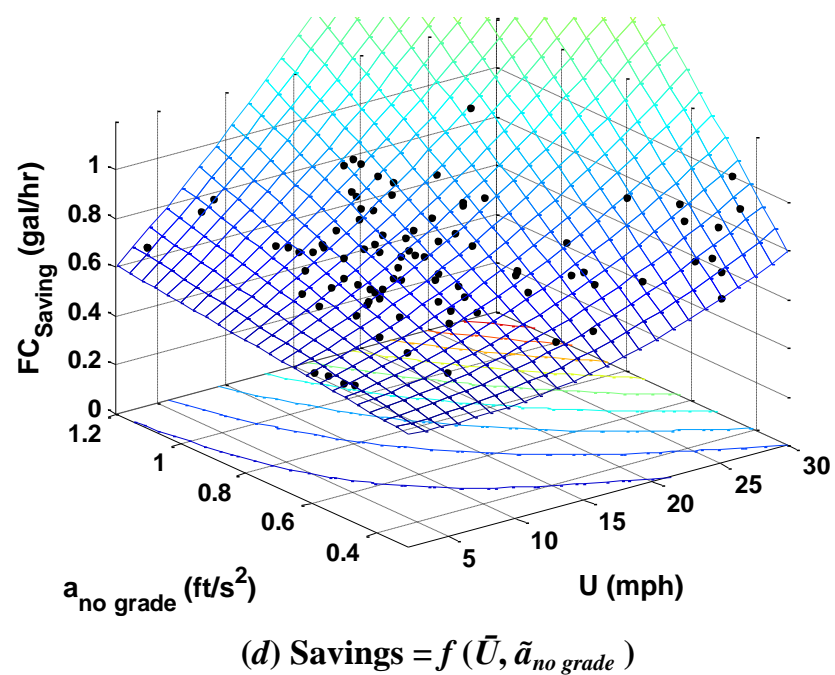

Figure 7-36 Fuel Consumption Rates and Fuel Saving Rates

Now, considering the reductions in distance specific $\mathrm{CO}_{2}$ emissions, it is concluded from Equation $7.8 a$ and Equation $7.4 b$ that the $\mathrm{CO}_{2}$ reduction is equal to the hybrid advantage. Also, from Equation $7.10 b$, the $\mathrm{CO}_{2}$ saving rate is directly proportional to the volumetric fuel saving rate: 
Equation 7.12 $\mathrm{CO}_{2}$ Savings Rate

$$
\begin{aligned}
& \stackrel{*}{C} \mathrm{O}_{2 \text { Savings }}(\mathrm{kg} / \mathrm{hr})=\text { F.C.Savings }^{*}(\mathrm{gal} / \mathrm{hr}) \cdot \rho_{\text {fuel }}(\mathrm{g} / \mathrm{gal}) \cdot \text { Fuel }_{\text {Carbon }} \cdot 44 / 12 \cdot(1 \mathrm{~kg} / 1000 \mathrm{~g}) \\
& \Delta \stackrel{*}{C^{O}}{ }_{2 \text { Savings }}= \pm \Delta F^{*} \cdot \text {.C.Savings } \rho_{\text {fuel }} \cdot \text { Fuel }_{\text {Carbon }} \cdot 44 / 12 / 1000
\end{aligned}
$$

\subsubsection{Uncertainty Figures}

The equations above defined the various results and their corresponding uncertainties. Recall that these uncertainties represent the statistical 95\% confidence on the mean response and are not meant to represent the error on individual observations. Figure 7-37 presents the confidence intervals for selected results. Figure 7-37 $a$ illustrates the confidence intervals for hybrid and conventional fuel economy predictions; there uncertainties are nearly equivalent to a $2 \%$ error in F.E. The uncertainties are adequate considering the wide range of operating conditions covered by the model. For the hybrid model, individual fuel economy relative uncertainties have the following 5 number summary (minimum, $1^{\text {st }}$ quartile, medial, $3^{\text {rd }}$ quartile, maximum): - $7.8 \%,-2.5 \%,-0.5 \%, 2.2 \%, 9.1 \%$.

Figure 7-37 $b$ shows the confidence intervals in hybrid advantage; the uncertainties can be as high as $20 \%$ with an average uncertainty of $7 \%$. Even though uncertainties in fuel economy were of the order of $2 \%$, error propagation works to increase the uncertainties and even more when the values are subtracted as in the case of H.A., fuel savings and $\mathrm{CO}_{2}$ savings.

Figure 7-37 $c$ presents the uncertainty in fuel savings. The 5-number summary for the distribution of fuel savings relative uncertainty was: $3.6 \%, 6.2 \%, 7.3 \%, 9.0 \%, 19.1 \%$. The data show that in every case the null hypothesis (there are not fuel savings) can be rejected.

Figure 7-37 $d$ shows the predicted fuel savings (and their uncertainties) when the model input is average speed alone; fuel savings with the full model are also shown. If one was to draw conclusions from the predicted values alone, it would look as if the highest savings were achieved at high speeds; but the uncertainty bars tell us that such conclusion is not accurate as the same levels of savings can be achieved at intermediate speeds as well. This emphasizes the importance of including margins of error in the predictions. It also signals that the three input parameters $\left(\bar{U}\right.$, Idle,$\tilde{a}_{n o}$ grade $)$ are necessary in order to predict fuel savings accurately. 


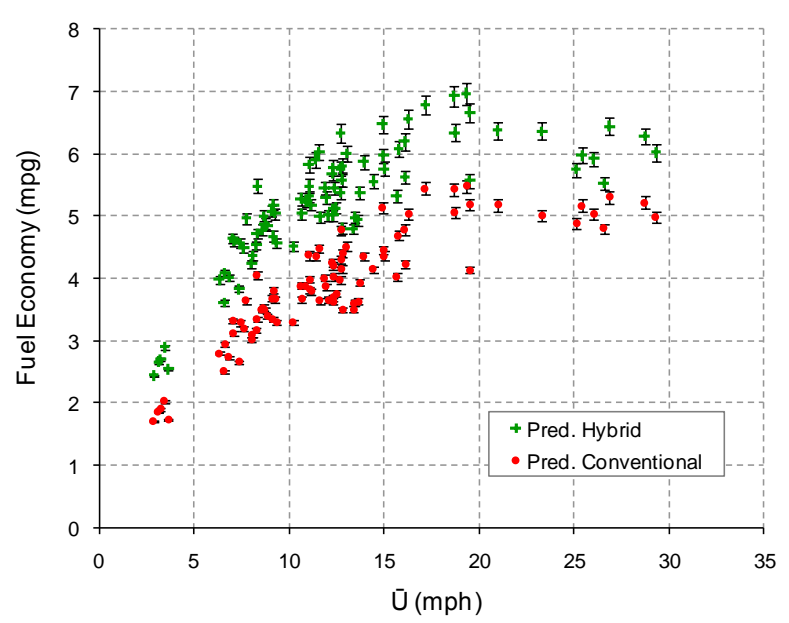

(a) Pred. Fuel Economy

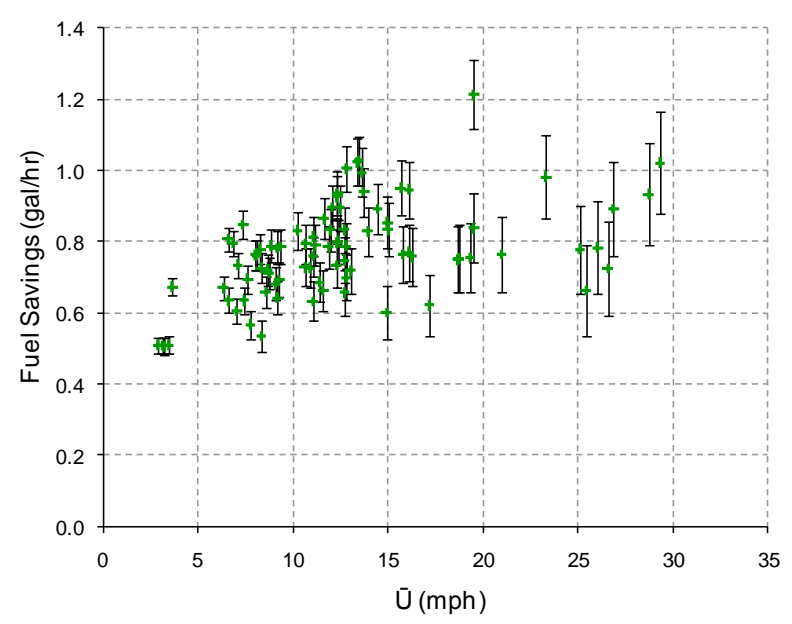

(c) Pred. Fuel Savings

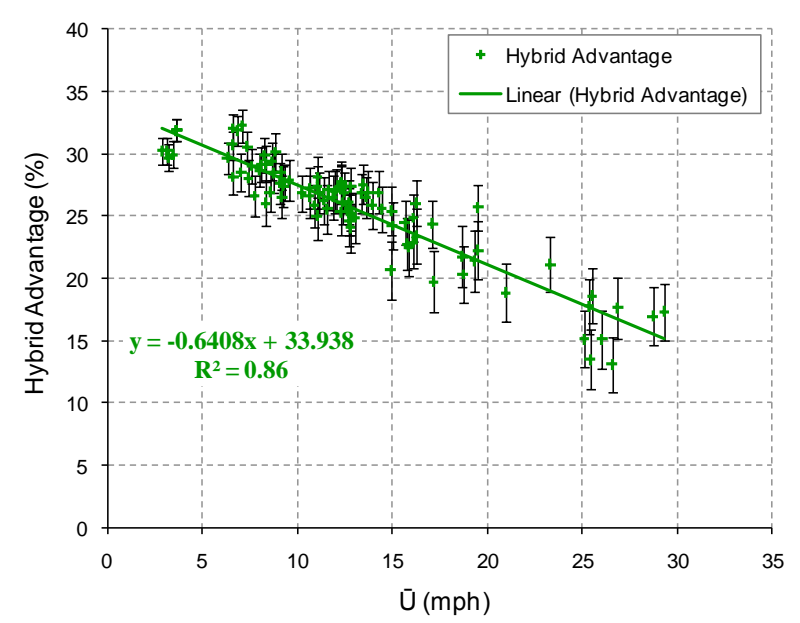

(b) Pred. Hybrid Advantage

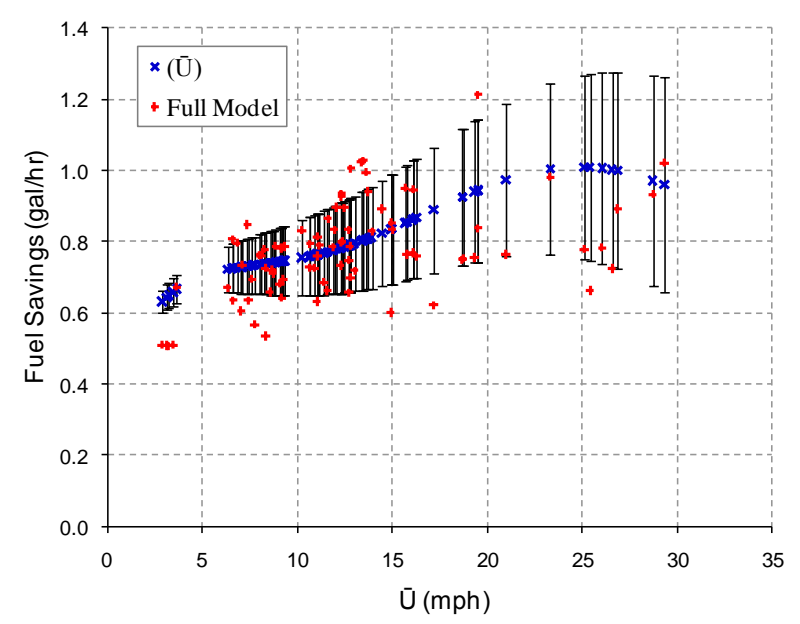

(d) Pred. Fuel Savings Input $\bar{U}$

Figure 7-37 Uncertainty Bars in Selected Results

\subsubsection{Transit Service Groups}

Four major categories of transit bus service were identified in Chapter 4, namely: Inner-City, Urban, Suburban, and Commuter. The duty cycle metrics that characterize an average route in these categories were presented in Table 4-7. Fuel economy and savings were predicted for the service type centroids and the results are shown in Figure 7-38. Part $a$ of the figure shows the conventional versus hybrid fuel economy parity plot while part $b$ shows fuel savings.

Prediction uncertainties are shown in the form of error bars. Unfortunately, overlapping of the error bars does not allow pointing to a single service category as the one best suited for hybrid buses. 
Contrary to the general belief that Inner-City service presents the highest room for fuel savings, InnerCity service shows the lowest savings potential among the four categories.

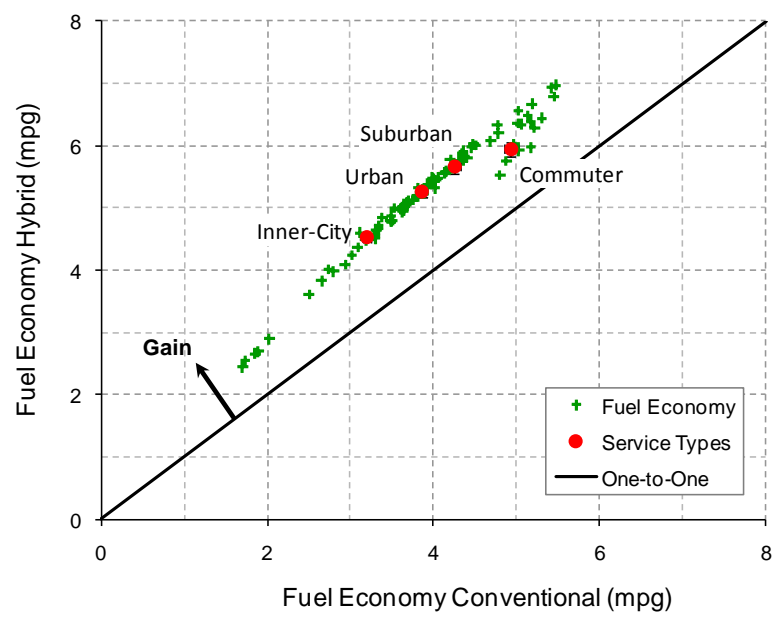

(a) Fuel Economy

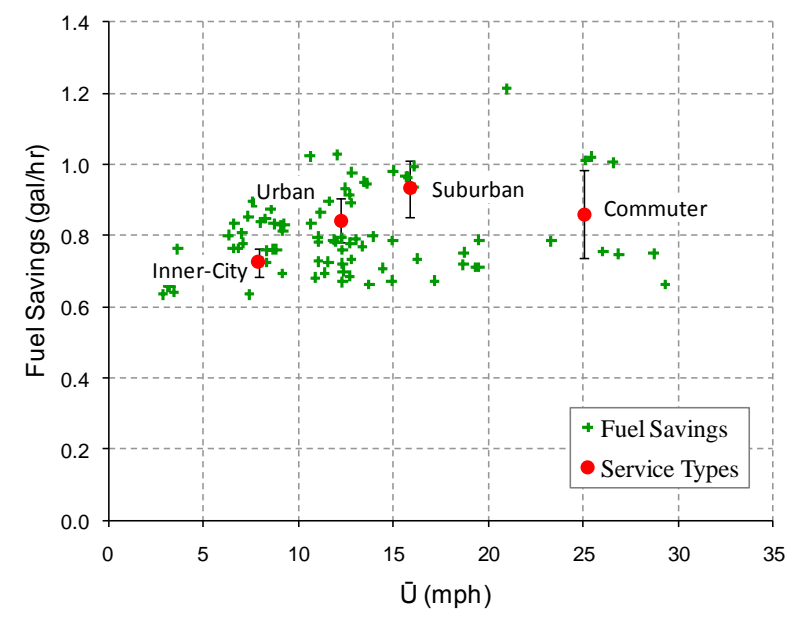

(b) Fuel Savings

Figure 7-38 Fuel Economy and Savings for the Four Bus Service Categories

What can be concluded is that not all routes in a particular service category, e.g. urban service, will get the same benefit from hybridization. The prediction tools developed in this dissertation give transit agencies the ability of exploring where their hybrids can be best exploited. The agencies will need to collect more information than average speed alone in order to obtain reliable results; for a given service category some routes showed savings as much as $60 \%$ higher than others.

\subsubsection{Effect of Automatic Engine Idle-Stop}

The proposed fuel economy and emissions predictive approach allows for a straightforward evaluation of the impacts of engine idle-stop strategies. Suppose that the vehicle had the ability to shut-off the engine whenever it came to a stop; for practical purposes, this is equivalent to setting $\overline{\dot{m}}_{f \text { Idle }}$ and $\overline{\dot{m}}_{N O_{x} \text { Idle }}$ to zero in Equation 7.2 and Equation 7.3, respectively.

Figure 7-39 shows the resulting F.E., H.A., fuel savings, and $\mathrm{NO}_{\mathrm{x}}$ emissions figures. As expected, low speed routes are the ones that benefit the most because they have the highest idle fractions. On the other hand, the benefit for commuter routes is marginal (Figure 7-39 $b$ and Figure 7-37 $b$ ). Fuel savings are increased from between 0.5 and $1.0 \mathrm{gal} / \mathrm{hr}$ (Figure 7-37 c) to between nearly 1.0 and 1.4 gal $/ \mathrm{hr}$, i.e. an average $50 \%$ gain and as much as $140 \%$ over the baseline savings. 


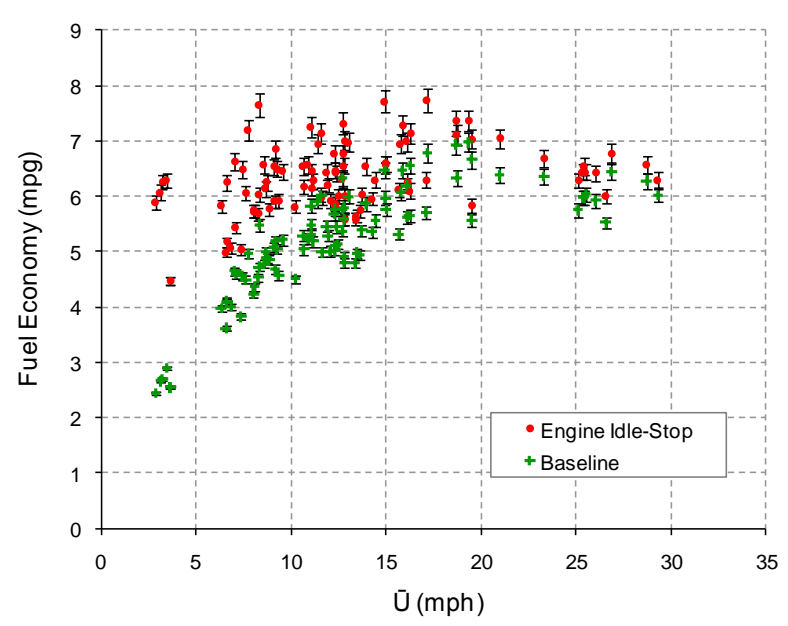

(a) Fuel Economy Hybrid Bus

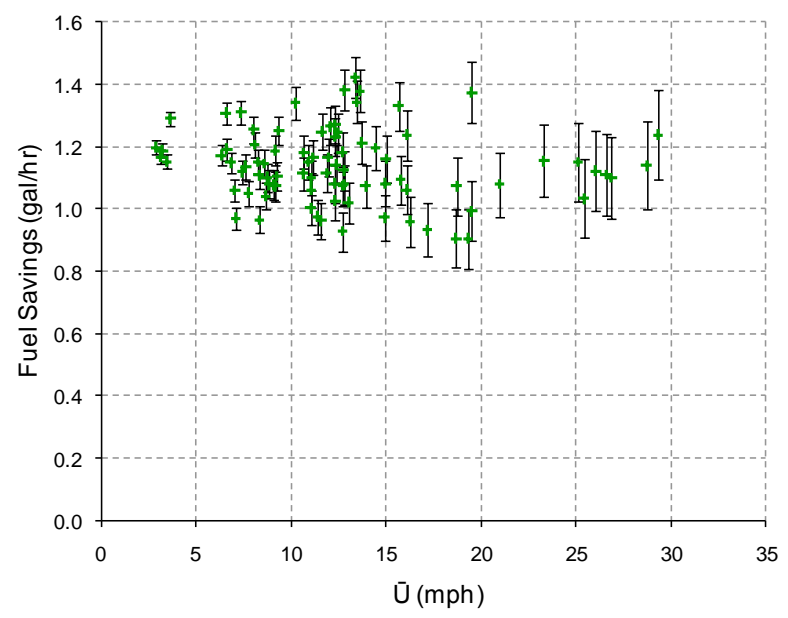

(c) Fuel Savings

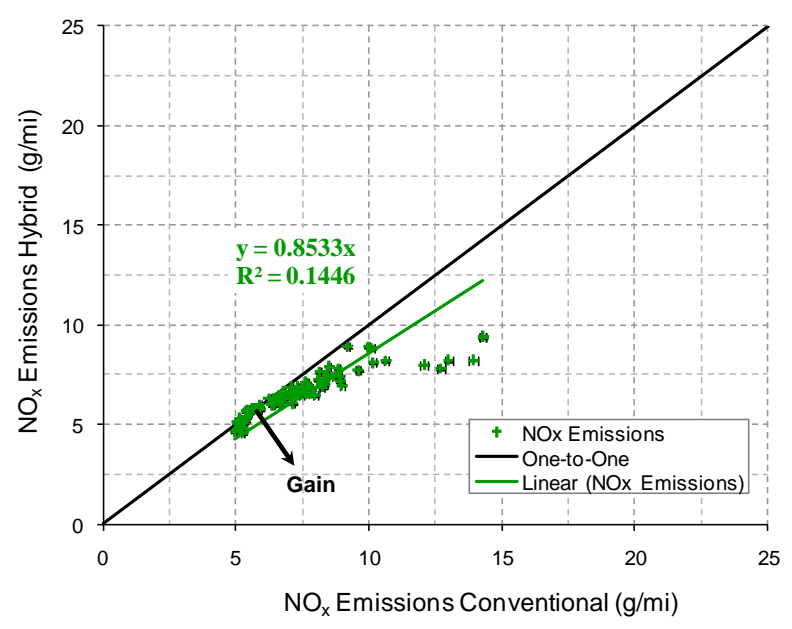

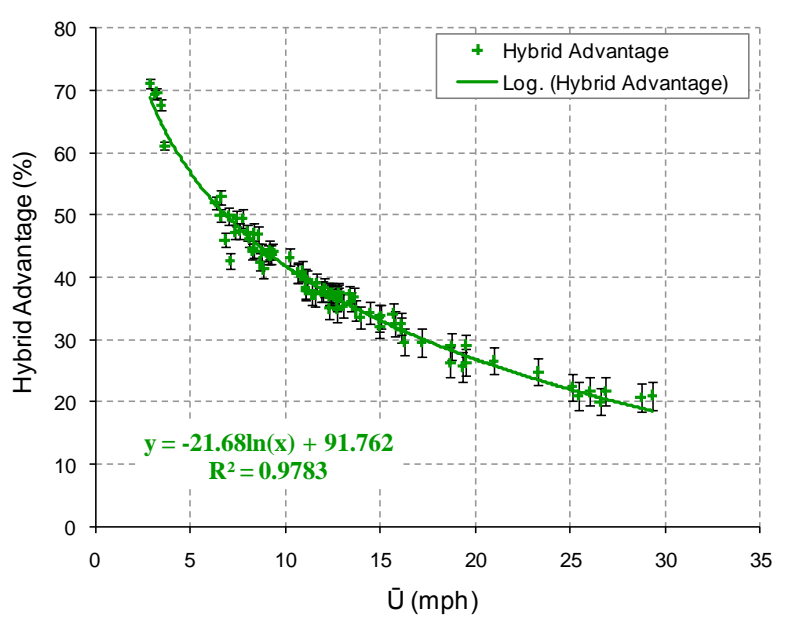

(b) Hybrid Advantage

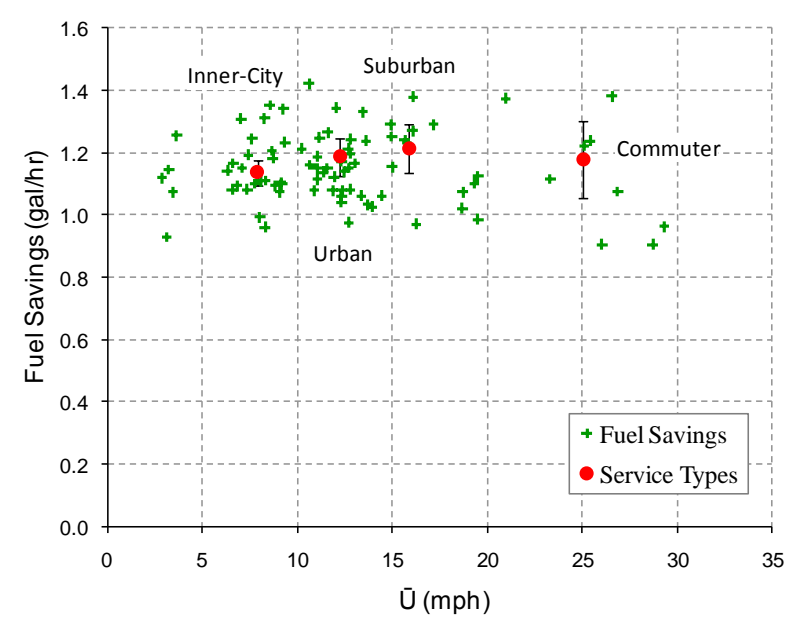

(d) Fuel Savings for Bus Service Categories

(e) $\mathrm{NO}_{\mathrm{x}}$ Parity Plot

Figure 7-39 Effect of Engine Idle-Stop 
Fuel savings of the four service categories are more similar with engine idle-stop (1.1 to $1.2 \mathrm{gal} / \mathrm{hr}$, Figure 7-39 $d$ ) than in the baseline scenario (Figure 7-38 b), as the error bars of the four categories overlap each other. Under this scenario there is a net reduction in $\mathrm{NO}_{\mathrm{x}}$ emissions (Figure 7-39 $e$ ).

These calculations neglect the presence of auxiliary loads such as lighting and hydraulic and pneumatic systems, which need to be sustained. As such, they represent a best case scenario for the impact of engine idle-stop on fuel consumption and $\mathrm{NO}_{\mathrm{x}}$ emissions.

\subsection{IBIS Tools}

Two tools were developed for IBIS. First, the GPS Data Cleaning Tool receives time-speed GPS logs of vehicle operation and performs data cleaning and filtering. A sample output from the GPS Data Cleaning Tool is presented in Figure 7-40 where noise in the GPS signal has been removed as well as the zero speed noise.

Second, the Hybrid Savings Calculator Tool is an implementation of the predictive models described in this chapter and allows transit agencies to evaluate their routes for hybridization fuel savings.

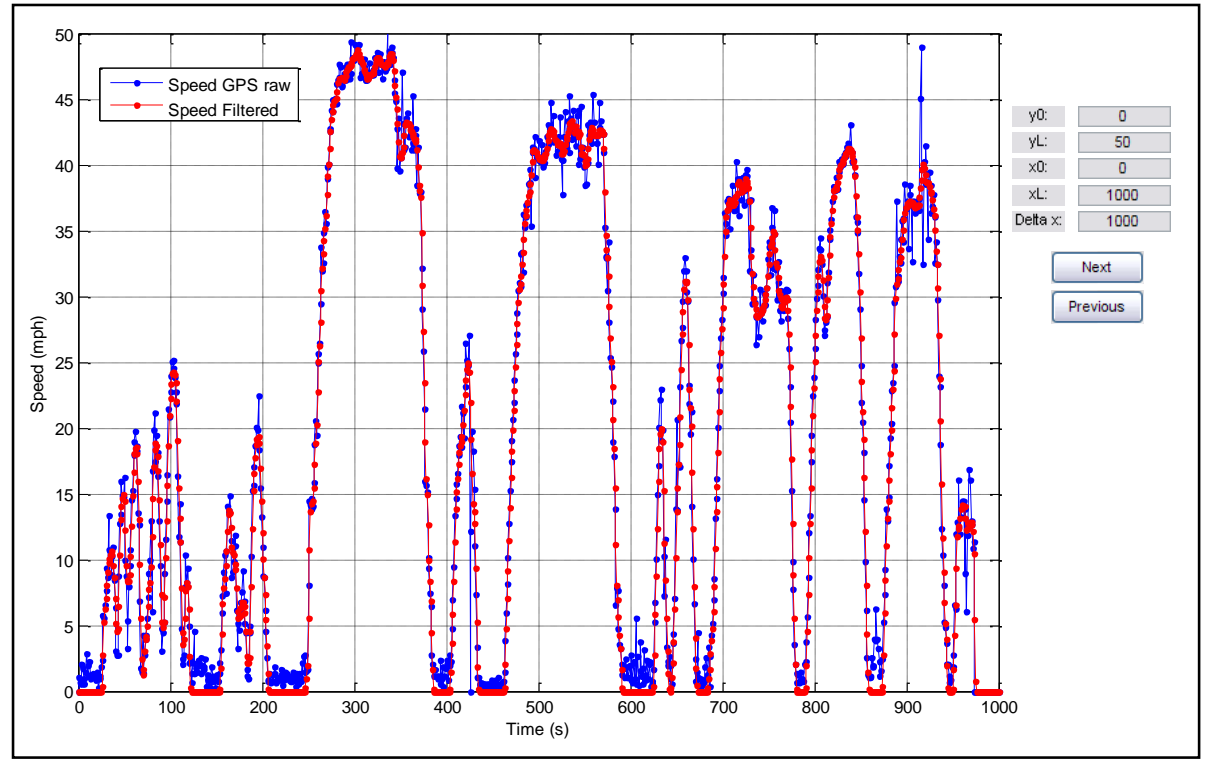

Figure 7-40 Sample Output from GPS Data Cleaning Tool

The input data menus of the savings calculator are shown in Figure 7-41. The user can upload a file such as the output file from the GPS data cleaning tool, type in his/her own cycle metrics or select from the four service categories. In the second option, the user could either type in the three inputs or enter route average speed and let the software approximate Idle and $\tilde{a}_{n o}$ grade. The uncertainties that 
correspond to the limited input cases are taken into account automatically. The user may enter as many cycles as desired.

A warning is issued when the inputs do not satisfy the requirements indicated in Section 7.2.5. After the user has finished entering data, the software proceeds to calculate hybrid and conventional fuel economy, hybrid advantage, fuel savings, and $\mathrm{CO}_{2}$ savings. Figure 7-42 shows a sample output. First, user inputs are plotted with the cycle database metrics to warn against inputs that are outside of the observed domain. Then, a summary is presented for each route and printed to screen (numeric results), and, finally, the results are given graphically plotted versus average speed.

\subsection{Summary}

This chapter analyzed the effect of duty cycle on performance of the hybrid bus with the purpose of predicting savings on fuel, $\mathrm{CO}_{2}$ emissions, and $\mathrm{NO}_{\mathrm{x}}$ emissions. The emissions and fuel economy prediction method was presented along with its corresponding uncertainties. The effect of limited inputs into the regression models was studied and the limits (ranges) of the acceptable input parameters were shown.

Chassis-dynamometer test data and the virtual cycles developed by Tu (2011) were used to build the regression models for fuel economy, $\mathrm{CO}_{2}$, and $\mathrm{NO}_{\mathrm{x}}$ of the conventional diesel transit bus. Comibint the predictions for conventional and hybrid, the benefits of hybridization were presented in terms of volumetric fuel savings rate and hybrid advantage. The potential effects of automatic engine idle-stop were evaluated.

The chapter closed with a brief discussion of the two tools developed for IBIS: the GPS Data Cleaning Tool and the Hybrid Savings Calculator Tool. 


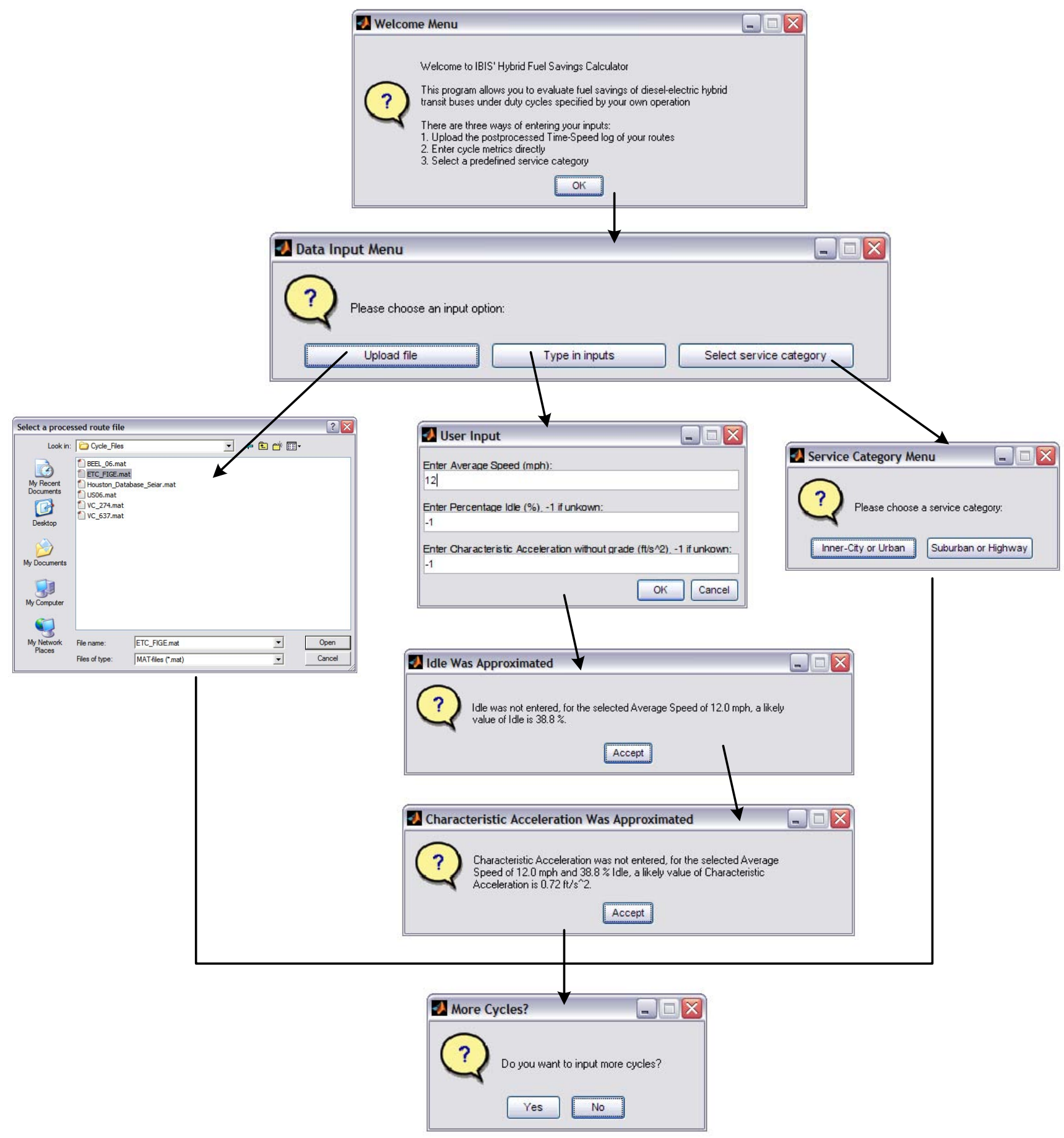

Figure 7-41 Data Input Menus for the Hybrid Savings Calculator Tool 


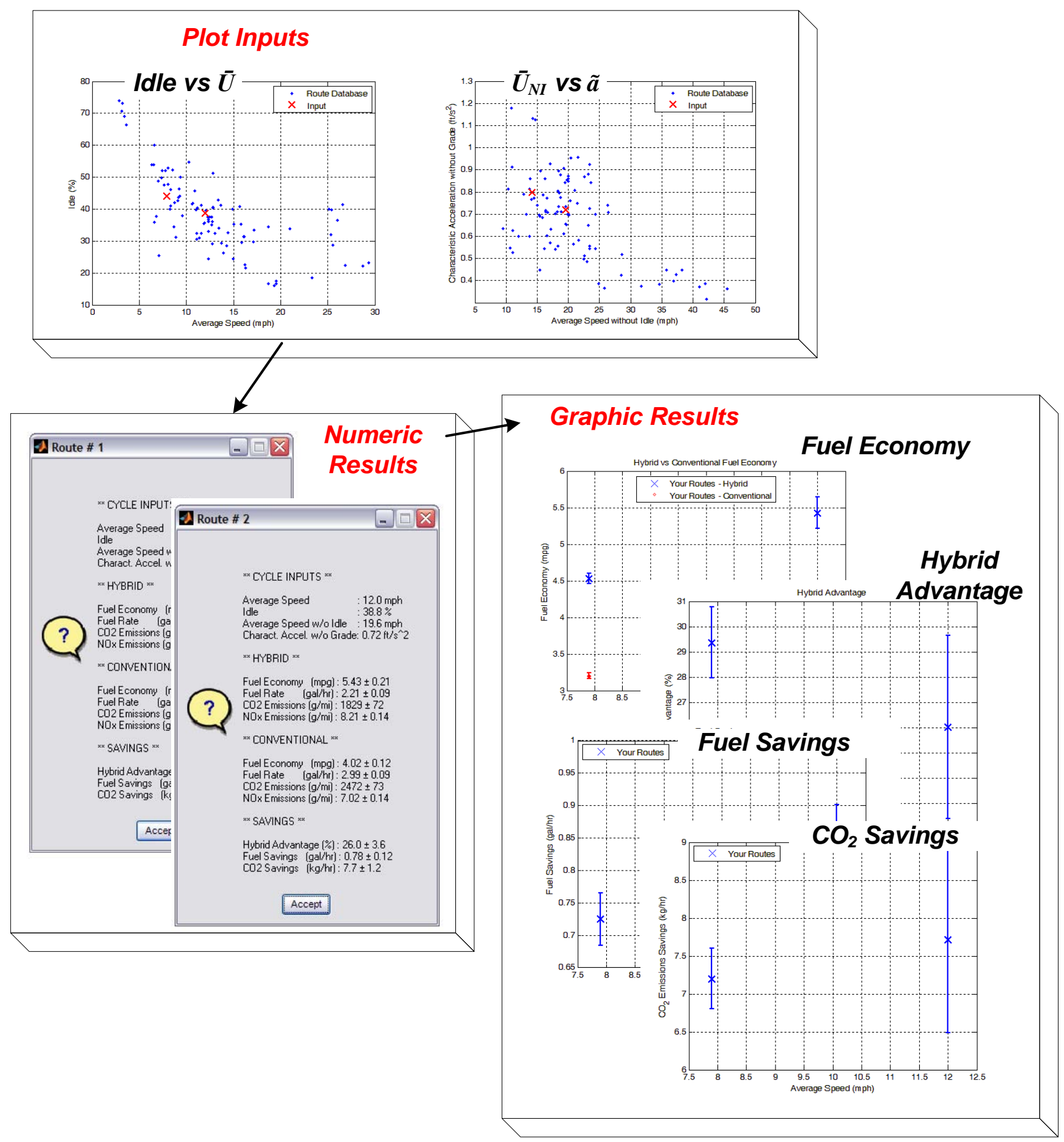

Figure 7-42 Outputs of the Hybrid Savings Calculator Tool 


\section{Chapter 8. CONCLUSIONS AND RECOMMENDATIONS}

\subsection{Summary}

During the last decade, many evidences were observed of the climate change effect resulting from anthropogenic activities. Substantial decline in arctic sea ice, rise in sea levels, and increase in average earth temperatures are making it harder for skeptics to prove their arguments. While the debate continues, government agencies throughout the world have introduced programs to significantly deploy alternative energy sources and reduce our carbon footprint. On the other hand, the price of crude oil has achieved levels of above 100 dollars per barrel, prices unseen since the petroleum crisis of the 1970s.

Of the total U.S. $\mathrm{CO}_{2}$ emissions, the power generation and transportation sectors accounted for 94\% in 2007 (Wayne et al., 2009). The transportation sector (public and private) was responsible for $31 \%$ of the national $\mathrm{CO}_{2}$ emissions in 2005 . Even tough, the contribution of public transit was only $1 \%$ of the transportation sector's share, it totaled 14.9 million metric tons of $\mathrm{CO}_{2}$.

Wayne et al. (2009) evaluated the potentials of alternative fuels and advanced technologies for reducing emissions in public transportation. They concluded that diesel-electric hybrid buses had the greatest potential to reduce $\mathrm{CO}_{2}$ emissions and fuel consumption; in the order of millions of diesel gallons within the U.S. transit fleet.

This dissertation studied transit bus routes and the effects of duty cycle on the fuel and emissions saving potential of the hybrid bus technology. The results of this dissertation will allow transit agencies to place hybrid buses in routes that take the most advantage of the hybrid-electric capabilities, maximizing fuel savings and reduction on $\mathrm{CO}_{2}$ emissions.

The project started with a preliminary analysis of the potential reductions in net tractive energy. Effects due to regeneration efficiency, vehicle weight, and road grade were studied. The final goal was the development of a regression based predictive model for fuel economy, $\mathrm{CO}_{2}$ and $\mathrm{NO}_{\mathrm{x}}$ emissions, and fuel savings for MY 2007-2009 diesel-electric hybrid buses. The predictive model had to be representative of the wide range of transit bus operation. 
With this goal in mind the following research approach was taken: (i) transit bus routes were studied considering routes from slow speed (congested traffic) to high speed (commuter service). (ii) A computational vehicle dynamic model was developed to represent the performance of diesel-hybrid buses. (iii) The vehicle model was simulated over the duty cycles determined in stage $i$. These simulations provided a database of continuous fuel consumption and emissions that served as the basis for the development of the desired predictive models. (iv) Predictive models were developed for F.E., $\mathrm{CO}_{2}$ and $\mathrm{NO}_{\mathrm{x}}$ emissions. The model breaks up fuel consumption and emissions into the idle and driving contributions, which are predicted based on user inputs, and aggregated to determine cycle F.E. and emissions.

A wide range of transit bus operation was evaluated using GPS and ECU logs of routes collected at Washington Metropolitan Area Transit Authority. These logs provided a good representation of the overall transit operation. Cycle metrics were computed and their relationships studied. Four service categories of transit operation were determined and classified by average speed: Inner-City ( $\bar{U} \leq 10)$, Urban $(10<\overline{\mathrm{U}} \leq 14)$, Suburban $(14<\overline{\mathrm{U}} \leq 20)$, and Commuter $(\overline{\mathrm{U}}>20)$.

Correlations between pairs of metrics were explored. It was determined that average speed is well correlated with most of the other metrics, except for characteristic acceleration and percentage idle. The author concluded that the three nearly independent metrics (average speed, characteristic acceleration, and percentage idle) should be explored as predictor metrics for emissions and fuel consumption. The emissions and F.E. predictive models were developed with this set of metrics and the results were remarkable; the coefficients of determinations were 0.963 for F.E, 0.986 for $\mathrm{CO}_{2}$, and 0.998 for $\mathrm{NO}_{\mathrm{x}}$.

The effects of grade were not analyzed since it is unlikely that transit agencies would have this information. Accurate determination of road grade poses a further obstacle than simply logging route speed to characterize the duty cycle.

Two tools were developed for IBIS. The first tool receives GPS route logs from the transit agencies and proceeds to filter the speed signal. The tool cautions the user when abnormal accelerations are found; these are mostly caused by the GPS losing satellites and presenting sharp speed steps. The second tool is an implementation of the F.E. and emissions predictive models. It receives user inputs from three possible sources: (i) post processed GPS logs (output from the first tool), (ii) cycle metric values, and (iii) selection from the four service categories. If the user does not have Idle or $\tilde{a}_{\text {no grade }}$ information, the program can estimate these parameters. The uncertainty on the predictions was evaluated and reported. 


\subsubsection{PSAT Model for Series Diesel-Electric Hybrid Transit Bus}

A PSAT model for a series hybrid transit bus was developed and validated. Test data was from a MY 2006 Orion bus powered by BAE Systems' HybriDrive ${ }^{\circledR}$ tested by WVU's TransLab; the bus was tested over six duty cycles. ECU broadcasts of fueling rate, engine speed and engine torque were combined with emissions measurements to develop and validate the engine fuel consumption and $\mathrm{NO}_{\mathrm{x}}$ emissions models.

The fuel consumption model was a $2 \mathrm{D}$ map or look-up table on torque and speed. The $\mathrm{NO}_{\mathrm{x}}$ emissions model was based on ANNs. Due to the computational efforts required to run the ANN in Simulink, the $\mathrm{NO}_{\mathrm{x}}$ ANN calculations were performed offline using the "calculate_user_perso" option in PSAT. $\mathrm{NO}_{\mathrm{x}}$ emissions were corrected from 2006 levels to 2007-2009 levels assuming a 15\% reduction independent of location in the map.

BAE Systems provided specifications for their hybrid system which allowed appropriate scaling and modification of PSAT default components to match the test vehicle specs. This process was developed in cooperation with Bell (2011). No validation data was available for individual hybrid components. The overall model was validated at the vehicle level evaluating its performance for fuel economy, $\mathrm{CO}_{2}$ and $\mathrm{NO}_{\mathrm{x}}$ when compared with chassis dynamometer test results.

It was acknowledged that the HybriDrive ${ }^{\circledR}$ control strategy did not match either of PSAT predefined controllers: thermostat and load following. As the purpose of the project was not to validate the HybriDrive ${ }^{\circledR}$ controller in PSAT but to validate the vehicle model as a system, it was decided to build upon PSAT controllers to achieve a vehicle model that had similar performance to the Orion bus.

The thermostat control was evaluated against the load following control and it was concluded that the latter was more similar to the HybriDrive ${ }^{\circledR}$ control. The similarity laid in engine operation varying as vehicle power demand varied whereas engine operation under thermostat control was fixed to the optimal engine efficiency point. Modified versions of PSAT's load following and braking controllers were used. The controllers were modified as follows:

$>$ A provision was established to recharge the battery whenever SOC was below a predefined threshold to bring SOC back to the SOC target. It was observed that the default controller did not monitor SOC directly and thus it would deplete the battery.

$>$ A provision was established to reduce traction motor performance if SOC was below a critical value and vehicle power demand was beyond the engine capabilities. Under such an event, the engine would recharge the battery to the target SOC value while output power from the traction motor would be restricted. It was observed that with the default controller, if the vehicle sustained high power demand (e.g. interstate driving at $70+\mathrm{mph}$ ), the engine maximum power may not be enough to continuously maintain such demand while the traction motor would still provide the 
power request depleting the battery without restriction. In other words, the original controller allowed full battery depletion when, for a sustained period, driver power request was beyond engine capabilities.

> Accessory loads: It was observed that electrical accessory loads were always engaged while mechanical accessory loads were disengaged at stop and engaged at driving. To produce a more realistic model, the braking control, which commands the engine during braking and stops, was modified to maintain mechanical accessory loads. It was also observed that the constant electrical loads acted to deplete the battery during idle segments. With this in mind, the controller was modified to maintain SOC constant during vehicle stop by having the generator load the engine and supply the required electrical load.

$>$ Electric only and engine stop options were disabled as these features were not observed in the Orion bus.

As the vehicle model was to be simulated under a variety of in-use transit routes, the model was brought offline of PSAT's interface once the development and calibration phases were finished. This allowed running a wide variety of routes in an automated manner. PSAT's continuous repeat option for SOC correction was implemented for the offline simulations. Only one repetition was necessary for short cycles as the modified hybrid control was able to maintain SOC at a stable level regardless of duty cycle. Longer cycles did not require repetitions.

A MATLAB ${ }^{\circledR}$ post processing script was developed to analyze offline simulation results. This script implemented SAE J2711 (SAE, 2002) calculations for $\triangle$ SOC to assure that no SOC corrections were necessary. Component efficiencies were calculated in the script, including the regenerative braking capture-redeployment efficiency. The script also evaluated fuel economy and distance specific -emissions and -fuel consumption.

\subsection{Conclusions}

The preliminary analysis of energy implications in hybrid vehicles suggested that fuel consumption (F.C.) should be predicted directly from cycle metrics and then translated to fuel economy. The transit route characterization study showed that three nearly independent cycle metrics were average speed, characteristic acceleration, and percentage idle, and they should be explored as the main predictors for cycle F.E. and emissions. These approaches were followed in developing the F.E. and emissions regression prediction models with successful results. 
When hybrid and conventional buses are compared, the reductions in distance specific fuel consumption (hybrid advantage, H.A.) were highest for low speed service. On the other hand, the highest fuel saving rates (gallons per hour) were observed at middle to high speed service. That is, even when at low speed the relative benefit of hybridization is highest that does not translate in the largest savings; this is because routes at higher speeds, which have a smaller relative advantage (H.A.), consume more fuel and their absolute advantage is higher. Unfortunately, overlapping of the error bars for fuel savings prediction did not allow pointing to a single service category as the one best suited for hybrid buses.

Contrary to the general idea that Inner-City (stop-and-go) service presents the highest room for fuel savings, the results showed that Inner-City service had the lowest fuel (and $\mathrm{CO}_{2}$ ) saving potentials among the four categories. This discussion only considers savings from fuel usage; further conclusions regarding which routes provide the biggest economic saving will require an assessment of the total bus operational costs, including maintenance and capital costs, among others.

It was concluded that for a given average speed, the highest fuel savings correspond to routes with the highest values of characteristic acceleration $(\tilde{a})$. This is because the higher the $\tilde{a}$, the more room there is for the hybrid system to recover regenerative braking energy and to balance engine operation.

The main concern for a transit agency when using the prediction model would be to obtain an accurate estimation of fuel economy and fuel savings. It is therefore necessary to use the full regression model (where $\bar{U}$, Idle, and $\tilde{a}_{n o}$ grade are the inputs) instead of using the model with $\bar{U}$ alone.

For a given service category some routes showed savings as much as $60 \%$ higher than others. Consequently, not all routes in a particular service category, e.g. urban service, get the same benefit from hybridization. The prediction tools developed in this dissertation give transit agencies the ability of exploring where their hybrids can be best exploited. As mentioned above, the agencies will need to collect more information than average speed alone in order to obtain reliable results. This implies instrumentation of some buses to collect route vehicle speed profiles with GPS or ECU monitors.

The benefits from engine idle-stop were briefly explored by setting idle fuel and $\mathrm{NO}_{\mathrm{x}}$ rates to zero. Fuel savings were increased from between 0.5 and $1.0 \mathrm{gal} / \mathrm{hr}$ to between nearly $1.0 \mathrm{and} 1.4 \mathrm{gal} / \mathrm{hr}$, i.e. an average $50 \%$ gain and as much as $140 \%$ over the baseline savings. These figures neglect the presence of auxiliary loads and are therefore equivalent to upper bound values for the series hybrid bus.

The data did not reveal a net benefit in $\mathrm{NO}_{\mathrm{x}}$ emissions from hybridization. On average hybrid $\mathrm{NO}_{\mathrm{x}}$ emissions predictions were $17 \%$ higher than for the conventional diesel. This fact can be explained by the lower loads experienced by the engine on the hybrid bus, as lower loads are associated with higher brake-specific $\mathrm{NO}_{\mathrm{x}}$; this would not be the case if considerable engine downsizing were observed in hybrid buses, but his is not the case since engines in conventional $40 \mathrm{ft}$ transit buses have a rating of $280 \mathrm{hp}$ while the ratings are $260-280 \mathrm{hp}$ for the hybrids. Finally, considering that EPA's $\mathrm{NO}_{\mathrm{x}}$ 
certification level dropped to $0.2 \mathrm{~g} / \mathrm{bhp} \cdot \mathrm{hr}$ in 2010 , the observed effect in $\mathrm{NO}_{\mathrm{x}}$ emissions should raise no concerns.

\subsection{Recommendations}

The developed fuel consumption ANN failed to produce accurate results when used with the PSAT vehicle model. ANN fuel consumption results were too low and engine efficiencies were unrealistic. The author looked back and found that a good part of the training data was corrupted in the sense that fuelling rates were too low for the given output power. This was translated into unrealistic efficiencies. Thus, the problem of the ANN performance could be attributed to issues with the training dataset. A recommendation for development of ANN engine fueling models is that training data should be filtered to remove such unrealistic efficiency points.

When characterizing in-use bus service routes, it was observed that vehicle speed logs from ECU were better than GPS values. It would then be advisable to log ECU vehicle speed when feasible rather than GPS speed. One advantage of GPS speed over ECU data is that it is accompanied by longitude and latitude position which may serve to estimate road grade in conjunction with a topographic map of the area.

The best estimations of road grade were determined with GPS location and topographic map information. GPS reported elevation profiles showed to be of little or no value for determining road grade and therefore are not recommended. Barometric inferred elevation presented inexistent elevation changes; the author believes these were due to gusts of wind that affected the static pressure reading by dynamic pressure. Barometer readings were good predictions of elevation changes at highway speeds (where the air flow is well oriented with the static pressure probe) but were poor predictors at low speeds.

\subsubsection{Route Data Logging Procedures}

When considering data logging systems for characterization of duty cycles, there are three basic options: a GPS logger, an ECU data logger (through an ECU J1939 interface), and the combination of the two. The GPS logger is the simplest to install because it does not require configuration with the test vehicles. GPS gives an advantage over ECU because it collects position (latitude and longitude), which can be used to evaluate road grade, as was done in this project, with the help of a topographic map. 
ECU vehicle speed is preferred over GPS speed because it contains much less noise. On the other hand, as GPS data are easier to collect than ECU data, a greater utilization of the tools developed in this project can be achieved if GPS data are used.

The length of the GPS antenna should be at least $20 \mathrm{ft}$. Depending on where the data logger is installed and the height of the bus you may encounter that there is not enough slack for the antenna to reach the roof. This was the case when the system was installed in articulated buses.

Raw log files were manually pre-processed to correct sections where the GPS lost all the satellites and produced rubbish data. When these sections were short, speed and position (longitude and latitude) were filled in assuming a linear variation between the nearest good points. When the sections were long they had to be removed. These procedures may serve as guidelines for pre-processing GPS data. The GPS Data Cleaning Tool can be used when pre-processing the data as it has built in warnings when accelerations are out of bounds; these peak accelerations are usually a result of the GPS losing satellites. A $1 \mathrm{~Hz}$ logging frequency is suggested for route logs. More details on the data reduction procedure can be found in Section 4.2.

Topographic maps for road grade estimation require a large amount of computational memory. To avoid this problem, it is recommended that the map is as small as possible, i.e. it contains no more than the latitudes and longitudes travelled by the test vehicles. For this study, the map covered 625 square miles and had a resolution of $1 / 3$ arc second (about $33 \mathrm{ft}$ ). The grade calculation MALAB® scripts developed for the project were not generalized as was the GPS Data Cleaning Tool; they could be modified upon request to incorporate topographic maps and provide road grade estimations to interested users.

\subsection{Suggestions for Future Research}

The effect of grade was not explored for the in-use logged routes. The vehicle dynamic model developed in this dissertation can be used with the in-use route logs to evaluate the effects of road grade on fuel economy, $\mathrm{CO}_{2}$ and $\mathrm{NO}_{\mathrm{x}}$ of the hybrid transit bus. The preliminary analysis of Chapter 3 showed increases of 5 to $10 \%$ in characteristic acceleration when geometric grade profiles were superimposed to existing drive cycles. On the other hand, analysis from the in-use routes of Chapter 4 revealed an increase in $\tilde{a}$ of up to $25 \%$ when true road grade was considered versus the route assuming a flat terrain. The true effect of grade on F.E. and emissions needs to be studied.

The tools developed for this dissertation can be expanded to build a tool for IBIS that receives route logs from transit agencies and determines road grade with topographic maps. This procedure was used when processing the WMATA route logs. 
Data reduction of chassis dynamometer test measurements is an involved process. Evaluation of the corresponding bias uncertainties is complex and has not been performed for WVU's TransLab Laboratories. A sponsored program that explores laboratory uncertainty as well as detection limits would be beneficial for assessments and comparisons of transit technologies. 


\section{REFERENCES}

APTA: American Public Transportation Association. "2009 Public Transportation Vehicle Database." Washington DC, June 2009.

An, F., and D. J. Santini. "Mass Impacts on Fuel Economies of Conventional vs. Hybrid Electric Vehicles.” SAE Technical Paper Series, 2004, SAE 2004-01-0572.

Andrei, P. "Real World Heavy-Duty Vehicle Emissions Modeling." Thesis (MS). West Virginia University, 2001.

Ang, B. W., and T. F. Fwa. "A study on the Fuel-Consumption Characteristics of Public Buses." Energy, 14 (12), (1989): 797-803.

ANL: Argonne National Laboratory. PSAT Documentation. Argonne, IL, 2008.

AutoSpeed.com, article by Julian Edgar, "Torque Overload: the GM Allison Hybrid Bus." AutoSpeed magazine, issue 384, June 62006.

BAE Systems. HybriDrive ${ }^{\circledR}$ Propulsion Overview. Source: Tom Webb, Director Business Development, Transport Systems, BAE Systems. 600 Main Street, Johnson City, NY 13790. July $6,2010$.

Barth, M., T. Younglove, and G. Scora. Development of a Heavy-Duty Diesel Modal Emissions and Fuel Consumption Model. California Partners for Advanced Transit and Highways (PATH). Research Report UCB-ITS-PRR-2005-1, Berkeley CA, 2005.

Bass, E., and T. Alfermann. "The Influence of Idle, Drive Cycle and Accessories on the Fuel Economy of Urban Hybrid Electric Buses - Chassis Dynamometer Tests." SAE Technical Paper Series, 2003, SAE 2003-01-3438.

Bedick, C. R. “Optimization of a Retrofit Urea-SCR System.” Dissertation (Ph.D). West Virginia University, 2009.

Bell, C. "An Investigation of Road Load Effects on Fuel Economy and $\mathrm{NO}_{\mathrm{x}}$ Emissions of Hybrid and Conventional Transit Buses.” Thesis (MS). West Virginia University, 2011. 
Buckley, F. T. Jr., C. H. Marks, and W. H. Walston Jr. "Analysis of Coast-Down Data to Assess Aerodynamic Drag Reduction on Full-Scale Tractor-Trailer Trucks in Windy Environments." SAE Technical Paper Series, 1976, SAE 760850.

Buckley, Frank T. Jr. “ABCD - An Improved Coast Down Test and Analysis Method.” SAE Technical Paper Series, 1995, SAE 950626.

CAFEE: Center for Alternative Fuels, Engines, and Emissions. Emissions and Fuel Economy from Diesel and Diesel Hybrid-Electric Transit Buses. Final Report presented to U.S. FTA / U.S. DOT. West Virginia University, 2007.

CAFEE: Center for Alternative Fuels, Engines, and Emissions. Evaluation of Emissions and Performance of Transit Buses Utilizing a Biodiesel Fuel Blend. Final report presented to Central Florida Regional Transportation Authority, Contract 10-C01. West Virginia University, October 2010.

CAFEE: Center for Alternative Fuels, Engines, and Emissions. Emissions Analysis of WMATA Transit Buses. Final Report presented to WMATA. West Virginia University, 2011.

Chiang, Peter K. "Two-Mode Urban Transit Hybrid Bus In-Use Fuel Economy Results from 20 Million Fleet Miles.” SAE Technical Paper Series, 2007, SAE 2007-01-0272.

Ciccarelli, T., and R. Toossi. Assessment of Hybrid Configuration and Control Strategies in Planning Future Metropolitan/Urban Transit Systems. California State University Long Beach, Los Angeles CA, 2002.

Clark, N. N., J. J. Daley, R. D. Nine, and C. M. Atkinson. "Application of the New City-Suburban Heavy Vehicle Route (CSHVR) to Truck Emissions Characterization." SAE Technical Paper Series, 1999, SAE 1999-01-1467.

Clark et al., 2007a. Clark, N. N., ABM S. Khan, S. W. Wayne, M. Gautam, G. J. Thompson, D. L. McKain, D.W. Lyons, and R. Barnett. "Weight Effect on Emissions and Fuel Consumption from Diesel and Lean-Burn Natural Gas Transit Buses." SAE Technical Paper Series, 2007, SAE 2007-01-3626.

Clark et al., 2007b. Clark, N. N., M. Gautam, W. S. Wayne, G. Thompson, D. W. Lyons, F. Zhen, C. Bedick, R. J. Atkinson, and D. L. McKain. "Creation of the "Heavy Heavy-Duty Diesel Engine Test Schedule" for Representative Measurement of Heavy-Duty Engine Emissions.” CRC Report No. ACES-1. July 16, 2007. 
CFR: Code of Federal Regulations. Title 40: Protection of Environment, Part 86: Control of emissions from new and in-use highway vehicles and engines. U.S. Government Printing Office, Washington DC, 2006.

CFR: Code of Federal Regulations. Title 40: Protection of Environment, Part 1065: Engine Testing Procedures. U.S. Government Printing Office, Washington DC, 2006.

Conley, J. J. "The Role of Power and Energy Demands in Hybrid Vehicles." Thesis (MS). West Virginia University, 2002.

Conlon, B. "Comparative Analysis of Single and Combined Hybrid Electrically Variable Transmission Operating Modes.” SAE Technical Paper Series, 2005, SAE 2005-01-1162.

CRC: Coordinating Research Council. Creation of the 'Heavy Heavy-Duty Diesel Engine Test Schedule' for Representative Measurement of Heavy-Duty Engine Emissions. CRC Report No. ACES-1, Alpharetta, GA, 2007.

Dembski, N., G. Rizzoni, A. Soliman, J. Fravert, and K. Kelly. "Development of Refuse Vehicle Driving and Duty Cycles.” SAE Technical Paper Series, 2005, SAE 2005-01-1165.

Duda, R.O., P.E. Hart, D.G. Stork. Pattern Classification. Second Edition. John Wiley \& Sons, New York, NY, 2001.

Duoba, M., H. Lohse-Busch, and T. Bohn. "Investigating Vehicle Fuel Economy Robustness of Conventional and Hybrid Electric Vehicles.” Electric Vehicle Symposium (EVS-21), Monaco, April 2-6, 2005.

Ergeneman, M., C. Sorusbay, and A. G. Göktan. "Exhaust Emission and Fuel Consumption of CNG/Diesel Fueled City Buses Calculated Using a Sample Driving Sample.” Energy Sources, 21, (1999): 257-268.

Fox, R.W., A.T. McDonald, and P.J. Pritchard. Introduction to Fluid Mechanics. Sixth Edition. John Wiley \& Sons, Hoboken, NJ, 2004.

Frey, H. C., N. M. Rouphail, H. Zhai, T. L. Farias, and G. A. Gonçalves. "Comparing Real-World Fuel Consumption for Diesel- and Hydrogen-Fueled Transit Buses and Implication for Emissions." Transportation Research Part D, 12, (2007): 281-291.

Friedman, J. H. "Multivariate Adaptive Regression Splines.” The Annals of Statistics, 19 (1), (1991): $1-67$.

Gajendran, P. "Development of a Heavy Duty Diesel Vehicle Emissions Inventory Prediction Methodology." Dissertation (Ph.D). West Virginia University, 2005.

Genta, G. Motor Vehicle Dynamics: Modeling and Simulation. World Scientific, London, 1997. 
Giechaskiel, B., M. Carriero, and G. Martini. "Heavy Duty Particle Measurement Programme (PMP): Exploratory Work for the Definition of the Test Protocol." SAE Technical Paper Series, 2009, SAE 2009-01-1767.

Gillespie, T. D. Fundamentals of Vehicle Dynamics. SAE International, Warrendale PA, 1992.

Grewe, T. M., B. M. Conlon, and A. G. Holmes. "Defining the General Motors 2-Mode Hybrid Transmission.” SAE Technical Paper Series, 2007, SAE 2007-01-0273.

Guensler, R., D. Sperling, and P. Jovanis. Uncertainty in the Emission Inventory for Heavy-Duty Diesel-Powered Trucks. Research Report, UCD-ITS-RR-91-02, June 1991.

Guzzella, L., and A. Sciarretta. Vehicle Propulsion Systems: Introduction to Modeling and Optimization. Second Edition, Springer, New York, 2007.

Hannon, R. E., N. A. Hinderer, and C. W. Greening Jr. "Dynamometer Simulation of Braking on Mountain Routes using Elevation Profiles." SAE Technical Paper Series, 2007, SAE 2007-014278.

Hayes, R. R., A. Williams, J. Ireland, and K. Walkowicz. King County Metro Transit: Allison Hybrid Electric Transit Bus Laboratory Testing. National Renewable Energy Laboratory, Technical Report NREL/TP-540-39996, 2006.

Heirigs, P., S. Delaney, and R. Dulla. MOBILE6 On-Road Motor Vehicle Emissions Model: 5-Day Training Course. Prepared for the U.S. Environmental Protection Agency, Contract No. 68-C70051, Work Assignment 3-06, 2001.

Holmén, B. A., Z. Chen, A. C. Davila, O. Gao, and D. M. Vikara. Particulate Matter Emissions from Hybrid Diesel-Electric and Conventional Diesel Transit Buses: Fuel and After-Treatment Effects. Report No. JHR 05-304. University of Connecticut, 2005.

Hucho, Wolf-Heinrich (Editor). Aerodynamics of Road Vehicles: From Fluid Mechanics to Vehicle Engineering. Fourth Edition, SAE International, Warrendale PA, 1998.

Jarrett, R. P., and N. N. Clark. "Weighting of Parameters in Artificial Neural Network Prediction of Heavy-Duty Diesel Engine Emissions.” SAE Technical Paper Series, 2002, SAE 2002-01-2878.

Kern, J. M. "Inventory and Prediction of Heavy-Duty Diesel Vehicle Emissions." Thesis (MS). West Virginia University, 2000.

Khan, ABM S. R. "Route and Grade Sensitive Modeling of Fuel Efficiency and Emissions for Diesel Buses.” Dissertation (Ph.D). West Virginia University, 2009.

Kim, N., Carlson, R., Jehlik, F., and A. Rousseau. "Tahoe HEV Model Development in PSAT." SAE Technical Paper Series, 2009, SAE 2009-01-1307. 
Kosto, M. E. "Modeling and Comparison of Transient Emissions Behavior of Hybrid and Conventional Vehicles.” Thesis (MS). Massachusetts Institute of Technology, 2001.

Krishnamurthy, M. "Development of Predictive $\mathrm{NO}_{\mathrm{x}}$ Model for On-Road Heavy-Duty Diesel Engines.” Dissertation (Ph.D). West Virginia University, 2006.

Madireddy, M.R., and N.N. Clark. "Sequential Inversion Technique and Differential Coefficient Approach for Accurate Instantaneous Emissions Measurement." International Journal of Engine Research 7(6), (2006):437-446.

Marlowe, C. L. "Development of Computational Tools for Modeling Engine Fuel Economy and Emissions.” Thesis (MS). West Virginia University, 2009.

Martini, G., B. Giechaskiel, and P. Dilara. "Future European emission standards for vehicles: the importance of the UN-ECE Particle Measurement Programme.” Biomarkers, 14 (S1), (2009): 2933.

McKain, D. L., N. N. Clark, T. H. Balon, P. J. Moynihan, S. A. Lynch, and T. C. Webb. "Characterization of Emissions from Hybrid-Electric and Conventional Transit Buses." SAE Technical Paper Series, 2000, SAE 2000-01-2011.

Miller, John M. "Hybrid Electric Vehicle Propulsion System Architectures of the e-CVT Type." IEEE Transactions on Power Electronics, 21 (3), (2006): 756-767.

Moynahan, N. A. "Development of a Vehicle Road Load Model for ECU Broadcast Power Verification in On-Road Emissions Testing.” Thesis (MS). West Virginia University, 2005.

Moore, David D., and George P. McCabe. Introduction to the Practice of Statistics. Fifth Edition, Freeman, New York, 2006.

NAVC: Northeast Advanced Vehicle Consortium. Hybrid Transit Bus Certification Workgroup: Engine Certification Recommendations Report. NAVC Report No. NAVC0599-AVP009903. Boston, MA, 2002. Page 10.

Nine, R. D., N. N. Clark, J. J. Daley, and C. M. Atkinson. "Development of a Heavy-Duty Chassis Dynamometer Driving Route.” Proc Instn Mech Engrs, 213 Part D, (1999): 561-573.

Nix, A. C., Principal Investigator. Co-Investigators: Wayne, W. S., Shade, B. C., Li, H., Clark, N.N., and M. Gautam. Emissions Analysis of WMATA Transit Buses: Technical Proposal. Morgantown, WV. Revision March 31, 2009.

Norton, Robert L. Design of Machinery: An Introduction to the Synthesis and Analysis of Mechanisms and Machines. Third Edition. McGraw-Hill, New York, 2004. 
O'Keefe, M. P., and K. Vertin. An Analysis of Hybrid Electric Propulsion Systems for Transit Buses. National Renewable Energy Laboratory, Milestone Completion Report NREL/MP-540-32858, 2002.

O'Keefe, M. P., A. Simpson, J. Kelly, and D. S. Pedersen. "Duty Cycle Characterization and Evaluation Towards Heavy Hybrid Vehicle Applications.” SAE Technical Paper Series, 2007, SAE 2007-01-0302.

Perhinschi, M. G., W. S. Wayne, N. N. Clark, and D. W. Lyons. "Neural Network Modeling of Emissions from Medium-Duty Vehicles Operating on Fisher-Tropsch Synthetic Fuel." $S A E$ Technical Paper Series, 2007, 2007-01-1080.

Rajagopalan, A., G. Washington, G. Rizzoni, and Y. Guezennec. Development of Fuzzy Logic and Neural Network Control and Advanced Emissions Modeling for Parallel Hybrid Vehicles. Subcontractor report from the Ohio State University to NREL, Technical Report NREL/SR-54032919, 2003.

Ramamurthy, R. "Heavy Duty Emissions Inventory and Prediction." Thesis (MS). West Virginia University, 1999.

Rawlings, J.O., S.G. Pantula, and D.A. Dickey. Applied Regression Analysis: A Research Tool. Second Edition. Springer, New York, 1998.

Ribeiro, S. K., and M. A. D’Agosto. “Assessment of Hybrid-Drive Bus Fuel Savings for Brazilian Urban Transit.” Transportation Planning and Technology, 27 (6), (2004): 483-509.

Rousseau, A., and M. Pasquier. "Validation of a Hybrid Modeling Software (PSAT) Using Its Extension for Prototyping (PSAT-PRO).” GPC 2001 Proceedings, 18, (2001): 83-90.

Sandoval, J. A., and W.S. Wayne. Characterization of WMATA Bus Duty Cycles. Report from WVU CAFEE to WMATA’s Bus Engineering Department, Aug. 10, 2010.

Schmidt, M. R. "Two-Mode, Compound-Split Electromechanical Vehicular Transmission." U.S. Patent 5,931,757. Aug. 3, 1999.

Sharer, P., R. Leydier, and A. Rousseau. "Impact of Drive Cycle Aggressiveness and Speed on HEVs Fuel Consumption Sensitivity.” SAE Technical Paper Series, 2007, SAE 2007-01-0281.

Simpson, A. G. "Parametric Modelling of Energy Consumption in Road Vehicles." Dissertation (Ph.D). University of Queensland, 2005.

Smith, J. B. “Optimum Hybrid Vehicle Configurations for Heavy Duty Applications.” Thesis (MS). West Virginia University, 2001. 
SAE International. "Chassis Dynamometer Simulation of Road Load Using Coastdown Techniques. SAE J2264. Surface Vehicle Recommended Practice." SAE International Publications, Warrendale PA, 1995.

SAE International. "Recommended practice for measuring fuel economy and emissions of hybridelectric and conventional heavy-duty vehicles. SAE J2711. Surface Vehicle Recommended Practice.” SAE International Publications, Warrendale PA, 2002.

SAE International. "Recommended practice for road load measurement using on board anemometry and coastdown techniques. SAE J2263." SAE International Publications, Warrendale PA, 1996.

SAE International. "Recommended Practice - Fuel Economy Measurement Test (Engineering Type) for Trucks and Buses. SAE J1376." SAE International Publications, Warrendale PA, 1982 (Cancelled Dec 1997).

SAE International. "SAE wind tunnel test procedure for trucks and buses, Recommended Practice. SAE J1252.” SAE International Publications, Warrendale PA, 1981.

SAE International. "Surface Vehicle Recommended Practice. Vehicle Application Layer. SAE J193971 (through December 2001).” SAE International Publications, Warrendale PA, 2003.

SAE UK. “Advanced GM-Allison Two-Mode Parallel Hybrid Technology Ready for Europe.” Vehicle Technology magazine, Spring 2007: 36-39.

Sovran, G., and D. Blaser. "A contribution to Understanding Automotive Fuel Economy and Its Limits.” SAE Technical Paper Series, 2003, SAE 2003-01-2070.

Sovran, G., and D. Blaser. "Quantifying the Potential Impacts of Regenerative Braking on a Vehicle's Tractive-Fuel Consumption for the U.S., European, and Japanese Driving Schedules." $S A E$ Technical Paper Series, 2006, SAE 2006-01-0664.

Strimer, C. M. "Quantifying Effects of Vehicle Weight and Terrain on Emissions, Fuel Economy, and Engine Behavior.” Thesis (MS). West Virginia University, 2006.

Thompson, G. J., C. M. Atkinson, N. N. Clark, T. W. Long, and E. Hanzevack. "Neural Network Modeling of the Emissions and Performance of a Heavy-Duty Diesel Engine." Proceedings of the Institution of Mechanical Engineers, Part D: Journal of Automobile Engineering, 214 (2), (2000): 111-126.

Thompson, G. J., N. N. Clark, M. Gautam, D. K. Carder, and D. W. Lyons. "Inference of Torque and Power from Heavy-Duty Diesel Engines for On-Road Emissions Monitoring.” SAE Technical Paper Series, 2002, SAE 2002-01-0614. 
Tóth-Nagy, C., J. J. Conley, R. P. Jarrett, and N. N. Clark. "Further validation of artificial neural network-based emissions simulation models for conventional and hybrid electric vehicles." Journal of the Air \& Waste Management Association 56 (7), (2006): 898-910.

Transit Resource Center and West Virginia University Center for Alternative Fuels Engines and Emissions. "Analysis of Tailpipe Emissions from Westchester County Transit Buses." Submitted to Liberty Lines Transit and Westchester County Department of Transportation. May $31,2007$.

Tu, J. "Investigation of Emissions and Fuel Economy for the Integrated Bus Information System." Dissertation (Ph.D). West Virginia University, 2011.

UITP: International Association of Public Transport. SORT-Standardised on-road tests cycles. Brussels, 2004.

UNECE: United Nations Economic Commission for Europe. Working Party on Pollution and Energy, GRPE. Suitability of Particle Number Measurement for Regulatory Use. Working paper number GRPE-PMP-17-1, 2006.

U.S. EPA: Environmental Protection Agency, Office of Transportation and Air Quality. Technical Guidance on the Use of MOBILE6.2 for Emission Inventory Preparation. EPA420-R-04-013, Washington DC, August 2004.

U.S. EPA: Environmental Protection Agency, Office of Transportation and Air Quality. EPA Releases "Draft MOVES2009” for Comment: Questions and Answers. EPA-420-F-09-019, Washington DC, April 2009.

U.S. FTA: Federal Transit Administration. Analysis of Electric Drive Technologies for Transit Applications: Battery-Electric, Hybrid-Electric, and Fuel Cells. Publication FTA-MA-26-710005.1, Washington DC, 2005.

U.S. FTA: Federal Transit Administration. Transit Bus Life Cycle Cost and Year 2007 Emissions Estimation. Washington DC, 2007.

U.S. Geological Survey, "National Elevation Dataset (NED)." <http://ned.usgs.gov/> ; accessed February 9, 2010.

Veness, Chris. “Calculate distance, bearing and more between Latitude/Longitude points.” Movable Type Scripts, 2010 <http://www.movable-type.co.uk/scripts/latlong.html>; accessed March 31, 2010.

Vora, K. A. "Cycles and Weight Effects on Emissions and Development of Predictive Emissions Models for Heavy Duty Trucks.” Thesis (MS). West Virginia University, 2006. 
Walston, W. H. Jr., F. T. Jr. Buckley, and C. H. Marks. "Test Procedures for the Evaluation of Aerodynamic Drag on Full-Scale Vehicles in Windy Environments." SAE Technical Paper Series, 1976, SAE 760106.

Wayne, W. S., R. Nine, N. Clark, and J. Schiavone. Diesel Engine Retrofit Program. Final report presented to Westchester County Department of Transportation by West Virginia University and Transit Resource Center, Mount Vernon NY, Oct 2002.

Wayne et al., 2004a. Wayne, W. S., N. N. Clark, R. D. Nine, and D. Elefante. "A Comparison of Emissions and Fuel Economy from Hybrid-Electric and Conventional-Drive Transit Buses." Energy \& Fuels 18 (1), (2004): 257-570.

Wayne et al., 2004b. Wayne, S., N. Clark, M. Gautam, G. Thompson, and D. Lyons. "Natural Gas Heavy-Duty Transit Bus Emissions Testing - Washington Metropolitan Area Transit Authority." Technical Report, Project Number ACL-4-33054-01, November 2004.

Wayne, W. S. "WVU Integrated Bus Information System (IBIS) Project.” APTA 2008 Bus Technical Sessions, October 6-8, San Diego CA, 2008.

Wayne, W. S., Sandoval, J. A., and N. N. Clark, "Emissions benefits from alternative fuels and advanced technology in U.S. transit bus fleet.” Energy \& Environment, 2009, 20(4), 497-515.

Wu, Y., D. Carder, B. Shade, R. Atkinson, N. Clark, and M. Gautam. "A CFR1065-compliant transportable/on-road low emissions measurement laboratory with dual primary full-flow dilution tunnels." Proceedings of ASME Internal Combustion Engine Division 2009 Spring Technical Conference, Milwaukee, WI, May 3-6, 2009.

Zia, S. A. "The Effect of Different Road Load Implementation Strategies on Fuel Economy of USPS Step Vans.” Thesis (MS). West Virginia University, 2009.

Zhang, D., J. Chen, T. Hsieh, J. Rancourt, and M. R. Schmidt. "Dynamic modelling and simulation of two-mode electric variable transmission" Proc Instn Mech Engrs 215 Part D, (2001): 1217-1223.

Zhou, M. "Neural Network Techniques and Their Engineering Applications." C. T. Leondes ed. Intelligent Systems: Technology and Applications. Volume II: Fuzzy Systems, Neural Networks, and Expert Systems. New York (2003): II-1 - II-19.

Zou, Z., S. W. Davis, K. D. Beaty, M. P. O'Keefe, T. J. Hendricks, R. L. Rehn, S. Weissner, and V. K. Sharma. "A New Composite Drive Cycle for Heavy-Duty Hybrid Electric Class 4-6 Vehicles." SAE Technical Paper Series, 2004, SAE 2004-01-1052. 